UNIVERSIDAD POLITÉCNICA DE MADRID

Escuela Técnica Superior de Ingeniería Agronómica, Alimentaria Y DE BIOSISTEMAS

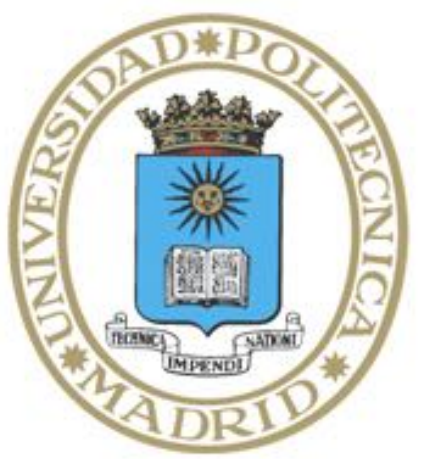

\title{
On STATistical Dynamics of Evolving MARKETS
}

TESIS DOCTORAL

Maximiliano Alfredo Fernandez

Máster en Física de Sistemas Complejos

Ingeniero Aeronáutico 



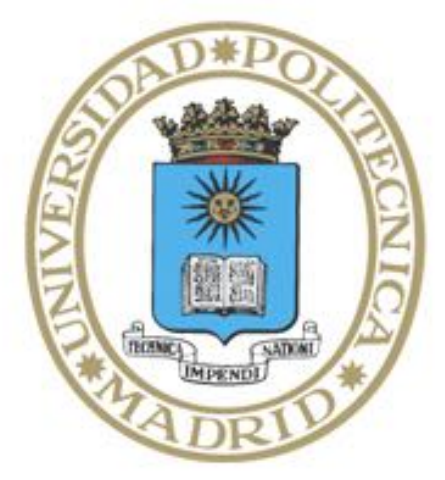

Grupo De Sistemas Complejos

DEPARTAMENTO DE INGENIERÍA AgROFORESTAL

Escuela Técnica Superior de Ingeniería Agronómica, Alimentaria y DE Biosistemas

\title{
On STATistical Dynamics of Evolving MARKETS
}

\author{
Maximiliano Alfredo Fernandez \\ Máster en Física de Sistemas Complejos \\ Ingeniero Aeronáutico \\ Director: Javier Galeano Prieto \\ Doctor en Ciencias Físicas
}





\section{Acknowledgements}

To my parents, for teaching how to be happy no matter what. To my wife, for teaching me how acomplish this in pragmatic way. To my academic director, for providing the wisdom to develop it further while having lots of fun.

I am grateful to have such a wonderful parents, Alfredo and Ada Beatriz, who always believe in me unconditionally. I am sure this will not change the way they feel about me, which makes me appreciate their support and teachings even more.

I am lucky to share my life with my wife, Bettina, who always supports me emotionally and beyond, in good moments and bad ones, always with a smile.

I would like to specially thank my thesis director, Javier, for his academic support and his friendship. It has been fun to learn from his experience, develop new concepts and explore interesting ideas. This has been a life changing experience to me and I am grateful for his guidance.

I would also like to thank my professors and classmates, for all the interesting debates and great moments. 



\section{Resumen}

Adam Smith es el padre de la economía moderna. Su trabajo sobre la riqueza de las naciones es la piedra angular de la teoría económica, la cual permanece como una referencia válida hasta hoy en día. Smith fue también uno de los primeros pensadores en introducir el concepto de fenómenos emergentes complejos al afirmar que las personas o las empresas pueden ayudar a la sociedad en su conjunto al perseguir sus propios intereses. Sobre la base de esta aparente contradicción, nuestro objetivo es estudiar la topología y la dinámica de los mercados de comercio internacional utilizando la teoría de redes complejas desde una perspectiva pesada y bipartita. Nuestros datos contienen registros de la actividad no agregada de comercio internacional de 5000 productos, 1000 categorías y 100 industrias a través de más de 15 años de historia, desde 1995 hasta 2009. Realizamos nuestro análisis de los mercados en base a la premisa de que los mercados de comercio internacional son una analogía económica de los ecosistemas mutualistas, donde una situación de beneficio mutuo es igualmente válida para las especies mutualistas (por ejemplo, las plantas y los polinizadores), así como lo son para los exportadores e importadores de un mercado. Sobre la base de esta analogía y el enfoque bipartito, aplicamos un conjunto de indicadores topológicos a las redes comerciales que es similar a aquellos que se usan comúnmente para estudiar las redes mutualistas. Sobre la base de los hallazgos y patrones estadísticos de estas mediciones topológicas, los cuales se asemejan en gran medida a los resultados de las redes mutualistas, también estudiamos la estructura anidada y el desempeño del anidamiento de las redes de comercio internacional para diferentes niveles de agregación de datos y para múltiples períodos. A continuación de este estudio topológico y estático, realizamos un análisis exhaustivo de fenómenos dinámicos específicos, a través de una metodología que hemos desarrollado recientemente y que aprovecha los datos históricos de comercio internacional, lo que conduce al descubrimiento de los mecanismos y patrones que explican la persistencia a lo largo del tiempo del anidamiento en las redes de comercio internacional. Con esto, recopilamos los todos hallazgos de la topología y de dinámica de los mercados y formulamos varios modelos estadísticos para describir el proceso de formación de mercados a través de un proceso de crecimiento de red. Probamos varios modelos, pero finalmente nos enfocamos en un modelo mutualista de mercados, que es un proceso de agregación de enlaces que combina el mecanismo de preferential attachment y el enfoque bipartido pesado. Este modelo es capaz de reproducir simultáneamente todas las características topológicas comunes encontradas en los mercados reales, a la vez que proporciona una mayor comprensión sobre cómo decisiones locales altamente sesgadas, que son consistentes con el concepto de interés propio, pueden conducir al bienestar del sistema en su conjunto, proporcionando una explicación alternativa a las afirmaciones de Adam Smith. 



\section{Abstract}

Adam Smith is the father of modern economics. His work on the wealth of nations is the corner stone of the economic theory, which remains a valid reference nowadays. Smith was also one of the earliest thinkers to introduce the concept of complex emerging phenomena when stating that persons or firms can help society as a whole by pursuing their own interest. Based on this apparent contradiction, our objective is to study the topology and dynamics of international trade markets using the theory of complex networks from a weighted and bipartite perspective. Our data contains records of non-aggregated activity of international trade for 5000 products, 1000 categories and 100 industries over 15 years of history, from 1995 to 2009 . We perform our analysis of markets based on the premise that markets are an economic analogy of mutualistic ecosystems, where a win-win situation is equally valid for mutualistic species (for instance, plants and pollinators) as it is for exporters and importers. Based on this analogy and the bipartite approach, we apply a similar set of the topological indicators to trade networks than those commonly used to study mutualistic networks. Based on the findings and statistical patterns of these topological measures, which highly resemble those of mutualistic networks, we also study the nested structure and the nestedness performance of trade networks across different level of aggregation of the data and for multiple periods. Following this topological but static study, we perform a thorough analysis of targeted dynamical phenomena, a newly developed methodology that takes advantage of the historical trade data, which leads to the discovery of mechanisms and patterns that explain the persistence over time of nestedness in trade networks. With this, we collect the findings of market topology and dynamics and formulate several statistical models to describe the process of market formation through a network growth process. We test several models but finally focus in a mutualistic model of markets, which is a link aggregation process that combines a preferential attachment mechanism and the weighted bipartite approach. The model is capable of simultaneously reproducing all the common features of the empirical data while providing further insights on how highly biased local decisions, which are consistent with self-interest, can lead to the well being of the system as a whole, providing an alternative explanation to the claims of Adam Smith. 



\section{Contents}

I Introduction 1

1 Thesis structure and objectives $\quad 3$

1.1 Thesis structure by parts . . . . . . . . . . . . . . . . 4

1.2 Objectives and relevance of the current approach $\ldots \ldots \ldots$

2 On Economic Complexity 7

2.1 International trade markets . . . . . . . . . . . . 8

3 On Complex Networks 13

3.1 Complex networks theory . . . . . . . . . . . . . . . 14

3.1.1 Types of complex networks _ . . . . . . . . . . 15

3.1 .2 Bipartite networks . . . . . . . . . . . 17

3.2 Mutualistic networks . . . . . . . . . . . . . . . . . . . 19

3.3 Previous related work on trade networks . . . . . . . . . . . . 25

3.3.1 Topology of the World Trade Web . . . . . . . . . . . . 25

3.3.2 Patterns of dominant flows in the world trade web . . . . . 27

3.3.3 The World-Trade Web: Topological Properties, Dynamics, and Evolution ..................... 31

II Materials and Methods 33

4 International Trade Data 35

4.1 Data availability . . . . . . . . . . . . . . . . . . 35

4.2 United Nations' COMTRADE dataset $\ldots \ldots$. . . . . . . . . . . 36

4.3 Data cleaning and consistency . . . . . . . . . . . . . . . . 40

4.4 Weighted Bipartite Trade Networks . . . . . . . . . . . . . . . . 41

4.4 .1 A simple example of WBTN . . . . . . . . . . . . . 45

4.4 .2 Some analytics on RSP and RPP . . . . . . . . . . . . 46

4.5 Big data process . . . . . . . . . . . . . . . . . 52

5 Topological indicators for WBTN 59

5.1 Degree distribution $\mathrm{P}(\mathrm{K}) \ldots \ldots \ldots \ldots$

5.2 Strength - degree correlation $\mathrm{S}(\mathrm{K}) \ldots \ldots$. . . . . . . . . . . . 61

5.3 End-point degree correlation . . . . . . . . . . . . . . . . . . 62 
5.4 Bipartite clustering $\mathrm{C} 4(\mathrm{~K}) \ldots \ldots \ldots 63$

5.5 Nearest Neighbor Degree distribution $\mathrm{Knn}(\mathrm{K}) \ldots \ldots$. . . . . . . . . . . . . 65

5.6 Summary of Bipartite Topological Indicators . . . . . . . . . . . 67

6 Nestedness $\quad 69$

6.1 Nestedness as a measure of order . . . . . . . . . . . . . . 69

6.2 Matrix Temperature Measure . . . . . . . . . . . . . . . . 71

6.3 NODF and WNODF Methods . . . . . . . . . . . . . . 73

6.4 WINE Method . . . . . . . . . . . . . . . . 76

6.5 Spectral Nestedness . . . . . . . . . . . . . . . . 79

6.6 Correlation between nestedness methods . . . . . . . . . . . . . 82

6.7 Nestedness considerations for this study . . . . . . . . . . . 85

$\begin{array}{lll}\text { III Results } & 87\end{array}$

7 Topological analysis $\quad 89$

7.1 Exploratory analysis of trade markets . . . . . . . . . . . . . . 90

7.2 Trade network results at HS2 industry level . . . . . . . . . . . . 95

7.2.1 Degree distribution, $\mathrm{P}(\mathrm{K}) \ldots \ldots \ldots$. . . . . . . . . . . . . . . . . . .

7.2.2 Strength-degree correlation, $\mathrm{S}(\mathrm{K}) \ldots \ldots \ldots$

7.2.3 Assortative properties of trade networks, $\mathrm{Knn}(\mathrm{K}) \ldots \ldots$

7.2.4 Clustering properties of trade networks, C4(K) . . . . . . . 111

7.2.5 Average weight vs. end-point degree, $<\mathrm{w}>\left(K_{i} . K_{j}\right) \ldots \ldots 118$

7.2 .6 Nestedness features . . . . . . . . . . . . . . . . . 120

7.3 Summary of Topological Features . . . . . . . . . . . . . . 125

8 Temporal analysis $\quad 129$

8.1 Nestedness across different markets . . . . . . . . . . . . . . . . 129

8.2 Dynamical phenomena . . . . . . . . . . . . . . . . . 131

IV On Market Models 143

9 Network models of trade markets $\quad 145$

9.1 On general market models . . . . . . . . . . . . . . . . . . . . . 146

9.2 Random network model . . . . . . . . . . . . . . . . . . . 149

9.3 Gravity models . . . . . . . . . . . . . . . . . . . . . 154

9.4 Radiation models . . . . . . . . . . . . . . . . . 165

10 A new model for ecosystemic analysis of markets 167

10.1 A mutualistic model for market formation . . . . . . . . . . . 168

10.1.1 Formal description of our model . . . . . . . . . . . . . 169

10.1.2 A simple example of market formation . . . . . . . . . . 172 
10.2 Model results and market simulation . . . . . . . . . . . . . 178

10.2.1 Highly-sparce market simulation . . . . . . . . . . . 187

10.3 Simulation of special cases of markets . . . . . . . . . . . 189

11 International Lending Markets 191

11.1 Some background on financial institutions . . . . . . . . . . . . . 192

11.2 Data analysis of interbank markets . . . . . . . . . . . . . 193

11.3 Networks properties of financial markets . . . . . . . . . . . . . . 195

11.4 A minimal model of interbank networks . . . . . . . . . . . . . 197

11.4.1 Assets and liabilities generation . . . . . . . . . . . . . 197

11.4.2 Random network generation . . . . . . . . . . . . . 199

11.5 Results of interbank networks . . . . . . . . . . . . . . . . 200

11.5.1 Theoretical predictions . . . . . . . . . . . . . 201

11.5.2 Comparison with empirical data . . . . . . . . . . . 206

11.6 Conclusions of interbank networks . . . . . . . . . . . . . . . . . 209

12 Conclusions $\quad 213$

12.1 A brief summary of the thesis . . . . . . . . . . . . . . 213

12.2 On statistical models of markets . . . . . . . . . . . . . . . . 217

12.3 List of conclusions . . . . . . . . . . . . . . . . . . . . . . 219

12.4 Areas of opportunity for future research . . . . . . . . . . . . 221

A Appendix A: References and tables of result 223

B Appendix B: Data visualization 247

B.1 Principles of Design . . . . . . . . . . . . . . . . . . . . . 248

B.2 Bipartite graph visualization . . . . . . . . . . . . . . 250

B.3 Geo-spatial graph visualization . . . . . . . . . . . . . . . . 254

B.4 Other network layouts . . . . . . . . . . . . . . . . 258

C Appendix C: Selected HS2 visualizations 263

D Appendix D: Market visualizations 293 

Part I

Introduction 



\section{Thesis structure and objectives}

Have a definite, clear, practical ideal - a goal, an objective.

\section{- Aristotle}

(Philosopher and scientist)

Social and economic phenomena are highly complex. Yet, in the age of information, the scientific community has shown an increased focus on approaching these problems from a multidisciplinary standpoint. Thus, the theory of complex networks has been positioned as a key methodology to understand the architecture and dynamics of these phenomena. In particular, interactions within a market, like those that take place between buyers and sellers of a product or service, are an opportunity to learn more about the way in which simple interactions may lead to complex emerging patterns. Within this study, we apply this concept to international trade markets for a variety of products, categories and industries based on actual data from 1995 to 2009. Based on the previous scientific work and our objectives pursued in this thesis, a structure of four parts has been selected. This organization is aligned to that of the majority of the scientific papers, providing a first part with an introduction, a second part that discusses materials and methods, a third part that provides a deep dive on results and discussions upon the meaning of them, and finally a fourth part with the main conclusions on what we have learned from the trade markets, including those derived from some statistical models of market. This structure has been modified several time over the research period, arriving to this format by a process of continuous improvement, one that potentially resembles the concept of evolution itself. The challenges we had in the beginning of our reserach were related to the massive volume of information we encounter and, hence, we had to make a decision on how to present such a large volume of results. For that, we focus on separating the different dimensions of our data, thus heavily depending on examples and randomly selected data and results, which can easily be extended to the rest of the data and markets. Repetitiveness has also been an issue during the research. Nevertheless, we address this potential problem by relying on, again, examples that can be extended to the rest of the data. 


\subsection{Thesis structure by parts}

In Part 1, we provide a conceptual introduction on international trade markets, both from an economic and a complex network standpoint. We have also decided to incorporate a specific chapter to formally introduce the theory of complex networks and provide example of networks in the real world together with a thorough review of the previous scientific work regarding bilateral trade. The main premise in this part is to find all the previously successful cases where a relevant scientific discovery has been made. Therefore, this study is planned to be able to benefit from those facts and findings, but will in turn propose an expanded bipartite implementation of the knowledge available in other disciplines such as ecology and genetics, disciplines that have extensively performed research based on bipartite networks.

Part 2 will focus on the availability and consistency of international trade data. A formal review of the bipartite network indicators will be provided as well. We have also decided to include an exclusive chapter to review the concept of nestedness as a measure of systemic internal organization, including a fully detailed analysis of the different way to measure this concept. Finally, in appendix A, we also argue about some considerations regarding data visualization, a critical tool to fully understand the complexity of markets, which is not formally included in this part, yet it is a of relevance. The key goal of this part is to provide the robust methodological background to study our large dataset with an absolutely rigorous approach that can point to new and innovative discoveries.

Part 3 will take care of the results obtained from the processing of the actual trade data. Initially, it will concentrate on basic network topological analysis. But later, we will incorporate an innovative network definition to reveal the hidden patterns, leading to the understanding of the key features found in trade markets. This is particularly important for our research since the bipartite and weighted apporach has never benn used before to describe trade networks. We think that our bold decision of building exporter-importer bipartite networks (country-country) can pay off after our finding proves to be revealing, at the very least, and innovative when considering our robust findings.

Finally, in Part 4, we will provide a detailed analysis of statistical models to describe the internal organization of markets and how they are formed. In this part, we rely deeply on the analogies we have found with mutualistic ecosystems and start from a naive null model, improving it over several iterations and presenting the final 3 models that can accomplish such a task. In the final part of the part, we will summarize the previous results in order to explain our results and the process of market formation together with the underlying patterns at the same time. In 
addition, an alternative model of financial markest will also be included as an example of how complex networks can be utilized to describe complex systems of economic nature. We end with a thorough review of our conclusions, a list of our key findings and future research opportunities.

\subsection{Objectives and relevance of the current approach}

Our research aims at describing international trade markets from a bipartite and weighted standpoint. In the last few years, after the global financial crisis of September 2008, there is a deep interest in the scientific community in order to understand the dynamics of complex problems in economics and international finance. At the same time, complexity science and complex networks have gained momentum mostly due to the revolution of big data and social networks as well. Hence, we believe that an innovative study on the international trade networks is of high importance and relevance in this context. Our approach to studying such markets is quite robust, which is inspired by biological ecosystems, and is targeted to provide a new view upon an old problem, that is, the concept of market itself and the dynamics within it.

Regarding our research objectives, these are some of the key ones, including some of the hypotheses and premises to be tested:

- Complex bipartite networks constitute an innovative framework to study international trade markets. By analyzing the topological properties of these markets, we will be able to find persistent patterns derived from the process of market formation at meso-scale.

- We use a bipartite approach based on the premise that importers and exporters of a product play a different role in the topology of a commercial trade system and therefore, we will be able to unveil the hidden dynamics and organizational patterns within trade markets.

- In order to formally implement a bipartite approach, where a country can be both an importer and an exporter of the same product, we separate this country in an exporter role and in an importer role as two different nodes. This is because the reported activity of a country as a whole does not separate firms that act as importers, which are mainly commercial companies, from those that act as exporters, which are industrial companies. Consequently, we believe that concept of the different roles is sufficiently supported by the reality. 
- A bipartite approach has not been implemented before. Hence, based on a combination of bipartite analysis and data visualization, we can prove the previous statements by using an analogy with mutualistic networks, a point that will be discussed across the study, and find revealing results that have not been studied in previous scientific work.

- One of our basic premises is that mutualistic ecosystems and trade markets constitute a close analogy of systems with similar internal dynamics. In the same way pollinators and plants cooperate with each other while pursuing their own interest simultaneously, so do exporters (sellers) and importers (buyers) of a product or service mutually benefit from the exchange where both parties end up in a better position, one by emptying its inventories and the others by satisfying a need.

- Finally, we will build several market models to explain the process of market formation and its behavior, maintaining a tight analogy with mutualistic ecosystems once again. 


\title{
On Economic Complexity
}

\author{
By pursuing his own interest, he frequently \\ promotes that of the society more effectually \\ than when he really intends to promote it.
}

— Adam Smith

(Father of modern economics)

\begin{abstract}
Adam Smith is the father of modern economics. His work on The Wealth of Nations [142] provided a groundbreaking undertanding on how markets behave at a macroscale, which was a disruptive concept at the time. Yet, Smith did not only concentrate on the behavior of markets itself, but also focused on the human forces that drive these behaviors. According to his arguments, people can behave in a selfish way, minding their own interest, and still be able to support the greater good of society as a whole, as evidenced by his work:
\end{abstract}

... By preferring the support of domestic to that of foreign industry, he intends only his own security; and by directing that industry in such a manner as its produce may be of the greatest value, he intends only his own gain; and he is in this, as in many other cases, led by an invisible hand to promote an end which was no part of his intention. Nor is it always the worse for the society that it was no part of it. By pursuing his own interest, he frequently promotes that of the society more effectually than when he really intends to promote it. I have never known much good done by those who affected to trade for the public good. It is an affectation, indeed, not very common among merchants, and very few words need be employed in dissuading them from it...

Many important concepts, such as that of the invisible hand that regulates markets or that of the self-interest attitude that helps society, are of critical importance to this study, since they provide the opportunity to explore innovative and alternative explanations from the perspective of complex network theory. Furthermore, these concepts have been an inspiration for this doctoral thesis, since they clearly suggest and imply that complex behavior may be a hidden feature of markets and that the complexity of these economic processes may lead to counter-intuitive outcomes, 
which are derived from simple, yet apparently opposite behavior. We believe this is the essence of complex systems, emerging complex phenomena can be originated by simple interactions. Moreover, we have decided that this should be one of the main goals in this study, that is, to understand such phenomena and, additionally, to put those concepts under stress by incorporating complex network theory and by testing results with advanced visual and analytics techniques.

\subsection{International trade markets}

Trade, as a human activity, has existed since the ancient ages. In this context, humans have always had the need to exchange goods and services in order to survive and grow as a civilization. And every time there was a need for a given product or service on the "consumers" side, so was there a will to satisfy this need on the "producers" side. The process to satisfy these necessities is, to these days, of great complexity though, which remains a valid claim regardless of the period in history. This is mainly because of several intangible variables, such as personal preferences, resource availability, bargaining power and alternative choices. These variables always play a fundamental role in the final decision. But markets, as we know them today, are a relatively new concept. In modern economics, a market is defined as the place where the supply, which is the total amount of a product (goods or services) available for purchase at any specified price, and the demand, which is the consumer's desire and willingness to pay a price for a specific good or service, converge together to perform trade activities. So, based on this definition, it would be quite interesting to understand whether macro- and meso-scale patterns may arise as a consequence of this micro-scale trade activity. In fact, the process of market formation will be one of the most important aspects of this thesis. The microeconomic theory commonly describes several types of theoretical markets, which are ideal or special cases of markets. These types of markets are:

A) The Monopoly, which is a market where there is one seller of the product (good or service) and several buyers. In this case, and because of the nature of its configuration, the seller has significant power over the market in order to dictate the selling price and quantities. They can be formed based on natural or artificial constraints. For example, Standard Oil, a company founded by John D. Rockefeller in 1882. By 1890, Standard Oil did not only control $88 \%$ of the refined oil flows in the United States, but also created Standard Oil Trust, a conglomeration that handled all oil production, transportation, refinement and marketing across the country.

B) The Oligopoly, which is a market where there are a few sellers of the product (good or service) and several buyers. For example, motion picture industry in the 
U.S. is dominated by seven companies that earn $87.4 \%$ of industry revenues as of 2013: Time/Warner, Disney, NBC/Universal, Sony, News Corporation, Lionsgate and Viacom.

C) The Monopsony, which is a market where there is one buyer of the product (good or service) and several sellers. For example, the labor market could be a monopsony depending the conditions. This is frequently common in some small towns, where only one large firm provides the majority of employment.

D) The Perfect Competition Market, which is a market where there are a several sellers of the product (good or service) and several buyers. For example, markets that involve basic products, such as agricultural ones, can be conceptualized as a perfect competition market, where no buyer or seller has power over the market. Later in this study, we will argue about the concept of perfect competition and how it may directly apply to international trade markets.
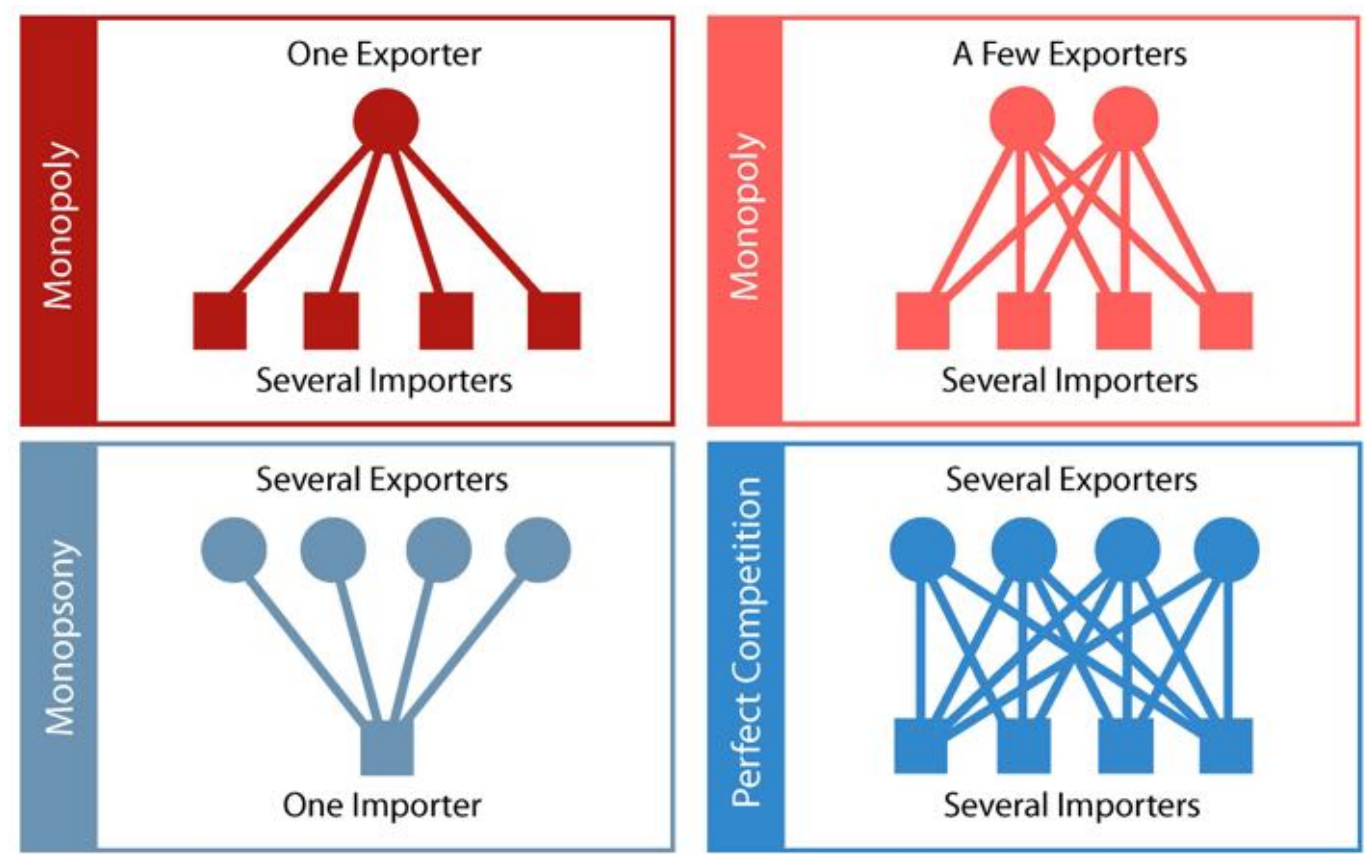

Fig. 2.1.: Types of market described by microeconomic theory. This figure shows the different types of markets described in the microeconomic theory. But they have been represented by using complex networks. We realize that complex networks can be a powerful tool to describe complex systems such as a market.

Now, most of the economic behavior can be derived from these 4 types of market, which we depict in figure 2.1. They serve as a testing field for the different market forces, which leads to powerful conclusions in terms of macro-scale behavior. Of course, the most important of this macro-scale behavior is the law of supply and demand. This concept states that the price of a product (good or service) is set by the equilibrium of the supply and demand functions, which we show in figure 2.2. 


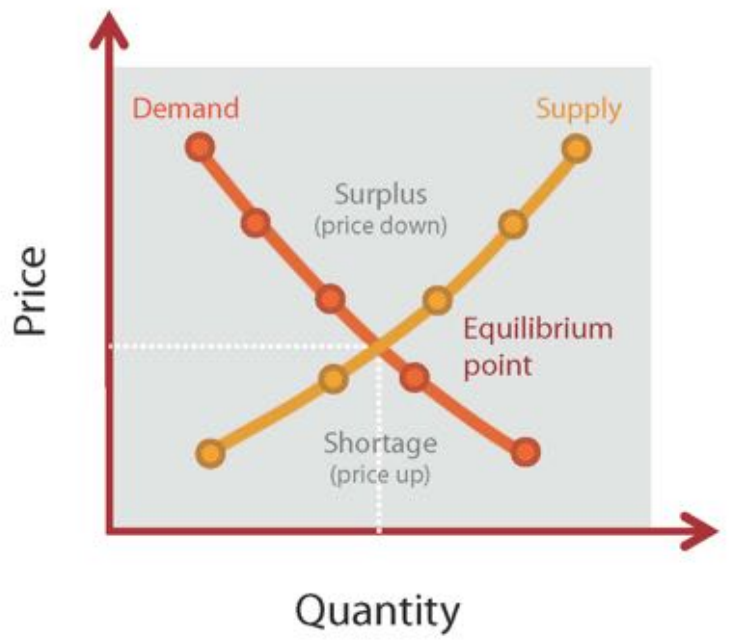

Fig. 2.2.: The law of Supply and Demand. This figure shows how the supply-demand law works. The demand function represents the behavior of the buyers, where they are willing to acquire higher quantities when the price is lower. In the supply function, the opposite is true. The market equilibrium is reached where the functions intersect. A different market price condition would generate either a shortage or surplus of inventories, which consequently will restore the equilibrium.

The demand function depicts the willingness of consumers to acquire a certain quantity of a given good as a function of its price, which is shown in figure 2.2 with a negative slope. Thus, the higher the price, the lower the quantity a reasonable consumer would buy. In contrast, the supply function works in the opposite direction for the producers of the same good, which is shown in figure 2.2 with a positive slope. In other words, the higher the price, the higher the quantity producers are willing to sell. Consequently, the equilibrium price will be determined by the intersection of these two functions. In figure 2.2, we observe the concept of supply and demand as well as the mechanism that takes place between them. The equilibrium between supply and demand is also a stable point because any variance in price would create either an inventory surplus when the price is higher than the equilibrium price or an inventory shortage in the opposite case, forcing the producers to reduce the price in the first case or giving the consumers no alternative but to buy at a higher price in the second one. By this logic, many classical economists have stated that such equilibrium of price and quantity could always be reached in a market regardless of the external conditions. But the appearance of a global crisis, like the great depression of 1929, has led economists to believe that markets can be flawed due to several reasons. The details of this claim are out of the scope in this study, but are still an inspiration to apply the complex network theory in order to characterize markets and find these relevant patterns.

At this point, we may realize that complex network theory is especially suitable to characterize these markets (see figure 2.1). By extension, we believe that complex 
networks are also suitable to depict and explain the process of market formation and their underlying patterns. As initial evidence, we have certainly described the special cases of markets from figure 2.1 through network graphs. Since the different types of market have now been introduced, we may have a question related to international trade markets and to which one of the four cases better describe them. Depending on the nature of the product, and especially for non-manufactured products or commodities, the international trade markets tend to be consistent with or, at least, are close to a perfect competition market. If we take a basic product, like wheat, which is also a homogeneous product, we will probably find several countries that sell this product and several countries that buy this commodity. In addition, the information about the quality of the product and its price is fairly well known for all the buyers and sellers, creating an environment of perfect information. But we may also argue that some barriers exist for countries to freely decide where to buy. This is mainly because of some regional agreements and some local government protection policies through higher taxes or duties, among others. Finally, and although this does not happen every year, we may find some exogenous effects over the market such as any natural disaster, like dry periods or hurricanes, which will put pressure on the market price, for instance, due to product scarcity. As previously stated, these types of market are mere idealizations. Still, we may think of international trade markets as one of the closest cases to perfect competition. 



\section{On Complex Networks}

The diversity of networks in business and the economy is mindboggling. There are policy networks, ownership networks, collaboration networks, organizational networks, network marketing-you name it. It would be impossible to integrate these diverse interactions into a single all-encompassing web. Yet no matter what organizational level we look at, the same robust and universal laws that govern nature's webs seem to greet us. The challenge is for economic and network research alike to put these laws into practice.

\section{— Albert-László Barabási}

(How Everything Is Connected to Everything Else and What It Means for Business, Science, and Everyday Life)

\footnotetext{
Any scientist who starts his or her career in the XXI century is irremediably biased towards dealing with and understanding the paradigm of big data and the complexity within real-world problems that have not been approachable until this point in the age of information. Thus, the complex network theory is nothing but a real need and a must-have tool to accomplish such a goal. Indeed, complex network analysis is unmistakably one of the most important tools developed to model the architecture of complexity, constituting a robust framework to understand the underlying structure of complex systems, to find hidden patterns in such systems, and to ultimately predict complex behavior in ways that have not been possible ever before.
} 


\subsection{Complex networks theory}

Complex networks are a highly effective tool to map and represent the relationships between the numerous components of a complex system. In a way, they depict the architecture of complexity. But complex networks are also mathematically known as graphs, which are better described as a combination of: a) a set of vertices or nodes, and b) a set of edges or links, where an edge will connect two vertices. Consequently, we can formally introduce:

A graph $G$ consists of a collection $V$ of vertices and a collection $E$ of edges, for which we write $G=(V, E)$. Each edge $e \in E$ is said to join vertices, which are called its end point. If $e$ joins $u, v \in V$, then these vertices are said to be adjacent and the edge is said to be incident with $u$ and $v$, respectively.

The connectivity features of a graph or network will be defined by the connection matrix, or more formally, the adjacency matrix $A$, which is the standard way of representing complex networks.

$$
a_{i j}= \begin{cases}1 & \text { if nodes } \mathrm{i} \text { and } \mathrm{j} \text { are connected } \\ 0 & \text { otherwise }\end{cases}
$$

And more generically for weighted networks, the weight matrix is defined as:

$$
w_{i j}= \begin{cases}w_{i j} & \text { if nodes } \mathrm{i} \text { and } \mathrm{j} \text { are connected } \\ 0 & \text { otherwise }\end{cases}
$$

The degree of a node is the number of links that are connected to it. Thus, for undirected networks:

$$
k_{i}=\sum_{j} a_{i j}
$$

This is somewhat different for directed networks, since accounting for the in-degree and out-degree is required: 


$$
\begin{gathered}
k_{i}^{\text {in }}=\sum_{j} a_{i j} \\
k_{i}^{\text {out }}=\sum_{j} a_{j i} \\
k_{i}^{\text {tot }}=k_{i}^{\text {in }}+k_{i}^{\text {out }}
\end{gathered}
$$

The strength of a node is the sum of weights of all links that are connected to it [20], thus:

$$
s_{i}=\sum_{j} a_{i j} w_{i j}
$$

Having introduced the definition of complex networks formally, we now need to concentrate on a very important aspect of these systems: the different types of networks

\subsubsection{Types of complex networks}

Having started over 3 centuries ago, the study of "graphs" or "graph theory" has provided a precise and complete framework to approach and tuckle complex realworld problems. It is, in a sense, the architecture of complex systems. Now, like in any discipline, we can classify complex networks based on the type of connectivity they present (figure 3.1). For example, we refer to undirected networks when the nodes of such graph are associated without any directionality. In this case, any pair of nodes are linked to each other so that the adjacency matrix will be symetrically formed. This means that node A and B are simultaneously linked together.

In contrast, a directed network does incorporate the directionality as its key feature. In this way, node A can be linked to node B, but the opposite is not necessarily true. This directionality will impact the way in which the node degree is understood and will create the need to consider both the in-degree and the out-degree in order to account for assymetric form of the adjacency matrix. The previous types of networks have been introduced by a mere discussion on whether or not to take in account the directionality features. Yet, in both cases, we are dealing with two binary or unweighted networks, where links are depicted by a one if the nodes are connected or a zero if the opposite is true. Let us consider a different type where links also 


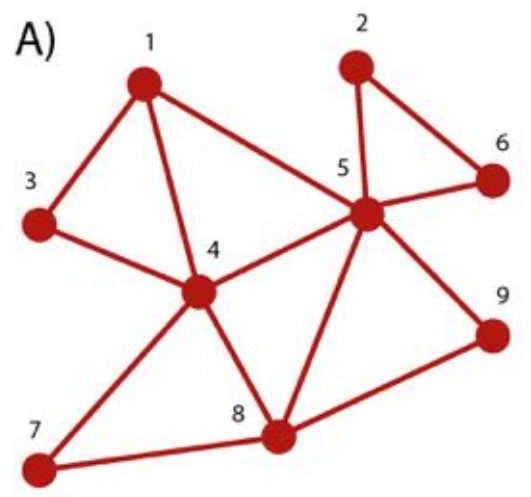

\begin{tabular}{llllllllll}
\hline n & 1 & 2 & 3 & 4 & 5 & 6 & 7 & 8 & 9 \\
1 & 0 & 0 & $\mathbf{1}$ & $\mathbf{1}$ & $\mathbf{1}$ & 0 & 0 & 0 & 0 \\
2 & 0 & 0 & 0 & 0 & $\mathbf{1}$ & $\mathbf{1}$ & 0 & 0 & 0 \\
3 & $\mathbf{1}$ & 0 & 0 & $\mathbf{1}$ & 0 & 0 & 0 & 0 & 0 \\
4 & $\mathbf{1}$ & 0 & $\mathbf{1}$ & 0 & $\mathbf{1}$ & 0 & $\mathbf{1}$ & $\mathbf{1}$ & 0 \\
5 & $\mathbf{1}$ & $\mathbf{1}$ & 0 & $\mathbf{1}$ & 0 & $\mathbf{1}$ & 0 & $\mathbf{1}$ & $\mathbf{1}$ \\
6 & 0 & $\mathbf{1}$ & 0 & 0 & $\mathbf{1}$ & 0 & 0 & 0 & 0 \\
7 & 0 & 0 & 0 & $\mathbf{1}$ & 0 & 0 & 0 & $\mathbf{1}$ & 0 \\
8 & 0 & 0 & 0 & $\mathbf{1}$ & $\mathbf{1}$ & 0 & $\mathbf{1}$ & 0 & $\mathbf{1}$ \\
9 & 0 & 0 & 0 & 0 & $\mathbf{1}$ & 0 & 0 & $\mathbf{1}$ & 0 \\
\hline
\end{tabular}

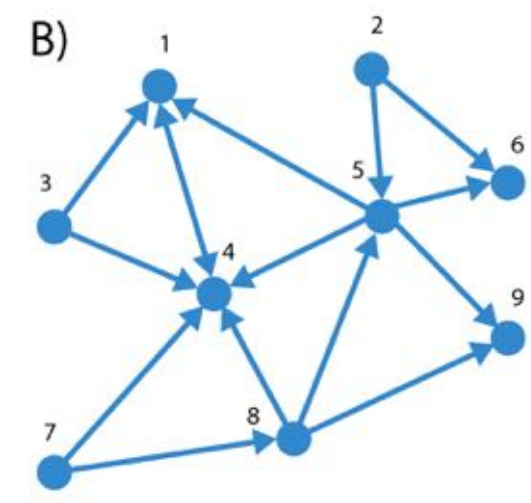

\begin{tabular}{llllllllll}
\hline $\mathrm{n}$ & 1 & 2 & 3 & 4 & 5 & 6 & 7 & 8 & 9 \\
1 & 0 & 0 & 0 & 1 & 0 & 0 & 0 & 0 & 0 \\
2 & 0 & 0 & 0 & 0 & 1 & 1 & 0 & 0 & 0 \\
3 & 1 & 0 & 0 & 1 & 0 & 0 & 0 & 0 & 0 \\
4 & 1 & 0 & 0 & 0 & 0 & 0 & 0 & 0 & 0 \\
5 & 1 & 0 & 0 & 1 & 0 & 1 & 0 & 0 & 1 \\
6 & 0 & 0 & 0 & 0 & 0 & 0 & 0 & 0 & 0 \\
7 & 0 & 0 & 0 & 1 & 0 & 0 & 0 & 1 & 0 \\
8 & 0 & 0 & 0 & 1 & 1 & 0 & 0 & 0 & 1 \\
9 & 0 & 0 & 0 & 0 & 0 & 0 & 0 & 0 & 0 \\
\hline
\end{tabular}

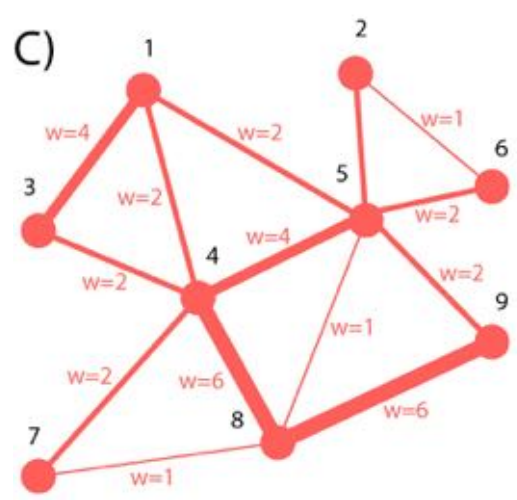

\begin{tabular}{|llllllllll}
\hline $\mathbf{n}$ & 1 & 2 & 3 & 4 & 5 & 6 & 7 & 8 & 9 \\
\hline 1 & 0 & 0 & 4 & 2 & 2 & 0 & 0 & 0 & 0 \\
2 & 0 & 0 & 0 & 0 & 2 & 1 & 0 & 0 & 0 \\
3 & 4 & 0 & 0 & 2 & 0 & 0 & 0 & 0 & 0 \\
4 & 2 & 0 & 2 & 0 & 4 & 0 & 2 & 6 & 0 \\
5 & 2 & 2 & 0 & 4 & 0 & 2 & 0 & 1 & 2 \\
6 & 0 & 1 & 0 & 0 & 2 & 0 & 0 & 0 & 0 \\
7 & 0 & 0 & 0 & 2 & 0 & 0 & 0 & 1 & 0 \\
8 & 0 & 0 & 0 & 6 & 1 & 0 & 1 & 0 & 6 \\
9 & 0 & 0 & 0 & 0 & 2 & 0 & 0 & 6 & 0 \\
\hline
\end{tabular}

Fig. 3.1.: Example of a undirected, directed and weighted networks. This figure shows an example of an undirected network (A), where nodes are linked together without any directionality, a directed network (B), where nodes are directionally linked together, and a weighted network (C), where links have a value different than one. The adjacency matrices for each typr of network are located to the right.

have different importance or weighted, thus allowing them to take any value. These networks are referred to as weighted networks. They can simultaneously be either directed or undirected. This clasification of complex networks has shown the most common types where the nature of the nodes is alike. But it is important to pay special attention to alternative cases, where the nature of the nodes may be different in several ways. This is the case of the bipartite networks, which we review in the following subsection. 


\subsubsection{Bipartite networks}

A bipartite graph can be defined as two subsets of nodes in which all links will connect two nodes from different subsets and none of them within the same subset. Then, we formally define:

A bipartite graph $G$ consists of a two disjoint collections $A$ and $B$ of vertices and a collection $E$ of edges, for which we write $G=(A, B, E)$. Each edge $e \in E$ is said to join two vertices such that $e=(a, b)$ where $a \in A$ and $b \in B$.

Bipartite networks, like any complex network, can also be classified as binary bipartite graph, when all links have a unitary value, and weighted bipartite graphs, when links can accept values different than 1 . These can be graphically visualized as shown in figure 3.2 .

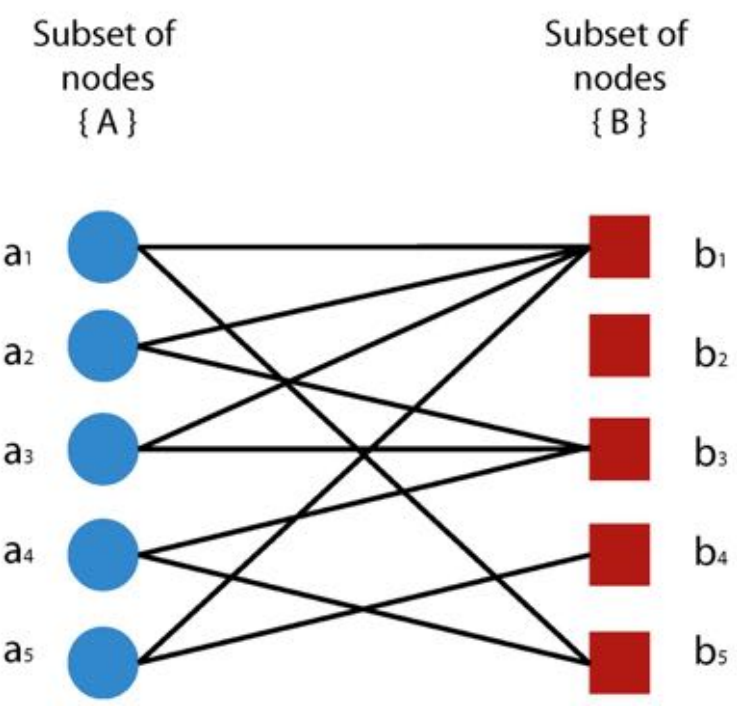

Fig. 3.2.: Bipartite network graph. This figure shows a bipartite network graph. We observe two clearly disjoint subset of nodes (blue circles and red squares). Therefore, the links can only connect two nodes of a different subset. This is a binary (unweighted) example. When the links have a specific weight, we find a weighted bipartite network.

Additionally, the topological measurements are applied to each subset. For example, the node degree will be:

$$
k_{i}^{A}=\sum_{j} a_{i j}
$$




$$
k_{j}^{B}=\sum_{i} a_{i j}
$$

When we deal with weighted bipartite networks, the same concept applies to the node strength, which is define as:

$$
\begin{aligned}
& s_{i}^{A}=\sum_{j} w_{i j} \\
& s_{j}^{B}=\sum_{i} w_{i j}
\end{aligned}
$$

This study will review more topological measures of bipartite networks on chapter 6. Nontheless, let us consider an interesting example of bipartite networks, which is that of Goh et al.[76]. This study provides a complete overview on the human disease network. In this case, the authors work upon the main premise in which the genetic origin of many diseases can be explained by building a bipartite network of human disorders and disease genes based on known disorder-gene associations, offering a platform to explore all known phenotype and disease-gene associations through complex network theory from a bipartite perspective.

With this methodology, the authors explore whether human genetic disorders and the corresponding disease genes might be related to each other at a higher level of cellular and organismal organization. Consequently, in figure 3.3, we fully appreciate the bipartite nature of the problem, where one node from the disease subset will always connect to nodes that belong to the gene subset. In this example, the authors actually use one of the properties of bipartite networks, which is the ability to project the bipartite graph into two unipartite graphs for each subset of nodes. Thus, they name these projections as the human "disease phenome" (HDN), with the complete list of disease genes, and the "disease genome" (DGN), a direct result of the global view of the human "diseasome", which is the bipartite combined set of all known associations of disorders and disease genes. Hence, a disorder and a gene will be connected by a link if the mutations in that particular gene are implicated in a particular disorder.

With these example of bipartite network show that they are highly suitable to approach real-world problems and provide a solid framework on the usefulness of bipartite graphs as a research tool. Let us review some of these real-world cases in order to build a broader notion of how to apply complex network theory. 


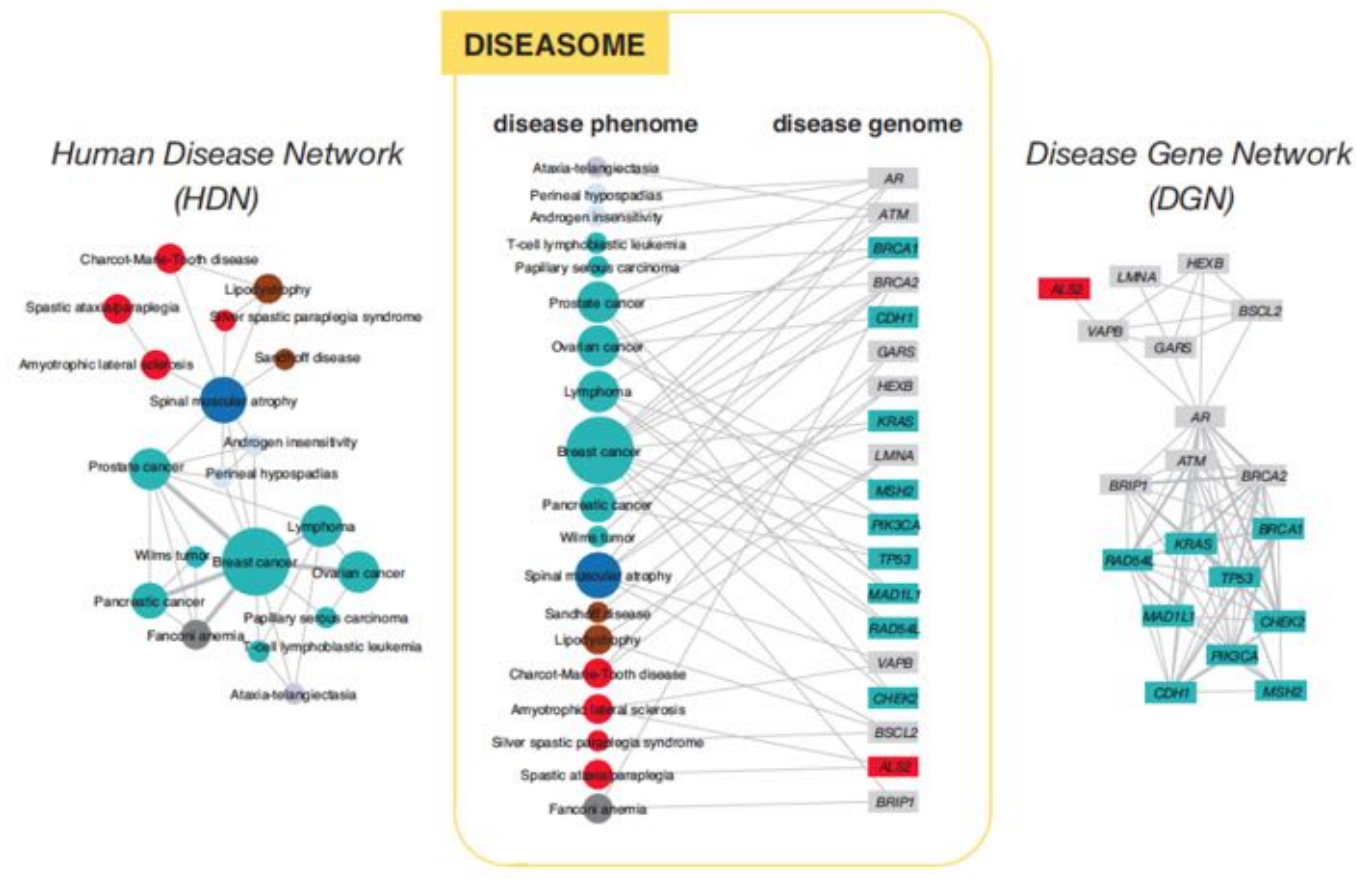

Fig. 3.3.: Bipartite network of phenome-genome (Diseasome). This figure shows the construction of the diseasome bipartite network as per Goh et al.[76]. The subfigure in the center is a small subset of disorder-disease gene associations, where circles and rectangles correspond to disorders and disease genes, respectively. A link will exist between a disorder and a disease gene if the mutations in that gene lead to the specific disorder according to the dataset. The size of any circle is proportional to the number of genes participating in the corresponding disorder, and the color corresponds to the disorder class to which the disease belongs. The subfigure on the left is the HDN projection of the diseasome bipartite graph, in which two disorders are connected if there is a gene that is implicated in both. The width of a link is proportional to the number of genes that are implicated in both diseases. Finally, the subfigure on the right is the DGN projection where two genes are connected if they are involved in the same disorder. The width of a link is proportional to the number of diseases with which the two genes are commonly associated.

\subsection{Mutualistic networks}

The study of mutualistic networks is a well-developed field across the community ecologists. It is mainly based on the assumption that mutualistic relationships between species can be described, studied and modeled by using bipartite networks and bipartite indicators. Thus, for instance, the existing relationships in an ecosystem of plants and pollinators can be portrayed as a bipartite graph (see figure 3.4).

This figure belongs to the work of Rezende et al.[127]. The structure of this network will be defined by the species that exist in the ecosystem, which are represented by nodes, depending on its nature. Each link between nodes will be the result of the existence of interactions observed in the ecosystem. Additionally, the number of 

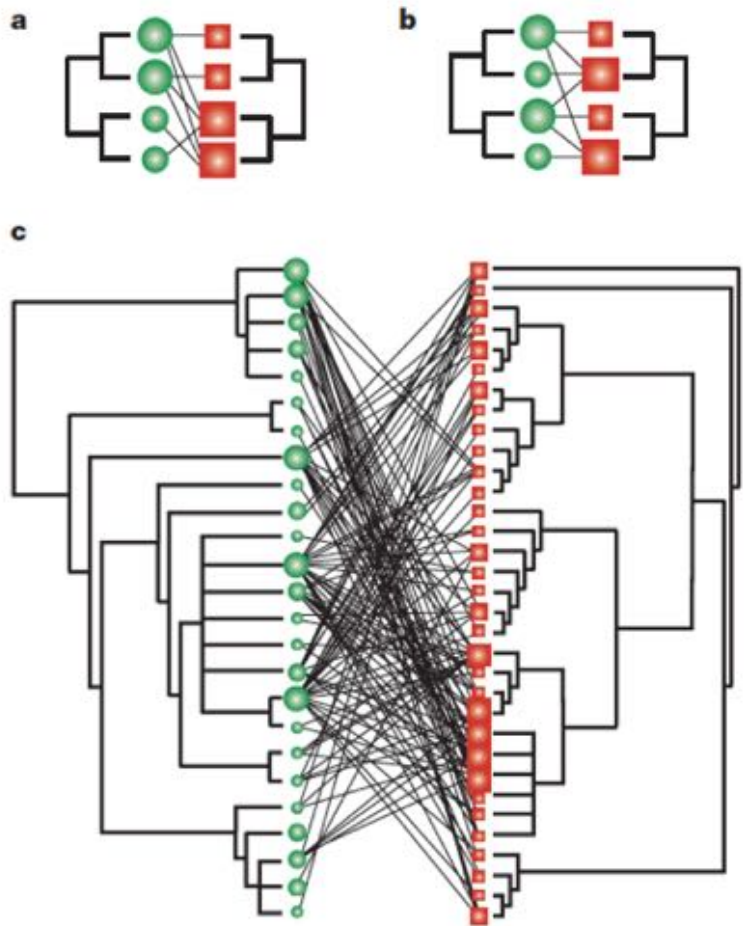

Fig. 3.4.: Bipartite (mutualistic) network of plants and pollinators. Rezende et al. [127]

interactions observed may also be used to depict the weight of each link, converting the representation into a weighted bipartite network. This approach has been proven to be extremely useful to understand the real dynamics in an real ecosystem. Furthermore, it enables a different form of analysis that leads to the discovery of the underlying structures in complex systems. On the other hand, a close analogy between mutualistic ecosystems and international trade markets may be found when zooming in into the dynamics of both systems. The resemblance in terms of competition and cooperation between the agents of both systems will be argued later in the study. Still, this is why the usage of the bipartite approach is of special interest to accomplish many goals of the study.

Now, despite the fact that we may deal with a mutualistic ecosystem or an international trade market, the presence of patterns resulting from the internal dynamics of the system is a fact that needs to be further explored. Another important paper on mutualistic networks is that of Bastolla et al.[25]. This study focuses on how the structure of a mutualistic network reduces the competition among agents which, in turn, will result in an increase of the biodiversity in the ecosystem (see figure 3.6). We may easily appreciate the close relationship between an ecosystem and a market in this aspect. If we can prove that a market behaves similarly to an ecosystem, then the same methodology could be used to test the claims of reduced competition in a market. This is not a minor claim, since it could provide an innovative framework to test the microeconomic theory and offer an alternative explanation of these phenom- 
ena based on a meso-scale approach. It would also make sense from an "economic biodiversity" standpoint. As previously defined, a perfect competition market takes place when a large number of sellers and buyers exist. As a result, no buyer or seller will be able to control the market, increasing the overall robustness of the system. To depict such situation from a mutualistic point of view, let us consider the figure 3.5 .
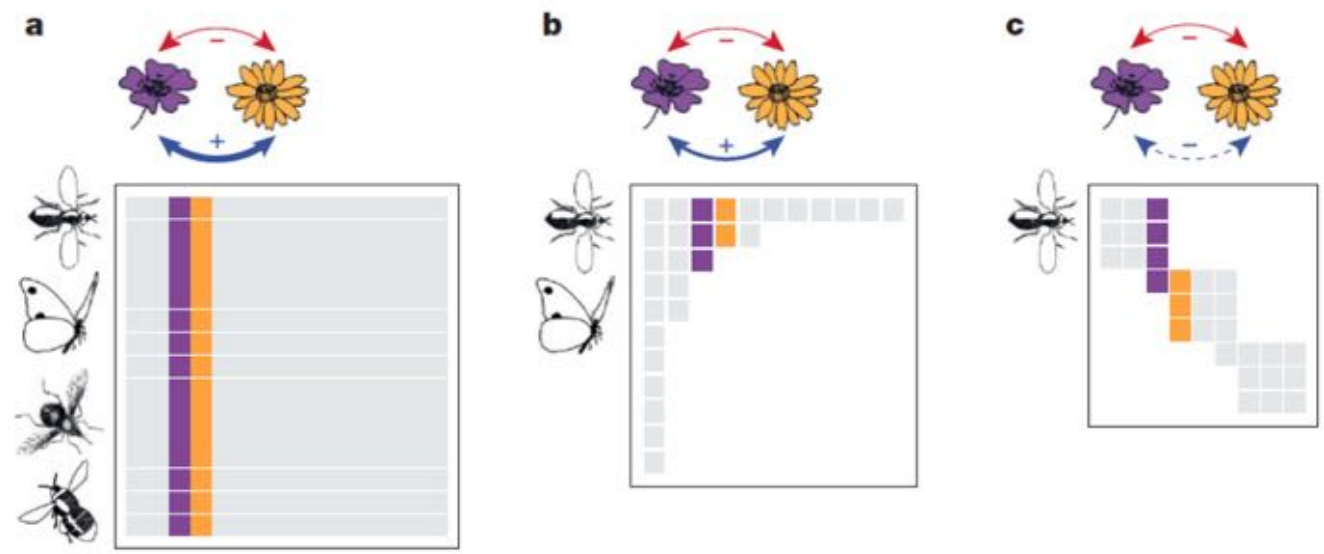

Fig. 3.5.: Number of coexisting species determined by the structure of the network. Bastolla et al.[25]. Each panel represents a plant-animal network with different structures. Subfigure A depicts a fully connected structure. Subfigure B depicts a nested structure. Subfigure C depicts a compartmentalized structure.

In these cases, the authors show the interactions of two plants in a) a fully connected network, b) a nested one and c) a compartmentalized matrix. In every one of these cases, the red arrows represent the competition for resources and the blue arrows represent the indirect interactions that take place via their common pollinators. The higher the number of common pollinators is, the bigger the mutualistic effect will be, which is depicted by the style of the arrow. Under these circumstances, the theory proposed predicts a higher number of coexisting species. By analogy, the same logic could apply to a market. If two sellers compete for market share and they sell to a higher number of common buyers, the higher diversity of supply and demand effects would ultimately turn into more robust market equilibrium, where the conditions of perfect competition may remain stable over time. In fact, the methodology of this paper proposes that a baseline biodiversity, which occurs in the absence of mutualistic effects, is required to account for the pure competitive interactions. This is performed by using the concepts from Bastolla et al. [24], which defines the maximum number of species in the ecosystem as:

$$
\tilde{S}^{(P)}=\frac{1-\tilde{\rho}^{(P)}}{\tilde{\rho}^{(P)}}
$$


where $\tilde{\rho}^{(P)}$ is the normalized effective interspecific competition parameter of the $P$ species (plants), which is computed based on the main eigenvalue of the competition matrix. Furthermore, the study proposes a dynamical equation to model the behavior of species:

$$
\frac{d N_{i}^{(P)}}{d t}=\alpha_{i}^{(P)} N_{i}^{(P)}-\sum_{j \in P} \beta_{i j}^{(P)} N_{i}^{(P)} N_{j}^{(P)}+\sum_{k \in A} \frac{\gamma_{i k}^{(P)} N_{i}^{(P)} N_{k}^{(A)}}{1+h^{(P)} \sum_{l \in A} \gamma_{i l}^{(P)} N_{l}^{(A)}}
$$

where the upper indices denote the type of species, either plant or animal, $N_{i}$ represents the number of individuals of the species $i$ and $P$ and $A$ represent the subset of plants and animals. Without getting into too much detail, we observe 3 terms in the equation. The first term describes an exponential growth, which is characterized by the intrinsic growth rate in absence of mutualism $\left(\alpha_{i}^{(P)}\right)$. The second one, a negative term, accounts for the interspecific competition through $\beta_{i} j^{(P)}$, a rate that measures the direct interspecific competition for resources between species $i$ and $j$. Finally, the third term describes the mutualistic interaction. From this expression, we may derive another aspect of the proposed analogy between ecosystems and markets, since the latter will indeed have an intrinsic growth term, another one to account for the competition between sellers of a good and finally, a term to account for the cooperation effect between sellers and buyers, a market force that should not be neglected when analyzing market dynamics and formation. The evidence provided by the simulations in this paper support such claims, introducing the concept of nestedness to measure the level of underlying organization within the ecosystem. For this purpose, the authors also introduce a measure of biodiversity increase in randomized networks in order to rule out the possibility of alternative sources of variance, such as other covariant variables (number of species and interactions).

The results are highly robust and statistically significant, concluding that the higher the nestedness of a mutualistic network is, the higher the number of expected species or biodiversity, as shown in figure 3.6. In addition, the authors find that a similar correlation takes place when comparing real networks to randomized ones (randomizations). These facts together prove that the theory is sound and that competition is directly and mainly influenced by the structure of the network, ruling out other causes of variance. The structure of mutualistic networks plays a central role in determining the dynamics of the system.

Finally, to conclude the review of mutualistic networks and the analogy with trade markets, it is appropriate to mention the study of Saavedra et al.[134]. In this paper, a strong argument regarding the probability of extinction turns out to be higher 


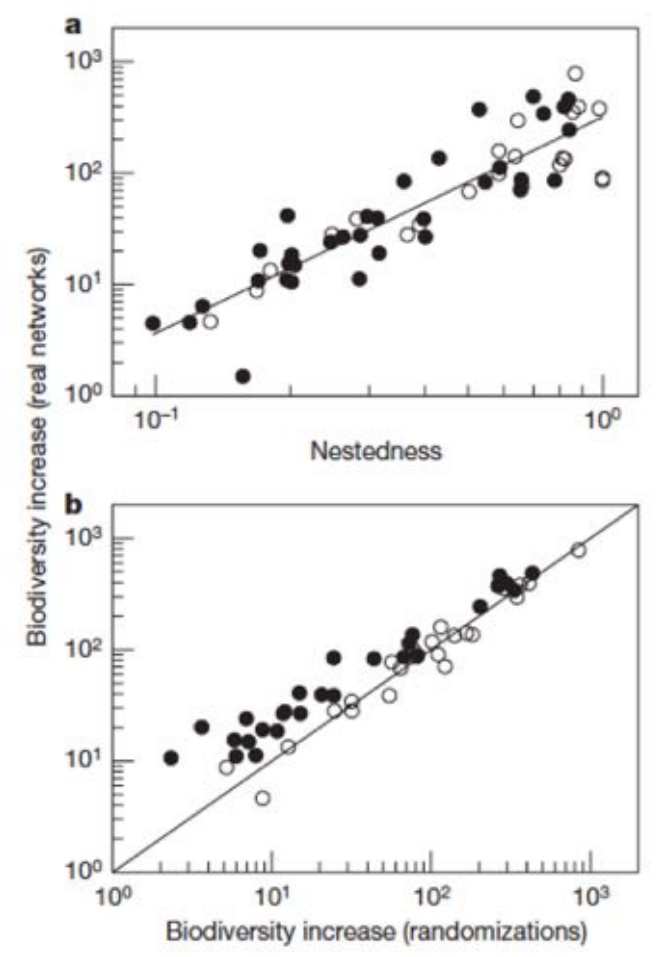

Fig. 3.6.: Correlations between nestedness, randomizations and biodiversity. Bastolla et al.[25]

among the species that contribute the most to network persistence. This assumes that there is a direct relationship between network persistence features and the concept of nestedness. It also implies that a measurement of node-level contribution to nestedness is required. To accomplish this, the authors have defined a procedure that once again relies on randomizations the elements of the bipartite matrix to infer such an indicator.

In figure 3.7, the process and results are clearly depicted. Subplot a) shows a perfectly nested bipartite matrix and the position of the elements of a given plant (in green). By calculating the nestedness of the actual matrix in addition to that of randomized cases, as shown in b), we could determine the individual contribution to nestedness. Formally, the authors define the measure as the degree to which the overall nestedness of the network compare with the values obtained after randomizing the interactions of the particular node. Subplot $b$ ) shows an example of such a process (in orange). It should be noted that the randomizations should only affect the column or row for the node under analysis only. The results suggest that the contribution to overall nestedness is not equally distributed across nodes. In contrast, there are a few nodes that contribute in a more significant way than others. At this moment, it is very difficult not to wonder whether a relationship between these results and the probability of extinction exists. 

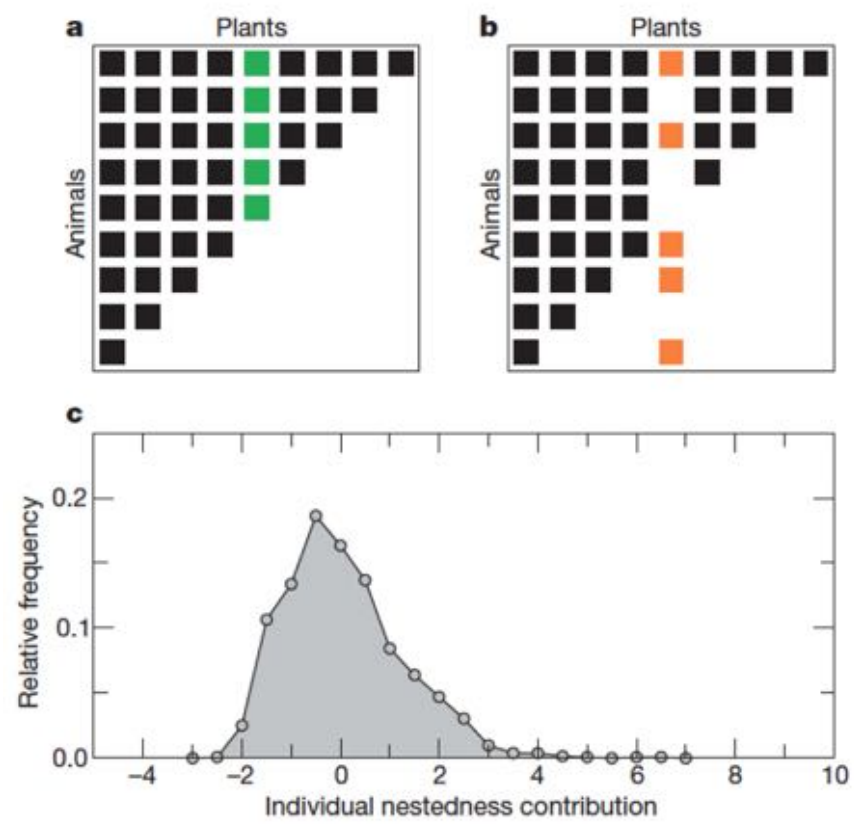

Fig. 3.7.: Process to determine individual contribution to nestedness and results after the randomizations. Saavedra et al.[134]

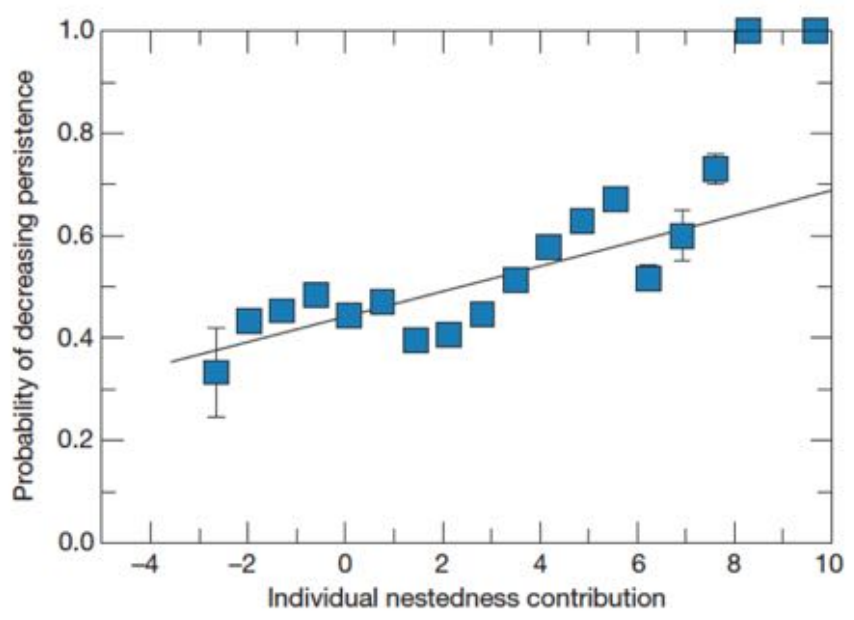

Fig. 3.8.: Extinction exposure as a function of individual contribution to nestedness. Saavedra et al.[134]

The simulations provide strong evidence that support an affirmative answer. Indeed, the higher the contribution of a node, the higher the probability of decreased persistence (see figure 3.8). This conclusion seems to be counter-intuitive, since an agent that allows the system to persist may well be an important one and hence, difficult to eliminate without affecting system at macro level. Nevertheless, the results show that the opposite holds, establishing a new paradox that will require further explanation. 
After reviewing these papers about mutualistic networks, relevant knowledge has been gathered and a vast methodological opportunity exists in order to apply such methodology to trade markets. If the analogy between systems holds, some of the conclusion could be a direct and mere reformulation of the same principles, pointing to universal patterns and universality classes.

\subsection{Previous related work on trade networks}

International trade networks, often referred to as bilateral trade networks, have attracted the attention of many researchers in the last decade, which on the one hand constitutes a solid background for references but, on the other hand, makes finding innovative results more challenging. As a general outline, we might find early efforts to depict the topology of trade networks based on a directed graph approach, both weighted and binary in nature. The most relevant findings are, as expected, the existence of heterogeneous degree distributions as well as a high clustering properties, which is an evidence of underlying organization in these networks.

\subsubsection{Topology of the World Trade Web}

This is the first published paper [139] that depicts world trade as a complex network. The authors completely rely on this modeling by arguing that trade itself is a fundamental part of human social organization and that they will introduce the first empirical characterization of this complex system. To support this claim, the authors also state that globalization processes lead to the liberalization of trade and, as a consequence, countries become more interrelated. Thus, the study the network of trade relationships between different countries in the world is a critical objective in the paper. Such characterization of the system is referred to as the world trade web (WTW). Furthermore, the study is targeted to show that the WTW is a complex network that shares many properties with technological networks. The actual data to build the WTW comes from the aggregated trade statistics published in the International Trade Center site, which are based on the United Nations' COMTRADE database. These statistics contain, for each country, an import and an export list of the forty more important goods in the year 2000. Therefore, the WTW can be constructed by considering imports as in-degrees and exports as out-degrees, enabling the consolidation of a directed network where vertices represent countries and directed links represent the importation or exportation relationships between countries. In addition, the import/export connections that are of little relevance for a given country are not considered in the datasets, but since the complete adjacency matrices must satisfy the symmetry relation $A_{i j}^{i m p}=A_{j i}^{e x p}$, the authors can then recover missing information from the original matrices. After this symmetrization 
procedure, the resulting directed network has 179 vertices (or countries) and 7510 directed links (or trade relationships) with a average degree of $\left\langle k_{\text {in }}\right\rangle=\left\langle k_{\text {out }}\right\rangle=30.9$, which now poses the highly relevant question of what to measure within such network.

To answer this question, the authors focus on 3 important properties of complex networks: a) the degree distribution, b) the clustering coefficient as a function of the degree, and c) the nearest neighbor degree correlation. These properties had already been used in several studies on complex network modeling, like the metabolic interaction network, and provided a solid framework for such studies. Consequently, these are the results obtained after computing such properties, as shown in figure 3.9 .
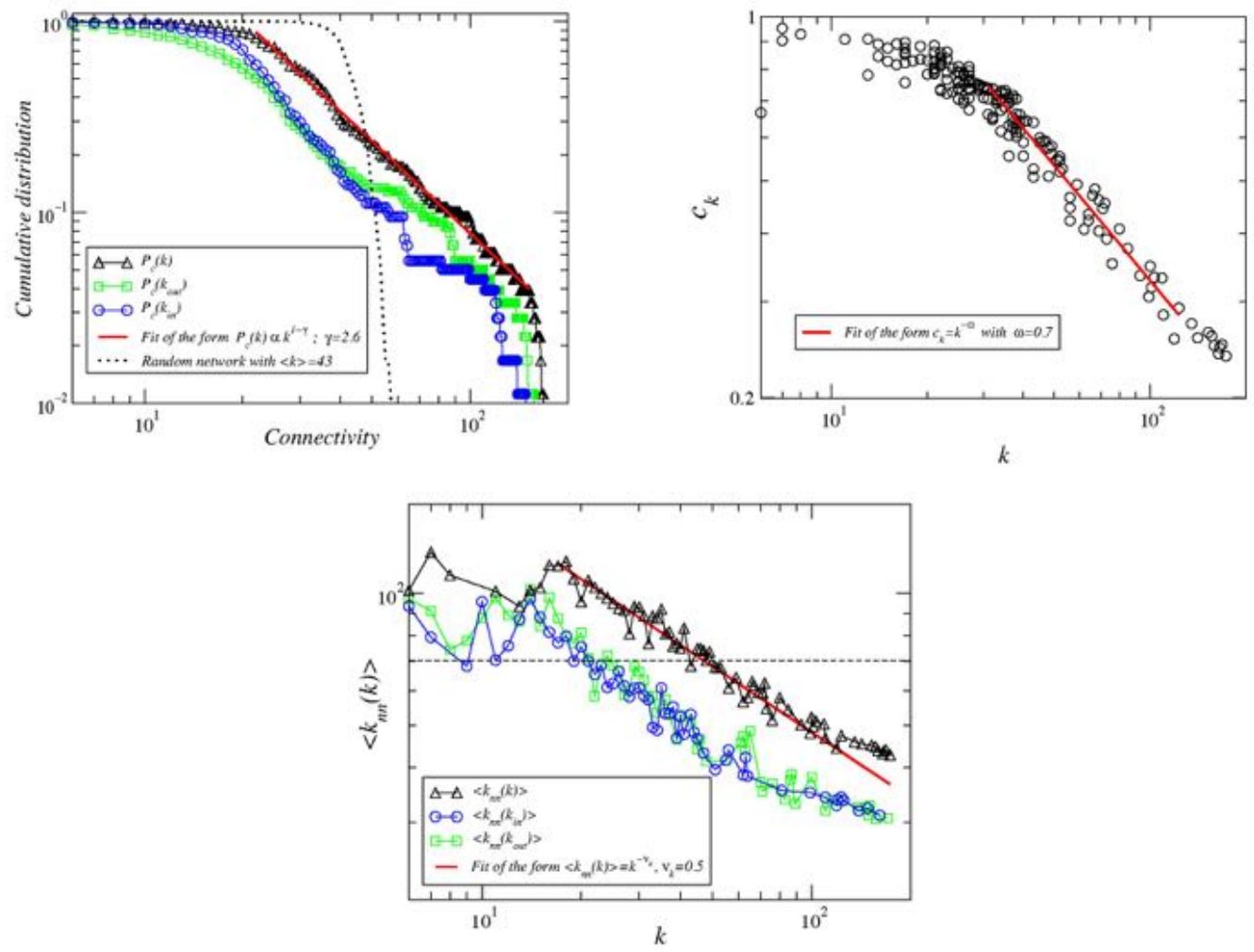

Fig. 3.9.: Results on the topology of the World Trade Web. Serrano et al. (2003)

The first (upper-left side) figure shows the degree distribution, $P(k)$, which measures the probability of a randomly chosen vertex to have $\mathrm{k}$ connections to other vertices and, since the network is directed in nature, the in- and out-degree distributions are also shown in blue and green, respectively. The red line shows the best fit of the form $P(k) \sim k^{-\gamma+1}$ with $\gamma=2.6 \pm 0.1$. This exponent was found to be within the range defined by other complex networks and, therefore, the WTW belongs to the class of Scale-Free (SF) networks, which implies an extremely high level of degree heterogeneity is present in the system. The second (upper-right side) and 
third (bottom) figures are mainly to evaluate how the organization within the WTW in terms of hierarchical structure. Such hierarchy is analyzed by the usage of the clustering coefficient and the nearest-neighbor degree correlation, as previously mentioned. Regarding the clustering properties, the authors use the definition of clustering coefficient in which the vertex $i$ of degree $k_{i}$ as $c_{i}=2 n_{i} / k_{i}\left(k_{i}-1\right)$, where $n_{i}$ is the number of neighbors of $i$ that are interconnected. If hierarchy were not present in the system, the clustering coefficient would be a random quantity independent of the degree or any other property. Yet, figure 3.9 (upper-right side) clearly shows the clustering coefficient of the undirected WTW is a function of the degree, $k$, displaying a power law behavior $c(k) \sim k^{-\omega}$ with $\omega=0.7 \pm 0.1$. Additionally, average clustering coefficient of the whole network is $C=0.65$, a value found to be greater, by a factor 2.7 , than that of an equivalent random network of the same size. Finally, hierarchy may also be reflected on the nearest-neighbor degree correlation, which is defined as $\left\langle k_{n n}(k)\right\rangle=\sum_{k^{\prime}} k^{\prime} P\left(k^{\prime} \mid k\right)$ ). Figure 3.9 (bottom) now clearly shows a behavior that is dependent on degree $k$. The black line depicts the results of the undirected network, whereas the blue and green lines represent the results for the in- and out-degree, respectively. The red line is best fit of the form $\left\langle k_{n n}(k)\right\rangle=k^{\nu_{k}}$ with $\nu_{k}=0.5 \pm 0.1$, which provides factual evidence, in combination with the finding on clustering, that hierarchical structure underlies the topology of the WTW.

On the conclusions side, the authors state that the topology properties of the WTW are similar to those of the Internet. They also propose that one of the reasons for this to happen is based upon the fact that both systems are competitive in nature. Yet, they do not forget about some constraints within the WTW, like the geographic ones, which introduce a different way of complexity as well. As stated by the authors:

... Our research suggests that the network's evolution is guided by collective phenomena, and that self-organization plays a crucial role in structuring the WTW scale-free inhomogeneities and its hierarchical architecture. It remains an open question if these properties could also be made apparent at other different scales, for instance, in the trade relations between regions, cities or even individuals. At the country level, the activity is driven by competition...

\subsubsection{Patterns of dominant flows in the world trade web}

This study [140] is a weighted analysis of the imbalances of the trade flows, aiming at uncovering the global emerging organization of the international trade network. For this reason, the authors analyze the world network of bilateral trade and characterize its overall flux organization in order to unravel local and global high-flux pathways 
that will ultimately define the backbone of the trade system. They also develop a general procedure capable to progressively filter out, in a consistent and quantitative way, the dominant trade channels. This procedure is completely general and can be applied to any weighted network to detect the underlying structure of transport flows. Then, a weighted directed network of merchandize trade imbalances between world countries is constructed. In this representation, each country appears as a node and a directed link is drawn among any pair whenever a bilateral trade imbalance exists. The direction of the arrow follows that of the net flow of money and it is weighted according to the magnitude of the imbalance between the two countries. The authors define $E_{i j}$ as the exports of country $i$ to country $j$ and $I_{i j}$ as the imports of country $i$ from country $j$. Consequently, the trade imbalance matrix is defined as $T_{i j}=E_{i j} I_{i j}$, which measures the net money flow from country $j$ to country $i$. Since $E_{i j}=I_{j i}$ and $I_{i j}=E_{j i}, T$ is an antisymmetric matrix with $T_{i j}=-T_{j i}$. The network of the net trade flows is therefore defined in terms of a weighted adjacency matrix $F$ with $F_{i j}=\left|T_{i j}\right|=\left|T_{j i}\right|$ for all $i, j$ with $T_{i j}<0$, and $F_{i j}=0$ for all $i, j$ with $T_{i j} \geq 0$. Thus, by using this procedure, the network of trade imbalances can be constructed. The data set contains the annual merchandize trade activity between independent states in the world during the period 1948-2000, in addition to the annual values of Gross Domestic Product per capita and population. The time span of the data allows the authors to study the change of trade flow networks with yearly snapshots, characterizing the time evolution of the trade system. Among the measures used in the study, we can find, as expected, the number of incoming and outgoing links, $k_{\text {in }}$ and $k_{\text {out }}$. Additionally, the magnitude of the fluxes carried by each trade relation can be retrieved by summing up all the weights of the incoming or outgoing links. These quantities are defined, both incoming and outgoing, as the strength. Formally, this is denoted as $s_{j}^{i n}=\sum_{i} F_{i j}$ and $s_{j}^{\text {out }}=\sum_{i} F_{j i}$. The trade imbalance will be $\triangle s_{j}=s_{j}^{\text {in }}-s_{j}^{\text {out }}$. It can be appreciated that, since one incoming link for a given country is always an outgoing link for another, the sum of all the countries' trade imbalances in the network must be zero. So, while the local balance is not conserved, we are therefore dealing with a closed system, which is globally balanced (the total flux is conserved). Merchandizes, or equivalently money, flows in the system from country to country with the peculiarity that there is a global flow of money from consumer countries to producer ones. The authors found that incoming and outgoing connections of a given country are positively correlated quantities, which means that countries with high $k_{i n}$ will also display high $k_{\text {out }}$. The same holds for incoming and outgoing strengths. Thus, applying these indicators to the actual data leads to the results in figure 3.10.

The scattered plots of $k_{\text {out }}$ versus $k_{\text {in }}$ and $s_{\text {out }}$ versus $s_{\text {in }}$ (upper-left and upper-right corners, respectively) for the years 1960, 1980 and 2000 are shown in the same subfigure. In both cases, the positive correlation is evident. A direct power law 

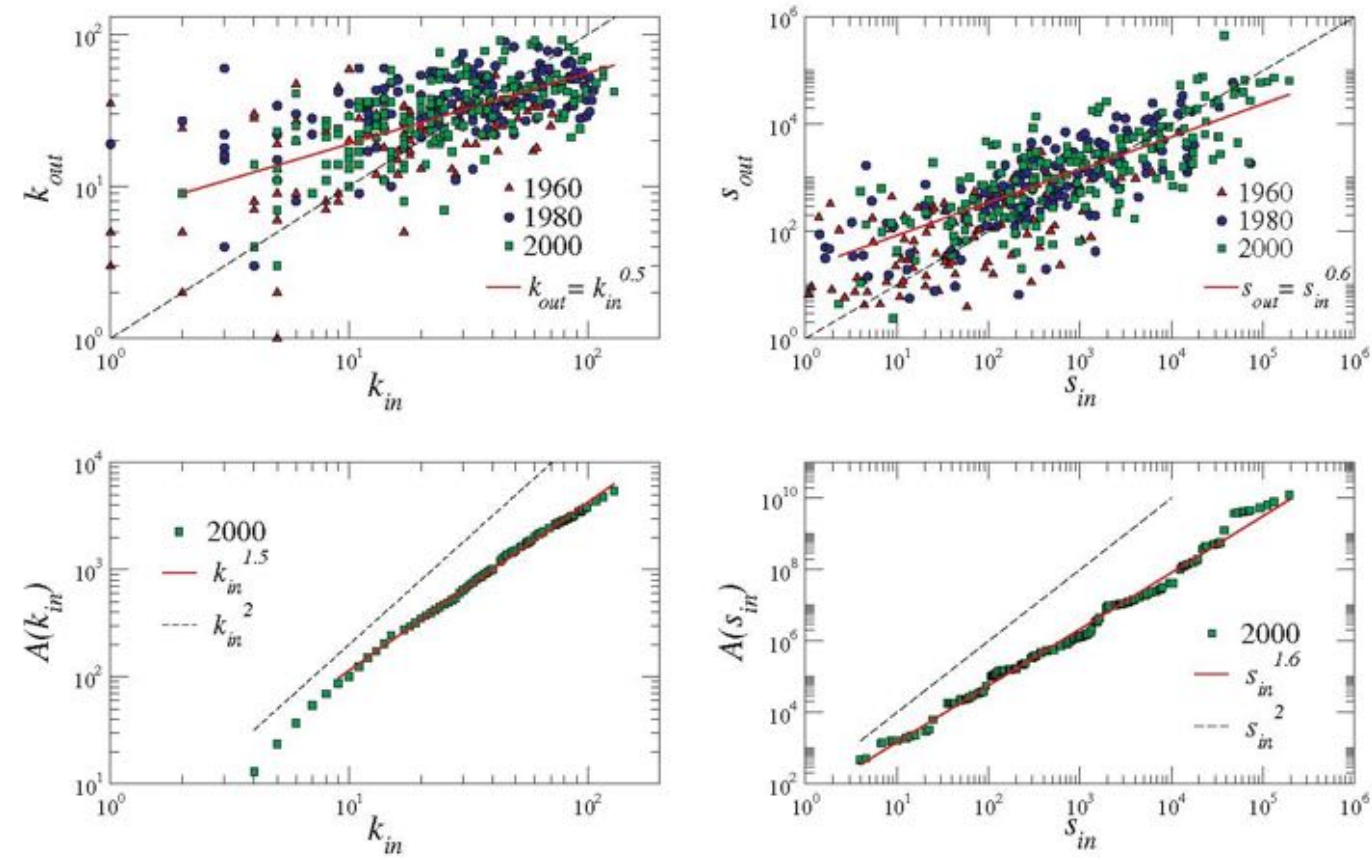

Fig. 3.10.: Topology weighted results on dominant flows in the WTW. Serrano et al. (2007)

regression on the 2000 set shows sub-lineal patterns of the form shown in equation 3.14 and 3.15 .

$$
\begin{aligned}
& k_{\text {out }} \sim k_{\text {in }}^{0.5} \\
& s_{\text {out }} \sim s_{\text {in }}^{0.6}
\end{aligned}
$$

However, the noise inherent to the data makes difficult to clearly appreciate this non-linear effect. To overcome this problem, the authors define integrated quantities instead of local ones as per equations 3.16 and 3.17 .

$$
\begin{aligned}
& A\left(k_{\text {in }}\right)=\int^{k_{\text {in }}} k_{\text {out }}\left(k_{\text {in }}\right) d k_{\text {in }} \\
& A\left(s_{\text {in }}\right)=\int^{s_{\text {in }}} s_{\text {out }}\left(s_{\text {in }}\right) d s_{\text {in }}
\end{aligned}
$$

By this logic, if the relation between the in and out quantities were to be lineal, then the integrated measure should scale quadratically. Nevertheless, in this case, it 
should scale with an exponent in the range between 1.0 and 2.0 if the relation were to be sub-lineal. The bottom graphs of figure show the integrated functions $A\left(k_{i n}\right)$ and $A\left(s_{i n}\right)$, which exhibit a clear scaling with exponents 1.5 and 1.6, a fact that is in perfect agreement with the results of the data regression. This sub-lineal behavior implies that countries with small $s_{i n}$ have small $s_{\text {out }}$, but for them the value of $s_{\text {out }}$ is on average bigger than that of $s_{i n}$. At the same time, countries with high $s_{i n}$ have high $s_{\text {out }}$, but for them the value of $s_{\text {out }}$ is on average smaller than that of $s_{i n}$. The same can be said from the results for the degrees $k_{i n}$ and $k_{i n}$ in the top graph of figure. All this indicates that small economies tend to be net consumers, hence with $\triangle s<0$, whereas big ones tend to be net producers, so with $\triangle s>0$.

In a second part of the article, the authors focus on measurement of local heterogeneity and the extraction of the backbone of the WTW. To perform such a task, they thought they could rely on the overall flux organization at the global scale, which can be characterized by the study of the flux distribution through the probability distribution $P\left(F_{i j}\right)$, denoting the probability that any given link is carrying a flux $F_{i j}$. Yet, given the expected large scale heterogeneity in complex networks, this might not be entirely suitable. For this reason, it would be more interesting to explore the characterization of the local heterogeneity in order to determine, given all the connections associated to each given country, how the flux distribution for each of them might be. Local heterogeneity implies that only a few links carry the biggest proportion of the country's total in-flow or out-flow. Such heterogeneity would define specific pathways within the network that accumulate most of the total flux. In order to assess the effect of inhomogeneities at the local level, for each country $i$ with $k$ incoming or outgoing trade partners, the authors compute this as indicated in equation 3.18 .

$$
k Y_{i}(k)=k \sum_{j=1}^{k} p_{i j}^{2}
$$

where $k$ can be either $k_{\text {in }}$ or $k_{\text {out }}$ in order to discern between inhomogeneities in incoming and outgoing fluxes, and where the normalized fluxes of node $i$ with its neighbors are calculated as $p_{i j}=F_{j i} / s_{i}^{i n}$ for incoming connections and as $p_{i j}=F_{i j} / s_{i}^{\text {out }}$ for the outgoing ones. The function $Y_{i}(k)$ is extensively used in economics as a standard indicator of market concentration, referred to as the Herfindahl-Hirschman Index, and it was also introduced in the complex networks literature as the disparity measure. In all cases, $Y_{i}(k)$ characterizes the level of local heterogeneity. If all fluxes emanating from or arriving to a certain country are of the same magnitude, $k Y_{i}(k)$ scales as 1 independently of $\mathrm{k}$, whereas this quantity depends linearly on $k$ if the local flux is heterogeneously organized with a few main directions. Increasing devia- 
tions from the constant behavior are therefore indicating heterogeneous situations in which fluxes leaving or entering each country are progressively peaked on a small number of links with the remaining connections carrying just a small fraction of the total trade flow. The authors also introduce a null model for the distribution of flows among a given number of neighbors in order to evaluate, in a case-by-case basis, whether the observed inhomogeneity can just be due to fluctuations or it is really significant. In conclusion, and to summarize the main conclusion, the authors have argued and proved that a) the trade fluxes properties of the world trade web determine a ranking of trade partnerships that highlights global interdependencies, b) the information is not accessible by simple local analysis, and c) this study provides new quantitative tools for a dynamical approach to the propagation of economic crises.

\subsubsection{The World-Trade Web: Topological Properties, Dynamics, and Evolution}

In order to provide an overview about this study, it is relevant to state that this is the first paper to tackle the dynamics within the WTW. Still, the main objective of studying the statistical properties of the web of import-export relationships among world countries using a weighted-network approach is not entirely different from the approach of the previous paper. Yet, the authors focus on analyzing how the distributions of the most important network statistics, like the connectivity, assortativity, clustering and centrality of the network, evolve over time. They use international trade data in order to develop a time-sequence of weighted directed networks from 1981 to 2000 for 159 countries. The network is later defined so that rows represent exporting countries and columns stand for importing countries. A trade relationship would exist if exports from country $i$ to country $j$ are strictly positive in year $t$ $\left(\tilde{e}_{i j}^{t}>0\right)$ so that, formally, ${\tilde{a_{i j}}}^{t}=1$. In addition, the authors also define the weight of a link from $i$ to $j$ in year $t$ as $w_{i j}^{t}=e_{i j}^{t}$. Thus, the sequence of $\mathrm{N}$ by $\mathrm{N}$ adjacency and weight matrices $\left[\tilde{A}^{t} ; \tilde{W}^{t}\right], \mathrm{t}=1981, \ldots ., 2000$ will depict the dynamics of the WTW.

Within the main conclusions, the authors find: a) that all node-statistic distributions and their correlation structure have remained stable in the 20 years period, b) that these properties are likely to do so in the future, c) that the distribution of link weights is slowly moving from a log-normal density towards a power law, d) that network-statistics growth rates are well-proxied by fat-tailed densities like the Laplace or the asymmetric exponential-power, and e) that all the results are reasonably robust to a few alternative, economically-meaningful, weighting schemes. 

Part II

Materials and Methods 



\section{International Trade Data}

Data! Data! Data!" he cried impatiently. "I can't make bricks without clay!"

- Sherlock Holmes

(The Adventure in the Copper Beaches, by Sir

Arthur Conan Doyle)

This chapter deals with the issue of reliable and accurate data about global trade. The main objective here is to address this issue in addition to introducing the large dataset used for the study, providing insights on big data techniques as well.

\subsection{Data availability}

The availability of data in the age of information is not really a problem. Yet, the availability of reliable and consistent data that is suitable for rigorous and robust research is, in contrast, a very different topic. Many countries publish their own international trade data either through their national statistics agencies or through some specific initiatives following a public information regulation, similar to the "freedom of information act"[159] in the United States.

However, the main problem with this information is related to completeness and consistency. On the one hand, only a few countries publish their complete trade data. On the other hand, the reported data turns out to be quite inconsistent in terms of common rules and definitions. Furthermore, some international organizations, like the International Monetary Fund (IMF) or the World Bank, share their statistics freely on the internet. But, they only provide high-level, aggregated data of macroeconomic indicators, a fact that addresses the issue of consistency. This data, however, does not help us analyze bilateral trade between countries at a product level. This is why we need to find a data source that is designed to overcome such important issues. After exploring several alternatives, the best option is a database from the United Nations called "COMTRADE" [158]. 


\subsection{United Nations' COMTRADE dataset}

The age of information requires a new standard of data availability, a concept that is often referred to as public transparency. The United Nations and its statistics department have opened their database on bilateral trade. The vision behind this initiative is as follows:

Free access to all of the trade data available in the UN Comtrade database. The UN Comtrade database contains more than 3.1 billion trade records starting from 1962.

As stated, this data is accessible at https://comtrade.un.org. In figure 4.1, we show an example of how to extract one record from this database. However, full access to this database is limited due to several reasons that range from purely technical to rights-related issues. The data, on the other hand, is structured in such a way that some reference tables are required. The data is also structured with standard product and country codes. The following is an example of our raw data within our database:

Figure 4.2 shows the first 20 lines of a data string that posses over 91 million records of 5039 products over 15 years of history from 1995 and 2009. The size of the data associated to this dataset exceeds 10 gigabytes of text files. These files are standard comma-separated values or CSV files. Data structure, however, seems to be relatively simple, since there are only 6 data fields. These data fields can be described as:

- Period: year in which the trade has been made.

- Product code: standard HS6 product code.

- Exporter code: standard ISO 3-digit country code of the exporter.

- Importer code: standard ISO 3-digit country code of the importer.

- Value: total amount of product in thousand dollars.

- Quantity: this amount is not used for the study. Yet, it is the quantity in volume, weight or other applicable unit.

The HS6 code is a 6-digit code defined by COMTRADE in order to standardize the reporting of trade activity at product level. The HS initials stand for "Harmonized 


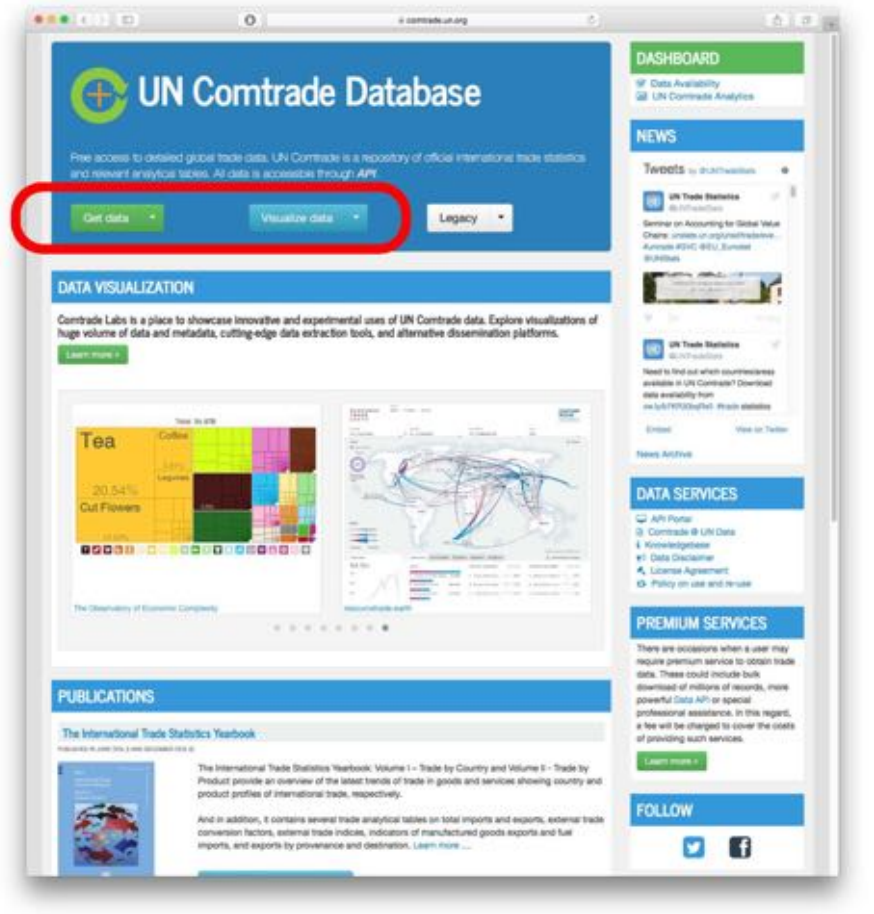

https://comtrade.un.org

This database from the United Nations allows for access to the data of international trade between countries.

It features an extraction interface and a data visualization one.

The raw data from this database need to be processed to ensure accuracy and consistency.

By clicking the "Get data" button, we access the extraction interface (below).

Here we show an example of how to extract a data record from COMTRADE.

In this case, we ask to retrieve the exports from Spain in the year 2016 for the industry HS2=84 (Machinery) on an annual basis.

The result of the query is shown at the bottom of the webpage.

Within our research, we manage about 91 million records like this one. Each record is later interpreted as a link in a trade network.

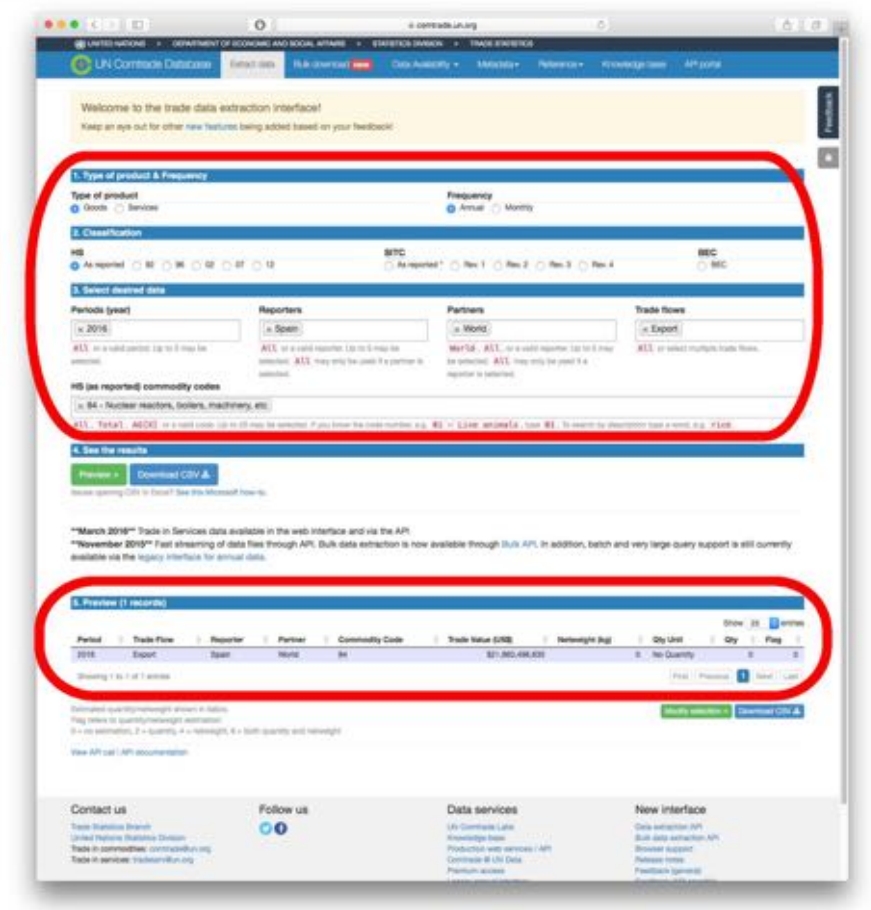

Fig. 4.1.: Example of data retrieval from COMTRADE. This figure shows the procedure to retrieve a record from the COMTRADE[158] database by accessing the extraction interface from the web portal. 
$1995,010111,251,012,16.046,0.597$

$1995,010111,251,020,6.8645719929,1.5623032045$

$1995,010111,276,020,2.727,0.398$

$1995,010111,724,020,1.178,0.898$

$1995,010111,058,032,60.188,0.902$

$1995,010111,076,032,39.481520592,10.008766888$

$1995,010111,124,032,22.632,1$

$1995,010111,152,032,7.0828230474,0.835565491$

$1995,010111,276,032,8,0.5$

$1995,010111,372,032,29.47,0.449$

$1995,010111,528,032,45.464080651,3.9486614551$

$1995,010111,600,032,10.988947348,1.5488280916$

$1995,010111,604,032,10.91,2.625$

$1995,010111,826,032,33.586396643,1.1935994674$

$1995,010111,842,032,468.58956204,25.539$

$1995,010111,858,032,11.391160796,2.5970664663$

$1995,010111,899,032,3.532,0.902$

$1995,010111,344,036,207.554,0.45$

$1995,010111,554,036,6427.8438051,90.382940686$

$1995,010111,702,036,42.679,2.7$

Fig. 4.2.: Example of non-aggregated trade data. This figure shows the first 20 records (out of a total of 91 million) of the international trade data from the COMTRADE database.

System". This is also the lowest level of aggregation on the product hierarchy. On the other hand, this information can also be consolidated at HS4 (4-digit code) and HS2 (2-digit code) levels. We may think of HS4 as a category and HS2 as an industry. In table 4.1, we show an example of product levels of aggregation. In this limited example of the fishing industry (HS2 code $=03$ ), we observe the different products at HS6 level. The consolidation of the HS6 products at HS4 or category level is the next step. Similarly, the live fish category (HS4 code $=0301$ ), the fresh fish category (HS4 $=0302$ ) and any other existing HS4 category will be consolidated into the fish industry at HS2. We will discuss later on this chapter the process used to perform the consolidation in the big data section.

Regarding country codes, they also follow a standard. We use the ISO 3-letter code standard. Regarding the country codes, they are shown in Table 4.2. Additionally, a detailed and complete list of country codes with their respective equivalences can be found in Appendix A.

Even though countries will not have any consolidation, it is important to notice other characteristics associated to them, namely the geographic location, the economy size and the importance of a country's exports as a porcentage of the total world trade. These aspects will also be later discussed in the visualization and gravity model sections. Given the nature of our data, which has a high resolution, is big in size and broad in scope, we conclude that it can easily be qualified as a big data problem. 


\begin{tabular}{lll}
\hline Level & Code & Description \\
\hline HS2 & 03 & Fish and crustaceans, molluscs and ... \\
HS4 & 0301 & Live fish \\
HS6 & 030110 & Live ornamental fish \\
HS6 & 030191 & Live trout \\
HS6 & 030192 & Live eels \\
HS6 & 030193 & Live carp \\
HS6 & 030199 & Live fish, n.e.s. \\
HS4 & 0302 & Fish, fresh or chilled, excluding fish fillets \\
HS6 & 030111 & Trout \\
HS6 & 030112 & Pacific salmon /Atlantic salmon \\
HS6 & 030119 & Salmonidae \\
$\ldots$ & & \\
\hline
\end{tabular}

Tab. 4.1.: Example of the level of aggregation of products (HS6), categories (HS4) and industries (HS6). This table shows the different levels of aggregation of the product hierarchy. Several products (HS6) are consolidated under a category (HS4). The same is true for categories (HS4) and industries (HS2).

\begin{tabular}{lll}
\hline Code & ISO-3 & Country name \\
\hline 004 & AFG & Afghanistan \\
008 & ALB & Albania \\
012 & DZA & Algeria \\
016 & ASM & American Samoa \\
020 & AND & Andorra \\
024 & AGO & Angola \\
028 & ATG & Antigua and Barbuda \\
032 & ARG & Argentina \\
036 & AUS & Australia \\
040 & AUT & Austria \\
044 & BHS & Bahamas \\
$\ldots$ & & \\
\hline
\end{tabular}

Tab. 4.2.: Example of country codes and equivalences. This table shows the standard coding for countries. A full list of country codes can be found in Appendix A. 


\subsection{Data cleaning and consistency}

Our dataset presents several advantages in terms of consistency. It is not actually a direct download from COMTRADE, but it is a harmonized dataset based on several data cleaning techniques.

The United Nations efforts to standardize the reporting of international trade started in the late 1980's with the progressive implementation of the product classification or the Harmonized System (HS). From that point on, the finest product classification of over 5000 products was an improvement over the previous Standard International Trade Classification (SITC), which only included about 1200 products. So, in a way, the product consistency issue was solved before the time in which our historical data starts (1995).

Another problem of the trade data is that countries report their importations and exportation in a different value base. While imports are reported in a CIF base, which includes the cost of the goods, the transportation insurance and freight cost, exports are reported at FOB values, which stands for Free On Board, including the cost of the goods up to the moment that is ready to be shipped only, where no insurance, dutties or freight are included. In this way, the reported data from the exporter country and the importer country would not match despite the fact that we are dealing with the exact same goods, however they are priced with a different base. A normalization is required. For this reason, a methodology to identify the CIF rates (incremental factor) as a function of the distance between countries, among other variables, has been put in place. In this way, imports and exports can now be mirrored in the dataset. This methodology is based on the following model:

$$
\ln R_{i j}=\alpha+\beta \ln D_{i j}+\delta \ln D_{i j}^{2}+\epsilon_{i j}
$$

where $R_{i j}$ is the CIF rate (incremental factor) for the exporter $i$ and the importer $j$ in the period $t, D$ is the distance between the exporter country $i$ and exporter country $j$ and $\epsilon_{i j}$ is the error term. The resulting regression will provide the base for CIF values normalization into FOB. Then, the distance is the variable that explains the majority of the variance. Yet, this model may also generate some distorsions for unit values of minor importers, which is not a concern for this study since we will later discuss a methodology to filter those links of very low importance. Additionally, our dataset does not include any trade record of less than 1000 US dollars. For example, in figure 4.3, we show the results of the model when applied to the case of France, obtaining a result with the form $\ln R_{i j}=-0.178 \ln d i s t+0.014 \ln d i s t^{2}+\epsilon_{i j}$. Then we observe that the evidence supports values of incremental rates $R_{i j}$ that 
vary from $57 \%$ for short distances, for exports to Belgium for example, and values of up to about $70 \%$ for long distances, for exports to Australia. Finally, we find an issue related to natural geopolitical changes. The worldmap is not a fixed system. Even though it does not happen every day, new countries are created, consolidated or even removed from existence. As a consequence, the world trade records will have some variability in the country code lists. This is not a big concern for our study as long as the regime change does not create a duplication of the country's activity in the same year. In fact, despite the different code, if the activity is entirely reported under one unique code, we can handle to perform our research without a problem, since the country code will still became a node in the trade network. This only creates some issues when analyzing historical trade evolutions, which we resolve by adopting the latest country code.

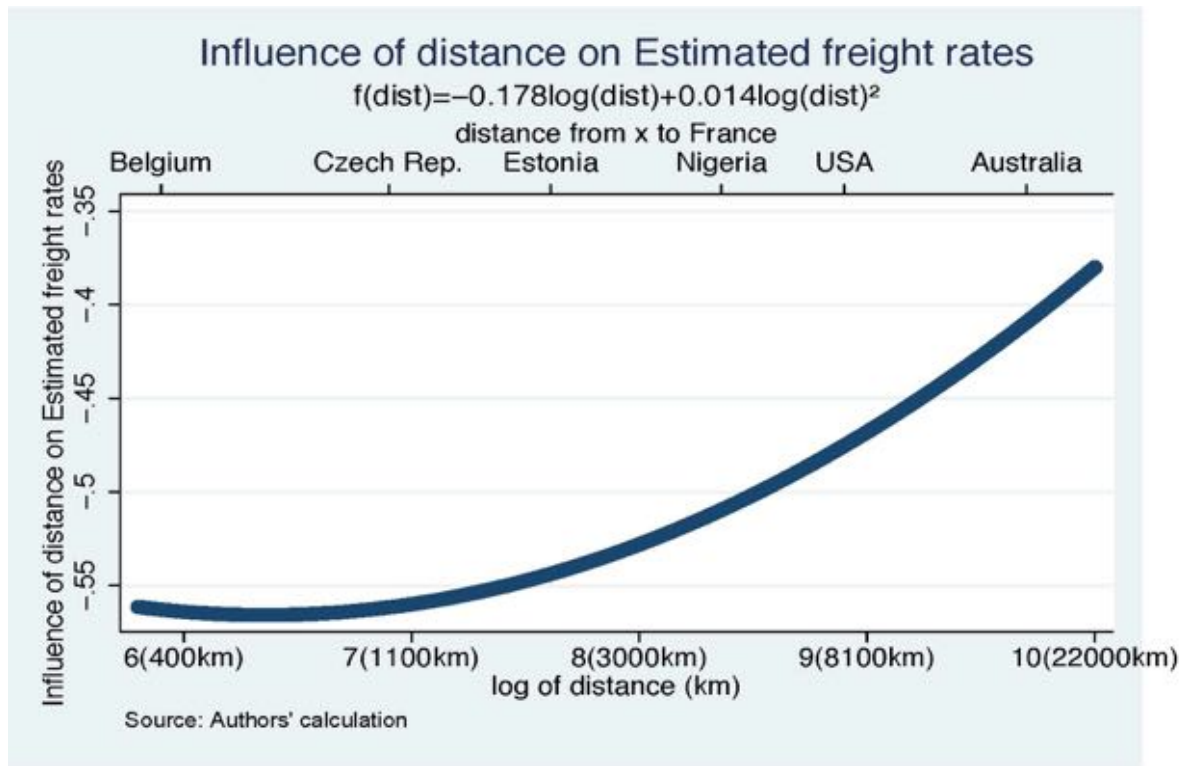

Fig. 4.3.: Example of the influence of distance on the estimated CIF rates. This figure shows results obtained after computing the model for the CIF rates for France. We show the logarithm of the distance on the $x$ axis and the value of the influence of distance on the $y$ axis, which is equal to the logarithm of $R_{i j}$. The evidence suggests that the incremental rates vary from 57 percent for short distances (for example, Belgium) up to about 70 percent for long distances (for example, Australia)

\subsection{Weighted Bipartite Trade Networks}

In the introduction, we have reviewed previous scientific work related to bilateral trade $[139,140,58,59,60,147,148,149,150]$ as well as other work of complex systems that may resemble international markets in their topological structure, the mutualistic ecosystems $[127,25,134]$. But our objective is to study international trade markets in a different way, hoping that we can obtain new insights on market 
topology and new explanations of how markets behave. For this reason, we use a weighted and bipartite approach, which is highly inspired by the mutualistic networks as a mean to unveil any potential hidden pattern within trade networks. We would like to present a solid argument to support such a decision, which is related to the role that both exporters and importers play in the system. It can be argued that the decisions of an exporter are different than those of the an importer since both parties will act so that their own benefits are maximized, which is a form of self-interest as defined by Adam Smith. Then, when an exporter makes a decision on where to sell its goods, it is highly likely that this decision will have little to do with that of an importer. In fact, when we consider a country as the sum of the economic activity of all enterprises that operate in it, we could also argue that, if a country play both the role of an exporter and an importer, it may not be the same companies that act as exporters and importers. This arguments, we think, can support a trade network definition where exporters and importers are represented by two disjoint subsets of nodes, that is, the bipartite approach. Moreover, the tradicional approaches to study networks have been successful in discovering many features of markets, yet we believe the power of bipartite matrices in combination with a weighted approach can provide further and deeper scientific findings. For this reason, a new definition of trade networks (country-country) that is both bipartite and weighted in nature is required and fundamental to this study (see figure 4.4).

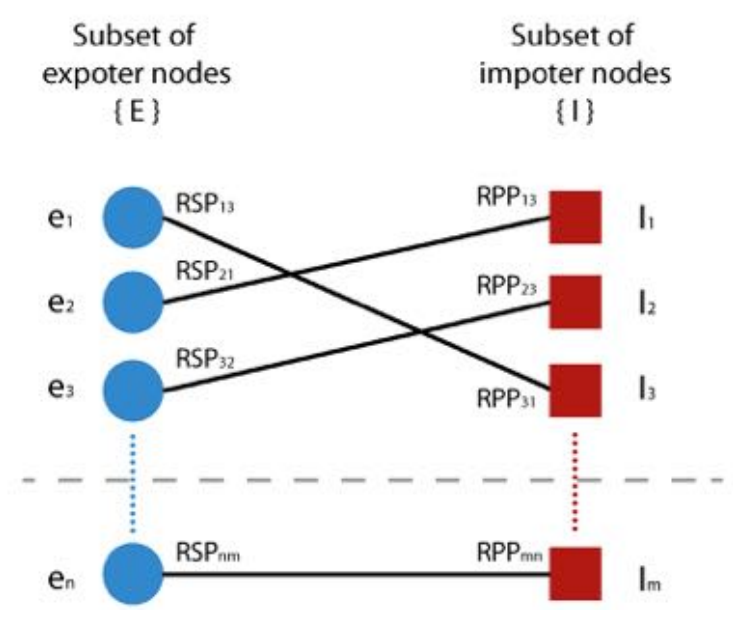

Fig. 4.4.: Definition of weighted bipartite trade networks. This figure shows the definition we adopt for this study. We incorporate the standard bipartite and weighted approach, but we also define additional quantitative parameters to depict relative importance of the trade between countries from both the exporter (RSP $=$ relative selling power) and the importer (RPP = relative purchase power) standpoints.

Therefore, we define a Weighted Bipartite Trade Network (WBTN) as a graph in which two disjoint subsets of exporters and importers will exist, as shown in figure 4.4. The nodes always represent a combination of countries and their roles in the trade activity. Thus, a country could be included in both subsets but, given the different roles that plays as exporter or importer, we consider it as a different node. 
For example, Argentina is both an importer and an exporter of fine polo horses. In this case, there will be a node in the exporters subset that represents the country in its exporter role and another node in the importer subset that follows the same logic. We adopt this specific approach based on two premises: a) the objective of our research is to study trade markets with a bipartite approach, and b) the roles played in the market by a country and the decision-making process are substancially different when acting as an exporter than it is when acting as an importer. Our bipartite approach requires that trade is performed between an expoter and an importer (and vice versa) but never between countries of the same subset. Also, we define the trade volume as the annual value in thousand US dollars of traded goods. Consequently, we define a WBNT link as a trade relationaship between an exporter $n$ and an importer $m$ that has a weight $w$,which is equal to the trade volume between countries in a year. In addition, we incorporate additional parameters to account for the relative importance of links. As we will discuss later on this chapter, the values of the links are quite diverse and heterogenous, going from a few thousand dollars up to several billion dollars. Also, the size of the economies of the exporter and the importer countries are quite heterogenous and hence, we need a way to determine how important a link is in its context, that is, how important it is in the market given the country size. For example, an exporter of a relatively small amount of a good may not have a large share of the global market, where large countries have the highest values of share, but this exporter may be highly important for its region, yet not relevant globally, or the exports of this particular good is highly important for the economy of this country. Another example of how parameters that indicate relative importance of links can be used is to separate the vital few from the trivial many, a concept that is inspired in the use of Revealed Comparative Advantage (RCA) within the Product Space $[14,82]$. Now, while the RCA is a node parameter, we could use a link parameter, for example, to filter the trade relationships of low relative importance, which would act as a topological filter of links. We will discuss more on this idea later on this chapter, but we now define this relative importance parameters formally, which is a new definition for link relative importance. The Relative Selling Power (RSP) is a link importance parameter from the exporter standpoint. It measures the relative share of the exportation from country $n$ to country $m$ in comparison to the average market share for the product, category or industry, respectively:

$$
R S P_{n m}^{i}=\frac{X(n, i)^{m} / \sum_{i} X(n, i)^{m}}{\sum_{n} X(n, i) / \sum_{n, i} X(n, i)}
$$

where $X(n, i)^{m}$ is the value of exports of product $i$ for exporter country $n$ towards importer country $m$. Similarly, we define the Relative Purchase Power (RPP) as the link importance parameter from the importers standpoint. It measures the relative 
share of the importations from country $n$ to country $m$ in comparison to the average market share for the product, category or industry, respectively:

$$
R P P_{m n}^{i}=\frac{Y(m, i)^{n} / \sum_{i} Y(m, i)^{n}}{\sum_{m} Y(m, i) / \sum_{m, i} Y(m, i)}
$$

where $Y(m, i)^{n}$ is the value of imports of product $i$ for importer country $m$ from exporter country $n$. It must also be noted that there is a close relationship between our RSP (Relative Selling Power) parameter and the RCA (Reveled Comparative Advantage) used in previous scientific work [82, 35, 61, 83]. Although the RCA is a node feature, which applies to the exports of a country, our RSP is a link level parameter that can be consolidated into the RCA. Therefore, the sum of the RSP values of all links of a given node will be equivalent to the RCA of the same node:

$$
R C A_{n}^{i}=\sum_{m} R S P_{n m}^{i}=\sum_{m}\left(\frac{Y(m, i)^{n} / \sum_{i} Y(m, i)^{n}}{\sum_{m} Y(m, i) / \sum_{m, i} Y(m, i)}\right)
$$

We have now defined our basic framework to tackle our trade networks, which include a large number of possible scenarios, a fact that may be an advantage and improvement over the previous scientific work, which usually was conducted for a unique dataset. Yet, we need to understand how the RSP and RPP behave and what purpose they may bring to this study. 


\subsubsection{A simple example of WBTN}

In order to depict the behavior of our network definition, we have created a simple market example for a given product with 4 exporters and 3 importers. We also set some random values to show the typical market behavior. Based on these values, we compute the parameters we proposed previously. The market configuration is as depicted in figure 4.5 .

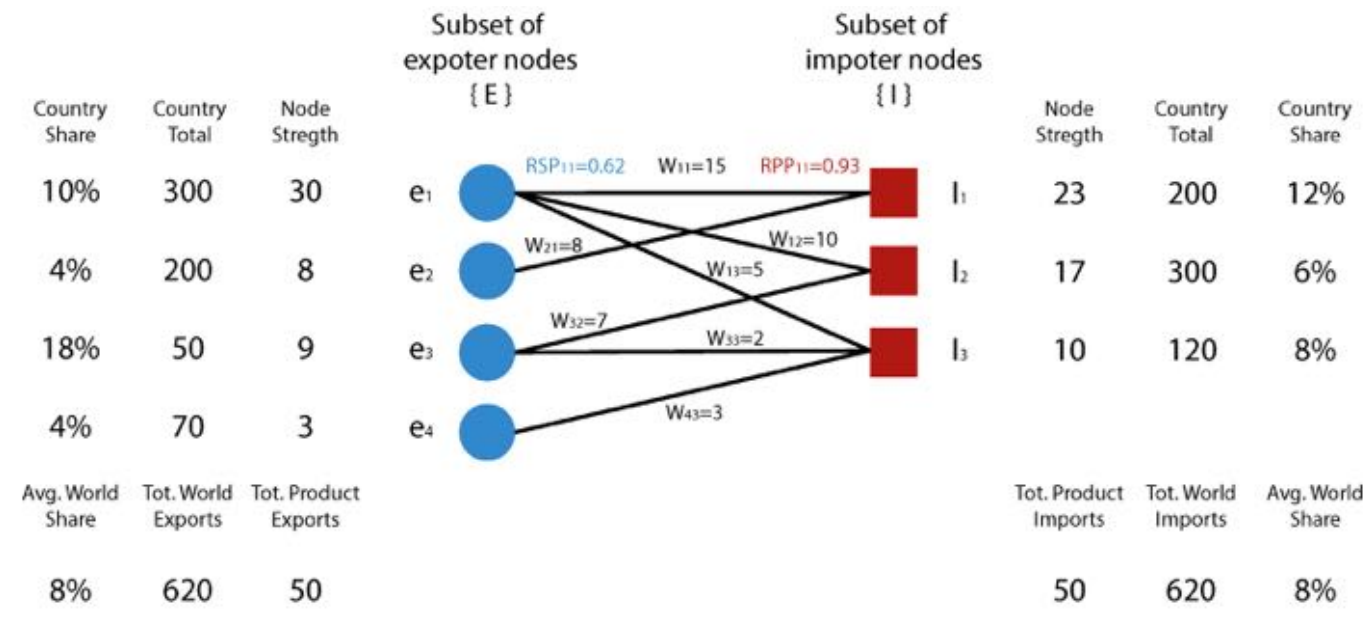

Fig. 4.5.: Example of a weighted bipartite trade network. This figure shows a simple example of a WBTN for a simple market with 4 exporters and 3 importers. The trade relationships and weights are represented in the center with weights $W_{i j}$. For each node, exporters and importers, we compute the total exports and importers, respectively, which are the node strengths. We also include additional values of the total country exporters and imports for all products. We compute the world totals as well, where the sum of the node strengths will be the total exports and imports of the product and the total world exports and imports for all products and all countries. With this, we finally compute the $R S P$ and $R P P$ values. For example, for the link between $e_{1}$ and $i_{1}$, we calculate the $R S P_{11}=\frac{15 / 300}{50 / 620}=0.62$ and $R P P_{11}=\frac{15 / 200}{50 / 620}=0.93$, where we observe that the same weight has a higher relative importance to the importer than it does to the exporter based on the fact that it represents a larger share of the total imports.

The strength has been added to each node, which shows the total exports or imports of the product for each country. The next column will show the total exports or import for all products and each country. Additionally, the share of product for each country is presented. Finally, the total product and world traded amounts are totalized, leading to the world's average share of product.

We build the bipartite matrix that is derived from the example market:

$$
B_{i j}=\left(\begin{array}{ccc}
15 & 10 & 5 \\
8 & 0 & 0 \\
0 & 7 & 2 \\
0 & 0 & 3
\end{array}\right)
$$


Now we compute the RSP for each link:

$$
R S P_{i j}=\left(\begin{array}{ccc}
0.62 & 0.41 & 0.21 \\
0.50 & 0.0 & 0.0 \\
0.0 & 1.74 & 0.50 \\
0.0 & 0.0 & 0.53
\end{array}\right)
$$

And the RPP for each link:

$$
R P P_{i j}=\left(\begin{array}{ccc}
0.93 & 0.41 & 0.52 \\
0.50 & 0.0 & 0.0 \\
0.0 & 0.29 & 0.21 \\
0.0 & 0.0 & 0.31
\end{array}\right)
$$

We observe that the trade volumes in the bipartite matrix can be better explained after computing the RSP and RPP parameters. Thus, for example, we show that trade volume between $E_{1}$ and $I_{1}$ is higher than that between $E_{3}$ and $I_{2}$. Nevertheless, when we compare the RSP values, we observe that opposite is true. This is due to the fact that RSP take in account the relative importance of the trade volume in the country and the proportion against the world average. In this way, the trade flow between $E_{3}$ and $I_{2}$ is quantitatively of more relative importance.

\subsubsection{Some analytics on RSP and RPP}

In this subsection, we perform an exploratory analysis of the parameters in our network definition by studying the distributions of such parameters. In addition, we also perform a correlation analysis among the 3 key variables (trade volume, RSP and RPP simultaneously). Finally, we show that both the RSP and the RPP can be utilized as an effective filter for the network topology, which is useful to separate the few vital from the trivial many, creating a base to analyze multiple scenarios of the WBTN.

We start with the higher aggregation level, the HS2 data. We compute the RSP and the RPP distributions by taking all the products, countries and links for a given year. We find that both the RSP and RPP have a similar distribution pattern. They are both log-normal distributions with similar mean and standard deviations. For the RSP and the year 2009, we compute the logarithm of RSP and RPP. In figure 4.6, we find a mean value of $\mu=-3.2$ and $\sigma=1.3$. For the RPP and the same year, we find a mean value of $\mu=-3.3$ and $\sigma=1.3$.

We only present the results for a single year, however, we also repeat the process for all years from 1995 to 2009. The results remain the same, which lead to the conclusion that the log-normal pattern does not change over time. Next, we also repeat the procedure for the HS4 aggregation level. In figure 4.7, we again find the 

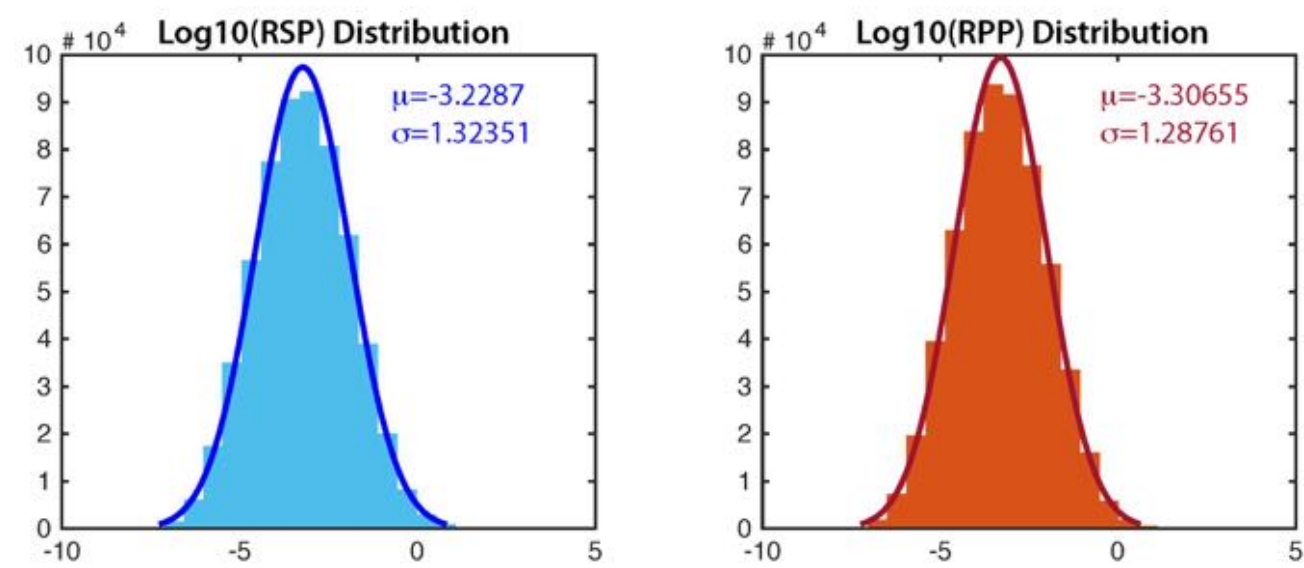

Fig. 4.6.: Probability distributions of the RSP and RPP (HS2 in 2009). This figure shows the probability distribution of the actual results of RSP (left) and RPP (right) with their respective best distribution fits. They are both log-normal distributions and their parameters are also shown. The data we use corresponds to all products at HS2 level in 2009. Similar behavior has been found from 1995 to 2009.

same pattern of log-normal distribution, but given the lower level of aggregation, we find different distribution parameters. For the RSP in 2009, we find a mean value of $\mu=-2.9$ and $\sigma=1.2$. For the RPP and the same year, we find a mean value of $\mu=-2.4$ and $\sigma=1.2$
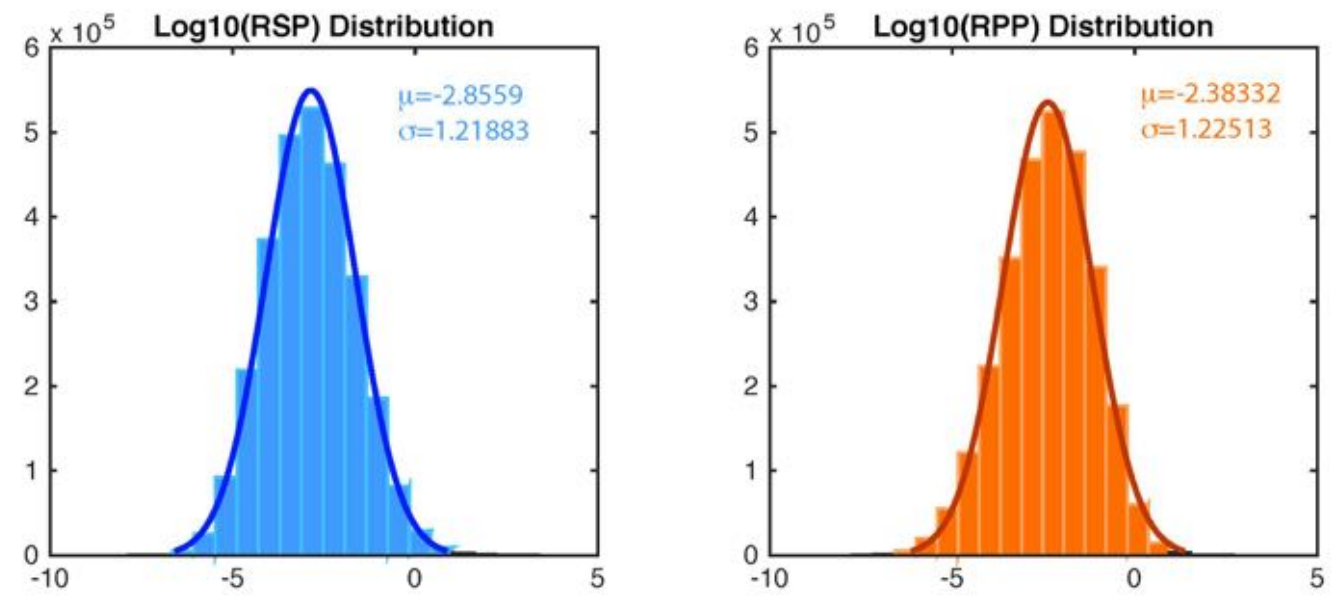

Fig. 4.7.: Probability distributions of the RSP and RPP (HS4 in 2009). This figure shows the probability distribution of the actual results of RSP (left) and RPP (right) with their respective best distribution fits. They are both log-normal distributions and their parameters are also shown. The data we use corresponds to all products at HS4 level in 2009. Similar behavior has been found from 1995 to 2009.

Regarding the HS6 level of aggregation, as expected, the same pattern appears as well. But since the log-normal pattern has been established, we now focus on the correlation between the RSP and RPP. We also include the trade volume as a third 
parameter for this part of the study. Starting with the HS2, we find that a strong correlation between RSP and RPP exists, as shown in figure 4.8.

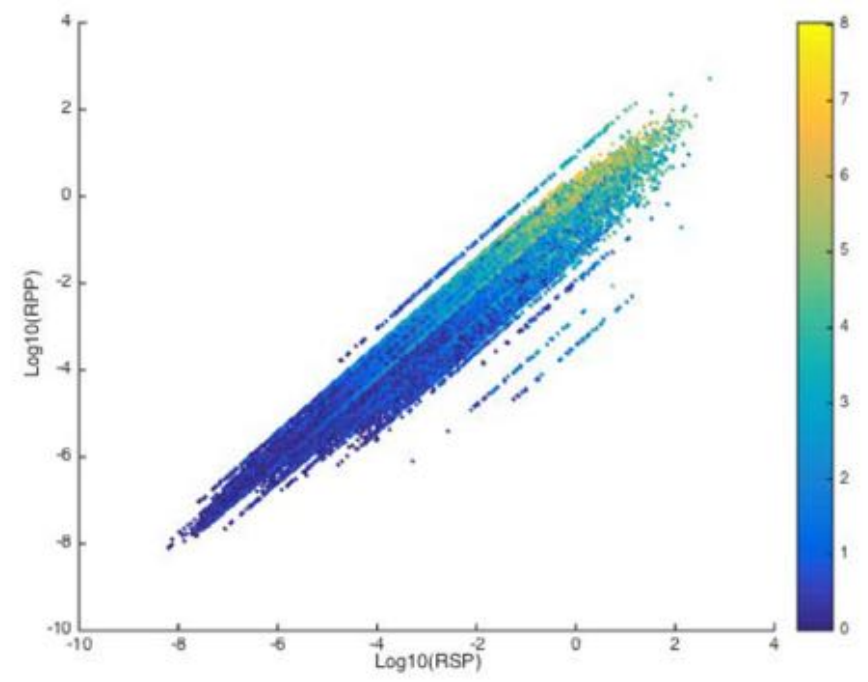

Fig. 4.8.: Correlation between RSP and RPP (HS2 in 2009).This figure shows the correlation between the RSP and the RPP. We observe a positive correlation among parameters including the trade volumes, which means that high relative importance and high volumes are also correlated. The colorbar depicts the logarithmic trade volume scale.

The figure (4.7) shows that a positive correlation between RSP and RPP can be found. This would show that links of high relative importance for the exporters are also of high importance to the importers. Additionally, we also observe a pattern for the high-volume transations, which mostly take place between countries with high relative importance simultaneously. So, the relevant question now is whether this pattern also stays the same over time, and for that we repeat the process again for all products at HS2 and for every year.

In figure 4.9, the evidence suggests that the pattern does not change over time, although some changes in the configuration of the system is observed. Despite the fact that the main pattern remains, the exact correlation still does change from one year to the next.

So let us perform an exercise to apply these RSP and RPP concepts to actual trade netwoks in order to visualize the effects of increasing the minimum RSP and RPP values of the links within a particular graph. We perform this by setting every link in two possible states, either active (not filtered) or inactive (filtered). Then, we perform a graph representation using the Gephi software and apply some usual visualization features: a) we set the node size proportional to the node degree, b) we apply a different color to highly interconnected subset of nodes, and c) a "ForceAtlas" layout is also applied so that it positions the high-degree nodes in the center while 

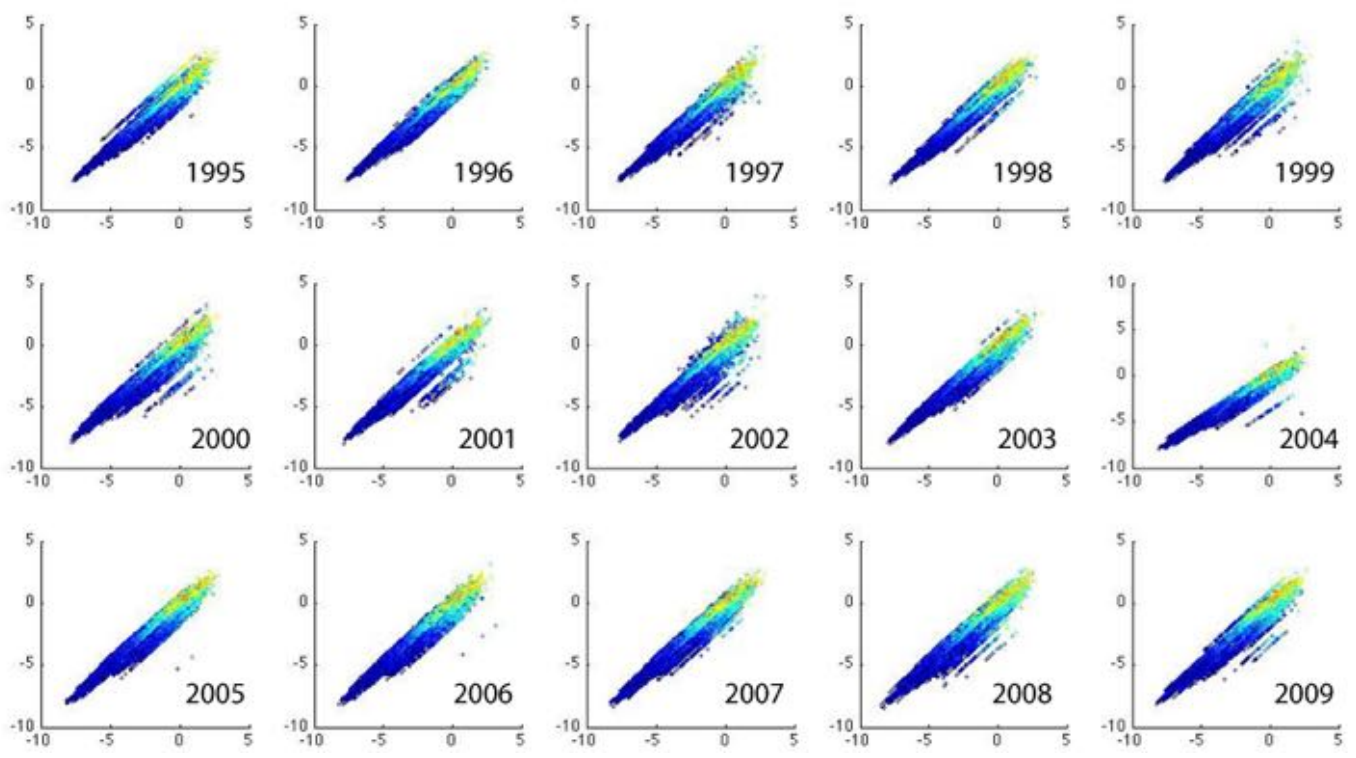

Fig. 4.9.: Correlation between RSP and RPP from 1995 to 2009. This figure shows the correlation of RSP and RPP for each year between 1995 and 2009. The y-axis shows the RPP values, whereas the $\mathrm{x}$-axis is for the RSP ones. The color of each point represent the trade volume in logarithmic scale. The aggregation level is HS2 in all cases.

pushing less connected nodes towars the periphery. We also run this procedure for one product at HS6 level of aggregation and repeat it for 7 cases: A) $R S P>0$ where all links are set to visible, B) $R S P>10^{-5}$, C) $R S P>10^{-4}$, D) $R S P>10^{-3}$, E) $R S P>10^{-2}$, F) $R S P>10^{-1}$, and G) $R S P>1$. The results can be visualized in figure 4.10.

Then, the evidence shows that a trade network can benefit from setting a RSP or a RPP threshold so that we can "filter" those nodes below this relative importance limit. We observe that the first network ("A" with $R S P>0$ ) is highly dense because all links (including those of very low relative importance) are visible at the same time, increasing the graph complexity in an unnecessary way and unabling us to extract conclusions by visualizing the graph. In contrast, the following graphs "B", "C", and " $\mathrm{D}$ " are more suitable to visualize the topology of the trade network. We are able to observe the structure of the network and the different clusters of interconnected nodes. However, in the case of "E" and "F", we realize that some important part of the topology is missing and that we might be losing important information about the markets. Finally, on the last graph "G", we no longer have network with the exception of a few nodes and virtually no interconnection between them.

So, in sum, the usage of a RSP and RPP parameters as a threshold can be useful to filter or eliminate the links that have a low relative importance in the market. Additionally, we could also argue that it may be benefitial to measure any topological 


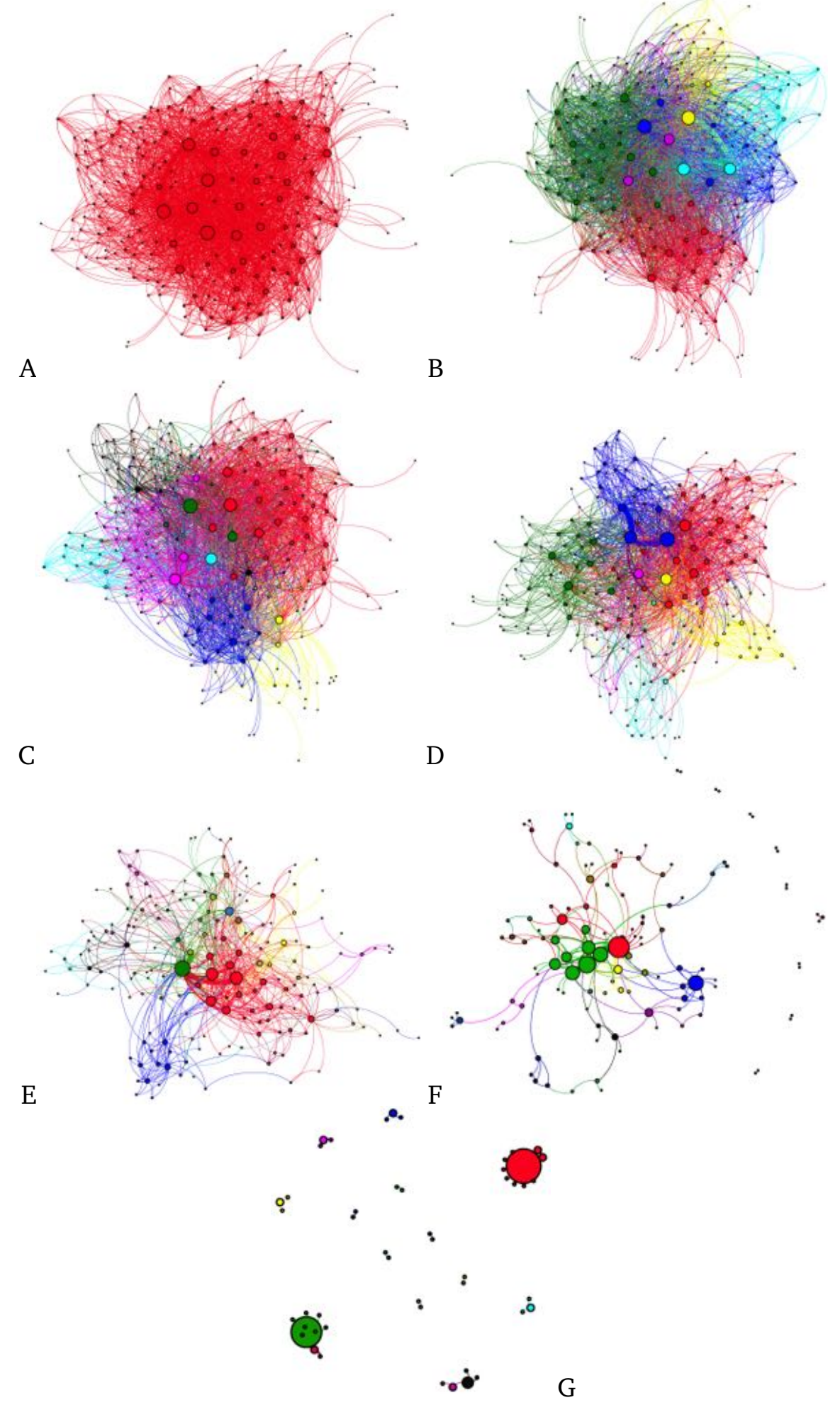

Fig. 4.10.: Effects of threshold values of RSP or RPP in our networks. This figure shows shows the graph representations for Beer products (HS6 $=220300$ ) in the year 2000. Thresholds for visualization are: A) $R S P>0$, B) $R S P>10^{-5}$, C) $R S P>10^{-4}$, D) $R S P>10^{-3}$, E) $R S P>10^{-2}$, F) $R S P>10^{-1}$, and G) $R S P>1$. 
property of a trade network in different scenarios, that is, with and without applying the RSP and RPP thresholds. Hence, we realize that the quantitative parameters that we have incorporated to our bipartite network definition can now be used as effective network or topological filters. Moreover, we can hypothesize that measuring the various network properties at different levels of filters may provide valuable evidence of how a trade network is fundamentally constituted. In practice, we only propose to use 2 scenarios: a) WITH RSP and RPP filters (normally $R S P>10^{-3}$ and $R P P>10^{-3}$ ), and $\mathrm{b}$ ) WITHOUT RSP and RPP filters.

Now we have to reflect about what the most convenient threshold value can be to set our filtered and unfiltered scenarios. The key to finding out this value might be linked to the probability distribution of RSP and RPP, but this is not a complete analysis. We actually need an additional variable that helps us set an objective methodology, and since we are dealing with weighted bipartite networks, we need to explore the role of the trade volume in this. For this reason, we compute the fraction of links and trade volume (links weights actually) that remain active when we increase the RSP and RPP thresholds. We also plot the results for several years (1995, 1999, 2004 and 2009) to test whether there is any changes of the patterns over time. We perform this analysis for all HS2 industry codes. The results are shown in figure 4.11 .

When analyzing the results we obtain, we find 3 important conclusions. First, the pattern formed (its probability distribution) by the links and the trade volume as functions of the RSP and RPP do not significantly change over time despite the numerous changes in the world trade year over year. Secondly, the links of highly relative importance in terms of RSP and RPP are also almost exclusively high trade volume links. And thirdly, the decay of active links take place much sooner than the decay of trade volume present in the network when we increase the RSP and RPP thresholds. One interesting effect takes place at about $R S P=10^{-3}$ and $R P P=10^{-3}$, which are coincidentally very close to the mean value of the probability distributions of each variable, where around only half of the links remain active, yet more than 99 percent of the trade volume is still active in turn. After this point, we observe that, if we continue to increase the RSP and RPP thresholds, the trade volume also start decreasing in a faster way. This is not only consistent with the visual conclusions from the analysis of figure 4.10, but also it can become our objective value of RSP and RPP threshold to finally set the filtered and unfiltered scenarios. 

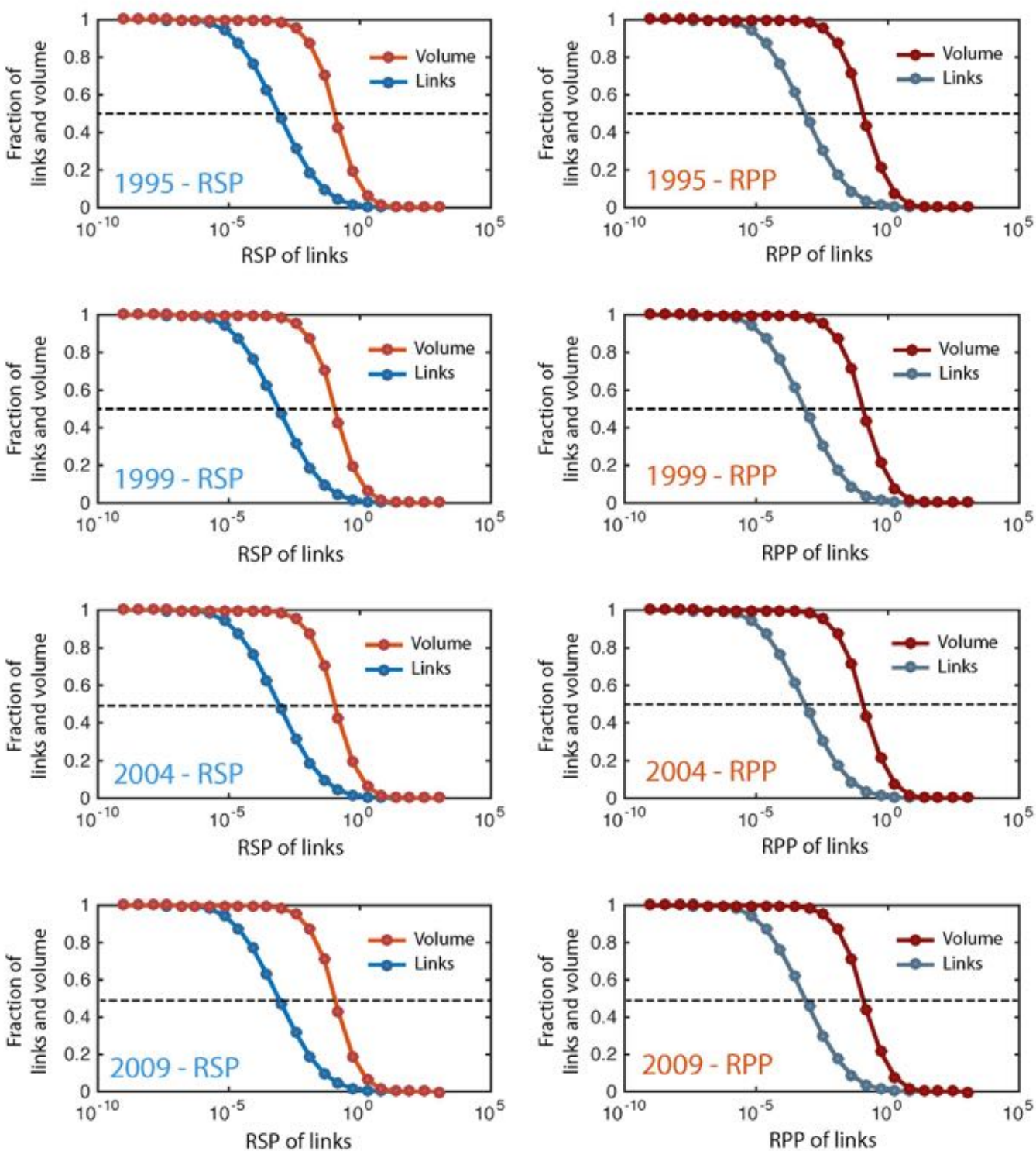

Fig. 4.11.: Using the RSP and RPP as efficient network filters. This figure shows the fraction of links and trade volume that remain active in the network as a funtion of the RSP and RPP respectively. We observe similar behavior across the periods at HS2 level for both RSP and RPP. While links rapidly become inactive when increasing the RSP and RPP (around $10^{-7}$ ), the trade volume does not decrease until much later (around $10^{-2}$ ). We also take the mean value of the distribution $\left(10^{-3}\right)$ and observe that, while virtually half the links have gone inactive, more than 99 percent of the trade volume is still active in the network, a clear sign of how to separate the trivial from the vital connections, which also remains valid regardless of the period.

\subsection{Big data process}

The major challenge to manipulate large datasets is related to the tool selection, since the variety of new technologies is quite broad and vast. The size of the datasets 
is considerably high, more than 10 gigabytes of text files, so its manupulation would be critical for the success of the study. Data processing of such amount of information requires a carefully planned procedure. The raw data, although having a consistent format and list of field, is not ready for direct use. Rather, some additional processing needs to be in place. For example, the consolidation of the several levels of aggregation needs to be programmed. Additionally, the calculation of the extended variables, such as the RSP and RPP, has to be included as well. In order to perform a pragmatic decision, we have selected one of the most popular open-source SQL server solutions, that is, MySQL server. This database engine is freely available in its basic version and works as a query builder and a data modeler so that we can easily consolidate and filter our data. It can also incorporate a direct link to most of the commonly used scientific programming languages. In addition, we can compute the required calculations for the extended variable by using relatively simple SQL commands. It is important to remember that the BACI process for data consistency is already applied to our raw data, ensuring that whatever input we upload to the MySQL server is absolutely ready for rigorous research. We also use Microsoft Excel from time to time to visualize some parts of the dataset. This preview actions are meant to verify that no changes in the data structure exists throughout the large dataset. The MySQL server setup process has been done as follows:

1. Raw data upload. The text files for each year, containing all international trade activity for all HS6 products, is uploaded to a temporary table.

2. Extended variables computation. The RSP and RPP is computed for the HS6 level of aggregation. The values are inserted in the corresponding fields.

3. HS6 activity table creation. The records from the temporary table are exported to the HS6 Activity table.

4. HS4 data consolidation. Based on the temporary table with HS6 data, we create a secondary table for HS4 raw data by using a SQL command.

5. HS4 activity table creation. Steps 2 and 3 are repeated and computed for the HS4 raw data from Step 4.

6. HS2 data consolidation. Based on the temporary table with HS4 data (Step 4), we create a another secondary table for HS2 raw data by using a SQL command.

7. HS2 activity table creation. Steps 2 and 3 are repeated and computed for the HS2 raw data from Step 6. 
These actions set our MySQL server up, which is at this point ready for query running. The technical details within the server functional setup will not be discussed in this study in order to retain the focus upon the scientific work, but extensive time had to be invested in these activities as well, especially those actions to determine the optimal database structure so that the total data size and the processing times are minimized. Up to this point, we have discussed everything that takes place before the database is fully finctional. In figure 4.12, we show the schematic data process, which is consistent with a data warehousing method, as commonly referred to by the industry. The flow of information is observed from the raw data requirements, accounting for the multiple inputs, to the various outcomes necessary for this study, where multiple tools have been used.

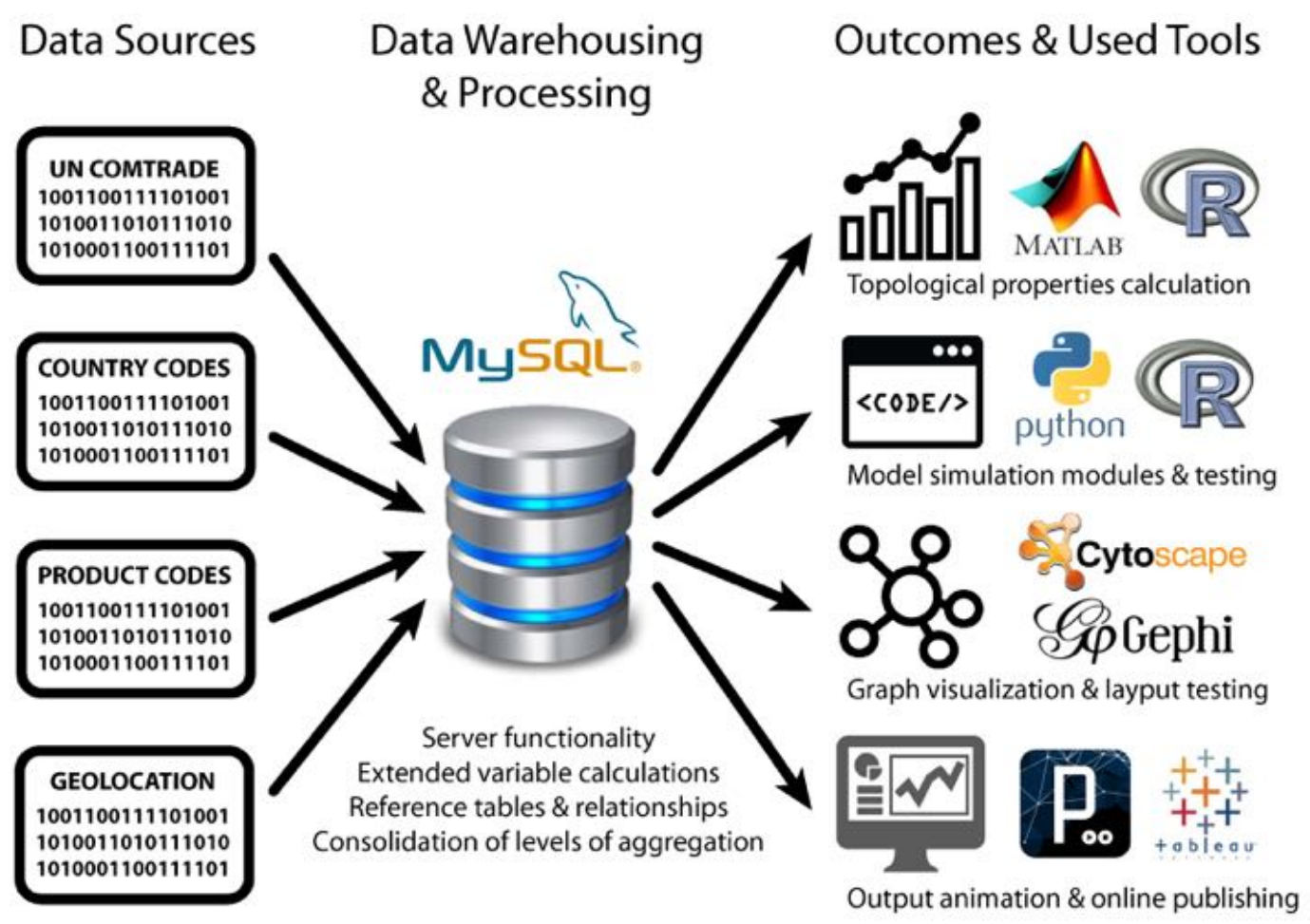

Fig. 4.12.: Big data process for this study. This figure shows technological architecture of our database and subsequent processing techniques.

Our data is multidimensional in nature. This means that every single piece of information is observed over and depends on three or more dimensions. Each dimension is, in turn, a part of the dataset that categorizes every fact or measure. Thus, our data dimension will include, for instance, time, products, importers, exporters and the RSP and RPP filters. In order to cleary define the different data units to which any computation will be applied, we will use the concept of scenarios. Any given scenario will be a combination of the several dimensions of our data. Let us review these dimension first: 
1. Time. This is direct reflection of the "Period" data field. Here we use this dimension to filter the year of activity or perform a time evolution of the indicators. An additional period will be added in some cases, that is, the cummulative "Total" for all periods.

2. Product. This is a dimension to filter the different data for each product, but it is extended across the 3 levels of aggregation: HS2, HS4 and HS6 (each level implemented in a different database table in our case). In contrast to the "Time" dimension, this one is hierarchically organized, where several HS6 products will consolidate at a HS4 category. The same is true for HS4 categories and a HS2 industry.

3. Filter Status. As discussed in the previous section, we adopt the $R S P \geq 10^{-3}$ and $R P P \geq 10^{-3}$ in order to separate the core of the network (filtered) and from the total network (unfiltered). Alternatively, a specific RSP or RPP value could also be used ocassionally. For example, if we refer to an "R5" filter, our nomenclature works so that the last digit represents the negative exponent in the expression $R S P \geq 10^{-5}$.

Therefore, as previusly defined, our scenario concept is a combination these dimensions, as shown in the following schematic:

$$
\text { Scenario = "Time" + "Product" + "Filter" }
$$

For example, a scenario string denominated as "1995+847120+Unfiltered" would refer to the data of HS6 product 847120 (Digital Automatic Data Processing Machines) in the year 1995 and all the network. Another example could be "2007+20+R2", which refers to the data of HS2 industry 20 (Preparations of vegetables, fruit, nuts or other parts of plants) in the year 2007, but only those records with $R S P \geq 10^{-2}$ and $R P P \geq 10^{-2}$. With this scenario structure, we can clearly appreciate the massive and exponential tasks to be performed. Considering the number of dimensions and the individual possibilities within each dimension, we find at least 195200 different scenarios (16 periods, 6100 products and at least 2 filter statuses).

Let us consider and discuss these various outcomes now. The first output from our big data process is the topological properties computation. Because of the nature of these properties, in addition to previously existing code, we implement these indicators, in its vast majority, in Matlab, a user-friendly software that provides a framework for relatively complex tasks. Our Matlab code is organized in different modules, which are linked to the data from our MySQL server. By running each module, we apply a standard set of topological indicators to each scenario as previously described 
(further discussion and details will be presented in Chapter 5). All vectors containing the results, exponents, distributions are saved as Matlab objects. We also perform basic visualization of the results with this software. There is one important caveat with Matlab, which is related to the memory resources that requires. From this perspective, Matlab present some problems when dealing with a large number of realizations. The "R" statistical software is an open-source tool with an extensive library of multidimensional data visualization. Ocassionally, we also perform some tasks exporting the data contained in vectors to $\mathrm{R}$, especially for histograms and probability distributions.

The second output is related to the implementation of model simulations and the proper testing of the results. For this purpose, we rely on the Python programming language, another open-source tool with an extensive adoption and usage from the scientific community. As we will discuss later in this work, we might require to run complex simulations of several models that we propose in order to replicate the behavior of empirical markets. The computation time required to run the multiple realizations of the model is quite high. Still, we need to carry on with such a large number of iterations so that we can provide quiality results that are highly rigorous as well as statistically significant. The way in which Python manages the memory and the fact that it does not require large computing resources from the processor makes this programming language an excellent alternative to other tools.

The third output from our big data process is the Graph visualization and layout testing. Given the nature of this work, the visualization of the highly complex relationships within a trade network is critical to conduct the study. Because of this, we use 2 tools to acomplish such a purpose, Cytoscape and Gephi. The first one is a more biology-oriented tool, but it has several layout options built upon that are highly suitable for our study. For example, the bipartite layout perfectly suits our kind of networks. Another examples are the various circular layouts, which can be ordered and drawn based on multiple network parameters. This is especially relevant to understand the roles and importance of each node of a trade networks. Gephi, on the other hand, is more generic type of tool. it does not allow a bipartite layout directly, but it can deal with, to some extent, the same type of adjacency matrix throught the directed layout. In this way, several algorithms can be specified to arrange the different layouts. These tools are functionaly used in 2 ways across this study: a) as an exploratory tool to visualize the overall structure of trade networks and, b) as a presentation tool to support the results we find.

Finally, the fourth output is related to animation and online publishing of the results. Visualization of multidimensional data is probably an academic topic by itself. But the benefits of experiencing a "visual storytelling" by means of animated visualizations are both tangible and desired for multidimensional datasets such as 
ours. For this reason, we have incorporated Processing as a fundamental tool. This tool can also help us create interesting applications to visualize and experience our data. Processing was developed by a community of computer scientists, artists and interaction designers, delivering a solution for a wide range of purposes. For online publishing, we use the Tableau software, a widely adopted tool both in science and in business. It is a very user-friendly tool that can automatically recognize the nature of the data, improving the development time. Our codes, visual outcomes and online material will not be of relevance for this study in particular, but it has been used during the development of this doctoral thesis in several scientific congresses and forums, providing an engaging way of communicating our complex phenomena. 



\section{Topological indicators for WBTN}

Physics is really nothing more than a search for ultimate simplicity, but so far all we have is a kind of elegant messiness.

— William McGuire Bryson

(Writter and author of "A Short History of

Nearly Everything")

The implementation of a system to measure the key bipartite topological properties is an imperiative at this point. Given the analogies between mutualistic ecosystems and trade markets described in the introduction, we use a set of indicator that is consistent with those used for mutualistic network analysis. Consequently, these indicators will fully described in the following sections. We also include some important comments of the challenges presented by our data in terms of finite size and other implications from the RSP and RPP filtered and unfiltered scenarios.

\subsection{Degree distribution $\mathrm{P}(\mathrm{K})$}

Having defined the concept of degree (chapter 3) as a property of nodes, the degree distribution, $P(k)$, is an indicator of the connectivity topological features of a complex network. It basically provides a measure of the probability that a randomly selected node has exactly $k$ links connected to it. In addition, the form or pattern displayed by the specific distribution will be of vital importance. For example, as shown in figure 5.1, we know that random networks will display a normal distribution pattern, whereas scale-free networks will display a power-law distribution pattern $[114,16]$.

In our WBTN, we could actually expect degree distributions that are consistent with the second case (see figure 5.1, subfigure on the right) of a power-law pattern of the form:

$$
P(k) \sim k^{-\gamma}
$$




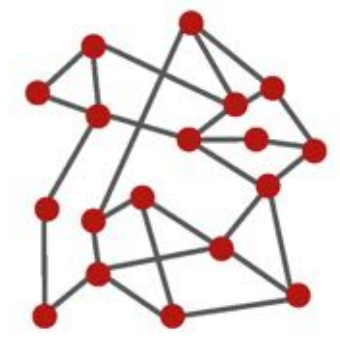

Random Network

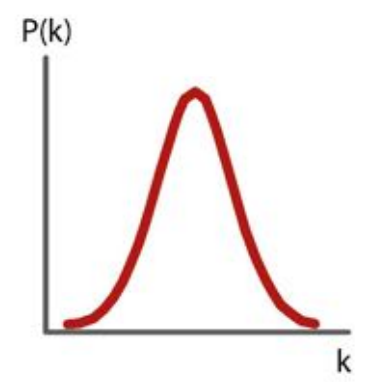

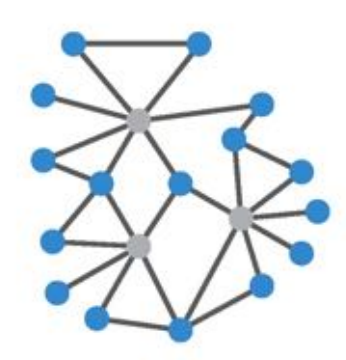

Scale-freeNetwork

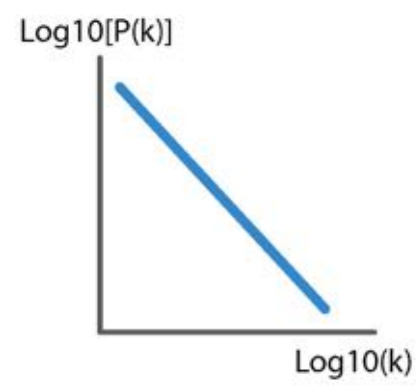

Fig. 5.1.: Degree distributions of random and scale-free networks. This figure shows the degree distributions of a random (left) and a scale-free (right) network. The first one follows a binomial distribution pattern on networks with small number of nodes and a Poison distibution otherwise, whereas the second one follows a power-law.

Yet, for our data and the resulting networks, there are a few aspects that we need to clarify. First, the degree distribution for bipartite networks needs to be managed in a different way than that of unipartitite ones. In fact, there will be two degree distributions, which apply to each subsets of exporters and importers. These distributions will share the same number of total connections or links, but the pattern of them will not necessarily be the same. This is mainly because of, for example, a different number of importers and exporters, which leads to an assymetric bipartite matrix. Another cause for such a difference may be related to the assymetric behavior of importers in comparison to exporters, where highly connected nodes may concentrate a higher number of links.

Secondly, and since we deal with finite size data, the tail of the distribution will not be without some variability, causing an apparent cut-off of the distribution or an apparent truncated power-law pattern. This discussion will take place later on the results chapters, but we hereby acknowledge the possibility of such an occurence. In this case, the pattern would be:

$$
P(k) \sim k^{-\gamma} e^{-k / k_{c}}
$$


Thirdly, and given the fact that some of the product level (Hs6) networks may be relatively small in size when we apply RSP and RPP filters, we will always use the cummulative degree distribution (normalized). In this way, we could observe the real pattern of the distributions and compute the $\gamma$ exponents in a more precise way [114]. Therefore, we have:

$$
P(K \geq k) \sim k^{-\gamma+1}
$$

Finally, we implement an iterative computation process to identify the valid range of $k$ where the $\gamma$ exponent regression provides the most accurate result.

\subsection{Strength - degree correlation S(K)}

In order to provide more information about the dependence of the strength $s_{i}$, previously defined in chapter 3 , upon the degree $k_{i}$, we propose to perform the strength-degree correlation as an standard measure of how weights are distributed in WBTNs $[74,20]$. This is important for our study since we are studying weighted bipartite networks, where the weighted portion needs to be accounted for because it is part of the objectives we have set as premises. Since we deal with economic problems, the principle of profit maximization is always present in the decisionmaking process of each country, either within the exporters or within the importers. This fact may lead to a concentration of power over the market, where countries with high trade volumes may not only be highly connected, but also be the ones with higher average weights per connection or per link than those of other countries. This phenomenon has been well known and defined for the number of links as "The rich get richer", where nodes with higher degree tend to attract more links as a consequence. Yet, this has not been defined within weighted networks, a situation that may be an opportunity to define a weighted equivalence of the concept. The expectation in a WBTN is that the pattern to be found in $S(k)$ is of the form:

$$
S(k) \sim k^{\beta}
$$

where the exponent $\beta$ will be equal to 1 if the node weights are randomly distributed across the network and higher than 1 if highly connected nodes have a higher average weight than that of the rest. This can be conceptualized as shown in figure 5.2 . 

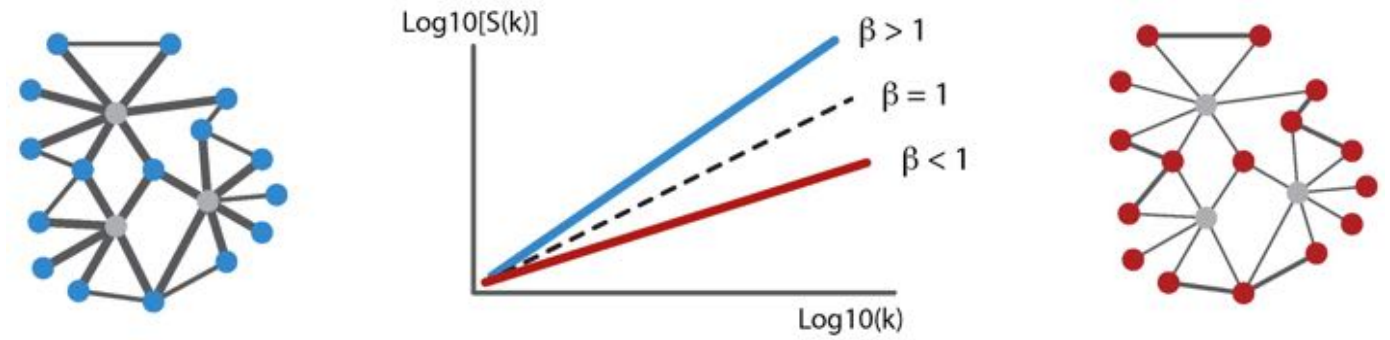

Fig. 5.2.: Strength-degree correlation and the $\beta$ exponents. This figure shows the strengthdegree correlation for weighted networks with the possible $\beta$ exponents. We show a network case (left graph) where highly connected nodes have a higher average weight, and consequently, the $\beta$ exponent is higher than 1 . The other network (right graph) is the opposite case, where highly connected nodes have a lower average weight, hence the $\beta$ exponent is lower than 1 .

In our study, we also plan to understand whether any dependency between exponents from the degree distribution and the strength-degree correlation exists within WBTN.

\subsection{End-point degree correlation}

The strength-degree correlation is vital to the understanding of how weights are distributed across the network. Yet, in order to provide additional information about this, we propose to use the average weight as a function of the end-point degree, which is defined as the product of degrees of end-point nodes, $k_{i}$ and $k_{j}$. This has a high importance, because it can provide further information about how the weights are allocated in the network. For example, a positive correlation of this topological indicator would imply that high weights are more likely to be allocated between nodes of high degree, respectively, and hence, with a high end-point degree. This would complement the information provided by $S(k)$, because it would suggest that larger weights are not only more likely to be found within high degree nodes, but also more likely to be found between a pair of nodes that have high degree individually and, by extension, high end-point degree collectively.

After computing this indicator, we expect to have a distribution with a power law pattern of the form:

$$
\left\langle w_{i j}\right\rangle \sim\left(k_{i} k_{j}\right)^{\theta}
$$

Based on the preliminary testing of this indicator, we forecast a significant sensitivity of this measure towards the RSP and RPP filters. This is mainly because the variance 

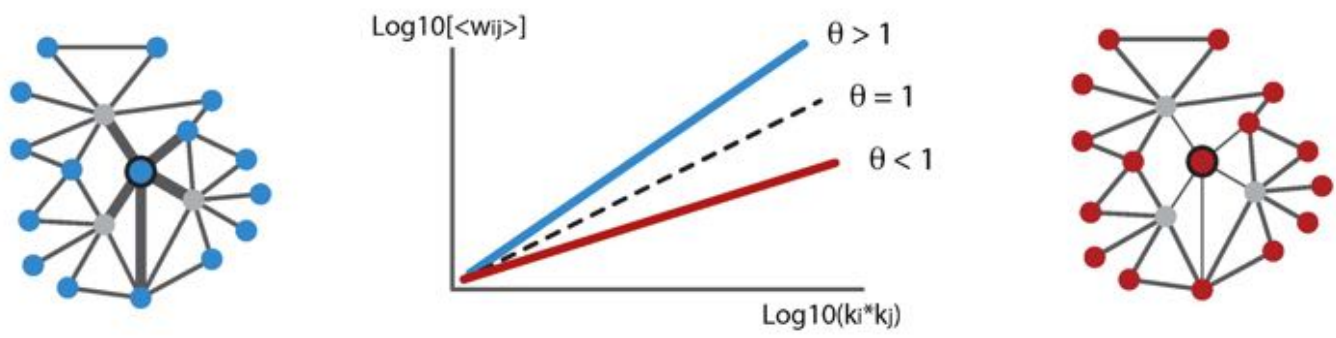

Fig. 5.3.: End-point correlation and the $\theta$ exponents. This figure shows the end-point degree correlation for weighted networks with the possible $\theta$ exponents. We show a network case (left graph) where highly connected nodes have a higher average weight when they are connected to other high degree nodes, and consequently, the $\theta$ exponent is higher than 1 . The other network (right graph) is the opposite case, hence the $\theta$ exponent is lower than 1 .

of low weight and low relative importance links will play an important role in the final correlation by adding noise within the low-end values of the end-point degree.

\subsection{Bipartite clustering C4(K)}

Previous indicators mainly provide information about how a network is connected and how the weights are distributed across the topology. But in order to understand the structural organization of our networks, we now need to study other aspects such as the hierarchy, the redundance or the cohesiveness within WBTNs. To measure this, we incorporate the clustering coefficient, which is defined for any node $i$ as the fraction of connected neighbors of $i$. In this way, we define $C_{i}^{w}$ as expressed in equation 5.6.

$$
C_{i}^{w}=\frac{1}{s_{i}\left(k_{i}-1\right)} \sum_{j, h} \frac{\left(w_{i j}+w_{i h}\right)}{2} a_{i j} a_{i h} a_{j h}
$$

where $s_{i}$ is the strength of the node, $k_{i}$ is the node degree, $w_{i j}$ is the weights matrix and $A_{i j}$ is the adjacency matrix. Now, this is the traditional definition of clustering coefficient [20], which is useful for weighted unipartite networks and measures the global density of interconnected node triplets in the network topology. Yet, for weighted bipartite networks, these triplets simply do not exist based on the fact that there are two disjoint subsets of nodes and these nodes are never connected to other nodes within the same subset, leading to a clustering coefficient $C_{i}=0$. To overcome this problem, there are two alternatives. The first one is to perform the projection of the bipartite network, where the fundamental topology is lost and the meaning of 


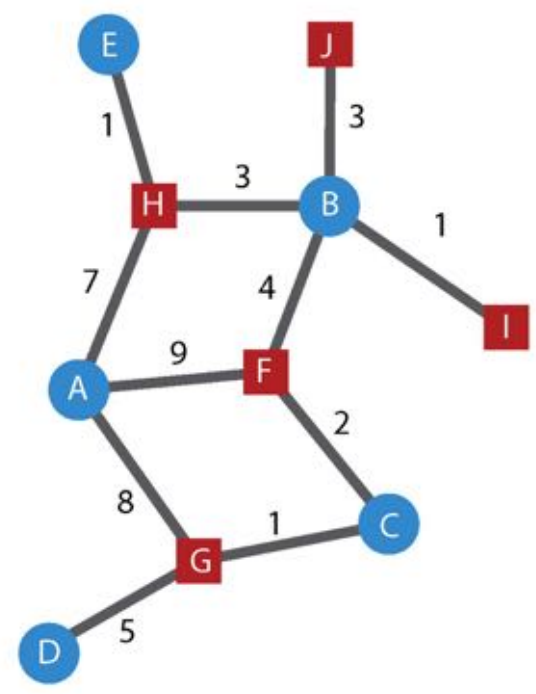

Fig. 5.4.: Example of bipartite network for clustering analysis. This figure shows an example of a bipartite network. We observe that no triplet can be found since nodes of the same subset do not connect between them. Hence, the tradicional clustering coefficient will always be zero $\left(C_{i}=0\right)$. But alternatively, we can find quadrilateral cycles like those formed by nodes A-H-B-F or A-F-C-G. Consequently, these quadrilaterals become the base concept for the bipartite $C 4_{i}$.

links will be different. The second alternative is to change to a bipartite clustering definition as proposed by Lind et al.[95]. In this way, the clustering coefficient is computed by searching for square cycles instead of triplets, avoiding the issues from the first alternative. We use the second option and the new bipartite unweighted clustering can be defined as shown in equation 5.7.

$$
C 4_{b}(i)=\frac{q_{i}}{Q_{i}}=\frac{q_{i}}{k_{i}^{n n} k_{i}\left(k_{i}-1\right) / 2}
$$

where $q_{i}$ is the number of observed quadrilaterals around node $i$ and $Q_{i}$ is the total number of possible quadrilaerals around the same node, which is equal to number of second-nearest neighbors $k_{i}^{n n}$ multiplied by the total number of pairs of neighbors $k_{i}\left(k_{i}-1\right) / 2$.

We also use the weighted bipartite clustering $C 4_{w} i$ [74] in order to account for the fact that quadrilaterals formed by strong interactions will play a larger role in the topology than those formed by weak interactions. Then, the weighted bipartite clustering will be as shown in equation 5.8 . 


$$
C 4_{w}(i)=\frac{\sum_{m, n} q_{i m n} \frac{\left(\tilde{w}_{i m}+\tilde{w}_{i m}\right)}{2}}{k_{i}^{n n} k_{i}\left(k_{i}-1\right) / 2}
$$

where $q_{i m n}$ is the number of quadrilaterals formed with nearest neighbors $m$ and $n$, and $\tilde{w}_{i m}$ and $\tilde{w}_{i m}$ are the normalized weights of the links between $i$ and $m$ and between $i$ and $n$, respectively; with $\tilde{w}_{i m}$ as described in equation 5.9.

$$
\tilde{w}_{i m}=\frac{w_{i m}}{\bar{w}_{i}}=\frac{w_{i m}}{\frac{s_{i}}{k_{i}}}
$$

With these clustering coefficients, we cover this aspect of the network structural organization and topology. Yet, it must be noted that the application of our RSP and RPP filters will have a highly significant effect upon the C4 performance, since removing non essential, low importance links helps us focus on the non trivial connections of the trade networks, but, at the same time, it limits the number of quadrilaterals by destructing the original topology that is relevant to the network cohesiveness. We deal with this issue by still measuring both filtered and unfiltered scenarios and later comparing both results while paying special attention to the unfiltered one as more representative reality scenario. Based on these concepts, we expect to find distributions of the unweighted and weighted clustering coefficients of the form shown in equations 5.10 and 5.11.

$$
\begin{gathered}
C 4_{b}(k) \sim k^{\sigma} \\
C 4_{w}(k) \sim k^{\nu}
\end{gathered}
$$

where $\sigma$ is the power law exponent of $C 4_{b}(k)$ and $\nu$ is the power law exponent of $C 4_{w}(k)$.

\subsection{Nearest Neighbor Degree distribution Knn(K)}

The last of the topological indicators that we adopt is the nearest neighbor degree distribution, which is a way of understanding the assortative features within WBTNs, a concept that can be found in complex networks and depicts a preference or bias for 

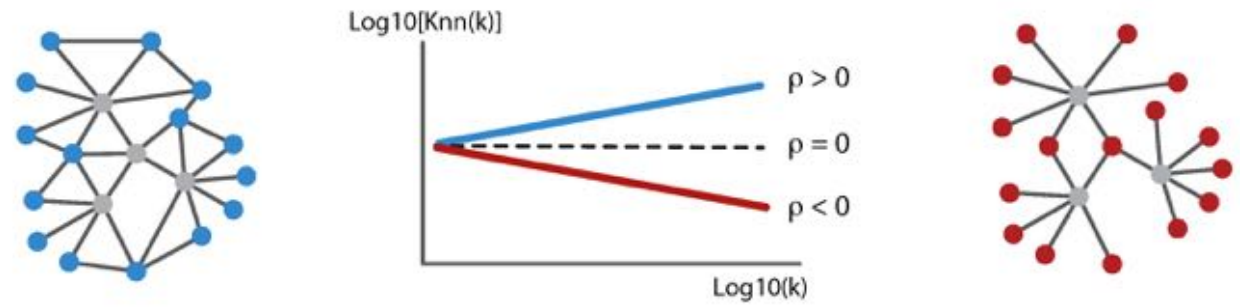

Fig. 5.5.: Nearest Neighbor Degree Distribution Knn(k). This figure shows an example of an assortative network on the left side (blue), where nodes tend to connect with other of similar degree. On the right side, we show a second example of a dissortative network, where nodes tend to connect with others of different degree. In the center, we observe the expected performance of both networks, where an assortative bahavior is depicted by an exponent $\rho>0$. The opposite is true for the dissortative case $(\rho<0)$.

nodes to connect to other of similar characteristics. For example, if highly connected nodes in a network tend to be linked to other nodes with high degree, this network is said to be assortatively mixed. Hence, $K n n(k)$ will be basically a measure of whether nodes are likely to interact with other nodes of similar degree. Thus, we can define this as shown in equation 5.12 .

$$
k_{n n}^{w}(i)=\frac{1}{s_{i}} \sum_{j} a_{i j} w_{i j} k_{j}
$$

Based on previous work and the typical behavior of complex networks $[20,139,140$, $58,60,147,148]$, we expect to find a power law pattern with an exponent $\rho$ that will provide evidence on whether a trade network displays assortative, where nodes tend to connect with other of similar degree, or dissortative properties, where the opposite is true. Based on this concept, we define this in equation 5.13.

$$
\left\langle k_{n n}^{w}(k)\right\rangle \sim k^{\rho}
$$

Moreover, in figure 5.5, we present the concept of assortativity by means of the $K n n(k)$ distribution, where the blue network (on the left side) depicts an assortative network in which nodes of higher degree tend to connect to other nodes with similar degree. In this case, a condition of $\rho>0$ would be expected. In contrast, the red network (on the right side) is a rather dissortative one, where higher degree nodes tend to connect to low-degree ones and therefore, a $\rho<0$ exponent would be expected. 
The nearest neighbor degree distribution will help us, in combination with the bipartite clustering, understand the connectivity features of trade networks and shed some light upon the preferences of countries to trade among either a limited or a broader set of countries depending on their position in the importance ranking, that is, based on trade volume, degree, strength, or a combination of these factors.

\subsection{Summary of Bipartite Topological Indicators}

After defining each of the bipartite topological indicators, we provide a consolidated summary of them in table 5.1.

\begin{tabular}{|c|c|c|c|}
\hline $\begin{array}{l}\text { Bipartite } \\
\text { Indicator }\end{array}$ & $\begin{array}{l}\text { Definition } \\
\text { (formula) }\end{array}$ & $\begin{array}{c}\text { Pattern } \\
\text { (hypothesis) }\end{array}$ & $\begin{array}{c}\text { Exponent } \\
\text { (hypothesis) }\end{array}$ \\
\hline Degree distribution & $k_{i}=\sum_{j} a_{i j}$ & $P(k) \sim k^{-\gamma}$ & $\gamma$ \\
\hline Strength-degree correlation & $s_{i}=\sum_{j} a_{i j} w_{i j}$ & $P(k) \sim k^{\beta}$ & $\beta$ \\
\hline Nearest neighbor degree & $k_{n n}^{w}(i)=\frac{1}{s_{i}} \sum_{j} a_{i j} w_{i j} k_{j}$ & $\left\langle k_{n n}^{w}(k)\right\rangle \sim k^{\rho}$ & $\rho$ \\
\hline Bipartite clustering & $C 4_{b}(i)=\frac{q_{i}}{Q_{i}}=\frac{q_{i}}{k_{i}^{n n} k_{i}\left(k_{i}-1\right) / 2}$ & $C 4_{b}(k) \sim k^{\sigma}$ & $\sigma$ \\
\hline Weighted clustering & $C 4_{w}(i)=\frac{\sum_{m, n} q_{i m n} \frac{\left(\tilde{w}_{i m}+\tilde{w}_{i m}\right)}{2}}{k_{i}^{n n} k_{i}\left(k_{i}-1\right) / 2}$ & $C 4_{w}(k) \sim k^{\nu}$ & $\nu$ \\
\hline $\begin{array}{l}\text { Average weight as a func- } \\
\text { tion of the end-point degree }\end{array}$ & $\left\langle w_{i j}\right\rangle\left(k_{i} k_{j}\right)$ & $\left\langle w_{i j}\right\rangle \sim\left(k_{i} k_{j}\right)^{\theta}$ & $\theta$ \\
\hline
\end{tabular}

Tab. 5.1.: Summary of bipartite topological indicators. This table shows the definition and power law exponents of each bipartite topological indicator as defined in the current chapter. 



\section{Nestedness}

Chaos is merely order waiting to be deciphered.

- Jose de Sousa Saramago

(1998 Nobel Prize in Literature)

For quite some time, ecologists have been trying to successfully measure the amount order or disorder that can be found in an ecosystem. In particular, Atmar and Patterson (1993) [13] applied such a concept to the distribution of species in fragmented habitat. Yet, several methodologies for measuring nestedness have been proposed over the years, and this continues to be an area for debate and improvement. Furthermore, the concept of nestedness has transcended the limits of ecological systems and became a generic indicator of underlying organization in a complex system. Studies like Bustos et al. (2009) and others [35, 154, 155] suggest that a highly nested structure can be found in many areas of the social sciences, economics and finance.

\subsection{Nestedness as a measure of order}

Nestedness is a concept that provides a sense of how much order or, by contrast, randomness we might find in a complex system. Patterson et al. (1993)[13] introduced a measure of nestedness in the context of interactions within species in ecosystems. Yet, it is difficult to describe a better indicator of system organization when working with bipartite networks. Let us consider a graphic approach to understanding this phenomenon.

In figure 6.1a, we show a random bipartite matrix. Clearly, no underlying structure can be found in this case. In its binary form, a bipartite matrix will have white cells, which denote the presence of an interaction between species, and black cells, which denotes the absence of such interaction. On the other hand, figure $6.1 \mathrm{~b}$ shows a perfectly nested matrix, where interactions can easily be characterized. When comparing both matrices, we observe that a clear pattern emerges in the second case. For instance, if this matrix represents a mutualistic ecosystem of plants and pollinators, we would conclude that the interactions between species do 
a)

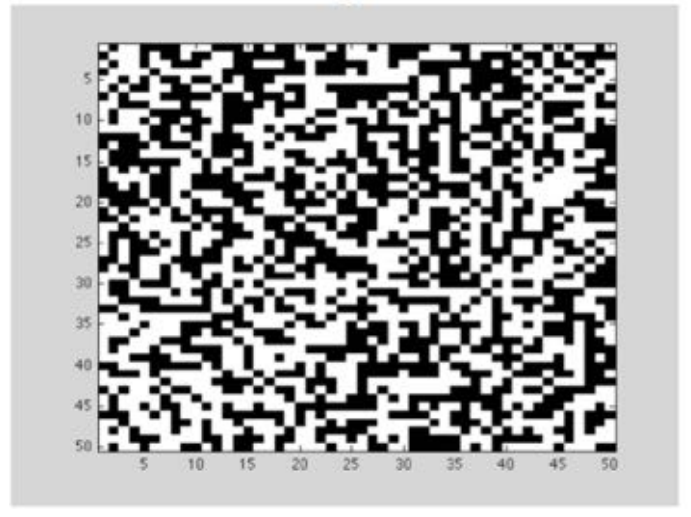

b)

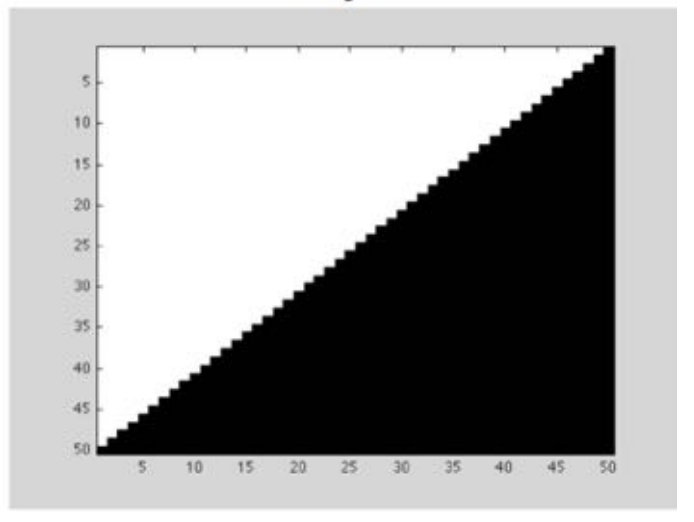

Fig. 6.1.: Basic nestedness concept. This figure shows two examples of bipartite binary matrices. This means that each white spot represents an link active link that connects the $i$ element of the first of nodes with the $j$ element of the other set. The first one (right) has been generated based on a random pattern. The second one (left) shows a perfectly nested bipartite matrix of the same size and number of links. Both matrices are equivalent in terms of network density and size, yet the amount of order is evidently higher in the second one.

not take place randomly, but in a way that can be described by understanding the different roles that species would play. Thus, we would observe that some species are specialists while others are generalists. A nested structure will be the consequence of generalist species interacting with either specialists or other generalists, whereas specialists will only interact with generalists exclusively. The previous work in this field $[21,74,127,25,134]$ as well as the empirical data support such a claim, which leads to very powerful conclusions and implications both at macro- and meso-scale. These implications are related to the persistence and robustness of such systems as well as the relationship between biodiversity and intra-species competition, two concepts that can clearly be adapted to the case of international trade markets. Let us consider the case of a perectly nested network (figure 6.2).

In this case, the decreasing order of the node degrees and the configuration of the system creates a bipartite matrix that is perfectly nested, where all values are organized towards the upper-left side of the matrix:

$$
B=\left(\begin{array}{lllll}
1 & 1 & 1 & 1 & 1 \\
1 & 1 & 1 & 1 & 0 \\
1 & 1 & 1 & 0 & 0 \\
1 & 1 & 0 & 0 & 0 \\
1 & 0 & 0 & 0 & 0
\end{array}\right)
$$




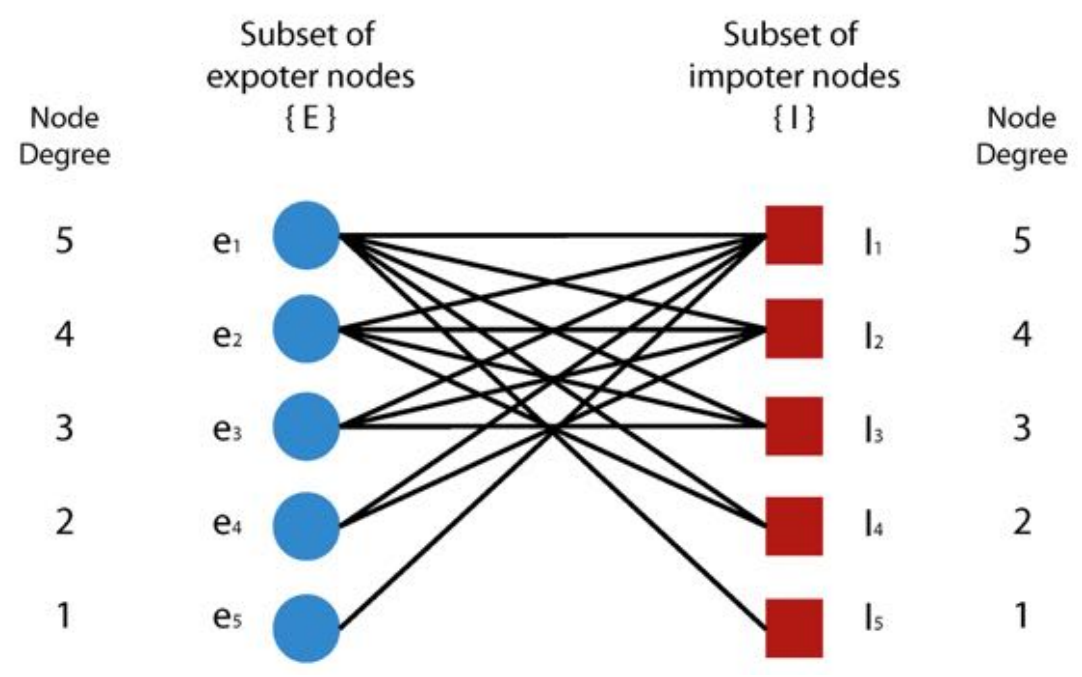

Fig. 6.2.: Example of a perfectly nested bipartite network. This figure shows an example of perfectly nested trade network of 5 exporters and 5 importers.

Now, there are many ways to measure nestedness in networks. Some methods, like the NODF and WNODF, are designed to look for organization patterns in bipartite matrices $[156,11]$. Other indicators, like WINE, are based on the weighted Manhattan distances between the elements of the bipartite matrix. And finally, the spectral nestedness approach uses eigenvalue analysis to determine the likelihood of a matrix to be nested in comparison to a set of randomized equivalent ones.

\subsection{Matrix Temperature Measure}

Altman and Patterson (1993) [13] introduced the concept of order and disorder in bipartite matrices as a consequence of the study of species distribution patterns in naturally fragmented ecosystems. Their argument on the reinterpretation of entropy of a system has led to a direct application of a thermodynamic variable in biology and, furthermore, in numerous other study fields. In order to overcome several problems and shortcomings from the previous existing methodology, they focused on the concept of the unexpectedness by either occurrence or absence of a given state of a system. This is another way of interpreting Shannon's information concept in which $I$ is a measure of surprise and defined as:

$$
I=-\log \left(p_{i}\right)
$$

where $p_{i}$ is the probability of a system state to occur. In this way, very rare states will have a high information entropy, whereas common states will have a low one. As a 


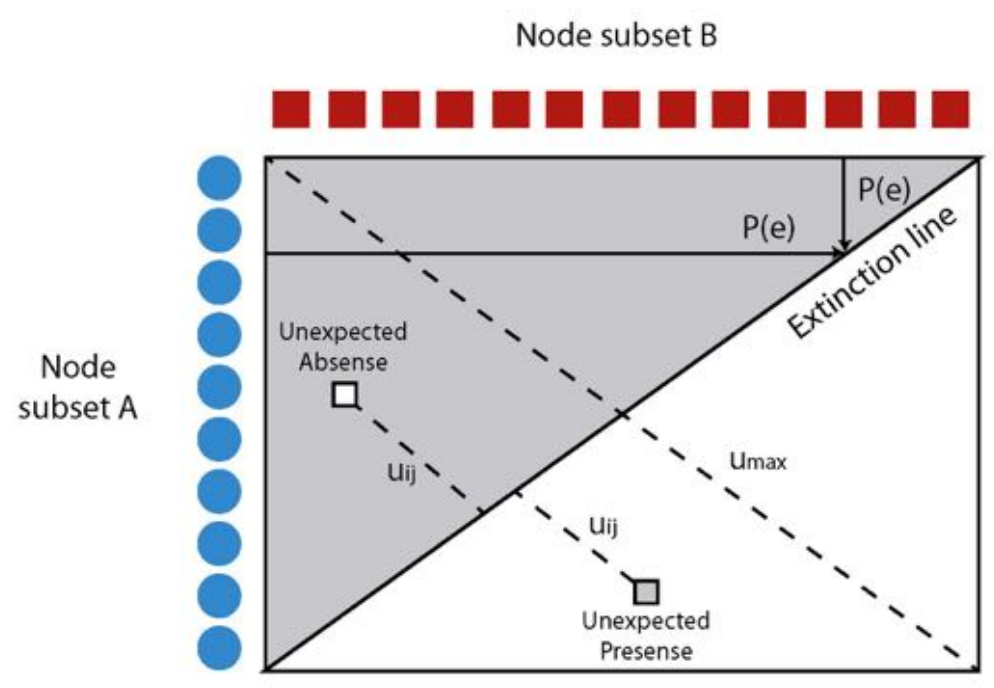

Fig. 6.3.: Bipartite matrix temperature and measure of expectedness. This figure shows schematic concept of how the unexpectedness can be measured in a packed bipartite matrix, in this case, very close to perfect nestedness. The extiction line is the boundary of what expected and what is not, from which the distance of the unexpected presence or absence can be measured. Nodes toward the upper-left corner are less likely to go extinct and have a high node degree; these are the generalist agents in the system.

consequence, this can be directly applied to bipartite matrices to determine the level of unexpected presences and absenses that in turn generate a higher disorder in the matrix itself. The process to perform this is by taking the matrix under study and rearraging it to its packed form, which is the state of maximum nestedness where the unexpected presences and absences are minimized. With this process, we could find the limit or threshold that separates the areas where presences and absences naturally take place. This is in fact the occurence boundary of the system, which can serve as a standard for measuring how unexpected presences or absences are. Given the biology nature of the paper, the authors have also named this boundary as the extinction line.

Even though the authors argue about the probability of extinction in the paper, which is higher for the right nodes of subset B and the bottom one of subset A, we need to focus on the way the unexpectedness is measured. Distances are critical for this discussion. Thus, any unexpected presence or absense that is far away from the extinction line increased the disorder of the system in a more significant way than does other close the line. Also, it is worth noting that the maximum unpecpectedness will be found in the case of specialist nodes connecting to each other ( $U_{\max }$ line). Furthermore, distances should be measured in the same direction. Consequently, the local unexpectedness is defined as: 


$$
u_{i j}=\left(d_{i j} / D_{i j}\right)^{2}
$$

where $D_{i j}$ is the length of the full line across the bipartite matrix and $d_{i j}$ is the specific length along that direction. The Total Unpectexpectedness will be:

$$
U=\frac{1}{(n m)} \sum_{i} \sum_{j} u_{i j}
$$

This is a normalized metric, which means that the resulting measure will be insensitive to the matrix size and shape. Hence, a perfectly ordered matrix will have $U=0$, whereas a matrix with maximum unexpectedness will have approximately $U_{\max }=0.04145$. Then, the system temperature is defined as:

$$
T=k U
$$

where $k=100 / U_{\max }$. Therefore, the system temperature will range from $0^{\circ}$ to $100^{\circ}$. At risk of being repetitive, it can be concluded that a highly nested matrix will display a low temperature (less disorder), whereas in the opposite case, high temperatures will appear.

\subsection{NODF and WNODF Methods}

The Nestedness based on Overlap and Decreasing Fill [9, 10, 11, 156, 157], or NODF, is another metric used to evaluate the order of disorder of a binary bipartite matrix. As suggested in its name, the metric relies on two properties: a) the decreasing fill of rows and columns, and b) the paired overlap. The author [10] applies this concepts to a $n$-by- $m$ matrix and defines the marginal totals (MT) as the sum of the values of any column or row. Then, the decreasing fill parameter for a pair of rows $i$ and $j$ is defined as:

$$
D F_{i j}= \begin{cases}100 & M T_{j}<M T_{i} \\ 0 & M T_{j} \geq M T_{i}\end{cases}
$$

The same process applies to the columns. In addition, the paired overlap for the columns, or $P O_{k l}$, is defined as the percentage of 1's in a given column $l$ that are 


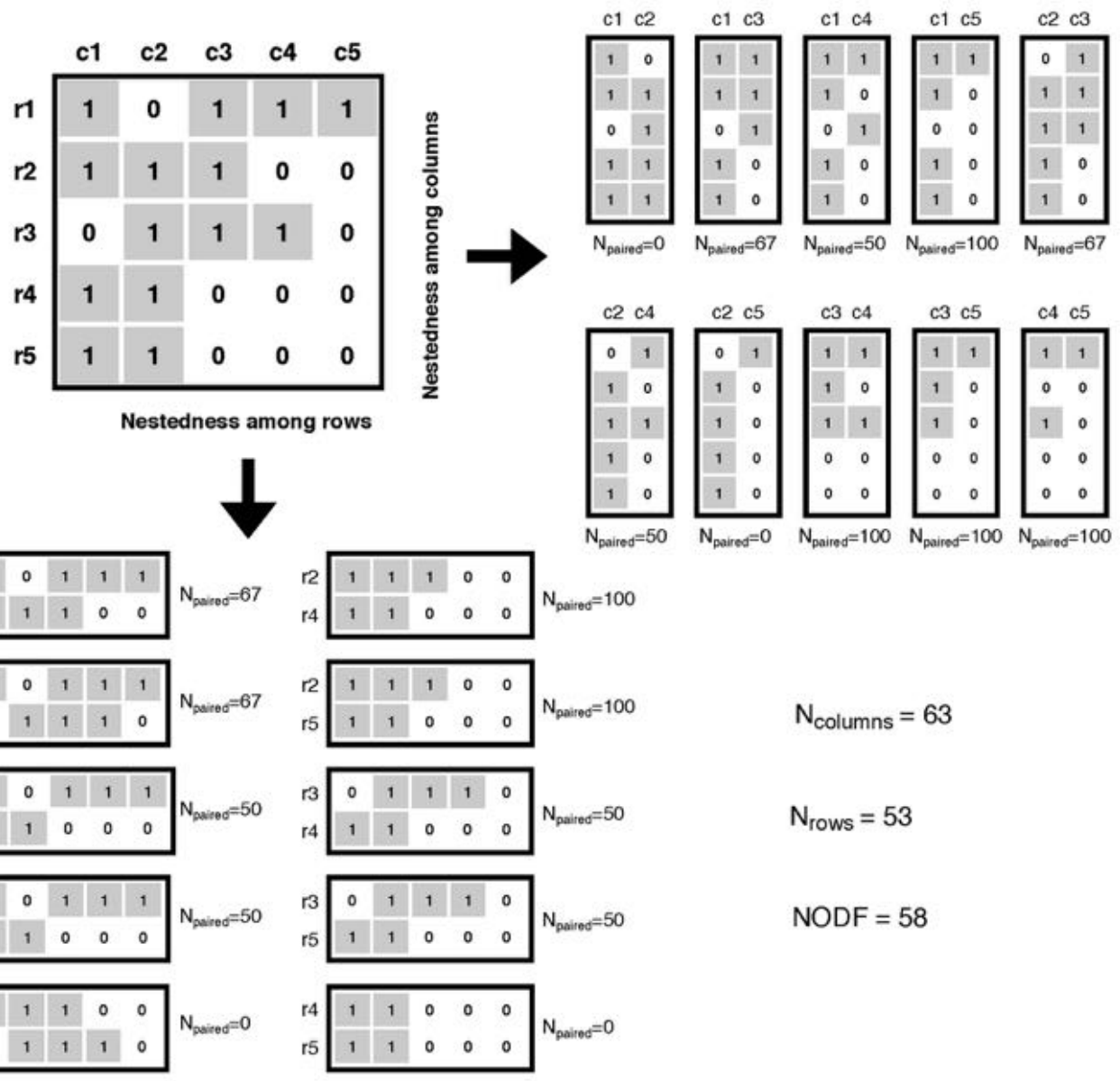

Fig. 6.4.: NODF calculation process, Almeida-Neto et al.[10]. This figure shows an example of the the process to calculate the NODF nestedness for the columns and the rows. The paired columns and rows are calculated separately to right and below the example binary bipartite matrix. The average nestedness for all columns $\left(N_{c o l}=63\right)$ and all rows $\left(N_{\text {row }}=53\right)$ are presented. Finally, the total nestedness for the whole matrix is computed $(N O D F=58)$.

located at identical row positions to those in column $k$. And, likewise, the same concept applies to rows again.

Consequently, for columns or rows, we can define the paired nesterness $\left(N_{\text {paired }}\right)$ as:

$$
N_{\text {paired }}= \begin{cases}0 & D F_{\text {paired }}=0 \\ P O & D F_{\text {paired }}=100\end{cases}
$$

Then, from the $n(n-1) / 2$ and $m(m-1) / 2$ paired degrees of nestedness for $n$ columns and $m$ rows, we can compute the nestedness among all columns $\left(N_{c o l}\right)$ and 
for all rows $\left(N_{\text {row }}\right)$ by taking the average of all paired values of columns and rows respectively. Finally, the total nestedness for the whole matrix can be defined as:

$$
N O D F=\frac{\sum N_{\text {paired }}}{\left[\frac{n(n-1)}{2}\right]+\left[\frac{m(m-1)}{2}\right]}
$$

We now have presented a quite popular metric for the ecology scientific community. Yet, one of its main disadvantages is that it only works with binary bipartite matrices. In order to overcome this, the author has also introduced the weighted version of the metric, the WNODF. With the ability to deal with quantitative matrices, this updated metric is calculated in a very similar way. Staying with the same matrix of $n$ columns and $m$ columns, the author defines $F$ as the number of cells with non-zero values for any column $c_{i}$ or row $r_{i}$. Then, the paired nestedness of any columns $c_{i}$ and $c_{j}$ $(j>i)$ will be greater than zero if $F\left(c_{i}\right)>F\left(c_{j}\right)$ and it will be zero otherwise. Next, the weighted value of paired nestedness for any pair of columns is computed as the percentage of cells in $c_{j}$ that have lower values than cells of the same row in $c_{i}$. Finally, the mean nestedness vale for all pairs of columns can be computed as:

$$
W N O D F_{c o l}=100 \sum_{i=1}^{n-1} \sum_{j=i+1}^{n} \frac{k_{i j}}{N_{j}}
$$

where $k_{i j}$ denotes the number of cells with lower values in $c_{j}$ and $N_{j}$ is the total number of non-empty cells in $c_{j}$. As it can be suspected at this time, the same applies to the rows and the WNODF for rows will be equivalent to that of the columns. This leads to the total nestedness calculation, which will be:

$$
W N O D F=\frac{2\left(W N O D F_{c o l}+W N O D F_{\text {row }}\right)}{m(m-1)+n(n-1)}
$$

\subsection{WINE Method}

The previous methods, as reviewed, use presence-absence adjacency matrices (binary matrices) as the basis for calculating nestedness. Indeed, that simple approach facilitates the description and characterization of the topology of a network. Nevertheless, these measurements do not incorporate other important aspects of the topology, which is the heterogeneity in the weight (or the intensity) of links, as defined by Barrat et al.[20]. So, if we characterize any link through the mere information of the presence $\left(M_{i j}=1\right)$ or the absence $\left(M_{i j}=0\right)$ of interactions, we are not 
taking in account the potential variance in the link intensities or weights. Instead, the usage of a new estimator from quantitative (or weighted) matrices that take in consideration this intensity, such as the number of visits in the plant-pollinator case or the amount of dollars traded in a given market, we would have a more interesting measure. Thus, Galeano et al.[68] have defined the Weighted-Interaction Nestedness Estimator (or WINE), which is the first estimator that allows for the characterization of weighted nestedness. Now, while previous measurements estimate nestedness through the comparison of pairs of columns or rows using indirect properties associated to nestedness, like the decreasing fill and overlap, the WINE process works upon rescaled, as well as weighted, Manhattan distances. In order to compute the indicator, the authors start with the weighted matrix, which is paked so that the row or the column with the greatest marginal totals is rearranged as the last row or column, repeating the procedure until the matrix is fully sorted. Thus, nestedness will be related to the proximity of existing links to one another in the packed matrix. By this logic, the most nested matrix will always be the one in which, after packing, a minimum mix of filled and empty cells is observed.

The process then starts by calculating two weighted adjacency matrices from the packed matrix, $P_{i j}^{c}$ and $P_{i j}^{r}$, in order to account for the effect of the weights upon the nestedness. These matrices depict the dependence of column element $j$ on row element $i$ and vice versa. Thus, the weights of element $M_{i} j$ over row $i$ and column $j$ are obtained:

$$
\begin{gathered}
P_{i j}^{c}=\frac{M_{i j}}{\sum_{j=1}^{N_{r}} M_{i j}} \\
P_{i j}^{r}=\frac{M_{i j}}{\sum_{i=1}^{N_{c}} M_{i j}}
\end{gathered}
$$

where $N_{r}$ and $N_{c}$ are the number of rows and columns of the matrix, respectively. Then, WINE is merely based on the concept of estimating nestedness through the calculation of a Manhattan distance from each of the matrix cells containing a link to the cell corresponding to the intersection of the row and columns with the lowest marginal totals (number of links). The distances in WINE are measured in reference to the opposite corner of the packed matrix. Additionally, in WINE, the Manhattan distance is replaced by a weighted Manhattan distance, which are introduced in the packed matrix by mapping it into a Cartesian space and rescaling it to the unit square to avoid distortions in asymmetric matrices. Finally, the elements of matrix $(\mathrm{i}, \mathrm{j})$ are assigned the positions $x_{i}$ and $y_{j}$ as follows: 


$$
\begin{aligned}
& x_{i}=\frac{i-1}{N_{c}}+\frac{1}{2 N_{c}} \\
& y_{j}=\frac{j-1}{N_{r}}+\frac{1}{2 N_{r}}
\end{aligned}
$$

With these new positions, the authors calculate a weighted-interaction distance, which estimates nestedness taking in account the number of events in the links:

$$
d_{i j}^{w}=P_{i j}^{r} x_{i}+P_{i j}^{c} y_{j}
$$

This equation shows the weighted distance of each link and provides a useful parameter for assessing the importance of each link in the network. The greater this distance is, the greater the contribution of this link to nestedness. The weightedinteraction nestedness (WIN) of the matrix will then be expressed by the mean weighted distance of all its non-zero elements:

$$
d^{w}=\frac{1}{N_{l}} \sum_{i, j} d_{i j}^{w}
$$

where $N_{l}$ is total number of links. This parameter takes into account both the relative position of links in the matrix and the number of events in each interaction, thus including more precise information than previous nestedness measurement.

Now, since the statistical significance of any nestedness index has to be tested against some null hypothesis, the authors use a null model that a) constrains matrix fill to observed values, b) retains the distribution of number of events in the links, but c) does not constrain marginal totals. By constraining matrix fill and replicating the distribution of number of events, the authors acknowledge that, in a given system, just a fraction of the links are feasible and that the differences in abundance of the interacting elements will condition the distribution of the intensity of the links. However, in terms of marginal totals, a equiprobable null model is followed, considering that there is no reason a priori to assume that certain links are less probable than others.

To generate random matrices, the events distribution of links are firstly computed in the original data matrix (see figure 6.5). Then, random matrices with the same size and intensity distribution are created as in the original data matrix. However, the algorithm does not preserve the number of links for each and every column and row. 

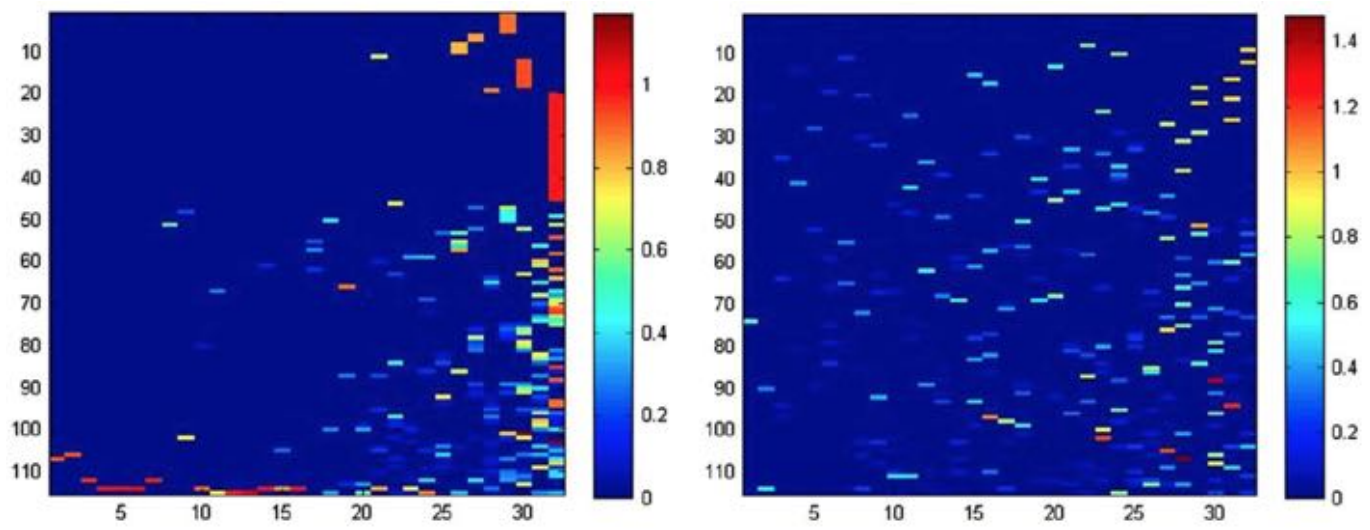

Fig. 6.5.: Weighted distances of the actual data (left) and random packed (right) matrices from the WINE Method. Example from Galeano et al.[68].

The random matrices are then packed and, subsequently, the weighted-interaction nestedness (WIN) for the random matrices is calculated according to previous equations, repeating the process and obtaining the distance of the random matrices $\left(d_{r n d}\right)$. The authors propose that they have chosen 100 repetitions or random matrices because increasing the number of replicates only delays the calculation of WINE with no significant improvement in accuracy. They later calculate the standardized static variable z-score, as $z=\frac{d^{w}-d_{r n d}}{\sigma}$, to assess the significance of WIN, where $\sigma$ is standard deviation. This score assesses how different WIN $d^{w}$ is from average random WIN $d_{r n d}$. It is important to remember that the original data is just one combination of all possible permutations of registered events. $\mathrm{z}$ values below -1.65 or above 1.65 indicate an approximate statistical significance at the 5 percent error level (one-tailed test). The WIN of the data matrix can then be normalized by comparing it to the average WIN of equivalent random matrices and to the WIN of the maximal nestedness matrix to obtain the weighted-interaction nestedness estimator as defined in equation 6.16.

$$
\eta_{w}=\frac{d^{w}-d_{r n d}}{d_{\max }-d_{r n d}}
$$

The value of this estimator approaches zero when the WIN of the original data matrix is close to the average WIN of the equivalent random matrices. It approaches 1 as it gets closer to the nestedness of the maximal nestedness matrix. 


\subsection{Spectral Nestedness}

Contrary to the approach of the previous methods, the spectral nestedness method [152] does not rely on any algorithm based on distances or individual contributions. Rather, it utilizes an universal property of any matrix, the eigenvalues of the matrix itself. In this way, a spectral study of nestedness of quantitative (weighted) and binary (unweighted) networks can be accomplish in time-efficient way from a computational standpoint, which is one of the main issues of the other methods when dealing with large matrices. In this paper [152], the authors define nestedness for both binary and quantitative bipartite networks, and present a general detection method that follows naturally from the matrix properties of nested graphs. Formally, a bipartite network is constituted by $S$ nodes, which can be divided into two distinct sets. For instance, for a mutualistic network, $|A|$ depicts the animals or pollinators in the network and $|P|$ denotes the set of plants. The links within the network are denoted by $|E|$, which connect a node of $|A|$ with another node of $|P|$. The adjacency matrix for the binary case will then be $\mathcal{A}$. For the quantitative case, $\mathcal{A}_{i j}$ can accomodate positive values different than 1 . The authors [152] state that the set of eigenvalues is an invariant property of a matrix, which does not change if rows and columns are permuted, and that because $\mathcal{A}$ is a symmetric matrix, all of its eigenvalues will be real, and because the graph is bipartite, the eigenvalues will be distributed symmetrically about 0 . The largest eigenvalue of $\mathcal{A}$, the dominant eigenvalue, is known as its spectral radius $\rho(\mathcal{A})$, and for binary matrices its value is bounded from above by $\sqrt{|E|}$. But since the adjacency matrix of a bipartite graph is symmetric, it is more efficient to use the incidence matrix $\mathcal{B}$, which will only have a size of $|P|$ by $|A|$. Then, nestedness can be defined as a property of $\mathcal{B}$.

Furthermore, the set of eigenvalues is an invariant property of a matrix, which does not change if rows and columns are permuted. If $\mathcal{B}$ is a perfectly-nested binary matrix, then there exists a permutation of rows and columns such that the set of edges in each row $i$ contains the edges in row $i+1$, while the set of edges in each column $j$ contains those in column $j+1$. More formally, the rows and columns of $\mathcal{B}$ can be sorted such that $\mathcal{B}_{i, j} \leq \min \left(\mathcal{B}_{i, j-1}, \mathcal{B}_{i-1, j}\right)$, a definition that can be extended to quantitative matrices as well. Now, from the mathematical literature we know that, among all the connected bipartite graphs with $|S|$ nodes and $|E|$ edges, the one yielding the largest spectral radius $\rho(\mathcal{A})$ will be a perfectly nested graph. Thus, the authors [152] are able to confirm numerically that among all the bipartite graphs with $|P|$ plants, $|A|$ animals and $|E|$ edges, the configuration leading to the largest spectral radius is a perfectly nested graph. In figure 6.6, it can be observed that the right tail of the spectral radius distribution contains either perfectly nested graphs or graphs that are very close to being perfectly nested, while the left tail contains graphs that are far from being perfectly nested. In this way, the spectral radius can 


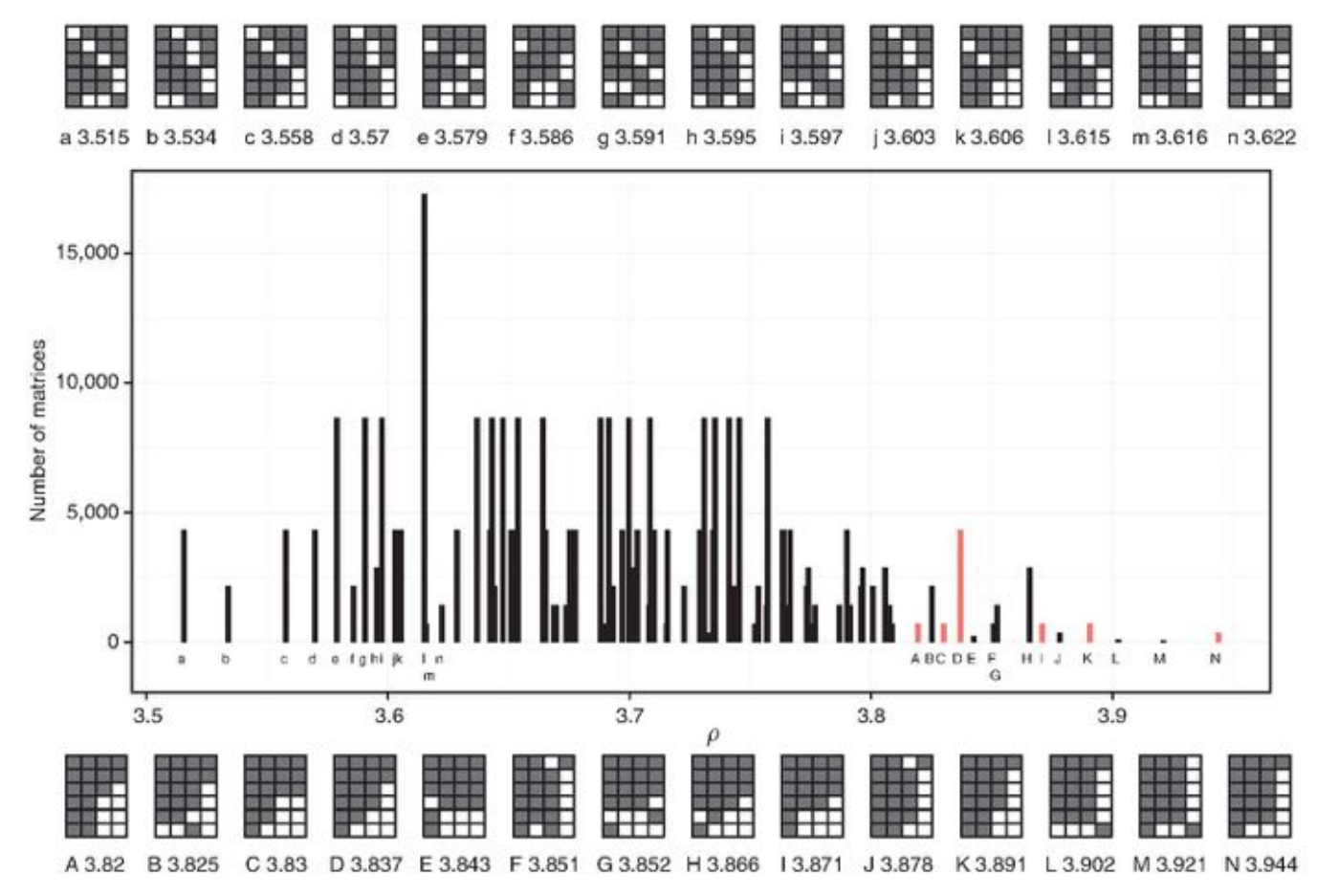

Fig. 6.6.: Binary matrices and spectral nestedness. Staniczenko et al.[152].

therefore be seen as a natural scale for nestedness. Larger values of $\rho(\mathcal{A})$ will be obtained for more nested matrices with the same size and number of edges. The same logic can then be applied to determine the nestedness of quantitative matrices. In figure 6.7, the authors [152] show how this concept works for such matrices.

So the validity of the concept has now been proven. Yet, the authors need to develop a set of statistical tests to determine the significance of nestedness for matrices that are not necessarily perfectly nested.

To perform this, they proposed a set of 4 statistical tests that can be applied depending on the cases, both binary and quantitative. As expected, all the tests are based on the spectral radius. Since perfectly nested binary and quantitative matrices are associated with large spectral radius, the authors propose to implement a strong tests that are suitable for real-world data sets. For each test, the probability $p$ that a randomly constructed incidence matrix $\mathcal{B}^{\prime}$ has spectral radius $\rho\left(\mathcal{A}^{\prime}\right) \geq \rho(\mathcal{A})$ should be computed, where $\mathcal{A}$ and $\mathcal{A}^{\prime}$ are the adjacency matrices of $\mathcal{B}$ and $\mathcal{B}^{\prime}$, respectively. For both binary and quantitative matrices, the authors define three null models for constructing $\mathcal{A}^{\prime}$ :

- a) to preserve $|P|,|A|$ and place $|E|$ edges at random within the matrix 


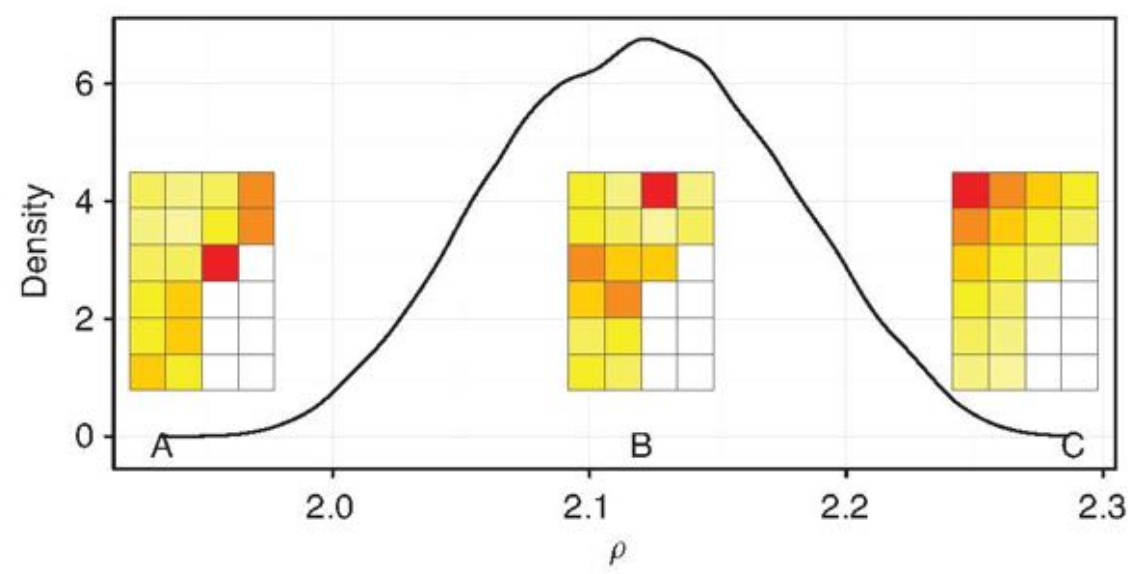

Fig. 6.7.: Quantitative matrices and spectral nestedness. Staniczenko et al.[152].

- b) to preserve $|P|,|A|$ and place $|E|$ edges at random within the matrix, but accepting only connected matrices

- c) same as in the previous cases ( $\mathrm{a}$ and $\mathrm{b}$ ), but conserving the degree distribution as well.

As measures of nestedness can be very sensitive to matrix size, fill and configuration, the authors have used null model implementations that preserve $|P|,|A|$ and $|E|$. For quantitative matrices only, they have introduced a fourth null model:

- d) to preserve $|P|,|A|$ and $|E|$, and shuffle the coefficient values of $\mathcal{B}$ but not their positions

This implies that the binary structure of the matrix remains the same, but the values of the elements are randomized within those positions. Using this last null model, the P-value will be equal to 1 when all the coefficients have the same value (for example, a binary matrix). In all tests, for all but the smallest of networks, $P \leq 0.05$ is a suitable general significance level for nestedness, with $P \geq 0.95$ indicating anti-nestedness.

\subsection{Correlation between nestedness methods}

We presented several measures of nestedness in this chapter. Still, when we implement these measures to our data, we realize that not all of them are suitable for our study. We work upon weighted bipartite networks. But the bipartite matrices that represent these networks are highly sparse. This has implications in the way 
some measures, like WNODF, would rate our bipartite matrices. In fact, WNODF is a nestedness measure that is designed to look for perfectly nested matrices and would unfairly penalize the nestedness results of naturally sparce matrices. This is because it tests for the decreasing fill, which is its most basic premise, relies on pairwise rows and columns that gradually decrease the number of presences and consequently increase absences as we move through the bipartite matrix. The sparse nature of our WBTNs would not allow for the WNODF measure to effectively score their actual nestedness performance. Therefore, the implementation of a unique nestedness indicator needs to be studied in a deeper manner. Let us consider a random market (HS2 $=01$ in 1995). We build a 3D bar chart of its bipartite matrix (figure 6.8). When we apply the WNODF measure, we obtain a value of 2.5 in a scale from 0 to 100, where the latter would be the score of a perfectly nested matrix. This finding is not consistent with what we actually observe in figure 6.8, where an organization pattern is clearly observed. We analyze the WNODF calculation step by step, and realize that the absences in the bipartite matrix of this market create a erroneous interpretation of whether the market is nested by severely penalizing the scores of every pairwise comparison of rows and columns. We conclude that WNODF is a method that is not suitable to evaluate nestedness within markets, where the link density is between 0.05 and 0.20 , or within any other naturally sparce system. WNODF could be useful to assess the nestedness of bipartite matrices with link density closer to 0.4 and higher.

In contract, when we apply the WINE indicator, we obtain a value of 0.78 in a scale from 0 to 1 , where the latter would be the score of perfectly nested matrix. This is mainly because its most basic premise is that the weighted manhattan distances of a highly nested matrix will be lower than those of a random matrix. It is evident that the WINE indicator does not penalize the absences of a naturally sparse matrix, but rather incorporates them in the process to only randomize the existing elements in the matrix. This approach is a more valid indicator of nestedness in trade markets.

Finally, the spectral nestedness is also a suitable indicator, although it is very expensive in terms of computational time since it requires a very high number of randomizations of the bipartite matrix in order to perform a statistically significant test. Nonetheless, it can be argued that the WINE indicator and the spectral nestedness are very similar process when it comes to concentrating only on the existing elements of a bipartite matrix. Yet, and despite the validity of the final results of each method, we now hypothesize that we should at least find some correlation of the results of 2 different indicators when applied to the same matrices, for instance, the WNODF and WINE. Let us consider a set of bipartite networks where both indicators are calculate for each network. We show the results on figure 6.9. 


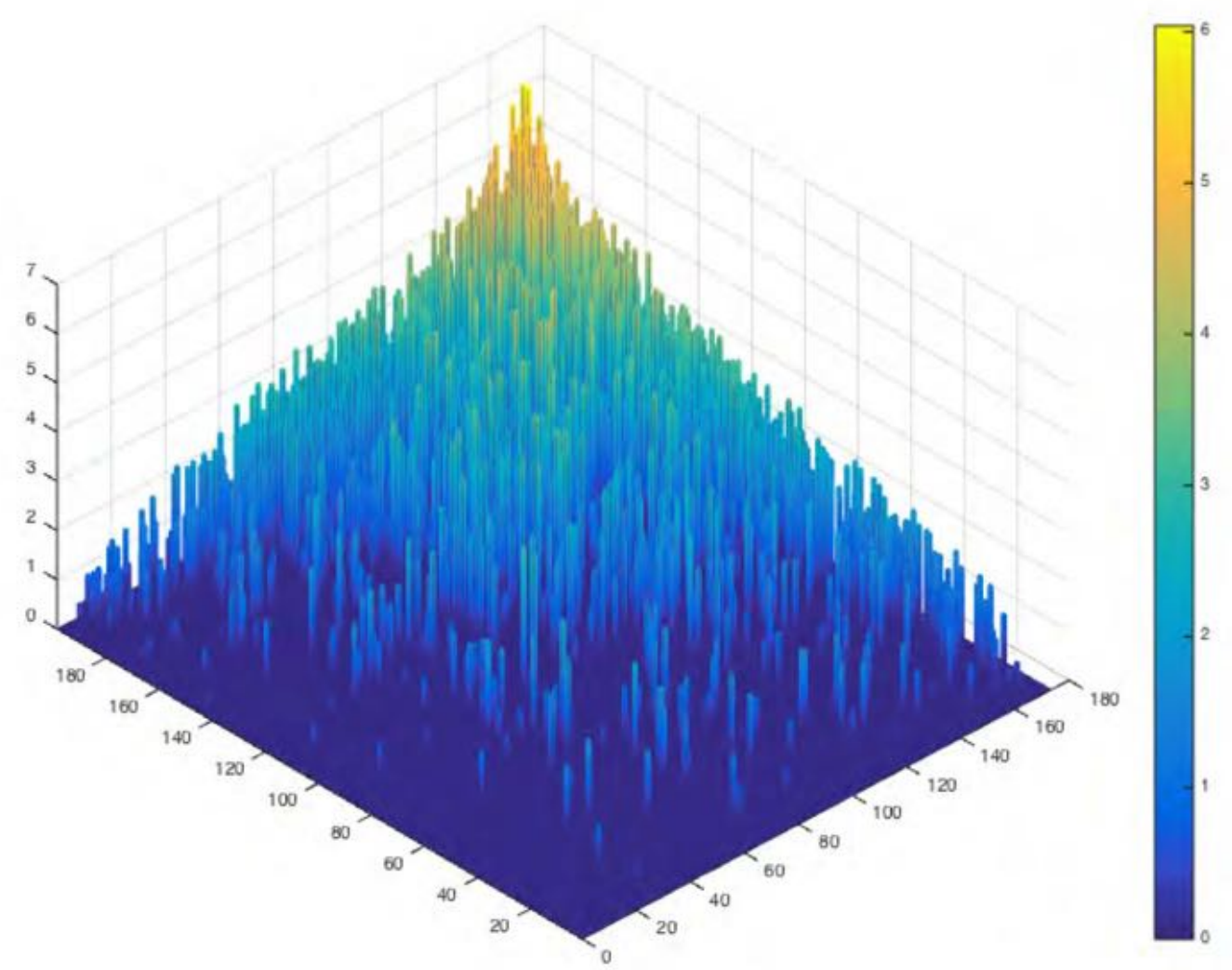

Fig. 6.8.: Weighted bipartite matrix visualization. This figure shows a 3D bar chart visualization of a bipartite matrix from a random product (HS2 $=01$ in 1995). The left axis shows the exporters, while the right one shows the importers, both of them ordered by their degree $k^{e x p}$ and $k^{i m p}$, respectively. The vertical axis shows the logarithm of the trade volume or Log 10 (Volume). The height of the bar is color-coded as referenced by the colorbar on the right. 

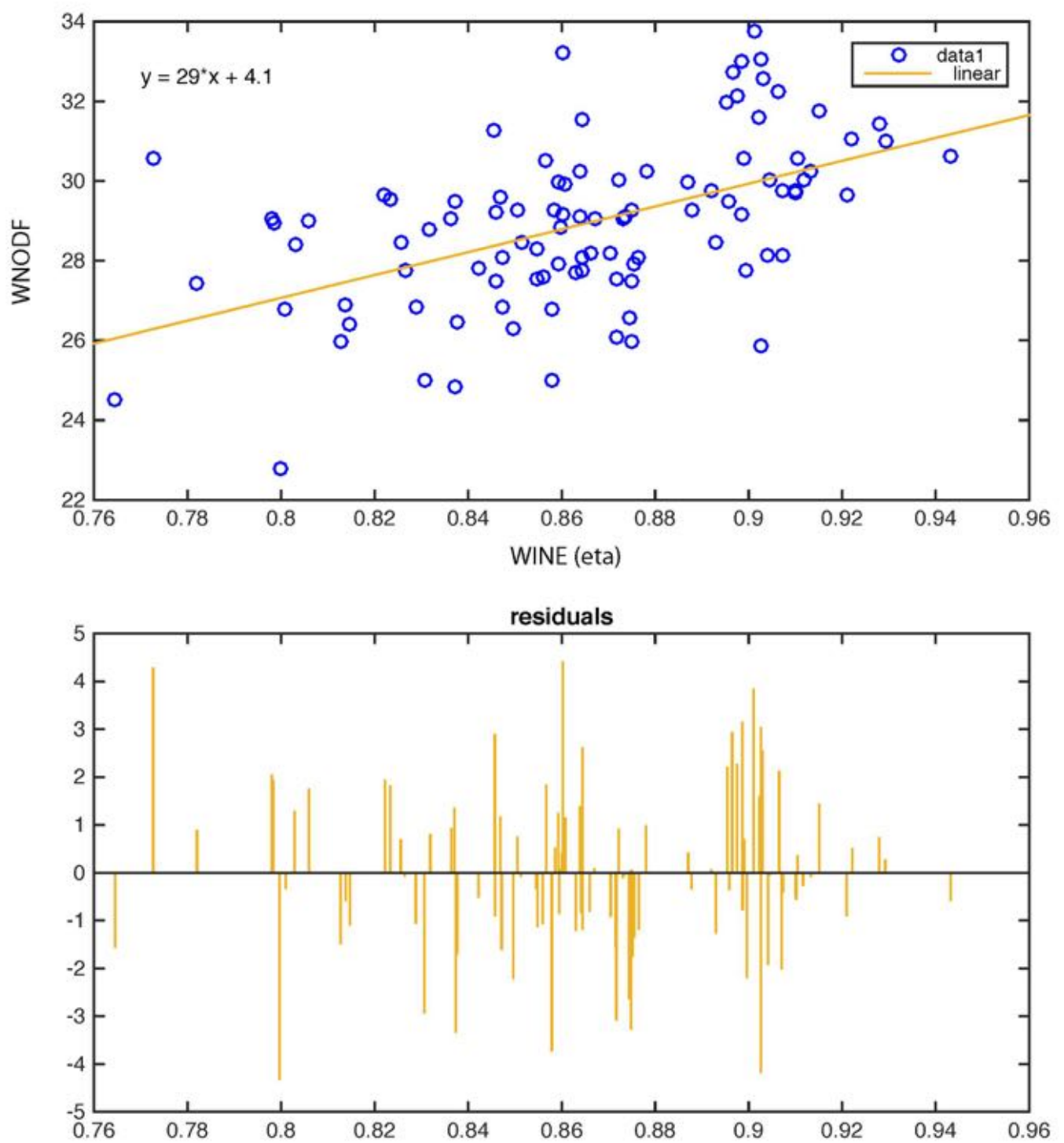

Fig. 6.9.: Correlation between WNODF and WINE for a set of bipartite networks. This figure shows the results obtained for a set of bipartite networks after applying the WNDF and WINE methods. Each dot in the figure represents a randomly selected market at HS6 level of aggregation. 


\subsection{Nestedness considerations for this study}

For this study, we test all nestedness measures. But since our WBTN are naturally and highly sparse, we are forced to adopt the Weighted Interaction Nestedness Estimator or WINE as the main indicator of how nested our markets are. In addition, we also adopt the spectral nestedness tests 2 and 4 for testing the statistical significance of the WINE results. The matrix temperature and the NODF indicator or its weighted form, the WNODF, are not suitable for this study. The main reason behind this claim is related to their fundamental objectives. They both naturally search for disorder and automatically interpret an unexpected presence or absence as a negative fact. In trade markets, the unexpected absenses and presences are not necessarilly bad news, since they may be natural restrictions due to the following facts: a) world trade works upon self-interest and there is no sufficient good and demand to reach a network density of about 50 percent, b) geographic cultural restrictions are part of the system and the overall nestedness performence should not be penalized for this reason, and c) unexpected presences (beyond the extinction line) have a very low weight (normally 4 to 5 levels of magnitude), they do not contribute in an extreme way to the anti-nested tests; yet the NODF method will severely penalize the results. 



\section{Part III}

Results 



\title{
Topological analysis
}

\author{
Too large a proportion of recent "mathematical" \\ economics are mere concoctions, as imprecise as \\ the initial assumptions they rest on, which allow \\ the author to lose sight of the complexities and \\ interdependencies of the real world in a maze of \\ pretentious and unhelpful symbols.
}

— John Maynard Keynes

(Economist whose ideas fundamentally changed the theory of macroeconomics)

On part two, a thourough discussion regarding the internacional trade data and our proposed bipartite methodology, highly inspired in the mutualistic ecosystems, has been reviewed. This part, however, focuses on the results we find after applying this methodology to the various levels of aggregation and periods, a total of 195,200 scenarios. On chapter 8, we will cover the results for the different levels of aggregation, industries (HS2), categories (HS4) and products (HS6), as well as the static comparison over different periods, but without analyzing any time variance per se. In order to minimize the repetitiveness that this chapter might include, we focus on some selected industry codes (HS2) to guarrantee that our results are representative of a wide spectrum of markets, still manageable within a reasonable number of pages. After this, we present a summmary for some selected categories (HS4) and selected products (HS6), again trying to avoid unnecessary, redundant or trivial repetition. Chapter 9, on the other hand, will focus on the temporal evolution of trade netwoks through the different periods, discussing how the key topological results change or remain unchaged over time. We will propose a simple and visual approach to analyzing dynamical phenomena and try to characterize it nature so that further conclusions can be drawn. Nonetheless, a key warning for the reader is that, although we worked hard to avoid it, some repetitiveness of the results can still be found across chapters 8 and 9. Our objective of charactirizing the features of international trade markets will deeply rely on this principle. Additionally, we also present several extended visualizations on the corresponding appendix ( $C$ and $D$ ) to further illustrate this point as additional information but without distracting the reader from our central goal. 

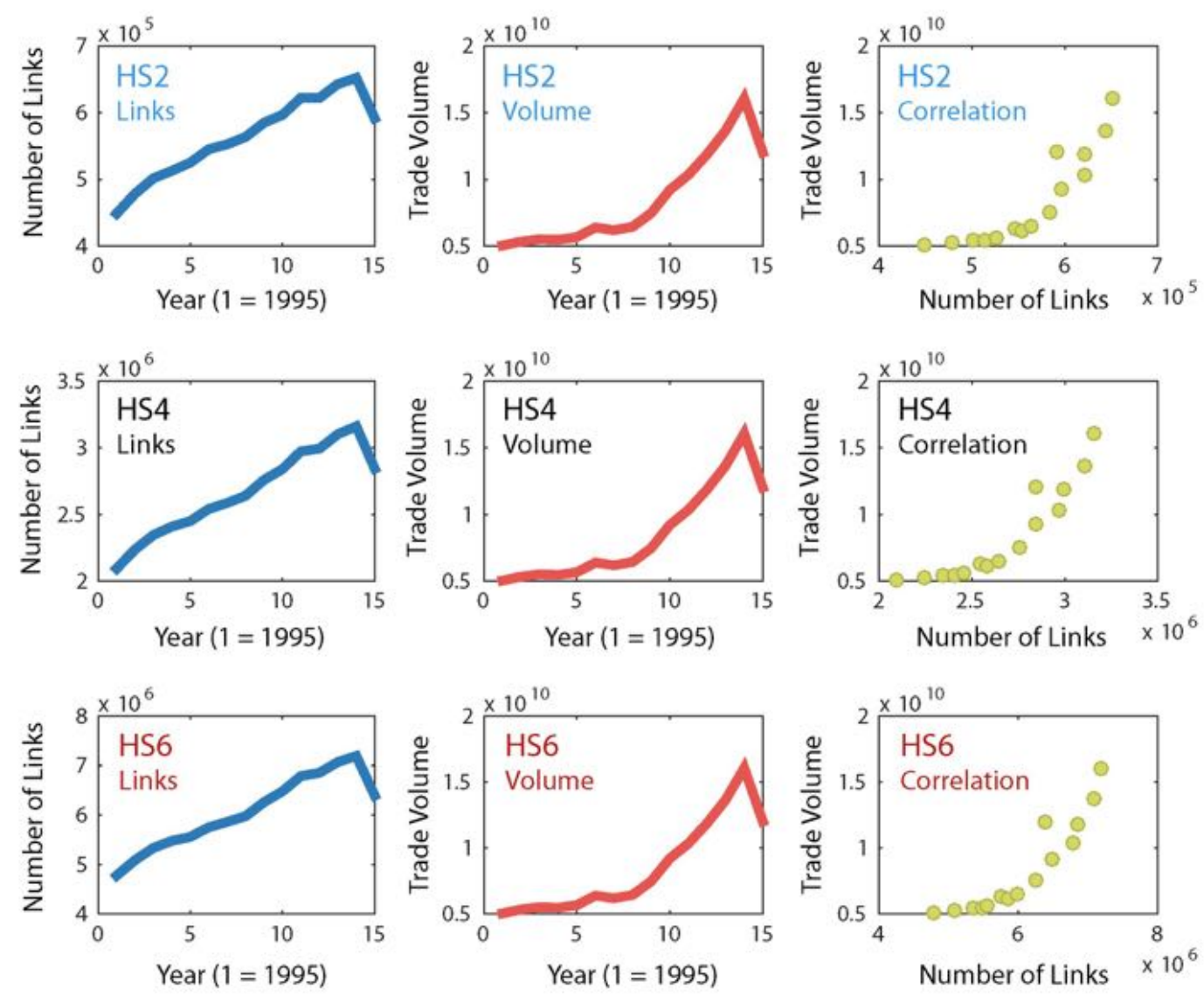

Fig. 7.1.: Market links and volume over time. This figure shows shows time evolution of the total number of links and the total trade volume for all products (HS6), all categories (HS4) and all industries (HS2) from 1995 to 2009. We also show a correlation between the two variables.

\subsection{Exploratory analysis of trade markets}

Markets are dynamic in their nature. They seem to evolve and adapt over time like an ecosystem. Our dataset contains over 91 million links, and this is just for the lowest level of aggregation (HS6). From that point, we can consolidate all data into the HS4 (category) and HS2 (industry), which incorporates further scenarios to our problem of understanding the topological properties of trade networks. Yet, the first relevant question about markets as a whole is related to the size of the system in terms of both number of links and trade volume over time. For this reason, we compute these total values for each period and for each level of aggregation, addition a correlation analysis between the number of links and the trade volume at the same time. We believe this is an important analysis to understand how markets behave at the macro-scale. In figure 7.1, we show the outcome for each level of aggregation for all periods from 1995 to 2009. The results are revealing in terms of overall patterns and outliers. We observe that both the number of links and 
the trade volume (in thousand dollars) grow consistently between 1995 and 2008 (periods 1 to 14 in the figure). Yet, in 2009 (period 15 in the figure), after the global financial crisis of September 2008, both the number of links and the trade volume show a significant decline in all levels of aggregation. We find a similar pattern for industries, categories and products, which is an expected situation since we are dealing with different consolidations of the same data. But still, 2009 is an interesting case since the magnitude of the trade volume decline is much higher than that of the links. This may also be consistent with the acceleration in world trade in terms of the average weight per link. Let us consider table 7.1 for annual trade activity details and its percentage variance year over year. The data basically shows that the annual changes, measures in percentage, reveal a phenomenon that starts after 2002 approximately. Starting 2003 and until 2008, the growth rates of trade volumes are significantly higher than those of the number of links. Until 2002, the behavior of both the trade volume and the number of links seem to be linked by a direct correlation, yet this is not the case from 2003 to 2008. Furthermore, between 2008 and 2009, we find that the decline of links is 9 percent, whereas the decline of trade volume is 25 percent, a percentage that is worth about 4 trillion dollars in terms of money value. Therefore, we observe that 2009 is actually an outlier in the correlation analysis. We do not try to explain this behavior in this study, this is beyond our scope. Nonethless, we believe it is relevant to show these facts for general context and in order to depict trade markets as an evolving system. In fact, some studies have pointed out that this type of behavior is consistent with situations that can work well as predictors of crisis. The arguments of the authors imply that temporal phenomena that exceed an exponential growth, which may frequently be observed in economic and financial systems, might lead to a crisis situation or financial bubles [146]. Yet, as previously stated, this is only for context purposes; it is not our intention to argue about this matter.

We now understand the general context of international trade markets in the period for which we have data. The next step of our exploratory analysis is to build and visualize our trade networks to determine what features can be found from a visual perspective. To this end, we utilize several network layouts so that any pattern may or may not be observed in the different visualizations, allowing us to confirm or reject many of our premises. We start by using a force-directed layout, which is recommended to explore central phenomena and patterns of complex networks. In figure 7.2, we observe for a random product, HS6 $=847120$ (digital computers) in 1995, which is also presented together with the network visualizations corresponding to the respective HS4 category (data processing machines) and the HS2 industry (machinery).

When visualizing the internal structure of a product-level trade networks, including the graphs for the corresponding category and industry, we realize that, although we 


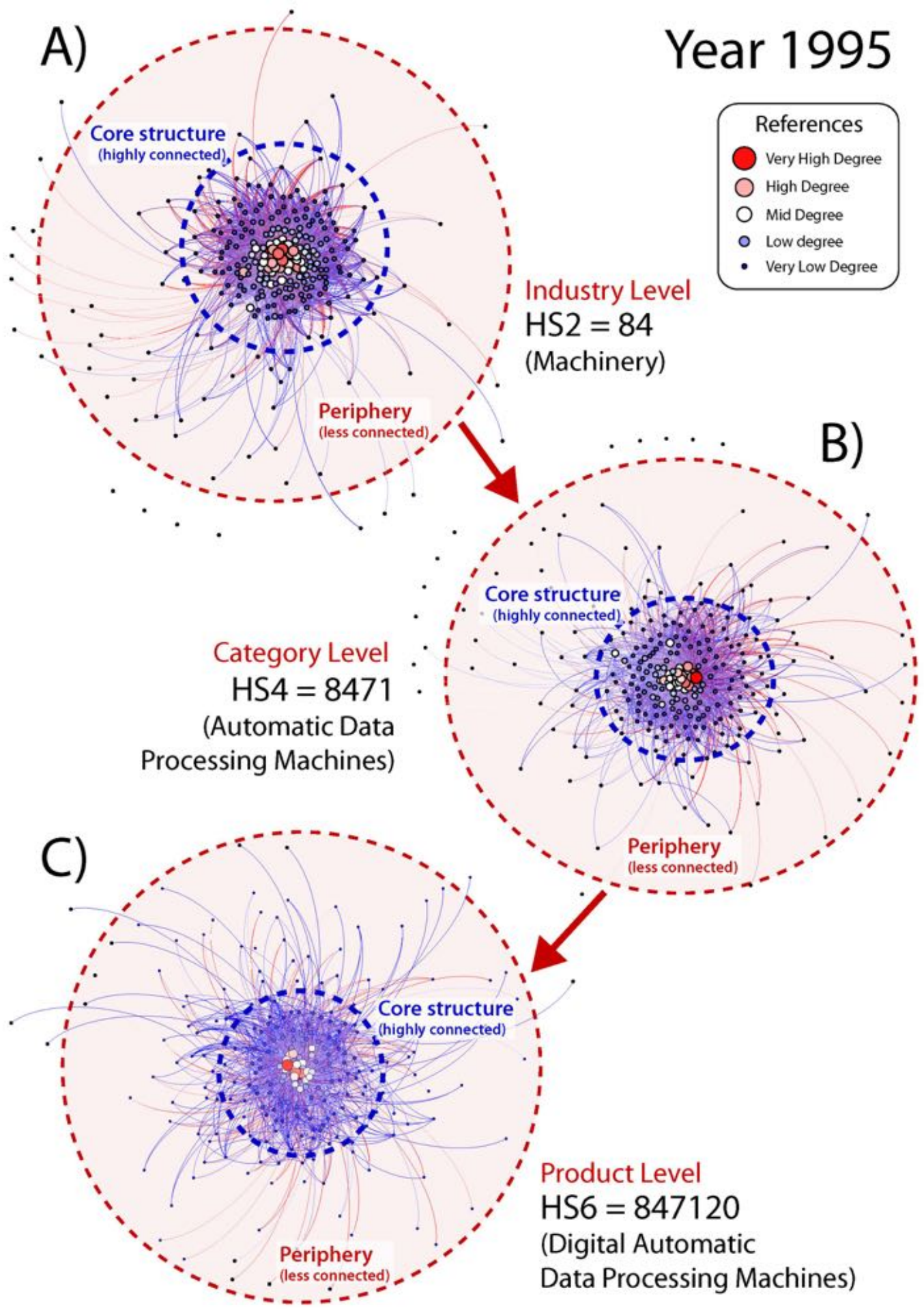

Fig. 7.2.: Trade networks at HS2, HS4 and HS6 levels of aggregation. This figure shows an example of a WBTN at HS2 level of aggregation. The network graph is for the 'Machinery' industry (HS2=84), for Data Processing Machines (HS4=8471) and for Computers (HS6 $=847120$ ). In each of the graphs, we observe two cleary differentiated parts of the topology, the core and the periphery. The core is formed by highly connected nodes, whereas the opposite is true for the periphery. This visualization does not provide information about the importance of the links and whether high degree nodes are connected by high-weight links, but we will investigate these topics throughout the chapter. 


\begin{tabular}{lllll}
\hline Year & $\begin{array}{l}\text { Links } \\
\text { (thousand) }\end{array}$ & $\begin{array}{l}\text { Volume } \\
\text { (billion) }\end{array}$ & $\begin{array}{l}\text { \% change } \\
\text { (links) }\end{array}$ & $\begin{array}{l}\text { \% change } \\
\text { (volume) }\end{array}$ \\
\hline 1995 & 4486.9 & 5043.9 & N/A & N/A \\
1996 & 4785.5 & 5337.7 & 6.7 & 5.8 \\
1997 & 5016.9 & 5512.5 & 4.8 & 3.3 \\
1998 & 5128.7 & 5467.1 & 2.2 & -0.8 \\
1999 & 5251.2 & 5667.2 & 2.4 & 3.7 \\
2000 & 5451.5 & 6393.9 & 3.8 & 12.8 \\
2001 & 5526.0 & 6187.5 & 1.4 & -3.2 \\
2002 & 5636.4 & 6444.9 & 2.0 & 4.2 \\
2003 & 5849.6 & 7485.4 & 3.8 & 16.1 \\
2004 & 5966.1 & 9210.3 & 2.0 & 23.0 \\
2005 & 6224.5 & 10347.8 & 4.3 & 12.4 \\
2006 & 6221.0 & 11872.1 & -0.1 & 14.7 \\
2007 & 6429.5 & 13658.7 & 3.4 & 15.0 \\
2008 & 6522 & 16069.1 & 1.4 & 17.6 \\
2009 & 5914.0 & 12016.1 & -9.3 & -25.2 \\
\hline
\end{tabular}

Tab. 7.1.: Links and trade volumes by year for HS2 industries. This table shows that number of links and trade volumes in the networks of all HS2 industries for each year. It also shows that year-over-year variance for the links and volumes.

cannot provide any certainty about the the key features of trade networks, we can however observe some characteristics that, once the evidence is provided about the bipartite indicators, can tell us directionally where and what to look for, in addition to hypothesize what specific features might be present in trade networks. These hypotheses are:

A) Core-Periphery Structure: trade networks are composed by two parts that are well defined in the topology. On the one hand, they present a core structure, which is highly-dense group of highly-connected nodes. On the other hand, the opposite is true for the periphery.

B) Similar structure across levels of aggregation: trade networks remain approximately with the same internal structure independently of the level of aggregatoin. Although this may appear a trivial fact, the evidence shall confirm, or not, that the coreperiphery structure remains present in trade networks regardless of their level of aggregation (product, category or industry). In fact, one of the hypotheses of this study is based on the premise that trade markets have statistically similar topologies because they are the result of a combination of persistent economic rules, like selfinterest and profit maximization, and rational decision-making, as well as perfect competition conditions.

C) Details are more evident for lower levels of aggregation: when comparing the machinery industry graph (for example, HS2 $=84$ ) with the computers product graph (HS6 $=847120$ ), we realize that the number of highly connected nodes is lower in the second case. Moreover, although the number of nodes that form the network 
core may be similar, the average degree seems to be much lower for the product level than that of the industry. Again, it may seem to be a trivial fact considering the product codes consolidation into categories and industries, but the key question will be whether the connectivity features, or degree distribution, vary from one level of aggregation to the next and, if so, in which way.

D) Trade networks have long-tailed, heterogeneous degree distribution: by visualizing the HS2, HS4 and HS6 graphs in figure 7.2, we observe that some nodes are distinctively more connected than others. We may think of this as a Pareto law, where a few nodes have the majority of the connections. The actual form of $\mathrm{P}(\mathrm{K})$ will be extensively discussed in this chapter.

E) The most important links are located within the network core: if we consider the previous point, we could hypothesize, by extension, that high values of RSP and RPP will be found almost exclusively at the core of the network, whereas the periphery will mostly be formed by low RSP and RPP links, thus creating a conceptual gradient of RSP and RPP towards the center of the network.

E) Clustering considerations about the core and periphery: we also observe that the core of the network is more dense in terms of number of links, leading to the idea of a higher clustering than that of the periphery. Yet, more links does not necessarily mean that these links will form a larger number of clusters. We need to test this through the bipartite clustering indicator.

F) Little nestedness perception: we have referred to topological properties, namely network connectivity and even clustering features. Yet, in contrast, this visualization does not provide much information about nestedness. This is another area to apply our methodology to gather more insights on evidence and results.

With these concepts as an initial assessment of how trade networks might look like, and also what their topological properties might be, we now need to set a framework to apply our bipartite methodology described in chapter 5 . At this point, we realize that it would be impractical, at least, to try to manage the complexity of 195,200 scenarios we have available from our data. Consequently, we define a set of selected HS2 codes or industries to apply our methodology throughout the study (and in the following chapters) as a representative sample of vast amount of trade data. These industries have been selected so that we present results from a broad and diverse type of markets. Some of them are markets with high-density networks, others are more sparse. Some industries represent a commodity market of basic products, others are markets with complex products. In table 7.2, we show the list of industries based on our selection criteria, which is a random selection across the 97 industries so that we make sure that all types of product are represented in the 


\begin{tabular}{|c|c|c|}
\hline HS2 code & Industry & Full description \\
\hline 01 & Live animals & Live animals \\
\hline 10 & Cereals & Cereals \\
\hline 20 & Vegetables and Fruit & $\begin{array}{l}\text { Prep of vegetable, fruit, nuts or other } \\
\text { parts of plants. }\end{array}$ \\
\hline 35 & Glues and Enzymes & $\begin{array}{l}\text { Albuminoidal subs; modified starches; } \\
\text { glues; enzymes }\end{array}$ \\
\hline 50 & Silk & Silk \\
\hline 62 & Apparel & $\begin{array}{l}\text { Articles of apparel and clothing access, } \\
\text { not knitted or crocheted. }\end{array}$ \\
\hline 75 & Nickel & Nickel and articles thereof. \\
\hline 84 & Machinery & $\begin{array}{l}\text { Nuclear reactors, boilers, machinery and } \\
\text { mechanical appliance; parts }\end{array}$ \\
\hline
\end{tabular}

Tab. 7.2.: Selected HS2 industries for the section. This table shows selected HS2 industries for the section to cover a broad range of different products.

sample, from basic commodities to complex industrial goods, and from minerals to high-end technology. Furthermore, we take the data for these selected industries and create visualizations for each of them with a bipartite and weighted layout. This layout is built with out cricular layout from the visualization chapter. In figures 7.3, we do not only show the trade network visualization for the first period (1995), but also, we include the visualizations for 1997, 1999, 2002, 2005, 2007 and 2009, in order to show depict how the networks evolve over time. In appendix $\mathrm{C}$, the full collection of visualizations for the HS2 selected industries can be found (figures C.1 through C.8). Once again, after visualizing the overall structure and patterns that arise from these selected industries and the new bipartite and weighted layout, we can observe that most of features previously hypothesized in this section may still be present in these 56 scenarios ( 8 industries and 7 periods). Yet, being an exploratory analysis, we lack the hard evidence to confirm or reject such statements. We now have to move forward to the formal implementation of our bipartite methodology so that results can speak for themselves.

\subsection{Trade network results at HS2 industry level}

We now focus on the results obtained from the level of aggregation HS2 of our dataset after computing the set of measures defined on chapter 5 . For this review of HS2 industries, we use the 8 selected codes. This will be recurrent across the sections and we will applied this to both scenarios, unfiltered and filtered, as well as the selected periods in particular. These selected periods have also been chosen so that we show the first year in our dataset and then we set a time step to cover homogeneously the 15 years of data, namely 1995, 1999, 2004 and 2009. 

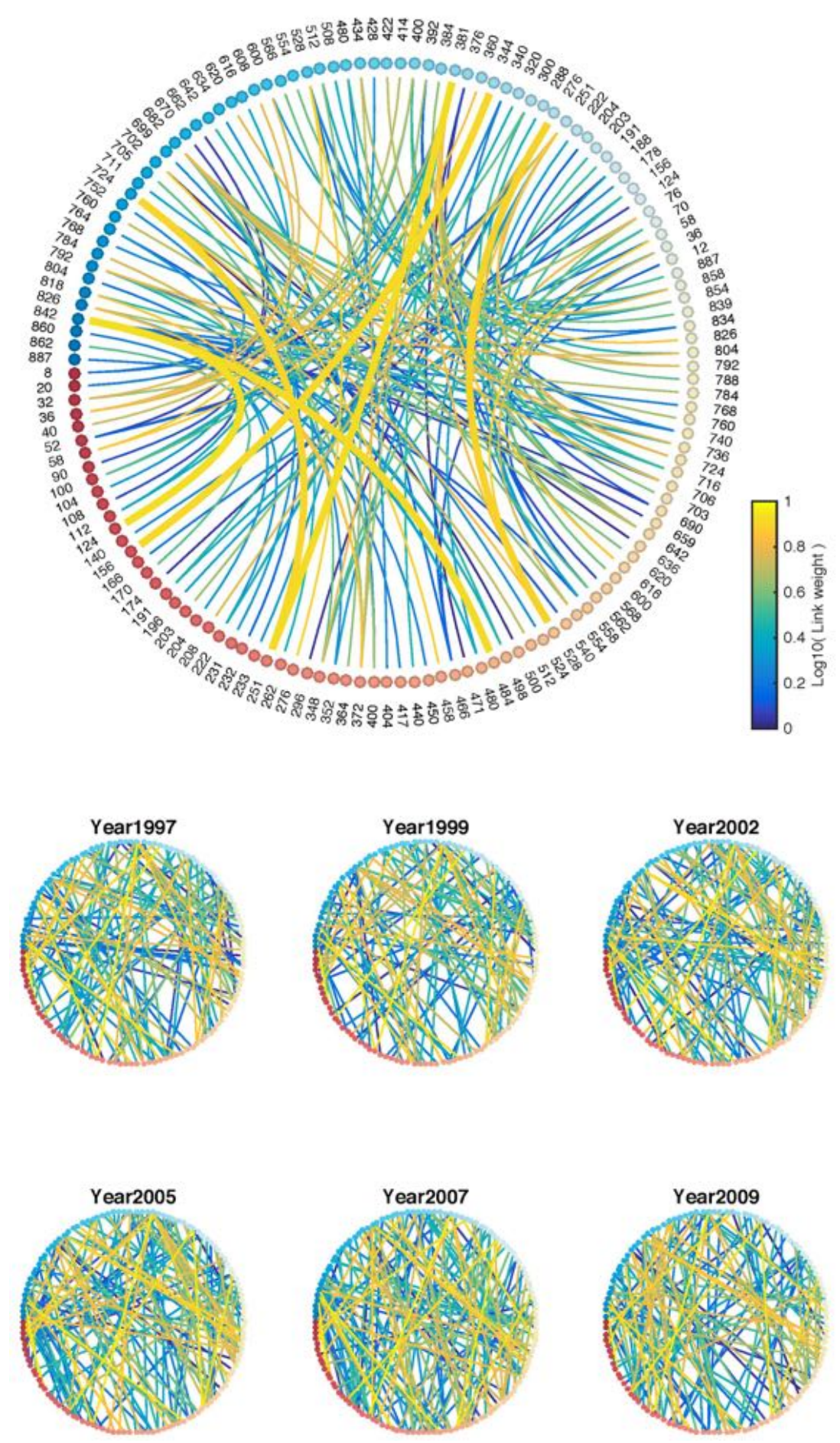

Fig. 7.3.: Selected HS2 $=01$ trade network visualization. This figure shows the circular layout visualization for the 'Live Animals' industry (HS2 $=01$ ). We set a threshold of $R S P>0.1$ and $R P P>0.1$ for visible links. Exporters are located at the bottom of the graph with a declining red color gradient. Importers are located on the top of the graph with a declining blue color gradient. The thickness of the line is proportional to the weight of the link. Note: the importers start at approximate 2 o'clock between the last exporter (887) and the first importer (12). The label of the node is the ISO country code. 

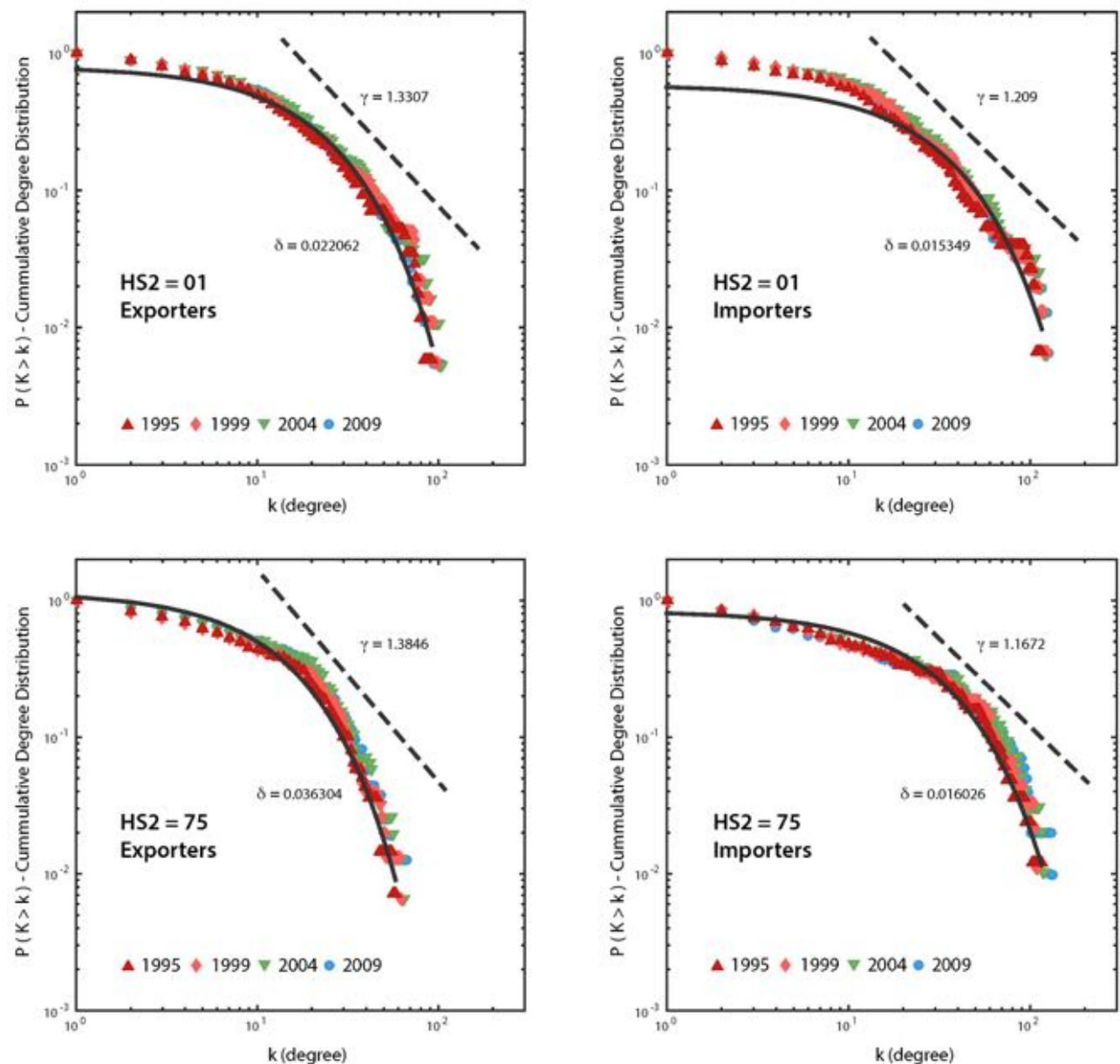

Fig. 7.4.: Cummulative Degree Distribution without RSP and RPP filters. This figure shows the $\mathrm{P}(\mathrm{K})$ degree distribution for 2 selected HS2 industries (01 and 75). On the right, we show the exporter subset results, whereas on the left, we show the importers performance. The $\mathrm{P}(\mathrm{K})$ performances include the results from 1995 , 1999, 2004 and 2009. We test 2 patterns: a) a power law of the form $P(K) \sim k^{-\gamma}$ and b) an exponential decay of the form $P(K) \sim 10^{-\delta k}$. Results for all selected industries HS2 can be found in Appendix C and D.

\subsubsection{Degree distribution, $\mathrm{P}(\mathrm{K})$}

The degree distribution has been defined previously in section 5.1 as a property of a network that characterizes the connectivity features of a network. We conduct a thorough examination of these features for the HS2 industries. We start with distributions of the selected industries HS2 during the selected periods. In this first analysis, we do not apply any RSP or RPP filter. Figure 7.4 shows the results for the cummulative degree distribution $P(K)$ for 2 example industries. In appendix $\mathrm{C}$ and $\mathrm{D}$, the results for all HS2 selected industries can be found.

We clearly observe that all the industries display heterogeneous probability distributions. We also realize that the connectivity patterns do not significantly change 


\begin{tabular}{ccccc}
\hline HS2 & $\begin{array}{c}\text { Exporters } \\
\text { Power Law } \\
\alpha \text { exponent } \\
\text { Industry } \\
\text { Code }\end{array}$ & $\begin{array}{c}\text { Unfiltered } \\
R^{2} \\
\text { (power law) }\end{array}$ & $\begin{array}{c}\text { Exporters } \\
\text { Exponential } \\
\delta \text { exponent } \\
P(K) \sim k^{-\alpha}\end{array}$ & $\begin{array}{c}\text { Unfiltered } \\
R^{2} \\
\text { (exponential) }\end{array}$ \\
\hline 01 & $1.33 \pm 0.07$ & 0.792 & $0.0221 \pm 0.0004$ & 0.964 \\
10 & $1.58 \pm 0.12$ & 0.745 & $0.0382 \pm 0.0006$ & 0.983 \\
20 & $1.43 \pm 0.08$ & 0.748 & $0.0214 \pm 0.0002$ & 0.987 \\
35 & $1.37 \pm 0.13$ & 0.651 & $0.0349 \pm 0.0011$ & 0.945 \\
50 & $1.48 \pm 0.12$ & 0.749 & $0.0433 \pm 0.0009$ & 0.977 \\
62 & $1.40 \pm 0.07$ & 0.718 & $0.0149 \pm 0.0002$ & 0.974 \\
75 & $1.38 \pm 0.10$ & 0.765 & $0.0363 \pm 0.0006$ & 0.984 \\
84 & $1.33 \pm 0.07$ & 0.689 & $0.0131 \pm 0.0002$ & 0.978 \\
\hline
\end{tabular}

Tab. 7.3.: Exporters $\mathrm{P}(\mathrm{K})$ regression model fitting, unfiltered. This table shows the power law and the exponential regression models for the exporters subset, including their $R^{2}$ performance.

\begin{tabular}{ccccc}
\hline $\begin{array}{c}\text { HS2 } \\
\text { Industry } \\
\text { Code }\end{array}$ & $\begin{array}{c}\text { Importers } \\
\text { Power Law } \\
\alpha \text { exponent } \\
P(K) \sim k^{-\alpha}\end{array}$ & $\begin{array}{c}\text { Unfiltered } \\
R^{2} \\
\text { (power law) }\end{array}$ & $\begin{array}{c}\text { Importers } \\
\text { Exponential } \\
\delta \text { exponent } \\
P(K) \sim 10^{-\delta k}\end{array}$ & $\begin{array}{c}\text { Unfiltered } \\
R^{2} \\
\text { (exponential) }\end{array}$ \\
\hline 01 & $1.21 \pm 0.05$ & 0.842 & $0.0153 \pm 0.0004$ & 0.931 \\
10 & $1.28 \pm 0.04$ & 0.834 & $0.0119 \pm 0.0001$ & 0.983 \\
20 & $0.97 \pm 0.04$ & 0.764 & $0.0099 \pm 0.0002$ & 0.963 \\
35 & $1.08 \pm 0.06$ & 0.693 & $0.0107 \pm 0.0002$ & 0.952 \\
50 & $0.82 \pm 0.04$ & 0.822 & $0.0129 \pm 0.0004$ & 0.906 \\
62 & $1.05 \pm 0.05$ & 0.716 & $0.0093 \pm 0.0002$ & 0.928 \\
75 & $1.17 \pm 0.06$ & 0.760 & $0.0160 \pm 0.0002$ & 0.984 \\
84 & $0.83 \pm 0.04$ & 0.687 & $0.0065 \pm 0.0002$ & 0.867 \\
\hline
\end{tabular}

Tab. 7.4.: Importers $\mathrm{P}(\mathrm{K})$ regression model fitting, unfiltered. This table shows the power law and the exponential regression models for the importers subset, including their $R^{2}$ performance. The first column is the industry HS2 code.

over time for any given industry. But a deeper discussion is required to determine the actual nature of the $\mathrm{P}(\mathrm{K})$ patterns. It can be argued that, in some of the industries, the form of $\mathrm{P}(\mathrm{K})$ is consistent with a power-law, but this is valid only in a limited range of the degree domain. In other cases, we may argue that the form of $\mathrm{P}(\mathrm{K})$ might be consistent with an exponential decay. For this reason, we test both regression models (power law and exponential decay) and select the best fit based on the highest coefficient of determination $R^{2}$. We perform this for the all selected industries HS2, and for the exporters subset, we show the results on table 7.3.

The results obtained for the exporter subset is consistent with those of the observed outcome of the $\mathrm{P}(\mathrm{K})$ performances on figure 7.4, where we do not find any linear pattern that leads to a pure power law on the log-log scaled diagram. Consequently, we find that the exponenial model is the best fit for this subset since the values of $R^{2}$ are mostly higher than 0.95 for all selected industries HS2. In a similar way, we perform the same process for the importers subset and show the results on the table 7.4 . 


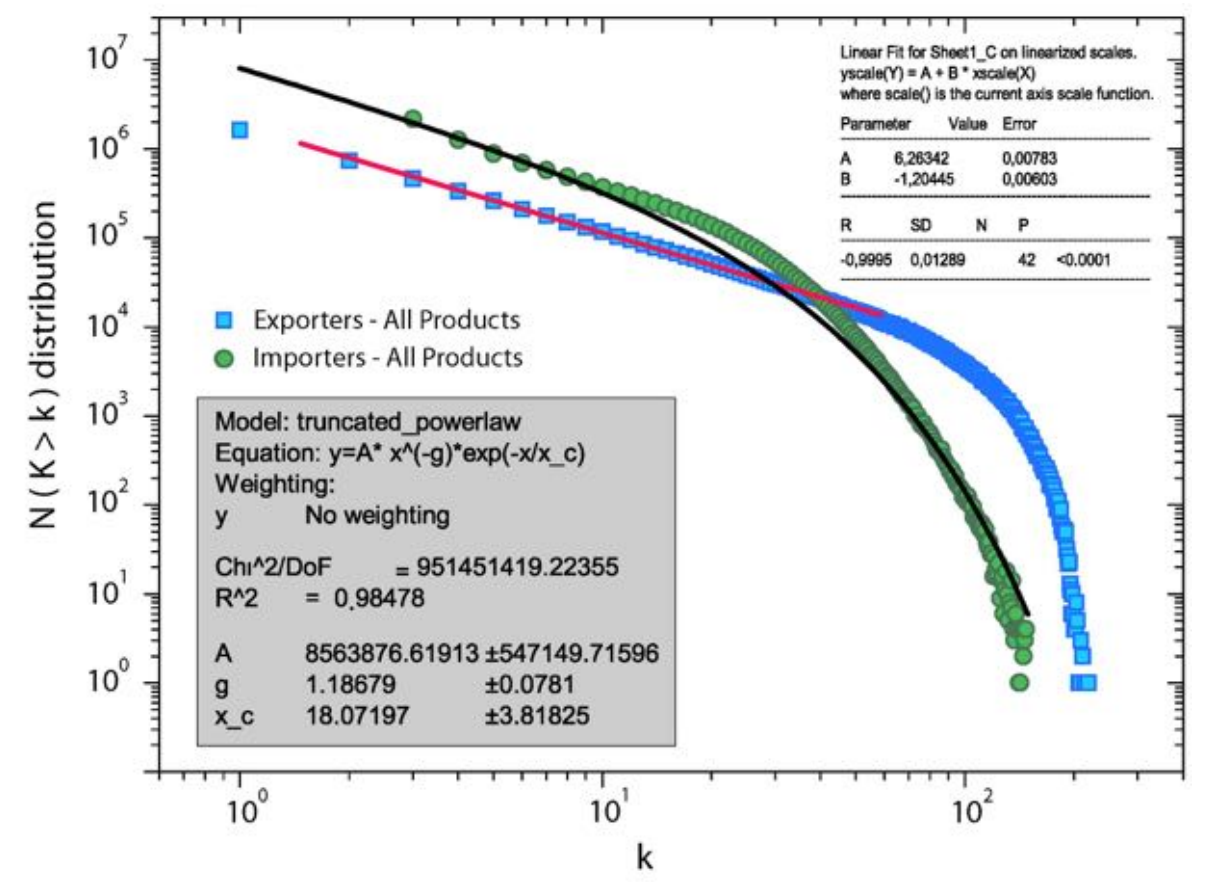

Fig. 7.5.: Degree distribution for all industries and periods (unfiltered). This figure shows the cumulative degree distributions for exporters and for all products. M. Fernandez et al.[61]

Once again, the importers subset also displays results that are consistent with an exponential decay model as the best fit. It is important to note that both the exporter and the importer subsets display different $\alpha$ and $\delta$ exponents. This means that the connectivity patterns of one subset are different than those of the other. We will later discuss how the assymetric structure of markets, where the number of exporters is in general different than the number of importers, may play a role in this finding. In sum, we conclude that, for the HS2 level of aggregation, the best fit for the cumulative degree distribution $P(K)$ is an exponential decay of the form $P(K) \sim 10^{-\delta k}$, where we find performances of $R^{2}$ that are typically higher than 0.90 for both the exporters and the importers subsets. Additionally, we can speculate that the issue of finite data may also play a role here since we do not have one very large dataset for any one particular industry. Nonetheless, we could still consolidate the results for all the industries and all periods to analyze a broader spectrum of networks and determine whether a different pattern of the "average industry" may still exist. In this case, we test again several models: a) the power law, b) the truncated power law, and c) the exponential decay. In figure 7.5, we show the results with the best fit for the exporters and the importers. For the exporters, the best fit is a power law of with an exponent $\gamma_{e}=1.20$. Nonetheless, for the importers, the best fit is a truncanted power law of the form $N(K>k) \sim k^{-\gamma} e^{-k / k_{c}}$, with $\gamma_{i}=1.19$ and $k_{c}=18.07$. 


\begin{tabular}{ccccc}
\hline HS2 & $\begin{array}{c}\text { Exporters } \\
\text { Power Law } \\
\alpha \text { exponent } \\
\text { Industry } \\
\text { Code }\end{array}$ & $\begin{array}{c}\text { Filtered } \\
R^{2} \\
\text { (power law) }\end{array}$ & $\begin{array}{c}\text { Exporters } \\
\text { Exponential } \\
\delta \text { exponent } \\
P(K) \sim k^{-\alpha}\end{array}$ & $\begin{array}{c}\text { Filtered } \\
R^{2} \\
\text { (exponential) }\end{array}$ \\
\hline 01 & $1.34 \pm 0.07$ & 0.828 & $0.0255 \pm 0.0006$ & 0.960 \\
10 & $1.54 \pm 0.12$ & 0.792 & $0.0480 \pm 0.0010$ & 0.982 \\
20 & $1.27 \pm 0.06$ & 0.828 & $0.0200 \pm 0.0002$ & 0.989 \\
35 & $1.33 \pm 0.10$ & 0.753 & $0.0356 \pm 0.0007$ & 0.980 \\
50 & $1.37 \pm 0.11$ & 0.812 & $0.0485 \pm 0.0014$ & 0.969 \\
62 & $1.28 \pm 0.06$ & 0.763 & $0.0144 \pm 0.0003$ & 0.953 \\
75 & $1.17 \pm 0.09$ & 0.786 & $0.0367 \pm 0.0006$ & 0.987 \\
84 & $1.42 \pm 0.06$ & 0.808 & $0.0160 \pm 0.0002$ & 0.986 \\
\hline
\end{tabular}

Tab. 7.5.: Exporters $\mathrm{P}(\mathrm{K})$ regression model fitting, filtered scenario. This table shows the power law and the exponential regression models for the exporters subset after applying the RSP and RPP filters, including their $R^{2}$ performance.

We have reviewed the results for the unfiltered scenario $(R S P>0$ and $R P P>0)$. We now focus on the cumulative degree distribution with RSP and RPP filters $\left(R S P>10^{-3}\right.$ and $\left.R P P>10^{-3}\right)$. This analysis excludes the non-vital links of low relative importance from the trade networks, which will then help us understand the $P(K)$ performance within the core of the network. Once again, we apply the process to 2 selected industries HS2 and the selected periods. We show the results on figure 7.6.

Here, we observe once again heterogeneous, long-tailed distribution patterns, similarly to the unfiltered scenario, but we test whether the core of the network presents a power law or exponential decay pattern. Then, for the exporters, we show the results on the table 7.5 .

As observed, we find a similar situation in comparison to the unfiltered scenario. The exponential decay remains as the best fit for the exporters subset. Yet, the $\alpha$ and $\delta$ exponent values also remain quite close to those of the total network. Now, we show the results for the importers in the filtered secenario in the table 7.6.

Once again, we conclude that the exponential decay is a better fit for the degree distribution of the importers subset in the filtered scenario. The results do not change significantly over time, but they do vary in the filtered scenario. As a matter of fact, $\alpha$ exponent values of the the importers have significant change, leading to the conclusion that the core of the network has a different connectivity performance than that of the whole network from the importers perspective. For the exporters, the $\alpha$ exponent values remain bearly unchanged. By implication, the connectivity features of the whole network and those of the core, in particular, may not necessarily be the same. This is revealing piece of evidence, which we will review in the conclusions. Our initial hypothesis for such a variance is that the importer countries have an increased power over the market when they become high volume buyers. Moreover, 

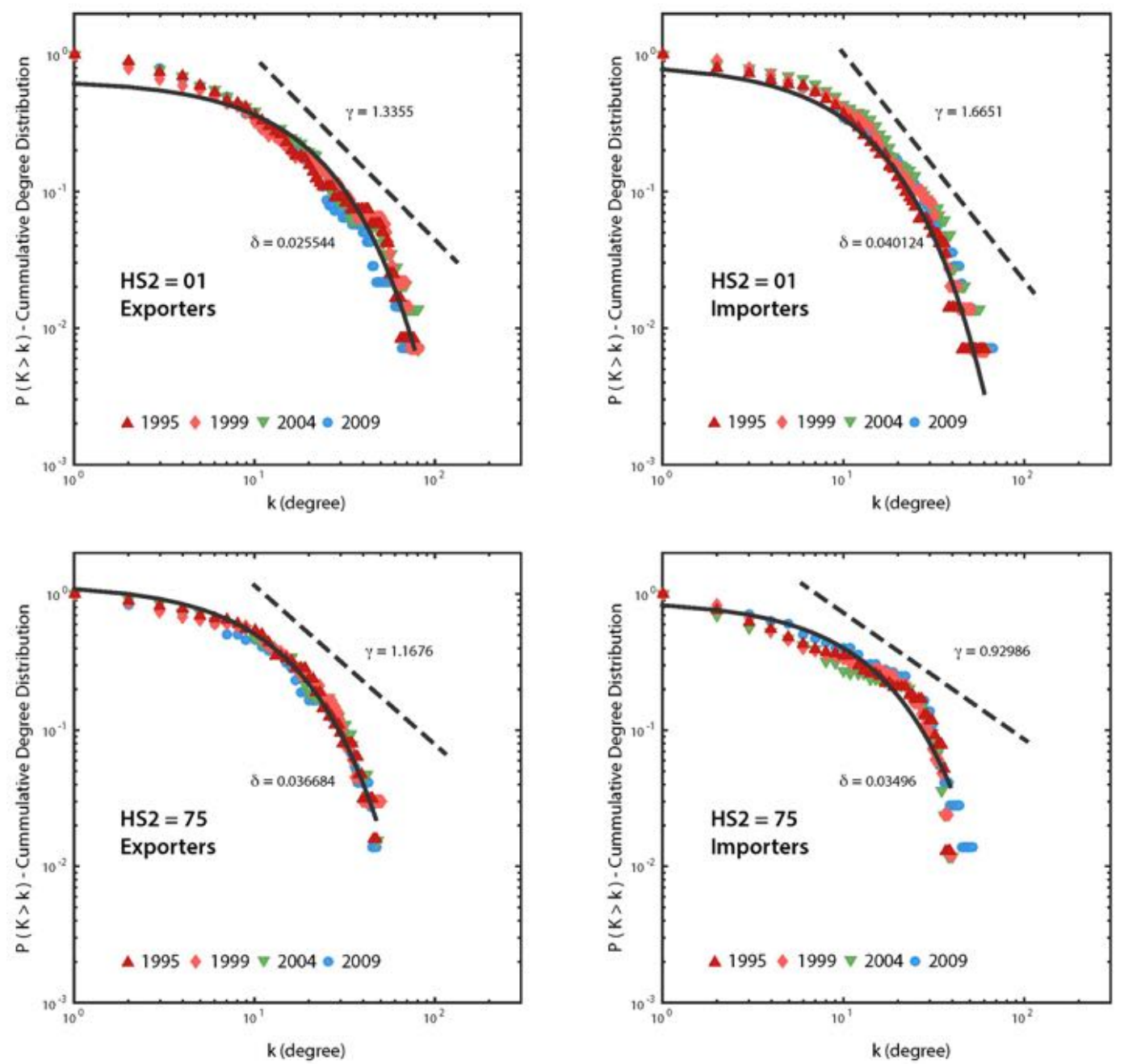

Fig. 7.6.: Cummulative Degree Ditribution with RSP and RPP filters. This figure shows the $\mathrm{P}(\mathrm{K})$ degree distribution for 2 selected HS2 industries (01 and 75). On the right, we show the exporter subset results, whereas on the left, we show the importers performance. The $\mathrm{P}(\mathrm{K})$ performances include the results from 1995, 1999, 2004 and 2009. We test 2 patterns: a) a power law of the form $P(K) \sim k^{-\gamma}$ and b) an exponential decay of the form $P(K) \sim 10^{-\delta k}$. Results for all selected industries HS2 can be found in Appendix C and D. 


\begin{tabular}{ccccc}
\hline $\begin{array}{c}\text { Importers } \\
\text { HS2 } \\
\text { Industry } \\
\text { Code }\end{array}$ & $\begin{array}{c}\text { Filtered } \\
\alpha \text { exponent } \\
P(K) \sim k^{-\alpha}\end{array}$ & $\begin{array}{c}R^{2} \\
\text { (power law) }\end{array}$ & $\begin{array}{c}\text { Importers } \\
\text { Exponential } \\
\delta \text { exponent } \\
P(K) \sim 10^{-\delta k}\end{array}$ & $\begin{array}{c}\text { Filtered } \\
R^{2} \\
\text { (exponential) }\end{array}$ \\
\hline 01 & $1.67 \pm 0.10$ & 0.839 & $0.0401 \pm 0.0009$ & 0.971 \\
10 & $1.31 \pm 0.05$ & 0.877 & $0.0202 \pm 0.0003$ & 0.977 \\
20 & $1.40 \pm 0.09$ & 0.757 & $0.0253 \pm 0.0003$ & 0.986 \\
35 & $1.45 \pm 0.09$ & 0.735 & $0.0256 \pm 0.0006$ & 0.949 \\
50 & $1.21 \pm 0.05$ & 0.896 & $0.0232 \pm 0.0007$ & 0.941 \\
62 & $1.47 \pm 0.08$ & 0.819 & $0.0276 \pm 0.0004$ & 0.985 \\
75 & $0.93 \pm 0.11$ & 0.649 & $0.035 \pm 0.003$ & 0.832 \\
84 & $1.28 \pm 0.10$ & 0.674 & $0.0273 \pm 0.0010$ & 0.916 \\
\hline
\end{tabular}

Tab. 7.6.: Importers $\mathrm{P}(\mathrm{K})$ regression model fitting, filtered scenario. This table shows the power law and the exponential regression models for the importers subset after applying the RSP and RPP filters, including their $R^{2}$ performance.

this behavior requires further in-depth evidence to either support or reject our initial hypothesis.

\subsubsection{Strength-degree correlation, $\mathrm{S}(\mathrm{K})$}

The strength-degree correlation is a fundamental network property that enables us to determine the actual weighted nature of our trade networks. In the introduction, we have hypothesize that we may find similarities between markets and mutualistic ecosystems. If this premise is found to be true, we would confirm that international trade markets, like mutualitic ecosystems, have a highly dense core of highly interconnected nodes and that these nodes also are connected to each other with high weights, a situation that is consistent with a weighted equivalence to the phenomenon known as "the rich club effect", which states that networks will have many connections between nodes of high degree. This effet is also known as "the rich gets richer" and, in our case, high-degree nodes would not only be connected between them, but also would do so with links of high weights. For this reason, it is critical that we tackle the issue and perform the analysis for the 2example industries HS2 (01 and 75) during the selected periods. We show the results of such analysis in figure 7.7.

We observe that a clear correlation is found for the 2 example industries. We also observe that the behavior of a given industry does not change significantly over time, leading to the idea of the $S(K)$ will remain virtually unchanged as network property. Yet, we still need to determine the nature of the correlation and whether weights are random distributed across the links. For this purpose, we propose a power law model fitting of the form $S(K) \sim k^{\beta}$. If weights are randomly distributed across the network, we should expect a performance of $\beta=1$ approximately. We show the results for the selected industries HS2 on table 7.7. 

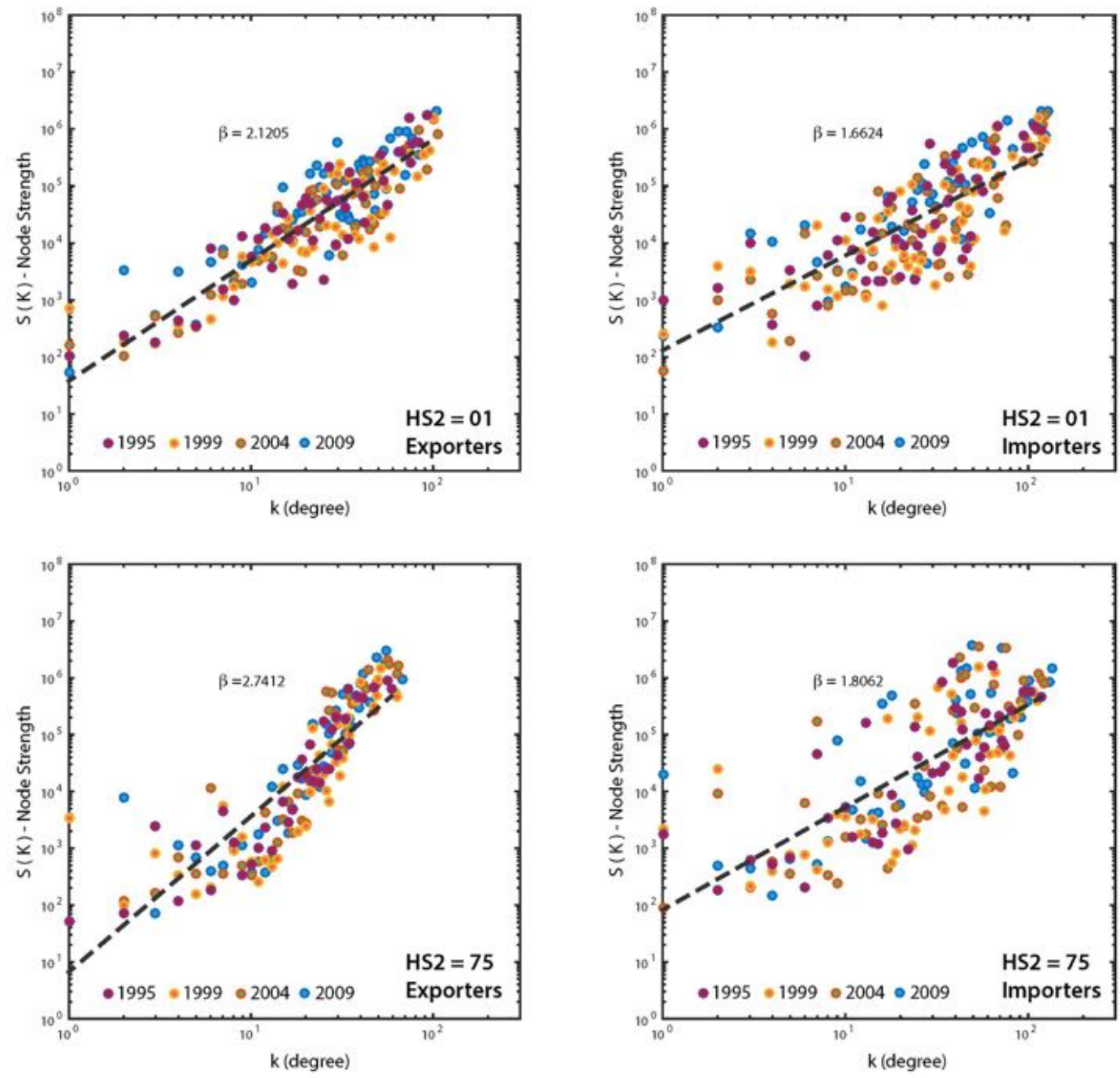

Fig. 7.7.: Strength-Degree correlation, unfiltered scenario. This figure shows the strengthdegree correlation for 2 selected HS2 industries (01 and 75) over the selected periods. We test a power law pattern of the form $S(K) \sim k^{\beta}$. Results for the rest of the selected industries can be found in Appendix $C$ and $D$. 


\begin{tabular}{ccccc}
\hline $\begin{array}{c}\text { HS2 } \\
\text { Industry } \\
\text { Code }\end{array}$ & $\begin{array}{c}\text { Exporters } \\
\beta \text { exponent } \\
S(K) \sim k^{-\beta}\end{array}$ & $\begin{array}{c}R^{2} \\
\text { (Exporters) }\end{array}$ & $\begin{array}{c}\text { Importers } \\
\beta \text { exponent } \\
S(K) \sim k^{-\beta}\end{array}$ & $\begin{array}{c}R^{2} \\
\text { (Importers) }\end{array}$ \\
\hline 01 & $2.12 \pm 0.15$ & 0.825 & $1.66 \pm 0.21$ & 0.582 \\
10 & $1.98 \pm 0.16$ & 0.763 & $1.94 \pm 0.14$ & 0.787 \\
20 & $2.23 \pm 0.11$ & 0.858 & $2.10 \pm 0.09$ & 0.890 \\
35 & $2.6 \pm 0.2$ & 0.762 & $1.88 \pm 0.10$ & 0.874 \\
50 & $2.4 \pm 0.2$ & 0.781 & $1.7 \pm 0.2$ & 0.685 \\
62 & $2.51 \pm 0.11$ & 0.868 & $2.23 \pm 0.10$ & 0.845 \\
75 & $2.7 \pm 0.2$ & 0.813 & $1.8 \pm 0.2$ & 0.622 \\
84 & $2.73 \pm 0.13$ & 0.826 & $2.76 \pm 0.12$ & 0.828 \\
\hline
\end{tabular}

Tab. 7.7.: $\mathrm{S}(\mathrm{K})$ regression model fitting, unfiltered scenario. This table shows the power law regression models for both the exporter and the importer subsets with no filters, including their $R^{2}$ performance. The first column is the industry HS2 code. The second column shows the exporters power law exponent with their margins of error. The third one is the determination coefficient of the exporters power law model. The fourth and fifth columns are the same as columns 2 and 3, but for the importers model.

We observe that $\beta$ exponent values that are higher than 1 , providing robust evidence that, in trade networks, highly-connected nodes will also connect to others by means of high-volume or high-weight links. This is valid for both the exporters and the importers with a coefficient of determination $R^{2}$ higher than 0.75 in the majority of the cases, which can reach values of up to 0.90 . We now perform the same analysis for the filtered scenario, where we display the behavior of the core of the network on figure 7.8 .

We observe similar patterns than we do in the unfiltered scenario. The power law is the best fit for the filtered scenario. On table 7.8, we show the model fitting for both the exporters and the importers.

We find that the exponents at the core of the network are still higher than 1, but the values for these exponents are slightly different than those of the whole network. Yet, in contrast to the observed behavior of the degree distribution, the strength-degree correlation show a significant decrease of the $\beta$ exponents of the exporters. Once again, we need to perform further analysis to determine the effect of the filters on $S(K)$. For this reason, and similarly to the $P(K)$ measure, we build a histogram for all industries HS2 over the selected periods to shed some light upon this issue. We show the results on figure 7.9.

The evidence points to a modified distribution of the $\beta$ exponents of all industries HS2 in the case of the exporters. However, we only observe a minimum variance of the importers histogram. We will review this finding in the conclusions, yet our hypothesis from the $P(K)$ behavior of importers may also play a role in the $S(K)$. 

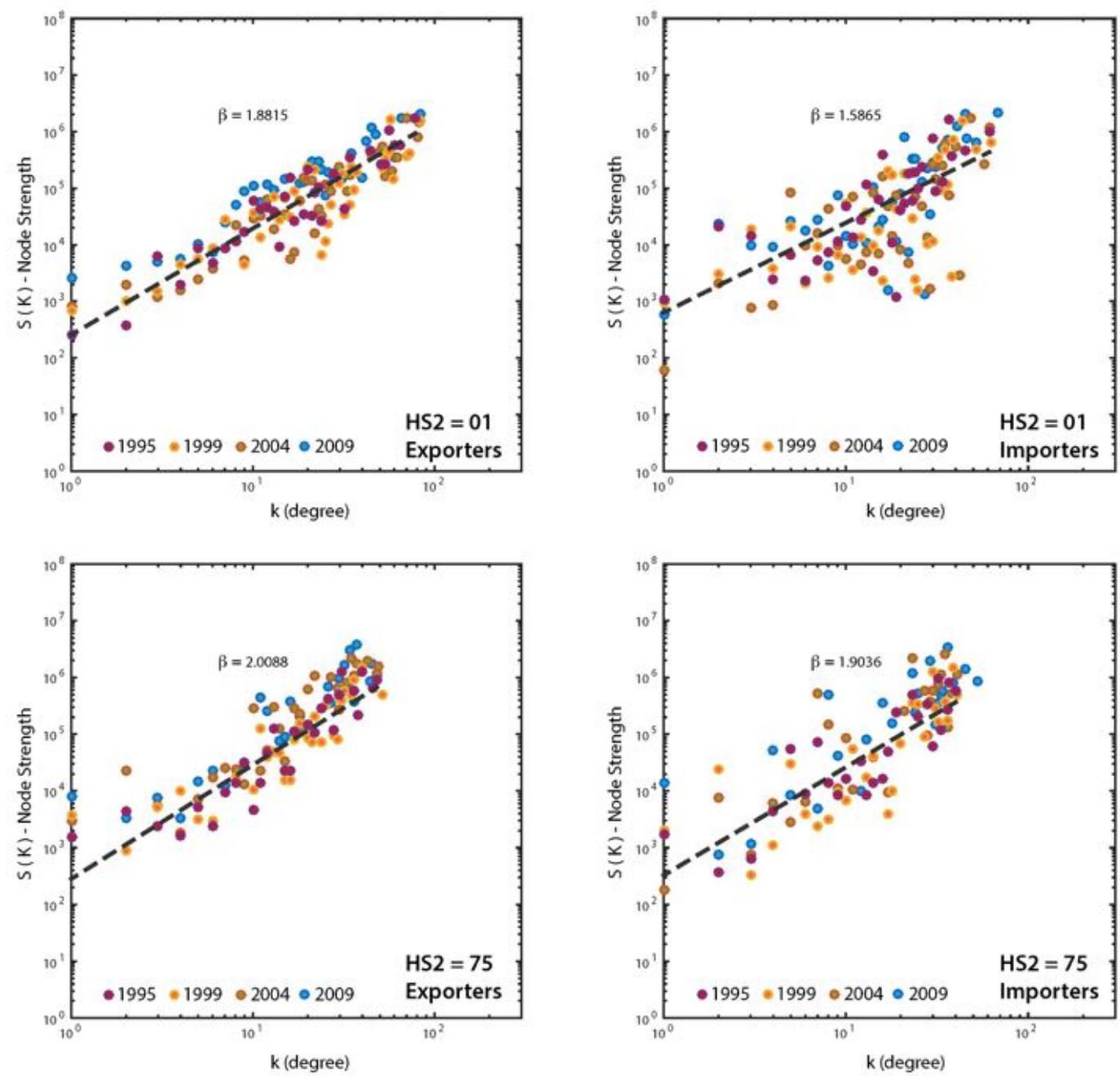

Fig. 7.8.: Strength-Degree correlation with RSP and RPP filters. This figure shows the strength-degree correlation for 2 selected HS2 industries (01 and 75) over the selected periods. We test a power law pattern of the form $S(K) \sim k^{\beta}$. Results for the rest of the selected industries can be found in Appendix C and D. 

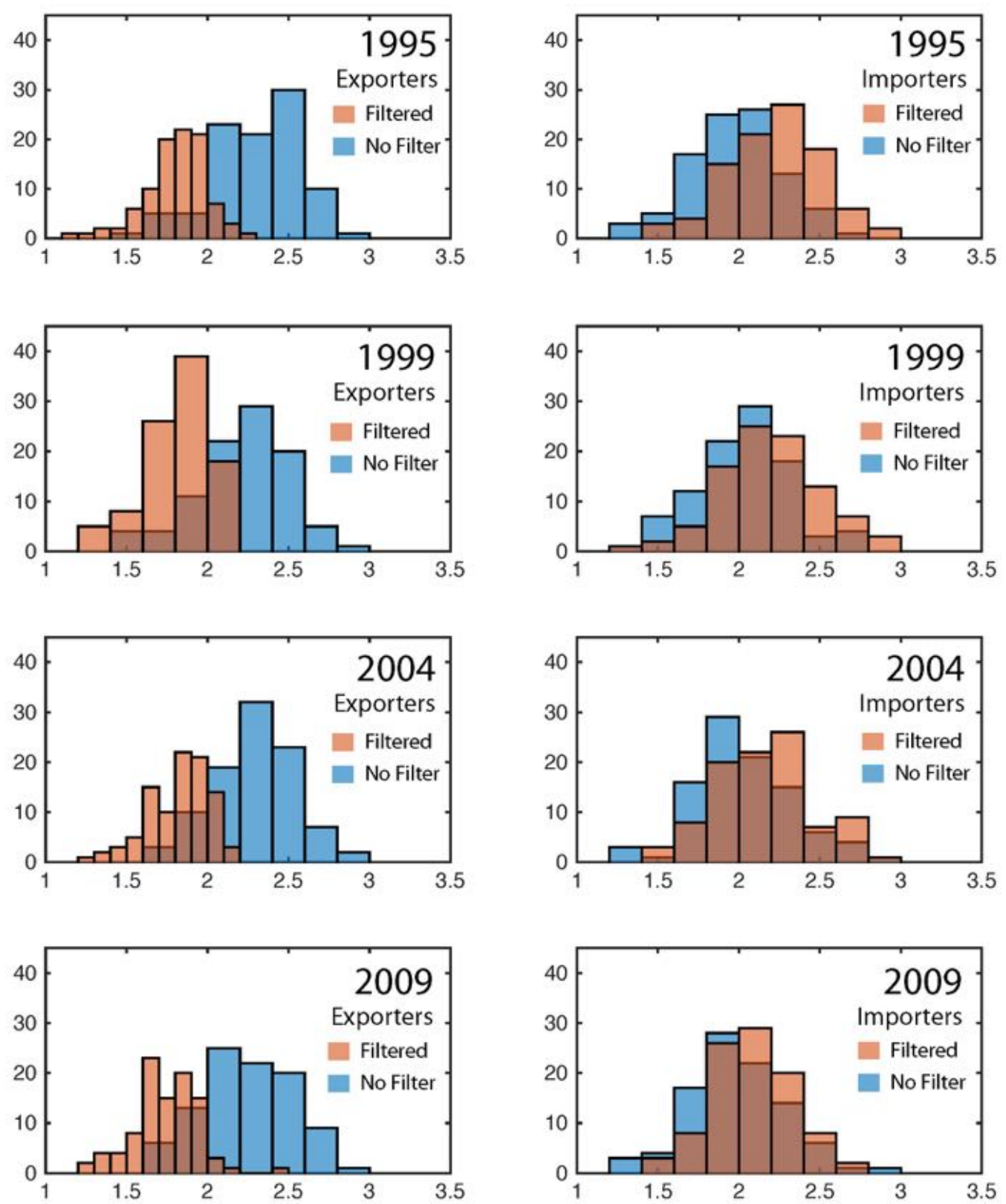

Fig. 7.9.: Beta exponent results for all HS2 industries. This figure shows the frequency of the $\beta$ exponents for all HS2 industries for the exporters (left) and importers (right). 


\begin{tabular}{ccccc}
\hline $\begin{array}{c}\text { HS2 } \\
\text { Industry } \\
\text { Code }\end{array}$ & $\begin{array}{c}\text { Exporters } \\
\beta \text { exponent } \\
S(K) \sim k^{-\beta}\end{array}$ & $\begin{array}{c}R^{2} \\
\text { (Exporters) }\end{array}$ & $\begin{array}{c}\text { Importers } \\
\beta \text { exponent } \\
S(K) \sim k^{-\beta}\end{array}$ & $\begin{array}{c}R^{2} \\
\text { (Importers) }\end{array}$ \\
\hline 01 & $1.88 \pm 0.13$ & 0.877 & $1.6 \pm 0.3$ & 0.545 \\
10 & $1.46 \pm 0.17$ & 0.725 & $2.01 \pm 0.14$ & 0.847 \\
20 & $1.89 \pm 0.10$ & 0.876 & $2.24 \pm 0.15$ & 0.823 \\
35 & $2.08 \pm 0.13$ & 0.867 & $2.29 \pm 0.13$ & 0.882 \\
50 & $2.13 \pm 0.15$ & 0.892 & $2.09 \pm 0.19$ & 0.850 \\
62 & $1.75 \pm 0.10$ & 0.874 & $2.29 \pm 0.16$ & 0.822 \\
75 & $2.01 \pm 0.18$ & 0.831 & $1.9 \pm 0.2$ & 0.743 \\
84 & $2.01 \pm 0.11$ & 0.856 & $2.8 \pm 0.2$ & 0.735 \\
\hline
\end{tabular}

Tab. 7.8.: $\mathrm{S}(\mathrm{K})$ regression model fitting, filtered scenario. This table shows the power law regression models for both the exporter and the importer subsets with RSP and RPP filters, including their $R^{2}$ performance. The first column is the industry HS2 code. The second column shows the exporters power law exponent with their margins of error. The third one is the determination coefficient of the exporters power law model. The fourth and fifth columns are the same as columns 2 and 3 , but for the importers model.

Having analyzed all aspects of the strength-degree correlation, we now focus on the assortative and clustering properties of trade networks.

\subsubsection{Assortative properties of trade networks, $\mathrm{Knn}(\mathrm{K})$}

The assortative properties in a complex network can provide evidence of a preference to connect with other nodes with similar characteristics. Thus, we implement this bipartite indicator to 2 selected industries HS2 (01 and 75) and for the selected periods, and present the results of this indicator with no RSP or RPP filters in figure 7.10. We inmediately observe an uncorrelated pattern for all selected industries HS2, a situation that does not change over time for a given industry. When we run a correlation analysis with a power law pattern of the form $K n n(K) \sim K^{\rho}$, we find values of $\rho$ that are close to zero. This fact provides evidence that trade networks are neither assortative $(\rho>0)$ nor dissortative $(\rho<0)$, leading to a condition where nodes can connect to each other with no preference in terms of node degree of the destination. This idea seems fairly acceptable in the context of an economic system, where all exporters and importers will try to maximize their own benefits in the presence of perfect competition. Thus, a node of any degree is equally likely to connect to other nodes without taking in account the degree of the destination node.

As per our procedure, we now incorporate the RSP and RPP filters, and apply this bipartite indicator to the core of the network. We show the outcome of this process in figure 7.11. Once again, we observe no correlation or pattern among the nearest neighbor degree of the 2 selected industries HS2. The results for the rest of the selected industries HS2 can be found in Appendix C and D. These patterns do not 

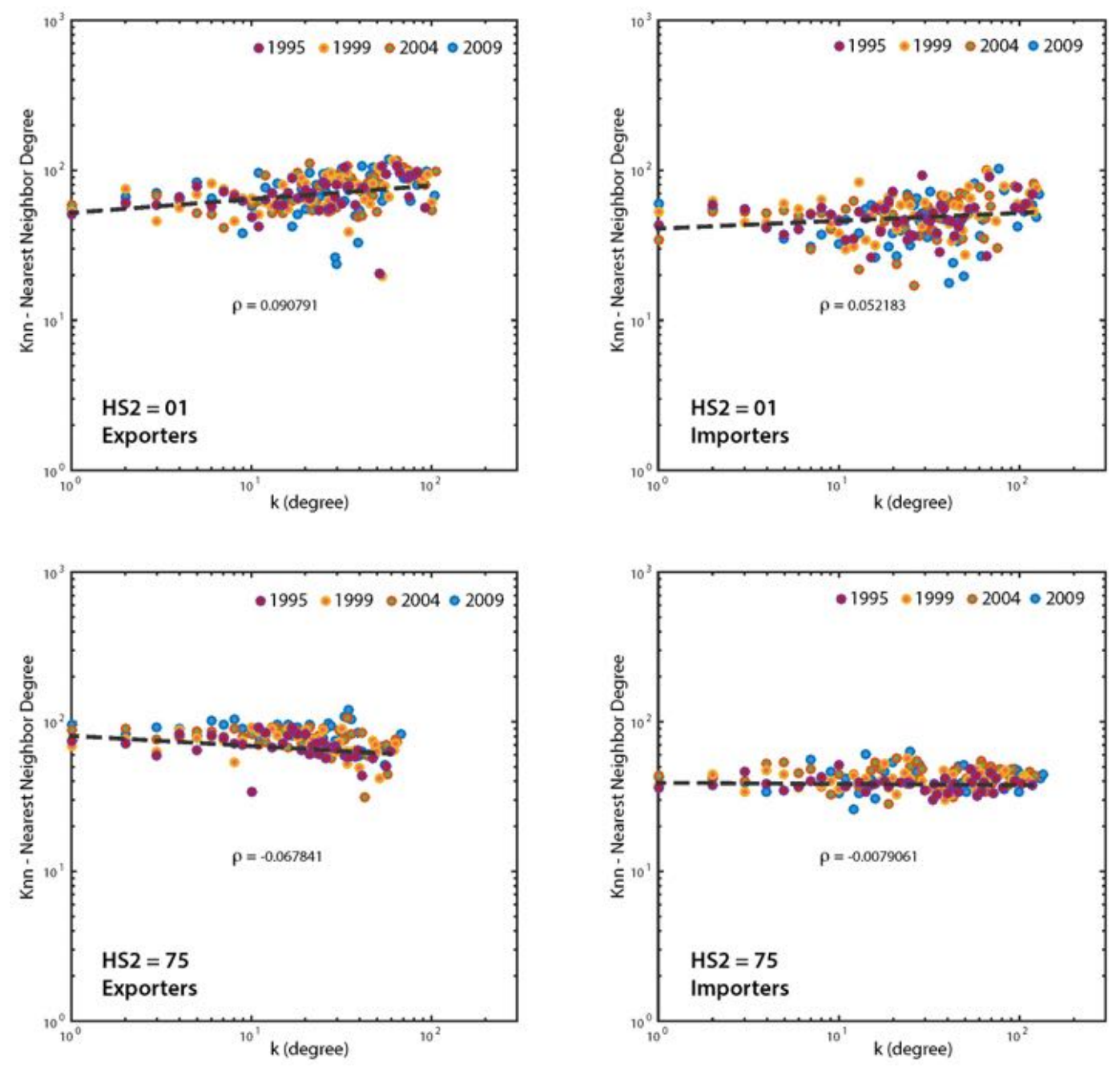

Fig. 7.10.: Nearest neighbor degree (weighted) without RSP and RPP filters. This figure shows the performance of $\mathrm{Knn}(\mathrm{k})$ for 2 selected HS2 industries (01 and 75) over the selected periods. We test a power law pattern of the form $K n n(K) \sim k^{\rho}$. Results for the rest of the selected industries can be found in Appendix C and D. 

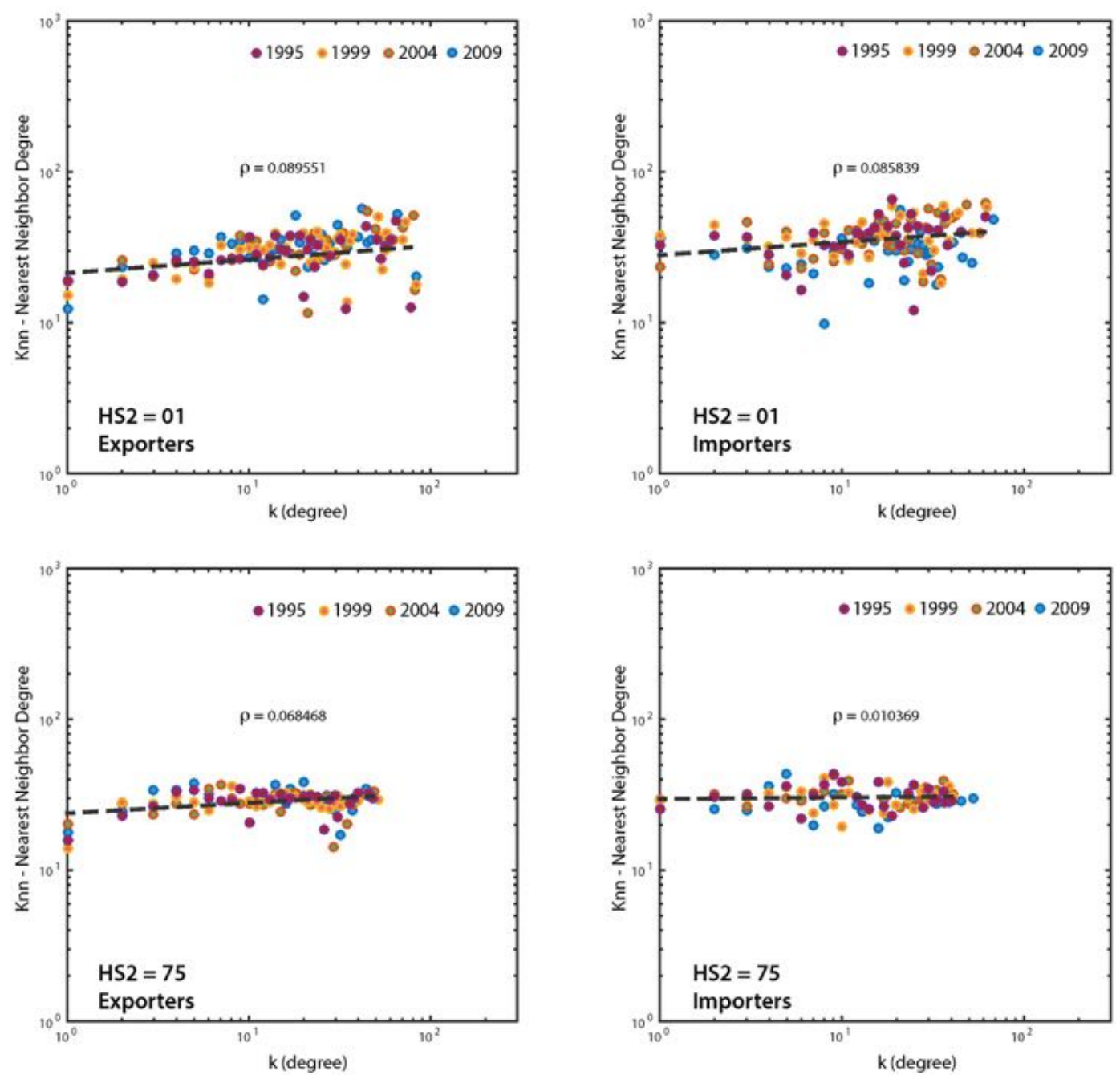

Fig. 7.11.: Nearest neighbor degree (weighted) with RSP and RPP filters. This figure shows the performance of Knn(k) for 2 selected HS2 industries (01 and 75) over the selected periods. We test a power law pattern of the form $K n n(K) \sim k^{\rho}$. Results for the rest of the selected industries can be found in Appendix C and D.

change significantly over time for any given industry. Consequently, the correlation analysis with a power law pattern of the form $K n n(K) \sim K^{\rho}$ have also found values of $\rho$ that are close to zero for the core of the network, showing no difference in comparison to the whole network. Still, we observe another finding for the $K n n(k)$, which is the fact that average value decreases in a significant proportion when we apply the RSP and RPP filters. This drop in the values of $K n n(k)$ is, in our opinion, also an expected situation since the topological filters remove about half of the links in the network, hence leaving a smaller number of connections between neighbors, yet without changing the uncorrelated pattern.

Finally, in order to increase the statistical sample and understand the actual nature of the assortative properties of trade networks, we now compute the $\rho$ exponents 

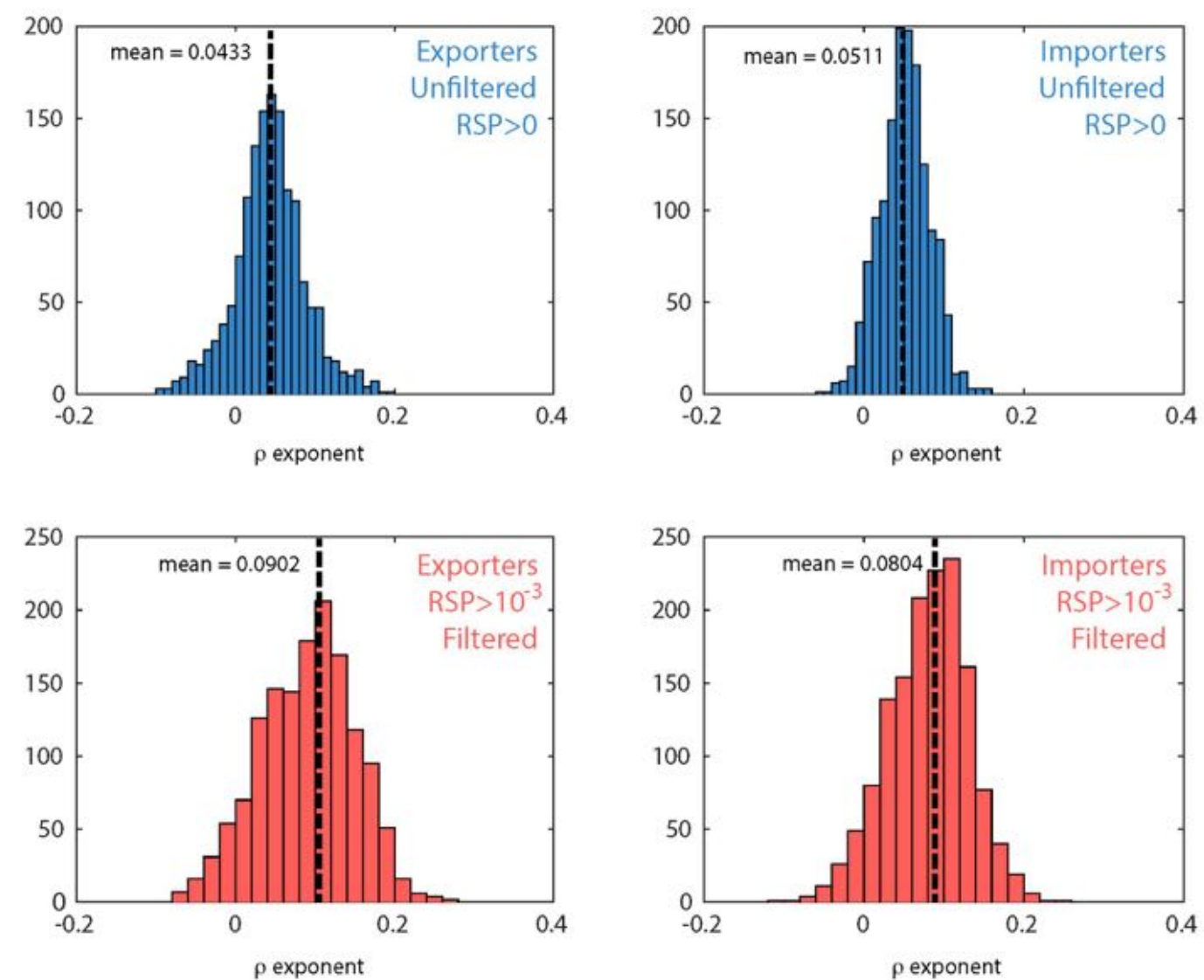

Fig. 7.12.: Exponents frequency for $\operatorname{Knn}(\mathrm{k})$. This figure shows the frequency of the $\rho$ exponents for all industries HS2 and all periods for the unfiltered scenario (top), filtered scenario (bottom), exporters (left) and importers (right).

for all industries HS2 and all periods. In figure 7.12, we show the results from this analysis. Overall, we can observe that the values for the $\rho$ exponents of all the industries HS2 have a probability function that resemble a normal distribution with a mean value that is close to zero and, more precisely, $\rho=0.04$ for the exporters and $\rho=0.05$ for the importers, values that reinforce the evidence toward trade networks that are neither assortative nor dissortative. Yet, when the the RSP and RPP filters are applied, the situation remains approximately the same, however, we do observe that the distributions for the exporters and the importer increase their mean values ( $\rho=0.09$ and $\rho=0.08$, respectively), moving the distribution towards the assortative side. Yet, we do not think that this values are large enough to justify a statement about the core of the network being more assortative than the periphery. We believe this is a tendency, yet the evidence does not support a conclusive fact. With these results in mind, we now move to the next bipartite indicator, the C4 clustering performance. 


\subsubsection{Clustering properties of trade networks, C4(K)}

In this subsection, we will perform an overview of the clustering behavior of trade networks, both unweighted and weighted. The clustering, as a concept, is a measure of redundancy of connectivity, which is of higher intensity when nodes of a complex network are more interconnected so that they form groups of a high density of ties among themselves, a.k.a. a cluster. The initial definition of clustering has not been applied to bipartite networks until it was absolutely required to depict this feature within network that can never form closed triplets due to the disjoint nature of the two subsets in it. But clustering may also be interpret as a measure of how hierarchical the internal structure of a network is. For example, in trade networks, the clustering properties can provide evidence of how high-degree nodes are connected in comparison to low-degree nodes, which would help us understand whether the most important exporters and importers tend to be more hierarchically and redundantly connected. Obviously, we have already determined that the core of the network is not only formed by high-degree nodes, but also that these nodes are connected through links of a higher weight than those of the periphery. But this fact do not provide any insight about how these connections are hierarchically organized; we just know that there is a large number of links with high weights. Consequently, we compute the bipartite clustering coefficient $C 4_{b}(k)$ for the importers and the exporters and then we present the results for 2 selected industries HS2 and the selected periods in figure 7.13.

From a direct observation, we realize that this bipartite indicator can be descrbed by a power law pattern of the form $C 4_{b}(k) \sim k^{\sigma}$, where $\sigma_{\text {exp }}$ is the exponent for the exporters and $\sigma_{i m p}$ for the importers. The evidence suggests that the clustering coefficients decrease with a larger degree for all selected industries HS2, making the core of the network, formed mainly by high-degree nodes, more hierarchical than the periphery, having less alternative paths for connection. This is an interesting finding, and perhaps an expected one to some extent, but the reality is that fact may also suggest that the most important nodes in a trade network are more difficult to reach by simply navigating the network, providing it a sort of systemic protection against other exporters or importers from outside of the core, a condition that can perfecty reflect and even confirm some of our premises. Like it happens in mutualistic ecosystems, where generalist species tend to form a highly connected and stable core, trade markets may also have the same internal organization, which, by extension, also provides robustness and resilience properties to the whole system. We will discuss this in the conclusions chapter, but, initially, we realize that the combination of several conditions of the bipartite indicators, taking place simultaneously, can indeed explain some of the ideas of Adam Smith from a systemic point of view. In this case, because exporters and importers act in a rational manner and they try to 

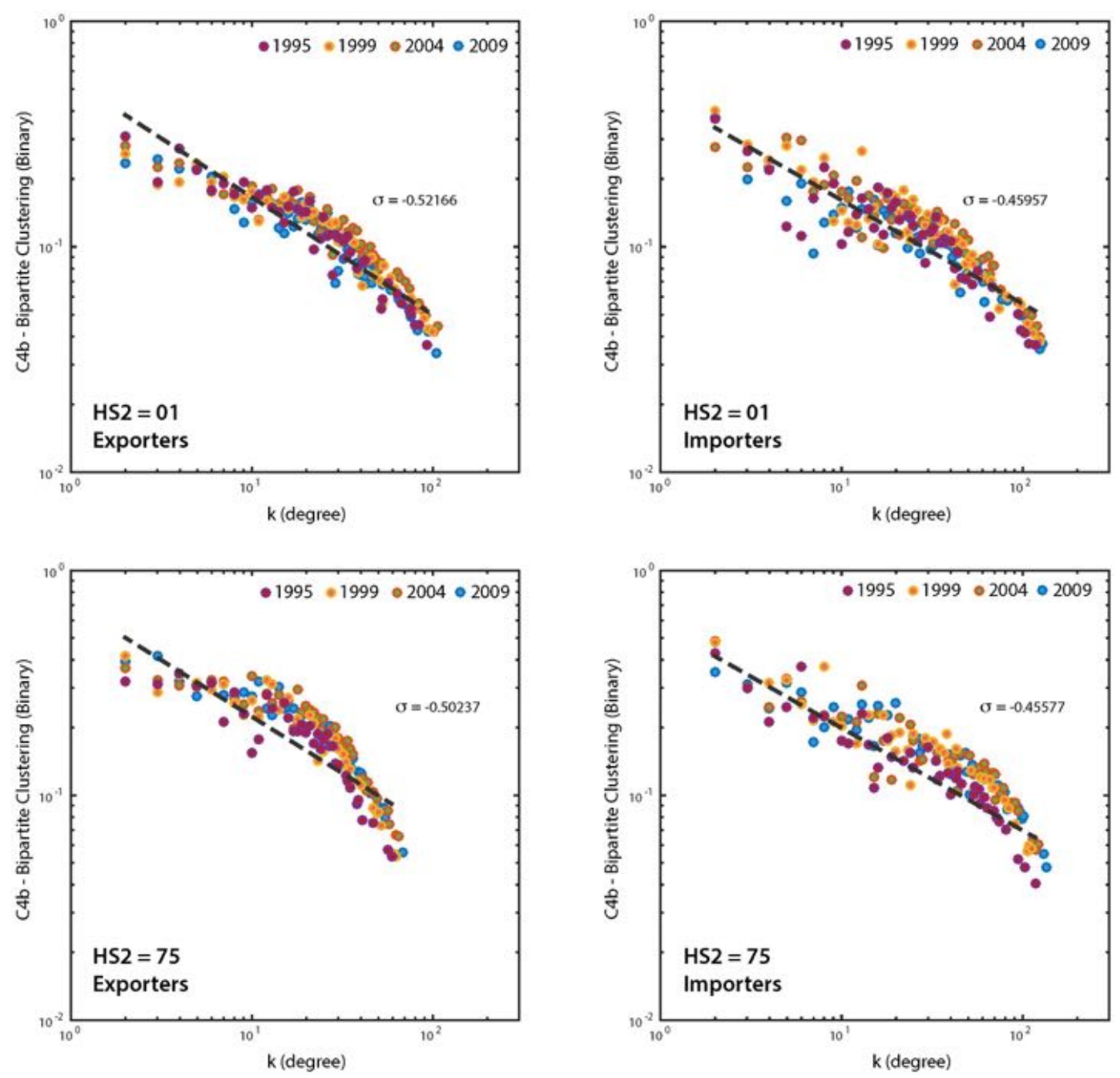

Fig. 7.13.: Bipartite clustering (unweighted) without RSP and RPP filters. This figure shows the performance of $\mathrm{C} 4 \mathrm{~b}(\mathrm{k})$ for 2 selected HS2 industries $(01$ and 75$)$ over the selected periods. We test a power law pattern of the form $C 4_{b}(K) \sim k^{\sigma}$. Results for the rest of the selected industries can be found in Appendix C and D.

maximize their own benefits (self-interest), the market then develops an emerging internal organization that provides robustness to the whole system, making it more sustainable in the long term and more resistent to externalities. Consequently, acting in a selfish way can indeed promote the well being of the whole market. On the other hand, the evidence also shows that the clustering behavior does not significantly change over different periods for any given industry.

We now apply the RSP and RPP filters to our data and repeat the same procedure for the core of the network. We need to state a special note for this point, which is related to a bias effect due to the nature of the clustering concept. We realize that applying a filter to a trade network can result in an unfair measurement of the clustering performance of the filtered scenario. Clustering relies deeply on 
the number of connections within the network, which, regardless of its weight or relative importance, can directly impact the chances for alternative paths to be formed, naturally creating a bias in the clustering results. We decide to still apply this measurement to the trade networks, since it can still provide some evidence about how the core is different, or not, from the periphery and, as a consequence, from the total market. We compute this and show the results in figure 7.14.
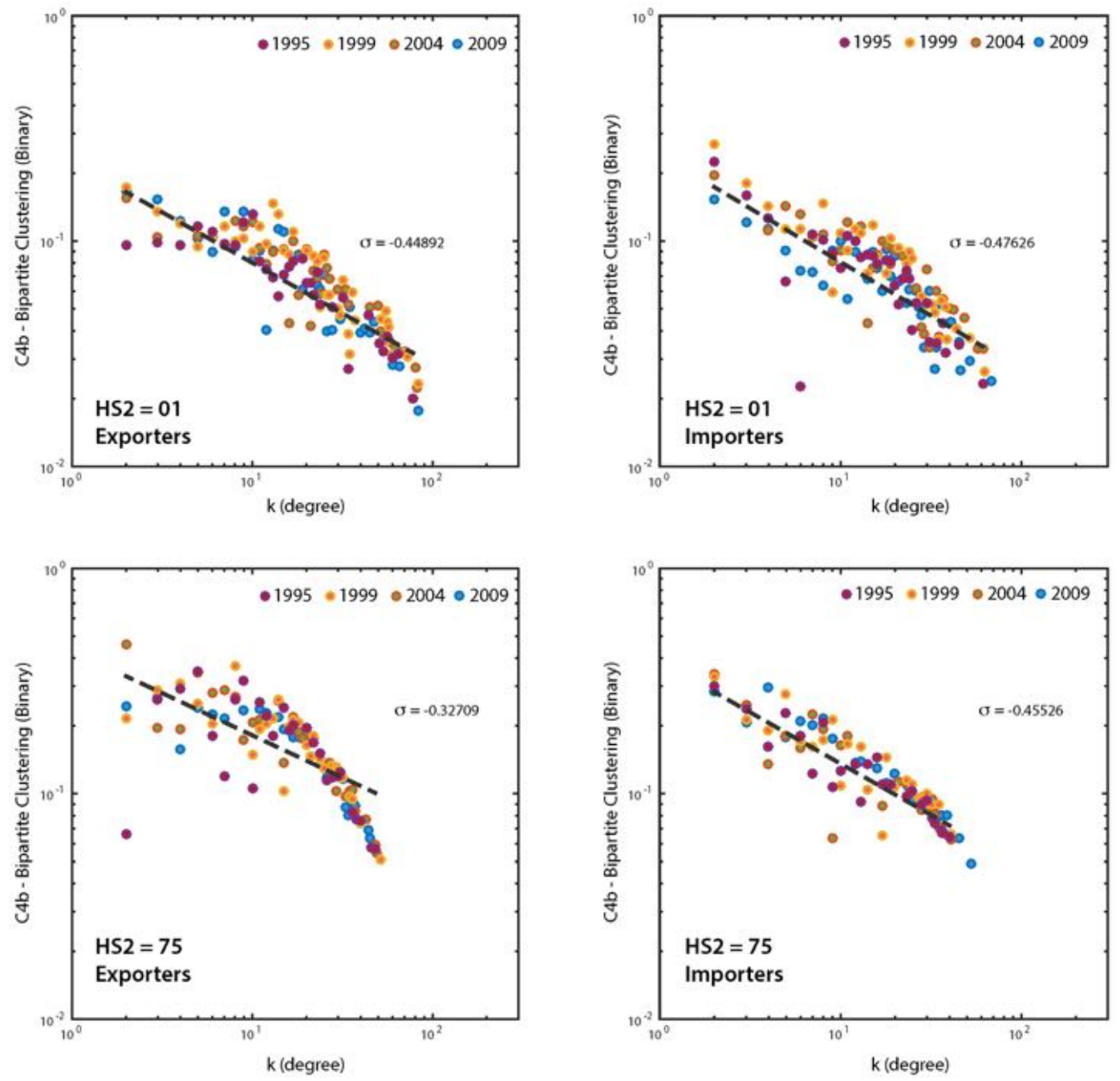

Fig. 7.14.: Bipartite clustering (unweighted) with RSP and RPP filters. This figure shows the performance of $\mathrm{C} 4 \mathrm{~b}(\mathrm{k})$ or 2 selected HS2 industries ( 01 and 75$)$ over the selected periods. We test a power law pattern of the form $C 4_{b}(K) \sim k^{\sigma}$. Results for the rest of the selected industries can be found in Appendix C and D.

The declining clustering patterns hold after the RSP and RPP filters are applied. This implies that the overall performance of the core and the whole network are similar from a conceptual standpoint. Nonetheless, we still need to test whether any variance can be found in the $\sigma$ exponents as a consequence of the filters. For this reason, we again compute the exponents for all the indutries HS2 and for all the 
periods to maximize the statistical sample. We show the results of this process in figure 7.15.
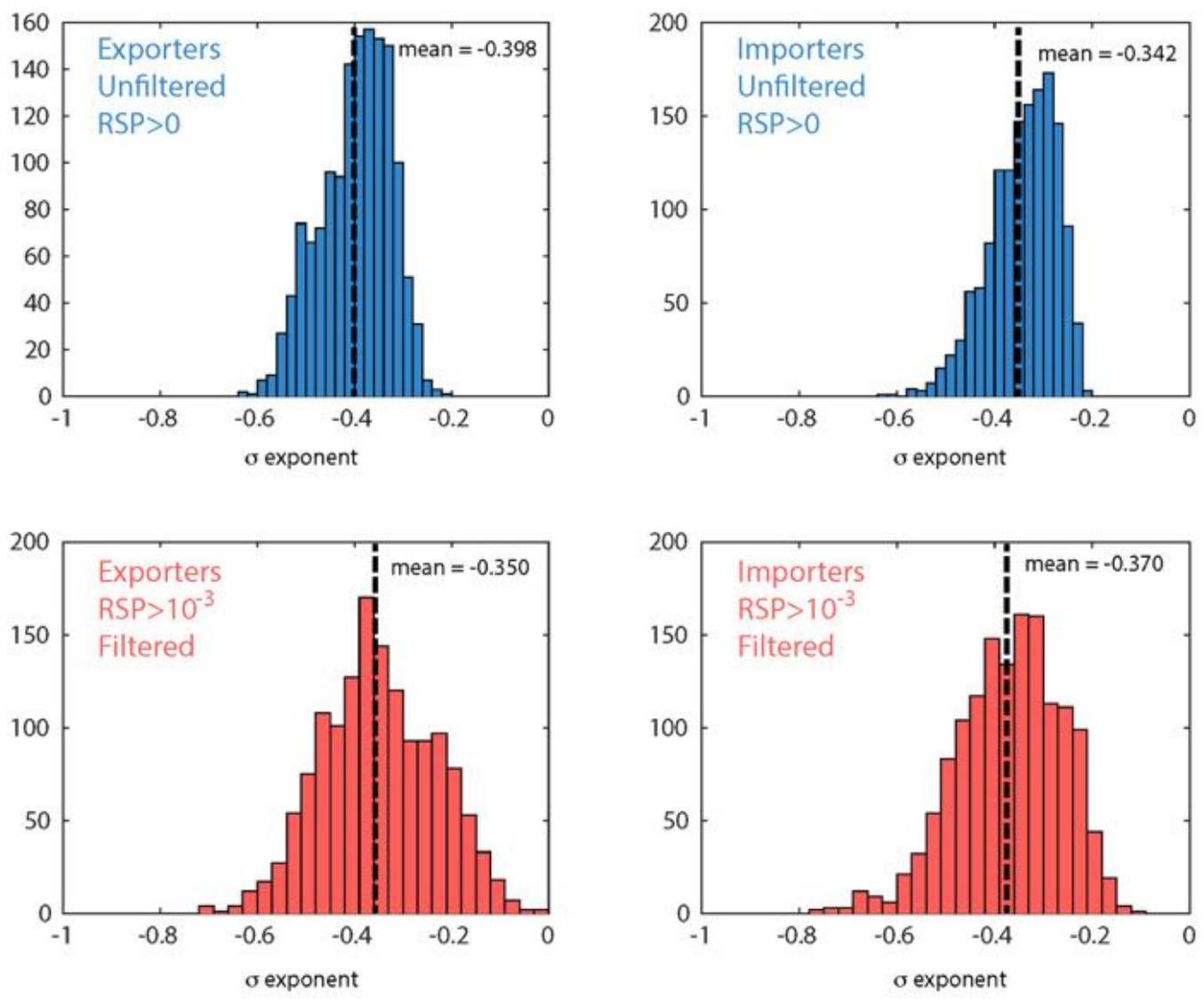

Fig. 7.15.: Exponents frequency for $\mathrm{C} 4 \mathrm{~b}(\mathrm{k})$. This figure shows the frequency of the $\sigma$ exponents for all industries HS2 and all periods for the unfiltered scenario (top), filtered scenario (bottom), exporters (left) and importers (right).

From the evidence of the process for all industries and all periods, we observe that the $\sigma$ exponents of $C 4_{b}$ are located in a range that goes from -0.62 to -0.2 with a mean value of -0.4 for the exporters and from -0.65 to -0.2 with a mean value of -0.34 for the importers in the unfiltered scenario. When the topological filters are applied, it is shown that the $\sigma$ exponents are now located in a range that goes from -0.72 to 0 with a mean value of -0.35 for the exporters and from -0.8 to -0.1 with a mean value of -0.37 for the importers. Despite the small changes in the average $\sigma$ exponents when the topological filters are applied, the main finding is actually related to the variability in the possible values of $\sigma$. The increased range of the values suggest that the core of trade networks have a broader spectrum in terms of clustering performance, where a larger exponent, in absolute value, implies that the differences between the high-degree nodes and the low-degree nodes are more intense, leading to a larger gap in terms of hierarchical organization. 

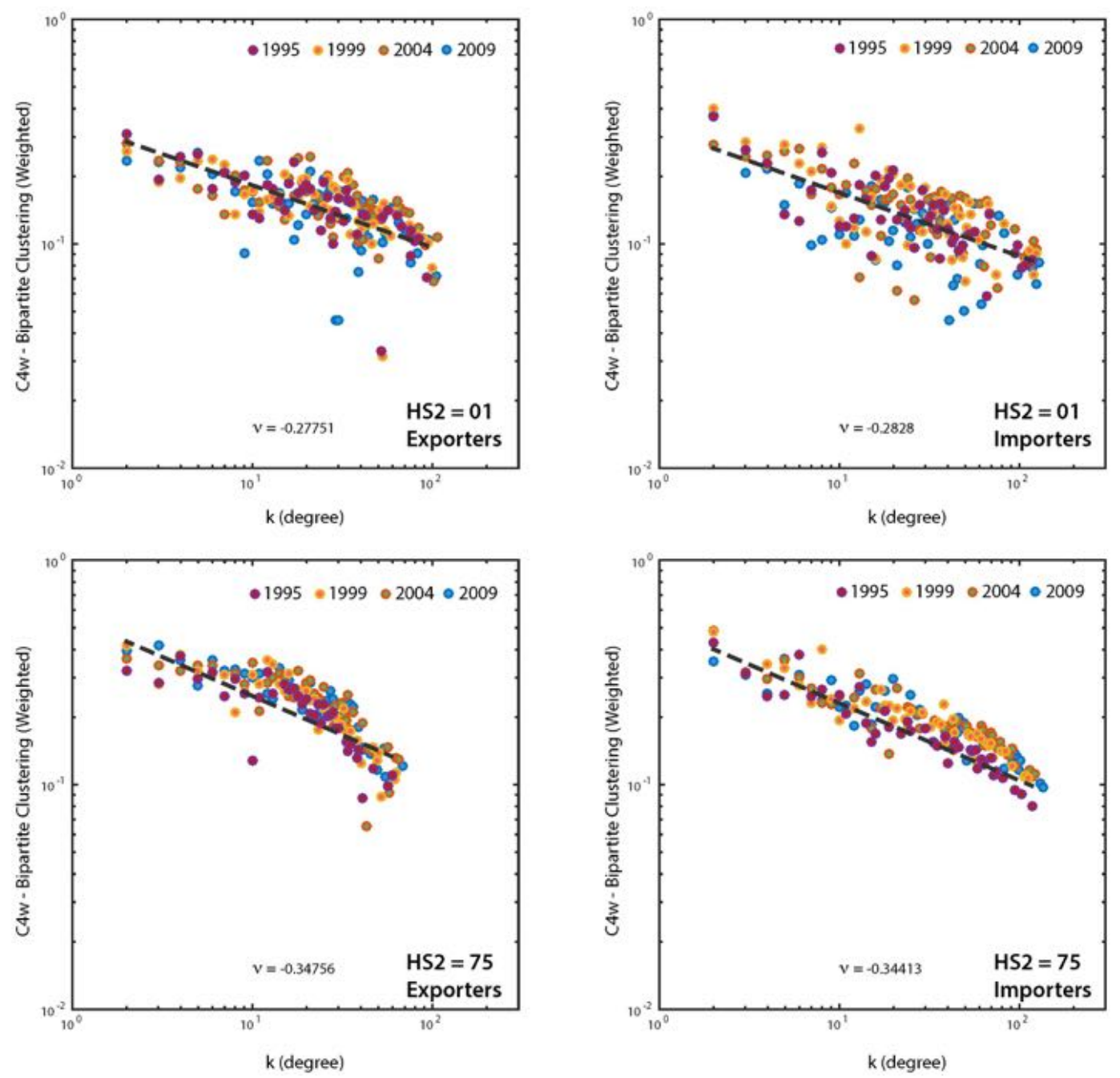

Fig. 7.16.: Bipartite clustering (weighted) without RSP and RPP filters. This figure shows the performance of $\mathrm{C} 4 \mathrm{w}(\mathrm{k})$ or 2 selected HS2 industries (01 and 75) over the selected periods. We test a power law pattern of the form $C 4_{w}(K) \sim k^{\nu}$. Results for the rest of the selected industries can be found in Appendix C and D.

We now repeat the whole process for the weighted version of the bipartite clustering $C 4_{w}(k)$. In this case, we compute the weighted bipartite clustering $C 4_{w}(k)$ for the 2 example industries HS2 and each selected period and show the outcome of this process in figure 7.16. We observe that the declining clustering performance is still valid for $C 4_{w}(k)$, in which we still find a power law pattern of the form $C 4_{w}(k) \sim k^{\nu}$, where $\nu$ is the exponent for the weighted clustering. We also observe that the patterns for $C 4_{w}(k)$ do not significantly change over time for any given industry.

We now perform the same analysis for the selected industries HS2 after applying the topological filters of RSP and RPP. We show the results in figure 7.17, which shows that the power law patterns remain and still, no siginificant changes over time for any given industry. 

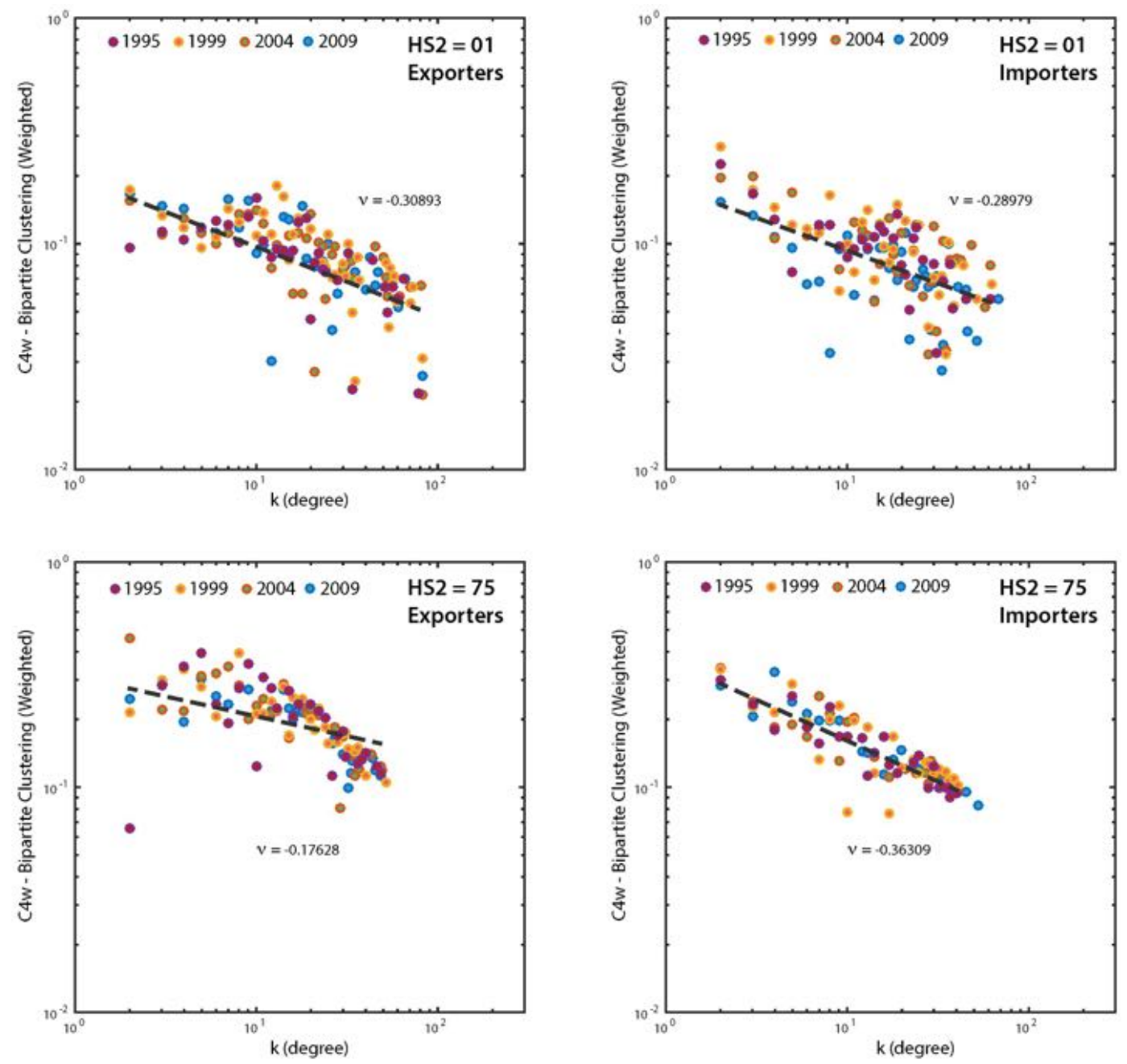

Fig. 7.17.: Bipartite clustering (weighted) without RSP and RPP filters. This figure shows the performance of $\mathrm{C} 4 \mathrm{w}(\mathrm{k})$ or 2 selected HS2 industries (01 and 75) over the selected periods. We test a power law pattern of the form $C 4_{w}(K) \sim k^{\nu}$. Results for the rest of the selected industries can be found in Appendix C and D. 
Finally, we extend the analysis to all industries HS2 and all period to increase the statistical sample and determine the range and mean value of the $\nu$ exponents. We display the results in figure 7.18 and observe that the $\nu$ exponents of $C 4_{w}$ are located in a range that goes from -0.40 to -0.03 with a mean value of -0.208 for the exporters and from -0.39 to -0.1 with a mean value of -0.22 for the importers in the unfiltered scenario. When the topological filters are applied, it is revealed that the $\nu$ exponents are now located in a range that goes from -0.5 to 0.05 with a mean value of -0.21 for the exporters and from -0.62 to 0.05 with a mean value of -0.22 for the importers. Once again, the mean value of the $\nu$ do not change significantly but the ranges are broader, with a small number of scenarios (industry in a given period) that actually show $\nu$ exponents that are either zero or positive. It is also important to state that the weighted clustering coefficients for any given product and period are larger than those of the unweighted indicator, revealing that $C 4_{w}(k) / C 4_{b}(k)>1$, a condition that has been also found in mutualistic ecosystems, for example. We will further refer to this findings in the conclusions section. Then, we move to the study of the next bipartite indicator, the average weight as a function of the end-point degree.
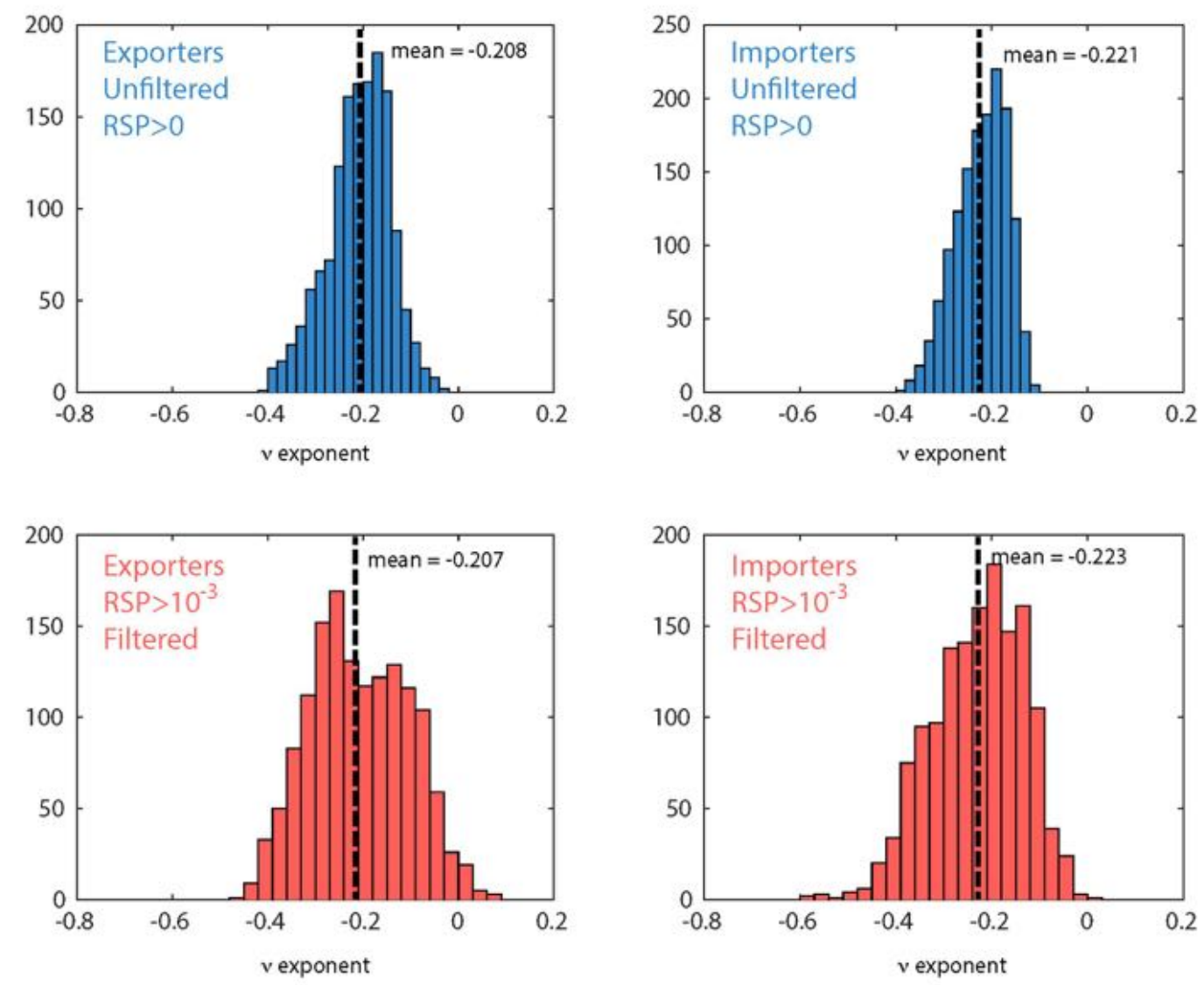

Fig. 7.18.: Exponents frequency for $\mathrm{C} 4 \mathrm{w}(\mathrm{k})$. This figure shows the frequency of the $\nu$ exponents for all industries HS2 and all periods for the unfiltered scenario (top), filtered scenario (bottom), exporters (left) and importers (right). 

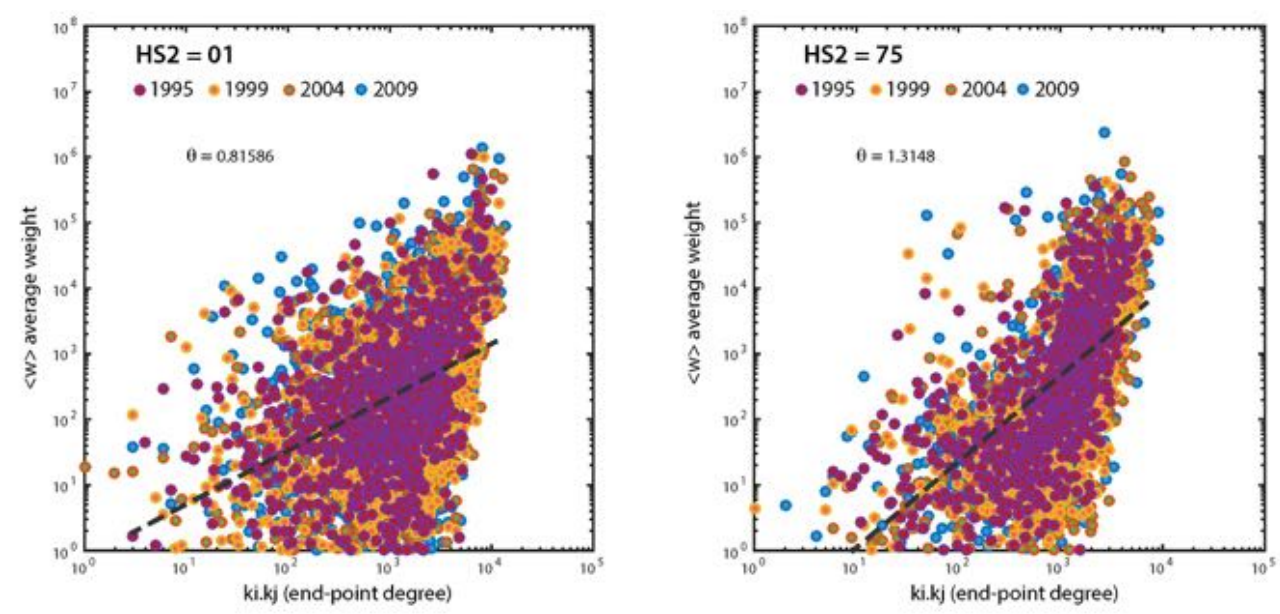

Fig. 7.19.: Average weight as a function of the end-point degree without RSP and RPP filters. This figure shows the performance of $\langle\mathrm{w}>(\mathrm{Ki} . \mathrm{Kj})$ for 2 selected HS2 industries (01 and 75) over the selected periods. We test a power law pattern of the form $\langle w\rangle \sim\left(k_{i} * k_{j}\right)^{\theta}$. Results for the rest of the selected industries can be found in Appendix C and D.

\subsubsection{Average weight vs. end-point degree, $\langle\mathrm{W}\rangle\left(K_{i} \cdot K_{j}\right)$}

As described in chapter 5 , we incorporate the measure of the average weight as a function of the end-point degree in order to determine how these parameters are connected. The benefits of performing this analysis is related to the issue of whether the end-point degree, which is the multiplication of the degree for the initial and destination nodes or $k_{i} * k_{j}$, have any influence on the average weight that connects such nodes. Certainly, it would be expected some correlation given the fact that the performance of $S(k)$ has already shown a strong and highly significant relationship of the strength and node degree. For this reason, we compute this bipartite indicator for the selected industries HS2 for the selected periods and show the results in figure 7.19 for the unfiltered scenario.

Similar conclusions to previous indicators apply from the direct observation of the evidence. The patterns remain virtually unchanged over time for any given industry. However, and in contrast to the previous indicators, we do not find a clear form to describe the resulting pattern. We actually observe two types of behavior, one for lower values of the end-point degree and another for higher values. We could chacterize this situation as a two-zone pattern where, for low values of the end-point degree, we observe a rather random, uncorrelated behavior whereas, for high values of the end-point degree, we observe a power law pattern of the form $\langle w\rangle \sim\left(k_{i} * k_{j}\right)^{\theta}$. 

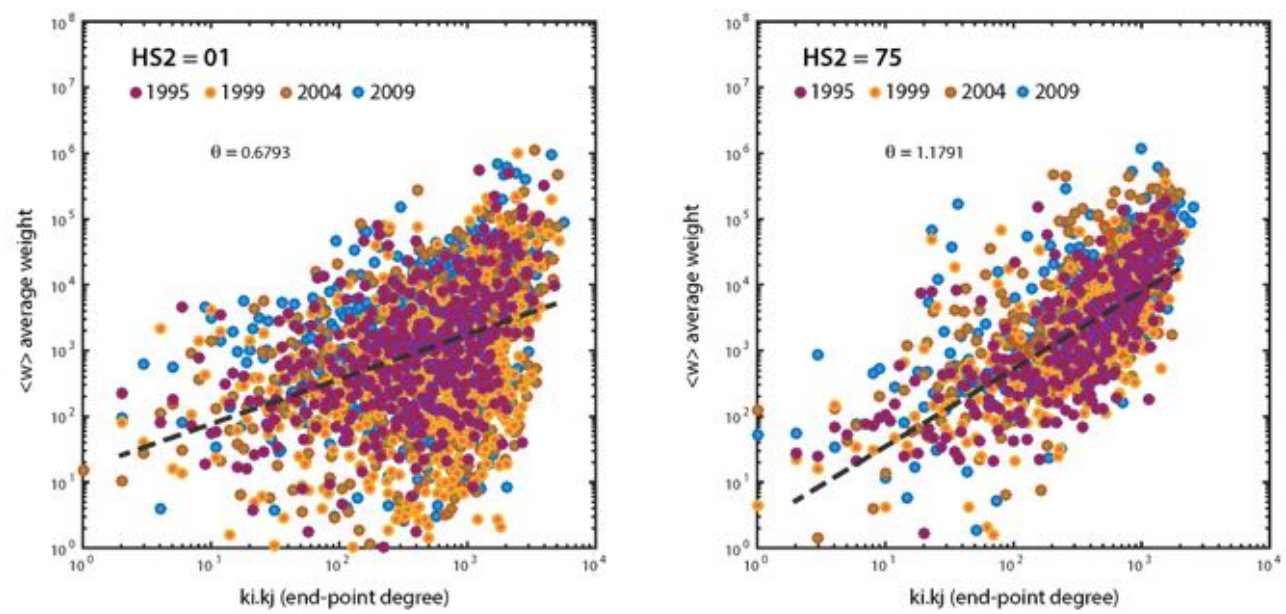

Fig. 7.20.: Average weight as a function of the end-point degree with RSP and RPP filters. This figure shows the performance of $<\mathrm{w}>$ (Ki.Kj) for 2 selected HS2 industries (01 and 75) over the selected periods. We test a power law pattern of the form $\langle w\rangle \sim\left(k_{i} * k_{j}\right)^{\theta}$. Results for the rest of the selected industries can be found in Appendix C and D.

Following the previous analysis, we also compute the same indicator for the filtered scenario (figure 7.20). Just to avoid unnecessary repetition, we observe the same findings from the unfiltered scenario (patterns do not change over time), however, when the links of low relative importance are removed, we do observe that the core the network display a more clear power law pattern than that of the total network. Repeating the same procedure than previous indicators, we also extend statistical sample by computing the results for all industries and periods. We show the outcome in figure 7.21.

In this case, and contrary to other bipartite indicators, we observe a reduction of the variability of the $\theta$ exponents when we apply the topological filters of RSP and RPP, leading also to a lower mean value of them. For the unfiltered scenario, the mean value of the $\theta$ exponent is 1.35 , whereas the mean goes down to 1.04 for the filtered scenario. We will discuss the implications of the findings in the conclusions section. With this, we conclude the review of the standard bipartite indicators as described in chapter 5. The next step in our research will focus on an innovative concept for international trade markets, which is the nestedness, a concept that has not yet been used to study bilateral trade between exporters and importers. 

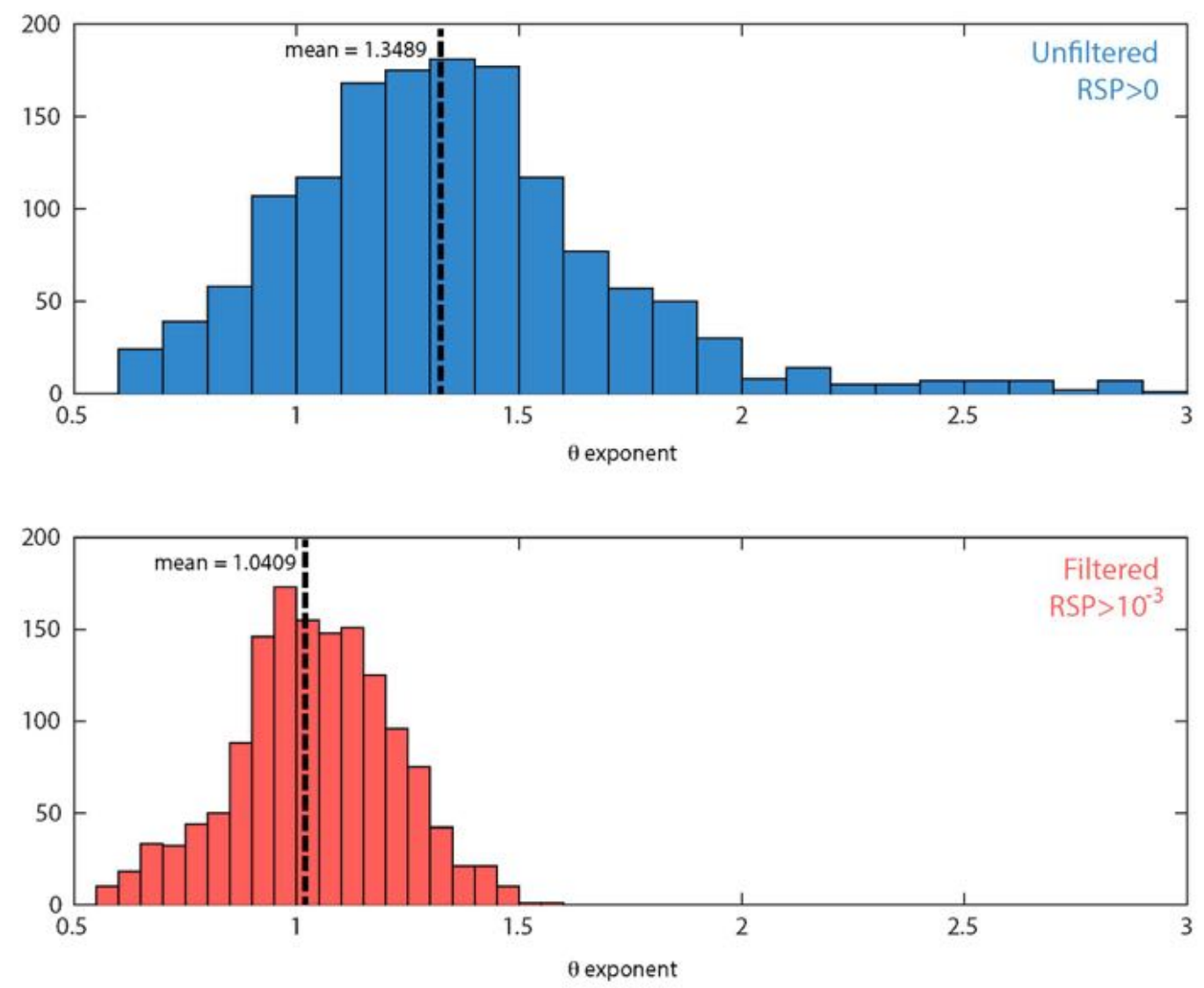

Fig. 7.21.: Exponents frequency for end-point degree. This figure shows the frequency of the $\theta$ exponents for all industries HS2 and all periods for the unfiltered scenario (top) and filtered scenario (bottom).

\subsubsection{Nestedness features}

We have hypothesized that weighted bipartite trade networks are highly nested. Since bilateral trade markets has not yet been studied as a complex bipartite network with an exporter-importer (country-country) approach, this idea is based on heteogenuous nature of markets, for which we have already provided extensive evidence previously in this chapter. Our hypothesis needs to be either confirmed or rejected at this moment. Therefore, and as argued on chapter 6 , we rely on the weighted interaction nestedness estimator, $\eta_{w}$, or WINE as our main measure. We compute the $\eta_{w}$ for each selected industry HS2 and for each selected period. We also perform this analysis for both scenarios (filtered and unfiltered). For every step of the computational process to compute the WINE estimator, we use 35 randomizations of the bipartite matrix, a number that is well balanced to provide quality results in terms of statistical significance, yet without unnecessarily increasing the processing time. We record the distances vectors, z-scores as well as the p-values. In particular, for the unfiltered scenario, we build a nestedness visualization and 
show the outcome of it in figure 7.22. This visualization includes a 2D view of the bipartite matrix, which is accompained by a 3D visualization of it. The weights of each link are shown in a logarithm scale, which have been color-coded for easier pattern detection. Let us review the results for the selected industries HS2.
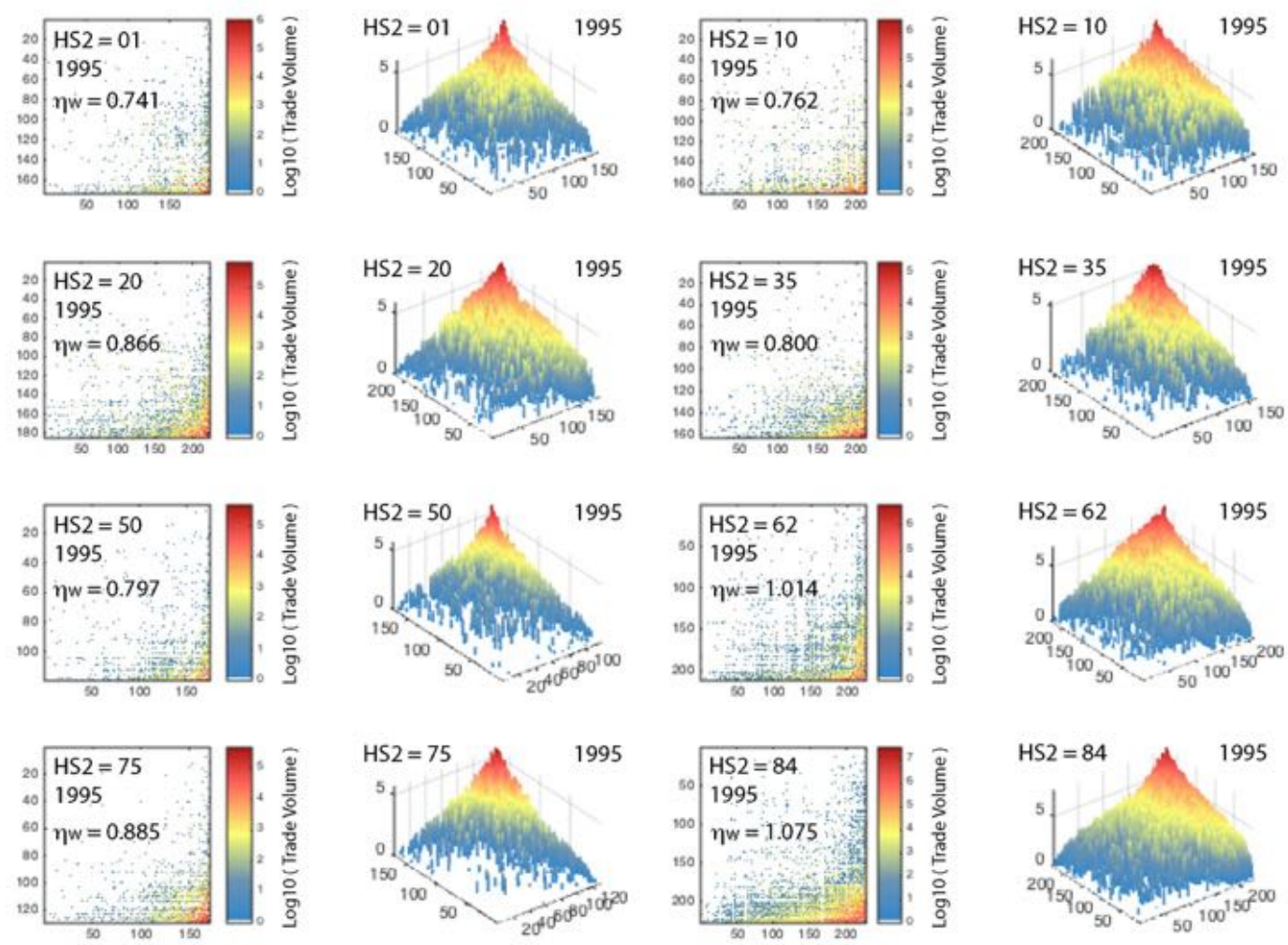

Fig. 7.22.: Nestednes results for the selected HS2 industries. This figure shows the bipartite matrix representation both in 2D (left) and in 3D (right) for the selected HS2 industries. The trade volume is color-coded in logarithm scale as showed by the colorbar. Unfiltered scenario is presented here.

From the outcome of our process, we observe that all selected industries HS2 display a highly nested pattern, where values of $\eta_{w}$ vary from 0.741 (HS2=01) to 1.075 (HS2 =84), a piece of evidence that confirm our initial hypothesis. We believe this is not a trivial finding if we consider the results we have found previously in this chapter. In fact, it might have been an expected one based on the combination of simultaneous results for $\mathrm{P}(\mathrm{k}), \mathrm{S}(\mathrm{k})$ and $\mathrm{C} 4(\mathrm{k})$, which reinforces the analogy with mutualistic ecosystems, but the reality is that the evidence suggests that some countries do form a highly dense core, holding the majority of the interactions between them (exporters and importers). This is consistent with the role of the generalists in the mutualistic ecosystems, where some species of both subsets have a higher connectivity and interact with a large number of species of the other subset. Here, we also observe that market interactions, like in ecosystems, tend to happen more frequently between a generalist exporter and a generalist importer or between a generalist exporter or importer and a specialist importer or exporter, respectively. 
We rarely observe interactions between a specialist exporter and a specialist importer or vice versa. We will discuss further in the conclusion about this from a conceptual standpoint but, initially, we remain satisfied with the current findings since they have not been stated or addressed by any previous scientific research. We now continue with the results for the filtered scenario, which can be found in figure 7.23.
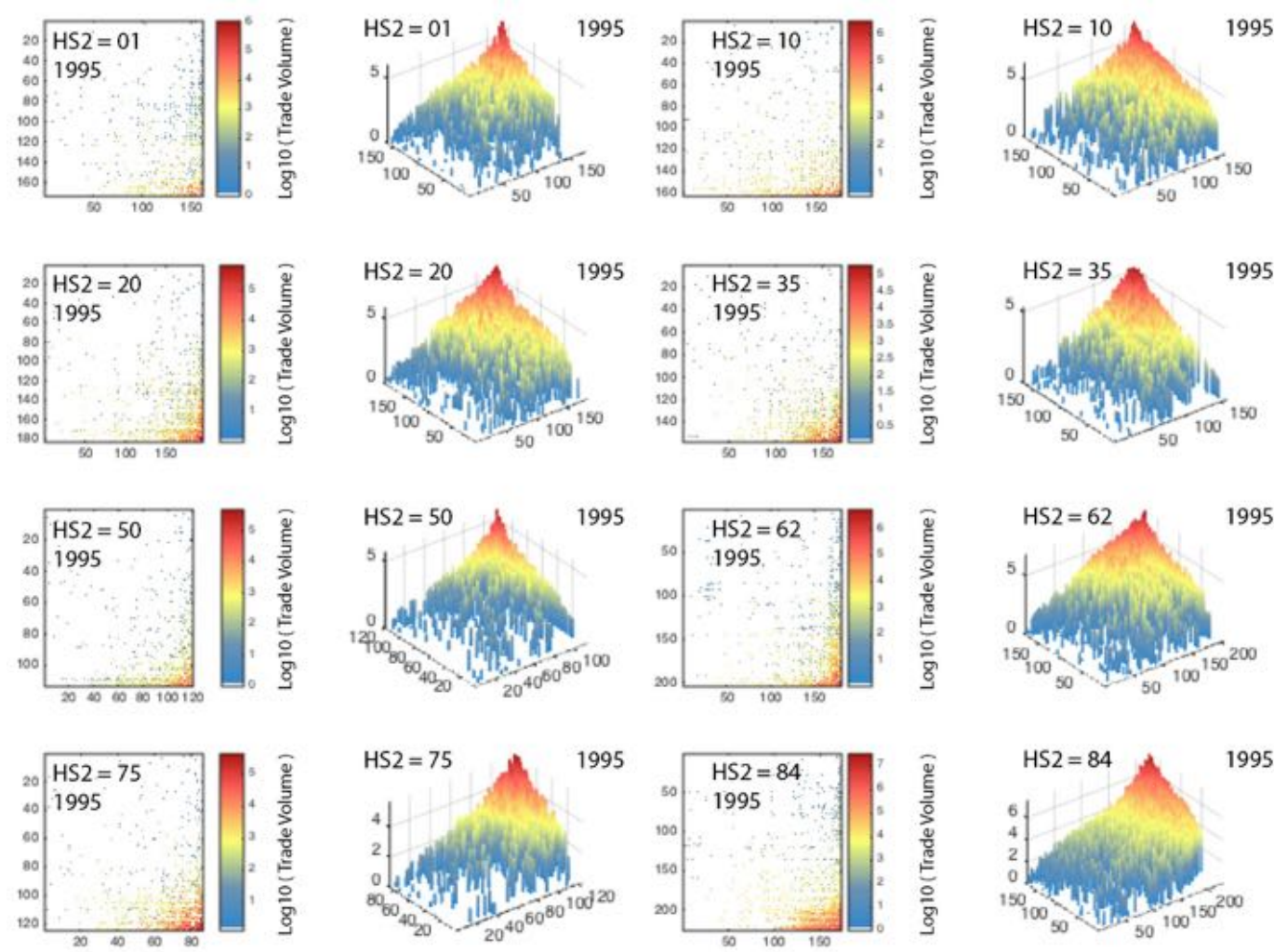

Fig. 7.23.: Nestednes results for selected HS2 industries (filtered). This figure shows the bipartite matrix representation both in 2D (left) and in 3D (right) for the selected HS2 industries. The trade volume is color-coded in logarithm scale as showed by the colorbar. Filtered scenario is presented here.

When the topological filters are applied, we observe that the interaction matrices do conserve the nested properties despite the removal of the links of low relative importance. On the one hand, it is true that these links were characterized before as being part of the periphery, which seems to still be the case from the nestedness perspective. In fact, this reinforces the idea of the correlation between the core of the network and the generalist exporters and importers. Yet, from a quantitive point of view, we realize that the core of the trade network tends to be more/less nested than the complete network. In this case, the values of $\eta_{w}$ are, after the filters have been applied, between 0.7 and 0.9 , a decline from the unfiltered scenario. As it happens with any measure, the fact that filters are applied intrinsically means incorporating some bias in the measure, but the evidence here shows that the core of the network still displays patterns of high nestedness, which is a important finding in order to understand the internal structure of markets. This means that the links that form the 
inner core of the network have not significantly been affected by the removal of low relative importance one and the nested configuration can and will probably survive against external attack, a situation that we have discussed before when considering the clustering properties.

On side note, we have also computed the alternative nestedness measure WNODF. The results we find for all industries HS2 are always less than 3 points in a scale from 1 to 100, which clearly fails to detect the type of nestedness that might be present in naturally sparse matrices. As argued before, this method is suitable for a relatively small size of the interaction matrix, where the nested patterns may tend to be less sparse. WNODF is also suitable for detecting nested patterns that resemble a perfectly nested matrix. In other words, WNODF is a highly efficient detector of perfect netedness. However, for larger (weighted as well) matrices that are naturally sparse, $\eta_{w}$ is a more suitable measure as it does not severely penalize unexpected absences at the core or presences at the periphery.

We now move to a more generalized way of analyzing nestedness in trade networks. For the unfiltered scenario only, where $R S P>0$ and $R P P>0$, we compute the results for all industries HS2 and for the selected periods, and show the outcome in figure 7.24. The pattern described of the results by HS2 industries remains constant over time, as we can observe, and also keeps relatively stable when zooming in industry by industry, a piece of evidence that further supports our premise. The distribution of the nestedness performance of industries HS2 also remain with a similar form over time, suggesting that indeed nestedness is a persistent property found in weighted bipartite trade networks. The mean nestedness for all industries HS2 is $\eta_{w}^{\text {mean }}=0.86$ with a standard deviation of $\sigma_{\eta}=0.08$ in 1995. If we take a look at the industries with lower nestedness results, they are: a) Explosives and Pyrotechnics (HS2 $=36$ with $\eta_{w}=0.66$ ), b) Fertilizers (HS2 $=31$ with $\eta_{w}=0.71$ ) and c) Milling products (HS2 $=11$ with $\eta_{w}=0.71$ ). In contrast, the industries with higher nestedness results are: a) Machinery, Nuclear reactors and Boilers (HS2 $=84$ with $\eta_{w}=1.08$ ), b) Electrical and electronic equipment (HS2 $=85$ with $\eta_{w}=1.05$ ) and c) Optical, photo and medical apparatus (HS2 $=90$ with $\eta_{w}=1.05$ ). Initially, we may argue that low nestedness results could be related to the restrictions that a market might be subject to. Network density could be a bias factor as well, where markets with a low density will tend to intrinsically show lower values of nestedness. For example, this can be the case for the explosive industry, a highly sensitive and regulated product. Strong regulation and control can explain both the restrictions, creating a scenario full of forbidden links (as it happens in ecosystems as well) and the low density of the trade network. But, on the other hand, this is not necessarily valid for fertilizers or milling products, so we cannot present a definitive statement on this matter. 


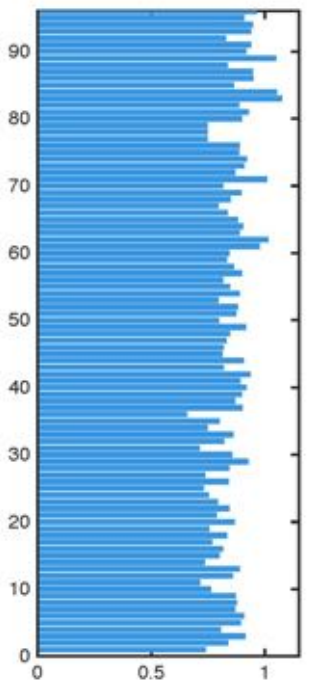

Histogram [ $\eta_{w}$ ] (all HS2 industries) 1995

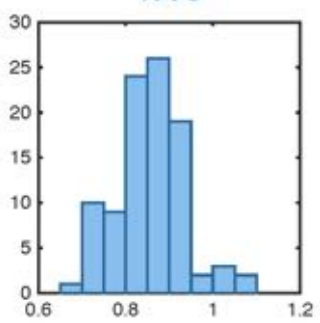

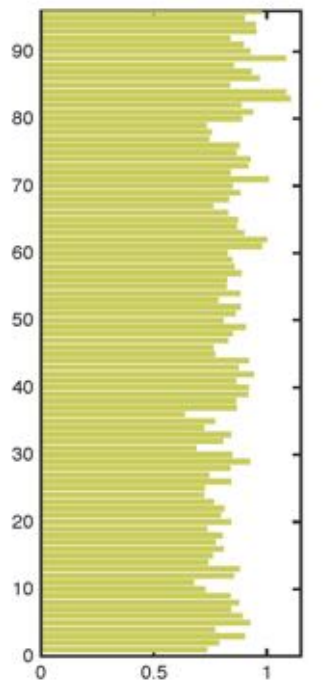

Histogram [ $\eta_{w}$ ]

(all HS2 industries)

1999

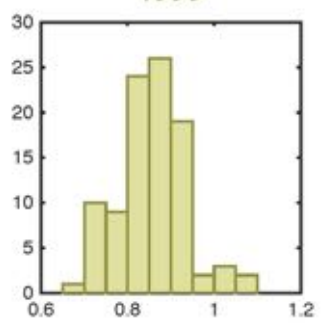

Nestedness [ $\rceil w$ ]

(by HS2 Industry) 2004

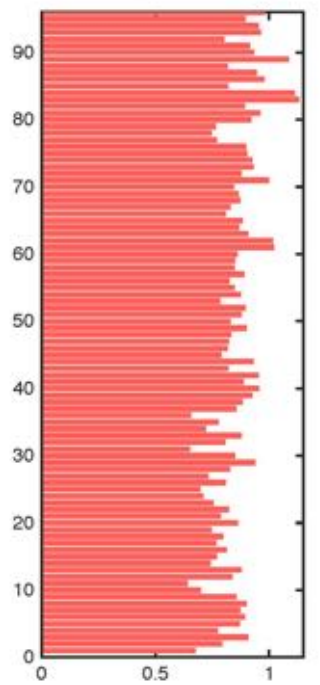

Histogram [ $\eta_{w}$ ]

(all HS2 industries) 2004

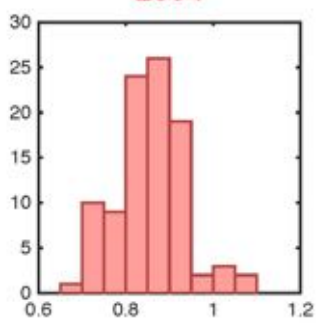

Nestedness [ $\left.\eta_{w}\right]$

(by HS2 Industry) 2009

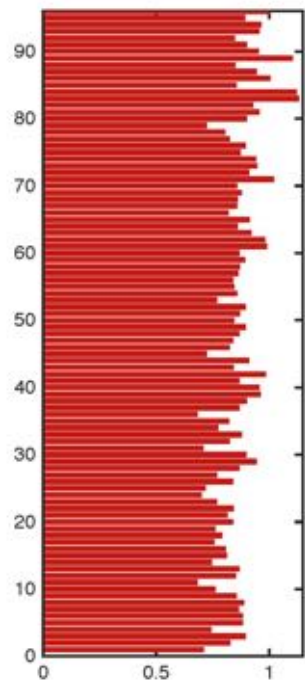

Histogram [ $\eta_{w}$ ]

(all HS2 industries) 2009

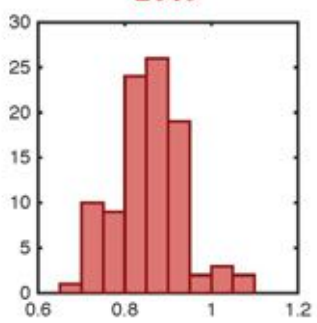

Fig. 7.24.: Nestednes results for all HS2 industries. This figure shows the results of nestedness $\eta_{w}$ for every HS2 industry (top) and for the selected periods (1995, 1999, 2004 and 2009). At the bottom, we show a histrogram of the frequency of $\eta_{w}$ for each selected period. We observe that the nestedness features do not significantly change from one period to another, suggesting that nestedness may be a persistent feature of trade networks.

In sum, it is now safe to say that nestedness is a feature that is present in all trade markets when analyzed with a weighted and bipartite methodology. We have also learned that some markets are more nested than others and that nestedness is a property that remains relatively unchanged over time for any given market. This fact is one that we have to explore further because we have already found that markets are quite dynamic and they do change from one period to the next. If nestedness is a property that does not change, we need to explore the reasons why this is the case. More on this subject will be discussed later in this chapter. 


\subsection{Summary of Topological Features}

The volume of information processed for this chapter and the outcomes that result from them can easily qualify as a big data problem. The size of the data, the scope of it and its detailed resolution is, considering any other study in this subject, beyond the threshold of small data and of traditional reseach. Because of this, we have decided that we need to consolidate the findings in a visual manner, so that the data itself, the results and the complexity of trade networks can be consumed in a simple way (see Appendix D for market visualizations).

Based on the various results of the bipattite indicators, and after computing them for a large number of scenarios, we conclude that trade networks have 8 common features. We create a visualization of these common features and show them in a format consistent with concept map in figure 7.25. We will not argue at this time about the meaning of these features taking place simultaneously for all markets, all periods and independently of the filter scenario. But the statement, we believe, comes from solid evidence presented throughout this chapter, which can be expanded in Appendix D (Market Visualizations).

We now ennumerate the 8 features and provide some comments about what we have learned in the current chapter. The order of these features is random; the order do not imply importance over the rest.

1. Long-tailed degree distribution. In this chapter, we have learned that trade markets have heterogenous, long-tailed $\mathrm{P}(\mathrm{k})$ distibutions. Depending on the level of aggregation, some markets are consistent with a power law pattern, other are consistent with exponential decays. We have also learned that the pattern for a given product does not significantly change over time, and that the core of the network has a similar pattern than that of the complete network. In this case, the exponents of the power law regression do not change for the exporters, but they do change for the importers. Furthermore, when we increase the statisical sample by aggregating all industries and periods, we find that the total market may present a truncated power law as main pattern. These findings are aligned to the evidence found in mutualistic networks.

2. High correlation of strength and degree. In this chapter, we have learned that weights are not randomly distributed across the network. In fact, highly connected nodes tend to connect with a higher weight than lower degree nodes, leading to a case where $\beta>>1$. With this, we could state that a situation similar to the "rich club effect" is present within trade network. We have also learned that the pattern for a given product does not significantly change over time, and that the core of the 


\section{Findings on Topological Features of Trade Markets}

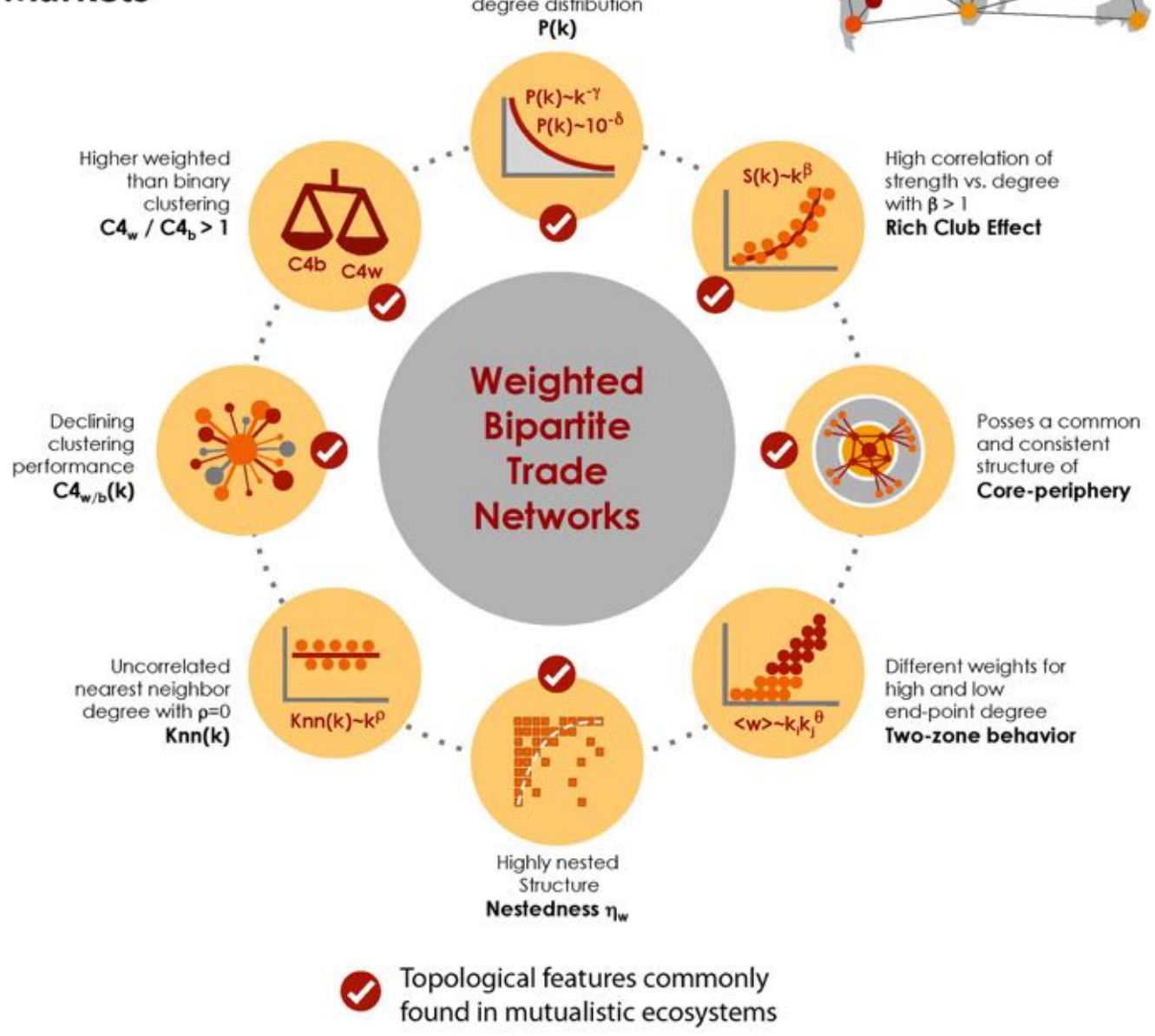

Fig. 7.25.: Common topological features of WBTN. This visualization shows a summary of the 8 common features of trade networks, as they have been observe for all levels of aggregation and all periods.

network has a similar pattern than that of the complete network. In this case, the exponents of the power law regression do not change for the exporters, but they do change for the exporters. These findings are aligned to the evidence found in mutualistic networks.

3. Trade networks have a core-periphery structure. In this chapter, we have learned that trade network have an internal organization that resemble a highly dense core, formed by highly connected nodes, and a periphery zone, formed by low connectivity nodes. Regardless of the product, category or industry, this pattern can be found consistently in all trade networks. These findings are aligned to the evidence found in mutualistic networks. 
4. Two-zone pattern of the end-point degree. In this chapter, we have learned that trade networks show two areas in the analysis of the average weight as a function of the end-point degree. The first zone is for the low connectivity nodes and low values of the end-point degree, where weights seem to have a random pattern. The second zone is for higher values of the end-point degree, where a more clear correlation can be found.

5. Highly nested structure. In this chapter, we have learned that the internal organization of internation trade markets is not random when conveniently sorting the interaction matrices. In fact, we find high values of nestedness for all markets (product, category or industry) and for all periods. This fact does not significantly change over time, making it a persistent feature of trade netwoks despite the considerable temporal changes in markets. These findings are aligned to the evidence found in mutualistic networks.

6. Uncorrelated nearest neighbor degree. In this chapter, we have learned that exporters and importers can connect to nodes of the other subset with no evident preference based on the fact that no correlation can be found in the empirical data. We have also learned that the pattern for a given product does not significantly change over time, and that the core of the network has a similar pattern than that of the complete network.

7. Declining clustering performance. In this chapter, we have learned that the closer a node is to the core of the network, and the higher its degree, the lower its clustering coefficient will be. We also find that the clustering, both unweighted and weighted, behave similarly. This fact does not significantly change over time, making it a persistent feature of trade netwoks despite the considerable temporal changes in markets. These findings are aligned to the evidence found in mutualistic networks.

8. Higher weighted than binary clustering. In this chapter, we have learned that, because of the different exponents of the unweighted (binary) and weighted clustering, a condition where $C 4_{w} / C 4_{b}>1$ is found. These findings are aligned to the evidence found in mutualistic networks.

Now that we have summarized and reviewed the 8 common features, we think it is relevant to point out that all of them take place simultaneously within any given trade network. The fact that they all take place at the same time is a matter that we will use later in this study to try to describe how markets are composed and formed. Moreover, in the conclusions chapter, we will provide a more econimic view of the meaning of these finding, trying to explain the initial premises of the study and, in particular, some of the ideas of Adam Smith regarding the complexity of markets and 
how a selfish attitude from elements of a market (persons, firms or even countries) can still lead to a situation that is mutually benefitial for all of them, an apparent contradiction that has fascinated me since before starting this study, becoming a secondary research objective, but no less important than others. 


\section{Temporal analysis}

But in my opinion, all things in nature occur mathematically.

— Rene Descartes

(One of the fathers of modern western

philosophy)

In the previous chapter, we have thoroughly studied the topological features of trade networks. We have also found that most of these these features do not change over time, leading to persisting behavior, which is may be a piece of evidence to support the idea that international trade markets have both a resemblance to mutualistic ecosystems and a set of persistent, universal properties. Within these properties, we have found that markets have a nested structure, a finding that has not been stated before for country-country trade networks. Yet, the persistence of nestedness is one aspect of trade markets that we have to study to get a deeper understanding of the reason or reasons why this is the case for all markets. In the following, we study the concept of nestedness across levels of aggregation and time.

\subsection{Nestedness across different markets}

The presence of nestedness in trade networks has now been established (chapter 7). Yet, in pursue of a better understanding of how consistently nestedness can be found in international trade markets, we now focus on analyzing how nestedness varies for different levels of aggregation and for different periods. This goal is not a random objective. When performing the exploratory analysis of trade markets for a large number of the 195,200 scenarios, nested patterns have appeared repeatedly with a similar structure when visualizing trade networks of the same product in different periods, for example. Therefore we want to extend this analysis in a formal way to determine whether hard evidence supports that initial perception. For this reason, we take an industry at random and compute the WINE nestedness from the lowest to highest level of aggregation. 

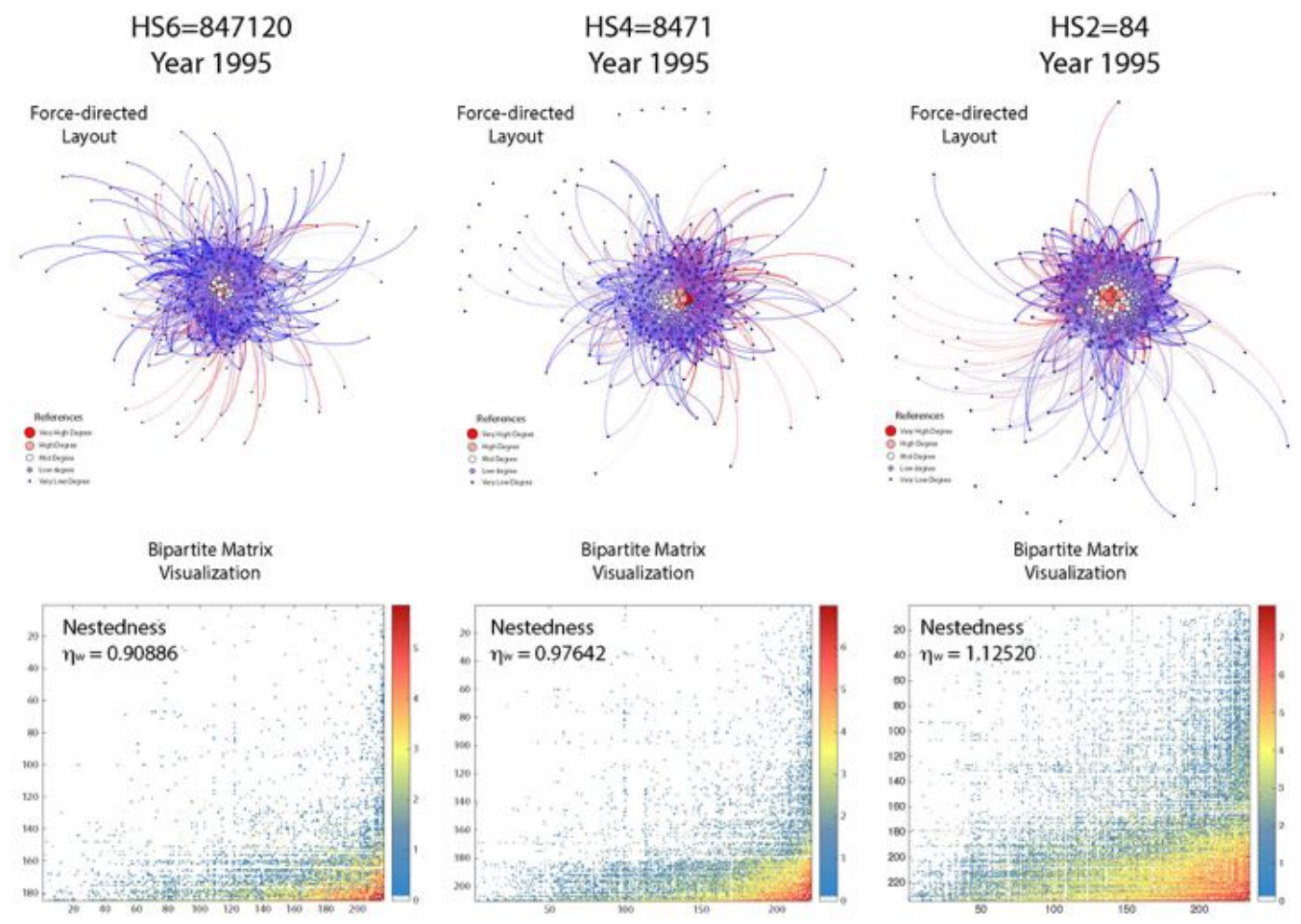

Fig. 8.1.: Nestednes results for different levels of aggregation. This figure shows the nestedness feature across different levels of aggregation. On the top, we present the network graphs with a force directed layout for HS6 $=847120$ (left), HS4 $=8471$ (middle) and HS2 $=84$ (right). We observe that the core periphery structure remains despite the level of agrregation while the core becomes more dense for the HS2 level over HS4 and HS6. On the bottom, we show the bipartite matrices for each case, where the evidence supports that nestedness, at least, remains a persistent feature.

In figure 8.1, we show the results of this process and include the visualization of the trade network, with a force-directed layout, in addition to the interaction matrix for each case. In this particular case, we use the product HS6 $=847120$ (digital computers), the category HS4 $=8471$ (data processing machines), and the industry HS2 $=84$ (machinery). From the results, we observe that the values of $\eta_{w}$ remain approximately constant for all levels of aggregation. Although this may seem to be a trivial fact, the reality is that nestedness is an omni-present feature that can be used to characterize international trade markets. We should not, in our opinion, pay an excesive attention to the variances of the nestedness value of each level, which are $\eta_{w}=0.91$ for the product HS6 level, $\eta_{w}=0.98$ for the category HS4 level, and $\eta_{w}=1.13$ for the industry HS2 level. These differences may be the consequence of the aggregation process itself, where several products are consolidated in a category, then several categories are consolidated in the industry, leading to a different condition, where links that do not exist in a product may exist in another, and naturally increasing nestedness due to larger data consolidation. We 
think that this finding works as an interesting concept to understand nestedness and the markets across markets and levels of aggregation. Let us consider the changes over time, or lack thereof. Similarly to the levels of aggregation, we perform the same procedure and build a visualization for a random product for the selected periods. We show this visualization in figure 8.2.

In this case, we use the product HS6 $=270900$ (oils, petroleum oils, crude), and track the nestedness across the periods, once again incorporating the visualization of the network, here in a geospatial layout, and the interaction matrix for the selected periods. We observe that the nestedness properties remain with minimum changes from one period to the next despite the several observed temporal variances in the trade network. When visually analyzing the geospatial network, we clearly observe that some links change in its intensity from one period to the next. Additionally, some links seem to disappear in one period and re-appear in the next, sometimes even with a different importance. The phenomema, which is dynamical in its nature, takes place in the network period after period, making it undeniable that, as we previously hypothesized, trade networks are not static, but quite the opposite in fact. But if markets are indeed quite dynamic and they constantly change its internal configuration, then we wonder why nestedness does not significantly change as a consequence of these temporal phenomena. For the oil product, we observe a value of $\eta_{w}=0.62$ in 1995, $\eta_{w}=0.70$ in 1999, $\eta_{w}=0.63$ in 2004, and $\eta_{w}=0.70$ in 2009. These changes are relatively minor in comparison of the number of changes (appearances, rewiring, and changes in volume) that we observe period over period. We think that a deeper analysis should be conducted in order to understand this situation. We also believe that the key to unveiling this is related to the tracking and characterization of the temporal changes. For this reason, we set another research objective to understand the effect of the dynamical phenomena in trade networks, a matter that we will discuss during the next chapter.

\subsection{Dynamical phenomena}

In chapter 7, we have analyzed the topological structure of trade networks for some selected industries, categories and products. In the previous section, we have analyzed how nestedness is a persistent feature of trade markets for different levels of aggretation and periods. Yet, these results have been shown from a stationary perspective. In fact, we did not account for the time evolution of networks and its effects upon topological indicators. Trade networks are not static and they certainly do not remain unchanged over time. In contrast, when observing the data at a low level of detail, we realize that various types of dynamical phenomena take place, from pure rewiring to link weigth changes and from link absences to appearances 

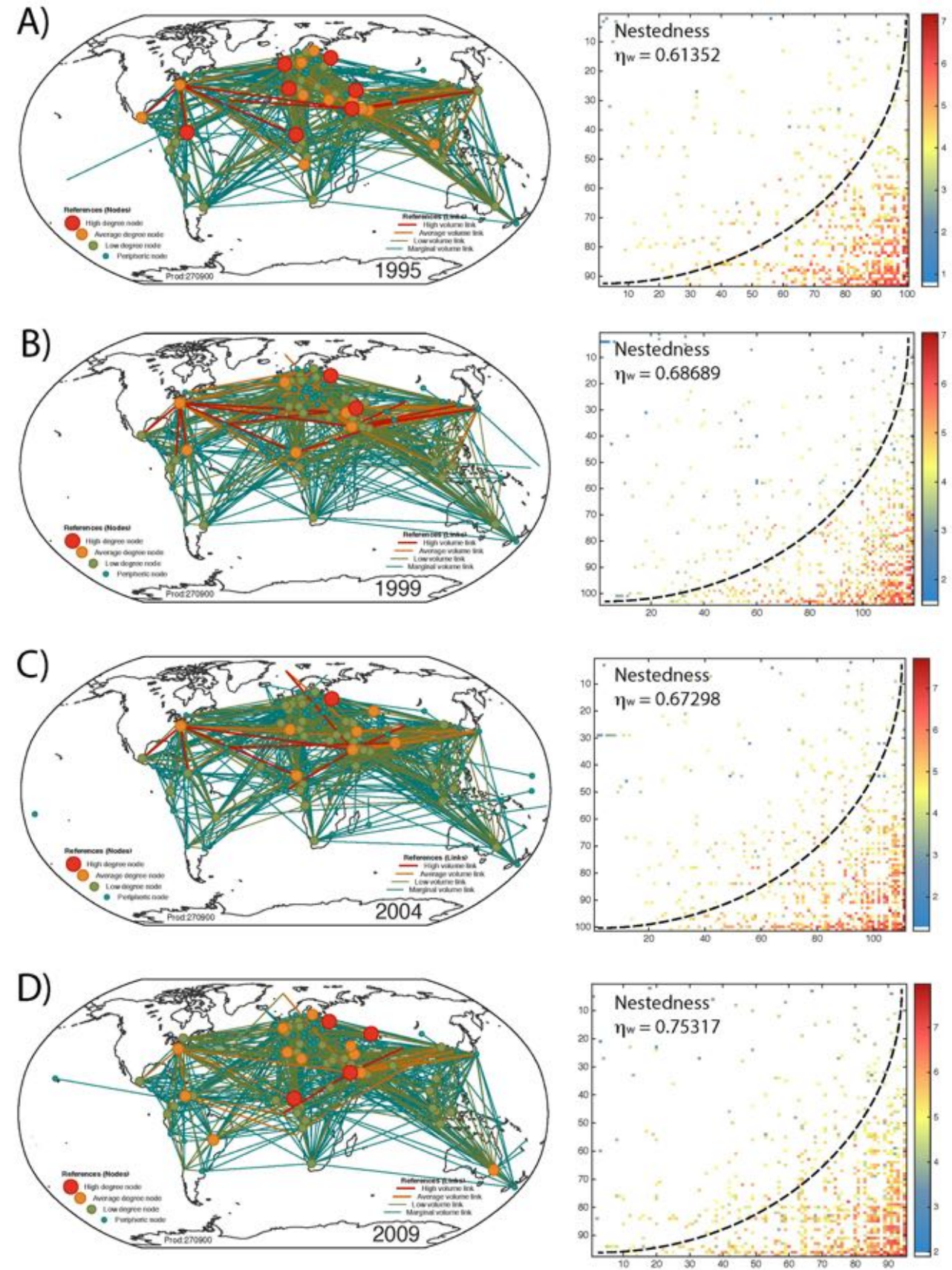

Fig. 8.2.: Nestednes results for different periods. This figure shows the nestedness feature across different periods for the same product (HS6 $=270900)$. On the left side, we present the network graphs with a geo-spatial layout in the years 1995 (A), 1999 (B), 2004 (C) and 2009 (D). We observe that the network has a high level of temporal changes over the presented periods. In the middle, we present a visualization of the topological results (exponents) for each period. The dark green dots are located in between the maximum and minimum values observed. On the rith side, we show we show the bipartite matrices for each period, where the evidence supports that nestedness remains a persistent feature.

of new links. The most relevant question in this chapter will be related to how the topological properties are affected by the dynamical phenomena and to the 
dynamical patterns we may find. We start by stating a premise in that markets are dynamic based on the assumption that countries may change who they buy from, leading to link appearance and absence, and/or they may also change how much they buy, leading to trade volume increases and declines. Hence, we propose a methodology that is both highly visual and deeply relies on the analysis of the variances of the interaction matrices of trade networks. Yet, these variances are only available for a short period, from 1995 to 2009, which gives us only 14 periods of variation. Another aspect to take in account is the formal definition of the dynamical phenomena, which is highly influenced by the overall market dynamics. For example, an increase of the trade volume in one country can merely be a side effect of the overall market trend. Hence, we define four types of dynamical phenomena as follows:

1. Volume Increase. This is a link weight variance that exceeds that of the market. For example, if the overall market volume increases 3 percent from one year to the next, a volume increase will take place when the link weight increases more than the total market rate.

2. Volume Decrease. This is a link weight variance that falls behind that of the market. For example, if the overall market volume increases 3 percent from one year to the next, a volume decrease will take place when the link weight shows a variance below the total market rate.

3. Link Appearance. This is a new link that did not exist in the previous period.

4. Link Absence. This is an lack of a link that did exist in the previous period.

In order to continue with our analysis, we propose a visual process to depict the dynamical phenomena. First, we compute the bipartite matrix for all periods and create a ranking of exporters and importers based on their node strength. This sets the positions of each exporter and importer in the matrix, which will remain a fixed position over the different periods. In figure 8.3, we present an example of this procedure, where we observe the different interaction matrices with the same position for each exporter and importer among all periods. Secondly, we measure the year-over-year variance for each element of the bipartite matrix and identify the dynamical phenomena, recording them in a variance matrix that is $n-b y-m$ in size. We repeat the procedure for all periods.

Thus, the variance matrices clearly show the dynamical phenomena in same position that takes place on the bipartite matrix. We set up a color code to identify each phenomenon as follows: a) BLUE represents a new link appearance, b) BLACK represents a link absence, c) GREEN represents a volume increase, and d) RED 
$\mathrm{t}=0$
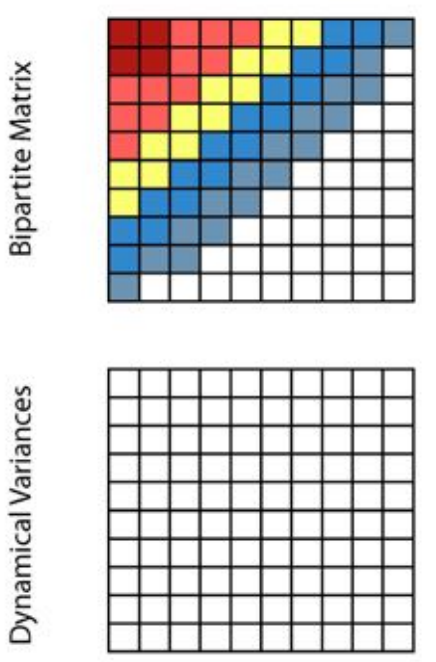

New Link Appearance $t=1$
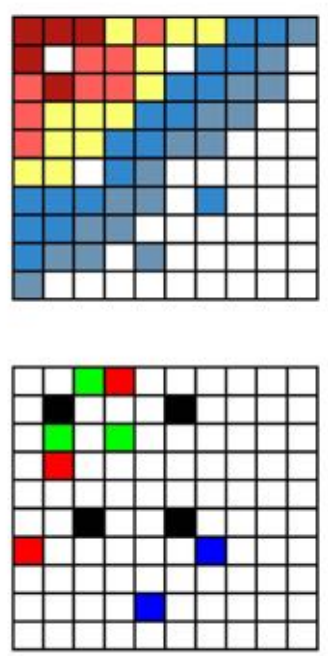

$\mathrm{t}=2$
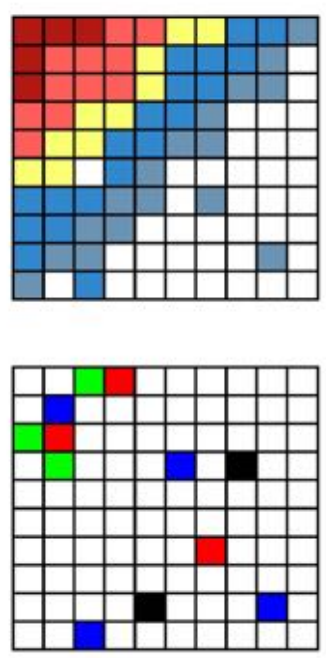

Volume Decrease

Fig. 8.3.: Concept of dynamical phenomena tracking method. This figure shows an example of our procedure to analyze and visualize the dynamical phenomena. For $t=0$, we observe the initial bipartite matrix, which is perfectly nested, but no dynamical variances. For $t=1$ and $t=2$, we observe the different variances based on the bipartite matrix changes.

represents a volume decrease. Hence, we present the bipartite matrices for all periods of an example product in figure 8.4.

It is not an easy task to visualize the dynamical phenomena in figure 8.4 and consequently, we cannot draw many conclusions from it. This is why, we now compute and display the visualization for the year-over-year variance matrices, as described by our process, in figure 8.5 , where the 14 year-over-year can be easily observed.

Now, we observe that most of the dynamical phenomena take place within certain exporters and importers, whereas others remain unchanged over time. For example, those countries with a high ranking as exporters and importers do not display dynamical changes. Dynamics of these highly important exporters and importers seem to be mainly explained by those of the market. This is an interesting fact because of the correlation between the highly connected nodes and the core of the network. At this point we may argue that the dynamical phenomena may not be equally distributed across the interaction matrix and that some exporters and importers are less likely to be affected by these dynamical phenomena. If this is the case, we should be able to find evidence of the internal dynamics of markets, which can ultimately help us better understand the relationship between the topology and 

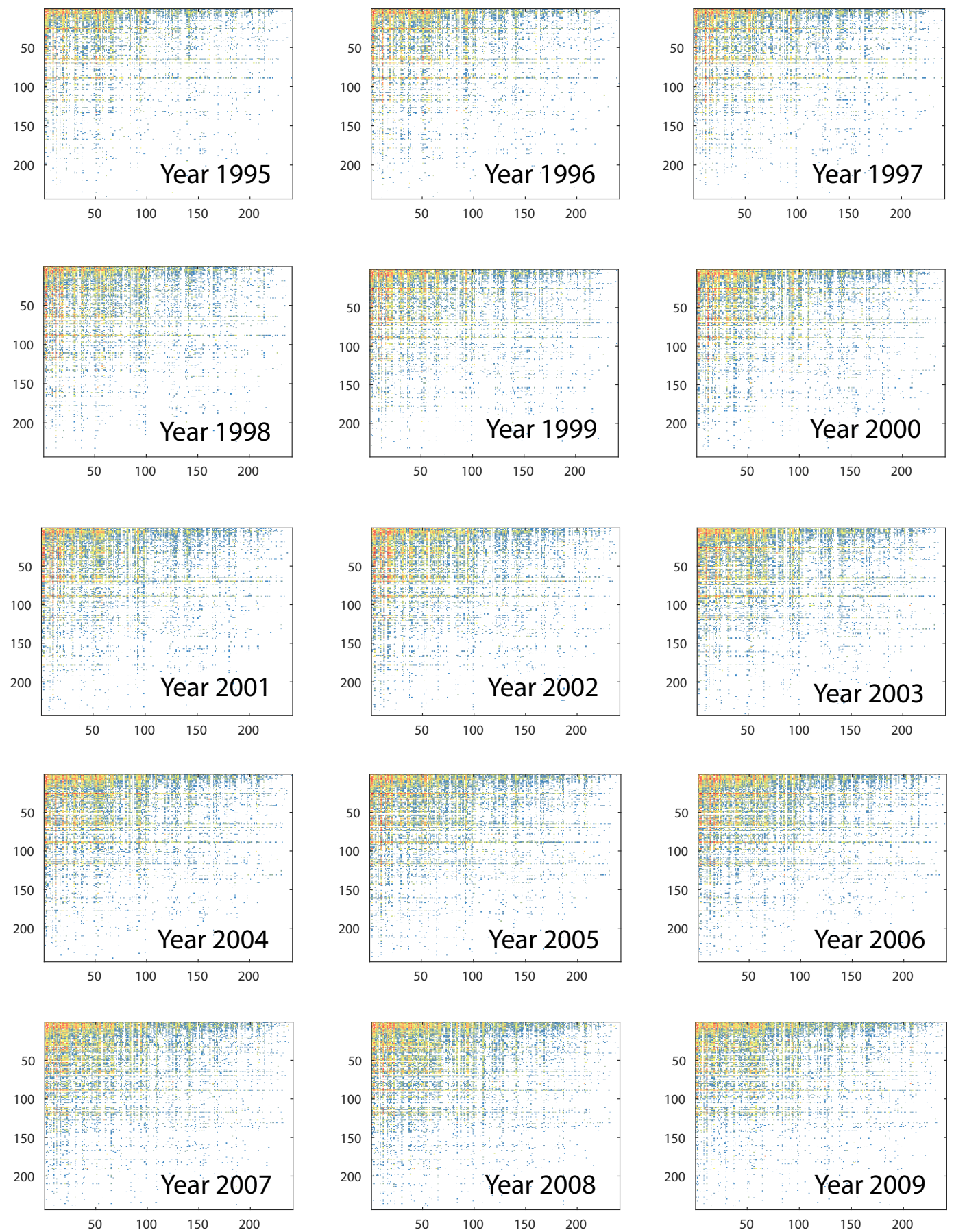

Fig. 8.4.: Bipartite Matrices for all periods from 1995 to 2009. This figure shows the interaction matrices for all periods for $\mathrm{HS} 2=84$ as an example of how these matrices evolve over time. In this format and due to their sizes, it is quite difficult to observe the changes from one period to the next. The exporters ( $\mathrm{x}$ axis) and the importers (y axis) are ordered so that the are ranked by cummulative strength over the 15 periods.

the temporal effects within trade networks. In order to depict the most common positions where the dynamical phenomena are more likely to appear, we define a parameter that allows us to measure the characteristic distance in which a temporal 

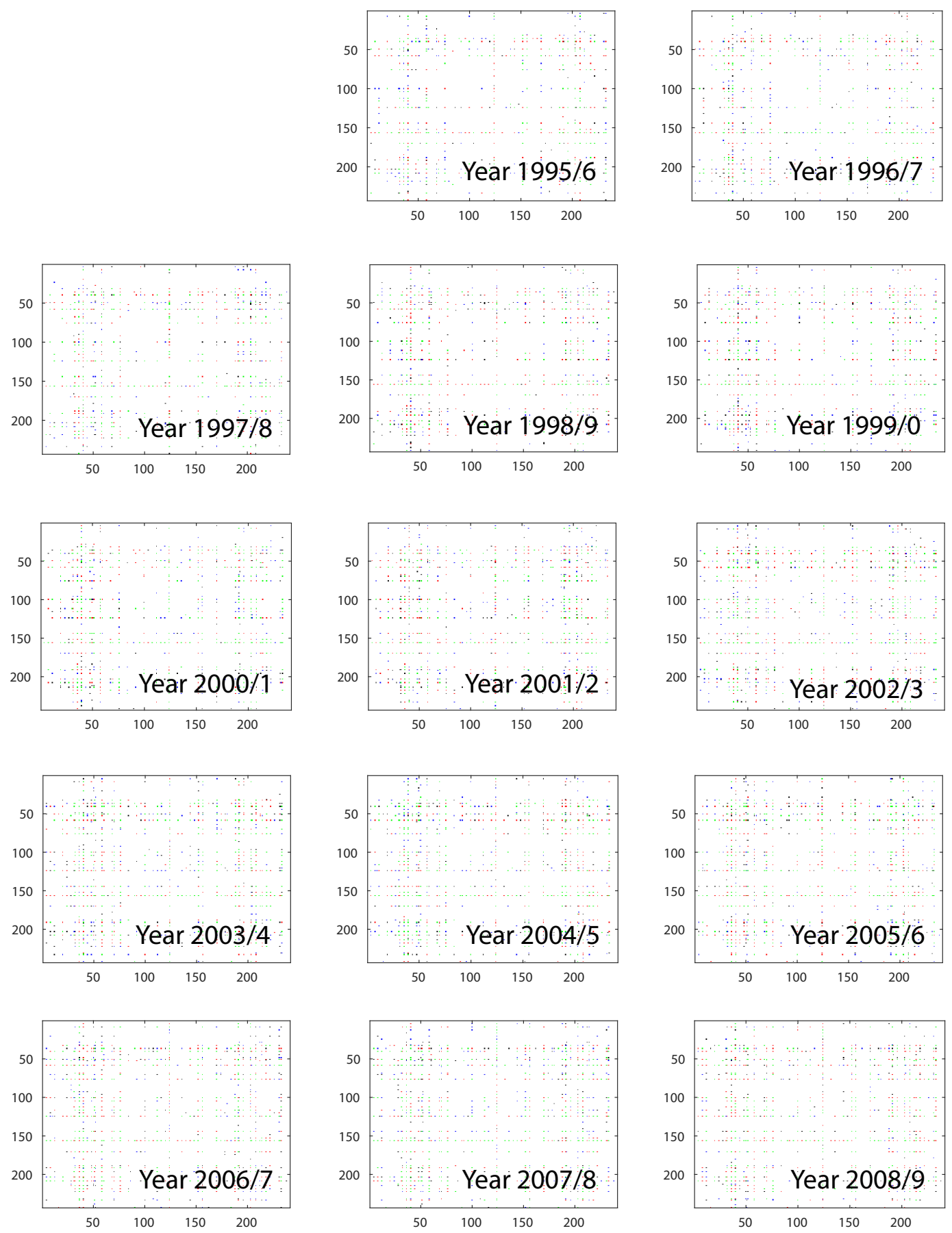

Fig. 8.5.: Dynamical variances for all periods from $1995 / 6$ to $2008 / 9$. This figure shows the dynamical variances matrices for all periods for HS2 $=84$ as an example of where the temporal phenomena appears in them. In this format, we have color-coded according to our convention. The exporters ( $\mathrm{x}$ axis) and the importers (y axis) are ordered so that the are ranked by cummulative strength over the 15 periods. Here, we observe that the dynamical phenomena is not equally distributed across the matrices. For example, the upper-left corner of each matrix, which is consistent with the core of the network, is rarely affected by these temporal changes, whereas the rest of the matrix does show areas of significant dynamical activity. 
variance takes place from the core of the network to the periphery. We call this parameter a occurrence distance $d$, as shown in figure 8.6. Then, this occurrence distance will be close to zero if a temporal variance takes place within the core of the network, whereas it will be close to a maximum value if it takes place in the periphery zone. With this occurrence distance $d$, we intend to describe the frequency in which the dynamical phenomena takes place as function of it and find evidence to either reject or accept our premise for dynamical phenomena not being equally distributed across the interaction matrix. We define $d$ as follows:

$$
d=\sqrt{\left(\frac{r a n k_{e}}{m}\right)^{2}+\left(\frac{r a n k_{i}}{n}\right)^{2}}
$$

where $\operatorname{rank}_{e}$ is the position ranking of the exporter in which the temporal variance takes place, $r_{a n k}$ is the position ranking of the respective importer, $m$ is the number of exporters for all periods and $n$ is the number of importers for all periods. In this way, the occurrence distance $d$ is defined as an dimensionless quantity that goes from 0 to $\sqrt{2}$, where 0 is consistent with the very core of the network and $\sqrt{2}$ is consistent with the farther away regions of the periphery.

$t=1$

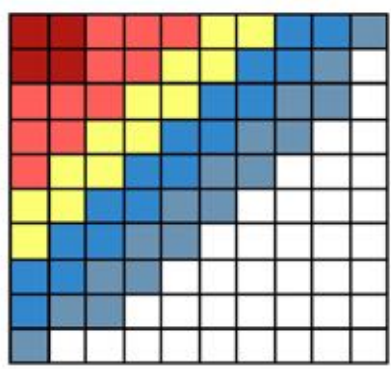

$t=2$

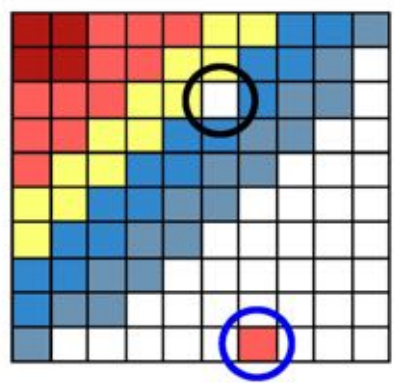

Dynamical Variances

$d_{1}=\sqrt{\left(\frac{\text { ranke }}{m}\right)^{2}+\left(\frac{\text { ranki }}{n}\right)^{2}}$

$d_{2}=\sqrt{\left(\frac{\text { ranke }}{m}\right)^{2}+\left(\frac{r a n k j}{n}\right)^{2}}$

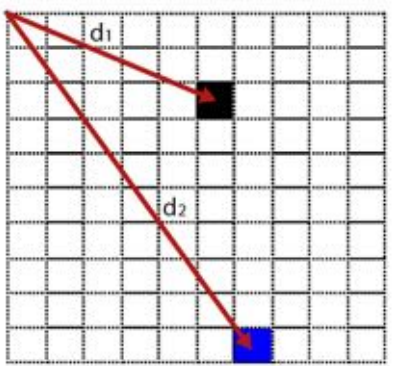

Core

$\mathrm{d}=0$

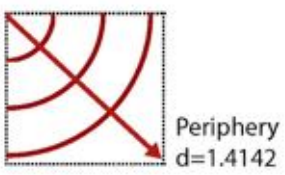

Variance Distributions

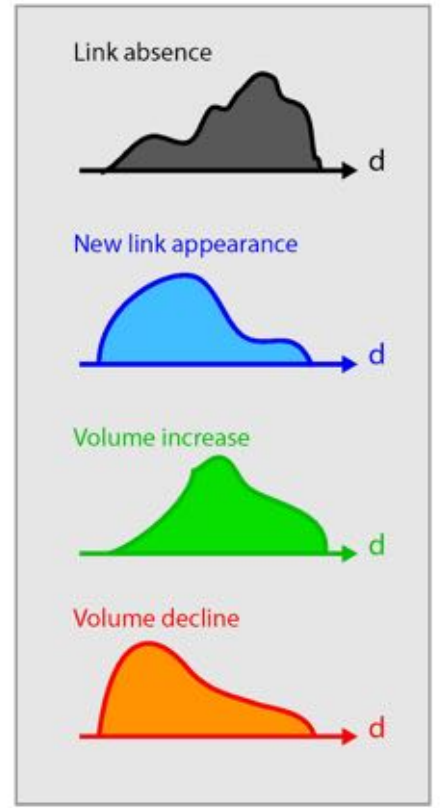

Fig. 8.6.: Dynamical distances for all type of variances. This figure shows the definition of the occurrense distance for an example matrix (right side), which leads to the variance frequency or distributions. 
We realize that dynamical phenomena is highly complex to analyze. This mainly because different types of variance take place at the same time and correlations among these types of variance are very difficult to determine. Consequently, we make a decision to analyze the 4 types of variance as independent and uncorrelated variables. Our hypothesis is that similarities between probability distributions as a function of the occurrence distance $d$ may provide some evidence about the process in which markets change and are re-configured over time. We believe that the form of the probability distributions are an enabler to the study of dynamical phenomena and to understanding why most of the bipartite indicators from Chapter 7 remain mostly unchanged over time, especially the nestedness features. We now display the results for the example for all periods in figure 8.7.
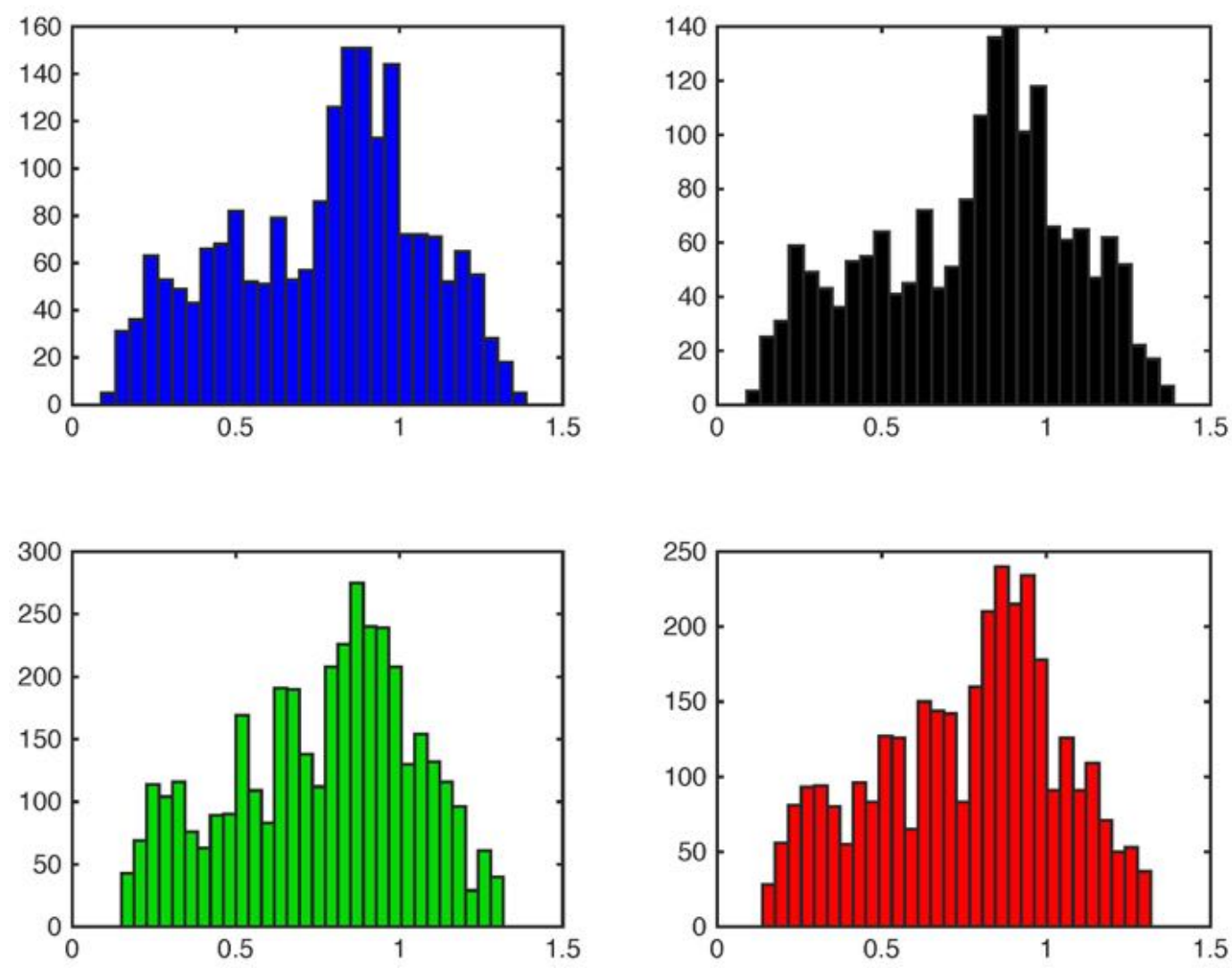

Fig. 8.7.: Dynamical distributions as a function of the occurrence distance. This figure shows the frequency of each type of dynamical variance as a function of the occurrense distance: link appearance (blue), link absence (black), volume increase (green) and volume decline (red).

We observe very clear similarities among the 4 distributions. In fact, the 4 probability distributions are quite alike in the form and values. To start, we first find little to no activity of temporal variance for small values of $d$ that are close to zero. This is valid for all the 4 distributions (appearance, disapperance, volume increase and volume decrease), pointing to a fact that we could already visually appreciate before, the fact that the core of the network is not affected by temporal variances or any 
dunamical phenomena. This is a revealing finding, since it may open the door for future research and some forecasting activity. If the core of a trade network is not affected by dynamical phenomena, we could argue that the top exporters and importer are solid, stable and unchanged group of highly inter-connected nodes that are only subject to global changes that affect the whole system, but they are not affected by local temporal phenomena. This is a confirmation of some of the premises in our research plan, where the analogy with mutualistic ecosystems was an important part of it. In fact, a high degree-high strength core is also present in these ecosystems, but due to lack of temporal data, it has not been possible to measure how the core and the periphery of the network was impacted by time evolution. Markets, on the other hand, do have temporal data and, if the analogy stands against hypothesis rejection, we could state that a robust core that is not affected by temporal variance is one of the reason why mutualistic ecosystems and international trade markets remain with similar topological features over time. Furthermore, it may suggest the activity of countries at the core are more predictable by only observing the market behavior. Another region of the bipartite matrix to observe is that of $0.7<d<1.1$, which seems to be a range of the occurrence distance where a high concentration of temporal variance takes place. On the one hand, we may interpret this region to be a transition zone that separates the core of the network from the periphery. On other hand, the evidence suggests that, for all 4 types of dynamical phenomena, the probability functions of temporal variances reach their maximum values simultaneously. This fact points to a highly active transition zone, where countries or nodes from the periphery seem to try to enter the core of the network. This idea may also be related to the self-interest and the profit maximization concepts. If one country in the periphery wants to access the core of the network, it has to implement strategies for growth at the expense of other countries of similar status and rank position in the market, provided that the overall bipartite features do not significantly change over the years. This may also be the case because the rational thinking process would tell the country not to risk a strategy that implies a direct competition with a much larger exporter or importer, but rather competition against another player of a similar size, where the chances for a positive outcome are higher. If we also take in account that, the closer to the core a country is, the lower the probability for temporal variances to appear, we may conclude that it is this range of the occurrence distance where a larger number of dynamical phenomema take place as a consequence of the periphery countries trying to reach the core. The emerging phenomenon is that this competition force may be a systemic protection to keep the core robust and stable, but without denying the access, or at least the possibility of access, to the periphery exporters and importers. If this is the case, trade markets would not be a case of perfect competition, but they would be an approximation to it, as described in the economics literatureSamu48,Smith76. 
We now extend the dynamical phenomena procedure to the rest of the HS2 selected industries. We show all the distributions for each HS2 selected industries and for all periods in Appendix C (see figure C.29). Yet, it is observed that the pattern described in the previous paragraph holds for the rest of the industries, suggesting that a steady, robust core and an active transition zone are still present in all cases. This evidence is both revealing and it confirms that our premises are not wrong from a factual standpoint. Nonetheless, we have not explained the reasons why the bipartite features, and the nestedness in particular, remain amost unchanged over time. After extending the dynamical procedure to all HS2 selected industries, we have now a solid framework to determine the actual cause for the bipartite indicators to remain almost unchanged over time despite the significant temporal variences and changes from one period to the next. The reality is that the form of the distributions, although resembling the previously described case of HS2 $=01$, are not of the same function across industries. However, we do observe a more stable similarity between opposite phenomena within the same industry. For example, for HS2 $=84$ (Machinery), the distribution of appearances and absences are of almost the same form and with a similar number of occurrences. The same is true for volume increase and decline distributions. Moreover, a similar situation is found repetedly across all industries. This fact suggests that, for every temporal variance that take place at a given occurrence distance, there will be an opposite variance that will take place at a relatively similar distance as well. If we consider more than one variance and think about the whole interaction matrix, we may state that there will be a similar number of opposite temporal variences taking place at a similar occurrence distance $d$. This provides solid evidence that several local and opposite variances taking place at the same distance from the core of the network would explain the reason why the global bipartite indicators do not change significantly over time.

But regarding nestedness in particular, the previous statement may be even more precise. Nestedness, in the way that is defined by the WINE indicator, also depends on the positions of the existing interactions and on the weights of such interactions. Thus, when a temporal variance takes place at a given occurrence distance and, at the same time, is accompained by an opposite type, that is, an appearance with an absence or a volume increase with a volume decline, the global WINE indicator will not significantly change its value despite the number of local and opposite changes or occurrences, hence leading to the conservation of the nested structure and its overall value as measured by $\eta_{w}$. Since this situation of similar opposite distributions does actually take place across the HS2 industries, this compensation mechanism is an important and fundamental feature of international trade markets. If we think for a moment in what this could mean in reality or from a geopolitical standpoint, for example, it might be a case where due to a new local regulation one importer can no longer buy a given product from an exporter. In this case, the importer country 
will try to satisfiy its needs for such a product by contacting and purchasing the good from a similarly-ranked exporter. Hence, the previous interaction would no longer exist, creating a link absence in the interaction matrix, but another link will appear as a consequence of the new deal with the new exporter. Since the importer will try to deal with an exporter of similar size and ranking, both the absence and the appearance will be located at a similar occurrence distance from the core of the network, leading to a similar outcome in terms of WINE nestedness $\eta_{w}$. This is probably the main finding of this chapter and, although it may seem to be trivial after some consideration, the relevance of procedure is, in our opinion, a step forward in the way that complex systems, and markets in particular, can be approached to perform solid research. With this finding in mind, we now can continue to Part 3 of this study and apply what we have learned to build statistical models of markets. 

Part IV

On Market Models 



\section{Network models of trade markets}

All models are wrong.

- Common aphorism in statistics...

(...generally attributed to the statistician

George Box)

Based on the thorough review of the results in Part 3, we now have a deeper understanding of the topological properties and the dynamics of Trade Networks. This knowledge leads us to the formulation of several hypothesis about the mechanism with which market agents command the forces and decisions that fundamentally shape international trade. However, we do not have any model to test our hypothesis. In Part 4, we provide a review of the models we have developed and tested to simulate international markets (chapter 9). After this, we formulate an innovative approach to modeling markets based on dynamics from and similarities with mutualistic ecosystems (chapter 10). This leads to a concept of mutualistic analysis of makets. Next, we also provide an additional model of financial markets, and more specifically, the interbank lending market. Finally, we conduct a detailed discussion of our conclusions and opportunities for future research. 


\subsection{On general market models}

When we consider our markets as complex systems and try to model their behavior, we also find ourselves in a point of our research where a framework has to be put in place to describe the market formation process. Particularly, we need to account for the bipartite nature of the problem in addition to the weighted nature of the interaction matrix that represents the complex networks of international trade markets. For this reason, and from a computational standpoint, we use a number of input parameters, which are directly related to the market size both quantitatively and qualititatively. We start by setting a restriction in the number of exporters $m$ and importers $n$ allowed to participate in the market model, leading to a maximum size of the interaction matrix $B$. However, this is a simple restriction in comparison to the quantitative or weighted properties. Any market has a amount of demand from the buyers or importers that has to be satisfied through a supply of the same size from the sellers or exporters. The issue of how to allocate this amount of supply and demand among exporters and importers is actually of special interest for the next few chapters.

We consider several alternatives to perform such a task. Yet, the different formulations can be summarized in two cases. The first one is to set a maximum amount of trade volume and incorporate a quantititive distribution across exporters and importers. This is quite an interesting approach but, however, it also incorporates too many restrictions to the model, especially when selecting the actual distribution among exporters and importers, which makes it highly dependent on the selected distribution functions themselves. The second one is a more pragmatic approach. We still consider a maximum amount of supply and demand, but we allocated this amount across exporter and importers by taking discrete units of demand and supply at each step of the process. This process is consistent with a unitary link aggregation procedure, where we may argue that the total trade volume within the market will ultimately be the aggregate of many decisions made over a small piece of the market at each step. Thus, we adopt the first ot the second case depending on which particular model we implement, and the market model can easily be described by the forms $M_{1}[m, n, W, f(W)]$ and $M_{2}[m, n, W]$, where $M$ is the model from the first or second formulation, $m$ is the maximum number of exporters, $n$ is the maximum number of importers, $W$ is the maximum trade volume and $f(W)$ are the demand and supply distribution functions across countries. By extension, the second model will be executed over $W$ steps of allocation of a unitary amount of trade volume in each step (link aggregation).

In figure 9.1, we show a summary of the two alternatives that we considered to develop our models. While both alternatives have some merit and can be implemented 
under the right conditions, it will be a matter of applicability that will eventually decide which one of the approaches we should use for each model. In this chapter and the next, we implement several models that share a common goal, which is to build complex networks with statistical properties that resemble those of the actual markets. In approaching this problem, we believe that the simplicity of the second approach may fall short in its prediction capabilities, whereas as the first one can improve this at the expense of introducing more complexity in terms of model restrictions and input. Balancing the simplicity and an additional restrictions will be key to find an optimum approach for each model. 


\section{A) Model 1}

$M 1(m, n, W, f[W])$

This model is based on the premise that both the demand and the supply have a pre-existing distribution across the nodes (exporter and importer countries).

M1 incorporates an additional restriction to the model. However, this may be of interest since it allows us to apply realistic constraints to each node to simulate actual market performance.

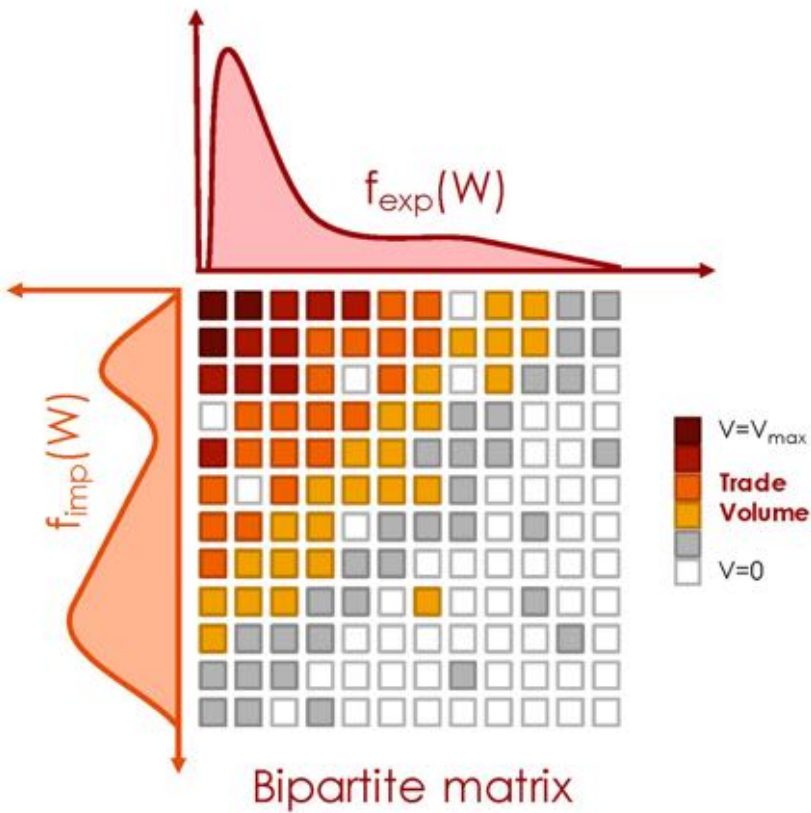

\section{B) Model 2}

$M 2(m, n, W)$

This model is based on the premise that both the demand and the supply have no pre-existing distribution across the nodes (exporter and importer countries).

M2 assumes that the trade volume is a discrete variable and that the decision to allocate each unit of trade is made in W steps. Each allocation is independently taken and follows the same process.
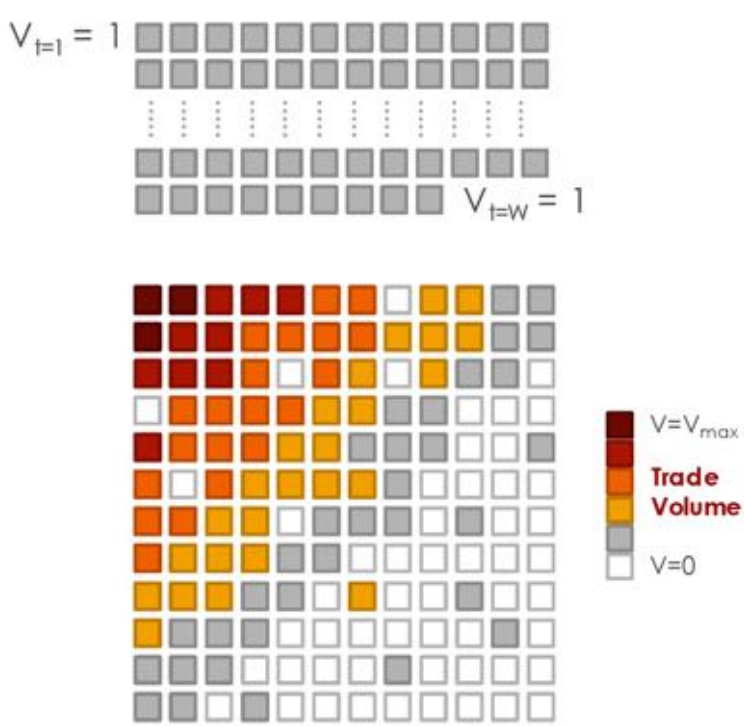

Bipartite matrix

Fig. 9.1.: Two approaches to market models. This figure shows the two alternatives considered to depict the market formation process. A) Model 1 relies on typical network size parameters in addition to the distribution of demand and supply across countries. B) Model 2 only relies on the network size parameters since it depicts a link aggregation process instead. 


\subsection{Random network model}

When analyzing network models, we start by considering the random network model. Widely known as the Erdos-Renyi model, this model can be formulated in two ways. The first formulation generates a graph $G(n, p)$ of $n$ nodes, where all posible links may or may not be present with a probability $p$. Alternatively, the second formulation of the model generates a graph $G(n, m)$, where a graph $G$ is generated with $n$ nodes and $m$ links, and therefore all possible graphs have the same the probability of being generated. In order to better describe the process of market formation, we select the second formulation of the random network model and we generalize it to fit the bipartite nature of trade networks. Thus, we build an algorithm to recreate the model computationally. Our algorithm works as follows:

First, we set up the model restrictions as indicated by the selected second formulation in section 9.1, where $m$ is the maximum number of exporters (type A nodes of the bipartite graph), $n$ is the maximum number of importers (type B nodes of the bipartite graph), and $W$ is the market size in terms of trade volume units, which is equal to the number of links in the network as well.

Secondly, we set up a rutine to allocate each link between an exporter and an importer over $W$ steps and record both the list of interactions for each link and the interaction matrix $B$ at each time step of the process. Thus, we do not only keep track of the final result of the process, but also we keep track of the network growth process itself. For each step of the process, we select an exporter and an importer to assign to the trade volume unit, or to the unitary link, at random with the same probability $p_{e}=\frac{1}{m}$ for the exporter nodes and $p_{i}=\frac{1}{n}$ for the importer nodes.

Thirdly, for each step, we allocated the unitary link for the selected exporter and importer and update the interaction matrix $B_{t=T}$ as a consequence, where $\mathrm{T}$ is the current time step. The value of the interaction matrix $B(a, b, t=T)$ is increased by 1 , where $a$ represents the selected exporters and $b$ represents the selected importer at that step $(t=T)$.

Finally, we also compute and display the process outome in terms of the interaction matrix $B$, which we order based on degree and strength to display the potentially nested structure, and also compute the bipartite indicartors discussed in chapter 5; namely, the degree distribution $P(k)$, the strength-degree correlation $S(k)$, the nearest neighbour degree $K n n(k)$, the bipartite clustering $C 4(k)$, the average weigth as a function of the end-point degree $\langle w\rangle \sim\left(k_{i} * k_{j}\right)$, and the nestedness $\eta_{w}$. 

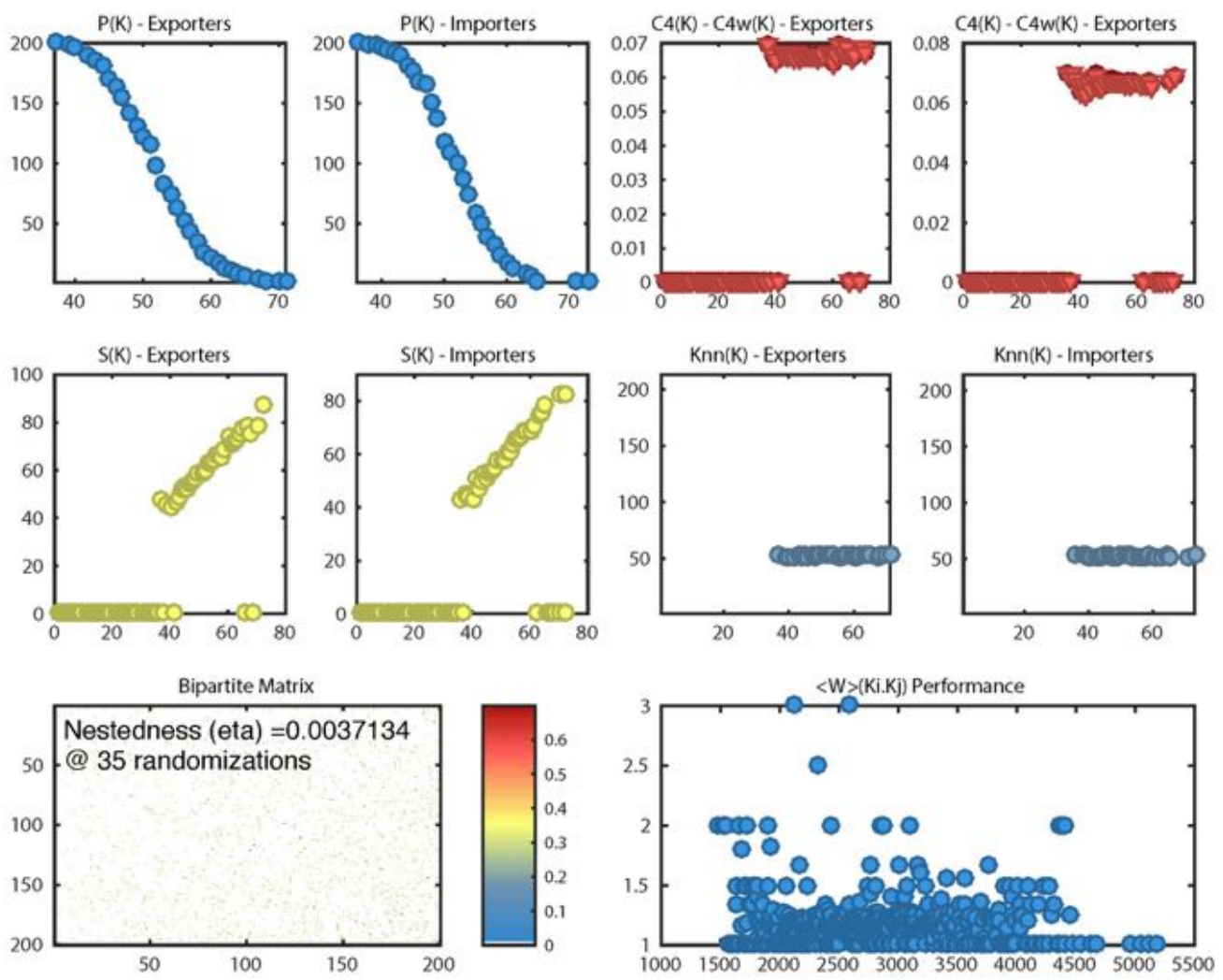

Fig. 9.2.: Example of the random bipartite weighted model. This figure shows the bipartite indicators of an example network created upon our random bipartite model. We observe that these properties do not resemble those of the actual markets in general and we find some expected behavior based on the theory. The degree distribution shows a normal distribution pattern for both the exporters and importers. The strength-degree correlation is shows a unitary $\beta$ exponent. The performance of both the $K n n(k)$ and the bipartite clustering $C 4(k)$ do not show a correlation with the degree $k$. Additionally, there is no correlation of the average weight as a function of the end-point degree. Finally, we find no nestedness in the interaction matrix.

We believe this process is an interesting point to start exploring the nature of the trade markets since it is both bipartite and weighted in nature at the same time and, because of that, the model is also capable of potentially reproducing the nature of international trade networks. In order to test this random bipartite model, we create an example of market formation. Figure 9.2 shows the process outcome for $M[200,200,12000]$, which we select mostly based on the typical parameters of actual markets. The total trade volume $W$ is expressed in million dollars.

After executing 300 realizations of the model, we find that the results are consistent and stable. Yet, when we analyze the performance of the bipartite indicators, the model shows significant deviations from the behavior of the empirical markets data. In figure 9.3, we show the aggregated indicators for the 300 realizations. Let us review each indicator one by one. 

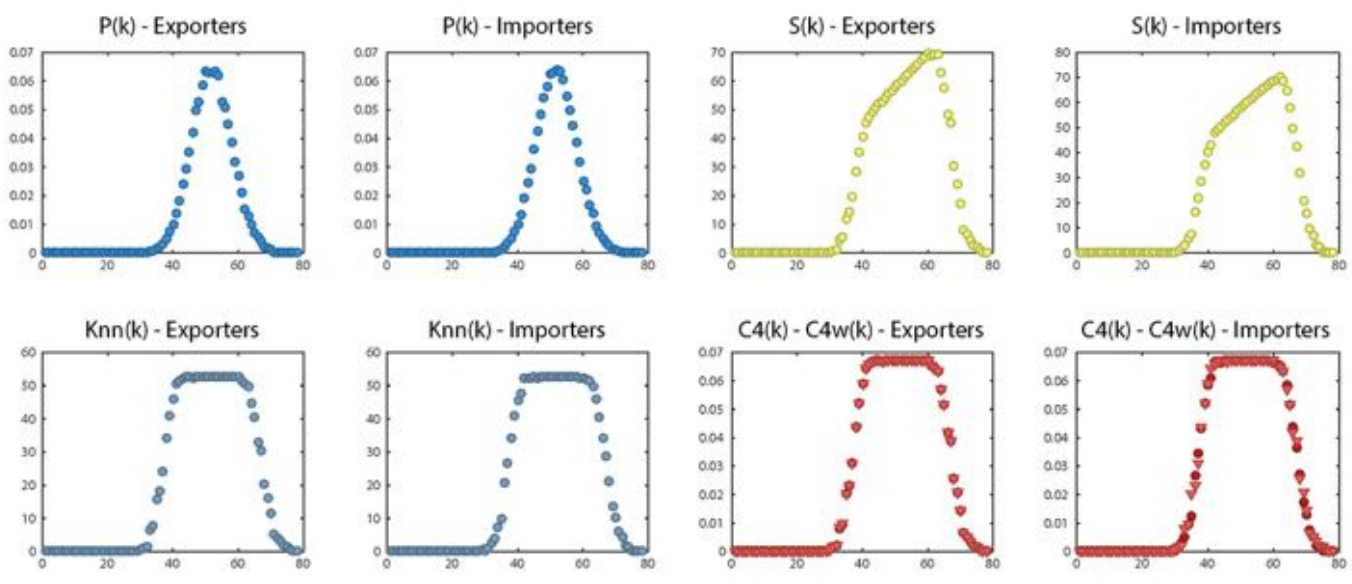

Fig. 9.3.: Results of the random bipartite model $M[200,200,12000]$ over 300 realizations. This figure shows the bipartite indicators of the random bipartite model over 300 realizations and after consolidating the average performance of each indicator. We continue to observe that most of properties do not resemble those of the actual markets. We find some expected behavior predicted by the theory. The degree distribution shows a normal distribution pattern for both the exporters and importers. The strength-degree correlation is shows a unitary $\beta$ exponent. The performance of both the $K n n(k)$ and the bipartite clustering $C 4(k)$ do not show a correlation with the degree $k$.

The degree distribution $P(k)$ resulting from this model is not a heterogeneous or long-tailed one, but a normal distribution for both the exporters and the importers. Similarly to what is expected from the pure, non-bipartite Erdos-Renyi model, our bipartite and weighted equivalent model behaves as expected. This pattern is not consistent with those of the empirical data, which makes it a challenge to depict actual market behavior.

The strength-degree correlation $S(k)$ shows a behaviour that is consistent with the case of randomly distributed weigths. That is, when weights are distributed with a random pattern, the $\beta$ exponent is equal to 1 , which is exactly what we find in our model. In this area, the model does not successfully replicate the behavior actual markets since they show a different condition where $\beta>>1$.

The nearest neighbor degree $K n n(k)$ does not display a correlation with the degree $k$, suggesting that neither an assortative nor a dissortative behavior is present within the outcome of our model. This finding is consistent with the performance we find within actual markets, but this may be a trivial coincidence since non-correlated variables are actually expected from a random model.

The bipartite clustering $C 4_{b}(k)$ and $C 4_{w}(k)$ also display a non-correlated pattern with the degree $k$, obtaining low values of the average clustering coefficient. This is again supported by the theory of random networks and expected as well. There 


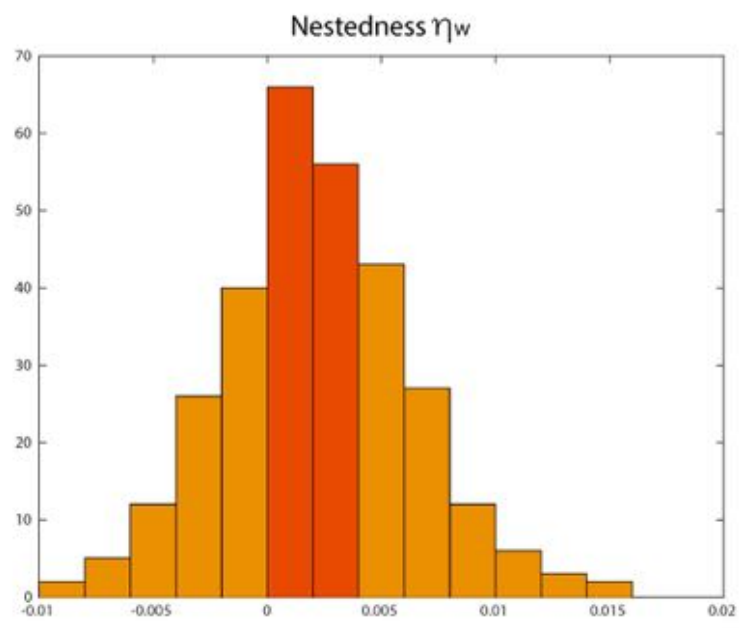

Fig. 9.4.: Nestedness results of the random bipartite model $M[200,200,12000]$ over 300 realizations. This figure shows the performance of the $\eta_{w}$ nestedness indicator calculated at 35 randomizations of the interaction matrix $B$ for each realization of the model. We observe an average value of $\eta_{w}=0.01$, which provides uncontroversial evidence that the random bipartite model cannot yield a nested structure of $B$, whereas actual markets show a nestedness performance $0.65<\eta_{w}<1.05$ depending on the product, category and industry.

is no evidence of distinct behavior between the weighted and non weighted performances and consequently, no evidence of $C 4_{w}(k) / C 4_{b}(k)>1$. These 3 findings also constitute another deviation from the empirical markets.

Regarding the average weight as a function of the end-point degree (as per figure 9.2), we do not observe a clear pattern of a two-zone behavior. In fact, we can only conclude that we obtain a random distribution in this indicator, yet again, a deviation from the actual market features.

Finally, and more importantly, we find no evidence of nestedness within this model. The $\eta_{w}$ values this model capable of generating are close to zero, showing once again a clear deviation from the features of actual trade markets. In figure 9.4, we show the distribution of nestedness values for the 300 realizations.

It is clear that the random model has fundamental gaps to reproduce both the topological properties and the dynamics of trade networks. First, it cannot generate a long-tailed degree distribution of any kind, since random networks typically display a normal distribution, and they actually do here. Secondly, it cannot reproduce the weighted condition in $S(k)$ similar to rich club effect. Finally, neither the clustering, assortative nor the nestedness features can ever be found as a result of implementing this random bipartite model. In this sense, it is safe to say that random networks are among the least suitable models to depict actual markets. 
Nonetheless, we do find a positive conclusion within this model. The random bipartite model is actually a quite suitable null model for our research, setting a solid foundation for the upcoming models and confirming that a link aggregation process offers a robust framework to understand how markets are formed. In the next section, we now explore a different approach to market models, which is based on the first formulation $M 1$ from section 9.1, the gravity models of trade. 


\subsection{Gravity models}

The weaknesses of the model from the previous section leaves us in a position that calls for further exploration for new models that can generate weighted networks. We now explore the $M 1$ formulation from section 9.1 and look for models that can accomodate to this approach. Contrary to the link aggregation process, the M1 formulation computes the final state of a market based on the increased restrictions to the exporter and importer nodes. This has some advantages, such as the fact that both the supply and demand are realistically distributed among countries. Yet, we still need to find a proper model to build complex bipartite and weighted networks. If we accept this, we also realize that analogies between physical and social phenomena are not new to the scientific community. In this section, we borrow a model from physics that can help us explain the weighted nature of international trade markets, that is, the law of gravity. Sir Isaac Newton's gravity principle has already been used in many fields to describe complex behaviors that are as different in nature as they are in the disciplines they belong to. For example, social scientists, (Lewer and Van den Berg, 2008

$$
G=\frac{M_{i} M_{j}}{D_{i j}^{2}}
$$

where $G$ is the attraction force between the objects of masses $M_{i}$ and $M_{j}$, and $D_{i j}$ is the distance between objects. We now rearrange this and incorporate logarithms:

$$
\log G=\log M_{i} M_{j}-2 \log D_{i j}
$$

Lewer and Van den Berg[94] proposed a similar formulation for human migration, where the migration flows $M_{i j}$ are a direct analogy to attraction force $G$. Furthermore, the population of cities $i$ and $j$, which are $P_{i}$ and $P_{j}$ respectively, play a similar role as do the masses $M_{i}$ and $M_{j}$ of objects attracting each other, whereas the distance between cities work similarly to the distance between objects. Formally, we have:

$$
\log M_{i j}=\beta_{0}+\beta_{1} \log \left(P_{i} P_{j}\right)+\beta_{2} \log \left(D_{i j}\right)+\epsilon_{i j}
$$

Thus, the migration flow of a person moving from city $i$ to city $j$ would be proportional to the product of their respective populations $\left(P_{i}\right.$ and $\left.P_{j}\right)$ and inversely 
proportional to the distance between them $\left(D_{i j}\right)$. Like objects with bigger masses attract each other with a stronger force, cities with larger populations would have larger migration flows. Additionally, cities that are closer to each other would also have a larger migration flow. This model requires the correlation constants $\beta_{0}$, $\beta_{1}$ and $\beta_{2}$ to be complete and they can be derived from the empirical data with a linear model $Y_{\text {pred }} \sim 1+x_{1}+x_{2}$. But this is not the only model for migration patterns. Other variables that affect people's decision on where to migrate to may be incorporated to the model. Model predictability can be improved in this way and, example, these variables could be: a) language $L_{i j}$, which takes in account whether the same language is spoken in both cities, b) cultural similarity $Q_{i j}$, which accounts for whether cities have a similar culture, and c) the stock of previous immigrants $S_{i j}$, which is the number of natives from city $i$ that are already living in city $j$. Hence, the improved model can be expressed as:

$\log M_{i j}=\beta_{0}+\beta_{1} \log \left(P_{i} P_{j}\right)+\beta_{2} \log \left(D_{i j}\right)+\beta_{3} \log \left(L_{i j}\right)+\beta_{4} \log \left(Q_{i j}\right)+\beta_{5} \log \left(S_{i j}\right)+\epsilon_{i j}$

In a similar way, other previous work has applied the same principle of gravity model to bilaterial trade $[94,43,34,97,12]$. In this case, an analogy has been drawn between the gross domestic product (GDP) and the mass of an object. Since the GDP is the economic output of a country in a year, it can also be intrepreted as the size of an economy in terms of the wealth generation. From a pragmatic standpoint, this seems a rather valid analogy, yet, distances between countries would also play a similar role for trade as it does for migration on the other hand. For example, the higher the distance between countries would be, the lower the "gravity effect" between them will be. This is supported by the fact that higher distances imply higher transportation costs and, consequently, a decreased attractiveness to perform trade activity will exist in comparison to countries that are closer to each other. Hence, we can write that:

$$
\log T_{i j}=\beta_{0}+\beta_{1} \log \left(Y_{i} Y_{j}\right)+\beta_{2} \log \left(D_{i j}\right)+\epsilon_{i j}
$$

where $T_{i j}$ is the trade flow between countries $i$ and $j, Y_{i}$ and $Y_{i}$ are the GDP for each country, and $D_{i}$ is the distance between countries. This is a robust apporach to understand the weighted nature of international trade, as evidenced by previous work ([94, 43, 34, 97, 12], but gravity models of trade do not come without some caveats either. First, we are trying to recreate complex bipartite networks with the features actual markets and, for any given product, a prediction based on solely the 
GDP and distance would yield a fully connected network, where all countries would have predicted trade flow that is higher than zero despite actual trade flows and the empirical restrictions. Despite the model predictions, some countries do not trade with each other due to many reason, and mainly due to geopolitical ones. Obviously, this would not be a realistic approach, a situation that calls for further consideration. Secondly, gravity models are normally applied and computed at country level, but not at product level. Because GDP represents the wealth generation of a whole country, this only variable may not be a good predictor for specific products. Thus, the total country GDP might not reflect the actual tendency to purchase or sell a specific type of product. For example, the number of nuclear reactors (HS6 = 840110) to be imported by a given country is not entirely related to the GDP of a country, but to many other factors, like energy policy, current energy requirement, number of domestic manufacturers of nuclear reactors, competitiveness of domestic manufacturers, and more. Finally, this calls for a reflection regarding the level of aggregation of the market to be simulated or predicted by this form of gravity model. It is evident that lower level of aggregation would potentially yield a less accurate prediction, whereas the opposite could be true for a higher level of aggregation. In this study, we will focus on the HS2 or industry level of aggregation to overcome such caveats.

Nonetheless, when considering further related work on gravity models of trade[12, $34,43,97]$, we realize that several additional variables can also be incorporated to the model so that predictability improves, similarly to the migration case. Being a higly complex human activity that involves complex decision making, international trade is susceptible of incorporating many statistically significant factors, hence, an improved trade model can be expressed as:

$\log T_{i j}=\beta_{0}+\beta_{1} \log \left(Y_{i} Y_{j}\right)+\beta_{2} \log \left(D_{i j}\right)+\beta_{3} \log \left(L_{i j}\right)+\beta_{4} \log \left(C_{i j}\right)+\beta_{5} \log \left(B_{i j}\right)+\epsilon_{i j}$

We observe the extended variables: a) $L_{i j}$, which represents whether the countries $i$ and $j$ have the same language, b) $C_{i j}$, which depicts whether country $i$ is a colony of country $j$, c) $B_{i j}$, which shows whether the countries $i$ and $j$ share a common border, d) $P_{i j}$, which tells us whether countries $i$ and $j$ have signed international trade agreements, and e) $F_{i j}$, which depicts whether countries $i$ and $j$ have a common colonizer. These variables can still be increased, but despite the efforts of the previous scientific work, gravity models have not yet been able to explain the restrictions that appear in actual trade networks, where their bipartite matrices are naturally sparse. 


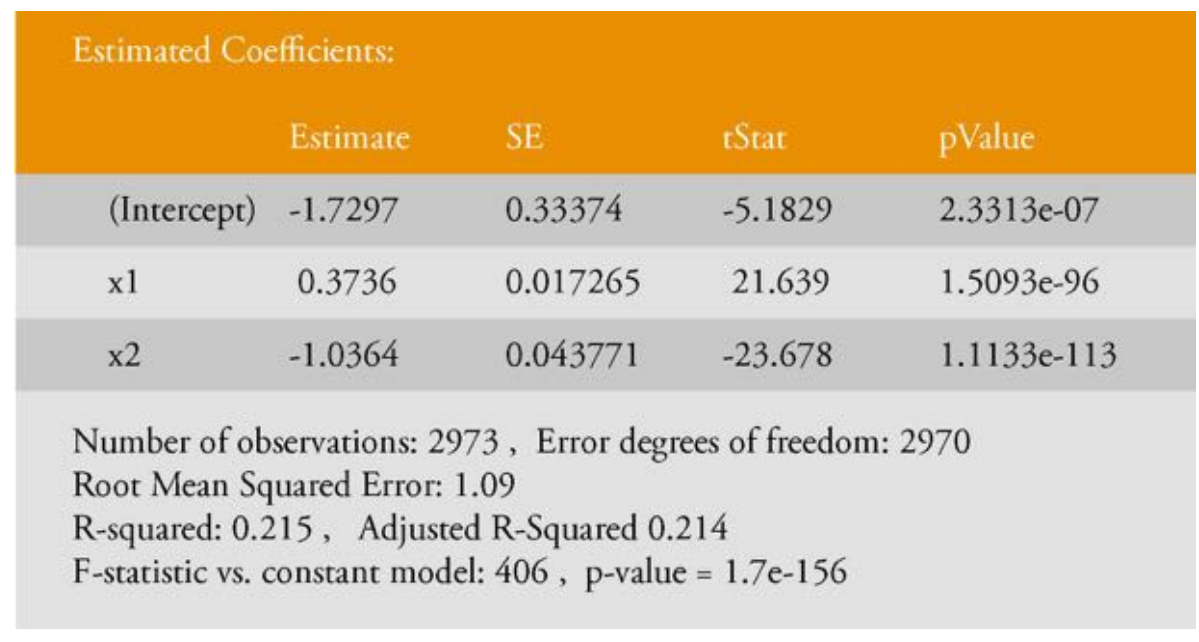

Tab. 9.1.: Estimated coefficients of our gravity model for HS2=01. This table shows the results of the regression analysis based on our gravity model for the total network.

So, instead of forcing a gravity model to mimic a highly sparse matrix based on randomness, we have decided to run a basic gravity model of trade that depends solely on GDP and distance, and incorporate the actual constraints of a market. Our gravity model can be formally expressed as:

$$
\log T_{i j}=\left[\beta_{0}+\beta_{1} \log \left(Y_{i} Y_{j}\right)+\beta_{2} \log \left(D_{i j}\right)+\epsilon_{i j}\right] R_{i j}
$$

In this model, $R_{i j}=0$ if country $i$ has no trade activity in a given year with country $j$ and $R_{i j}=1$ otherwise, hence, acknowledging the fact that the interaction matrix will display no trade activity between them and updating our gravity model to zero. Consequently, the resulting complex bipartite network and its interaction matrix $B$ will be as equally sparse as that of the actual market in given period. We also apply our gravity model definition to the selected industries HS2. In figure 9.5, we show the results of our gravity model in the industry HS2 $=01$. We perform this study for all the network links and use no RSP or RPP filters, which we defined in chapter 4 . In this case, we observe a similar pattern than that of the $\langle w\rangle\left(k_{i} k_{j}\right)$ end point degree distribution, where high volume links on the upper-righ quadrant of the graph seem to have a different behavior than those on bottom-left quadrant. As a consequence, we have a weak correlation $\left(R^{2}=0.215\right)$. These are the details on the linear regression model:

Because of the apparent different behavior in the regression graph, we also decide to implement a filter $\left(R S P>10^{-1}\right)$ to run a separate gravity model for the network core. Figure 9.6 shows the model for the filtered scenario, where we observe a stronger correlation $\left(R^{2}=0.46\right)$. Details on the filetered scenation regression are: 


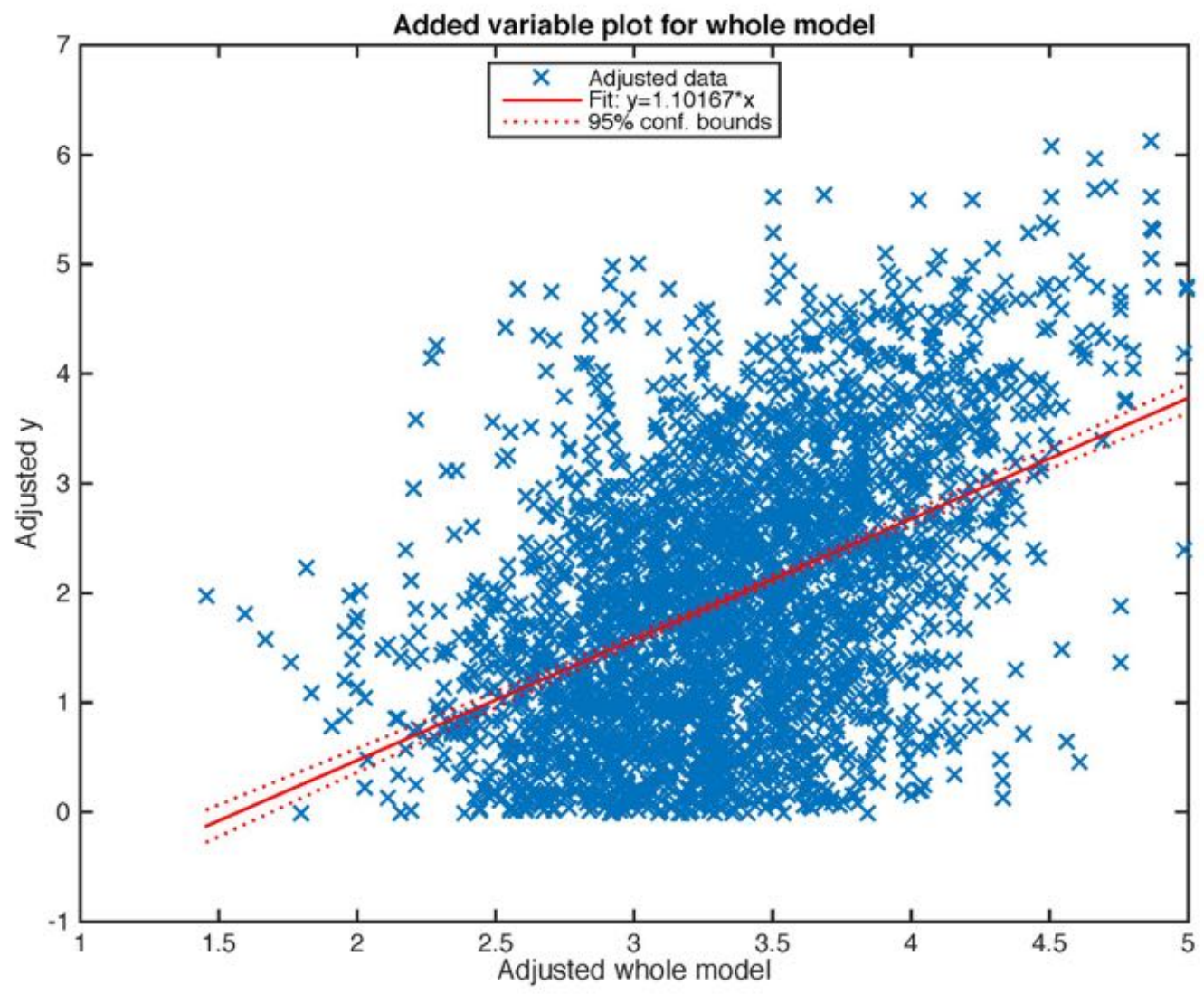

Fig. 9.5.: Gravity model fitting for HS2 $=01$. This figure shows model fitting performance of our gravity model for an example industry (HS2 $=01$ ). This is an added variable plot, which illustrates the incremental effect on the response of specified terms. The slope of the fitted line is the coefficient of the linear combination of the specified terms projected onto the best-fitting direction. The adjusted response (y) includes the constant (intercept) terms, and averages out all other terms.

\begin{tabular}{|c|c|c|c|c|}
\hline \multicolumn{5}{|c|}{ Estimated Coefficients: } \\
\hline & Estimate & SE & tStat & pValue \\
\hline (Intercept) & -3.4226 & 0.74517 & -4.5931 & $7.47 e-06$ \\
\hline $\mathrm{x} 1$ & 0.47315 & 0.037562 & 12.596 & $1.4787 e-27$ \\
\hline $\mathrm{x} 2$ & -0.56427 & 0.089662 & -6.2933 & $1.7437 \mathrm{e}-09$ \\
\hline \multicolumn{5}{|c|}{$\begin{array}{l}\text { Number of observations: } 216 \text {, Error degrees of freedom: } 213 \\
\text { Root Mean Squared Error: } 0.744 \\
\text { R-squared: } 0.46 \text {, Adjusted R-Squared } 0.454 \\
\text { F-statistic vs. constant model: } 90.6, \quad \text { p-value }=3.46 \mathrm{e}-29\end{array}$} \\
\hline
\end{tabular}

Tab. 9.2.: Estimated coefficients of our gravity model for HS2 $=01$ with $R S P>10^{-1}$. This table shows the results of the regression analysis based on our gravity model for the high importance links. 


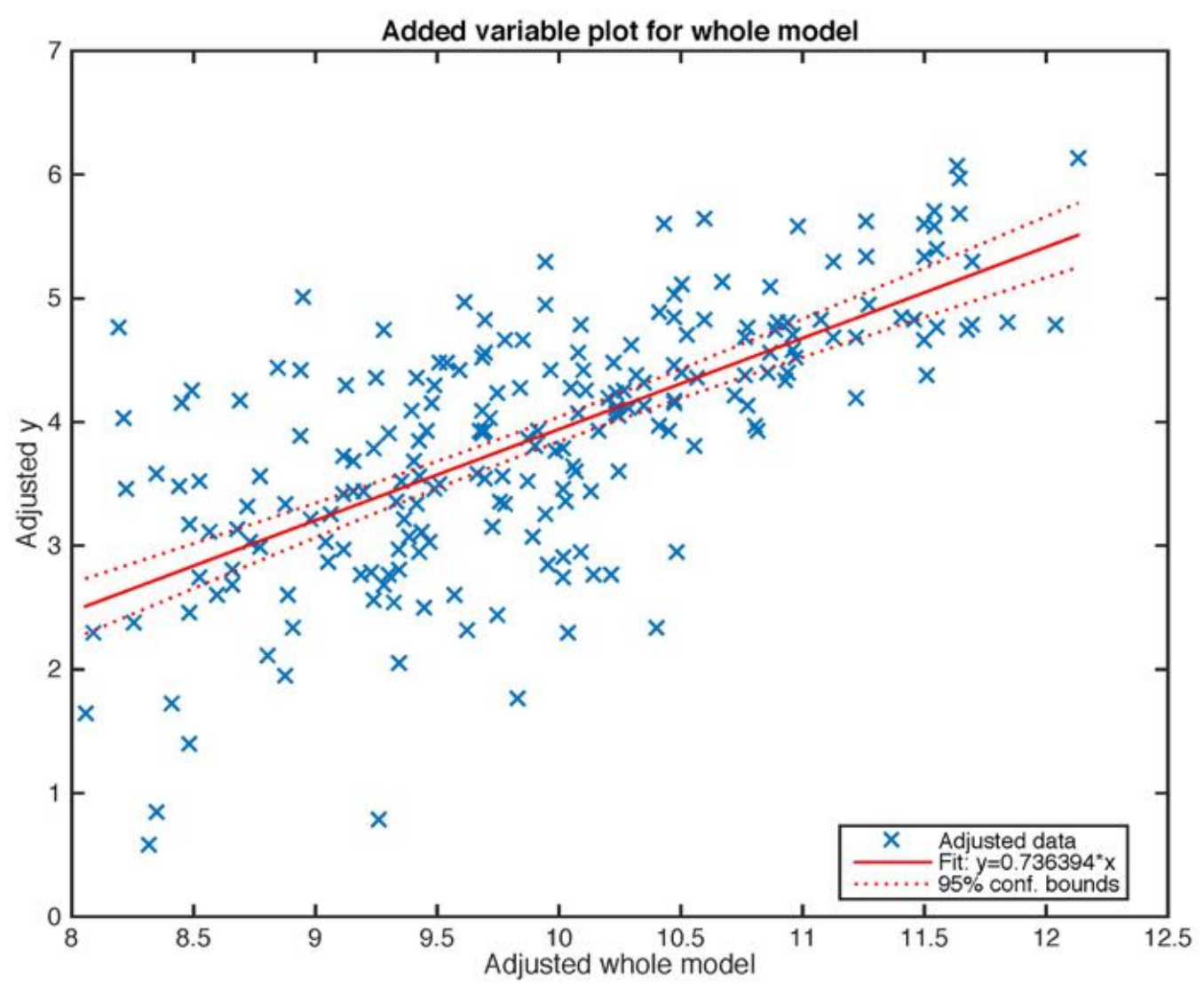

Fig. 9.6.: Gravity model fitting for HS2 $=01$ with $R S P>10^{-1}$. This figure shows model fitting performance of our gravity model for the high weight links of the example industry $(\mathrm{HS} 2=01)$. This is an added variable plot, which illustrates the incremental effect on the response of specified terms. The slope of the fitted line is the coefficient of the linear combination of the specified terms projected onto the best-fitting direction. The adjusted response (y) includes the constant (intercept) terms, and averages out all other terms. 
Furthermore, there is an additional phenomenon to comment on. When comparing the results of the gravity models for the unfiltered and filtered scenarios, we find a dimished effect of and contribution from the distance variable. Let us compare the models:

$$
\begin{gathered}
\log T_{i j}(\text { unfiltered })=-1.73+0.37 \log \left(Y_{i} Y_{j}\right)-1.04 \log \left(D_{i j}\right)+\epsilon_{i j} \\
\log T_{i j}(\text { filtered })=-3.42+0.47 \log \left(Y_{i} Y_{j}\right)-0.56 \log \left(D_{i j}\right)+\epsilon_{i j}
\end{gathered}
$$

For this case, the distance contribution to the total trade prediction turns out to be less important once the RSP filter has been applied. Yet, the overall performance of the model improves dramatically as previously reviewed. The combination of both facts may shed some light on the dynamics taking place at the core of the network, where distance is a less relevant predictor for trade than is the mass effect of the GDP or the size of the economies, still making it a more globally accurate model.

Now, this is only one case that we have examined so far. We now extend the same analysis to the rest of the selected HS2 industries. All models are consistent based on $Y_{\text {pred }} \sim 1+y_{1}+y_{2}$. In table 9.1, we show the consolidated results for these HS2 industries, where each industry has 2 cases, the unfiltered and the filtered scenarios. Also, in figure 9.7 and 9.8, we show the results of the regressions for all selected industries. Based on the results of the various scenarios, we can start validating some of the premises and hypothesis we mentioned earlier in this section and with very limited information. First, by reviewing table 9.1, we repeatedly observe the same pattern in terms of accuracy and contribution of each variable. Thus, we exception of the HS2 $=75$, all industries display an increased predictability of the gravity model at the core of the network in comparison with the total network. This reflected in the considerable improvement of the coefficient of determination $R^{2}$, reinforcing our premise in that the trade activity of the network core can be better explained by the size of the economy or GDP. This is a mass effect that is not unique to international trade markets, but also present in other type of complex system, such as ecosystems, social networks, and more. Additionally, the diminished effect of the distance is also confirmed for all industries. This support the hypothesis in which the trade activity at the network core is less affected by distance itself and the respective increased cost of transportation. One of the reasons for this to take place is potentially related to the need of satisfying the demand of a given good or product. The fullfilment of an economic need may help countries overcome the challenges of distance and transportation cost so that the supply chain that fuels an economy is not interrupted by operational or cost constraints. 


\begin{tabular}{|c|c|c|c|c|c|}
\hline $\begin{array}{c}\text { HS2 } \\
\text { Industry } \\
\text { Code }\end{array}$ & $\begin{array}{c}R^{2} \\
\text { coefficient } \\
\text { of determ. }\end{array}$ & $\begin{array}{c}\text { Regression } \\
\text { parameter } \\
\beta_{0}\end{array}$ & $\begin{array}{c}\text { Regression } \\
\text { parameter } \\
\beta_{1}\end{array}$ & $\begin{array}{c}\text { Regression } \\
\text { parameter } \\
\beta_{2}\end{array}$ & $\begin{array}{l}\text { p-value } \\
\text { gravity } \\
\text { model }\end{array}$ \\
\hline 01 unfiltered & 0.215 & $-1.7 \pm 0.3$ & $0.37 \pm 0.02$ & $-1.04 \pm 0.04$ & 0.0 \\
\hline 01 filtered & 0.460 & $-3.4 \pm 0.8$ & $0.47 \pm 0.04$ & $-0.56 \pm 0.09$ & 0.0 \\
\hline 10 unfiltered & 0.094 & $-1.2 \pm 0.3$ & $0.30 \pm 0.02$ & $-0.59 \pm 0.05$ & 0.0 \\
\hline 10 filtered & 0.589 & $-3.7 \pm 0.4$ & $0.43 \pm 0.02$ & $-0.03 \pm 0.06$ & 0.0 \\
\hline 20 unfiltered & 0.311 & $-4.5 \pm 0.2$ & $0.51 \pm 0.01$ & $-0.82 \pm 0.02$ & 0.0 \\
\hline 20 filtered & 0.661 & $-4.4 \pm 0.4$ & $0.54 \pm 0.02$ & $-0.63 \pm 0.05$ & 0.0 \\
\hline 35 unfiltered & 0.397 & $-7.8 \pm 0.2$ & $0.66 \pm 0.01$ & $-0.82 \pm 0.03$ & 0.0 \\
\hline 35 filtered & 0.764 & $-5.2 \pm 0.4$ & $0.50 \pm 0.02$ & $-0.22 \pm 0.06$ & 0.0 \\
\hline 50 unfiltered & 0.240 & $-7.2 \pm 0.4$ & $0.51 \pm 0.02$ & $-0.39 \pm 0.05$ & 0.0 \\
\hline 50 filtered & 0.661 & $-8.8 \pm 1.2$ & $0.68 \pm 0.06$ & $-0.46 \pm 0.16$ & 0.0 \\
\hline 62 unfiltered & 0.380 & $-6.4 \pm 0.2$ & $0.65 \pm 0.01$ & $-1.05 \pm 0.03$ & 0.0 \\
\hline 62 filtered & 0.642 & $-8.1 \pm 0.6$ & $0.70 \pm 0.03$ & $-0.34 \pm 0.06$ & 0.0 \\
\hline 75 unfiltered & 0.365 & $-12.7 \pm 0.5$ & $0.84 \pm 0.02$ & $-0.59 \pm 0.05$ & 0.0 \\
\hline 75 filtered & 0.341 & $-2.6 \pm 1.4$ & $0.39 \pm 0.06$ & $-0.21 \pm 0.13$ & 0.0 \\
\hline 84 unfiltered & 0.492 & $-10.1 \pm 0.2$ & $0.90 \pm 0.01$ & $-1.07 \pm 0.02$ & 0.0 \\
\hline 84 filtered & 0.805 & $-7.2 \pm 0.8$ & $0.67 \pm 0.03$ & $-0.13 \pm 0.10$ & 0.0 \\
\hline
\end{tabular}

Tab. 9.3.: Estimated coefficient of our gravity model for selected industries. This table shows the results of the regression analysis based on our gravity model, where $\beta_{2}$ is regression coefficient associated with the distance and $\beta_{1}$ is the regression coefficient associated with the size of the economies while $\beta_{0}$ is the intercept coefficient. We observe that the $R^{2}$ or coefficient of determination increases when considering the filtered scenario in comparison to the unfiltered scenario. In addition, we observe a decrease in the dependence of the distance when performing the same comparison for $\beta_{2}$. The last column shows p-value, which is zero in all cases, and shows a high statistical significance of the gravity model. 
A)
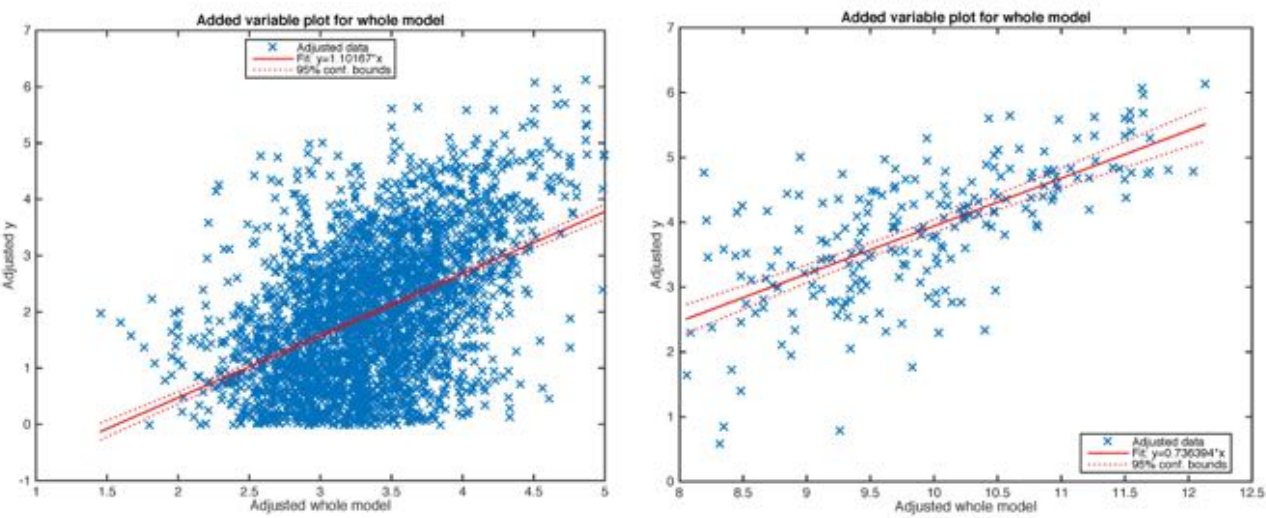

B)
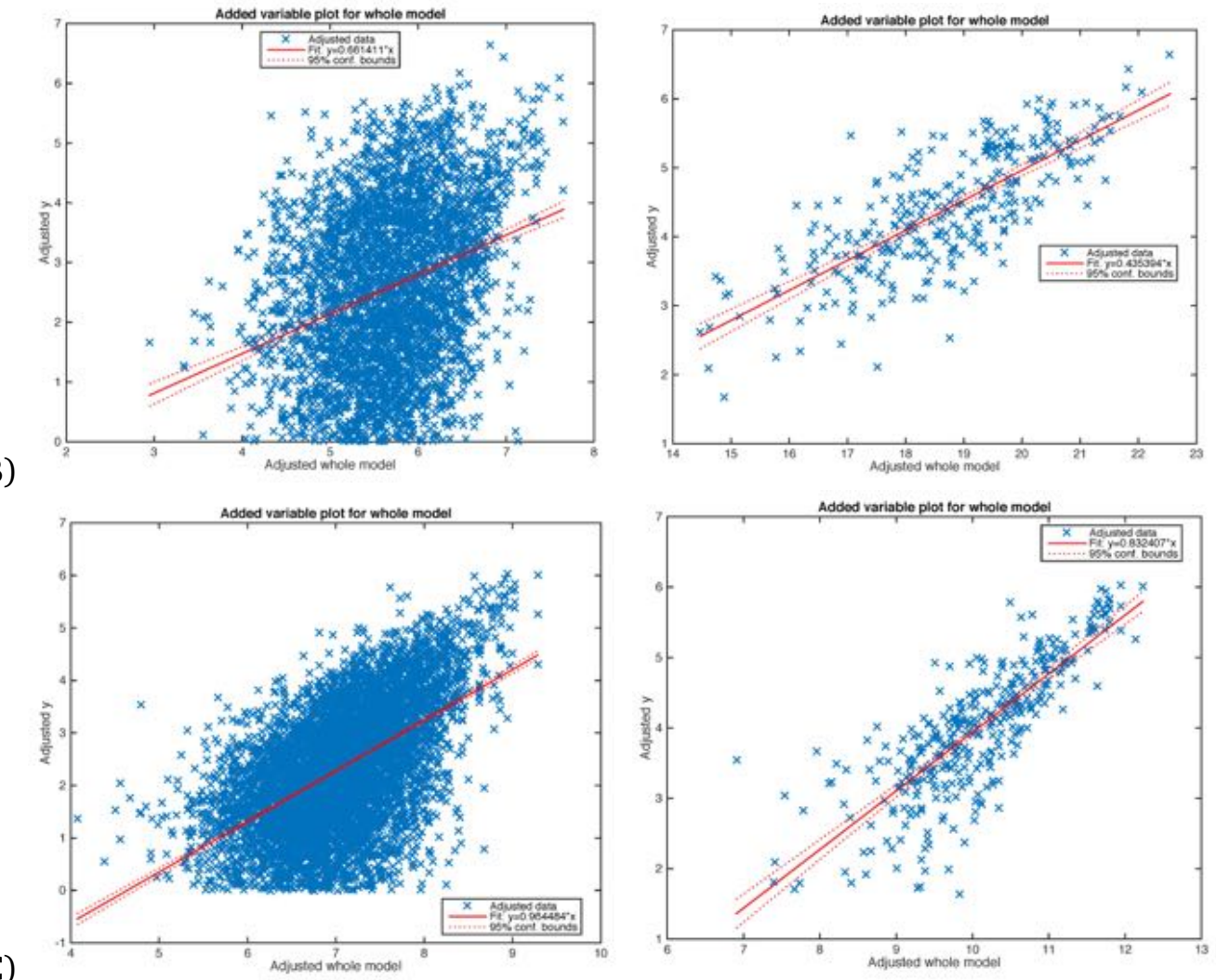

C)
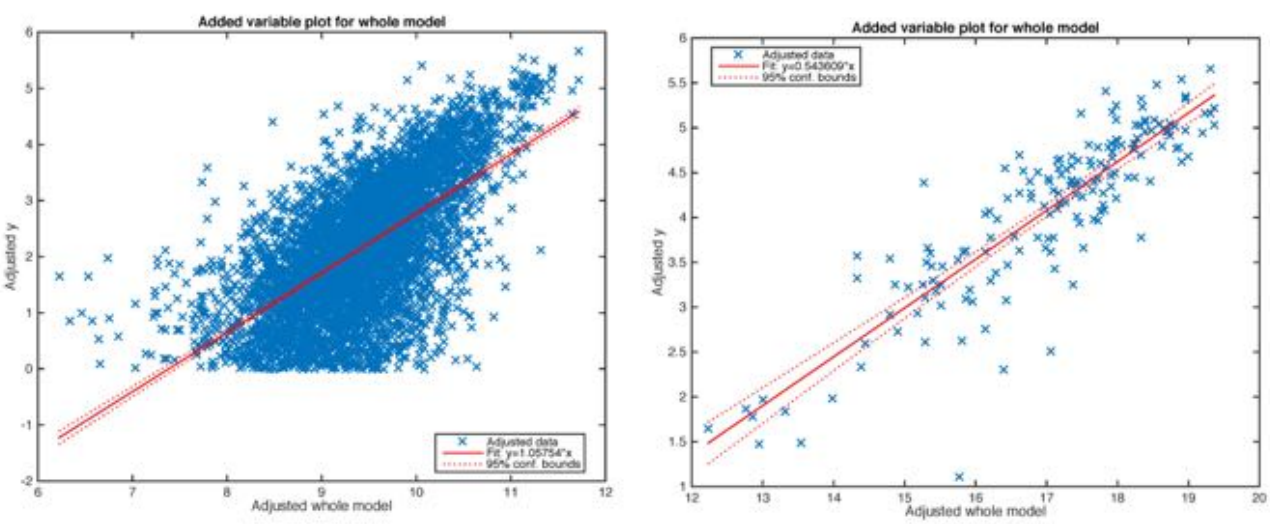

Fig. 9.7.: Results of gravity models for selected HS2 industries. This figure shows the gravity model fitting for the total network (left) and the high relative importance links (right) for each selected industry HS2. A) HS2 $=01$, B) HS2 $=10$, C) HS2 $=20$, and D) $\mathrm{HS} 2=35$. 
E)
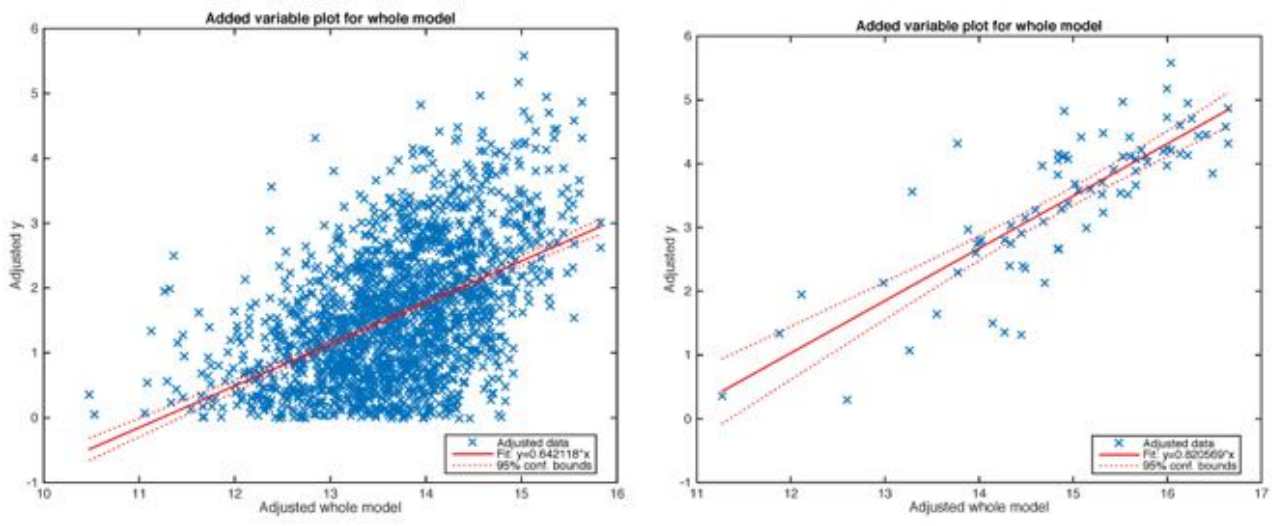

F)
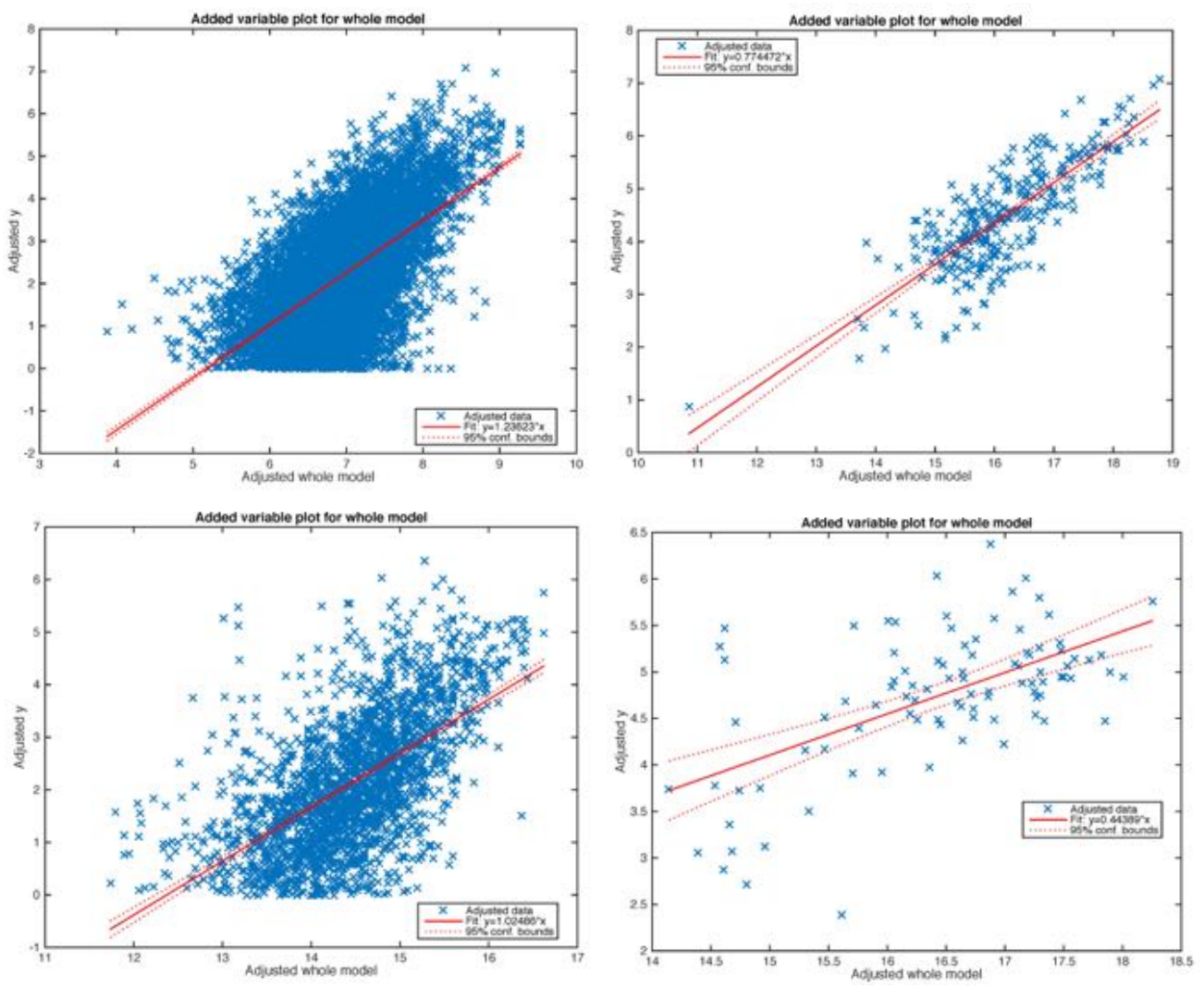

G)
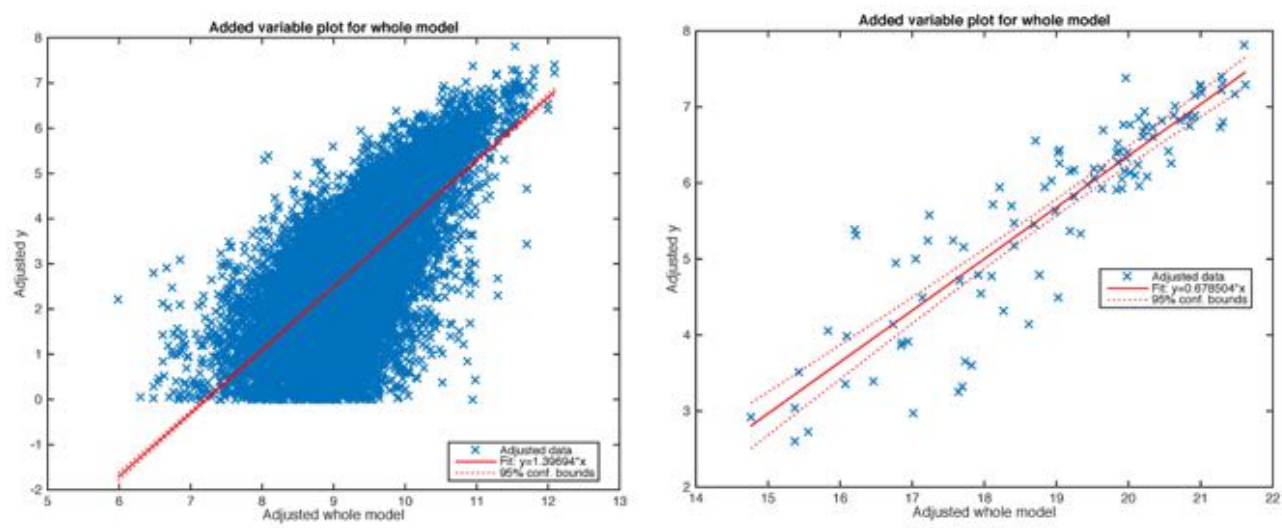

Fig. 9.8.: Results of gravity models for selected HS2 industries. This figure shows the gravity model fitting for the total network (left) and the high relative importance links (right) for each selected industry HS2. E) HS2=50, F) HS2=62, G) HS2=75, and H) $\mathrm{HS} 2=84$. 
Another recent research is that of Simini et al.[141], which introduces an alternative approach to gravity models that analyze not only the distance between locations, but also the competing opportunities within the same radius. This is a rather improved model, called the Radiation model, which solves virtually all the known disadvantages in the "pure" models based solely on gravitation.

A final consideration regarding the gravity model was inspired by the similarities between this model and the average weight vs. end-point degree distribution from subsection 7.2.5. We find that this similarity might be based on the following logic. First, the end-point degree could be interpreted as another way of defining a gravity model with a network parameter. Hence, we have:

$$
\langle w\rangle \sim\left(K_{i} K_{j}\right)^{\theta}
$$

Our model is similar to the end-point degree distrbution in that it is a link-level indicator of the average weight of the links themselves, which is fundamentally what our gravity model tries to predict. they are also consistent regarding the constraints among countries, in which only those countries with actual trade flows will have a prediction as well. We could write:

$$
T \sim\langle w\rangle \sim\left(K_{i} K_{j}\right)^{\theta} \sim \frac{\left(Y_{i} Y_{j}\right)}{D_{i j}^{\delta}}
$$

Assuming that the GDP of a country is proportional to the country's demand of a good, we can replace it with the trade volume $V_{i}$ and $V_{j}$, which as shown in subsection 7.2.2 is also highly correlated with node degrees $V_{i} \sim\left(K_{i}\right)^{\beta_{1}}$ and $V_{j} \sim\left(K_{i}\right)^{\beta_{2}}$. In trade networks, $\beta$ exponents are quite similar so that we can assume $\beta_{1} \simeq \beta_{2}$, then we have:

$$
\langle w\rangle \sim\left(K_{i} K_{j}\right)^{\theta} \simeq \frac{\left(V_{i} V_{j}\right)}{D_{i j}^{\delta}} \simeq \frac{\left(K_{i} K_{j}\right)^{\beta}}{D_{i j}^{\delta}}
$$

to which we apply logarithms and that yields:

$$
\log \langle w\rangle \sim \theta \log \left(K_{i} K_{j}\right) \simeq \beta \log \left(K_{i} K_{j}\right)-\delta \log D_{i j}
$$


In this way, we observe that the similarities are indeed based on a structural condition of trade networks. It is not a perfect similarity, because the end-point degree has a different exponent $(\theta)$ than does the gravity model by implication $(\beta)$ and, additionally, does not incorporate the distance variable. Still, this logic partially explains the similar pattern.

\subsection{Radiation models}

For the sake of completeness, we also provide a theoretical overview of the radiation model. Inspired in the processes of radiation emission and absorption, this model is a direct analogy with this physical process where particles are emitted at a given location and have a certain probability $p$ of being absorbed by surrounding locations. But, as explained in related works [141], it comes out that the probability for a particle to be absorbed is independent of $p$, but it depends only on the origin population $m_{i}$, the destination population $n_{j}$ and on the population in a circle whose centre is the origin and radius the distance between the origin and the destination, but discounting both the population at the origin and the population at the destination, $s_{i j}$. Then the number of commuters, that we call $T_{i j}$, from location $i$ to location $j$ is estimated to be a fraction of the commuters from population $i, T_{i}$, then we have:

$$
T_{i j}=T_{i} \frac{m_{i} n_{j}}{\left(m_{i}+s_{i j}\right)\left(m_{i}+n_{j}+s_{i j}\right)}
$$

This model has been successful in predicting the commuting and mobility flows across counties in the US, for example. There are several reason why the radiation model is more consistent than the gravity model. First, the radiation model, unlike gravity models, does not depend on previous historical activity, since the current local properties are sufficient. Secondly, it does not have the same level of predictability issues in comparison to gravity models, which are deterministic one and consequently will not accept local variances beyond what the model variables would allow. And finally, the radiation model can also handle fluctuations between two locations, making it a more suitable model to predict social and economic phenomena. We do not formally apply this model in this study, which we present merely in order to propose an academic argument on alternatives to the gravity model. But the main reason for this is related to the fact that we will now proposed a different bipartite and weighted model in the next chapter. 



\section{A new model for ecosystemic analysis of markets}

Cooperation for mutual benefit, a survival strategy very common in natural systems, is one that humanity needs to emulate.

\section{— Eugene Odum}

(Father and pioneer of the ecosystem concept)

After evaluating and testing several approaches, based on the different models described in the previous chapter, we find that there is no single model that can simultaneously explain and reproduce all the key features found in international trade markets, both the topology and the internal organization. First, the random bipartite model has shown significant deviations from the network indicator results found in empirical data. Then, our modified gravity model has been an improvement over the previous model, yet it depends on deep restrictions to the nodes as well as it only yields the final state of the network, hence lacking a growth process to demostrate market formation. However, the origin of trade networks itself is not well understood in the scientific community. This is why, we take advantage of the lessons learned from the previous models and, once again, of the analogy with mutualistic ecosystems and their dynamics in order to find a theory that may explain how markets are fundamentally formed, a significant challenge when considering all the topological properties and internal organization simultaneously. In our thought process, we pay special attention to identify the most basic element of a market. The economic theory defines that markets are the places, both literally and figuratively, where the supply and demand converge, that is, the place where the barganing process between buyers and sellers takes place. But this definition may not be helpful for our purpose. Alternatively, we could also think of markets as the result of the economic activity of all agents in the market, which is actually true, but this is too undetermined to formulate an innovative market formation process. Ludwig Wittgenstein has argued in his Tractatus Logico-Philosophicus that "The world is the totality of facts, not of things"[167]. Therefore, as a direct analogy, it can be argued that markets are fundamentally formed by decisions, and not by products. These decisions are merely the result of a complex thinking process to determine: a) what to buy, b) where to buy from, and c) how much to buy; so that benefits can be 
maximized for the buyers. The same is true from the seller perspective. Hence, again, we arrive to the same point of self-interest being the engine of an economic process, the same idea that Adam Smith embraced and formulated more than 2 centuries ago to support his research and conclusions [142]. Now, the critical question at this point is whether decisions may or may not be incorporated as the most basic element of a market model. Let us now consider a new model of ecosystemic analysis of markets.

\subsection{A mutualistic model for market formation}

While we have established the properties of international trade markets, both topological, structural and dynamical, in chapters 7 and 8, we have not yet explained how these market structures are formed.

First, we remind ourselves the network features that we are trying to reproduce: a) heterogeneous degree distribution, b) highly correlated strength and degree with $\beta>1$, c) declining clustering performance as a function of the degree, d) $C 4_{w} / C 4_{b}>1$ pattern, e) uncorrelated nearest neighbor degree distribution, f) a coreperiphery structure, $g$ ) high nestedness, $\mathrm{h}$ ) two-zone end-point degree correlation. Thus, our challenge is to find a market formation model that explains all the features, from a to h, simultaneously.

Secondly, markets present internal dynamics that resemble those of, for example, an ecosystem of plants and pollinators. Interactions (visitations) in an ecosystem may be viewed as the biological equivalent to trade activity. But unlike ecosystems, interactions in an trade market are based on some rational thinking, but mostly by self-interest in particular. Here, we find the fundamental difference between ecosystems and markets, which is the decision making process of sellers and buyers. However, we propose that, despite this significant gap, a similar mechanism can still be formulated. This is due to fact that, with or without rational thinking capabilities, both systems will still display a delicate balance between competition and cooperation. Exporters will compete against other exporters to capture a higher amount of market demand, but they will cooperate with the importers to sell them a higher portion of its offering. Certainly, this last idea may be somewhat speculative, but it can help us identify the fundamental forces in the process of market formation, which has to be a network growth process by definition.

Then, in order to formulate a statistically valid network growth model, we first need to reconsider our premises more formally. As a consequence, we focus on the concept of individual neutrality hypothesis[162, 163], which states that simple interactions 
between agents of a system can occur randomly and still lead to a complex emerging outcome. Consequently, we propose a simple process for market formation based on a link aggregation process based on the assumption that the world trade is the sum of the decisions that each person, firm and/or country make to acquire the products required to satisfy their needs (imports) or sell the goods required to empty their inventory or stock (exports). Thus, we can associate each link that exists in a trade network as a unitary element of demand or supply. This is actually consistent with the M2 formulation discussed in section 10.1 and, even though decisions in a market is the results of complex economic thinking that takes in account several variables (such as price, quality, distance between countries, country affinity, international trade agreements, country policy, regional trade incentives and existing stocks, just to name a few), the link aggregation process will be based on random interaction probabilities as a consequence of the individual neutrality concept. Another important assumption is related to the perfect competition conditions within international trade activity, in which a market will have: a) a fairly large number of sellers and buyers so that no one party has control over the market, b) product information and terms that are publicly and easily available so that every buyer or seller can make informed decisions (a.k.a perfect information condition), c) no barriers for entering or exiting from the market (specially biased penalties or selective fees), and d) products that are homogeneous so that no preferences arise from the product features (quality, price, availability, payment terms, and more). These concepts allow us to formulate our mutualistic model of markets.

\subsubsection{Formal description of our model}

For each step in the process, we add one unitary link $\left(w_{i j}=1\right)$ and select an exporter country $E_{i}$, which provides this unitary quantity of goods from its existing inventory, and an importer country $I_{j}$, which buys this quantity of goods to cover its needs or, at least, a portion of them. The end-points of the newly aggregated link are selected independently and with a probability proportional to the strength of each node within each disjoint subset (exporters and importers). We also allow the selection of a disconnected node with probabilities $P_{E}^{\text {new }}$ for the exporters and $P_{I}^{\text {new }}$ for the importers, which represent the arrival of a new competitor to the market. The market size control is set by means of 3 input parameters: a) $N_{E}$, which is the maximum number of exporters, b) $N_{I}$, which is the maximum number of importers, and c) $W_{t}$, which is the total trade volume and the total number of unitary links at the same time. We also set the time-step so that every time a new unitary link is added $w(t)=\sum_{i j} B_{i j}(t)=t$, where $w(t)$ is total trade volume of the market for the current time-step and $B_{i j}(t)$ is the interation matrix at the current time-step. We show our basic process of link aggregation in figure 10.1. 


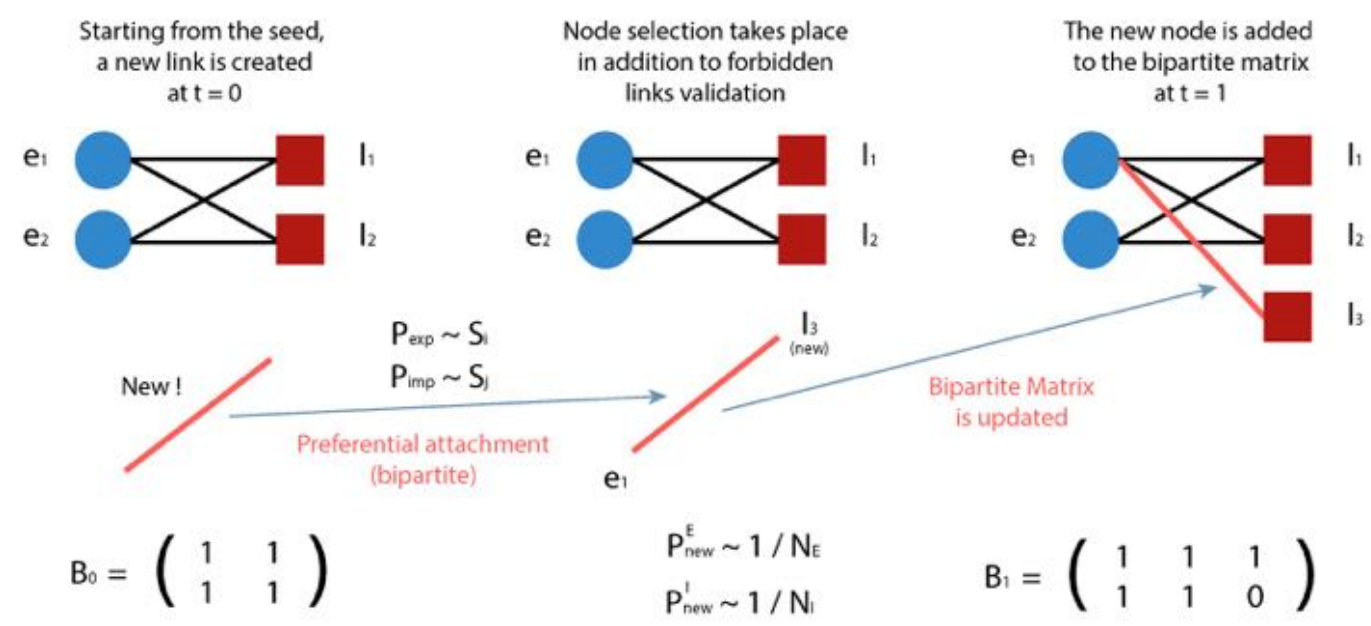

Fig. 10.1.: Step-by-step process of the mutualistic model. This figure shows the process of creating a new link and selecting the nodes. First, the new link is crated. Secondly, the decision is made on whether connecting to a new or an existing node. Thirdly, if it is not a new node, the node selection takes place based on a preferencial attachment mechanism. Finally, the bipartite graph and matrix get updated and the network is ready for the following iteration. The process continues until $W_{t}$ is reached, taking $n$ and $m$ as the limit size of the system.

It is important to note that the model we are proposing is a bipartite and, at the same time, a weighted version of the preferential attachment mechanism proposed by Barabasi et al.[16]. Then, our algorithm works as follows:

First, we start with a bipartite matrix of size $N_{E} \times N_{I}$, where all interactions are zero.

$$
B_{i j}(0)=0 ; i=1,2, \ldots, N_{E} ; j=1,2, \ldots, N_{I}
$$

Secondly, we set a seed of fully connected nodes of size 2-by-2:

$$
B(t=4)=\left(\begin{array}{ll}
1 & 1 \\
1 & 1
\end{array}\right)
$$

Thirdly, for every time-step $\Delta t$, a new link with $w_{i j}=1$ is added to the bipartite matrix, as previously described.

Finally, every time-step also has to follow a protocol to locate the link in its position by checking the arrival of new competitor or a new buyer to the market with probabilities $P_{E}^{\text {new }}$ and $P_{I}^{\text {new }}$ defined as: 


$$
\begin{gathered}
P_{E}^{\text {new }}=\frac{\lambda_{E}}{N_{E}(t)} \\
P_{I}^{\text {new }}=\frac{\lambda_{I}}{N_{I}(t)}
\end{gathered}
$$

where $\lambda_{E}$ and $\lambda_{I}$ are parameters with values of $\lambda_{E}=N_{E}(t)\left(N_{E}(t)-1\right) / 2 t$ and $\lambda_{I}=N_{I}(t)\left(N_{I}(t)-1\right) / 2 t$, as described by M. Jimenez et al.[89]. We define this form of a new competitor or a new buyer entering the market with a decreasing probability throughout the entire process to account for the increased competition derived from a larger number of existing exporters and importers. But when no new competitor or buyer is selected at the time-step, we use a strength-preferential attachment concept as the only market force that determines the interaction between exporters and importers, which accounts for the cooperation between them, and consequently the selection probabilities are:

$$
\begin{aligned}
& \left(1-P_{E}^{n e w}\right) P_{E}(t)=\frac{S_{i}(t)}{w(t)} \\
& \left(1-P_{I}^{n e w}\right) P_{I}(t)=\frac{S_{j}(t)}{w(t)}
\end{aligned}
$$

where $S_{i}(t)$ is the strength of the exporter nodes at the current time-step, $S_{j}(t)$ is the strength of the importer nodes at the current time-step, and hence the value of the bipartite matrix $B$ will be updated after the exporter and importer have been selected according to the previous mechanism, therefore having $B_{i j}(t+1)=B_{i j}(t)+1$ and repeating this until $t=W_{t}$. In sum, the formal definition of our mutualistic model of market formation can be defined as:

Let $i$ be a new link of a bipartite graph $G$, where there are two disjoint subsets of nodes $E$ and $I$ of size $n$ and $m$, respectively. $G$ also has a bipartite matrix $B$, which is $n$-by- $m$ in size, leading to its adjacency matrix $A_{i j}$. The new link $i$ will connect either to a new node with probability $p_{\text {new }}^{E}$ for $E$ and $p_{\text {new }}^{I}$ for $I$ or to an existing node with probability (1 $\left.P_{\text {new }}^{E}\right) P_{E}=S_{i}(t) / w(t)$ and $\left(1-P_{\text {new }}^{I}\right) P_{I}=S_{j}(t) / w(t)$, for $E$ and $I$, respectively. 

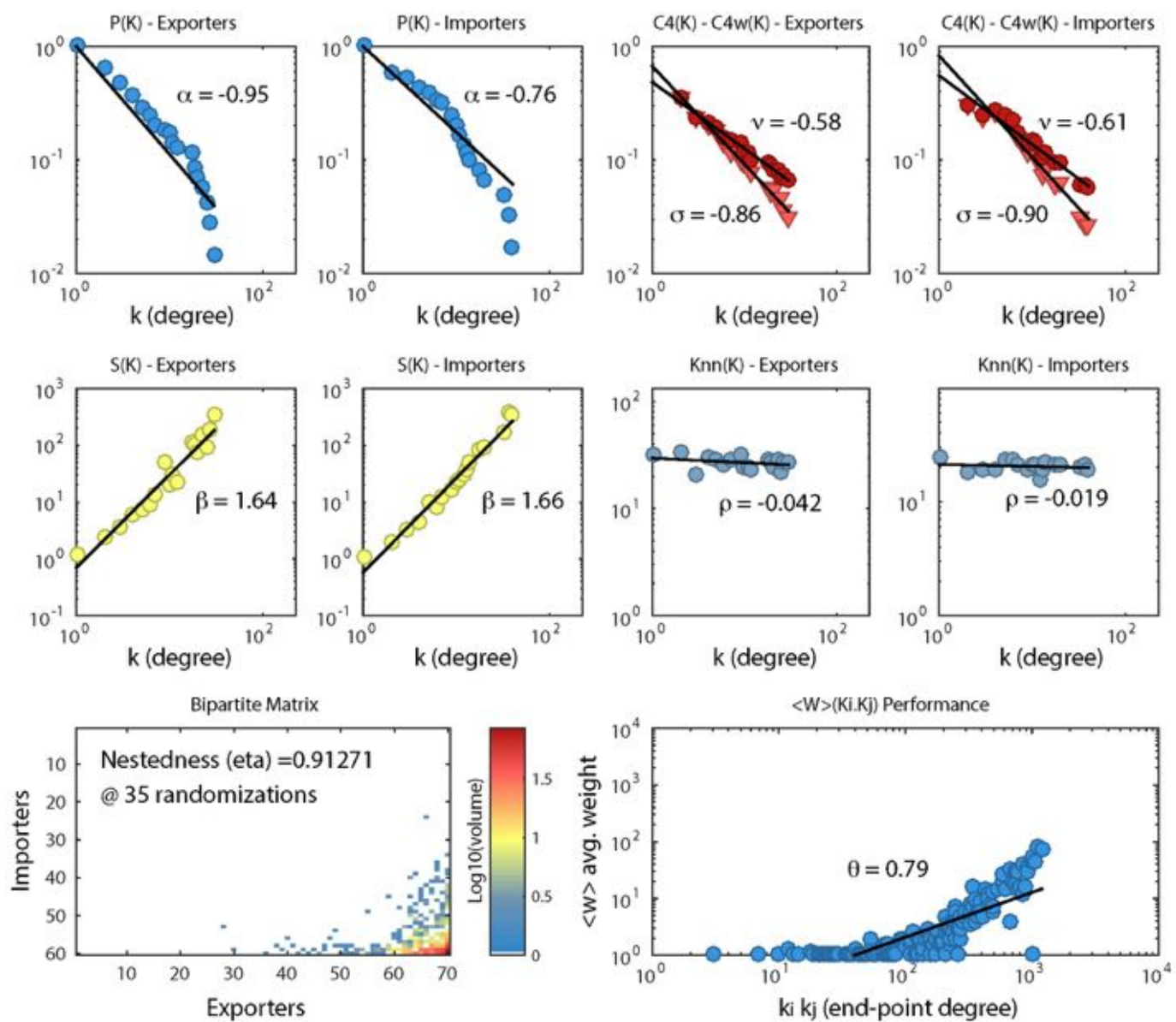

Fig. 10.2.: Example of a market simulation with our mutualistic model. This figure shows a simulation example of a market with $\mathrm{n}=70, \mathrm{~m}=60, \mathrm{w}=1500$ and $\mathrm{p}(\mathrm{fl})=0.20$. We observe that all the topological indicators are consistent with those utilized in chapter 7 for the actual data.

\subsubsection{A simple example of market formation}

In order to depict the overall outcome of our market formation process, we simulate a dummy market of 70 exporters and 60 importers with a trade volume of $W_{t}=1500$, which is a computationally manageable matrix size that is close to one half the maximium amount of exporters and importers in actual markets in addition to selecting the maximum weight so that the density of the simulated market is consistent with that of empirical ones. This decision is in order to perform an exploratory test as well as an initial implementation of our mutualistic model of markets. Also, it allows us to optimize our code for minimum processing time. Additionally, when we implement this model, we incorporate an extra parameter, which is the probability of forbidden links, $p(f l)$. This probability represents, in ecosystem, the biological constraints for polinators to interact with plants. However, in our mutualistic model of markets, it represents the geopolitical constraints between any given pair of 
exporter and importer. We think this parameter is a realistic part of the model since some countries do have biased rejections for others. The typical sources of a biased rejection can be: a) international embargo policies, b) differential duties and taxes from protectionist policies, c) quotas to specific products, d) local health regulations affecting imports, and more. We also test the effect of forbidden links in the current subsection and evaluate the changes in topological features as a consequence. But after implementing and perfecting our code, we execute the algorithm and show the results in figure 10.2. We observe that most of the features found in actual trade networks are clearly present in the simulated market, an encouraging fact towards developing our theory. The degree distribution displays a long-tailed pattern that is consistent with a power law pattern $\left(P(k) \sim k_{\text {exp }}^{-0.5}\right.$ and $\left.P(k) \sim k_{i m p}^{-0.6}\right)$. Regarding the strength-degree correlation, we find a power law pattern with $\beta>1\left(S(k) \sim k_{e x p}^{2.1}\right.$ and $S(k) \sim k_{i m p}^{2.0}$ ), suggesting that a core-periphery structure is present as well as the rich club effect. The clustering performance is a declining one, making its internal structure more hierarchical at the core, with a condition of $C_{w}^{4} / C_{b}^{4}>1$. The nearest neighbor degree distribution is a uniform one, and hence uncorrelated with the degree. The nestedness performance has a high value $\left(\eta_{w}=0.806\right)$, aligned to the typical values of actual markets. Finally, the end-point degree displays a two-zone pattern, with a positive exponent $\theta=0.7$, consistent with values observed in actual markets. At the very minimum, our mutualistic model of markets looks promising so far. With one single simulation, we are able to repreduce the all the key network features from trade markets simultaneously and, in addition, they seem to be consistent with those of the empirical networks. In sum, and based on this initial outcome, we proceed to perform further and more rigorous analysis of our mutualistic model. For that, we perform 100 realizations in our dummy market expressed as $M(m=70, n=60, w=1500, p(f l)=0.20)$. In this case, we select a value of $p(f l)=0.20$ based on a empirical testing to match the density of actual markets. We take the results of all the realizations (exponents for exporters and importers) and build a parallel axis visualization. In figure 10.3, we show the results of the 100 realizations of our model, depicting the resulting exponents in a parallel axis visualization, where each axis represents one exponent and each line represents the combined exponent path across variables.

Clearly, our model reproduces consistently all the key features of the actual markets. Yet, being a stochastic model, there is an expected variability in the value of the exponents of each indicator for the exporters and importers. Nonetheless, the probability distribution of the each exponents $(\gamma, \alpha, \beta, \nu, \sigma, \rho$ and $\theta)$ resemble a normal distribution, but given the limited sample, we are not able to prove this conclusively nor are we trying to do so. The purpose of this exercise is merely to understand how stable the outcome of the model is. We observe that the model consistently reproduces the same outcome and patterns across all the realizations, 


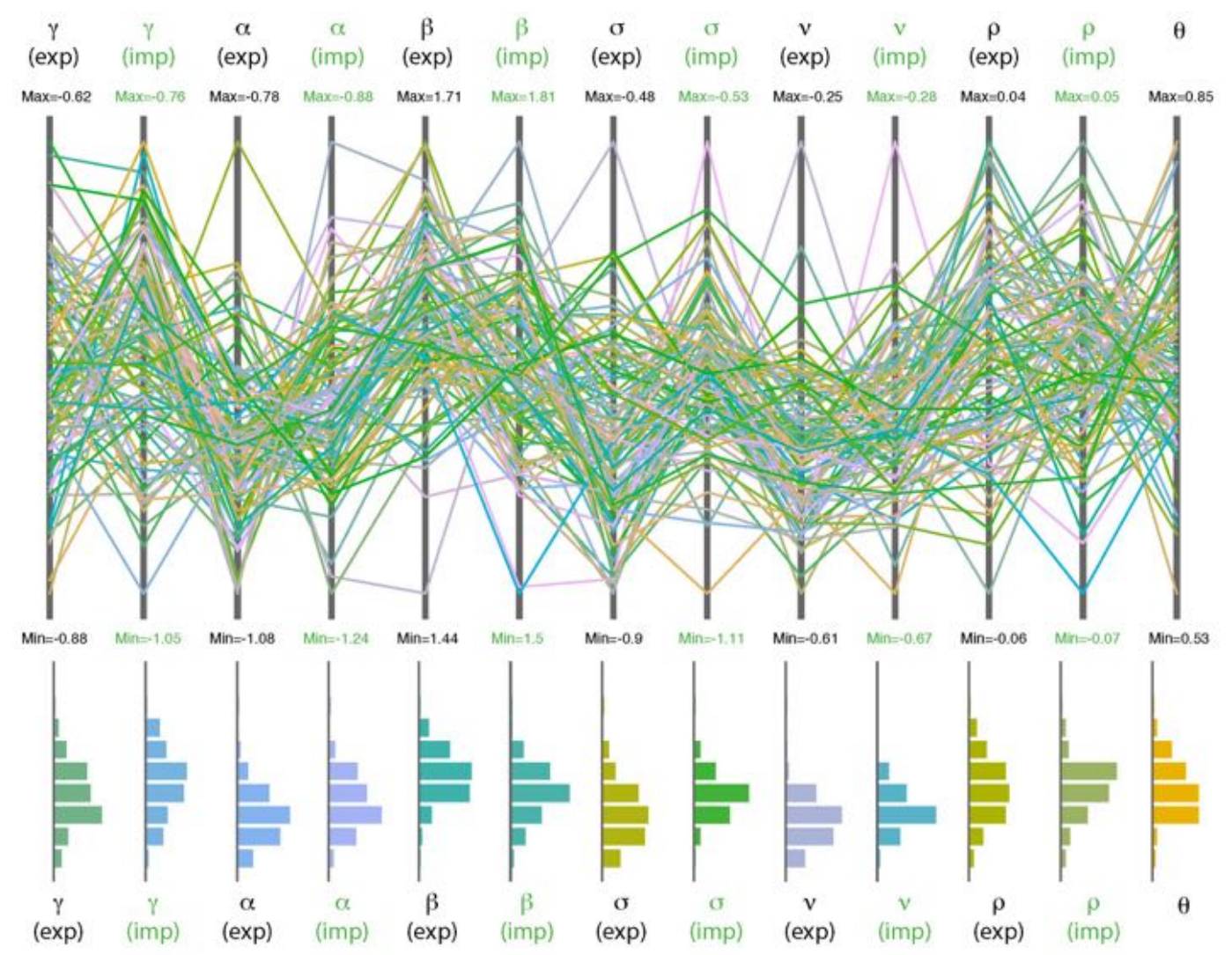

Fig. 10.3.: Topological results of all 100 realizations. This figure shows the results of each exponent from the 100 simulations of a market with $n=70, m=60, w=1500$ and $\mathrm{p}(\mathrm{fl})=0.20$. We create a parallel axis visualization, where each axis is depicted between a maximum and minimum value of each result obtained. In this way, we locate a point on each axis for a given set of results obtained and assign a different color at random. This visualization is designed to unveil any pattern and/or correlation between the multiple variables. Furthermore, we observe that no correlation can be found across the variables, which would indicate that the variables are independent of each other. On the bottom, we also build a vertical histogram for each of the variables so that we can visualize how the results are distributed between the minimum and maximum values obtained over the 100 realizations.

where the exponents derived from regression analysis do have some variability but this never falls far from overall pattern of markets from chapters 7 and 8 .

But so far, we have only incorporated the effect of one condition of forbidden links in the model. We now run our model for the dummy market at different values of probability $p(f l)$ of forbidden links. We select the values to depict the full range of $\mathrm{p}(\mathrm{fl})=(0.0 ; 0.01 ; 0.02 ; 0.05 ; 0.10 ; 0.15 ; 0.20 ; 0.25 ; 0.30 ; 0.35 ; 0.40 ; 0.50 ; 0.60$; $0.70 ; 0.80)$. After running the simulations, we find how the probability of forbidden links affect the connectivity patterns in terms of degree distribuion exponents. In figure 10.4, we show the degree distribution for different values of $p(f l)$. We perform this by averaging the 100 realizations for each condition of $p(f l)$. As a result, we 
observe how the exponent value changes. When the probability of forbidden links increases, the absolute value of the $\gamma$ exponent decreases, suggesting that a higher level of constraints can lead to a more concentrated connectivity across the nodes and a higher probability of hubs.
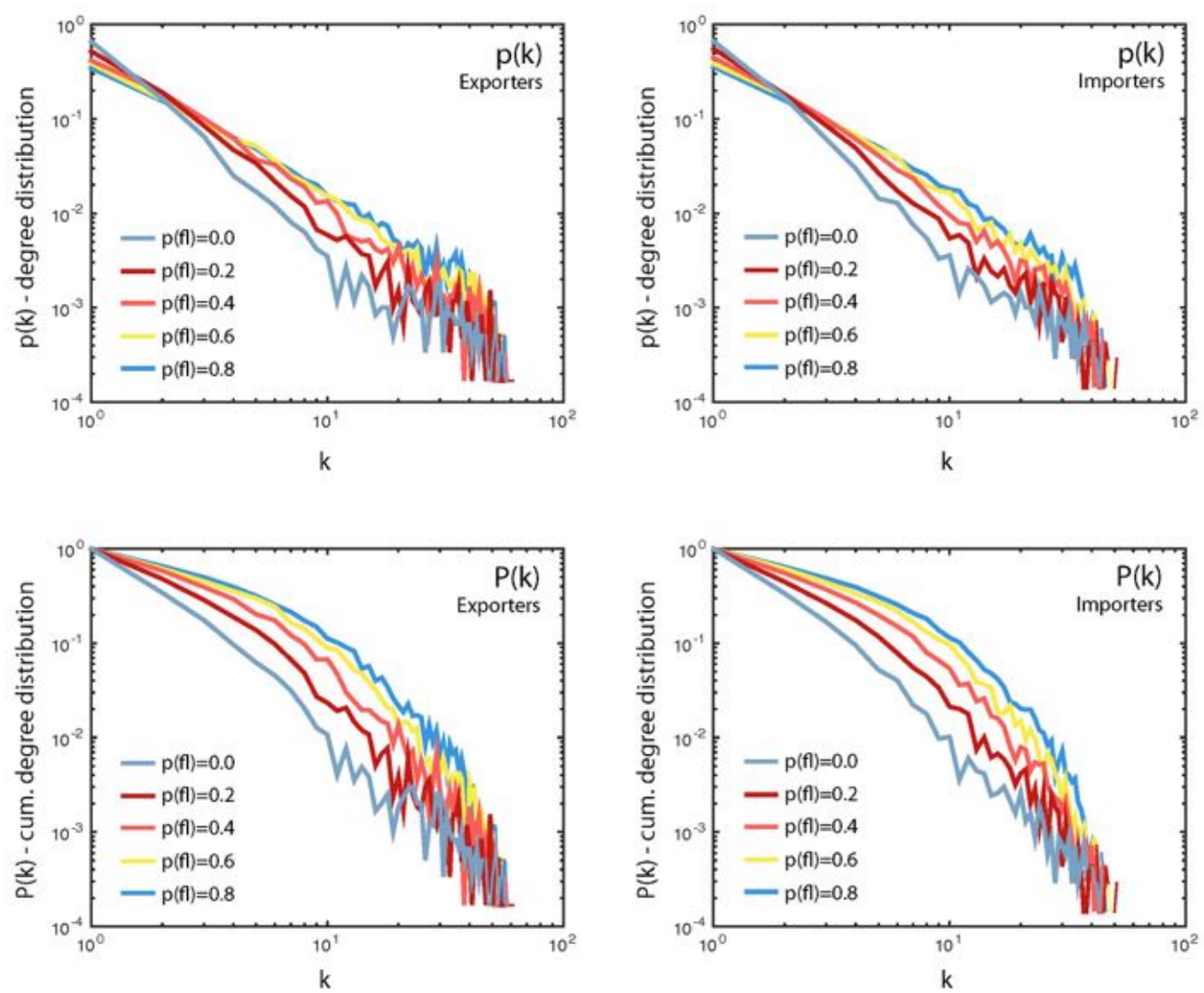

Fig. 10.4.: Degree distributions $\mathrm{p}(\mathrm{k})$ and $\mathrm{P}(\mathrm{K})$ for various $\mathrm{p}(\mathrm{FL})$. This figure shows the results found after executing 100 realizations of our mutualistic model of markets for each value of $\mathrm{p}(\mathrm{fl})$. At the top, we show how the degree distribution $\mathrm{p}(\mathrm{k})$ varies when $\mathrm{p}(\mathrm{fl})$ is increased from $\mathrm{p}(\mathrm{fl})=0.0$ to $\mathrm{p}(\mathrm{fl})=0.8$, depicting the average performance of the 100 realizations. On the bottom, we show how the cumulative degree distribution $\mathrm{P}(\mathrm{k})$ varies when $\mathrm{p}(\mathrm{fl})$ is increased from $\mathrm{p}(\mathrm{fl})=0.0$ to $\mathrm{p}(\mathrm{fl})=0.8$.

We propose a methodology to evaluate the variance of the topological features with $p(f l)$. In figure 10.5, we observe a visualization of the $\gamma$ exponent for the exporters as well as the distributions for each condition of $p(f l)$ for the 100 realizations. In sum, when the probability of forbidden links increases, the $\gamma$ exponents increases in absolute value and the interval of the results become broader (higher variability). In this sense, we will argue in the conclusions about how the existence of forbidden links may work as a systemic way to create or avoid monopolies.

We repeat the same procedure for the rest of the topological indicator and show the results in figure 10.6. In this figure, we observe that: a) the strength-degree 


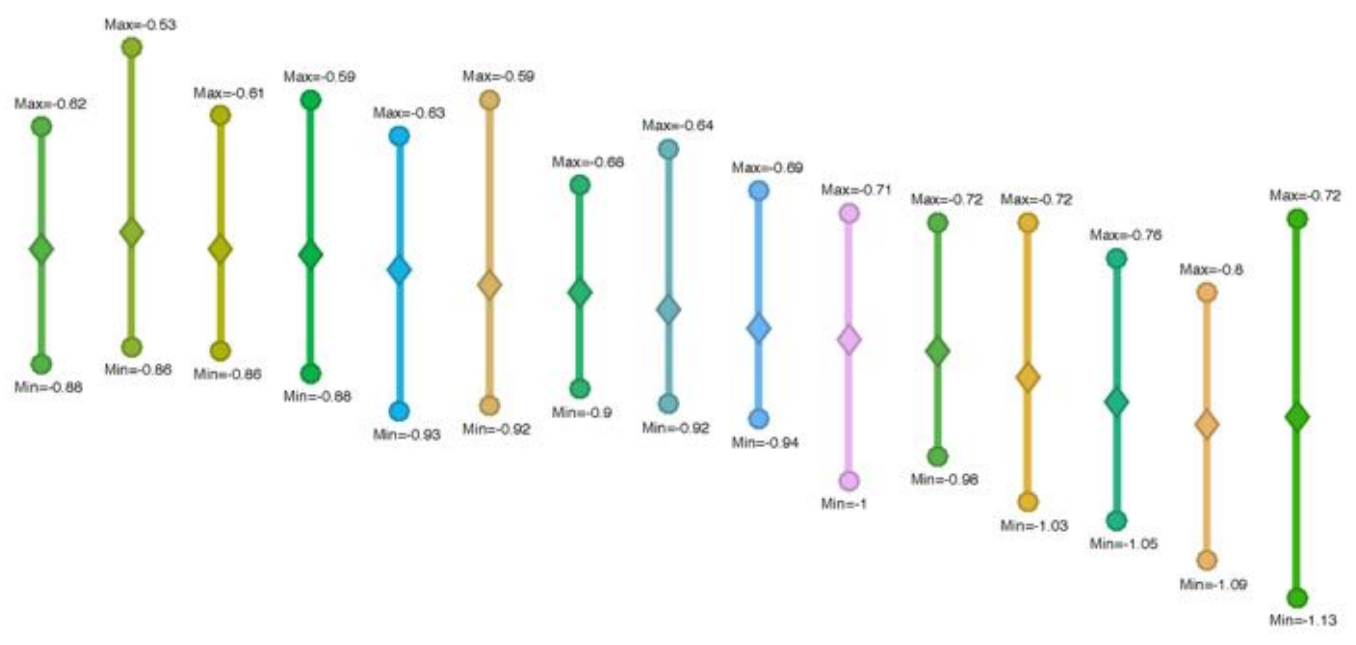

$p=0.0 \quad p=0.01 \quad p=0.02 \quad p=0.05 \quad p=0.10 \quad p=0.15 \quad p=0.20 \quad p=0.25 \quad p=0.30 \quad p=0.35 \quad p=0.40 \quad p=0.50 \quad p=0.60 \quad p=0.70 \quad p=0.80$

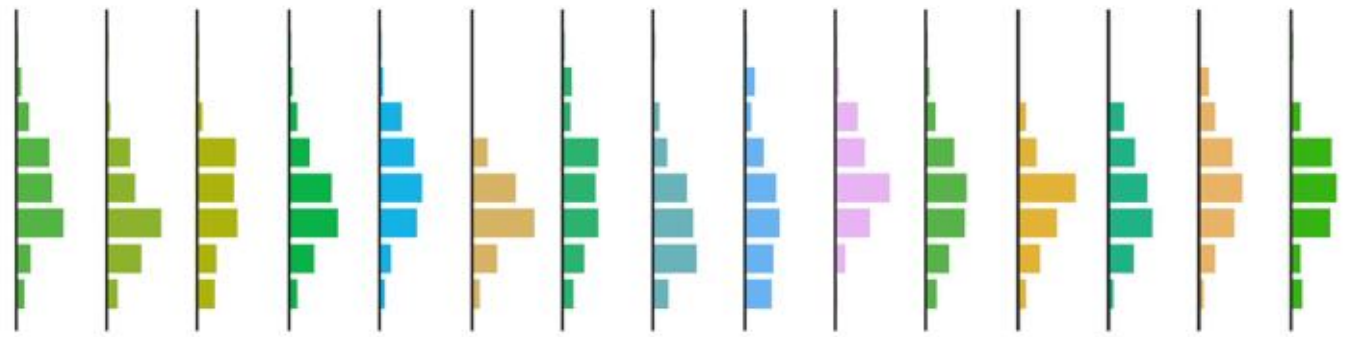

Fig. 10.5.: Exponent $\gamma_{\exp }$ of $\mathrm{P}(\mathrm{K})$ for each $\mathrm{p}(\mathrm{FL})$. This figure shows the results found after executing 100 realizations of our mutualistic model of markets for each value of $\mathrm{p}(\mathrm{fl})$. At the top, it shows how the values vary when $\mathrm{p}(\mathrm{fl})$ is increased from $\mathrm{p}(\mathrm{fl})=0.0$ to $\mathrm{p}(\mathrm{fl})=0.8$, depicting the maximum and minimum values obtained across the 100 realizations (circular dots) as well as the average value (diamond dots). On the bottom, it shows the distribution of the results for the 100 realizations between the maximum and minimum values.

correlation $\mathrm{S}(\mathrm{k})$ shows an increased value of the $\beta$ exponent when the probability of forbidden links is higher for both the exporters and the importers, creating a stronger rich club effect, b) the bipartite weighted clustering $C 4_{w}$ displays a bigger exponent in absolute value, creating an even more hierarchical core in the trade network for larger values of $p(f l)$, c) nearest neighbor degree distribution $K n n(k)$ is observed with an uncorrelated pattern based on exponents close to zero independently of the forbidden link probability. We observe one general phenomenon, which is related to the level of variability in the resulting exponents. When $\mathrm{p}(\mathrm{fl})$ is increased, the range of the resulting exponents also increase significantly in comparison with the $\mathrm{p}(\mathrm{fl})=0$ condition. In the case of $\mathrm{P}(\mathrm{K})$, we observe that the average exponent $\gamma$ decreases when $\mathrm{p}(\mathrm{fl})$ is increased. In contrast, $\mathrm{S}(\mathrm{k})$ shows the opposite condition since the exponent $\beta$ increases when $\mathrm{p}(\mathrm{fl})$ is larger. Regarding the weighted bipartite clustering $\mathrm{C} 4 \mathrm{w}(\mathrm{k})$, we observe that the exponent $\sigma$ decreases for a large value of $\mathrm{p}(\mathrm{fl})$, whereas $\mathrm{Knn}(\mathrm{k})$ shows a slight increase as well, with values that are still close to 0 , maintaining the uncorrelated pattern despite the change in $\mathrm{p}(\mathrm{fl})$. 
$P(k)-\gamma$ exponent for various $p(f)$ - Exporters

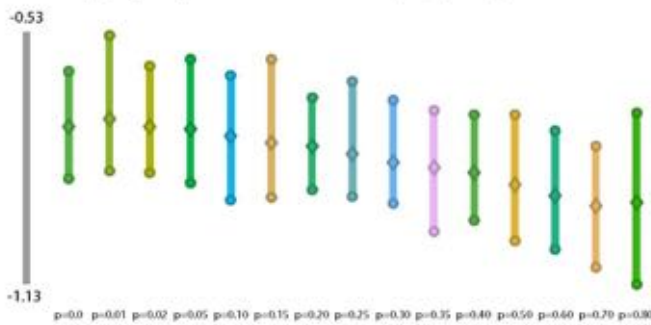

$S(k)$ - $\beta$ exponent for various $p(f)$ - Exporters

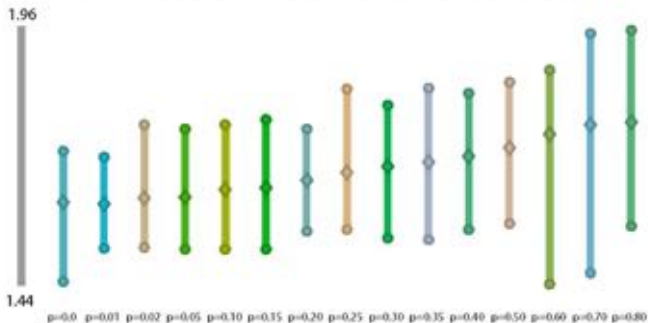

$C 4 w(k)-\sigma$ exponent for various $p(f l)$ - Exporters

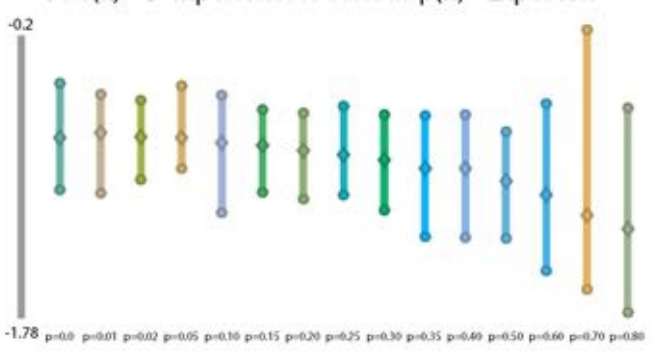

$K n n(k)-\rho$ exponent for various $p(f)$ - Exporters

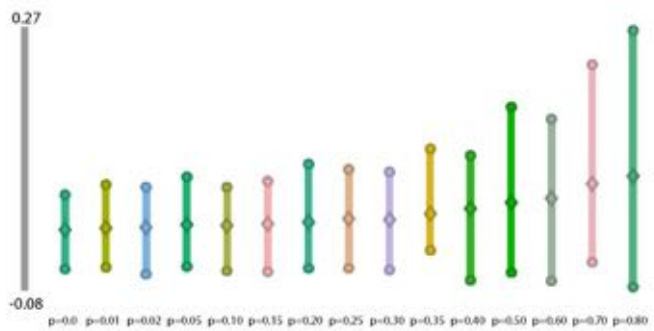

$P(k)$ - $\gamma$ exponent for various $p(f l)$ - Importers

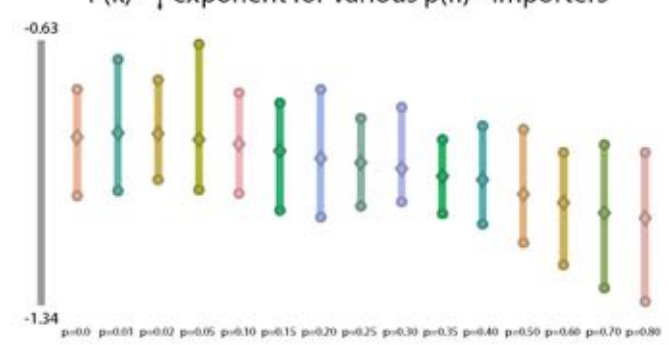

$S(k)-\beta$ exponent for various $p(f)$ - Importers

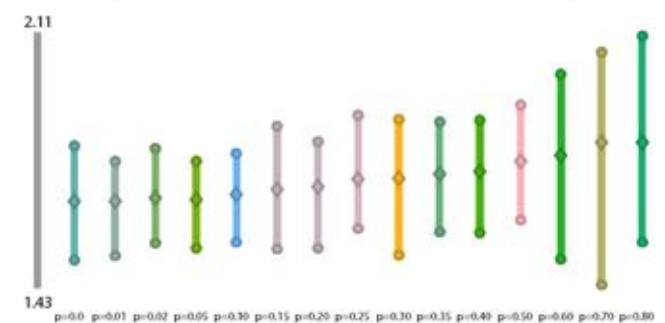

$C 4 w(k)-\sigma$ exponent for various $\mathrm{p}(\mathrm{fl})$ - Importers

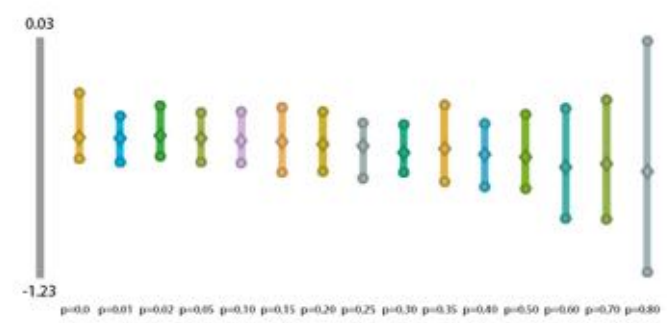

$K n n(k)-\rho$ exponent for various $p(f)$ - Importers

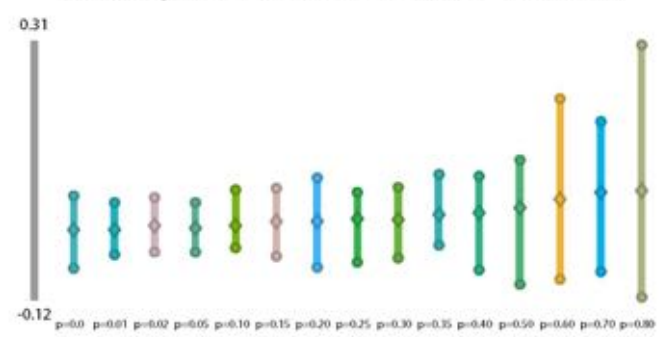

Fig. 10.6.: Exponents of selected mutualistic indicators for various $\mathrm{p}(\mathrm{FL})$. This figure shows how the exponents $\gamma, \beta, \sigma$, and $\rho$ (for the exporters and the importers) are affected by the increase of $\mathrm{p}(\mathrm{fl})$ from 0.0 to 0.8 , displaying the maximum and minimum values within the 100 realizations (circular dots) and the average value (diamond dots) at the top of each diagram. At the bottom, we show the distribution of the exponents found for each case between the maximum and minimum values.

Finally, we need to account for the nestedness performance of the model and still determine how the probability of forbidden links affects it. For that reason, we show in figure 10.7 the distribution of our weighted interaction nestedness indicator (WINE) by means of the $\eta_{w}$ parameter. Previously in this section, we have already determined that our mutualistic model does generate a nested structure as part of its outcome, but we are now extending the analysis to the 100 realizations of 
the model. We observe in the first case of $p(f l)=0$ that all simulated matrices have resulted in high values of nestedness with a mean $\eta_{w}=0.88$ and a standard deviation of $\sigma_{\eta}=0.03$, leading to a range between $\eta_{w}=0.80$ and $\eta_{w}=0.95$. The evidence then supports the fact that our model generates high values of nestedness in a consistent way. Furthermore, we now analyze the effect of the forbidden links in the model. Our working hypothesis was, up to this point, that a higher probability $p(f l)$ would lead to a more restricted interaction matrix, decreasing the nestedness due to the restrictions of the posible interaction and making the matrix itself more sparse. The evidence once again supports our premise, observing a decrased mean nestedness value when $p(f l)$ is increased. We also observe our nestedness parameter $\eta_{w}$ going from 0.88 down to 0.81 when $p(f l)$ is set to 0.80 in our model. But this is not the only finding. In fact, we observe that the variability of the results is also increased in terms of its standard deviation, causing a broader range of nestedness parameters results. For example, for $p(f l)=0.80$, the range of values of our nestedness parameter $\eta_{w}$ goes from 0.6604 to 0.9184 , both values being the lowest across the different cases of $p(f l)$. We may argue that this is an expected outcome as well, since the restrictions are playing again a role in the variability of the outcomes in the same way that happens with the exponents of the previously described bipartite indicators. Yet, even with extreme values of $p(f l)$, our model keeps generating highly nested network structures. This, in turn, encourages us to continue to explore the model and apply it to reproduce actual markets in the next section.

But regardless of the effect caused in the outcomes of the model as a consequence of the variation of the probability of forbidden links $p(f l)$, and just to reinforce the point, there indeed is a common feature for large values of $p(f l)$ probability. The majority of the exponents from the bipartite indicators do show an increased variability. This is due to the fact that, when more positions of the bipartite interaction matrix are restricted to exist, the model will allocated the unitary weights or links among a more limited set of posible interactions, hence contradicting and constraining the individual neutrality hypothesis. In this way, it is important to discuss the role of geopolitics within actual markets in the discussion chapter and how they may influence the market both voluntarily and involuntarily.

\subsection{Model results and market simulation}

After testing our mutualistic model of markets, we now know the model itself is highly suitable to reproduce the topological features of trade markets. We also understand the role of the forbidden links. Now the next challenge is to implement it so that we can simulate actual markets. But this task may require some adaptation 

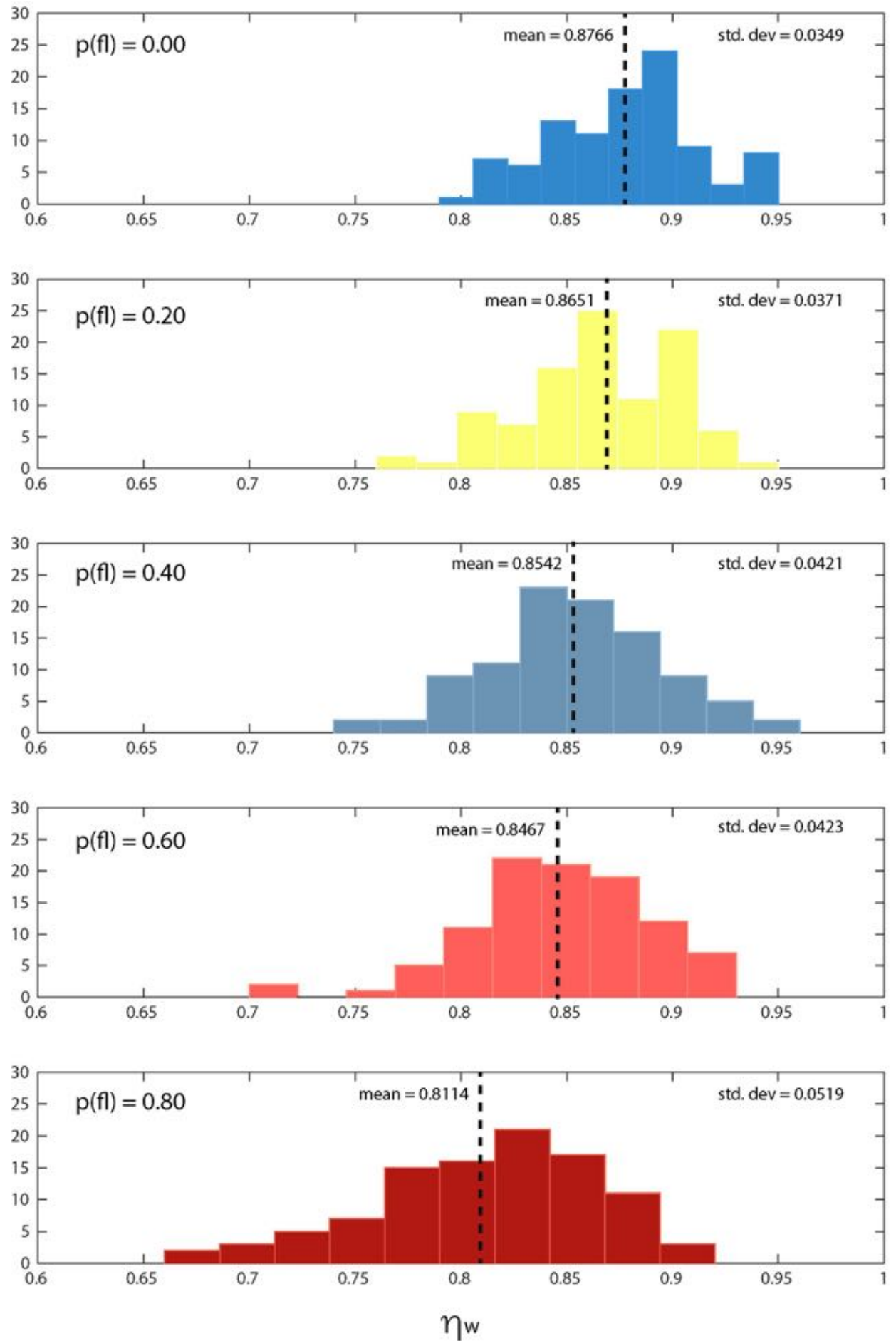

Fig. 10.7.: Nestedness performance for various $\mathrm{p}(\mathrm{fl})$. This figure shows the distributions of the weighted interaction nestedness estimator (WINE) for different values of $\mathrm{p}(\mathrm{fl})$ for the 100 realizations of our mutualistic model of markets. We observe that the mean value of $\eta_{w}$ decreases when $\mathrm{p}(\mathrm{fl})$ is increased from 0.0 (top) to 0.8 (bottom). Additionally, the variance of the values is also larger as denoted by an increased standard deviation. 
of market parameters. For example, if we start with the first industry HS2 $=01$, we observe that this industry has an average of 173 exporters and 162 importers, with an average trade volume of 10,384.2 million dollars annually. Since running our mutualistic model with $w_{a}=10,384,175,799.0$ (US dollars) would not be practical or time-effective since the total weight is equal to the time-steps of the process. Then, we are forced to set some rules to scale down the model in order to avoid computational constraints. For this, we define that the actual market will be simulated with our mutualistic model by incorporating the parameters $m=173$, $\mathrm{n}=162, w_{t}=10384$ and $\left.p(f l)=0.2\right)$, where $w_{t}=w_{a} \times 10^{-3}$. In this way, our model would be allocating 10384 demand units or unitary links, which is a manageable number of links to be processed by our algorithm. There is an evident caveat related to this volume scaling. By implementing this modification, the minimum volume to be allocated at each time-step will be one million dollars of demand since $w_{a}$ is already expressed in US dollars. This obviously creates a scale bias in the process, where any country with a trade volume of less than a million dollars will be inaccurately represented by the algorithm. We believe this inaccuracy is one that we can assume as a compromise to facilitate the computational process and to avoid extremely long processing time. Hence, in figure 10.8, we show the result of the simulation for the industry HS2 $=01$. We observe that the topological indicators are consistent with those of actual data. The degree distribution of exporters and importer are both long-tailed and heterogeneous in shape. The strength-degree correlation of exporters exporters and importers both show a power law pattern with $\beta>>1$. The weighted bipartite matrix shows a high value of nestedness. The bipartite clustering, both weighted and unweighted, for the exporters and importers display a declining performance with a $C 4_{w}>C 4_{b}$ condition. The Knn(k) indicator shows an uncorrelated pattern with the degree. Finally, the average weight as a function of the end-point degree show a two-zone pattern with positive correlation.

The first conclusion of our model implementation for an actual market is that, once again, all the key features found within empirical data are present in the outcome of the model. Let us compare the results in a more detailed way. We start with the degree distribution, which displays a power law pattern of the form $P(k) \sim k^{-\gamma}$. Our simulation shows $P(k)$ exponents $\gamma_{\text {exp }}^{\text {sim }}=0.94$ and $\gamma_{i m p}^{\text {sim }}=0.88$, which compare against those of the empirical data $\gamma_{e x p}^{a}=1.33$ and $\gamma_{i m p}^{a}=1.21$. Secondly, the strength-degree correlation is also depicted by a power law pattern of the form $S(k) \sim k^{\beta}$ with exponents $\beta_{\text {exp }}^{\text {sim }}=1.79$ and $\beta_{i m p}^{\text {sim }}=1.76$. These exponent are higher than 1.0 similarly to those we find in the emprirical data (2.12 and 1.66, respectively). Thirdly, we also find an uncorrelated nearest neighbor degree of the form $K n n(k) \sim k^{\rho}$, with exponents $\rho_{e x p}^{\text {sim }}=0.004$ and $\rho_{i m p}^{\text {sim }}=-0.002$, values that are again aligned to those found in the actual market ( 0.09 and 0.05 , respectively). Then, the clustering performance, both unweighted and weighted, are found to display power law patterns of the form $C 4(k) \sim k^{\sigma}$ and $C 4^{w}(k) \sim k^{\nu}$, respectively. In the 

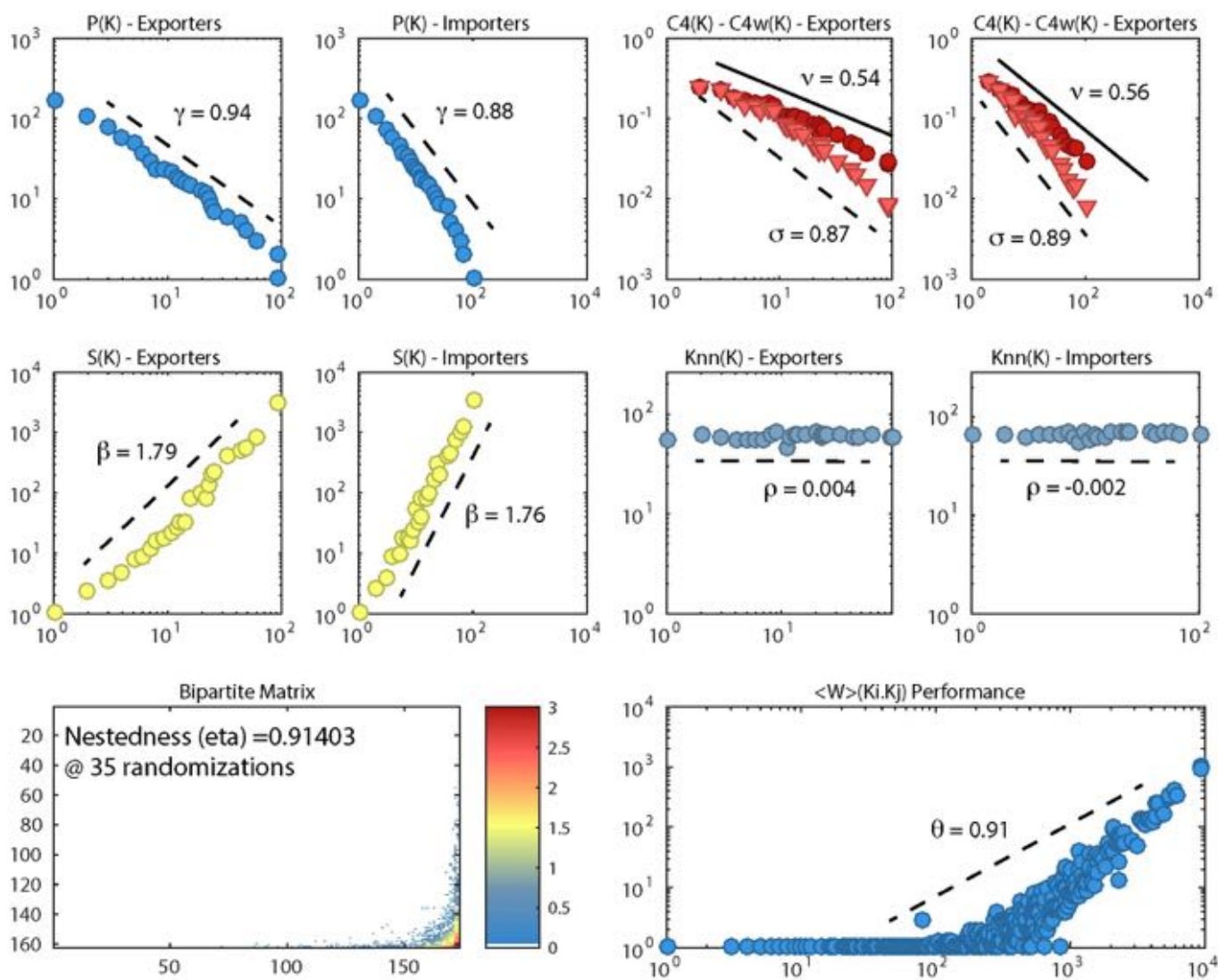

Fig. 10.8.: Actual market simulation with our mutualistic model for HS2 $=01$. This figure shows a simulation of the HS $2=01$ market with $n=173, m=162, w=10384$ and $\mathrm{p}(\mathrm{fl})=0.20$.

first case, the unweighted clustering, we find exponents of values $\sigma_{e x p}^{\text {sim }}=-0.87$ and $\sigma_{i m p}^{\text {sim }}=-0.89$ (the actual markets show -0.52 and -0.46 , respectively), whereas the weighted clustering shows that $\nu_{e x p}^{\text {sim }}=-0.54$ and $\nu_{i m p}^{\text {sim }}=-0.56$ (the actual markets show -0.28 and -0.28 , respectively). This values, although quite repetitive, are aligned again to those we find in actual markets. Although they are not exactly the same, they remain very close to them. Finally, the average weight as a function of the end-point degree shows a power law pattern of the form $\left\langle w_{i j}\right\rangle \sim\left(k_{i} k_{j}\right)^{\theta}$ with $\theta^{\text {sim }}=0.91$ (the actual market is 0.82 ).

On the other hand, we now need to analyze the nestedness in a separate way. The simulations of the actual market based on our mutualistic model of markets does yield a highly nested structure, which is supported by the evidence of $\eta_{w}=0.96$. This value implies that our model is capable of generating an interaction matrix that is almost perfectly nested. Yet the reality of actual markets, although displaying high values of nestedness, are not quite at the same range. In fact, actual markets show values of nestedness in a range that goes from $\eta_{w}=0.60$ to $\eta_{w}=0.9$ for HS2 industries based on the results from Chapter 7. In particular, the empirical 
data shows that the market for HS2 $=01$ has an average value of $\eta_{w}=0.72$ over 15 years of historical data, suggesting that our model generates a structure that is more nested than the reality despite observing similar exponents in the rest of the bipartite network indicators.

Nontheless, this is only the first selected industry. We now extend the simulation with our model to the rest of the selected industries (HS2 $=01,10,20,35,50$, $62,75,84)$. In figures $10.9,10.10,10.11$ and 10.12 , we show the outcomes for each industry based on the $M(m, n, w)$. For all the industries, the results are consistent with those of the empirical data. Yet, the issue of extremely nested structures is still present in each industry, which suggest that the finding of values of nestedness higher than those of the actual markets may be a systemic deviation from the reality. In this way, we could argue that our mutualistic model of markets is an algorithm that generates virtually perfectly nested markets, which are quite hard to find in reality despite our efforts to account for the geopolitical constraints through forbidden links. The model can, on the other hand, consistently generate network structures and mutualistic feature simultaneously that resemble those of the empirical data, which is not a trivial fact. After analyzing this situation, we have confirmed that the main factor for this to happen is related to the way we set up the decreasing probability for new nodes (exporters or importers) to arrive or appear in the market. But this may be an interesting fact in itself since the very definition of the model intrinsically, and also inadvertely, has incorporated a restriction that can be interpreted as a barrier to access the market, a clear deviation from the perfect competition concept. Although it may make sense for ecosystems, markets do not necessarily need to have a lower probability of a new player appearing when a large number existing players are in place. There is also a numerical consideration to account for. Since our model is highly inspired by ecosystems, where the usual size of the interaction matrix is between 20 to 50 species, the size of international trade markets is normally between 120 and 250 importer or exporters. This means that, as the algorithm starts allocating unitary links to a fairly large number of existing exporters or importers, the unitary links at the end of the process have a probability of being allocated to a new node that is about 250 times lower than that of the initial step, or formally, $p_{\text {new }}(t=W) \sim \frac{p_{\text {new }}(t=1)}{250}$. Clearly, the higher the market size, the lower the probability of new nodes appearing in the market.

In the next section, and just for the sake of providing evidence to our hypothesis, we will provide an alternative definition of our mutualistic model of markets, but we change the definition of the probability of new node for an arbitrary one. 

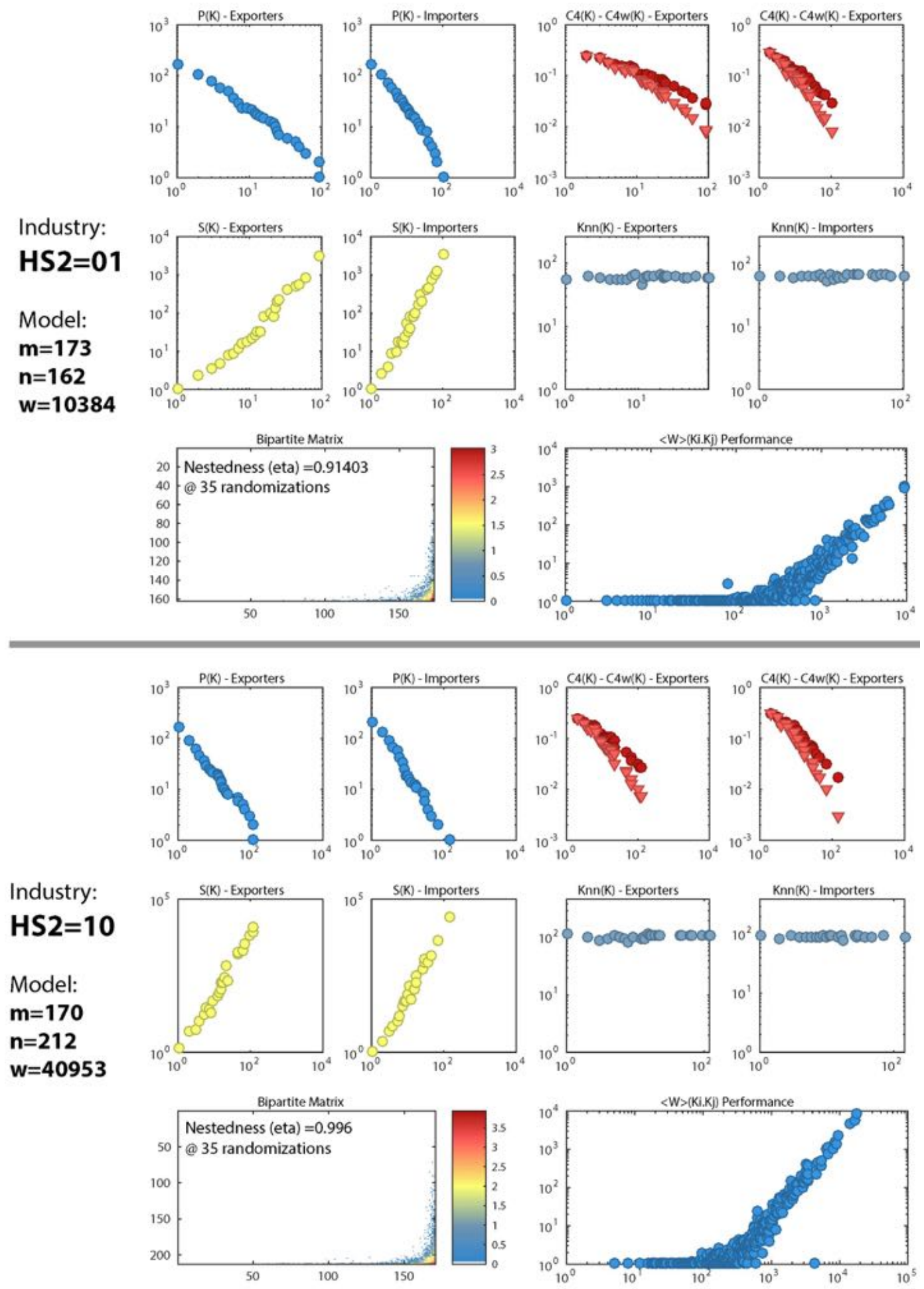

Fig. 10.9.: Actual market simulations with our mutualistic model (HS2=01; HS2=10). This figure shows the results of the simulation based on our mutualistic model of markets for the industries HS2 $=01$ (top) and HS2 $=10$ (bottom). Both models show similar patterns as described in chapter 7 and all common features of trade networks are present here. 

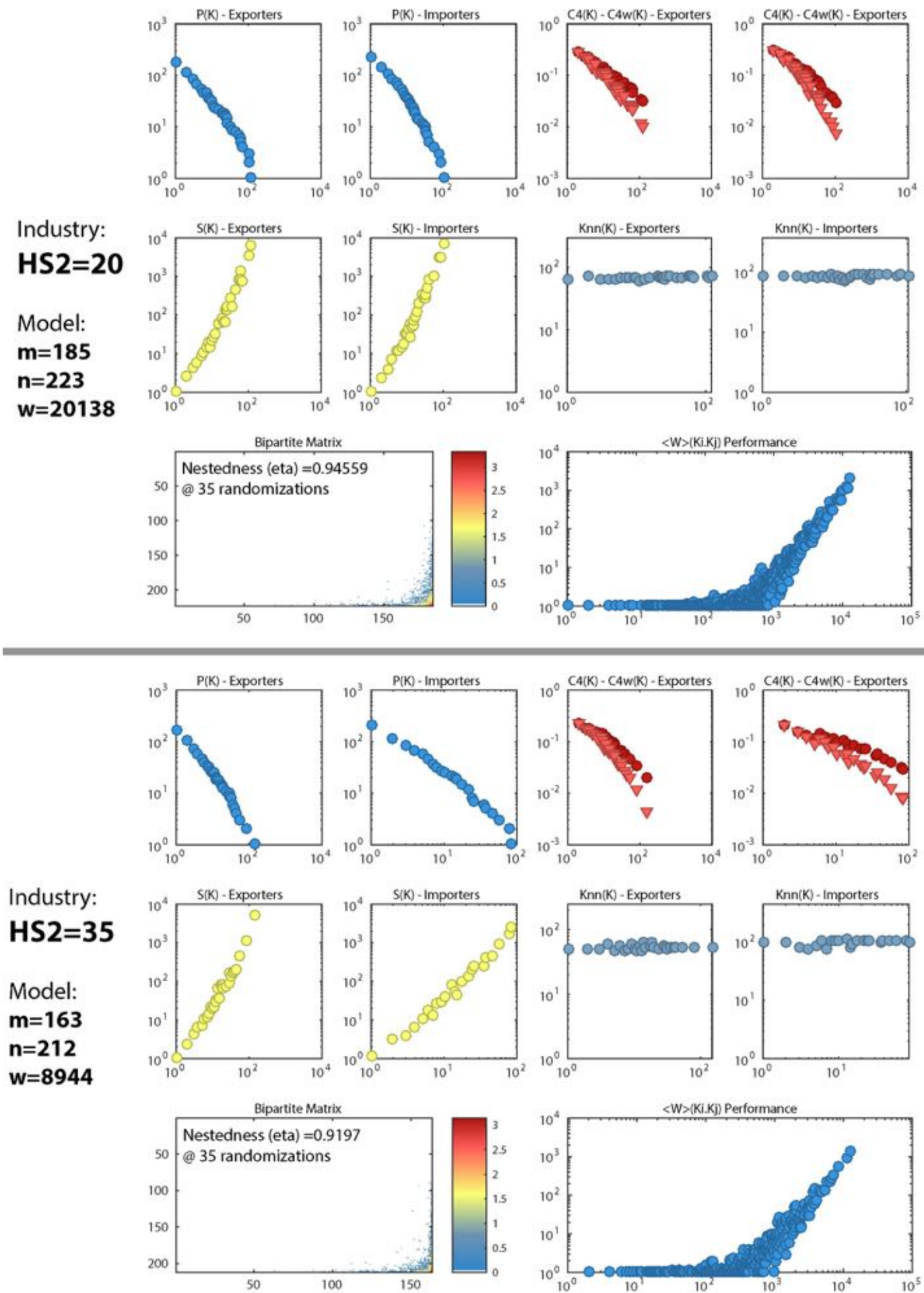

Fig. 10.10.: Actual market simulations with our mutualistic model (HS2=20 ; HS2=35). This figure shows the results of the simulation based on our mutualistic model of markets for the industries HS2 $=20$ (top) and HS2 $=35$ (bottom). Both models show similar patterns as described in chapter 7 and all common features of trade networks are present here. 

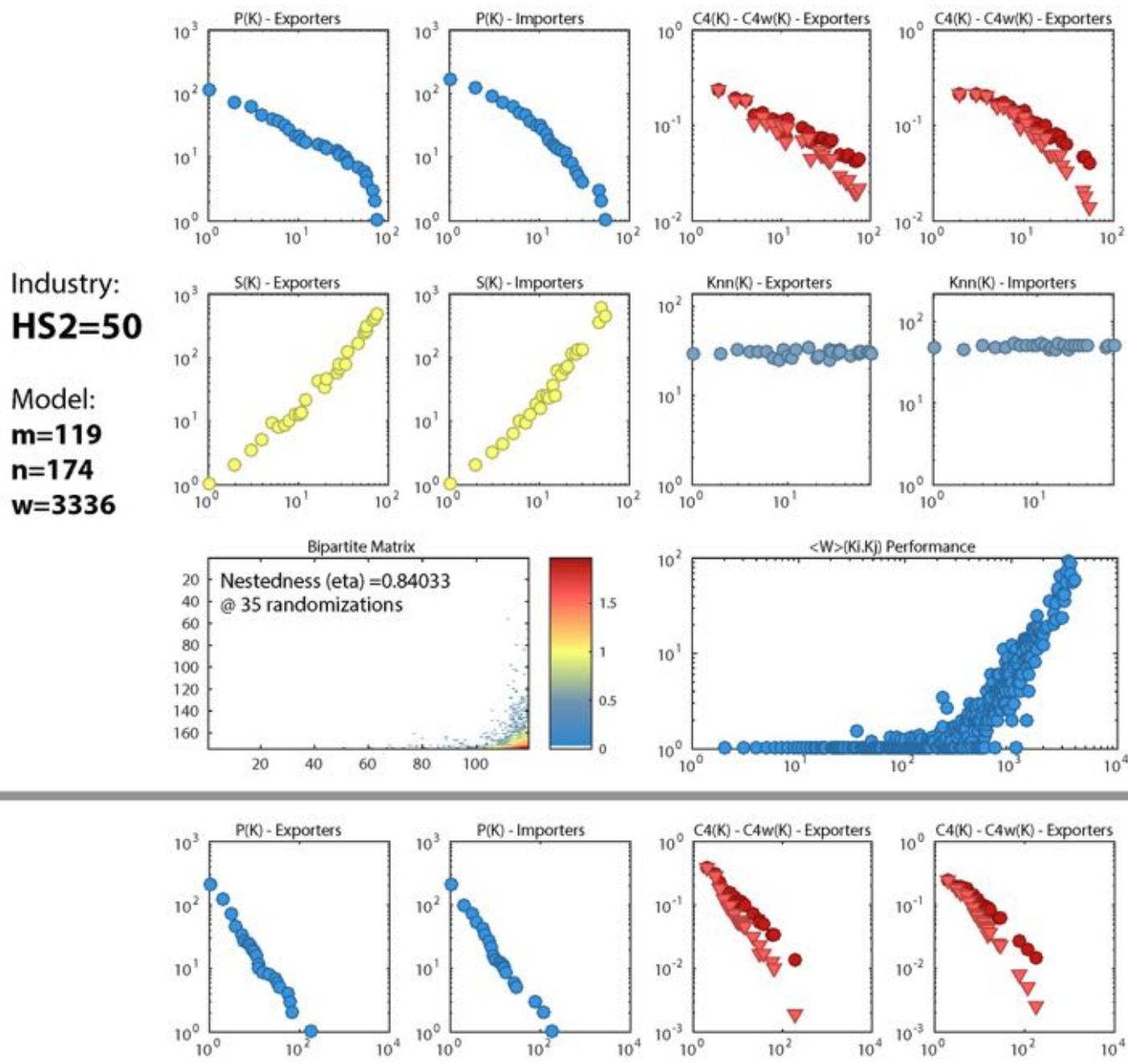

Industry:

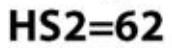

Model:

$\mathbf{m}=\mathbf{2 1 1}$

$\mathrm{n}=\mathbf{2 2 5}$

$w=95415$
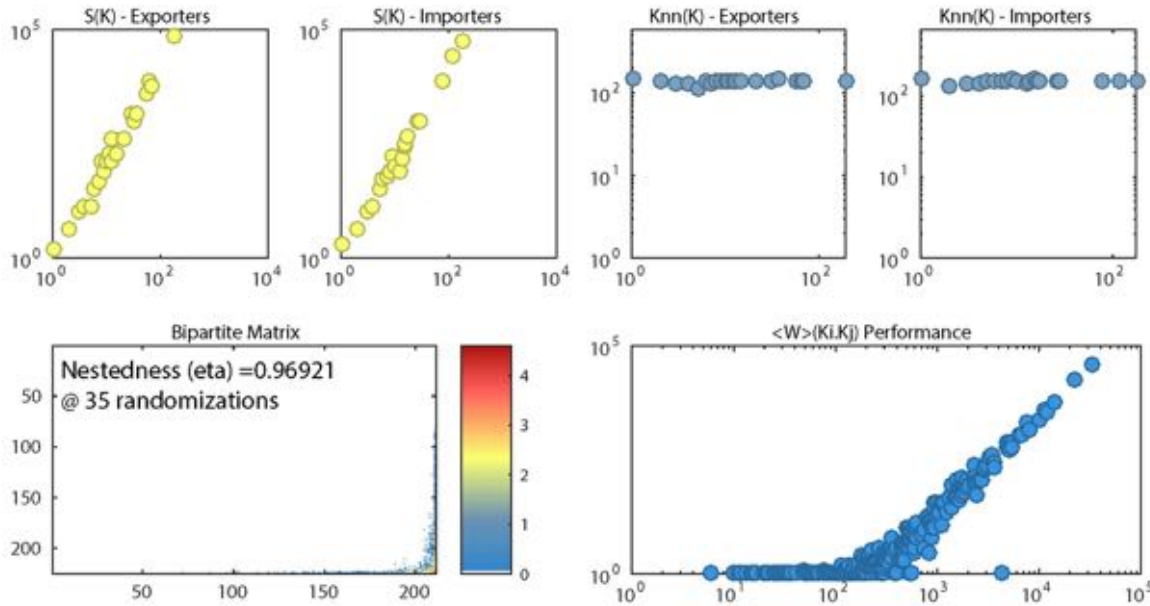

Fig. 10.11.: Actual market simulations with our mutualistic model (HS2 $=50$; HS2 $=62$ ). This figure shows the results of the simulation based on our mutualistic model of markets for the industries HS2 $=50$ (top) and HS2 $=62$ (bottom). Both models show similar patterns as described in chapter 7 and all common features of trade networks are present here. 

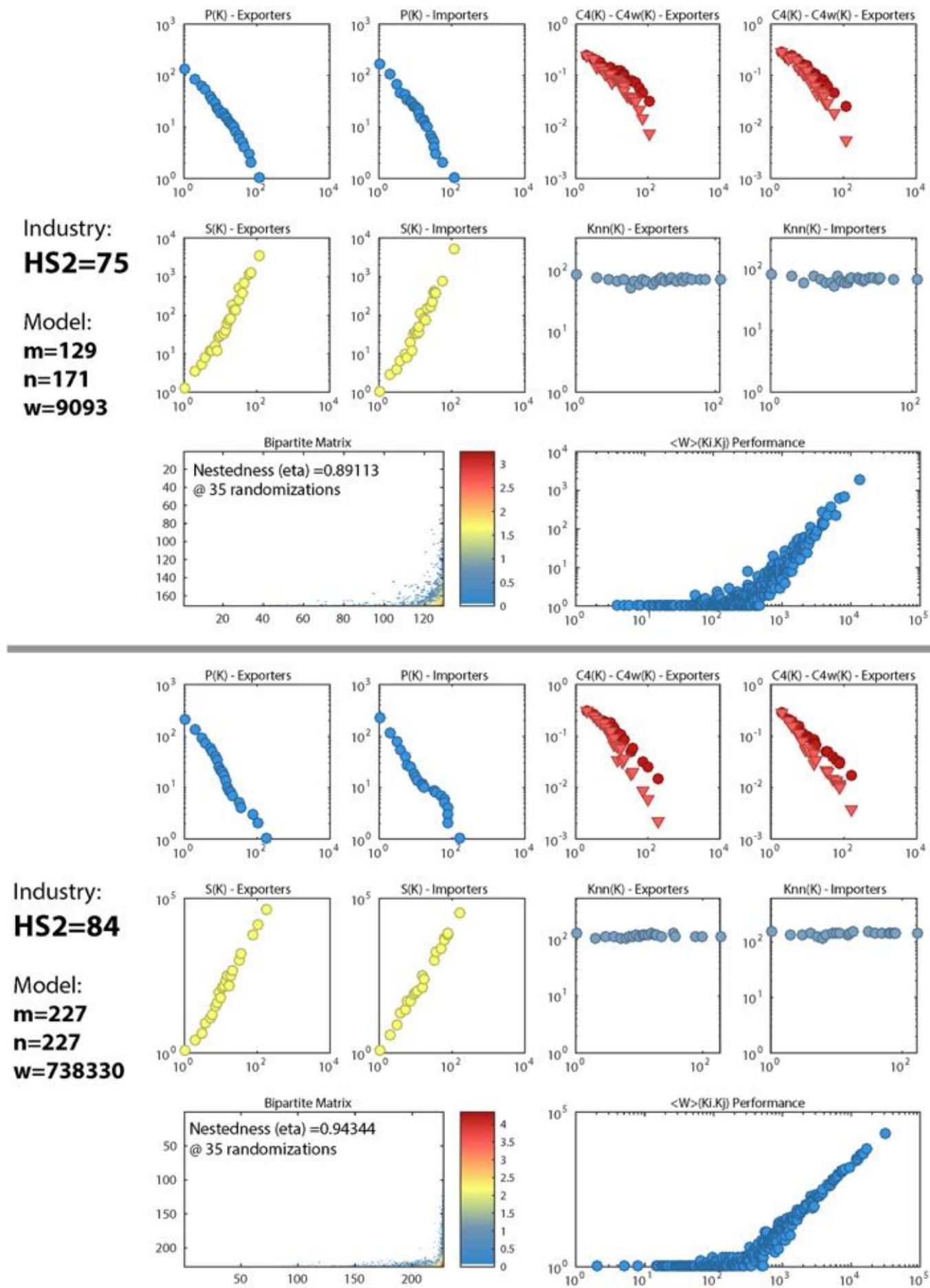

Fig. 10.12.: Actual market simulations with our mutualistic model (HS2 $=75 ;$ HS2 $=84$ ). This figure shows the results of the simulation based on our mutualistic model of markets for the industries HS2 $=75$ (top) and HS2 $=84$ (bottom). Both models show similar patterns as described in chapter 7 and all common features of trade networks are present here. 


\subsubsection{Highly-sparce market simulation}

As argued in the previous subsection, we find only one down side of our mutualistic model of markets. That is, because we have defined a decrasing probability of new nodes with the number of existing nodes at any time step of the process, we observe that the outcome of the model is more highly nested than the markets from the empirical data. We have a premise to test a few arbitrary values of probability of new nodes in this subsection as a way to approximate the actual behavior of markets. However, we believe that this modification to the model will not change the academic value of our model. This is based on the fact that a modification of a thoeretical model through trial and error will not diminish the elegance and simplicity of it. Rather, the modification should be viewed as an applied exersice to approximate reality from a pragmatic point of view. This being clarified, we perform the experiment with several functions, yet we realize that a constant probability can provide a suitable contrast to the outcomes of our formal model. We set a $p_{\text {new }}=0.2$ for both the exporters and the importers, which implies that approximately 1 out of 5 time steps will result in a new node (either an exporter or an importer, or both) entering the market. This value was empirically determined with a large number of iterations, concluding that this value is the most suitable to recreate the sparse properties found within empirical data. In figure 10.13 we observe the outcome with a simulation of the modified mutualistic model of markets for the industry HS2 $=01$.

The first and main conclusion is that the nestedness is now at a value of $\eta_{w}=0.80$, which is aligned to the values of the empirical data. We observe that a constant probability of new nodes works in such a way that allows that interaction matrix to incorporate more interactions beyond the core of the network, enabling potential links on the periphery despite the forbidden links. In a way, we find that the forbidden links are not as efficient in our formal model because the decreasing probability of new node does allow the near periphery links to appear, hence only allowing for links close to the core to be reached. This finding is a fine complement to the internal structure of the markets, thus sheding some light to how the mechanism of self-interest might formally be found in the process of market formation. Indeed, some level of promiscuos connectivity can help markets reach a level of nestedness where the whole network is still robust overall, but there is still room for strong competition between a larger number of players, either exporters or importers, so that the concept of perfect competition remains the main force that shape the market formation process. As speculated in our premise, we now understand in a better way how the correct balance between cooperation and competition in markets as a complex system will determine the internal structure of trade networks. 

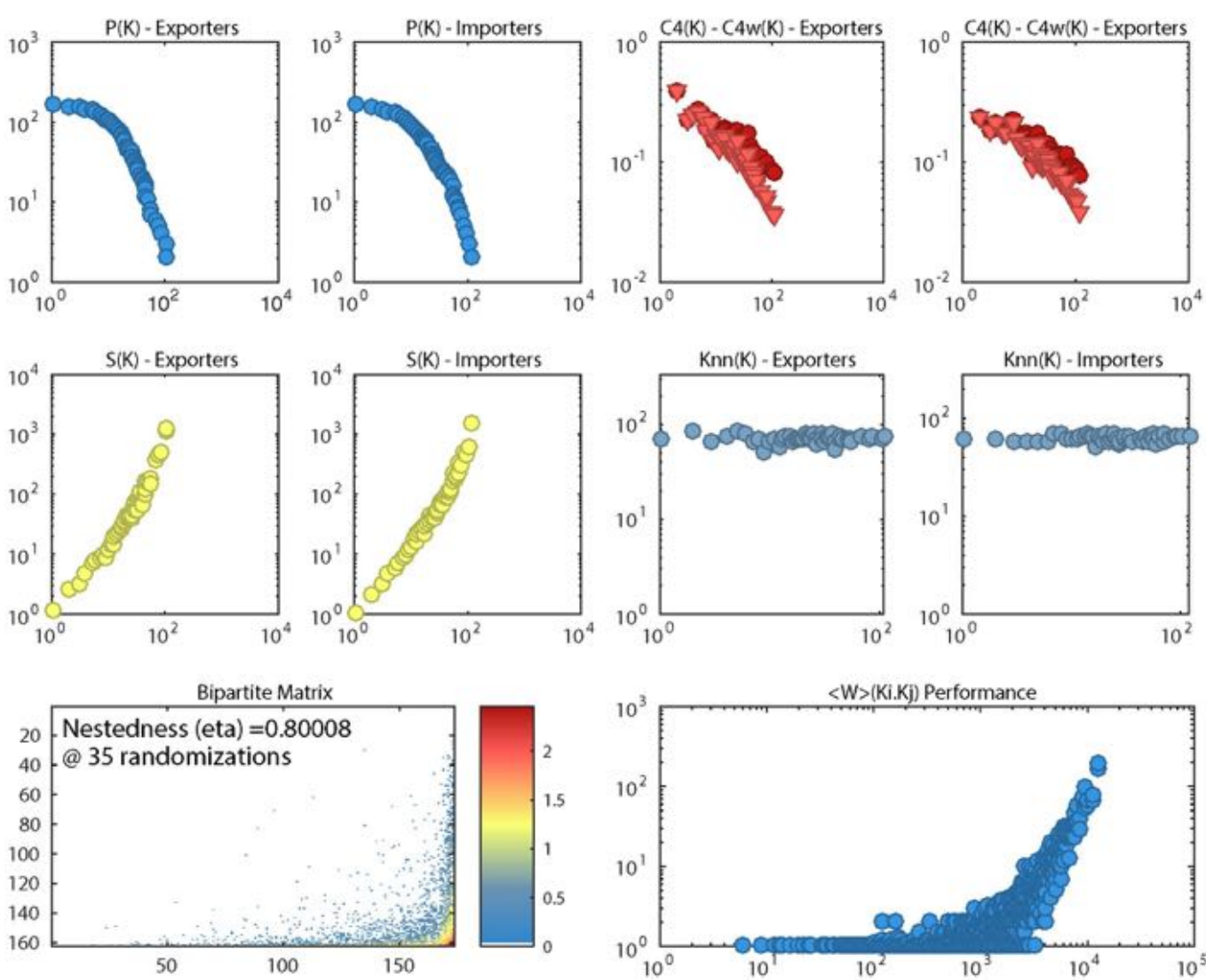

Fig. 10.13.: Actual market simulation with a modified mutualistic model for HS2 $=01$. This figure shows a simulation of the HS2 $=01$ market with $n=173, m=162$, $\mathrm{w}=10384$ and $\mathrm{p}(\mathrm{fl})=0.20$, yet with a constant probability $p^{\text {new }}=0.2$ to recreate the sparsity of actual markets. We observe that the topological indicators are consistent with those of actual data. Additionally, the nestedness value are closer to that of the empirical data. The degree distribution of exporters and importer are both long-tailed and heterogeneous in shape. The strength-degree correlation of exporters exporters and importers both show a power law pattern with $\beta>>1$. The weighted bipartite matrix shows a high value of nestedness. The bipartite clustering, both weighted and unweighted, for the exporters and importers display a declining performance with a $C 4_{w}>C 4_{b}$ condition. The $\mathrm{Knn}(\mathrm{k})$ indicator shows an uncorrelated pattern with the degree. Finally, the average weight as a function of the end-point degree show a two-zone pattern with positive correlation.

In sum, the form of the probability function for new nodes can account for two critical points: a) the incorporation or the lack of barriers to access the market, a basic condition for the existance of perfect competition or a quasi-perfect competition, and b) the control of the outcome of the model as a highly nested network (in the case of the formal model with increasing barriers with the size of the market) or a more realistic and sparse network with high values of nestedness (in the case of constant probabilities and minimum barriers to access the market). 


\subsection{Simulation of special cases of markets}

In this section, our goal is to simulate the special cases of markets from chapter 2 by adding the proper constraints in our model. These cases are A) the monopsony, and B) the monopoly. The way to accomplish this is to simply set a number of exporters or importers in such a way that the outcome resembles the special case itself. For example, if we try to simulate a monopoly, our model should be set to $M(m=1, n, w)$. For a monopsony, the model would be $M(m, n=1, w)$. However, from a computational standpoint, although using one exporter or one importer is possible, we will start by constraining the number of exporters and importers with a small value, which can be interpreted as a quasi-monopsony or a quasi-monopoly. We implement this idea by using 10 importers or exporters and 200 exporters or importers, respectively. In figure 10.14 we show the results of both cases together with the key results and exponents for the mutualistic indicators.

We observe that the quasi monopsony and the quasi monopoly display a very similar behavior, where a very high level of nestedness has been achieved in both cases. Yet, regarding the exponents of the mutualistic indicators, we observe a mirror effect across all exponents. The constraint in the number of importers for the monopsony and the number of exporters for the monopoly has created an assymetric behavior of the degree distribution, where the exponent for the importers and the exporters are not similar like those of the actual markets. Rather, the exponent corresponding to the constrained subset is abnormally smaller in absolute value than the average exponent found in empirical data. In contrast, the exponent corresponding to the non-constrained subset is abnormally larger in absolute value than the average exponent found in empirical data. Regarding the strength-degree correlation, the $\beta$ exponent of the constrained subset displays a value closer to one, whereas the value of the non-constrained subset larger than the average exponent of the empirical data. The performance of the nearest neighbor degree distribution is still uncorrelated with the degree, but we cannot draw a conclusion in this case since this may be a trivial case of coincidence. The bipartite clustering is not an exception in terms of assymetric behavior between the constrained and the non-constrained subsets, displaying both smaller and larger than average exponents, respectively. Finally, the average weight as a function of the end-point degree is within the range of results found in the empirical data.

With these results, we finalize our detailed analysis of how the mutualistic model of markets can be utilized to simulate trade networks that resemble those of the actual markets. In the conclusions chapter, we will argue further about the collective behavior of the model and the lessons learned from the different exercises we performed. 


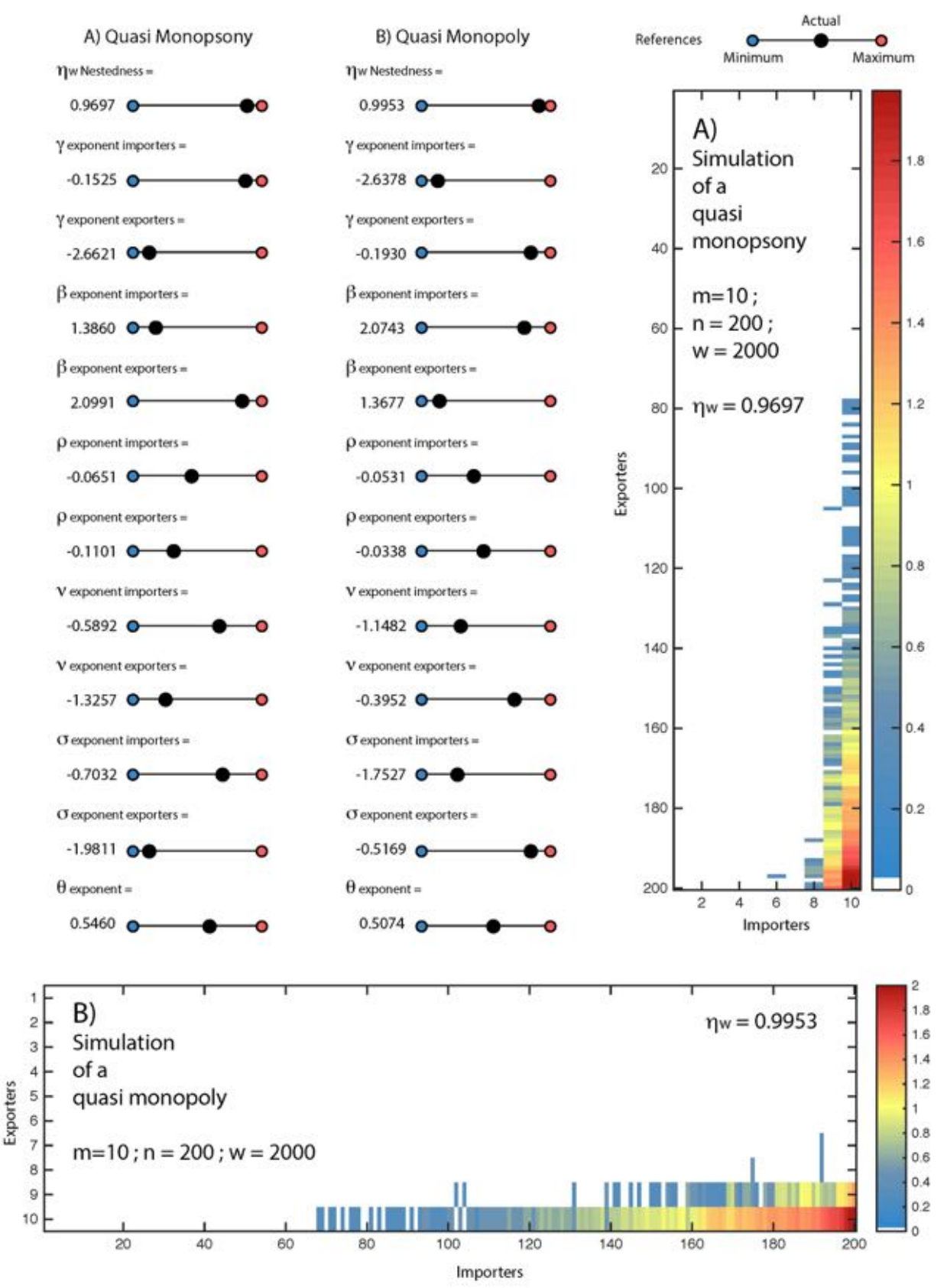

Fig. 10.14.: Simulations of the special cases of markets. This figure shows the results of an approximation to the special cases of market, namely, a monopoly (A) and a monopsony (B). We observe that most of the common features of trade networks are still present. Yet, the constraints imposed to the size of subsets of exporters and importers generates assymetric results in terms of the power law exponents for $\mathrm{P}(\mathrm{k}), \mathrm{S}(\mathrm{k}), \mathrm{Knn}(\mathrm{k}), \mathrm{C} 4 \mathrm{~b}(\mathrm{k}), \mathrm{C} 4 \mathrm{w}(\mathrm{k})$ and the end-point degree performance. In these cases, we also observe that the exponents of both the contrained and the uncontrained subsets display a deviation from the typical values found in actual markets with the exception of $\rho$ exponent, which may trivially show a value close to zero. 


\section{International Lending Markets}

What we know about the global financial crisis is that we don't know very much.

- Paul Samuelson

(Nobel Memorial Prize in Economic Sciences, 1970)

After gaining a deep understanding of international trade markets, a topic in which a great deal of data is publicly available, we now propose another exercise to study a different type of market, but with a similar complex network approach. Financial markets, such as stock markets, are highly complex, dynamical and sometimes even difficult to analyze. However, other financial markets are more approachable and researchable with a more manageable, still rigorous methodology. This is the case of the international lending markets and, more precisely, the interbank market. Every day, banks around the world perform transactions of both commercial and financial nature. Whenever a bank grants a loan to a person to buy a house, a car or simply to pay for his or her next vacations, a commercial transaction takes place. As a consequence of the commercial transactions, banks are continuously forced to manage their assets, which are everything they own, as well as their liabilities, which are everything they owe. Therefore, any imbalance between the assets and the liabilities calls for a corrective action to regain an equilibrium between them. Thus, it would be against a bank's best interest to hold an excess of debt, which could put the bank in a weak position. The opposite is also true in the case of a cash excess, which could be interpreted as an idle asset and hence, a loss of oppotunity. In order to resolve this problem of cash or debt excess, financial institutions normally use the interbank market, enabling any bank with a cash excess to lend this excess money to other banks with a debt excess. Additionally, it is worth pointing out that the interbank market has special regulations and conditions, such as preferential interest rates, making it the preferred choice to cover any imbalance. Given the large number of banks and financial transactions, in addition to a lack of public data, this markets involve a great deal of complexity. In this chapter, we tackle such a problem and try to find a statistical model that can explain and reproduce the complex networks that arise from this market. 


\subsection{Some background on financial institutions}

The scientific community has demonstrated an increased interest in studying the systemic risk derived from financial crisis such as the one that started in September 2008. In that moment, and after Lehman Brothers bankrupcy was declared, a large chain of events has taken place, leading to the deepest financial and economic crisis since the big depression of 1930's. In this scenario, the study of the structure, stability and resilience of financial systems became highly relevant in order to prevent future financial shocks that may lead to a new economic crisis. Furthermore, a particularly important topic was identified, that is, the liquidity shocks of the interbank market as well as the contagion phenomena that may arise from it. The interbank market is a fundamental mechanism to sustain the backbone of the financial system. This market is not only complex and critical in nature, but also large in size. The evolution of financial institutions can be depicted by the way in which payments were managed in the past and the way in which they are currently. Clearing houses were the main resource during most of the last century. Nevertheless, in the last decade, electronic markets were created and banks have been able to access these modernized markets. Since then, the large-value payment systems, or LVP, have evolved, leading to simplified ways of managing interbank transactions and creating a reliable data source. Based on the LVP data, the interbank market becomes susceptible of study through the complex network theory. Several empirical studies have been published with this approach. Boss et al.[31] has achieved the goal of performing empirical analysis of real-world interbank networks. The lessons learned from this and other studies $[66,79,7,105,1]$ show a promising and robust field of study. The logic to build interbank networks require some attention however. On the one hand, transactions between banks can be conceptualized as direct links, where the amounts involved will be the link weights. Then, the aggregation of the intraday transactions over a large period will be the interbank networks. From the empirical studies [31, 145, 65], we know that long-tailed degree distributions can be found in these networks. But they are often approached as static networks, since they are fixed in time. This can lead to potential errors due to the fact that any interbank network is the result of the aggregation of high frequency trading. Consequently, we propose a consistent model to reproduce the topology of empirical interank networks. Our fundamental premise is that the interbank market structure emerge endogenously and, hence, it can be modeled with agent-based dynamic process. Since we do not have access to transaction databases, we perform a detailed statistical analysis of the balance sheets of more than 7000 global banks, extracting their actual position in terms of assets and liabilities. For this purpose, we use the Bankscope database [33]. Ultimately, the combination of the statistical analysis and the agent-based process will lead our minimal and stochastic model. It is minimal because it makes simple assumptions and does not define complicated actions between agents, which 
is simply that any imbalance of assets or liabilities need to be compensated for every step of the process. It is also stochastic because we have no insights on the strategies of each agent, which we replaced with randomness.

\subsection{Data analysis of interbank markets}

The Bankscope database [33] gathers information of the financial statements, ratings and intelligence of over a dozen of thousands of banks globally. Our raw data contains historical records of 32505 banks, from 2008 to 2015. We extract specific data in order to depict the size of banks, or total assets (TA), the interbank assets, depicted by loans and advances to banks (LAB), and the interbank liabilities, depicted by deposits from banks (DB). We filter and average the data from around 7000 entities that are consistently active throughout the period, excluding all central banks and clearing houses, since they are driven by different dynamics than are regular banks, and taking strictily positive values only.

In figure 11.1, we show the distribution of interbank assets and liabilities and the correlation as a function of total assets. Thus, since the interbank assets and liabilities are part of the total assets, the conditions of both $L A B \leq T A$ and $D B \leq T A$ must hold for each entity. As observed, they are both far from being bivariate log-normal distributions but still, the marginal distributions seem to be very close to log-normal functions. We also introduce the relative variables $x=L A B / T A$ and $y=D B / T A$ and their respective distributions are shown in figures 11.2 and 11.3. We find a side effect, which is the truncation of bivariate distributions of $x$ and $y$. More importantly, we find that the correlations of the variables $x$ and $y$, and $T A$ are weak. The linear correlation between the relative variables is shown in Table 1.

\begin{tabular}{lll} 
Linear model in log-log scale & $r^{2}$ & $p$-value \\
\hline Relative IB assets $(x)$ vs. total assets & 0.042 & 0 \\
Relative IB liabilities $(y)$ vs. total assets & 0.012 & 0 \\
Relative IB assets $(x)$ vs. relative IB liabilities $(y)$ & 0.00 & 0.051 \\
\hline
\end{tabular}

Tab. 11.1.: Linear regression analysis of relative variables $x$ and $y$. Linear regression analysis for pairwise bank relative positions and total assets. The explained variance by linear models is very poor, although correlations with total assets are significant due to the large size of the dataset. As confirmed by a small $r^{2}$ value, statistical correlation is significant but irrelevant in practice (in particular, the consideration of correlations influences network model results only marginally). The coefficients of the linear model are statistically significant in both cases. The correlation between variables $x$ and $y$ is not significant at a 1 confidence level, for example (the slope is compatible with zero at such confidence level). 

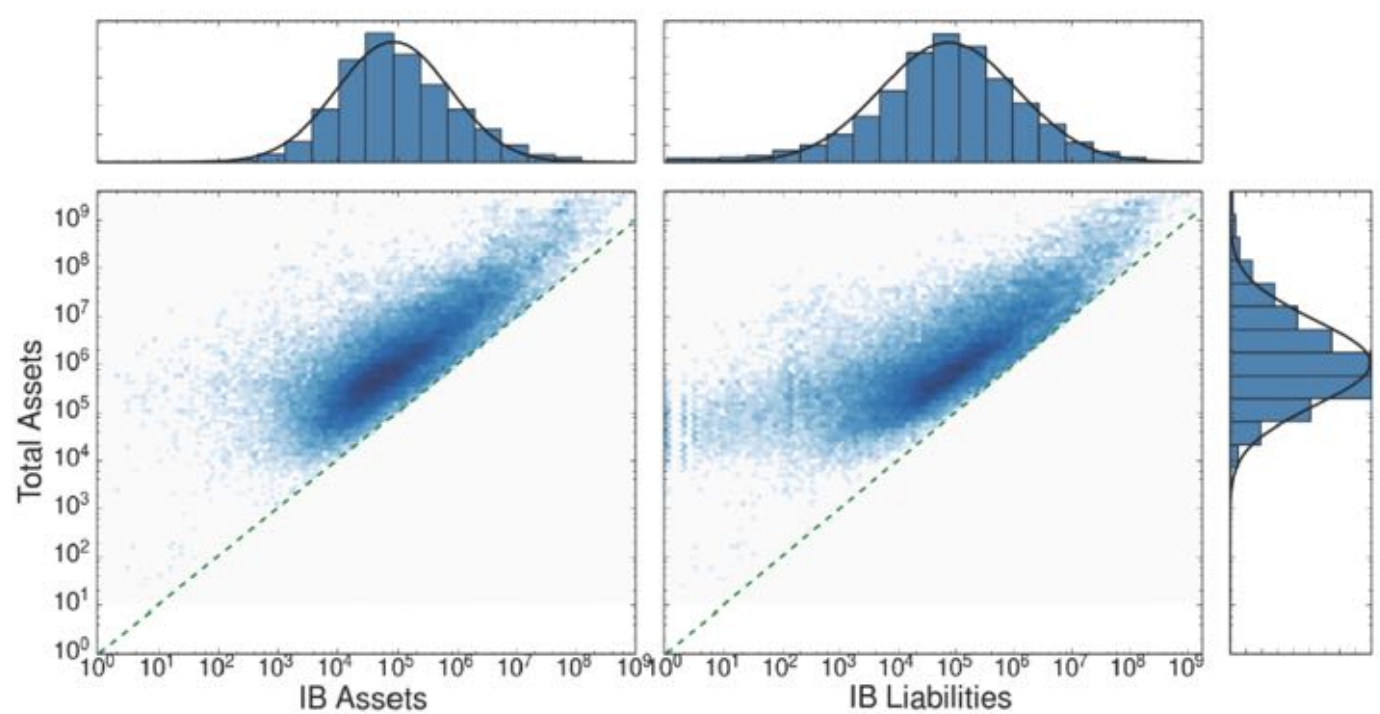

Fig. 11.1.: Analytics based on global bank positions. This figure shows the marginal and bivariate distributions of TA vs. LAB and TA vs. DB and the associated marginal 1D-distributions. The straight (green, dashed) lines represent the limits LAB = TA (left) and DB $=$ TA (right). Log-normal functions $f(x)=e^{(\log x-\mu)^{2 / 2 \sigma^{2}}} / \sqrt{2 \pi} \sigma x$ are fitted to marginal aggregated data (black lines, non-linear least squares fit from marginal cumulative distributions).

At this moment, we find an additional issue to discuss that could directly affect our model, that is the maturity of the loans recorded in the balance sheets of banks (based on the loan start date for assets or due dates for liabilities). Our simulations should be oriented to understanding and depicting the short-term interbank market and, more precisely, the overnight loans, the most common type of loans in the interbank market. The main reason is that these loans account for the majority of the transaction volume, making them the backbone of the interbank liquidity system, which is exactly the environment where a crisis would start. Therefore, we also perform a similar analysis for the same variables in the short-term, namely $L A B_{s}$, $D B_{s}$, and the relative values $x_{s}$ and $y_{s}$.

\begin{tabular}{lll} 
Linear model in log-log scale & $r^{2}$ & $p$-value \\
\hline Relative IB assets $\left(x_{s}\right)$ vs. total assets & 0.042 & 0 \\
Relative IB liabilities $\left(y_{s}\right)$ vs. total assets & 0.012 & 0 \\
Relative IB assets $\left(x_{s}\right)$ vs. relative IB liabilities $\left(y_{s}\right)$ & 0.00 & 0.051 \\
\hline
\end{tabular}

Tab. 11.2.: Linear regression analysis of short-term variables $x_{s}$ and $y_{s}$. Linear regression analysis for pairwise bank relative positions in the short-term and total assets. The explained variance by linear models is very poor, although correlations with total assets are significant due to the large size of the dataset, similarly to Table 11.1 

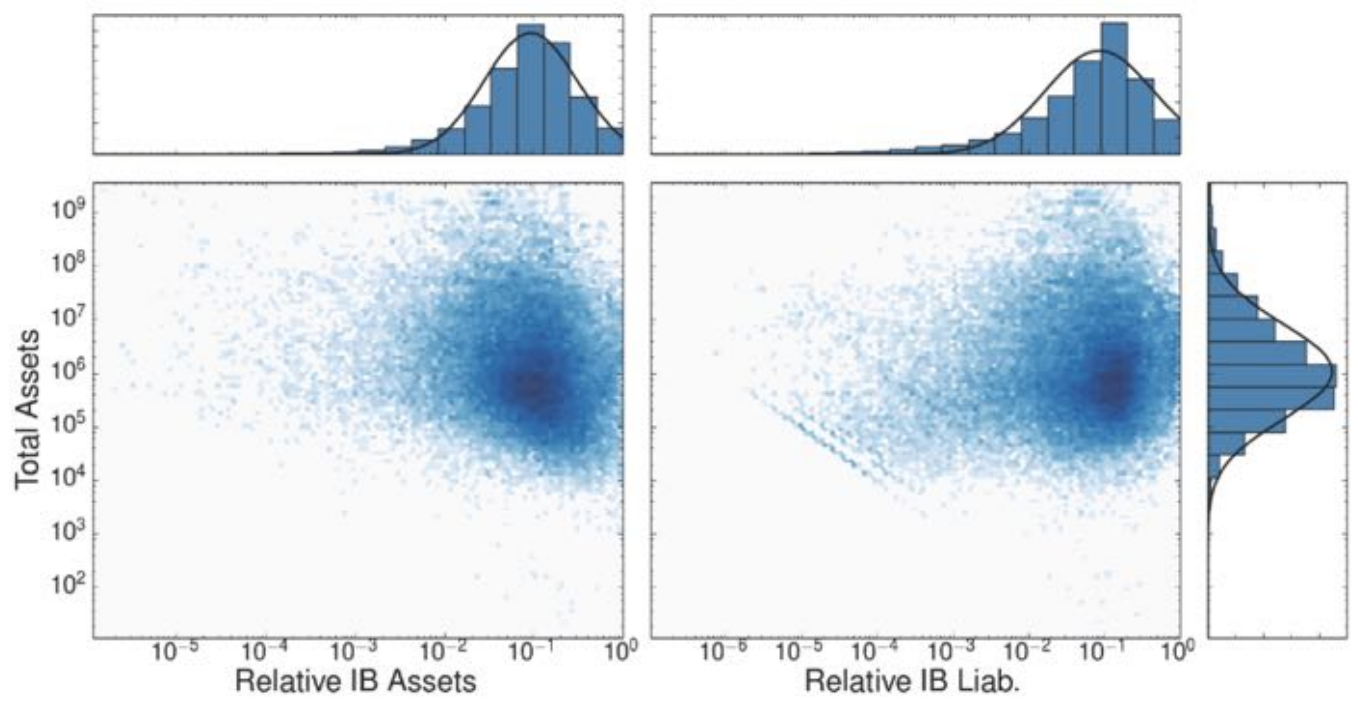

Fig. 11.2.: Analytics based on relative variables $x$ and $y$. This figure shows the marginal and bivariate distributions using the relative variables $x$ and $y$, and the associated marginal 1D-distributions. Truncated log-normal distributions with mean $\mu<0$ are now fitted against the marginal distributions of these variables (black lines). Log-normal functions $f(x)=e^{(\log x-\mu)^{2 / 2 \sigma^{2}}} / \sqrt{2 \pi} \sigma x$ are fitted to marginal aggregated data (black lines, non-linear least squares fit from marginal cumulative distributions).

\subsection{Networks properties of financial markets}

Provious scientific work [31, 85, 26, 96, 65] that tackled the topic of interbank markets and its network structure has incorporated a diverse set of topological indicators. Here we briefly define those quantities for the sake of completeness. Topological properties of interbank networks include:

Degree distribution. For a bank $i$, its out-degree $m_{i}$ is the number of banks in the network for which at least a loan from $i$ has been recorded. Multiple loans from bank $i$ to bank $j$ can occur but only a single directed link from $i$ to $j$ is considered for out-degree computations. Similarly, the in-degree $n_{i}$ of bank $i$ is the number of banks from which it has received at least one loan. The probabilities $P_{i}(m)$ and $P_{o}(n)$, measured over the network, are the in- and out-degree distributions, respectively. Empirical studies for different regional interbank networks show that in- and out-degree distributions are long tailed [31, 145, 65].

Distribution of transactions by volume. For a bank $i$, the number of out-transactions by volume $s_{i}$ is the number of loans that $i$ has performed. The number of intransactions $t_{i}$ of bank $i$ is the number of loans it has received. The probabilities $P_{o}(s)$ and $P_{i}(t)$ stand for the in- and out-transactions distributions, respectively. Reported empirical transaction volumes for the USA interbank market display a power-law 


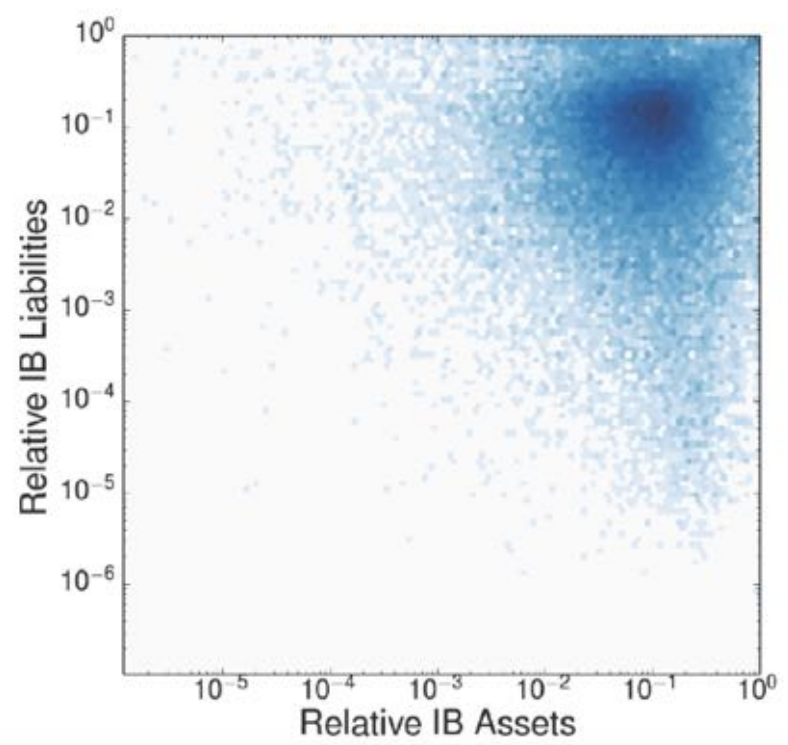

Fig. 11.3.: Bivariate distribution of relative variables $x$ and $y$. This figure shows bivariate distribution of relative variables $\mathrm{DB} / \mathrm{TA}$ (vertical axis) vs. LAB/TA (horizontal axis).

distribution [145]. However, other studies [85] find exponential distributions for transactions by volume.

Distribution of transactions by value. Transactions by value take into account the amount lent in each interbank interaction. For a bank $i$, the strength of outtransactions by value $\tilde{s}_{i}$ is the total sum of amounts corresponding to the $s_{i}$ loans recorded for the institution i as a lender. Analogously, the strength of in-transactions by value $\tilde{t}_{i}$ is the overall value of the $t_{i}$ loans received by bank $i$. The corresponding in- and out-transactions distributions are denoted as $P_{o}(\tilde{s})$ and $P_{i}(\tilde{t})$, respectively. Some studies report log-normal distribution functions for transactions by value [145].

Distribution of exposures. Since a transaction value can be associated to each link in interbank networks, the weight of all interactions between two banks can also be calculated. The exposure $e_{i j}$ (which is the technical term to refer to the balance of assets and liabilities) between banks $i$ and $j$ is thus defined as the sum of all the transaction values for all the loans that have occurred with $i$ as a lender and $j$ as a borrower. Some theoretical studies analyzed the distribution of exposures in empirical networks and reported long-tailed distributions for this quantity $[31,145,26,96]$.

Correlations with nearest-neighbor averaged quantities. For each one of the quantities defined above, we also calculate how the average value over nearest neighbors 
correlates with the property itself. This is a measure of assortativity, a concept defined in chapter 5 as the preference of nodes to connect to others of similar characteristics, and has been analyzed here for degree, transactions by value and transactions by volume. References like Iori et al.[85], Soramäki et al.[145] and Martínez-Jaramillo et al.[96] have studied degree-degree correlations in empirical data, showing that networks can be classified as disassortative.

Clustering coefficient. To measure the clustering coefficient, we ignore the directionality of links and regard the network as undirected. Empirical studies proceeded in this way[26]. For an undirected graph, the clustering coefficient of node $i$ is defined as the number of edges observed between pairs of neighbors of $i$ standardized to the maximum value this quantity may take, $k_{i}\left(k_{i}-1\right) / 2$, where $k_{i}$ is the degree of node $i$. It has been shown that empirical clustering coefficient declines with node degree [26].

\subsection{A minimal model of interbank networks}

In this section, we define the model that generates interbank networks. The set of TA, LAB and DB data described in Section 11.2 allows for the definition of our model of the interbank system as follows. We consider the (world) banking system formed by $N$ banks. As mentioned, we use here $N=7000$ as a representative number of institutions each year. The position (technical term to refer to the cash status, either with a surplus or with short fall) of bank $i(i=1, \ldots, N)$ at time $t(t$ in days) in the interbank market is defined by variables $\left(a_{i}(t), l_{i}(t)\right)$, where $a_{i}(t)$ stands for the interbank assets (LAB) and $l_{i}(t)$ is the amount of interbank liabilities (DB) of the bank, both at time $t$. As shown in Figure 11.1, these variables cannot be drawn independently of the size (TA) of bank $i, z_{i}(t)$. We can, however, assume that $z_{i}(t)$ is approximately constant for all the simulation time, $z_{i}(t) \simeq z i$, since the time aggregation window is only a quarter of the year ( $1 \leq t \leq 60$ working days).

Thus for each quarterly aggregated network we must: a) randomly draw the sizes $z_{i}$ of all banks from an arbitrary marginal distribution $f(z)$; b) for each $t$, randomly generate the relative variables $\left(x_{i}(t), y_{i}(t)\right)$ and randomly create the daily interbank network; and c) we have to finally aggregate all the resulting networks by merging the links and superposing all transactions.

\subsubsection{Assets and liabilities generation}

The triplet $\left(x_{i}(t), y_{i}(t), z_{i}\right)$, with $x_{i}(t)=a_{i}(t) / z_{i}$ and $y_{i}(t)=l_{i}(t) / z_{i}$, is the set of random variables used to generate $a_{i}(t)$ and $l_{i}(t)$. We use relative variables instead 
of drawing directly $a_{i}(t)$ and $l_{i}(t)$ because relative variables are weakly correlated to bank sizes. As shown below, we have checked the implications of ignoring pairwise correlations in our model. Since our dataset lacks of time-dependent information, we assume that these pairs are uncorrelated in time, i.e., $\left(a_{i}(t), l_{i}(t)\right)$ and $\left(x_{i}(t+h)\right.$, $\left.l_{i}(t+h)\right)$ are uncorrelated for any $h>0$.

However, since we do have information about actual variable distributions, and in order to assess the importance of the distributions themselves, we used in the simulations four different ways of generating the triplets $\left(x_{i}(t), y_{i}(t), z_{i}\right)$. In any case, the size $z_{i}$ of bank $i$ is drawn from a marginal distribution $f(z)$, which is arbitrary based on the several ways of generating it:

Full correlation (FC). Here we choose $f(z)$ as the empirical marginal distribution of bank sizes, $P(z)$. For each bank $i$, which has size $z_{i}$, we calculate empirical conditional probabilities $P\left(x, y \mid z_{i}\right)$ and, from them, draw the pairs $\left(x_{i}(t), y_{i}(t)\right)$ for each $t$. This method generates samples of the joint distribution $P(x, y, z)$ of the original data.

Half correlation (HC). This case also uses the empirical marginal distribution of bank sizes and, for each $z_{i}$, we calculate conditional empirical probabilities $P(x \mid z i)$ and $P(y \mid z i)$ to independently draw variables $x_{i}(t)$ and $y_{i}(t)$. Here we are assuming zero correlation between variables $x$ and $y$. This method generates samples with the same joint, marginal distributions $P(x, z)$ and $P(y, z)$ of the original data.

No correlation I (NC1). In this case, $x_{i}(t), y_{i}(t)$ and $z_{i}$ are independently drawn according to the marginal empirical distributions $P(x), P(y)$, and $P(z)$. Here we assume zero correlation between all variables.

No correlation II (NC2). Here $x_{i}(t), y_{i}(t)$ and $z_{i}$ are independently drawn from the fitted log-normal distributions (truncated for $x$ and $y$ ) obtained from the marginal empirical distributions. This assumption reduces the information of the entire dataset to the parameters of the log-normal functions representing the three marginal distributions. In the absence of available asset/liability data, our model still remains useful, having six free parameters to be fitted to network data through a maximum likelihood procedure, for instance.

In Section 11.5 we show that simulation results depend weakly on the correlation between the variables, but strongly on the marginal distributions used to generate them. 


\subsubsection{Random network generation}

After generating the pair $\left(a_{i}(t), l_{i}(t)\right)$ for each bank with one of the methods mentioned above, daily interaction networks are generated as follows. Let $L(t)$ be the set of lending banks at time $t$, i.e., banks with strictly positive interbank assets, and $B(t)$ the set of borrowing banks, i.e., those with $l_{i}(t)>0$. These quantities will vary throughout the algorithm, as we show below.

The rationale behind the way that links from lenders to borrowers are drawn tries to approximately compensate assets and liabilities through a number of loans at each time step. While sets $B(t)$ or $L(t)$ are not empty:

1. Pick uniformly at random a borrower bank $i$ from set $B(t)$.

2. While $l_{i}(t)>0$ :

(a) Pick uniformly at random a lender bank $j$ from set $L(t)$.

(b) If $a_{j}(t) \geq 1_{i}(t)$, then there is a new loan of size $l_{i}(t)$ from bank $j$ to bank $i, a_{j}(t)$ is set to $a_{j}(t)-l_{i}(t)$ and $l_{i}(t)=0$. Borrower's debt is fully compensated with a single loan from lender $j$.

(c) If $a_{j}(t)<l_{i}(t)$, then there is a new loan of size $a_{j}(t)$ from bank $j$ to bank $i, a_{j}(t)$ is set zero and $l_{i}(t)$ updates to $l_{i}(t)-a_{j}(t)$.

(d) After the transaction, if $l_{i}(t)=0$, then bank $i$ is removed from set $B(t)$ and, if $a_{j}(t)=0$, then bank $j$ is removed from set $L(t)$.

\section{Go to step 1.}

Each new loan from bank $j$ to $i$ sets a new directed link $j \rightarrow i$ if it did not existed previously and adds a new transaction between them whose value equals the amount of the loan. The process replicates when aggregating daily graphs into a quarterly network.

Since positions $\left(a_{i}(t), l_{i}(t)\right)$ are randomly drawn, the probability that $\sum_{i=1}^{N} a_{i}(t)=$ $\sum_{i=1}^{N} l_{i}(t)$ is close to zero. That means that, when set $L(t)$ is empty but $B(t)$ is not, we assume that the banks left must trade from other banks outside our interbank network (central banks, clearing houses, etc., which act as a liquidity "reservoir" for our model). The same happens when $B(t)$ is empty but $L(t)$ is not. See Figure 11.4 for a visual representation of small networks generated by these rules. 


\section{A) One day network snapshot.}
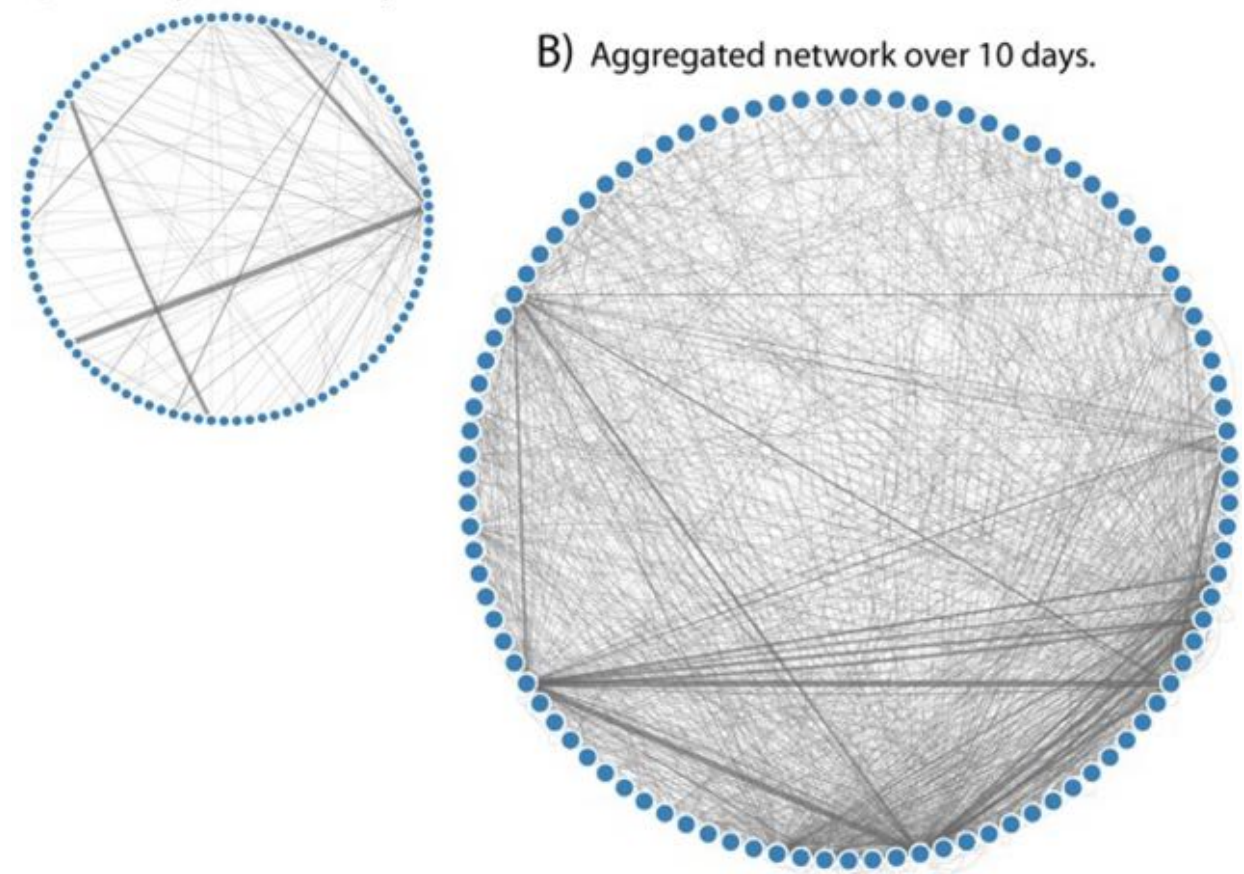

Fig. 11.4.: Circular layout graphs of interbank network examples. Example of a network generated by our model. We directly sampled 100 banks (including their positions) from the Bankscope data and generated 10 daily networks. Panel (a) shows a one-day network snapshot, whereas panel (b) represents the aggregated network over the 10 time steps. The thickness of links is proportional to the overall value of the transaction conducted between entities. To easily visualization, we have not made explicit the directionality of links.

This algorithm makes several unrealistic assumptions. Borrower banks choose at random lender banks, regardless the loan interest, proximity, or previous lenders they chose. And lenders always accept the loans with all their necessary resources regardless the amount or the borrower rating. Our model, therefore, considers no prices, no regional preferences, no time correlation, nor risk aversion.

In the following sections we analyze the topological properties of quarterly-aggregated model networks and their similarities with empirical network magnitudes measured in the interbank literature.

\subsection{Results of interbank networks}

A thourough review of the results found with the interbank networks created with our model takes place in this section. Yet, we have decided to split the section into two subsections. The first one deals with the theoretical features that might be expected based on our premise that the model can replicate the topology of real- 
world interbank networks. The second one, on the other hand, explicitly compares the features against the previous empirical results from other authors.

\subsubsection{Theoretical predictions}

In this section, we derive model predictions for the network properties studied in empirical networks (Section 11.3). Analyzed properties include distributions of inand out-degree, transactions by volume, transactions by value and exposures. We also compare model results with reported network assortativity and the relation between clustering coefficient and degree. A first conclusion of this analysis is that the simple assumptions underlying our model are able to reproduce, at least qualitatively, the distributions and correlations found in empirical studies.

Model simulations were performed for interbank systems of $N=7000$ financial institutions, which is approximately the number of active banks per year found in the Bankscope database in the period between 2008 and 2015. Network properties were averaged over 100 model realizations.
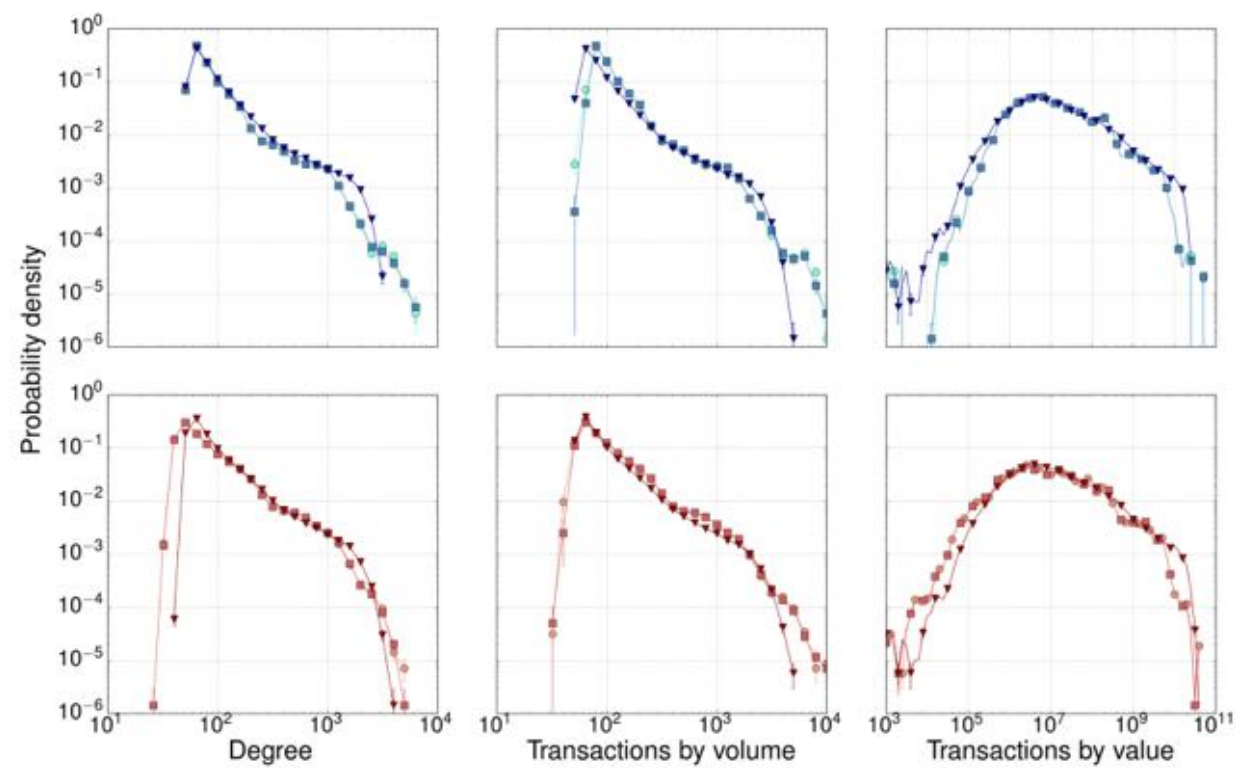

Fig. 11.5.: Network properties of our model. Top panels: Probability densities of (from left to right, blue curves and symbols) in-degree, in-transactions by volume and intransactions by value obtained using the full dataset. Bottom panels: Probability densities of (from left to right, red curves and symbols) out-degree, out-transactions by volume and out-transactions by value using the complete dataset. 100 model realizations have been used to compute averages and error bars. Simulations are generated using FC variables $(O)$, HC variables () and NC1 $(\nabla)$. The generating methods FC, HC and NC1 are described in Section 11.4.1 
Effect of correlation. In order to study the importance of the empirical correlation observed for interbank variables (Section 11.2), we constructed several realizations of quarterly networks for each of the random data generation methods described in Section 11.4.1. Figures 11.5 and 11.6 show that these correlations do not condition significantly the network topology, not at least for the properties usually studied in the literature. Although we found a weak (but statistically significant) correlation between relative interbank assets $(x)$, liabilities $(y)$ and total assets $(z)$, such degree of correlation is blurred in global averaged properties such as the degree, transactions by volume and transactions by value distributions (Figure 11.5). The pattern of network disassortativity in these three properties (Figure 11.6) turns out to be conserved and almost independent of the degree of correlation reported for interbank variables.
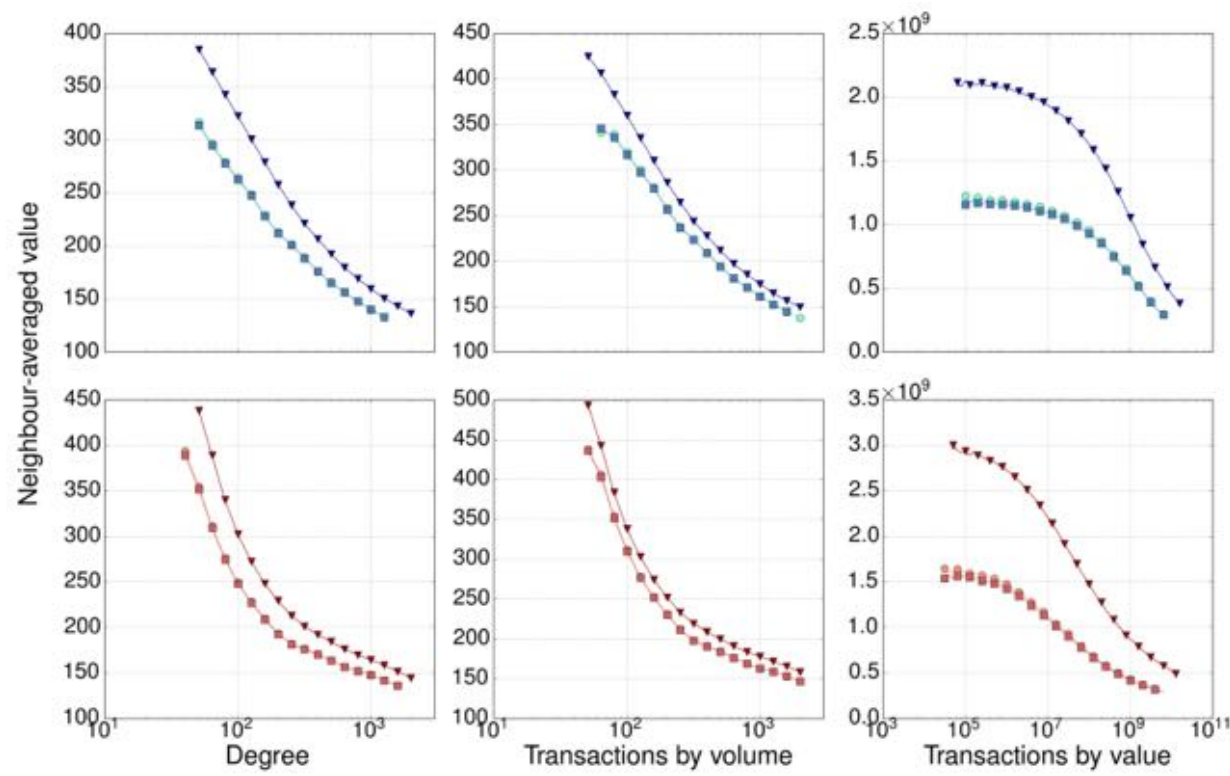

Fig. 11.6.: Network properties of our model. Same as Figure 11.5, but for (top) the averaged in-magnitude of incoming neighbors and (bottom) the averaged out-magnitude of outgoing neighbors, where magnitude can be degree, transactions by volume or transactions by value. Although FC (O) and HC () results overlap, curves generated by the $\mathrm{NC1}(\nabla)$ procedure preserve disassortativity.

Effect of marginal distributions. Here we analyze, for networks with uncorrelated $x, y$ and $z$, the influence of marginal distributions $P(x)$ and $P(y)$ on network properties. To this end, we studied networks generated using the empirical marginal distribution (through the NC1 procedure, see Section 11.4.1) and with several lognormal distributions (using NC2). Results are shown in Figures 11.7 and 11.8. We observe that network properties depend on the width of marginal distributions. That behavior is notably relevant in the correlations with neighbor-averaged properties (Figure 11.8). 

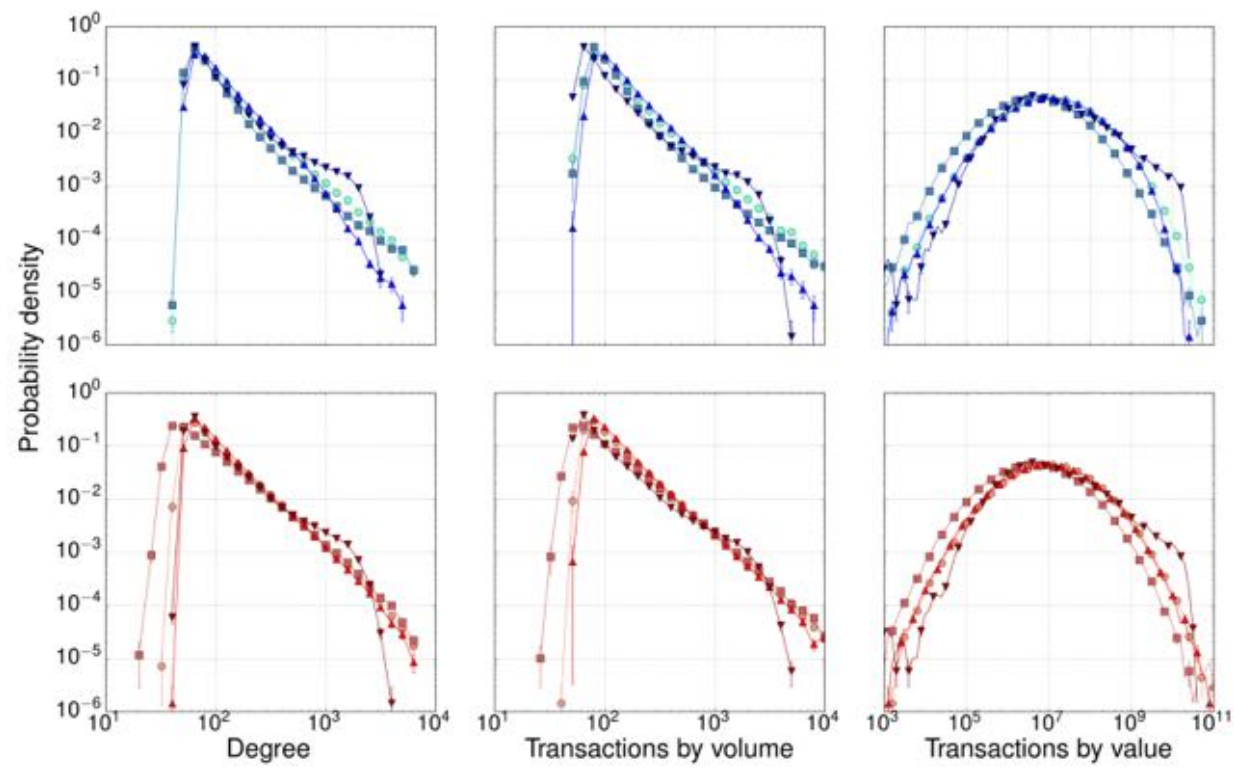

Fig. 11.7.: Network properties of our model. Same as Figure 11.5, but comparing different marginal distributions: best fit of truncated log-normal functions to empirical marginal distributions, with $\log x \sim N_{t}(-1.03,0.52)$ and $\log y \sim N_{t}(-1.06,0.72)$ (O); shifted distributions, with $\log x \sim N_{t}(-1.5,0.5)$ and $\log y \sim N_{t}(-1.5,0.75)$ (); wider distributions, with $\log x \sim N_{t}(-1.0,1.0)$ and $\log y \sim N_{t}(-1.0,1.5)$ $(\nabla)$; and empirical marginal distributions $(\triangle)$. Here $x \sim N_{t}(\mu, \sigma)$ stands for a truncated $(x \leq 0)$, normally-distributed variable with mean $\mu<0$ and standard deviation $\sigma$. Simulations are generated using NC1 algorithm for the empirical, marginal distributions and NC2 for marginal log-normal distributions. See Section 11.4.1 for a full description of the generating methods NC1 and NC2.

Effect of subnetwork size. All interbank networks reported in empirical works only have access to a small fraction of the total, world-wide interbank network. Our simulations, however, consider all banks of the Bankscope database that have listed their interbank assets and liabilities. In order to generate networks with a number of banks comparable to reported empirical network sizes, we should consider only banks belonging to those regions studied in the literature. In doing so, we face two related problems, though. First, Bankscope offers only world-wide, balance sheet aggregated data, which contain the overall contribution from multiple countries and cannot be disaggregated. Second, to restrict model simulations to smaller networks, we would also have to lower interbank positions (with respect to the reported values in Bankscope) in order to correct the excess of liquidity that the system would have as a whole. We have to take into account size effects in order to confront model predictions against available data. To circumvent these problems, we generate networks with exactly $N_{s}$ nodes by taking samples of size $N s$ from complete model networks (i.e., those generated with $N=7000$ financial institutions) and measure network properties for the corresponding subgraphs. This way we can study network properties for different subnetwork sizes. Results are shown in Figures 11.9 and 11.10. We observe that the effect of taking samples in model networks displaces 

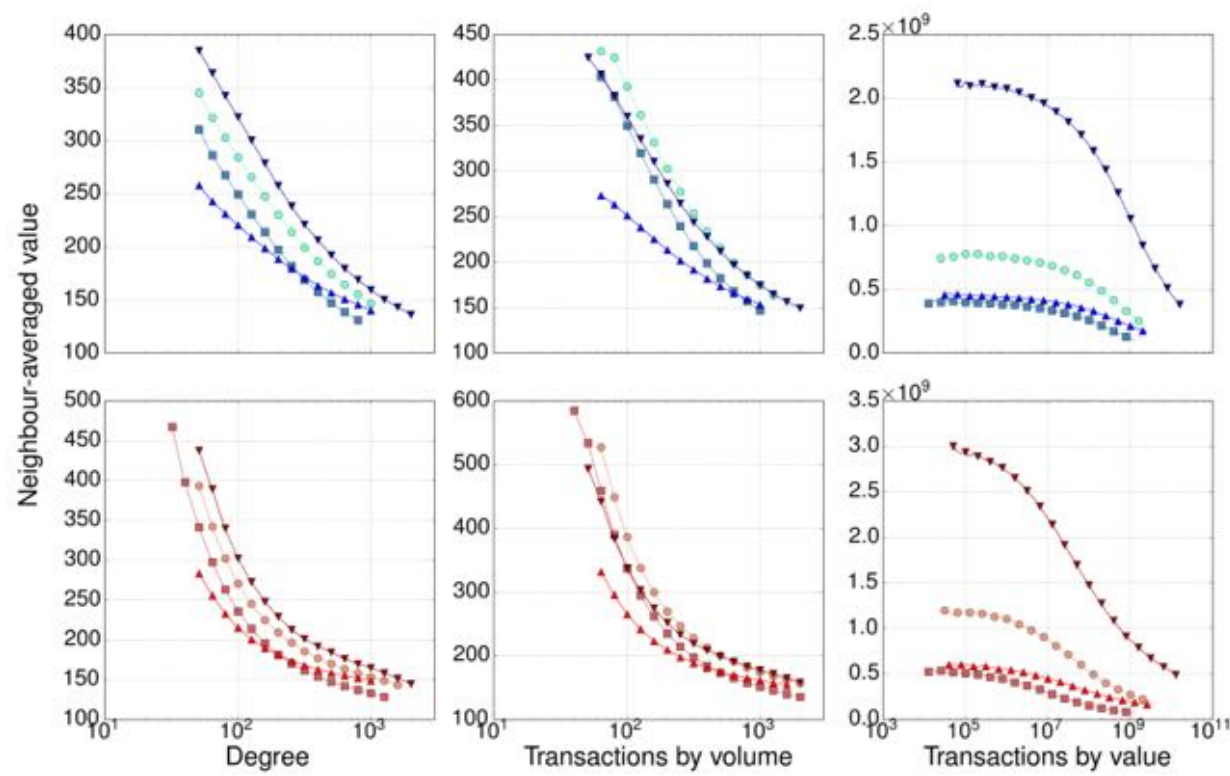

Fig. 11.8.: Network properties of our model. Same as Figure 11.7, but for (top) the averaged in-magnitude of incoming neighbors and (bottom) the averaged out-magnitude of outgoing neighbors, where magnitude can be degree, transactions by volume or transactions by value.

degree and transactions distributions to the left (which is not surprising), but does not significantly change the form of the distributions (degree and transactions by volume maintain a long-tailed form, and transactions by value are displaced lognormal functions, see Figure 11.9). Assortativity results almost remain unchanged except for the distribution ranges: whereas distributions move towards smaller values of degree and transactions by volume as $N_{s}$ decreases, distribution ranges for transactions by value are almost independent of sampling sizes (Figure 11.10).

Exposures. As for transactions by volume and degree, exposures exhibit a power-law distribution, even when bank positions are sampled according to different correlation assumptions (FC, HC and NC1 procedures) or when bank positions are uncorrelated and drawn from truncated lognormal distributions (NC2 method), see Figure 11.11. The effect of subnetwork sizes remains unchanged: the tail of the distribution displaces towards smaller exposure values as long as the sampled network size decreases (Figure 11.11).

Clustering coefficient. Empirical works have shown that node clustering coefficient is negatively correlated with degree[26]. Our model captures such behavior under the FC, HC, NC1 and NC2 sampling methods, see Figure 11.12. However, when subnetworks are randomly sampled the clustering coefficient significantly decreases. Since nodes in subnetworks are sampled at random, it is likely that many of the links that formed local triangles are no longer present in the subnetwork. This 

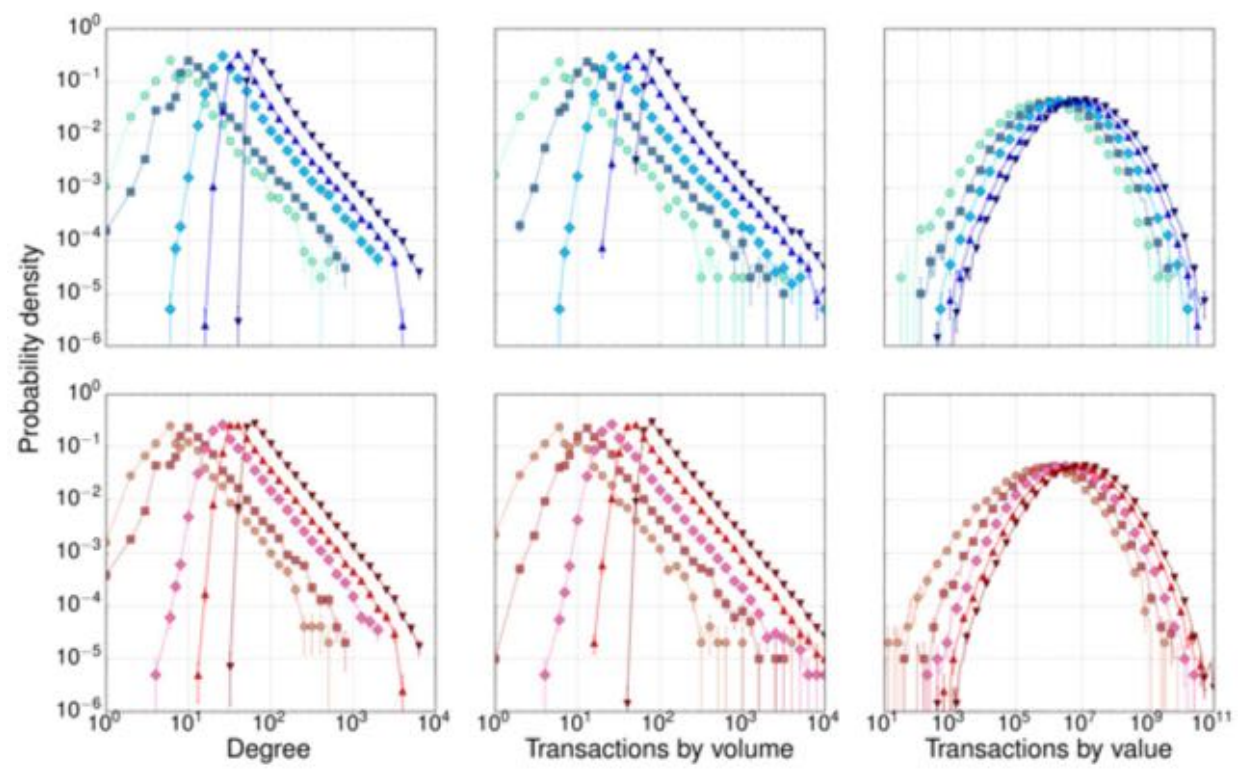

Fig. 11.9.: Network properties of our model. The same as Figure 11.5, but comparing different sampled network sizes Ns: Ns $=500(\bigcirc)$, Ns $=1000(), \mathrm{Ns}=2000$ $(\diamond)$, Ns $=4000(\nabla)$ and the total network with $N=7000(\triangle)$. Simulations are generated using the NC2 algorithm with the best fit (truncated) log-normal distributions.
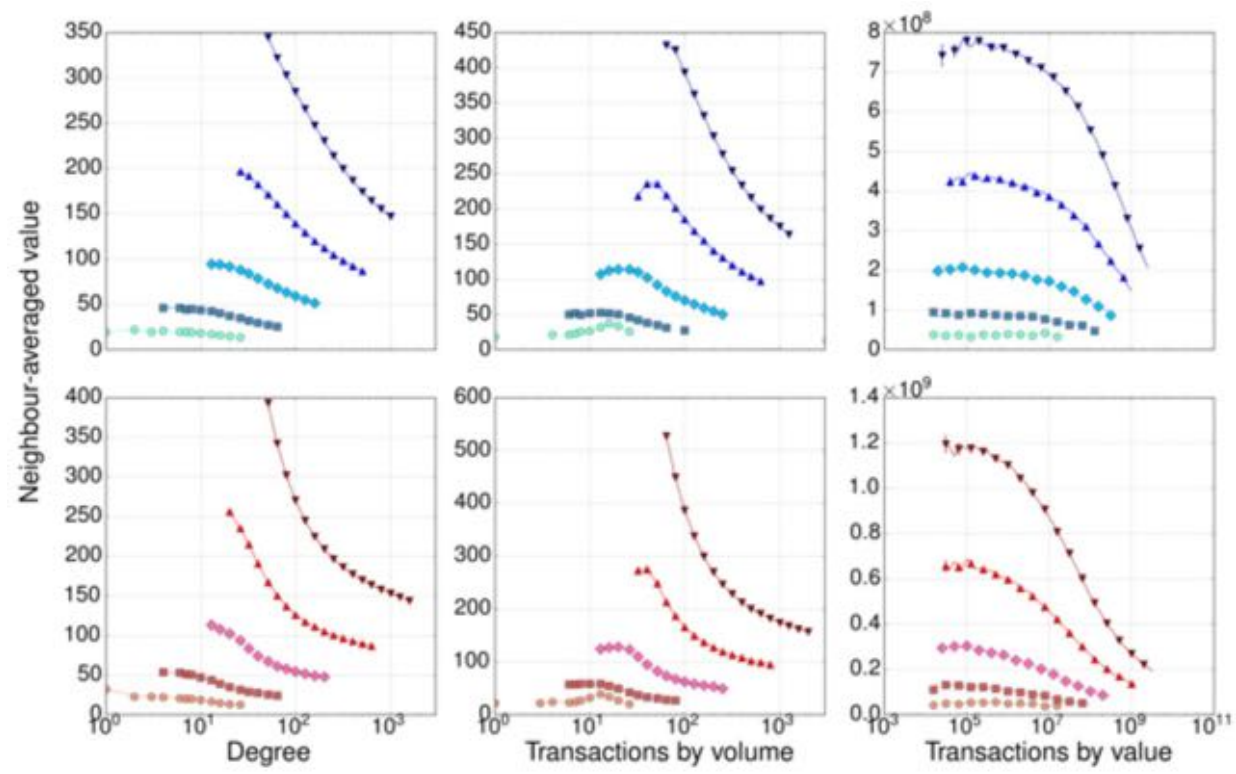

Fig. 11.10.: Network properties of our model. The same as Figure 11.9, but for (top) the averaged in-magnitude of incoming neighbors and (bottom) the averaged outmagnitude of outgoing neighbors, where magnitude can be degree, transactions by volume or transactions by value.

explains intuitively why clustering decreases under network sampling. As we will see below, our model is not able to capture quan-titatively the functional dependence 

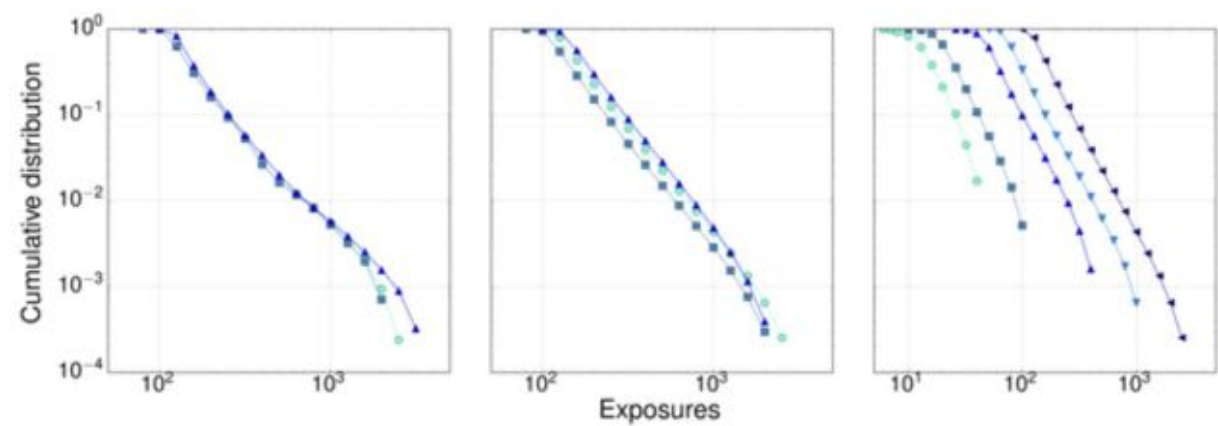

Fig. 11.11.: Network properties of our model. Complementary cumulative distribution of exposures: (left) using reported data under FC $(\bigcirc)$, HC $($ ), and $\mathrm{NC1}(\nabla)$ assumptions; (center) exposures distribution using synthetic asset and liability data generated with a best fit of truncated log-normal distributions $\left(\log x \sim N_{t}(-1.03,0.52)\right.$ and $\left.\log y \sim N_{t}(-1.06,0.72), \bigcirc\right)$, shifted distributions ( $\log x \sim N_{t}(-1.5,0.5)$ and $\log y \sim N_{t}(-1.5,0.75)$, ); wider distributions ( $\log x \sim N_{t}(-1.0,1.0)$ and $\left.\log y \sim N_{t}(-1.0,1.5), \nabla\right)$; (right) variability of distributions with network size: $N_{s}=500(\bigcirc), N_{s}=1000(), N_{s}=2000(\nabla)$, $N_{s}=4000(\diamond)$ and the total network with $\mathrm{N}=7000(\triangle)$. In the right-hand side panel, bank positions are generated with the NC2 algorithm with the best fit (truncated) log-normal distributions.

of clustering vs. degree observed in empirical networks. Likely, regional processes inducing preferential loans between banks could help to explain this discrepancy.

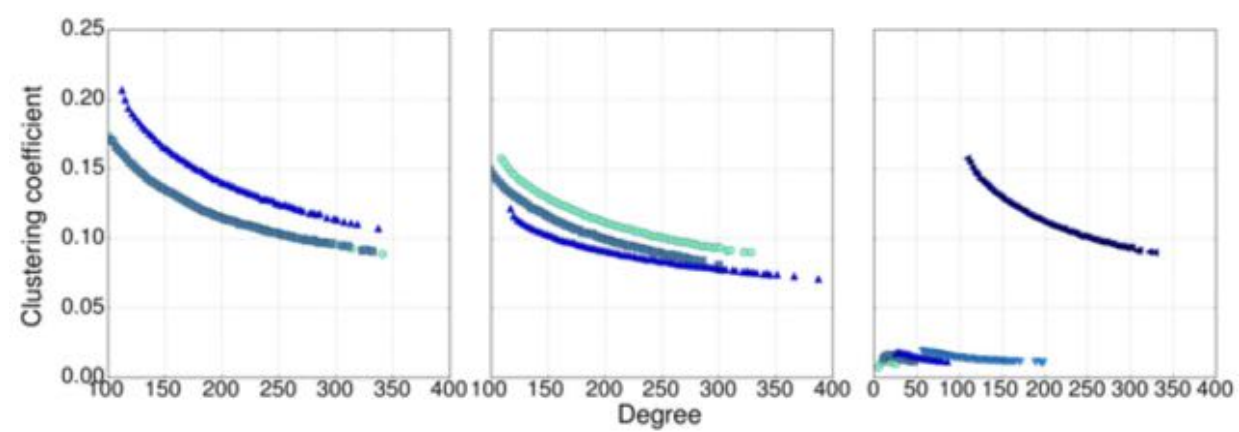

Fig. 11.12.: Network properties of our model. Clustering coefficient vs. degree: (left) using reported data under FC $(\bigcirc)$, HC () , and NC1 $(\nabla)$ assumptions; (center) using synthetic asset and liability data generated with a best fit of truncated $\log$-normal distributions $\left(\log x \sim N_{t}(-1.03,0.52)\right.$ and $\log y \sim N_{t}(-1.06,0.72)$, $0)$, shifted distributions $\left(\log x \sim N_{t}(-1.5,0.5)\right.$ and $\log y \sim N_{t}(-1.5,0.75)$,); wider distributions (log $x \sim N_{t}(-1.0,1.0)$ and $\left.\log y \sim N_{t}(-1.0,1.5), \nabla\right)$; (right) variability of distributions with network size: $N_{s}=500(\bigcirc), N_{s}=1000()$, $N_{s}=2000(\nabla), N_{s}=4000(\diamond)$ and the total network with $\mathrm{N}=7000(\triangle)$. In the latter case, bank positions are generated with the NC2 algorithm with the best fit (truncated) log-normal distributions 


\subsubsection{Comparison with empirical data}

We now compare model predictions against data reported for empirical interbank networks. It is important to remark that we have not tried to find the set of model parameters that better fit simultaneously a subset of empirical network properties. Instead, we show how our minimal model can reproduce qualitatively and, sometimes quantitatively, some of the properties observed in empirical works. For that purpose, we have chosen a single set of model parameters using the NC2 simulation scheme and compared model outcomes with empirical data, which have been captured from published figures using a digitization tool (Rohatgi 2015). Figure 11.13a shows how our model can recover reported in-degree distributions. Most of the empirical interbank networks exhibit a long-tailed degree distribution, which is recovered by our model after network sampling. Other authors (Iori et al. 2008 [85]; Fricke \& Lux 2015 [65]) report distributions with shorter tails (Figure 11.13a); in those cases our model could be used to reproduce in-degrees at certain ranges. The same comments apply for out-degree distributions (Figure 11.13b).

In- and out-transactions by volume are underestimated by our model for the Italian e-MID interbank market (Iori et al.[85]; Fricke \& Lux 2015[65]), see Figures 11.13a,b. Soramäki et al.[145] only report out-transactions by volume, which are well reproduced for model sampled networks (Figure 11.13b). The discrepancy in this case could be due to the assumptions of our model. Links are drawn in our model in a way that, if a lender has liquidity enough to compensate the full debt of a borrower, the transaction takes place so that the borrower cancels its debt. However, consideration of partial loans in our modeling approach would tend increase the number of transactions. An additional parameter accounting for the average number of partial loans would eventually correct the mismatch. The distribution of transactions by volume in empirical works have sometimes been reported as exponential (see Figure 11.13 and Iori et al.[85]). Our model yields long-tailed distributions for this quantity, though, and cannot account for short-tailed distributions in this case. However, this is the only network property for which a short-tailed distribution has been found among all the empirical studied used here for model validation. Soramäki et al.[145] report distributions for out-transactions by value. Figure 11.14a shows that our model overestimates transaction values. However, this magnitude corresponds to one of the inputs of our model, LAB, whose distribution is replicated in the simulations. The discrepancy is probably the result of two different empirical distributions of the lending positions of banks in the interbank market.

The distribution of exposures is partially captured by our simple model (Figure 11.14b). The ranges of exposure values yielded by our model are narrower than the ones observed empirically. An extensive exploration of the parameter space, which is 

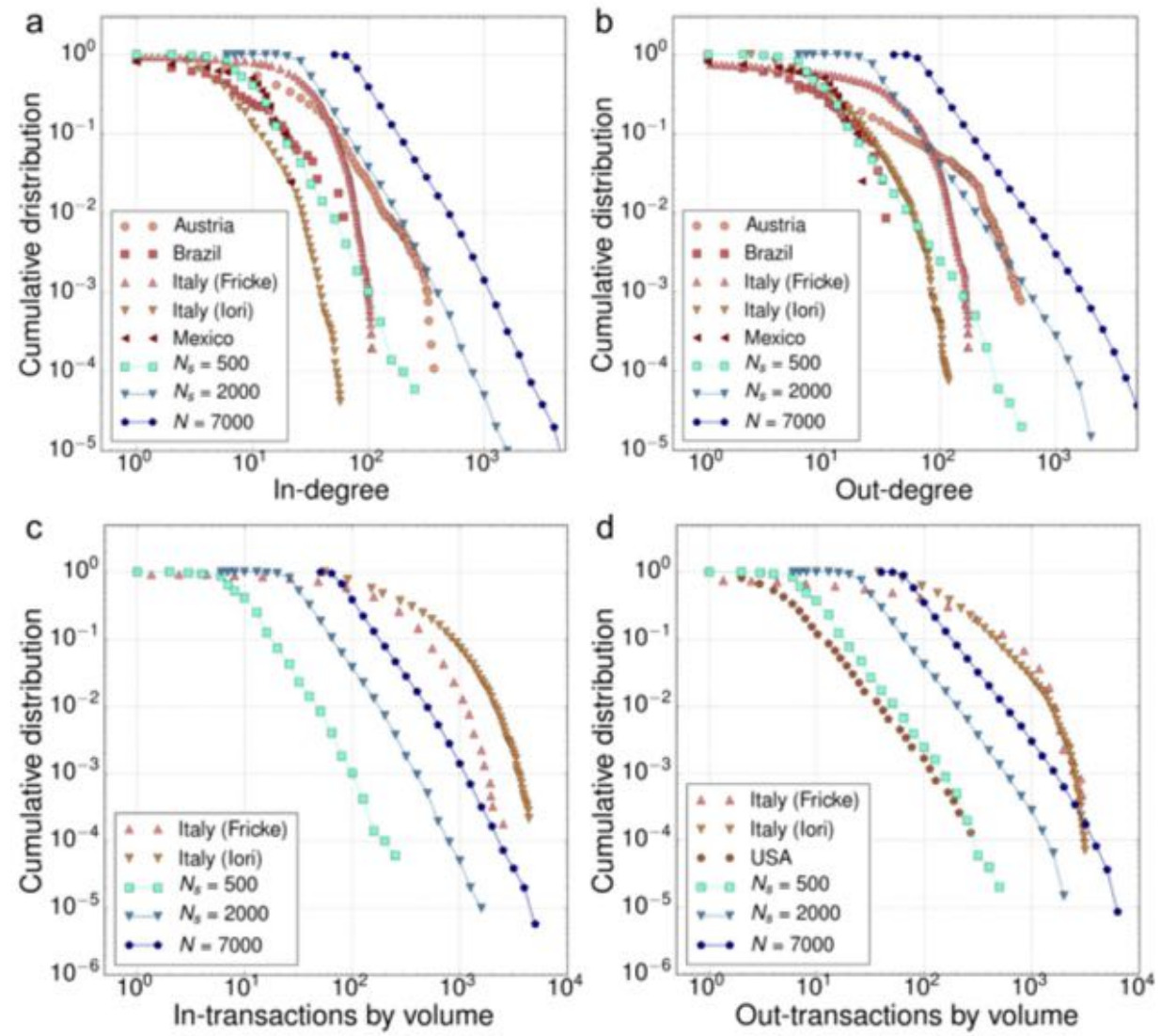

Fig. 11.13.: Network properties of our model. In-degree (a) and out-degree (b) distributions reported for 5 empirical works compared with model-generated distributions. Model distributions compare qualitatively well with empirical ones. Panels (c) and (d) depict the distributions of in- and out-transactions by volume for three empirical networks. The number of transactions is underestimated by our model. Bank positions in all cases have been performed under the NC2 algorithm with truncated log-normal distributions with $\log x \sim N_{t}(-1.0,1.0)$ and $\log y \sim N_{t}(-1.0,1.5)$. Empirical data have been recovered from published references $[31,26,85,65,96,145]$ using the digitization tool provided by Rohatgi[130]

beyond the scope of this contribution, would be necessary to evaluate whether our model can reproduce empirical exposure values or not. Network assortativity in node out-degree is qualitatively reproduced by our simple rules (Figure 11.14c). Model and empirical networks show negative correlations between out-degree and the average out-degree of nearest neighbors, so our assumptions lead to disassortativity. However, the agreement of model predictions and empirical is qualitative for the set of parameters chosen, and sometimes can be even quantitative (Figure 11.14c). Our model captures the negative correlation between clustering coefficient and node degree (Figure 11.14d). However, sampling procedures that decrease the average degree of nodes are accompanied by a drastic reduction of the clustering coefficient. Random node selection implies breaking existing links and, as a consequence, leads to a reduction of the number of triangles in sampled subnetworks. Preferential 

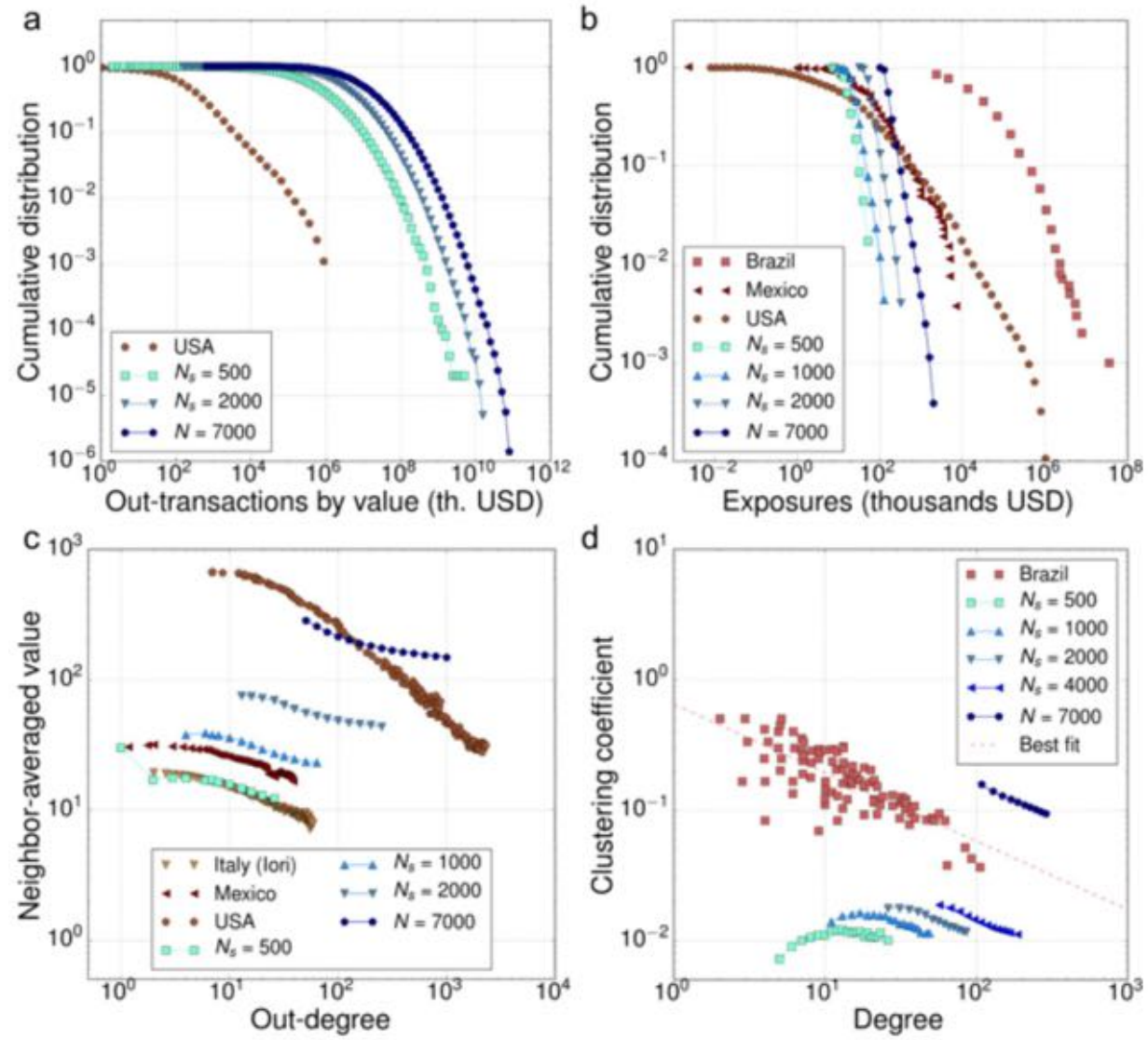

Fig. 11.14.: Network properties of our model. (a) Distribution of out-transactions by value. Our model tends to overestimate transactions values. (b) Empirical distribution of exposures compared to model results. Selected simulation parameters predict larges slopes for distribution tails. (c) Empirical networks are found to be disassortative, and model results predict that result as well. The agreement with empirical data can be sometimes quantitative. (d) Clustering coefficient declines with node degree. Model networks lead to a similar pattern, but subnetwork sampling reduces significantly clustering values. Bank positions in all cases have been performed under the NC2 algorithm using truncated log-normal distributions with $\log x \sim N_{t}(-1.0,1.0)$ and $\log y \sim N_{t}(-1.0,1.5)$. Empirical data have been recovered from published references [26, 85, 96, 145] thanks to the digitization tool Webplotdigitizer (Rohatgi 2015)[130].

transactions between closer entities can be a plausible explanation of this discrepancy. Regional preferences are probably the rule in reported interbank networks ([50]), and our model is based on simulations at global scales. Including bank preferences to borrow and lend would lead to a community structure in model networks, and sampling banks according to those groups could help to increase node clustering. 


\subsection{Conclusions of interbank networks}

In this contribution we have introduced a network model for interbank markets using a simple agent-based algorithm to generate daily, temporal transaction networks, which have been aggregated into quarterly networks. To that end, we have used interbank positions of financial institutions from end-of-year balance sheets of the Bankscope database, which is available for researchers in many institutions world wide. We have also gathered quantitative information regarding topological properties of empirical interbank networks, reported in the literature, to compare with our model. The basic assumption we made to draw links in model networks is the requirement of interbank assets and liabilities compensation at each time step. In other contexts, a simple rule like this has been used, for example, to generate good approximations for predator-prey interaction networks in ecology [166, 38, 153, 50] or contact networks in language biogeography $[42,41]$.

Our model can be used as a benchmark to compare with network and agent-based properties from empirical and theoretical interbank data. Here we have analyzed basic topological properties and we have shown that the model qualitatively reproduces them on the basis of very simple rules: a random choice of lenders and a cumulative compensation of the debt of a given bank through repeated loans is enough to capture those topological quantities. This methodology would allow to discriminate magnitudes and mechanisms that are relevant to interbank systems from others that can be explained by our simple model. For example, we have not analyzed how our model accounts for the local, motif structure of empirical interbank networks[151]. Given that our model captures basic properties itself, it could be used as a null model to test the degree of significance of the observed frequencies of motifs in real networks. Be believe that this benchmark is of paramount importance in the development of interbank network modeling, as it rules out models that may comply with some data from real LVP systems whose results do not significantly differ from those of our model. We have shown that model outcomes are almost independent of the way in which assets and liabilities are drawn, and we have compared four methods of random generation of these variables. In terms of the applicability of our results in the absence of asset, liability, and bank size data, we have shown that these quantities can be drawn as independent trials of truncated log-normal distributions to a good degree of approximation. In this case, our model depends on six free parameters that can be fitted to data by maximum likelihood estimation. This amounts to the calculation of the log-likelihood function of this model. Due to similarities with resource-based models for ecological network structure, presumably this likelihood function could be explicitly calculated or, at least, analytically approximated $[38,41]$. 
Our model lacks several realistic features, such as profit maximization, regional preferences, time correlation, or risk aversion. However, the networks generated fit qualitatively basic empirical properties reported in the literature. This result yields important implications. First, as we have pointed out, our model could be used as a benchmark of the interbank market, a null model that can be compared with more realistic models. Second, the properties usually measured in empirical networks can be accounted through a minimal set of basic rules, so other magnitudes are to be analyzed in forthcoming studies. More complex, realistic models replicating the properties exposed in this manuscript with the same accuracy as ours cannot be considered better, unless additional quantities are further considered. These new properties could be used to reject our model and to test more realistic assumptions. Either way, it would mean a step forward in the knowledge of interbank networks as a way to study, for instance, important aspects that cause systemic or liquidity risks.

An important prospect of our work is related to data sources. It seems reasonable that any modeling approach to describe banking networks should be based on reliable data from financial transactions. However, transactions data in electronic markets are not publicly available, not even for most of the researchers. The few people that can access these data sets are bound by the rules of professional conduct and secrecy to ensure the confidentiality of the data. This constitutes a drastic limitation when it comes to devise data-driven models useful to derive reliable predictions regarding the resilience of interbank markets and the assessment of potential contagion. We have overcome this problem using the Bankscope database, but a modeling approach based on daily transaction data (not only end-of-year balances) of real systems would be optimal.

Cross-disciplinary researchers are used to introduce or improve models to explain real data whenever the theory or the numerical results fit some expected behavior. Scientific method, however, proceeds the other way round. It is not only about the ability to explain some empirical data. It is also the capability of testing the predictions of our models, and this can only be done if appropriate data are available. If we want to understand the underlying drivers that shape interbank networks and ensure the stability of the banking system, additional empirical information should be made public and available to researchers. 



\section{Conclusions}

By pursuing his own interest, he frequently

promotes that of the society more effectually

than when he really intends to promote it.

— Adam Smith

(Father of modern economics)

\subsection{A brief summary of the thesis}

The story of our research, like most cases, is not a straight line, but rather a continuous road in the right direction with a lot of wrong turns and direction changes. Yet, we have gone through an evolutionary process in order to develop a innovative story to understand markets and their dynamics in a different way; that is, from the complex bipartite networks perspective. Our decisions could have probably been improved in many ways, but every mistake has fortunately taken us one step closer to our final objective. For us, everything has started with Adam Smith's statement on how sef-interest should lead to the well being of the society, an idea that initially sounded, at the very least, a contradiction. This idea later became the corner stone of our research goal: to understand the behavior of markets at a meso-scale while providing deeper explanation Smith's statement. At that point, we have also discovered the theory of mutualistic networks. From this theory, we have learned about the elegance and complexity of the interactions of species, which, in my mind, seemed to resemble that of a seller and a buyer in a market. Now, because international trade markets are a special case of the economic theory, known to approach closely the conditions of perfect competition, and because their data is widely available, it was clear to us that the combination of complex network theory and these markets could lead to innovative scientific findings. Our premise that a bipartite and weighted approach is a suitable framework to understand the behavior of markets was not only a valid methodology, but also one that helped us establish the close analogy between mutualistic ecosystems and international trade markets. Then, we also knew that a visual approach was required in order to analyze and understand the large dataset we had as well as the massive volume of results that we would later obtain after applying our methodology, which, highly inspired 
by mutualistic networks, paid off in the long term based on the solid findings of our study. We have found the 8 common features and patterns that are present in the topology of any international trade market. We have also learned that these patterns do not significantly change over time. Some of these patterns may even be classified as a sort of universal behavior. For example, nestedness has never been defined or found as a key feature of markets as bipartite trade networks of exporters and importers (country-country), a concept that could only be discovered when our methodology is implemented. We believe our study changes the way in which markets may be perceived. Thus, markets might not only be conceptualized as a unipartite, directed networks that account for the flows of goods between countries, but also as bipartite systems where the players in these markets do not share the same interests and make decisions with a different logic, hence justifying our premise and unlocking the potential for innovative findings, which is a new contribution to the existing research. Long-tailed degree distributions, highly correlated strengthdegree patterns, uncorrelated performance of $\mathrm{Knn}(\mathrm{k})$, and declining clustering are only among these key findings. Repetition, however, has been both an enemy of and an advantage to our study, providing us with a challenges to minimize the number of time we show the same result, but also leading to the findings themselves by displaying the same patterns over and over again across all periods and all levels of aggregation of our data. Nestedness itself has actually been the key to connecting the dots between our static findings in the topology of trade networks and the dynamical phenomena that result from these temporal changes, period after period. The fundamental question about how topological properties do not significantly change over time while the interactions of exporters and importers do show a significant activity of temporal variance has led us to find the very nature of how the core of a trade network is less likely to be affected by any dynamical phenomena, whereas there will be a transition zone located between the core and the periphery of a trade network in which the majority of the temporal variences will take place. After defining four types of temporal phenomena and measuring the distance from the core of the network at which these changes occur, we have found that the similarity in shape and size of the distributions of opposite phenomena (appearances and absences, volume increases and declines) is the most plausible explanation for the invariance of the topological properties of all bipartite indicators, but is the actual reason why nestedness does not change over time. We have a long list of revealing findings that are interconnected between them and can, from all different perspectives, explain the economic behavior of markets, both dynamically and statically. Nonetheless, we could not affirm with a high level of confidence that we understand the essence of market without a statistical model that could replicate simultaneously all the key features found in trade networks. For this reason, we have designed and tested 3 models, which are actually a selection from the many prototype models we have tried along the way, and learned extensively and vastly from them. All these models had to be defined and described as weighted bipartite 
networks. We have started with a random model of markets, which, despite the fact that has failed to replicate the actual features of international trade markets, has provided us with a solid framework to build networks that satisfy both the bipartite and the weighted approach requirements. Following the random model, we continued with the exploration of gravity models, which was one step closer to describing the actual features of trade networks, but the model required too many restrictions to work. Finally, and again taking advantage of the analogy between trade markets and mutualistic ecosystems, we have created a mutualistic model of markets. This model does not only replicate all the actual features of actual markets simultaneously, but also it does so with the elegance of a proper network growth model, unveiling the hidden patterns of statistical process behind market formation. The only and main issue with this model is related to the nestedness performance of the simulated markets. Actual markets have a naturally sparse interaction matrix as a consequence, among other reasons, of geopolitical restrictions to trade bewteen countries. Our model generates simulations of actual markets that display values of nestedness that are much higher than those found in the empirical data. Yet, our mutualistic model generates stable results while being capable of simulating any special market cases, such as quasi-monolopies and quasi-monopsonies, revealing how the balance between competition and cooperation forces is key for markets to reach a sustainable equilibrium. Additionally, we have also worked in parallel to generate alternative models for the financial markets, a study that we also present in this thesis as an alternative view of powerful statistical models based on complex networks. Finally, we believe we have reached a point where we can provide an explanation to the initial statement from Adam Smith. Because both the exporters and importers act in selfish manner, caring only for its own benefit, the emerging topology of a market is structured in such a way that the market itself becomes a globally robust system, which is protected by the high nestedness and the declining clustering of a highly connected core. This configuration of the market works, as an extension of the known complex network properties, as a natural protection against externalities, securing the persistence of the system, a mutually benefitial situation in which the interests of most of the exporters and importers are protected, despite some extintions of minor players in the periphery (which are the economic equivalent to specialist species). It is not an invisible hand that self-regulates a market, but an emerging complex phenomena that can easily be understood by embracing complexity science and complex network theory. No dogma here, just disciplined research.

On figure 13.1, we show a visualization of the key milestones in this study. From the concepts that Adam Smith introduced more than 2 centuries ago, passing through the topological analysis of trade networks and the design and implementation of market models, until the explanation of the apparent contradiction, from self-interest to mutual benefits. Yet, there are 3 major findings we think it stands out from the rest, 


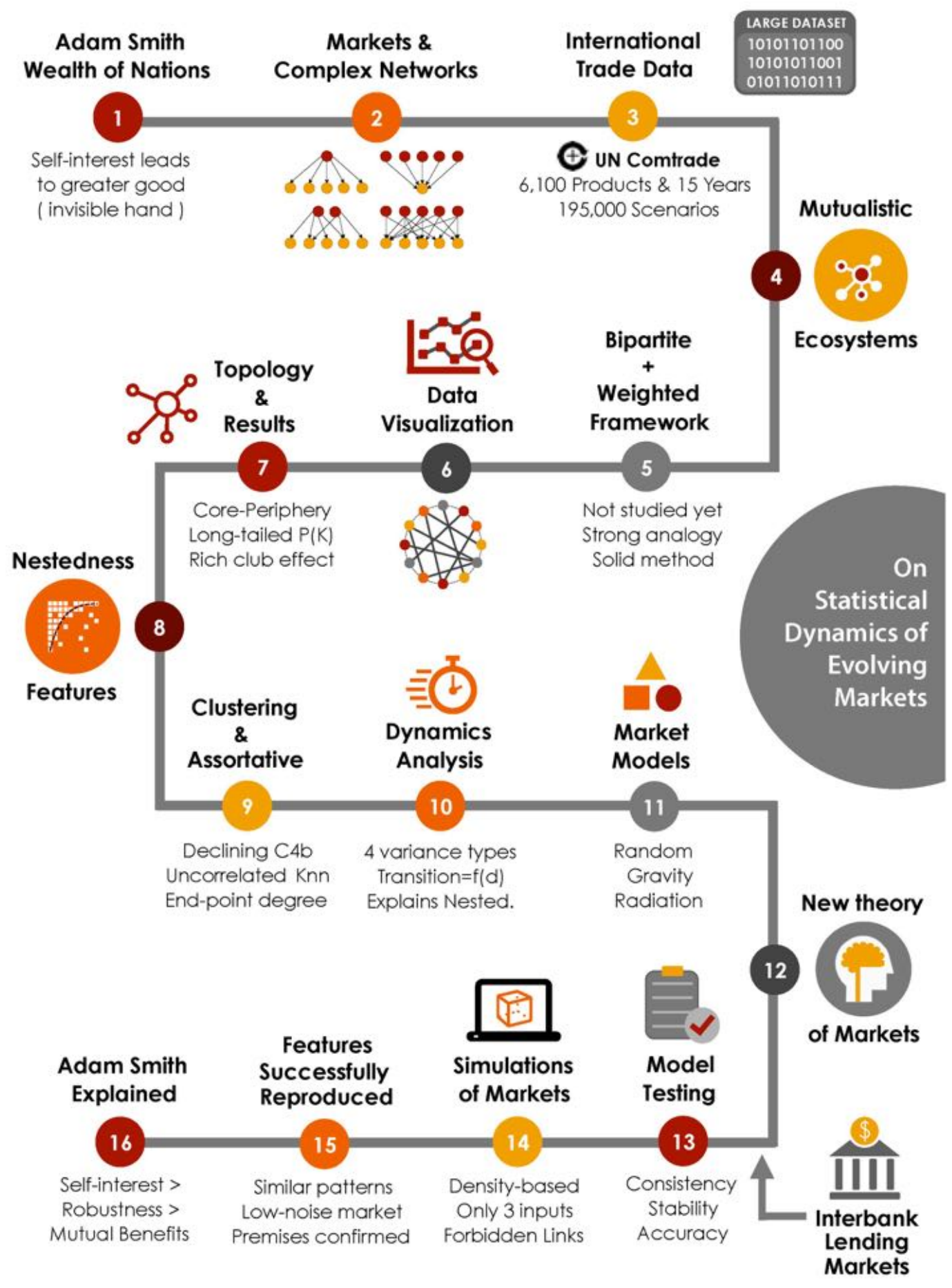

Fig. 12.1.: Visual timeline of the thesis.

namely, a) our premise of the analogy between markets and mutualistic ecosystems, b) the discovery of nestedness as a persistent feature of markets and c) our new mutualistic model of markets. 


\subsection{On statistical models of markets}

During the design and implementation of our statistical models of markets, we think we have made a contribution to science by creating bipartite models that lead to network growth process. Our objective was to describe the process of market formation and, by providing 3 alternatives in this area, we have shown an evolution towards explaining the fundamental mechanism upon which markets are formed. The random bipartite model has demonstrated that it cannot reproduce the features of the empirical markets in a successful way. Yet, it has provided a solid base to build further models. Then, the gravity model has shown two major findings. First, we realized that, even if we neglect the fact that it is not really a network growth process, but rather a projection of the final time-step only, there is a high dependency on the constraints between countries that do not have trade relationships in reality. Failure to add these constraints would result in a full matrix with a forecasted value of bilateral trade for all exporter-importer country pairs. Secondly, we realized that gravity models are more precise to depict trade activity between large countries than they are for small ones while revealing that the influence of the distance is less relevant within these links.

These lessons learned have taken us down to the path of revisiting the analogy existing between mutualistic ecosystems and international trade markets. Consequently, we have designed and implemented our mutualistic model of markets, which allowed us to recreate all features found within actual markets simultaneously. Despite the fact that one of our key research objectives was accomplished, we extended the study of markets through this model, studying the influence of the forbidden links on the topological properties, which adds a higher variability of the resulting topological exponents, so that we could ultimately simulate actual markets in a precise way. Yet, we have found one specific short fall within our model. The nestedness performance of the mutualistic model of markets are consistently higher than that of the empirical data. In other words, our model generates interactions matrices that are "too nested to be realistic". We have found a way to overcome such a short fall, which led us to a modified model. By changing the decreasing probability for new nodes to appear, a clear contradiction to the perfect competition concept in retrospective, for a constant probability, we have been able to reproduce the nestedness values of actual markets while keeping the rest of the topological features of trade networks. Finally, we extended the study of the mutualistic model of markets to some special cases of markets such as the monology and the monopsony.

Throughout this journey, we have learned how to influence the variables and parameters to approximate the process of market formation in such a way that we have also learned how markets work at the meso-scale and the micro-scale. In this way, 
our models can support the ideas of Adam Smith in that self-interest could lead to the greater good and the well being of the society. Our model does support the fact that even if the agents in the system act in a selfish and biased way on an individual basis, the process of market formation will still create an internal organization with the 8 common features of markets, including high nestedness, long-tailed degree distributions and a condition similar to the rich club effect in a weighted context, which together with a declining clustering performance and an uncorrelated nearest neighbor degree distribution, will lead to a core-periphery structure where the core is rarely affected by externalities, as discovered and evidenced in the dynamical analysis. All these factors simultaneously can lead to a condition of robustness of trade networks with persisting topological properties that, indeed, will benefit the society as a whole. In this way, we believe the concept of invisible hand that self-regulates markets, as stated by Adam Smith, is merely a complex emerging phenomenon derived from the simple and selfish interactions between buyers and sellers.

Regarding the minimal model of interbank lending markets, we have implemented a simplistic algorithm that is capable of reproducing the features found within empirical data, which is extremely difficult to get. The fact that it is not the algorithm what determines the outcome, which is a random selection over a set of possible financial partners, but the heterogeneous distribution of cash necessities and cash excesses what makes this study a revealing one. In reality, banks may have a natural propensity to work (borrow and lend money) with other specific institutions, based on past experiences and other variables, but our model shows that even if this process is performed in complete random way, the emerging outcome in terms of topological features would still resemble those of the actual financial markets. Our evidence suggest that the heterogenous boundary conditions of the problem are more relevant than the decision making process itself. 


\subsection{List of conclusions}

As a summary of the key conclusions of this study, we would like to propose the following list:

1. The statistical patterns we find in international trade markets greatly resemble those of mutualistic ecosystems. Besides the number of topological indicators that show a similar pattern, our premise that an analogy exists between markets and ecosystems turned out to be a realistic assumption.

2. The internal structures of international trade markets are highly nested. By using a weighted and bipartite methodology, we discovered that trade networks show a great deal of nestedness as a key feature, and that these properties do not significantly change over time, making the high nestedness condition a persistent one. Since our study is applied to a large number of industries, categories and products, the evidence suggests that all international trade markets have also high nestedness regardless of the level of aggregation.

3. The topological features of international trade markets are deeply connected to the economic theory. Our findings, from a complex network perspective, provide a deeper understanding of how the international trade markets are organized and how exporters and importers are interconnected so that they create together a core-periphery network structure, as evidenced by the long-tailed nature of the degree distribution and the way high weights are more likely to be allocated to nodes of high degree based on exponents that are greater than one among the strength-degree correlations. We combine the previous findings with a declining clustering performance, making the network more hierarchical at the core, and an uncorrelated pattern of the nearest neighbour degree distribution, it is not difficult to realize that markets, like ecosystems, are an elegant symphony of complex behavior derived from simple interactions, which are all consistent with concepts described in the economic theory, those are, self-interest, profit maximization and perfect competition.

4. International trade markets are highly dynamic and the temporal phenomena in them are not equally distributed across the network. After analyzing targeted temporal dynamics of international trade markets, we found that the dynamical phenomena do not significantly affect the core of trade networks, making these cores a highly stable part of the system, and that the majority of temporal activity is more likely to occur in a transition zone between the core and the periphery. This, as a consequence, supports the idea that the static topological properties of trade networks work as a natural protection against temporal variances, where 
an exporter or importer at the periphery is unlikely to reach the core in an easy way. It also means that the core of a trade network is not affected by temporal dynamics, prodiving a methodology to forecast the total market performance by tracking the trends of the countries at the core. By extension, the transition zone is also a protection to the whole system, creating a barrier against externalities.

\section{Neither the random bipartite nor the gravity models are capable of ex- plaining the process of market formation. We have proposed two initial models in order to reproduce the features of trade networks. Yet, neither of them is capable of simultaneously reproducing the common features found in the empirical data and explain the process of market formation with a network growth process. Still, the lessons learned from these models allowed us to pursue further models to accomplish such a task.}

\section{Our mutualistic model of markets is capable of reproducing all features} of the empirical data simultaneously. All 8 common features observed in the empirical data can simultaneously be repreduced by our third model. In a way, this model provides a glimpse of what the perfect market may look like since, from an academic standpoint, it provides topological indicators with a low level of perturbances or noise. The only minor caveat within this model is that it generates extremely nested interaction matrices of trade networks, which are evidenced by the values of nestedness that are significantly above the range of actual markets.

\section{Our mutualistic model of markets provides deeper insights on the process} of market formation. When executing the algorithm of our mutualistic model of markets, we realize that the process of market formation is a highly biased one and that this condition leads to the topological properties of actual markets. Simple interactions between exporters and importers in order to allocate a demand unit include the concept of self-interest as a mean to maximize benefits. For each step of the process, there is a higher probability that a demand unit will be allocated between nodes of high strength in the previous step. This is equivalent to stating that large exporters will try to sell their good to large importers with a higher propensity, leading to a core-periphery structure with long-tailed degree distributions and strength-degree correlation with $\beta>>1$ (a condition similar to the rich club effect). In this way, we have a tangible idea of how decisions are made in the real world and how this decision making process leads to the consistent and persistent organization of international trade markets.

\section{The complex phenomena in financial markets can be explained by simple} interactions, leading to realistic results. In parallel to international trade markets, we have also studied other types of markets. The results we obtained from the 
implementation of a simple and minimal model of interbank lending markets has reinforced the idea of complex phenomena emerging from simple interactions. This study has show that, even if banks act in a random way, the constraints of the system in terms of cash shortage and cash excess, which are heterogeneously distributed across financial institution, can lead to complex networks that resemble those of the empirical data too. Consequently, a minimal model with the right boundary conditions can reproduce the organization of actual data based on simple interactions.

9. The boundary conditions of the problem could be more relevant to determine the outcome than the form of the interactions. Because the intrinsic constraints that exist within a system may influence or systematically condition the interactions between the agents of the system, a minimal model of random interactions can still reproduce the complex emerging outcome found in the empirical data, a fact that opens new possibilities to simulate complex systems.

\section{The concept of an invisible hand that self-regulates markets can be} explained the complex emerging phenomena within trade networks. If a selfish and highly biased behavior can lead to decision that create a specific topological structure of the system, the robustness of that system and the persistence of its features can work as benefitial situation for most of the agents within that system as a whole, providing an alternative explanation of a known phenomenon from a complexity science perspective.

\subsection{Areas of opportunity for future research}

We are delighted to have learned that the analogies between international trade markets and mutualistic ecosystems are a realistic assumption and that, in many ways, we can use what we know about one systems, apply this to the other, and find new insights of how the latter work, which is also benefitial for mutualistic ecosystems since they have a general lack of historical data, which is not the case for markets. In this way, we believe we can use the models developed for this study to continue to monitor the evolution of markets and determine their validity with a broader time window. Without a doubt, there is still room for improvement of the mutualistic model of markets, which we have already started by incorporating a constant birth probability function in subsection 10.2.1. Yet, we believe there is more work to be done in this are. To start, the birth probability function could be thought as a control mechanism upon which an international organization may have some influence so that imbalances can be corrected. Next, the concept of mutualistic markets could also be applied to more local markets and test whether the same 
rules apply to these local markets (domestic consumption of local products and services, for instance). Finally, and once modified for "pure" perfect competition, the resulting topology of our model can be used as a benchmark to detect deviations in any market. This can be useful for policy makers in order to pass anti-monopoly regulation and other fair market laws (in this case, the concept of fair market can be approached from an ecosystemic standpoint with mutual benefits for all players).

We could also develop and implement some new measures to categorize the role of some key exporters and importers to determine the influence they have over the total system. But this does not stop there, we could also move to other areas of the interdisciplinary research. For instance, taking advantage of the proven effectiveness of the analogies between socio-economic and biological systems, we could explore the similarities of the productive process in a economy as an analogy of a food web. The economy of a country could be conceptualized as a very large food web where, starting from the raw materials, there will be a number of firms that will take and consume these materials and consequently will create an emerging semimanufatured product of a higher value. From there, there will be another number of firms that will take and consume the semi-manufatured products and, once again, will create an emerging manufatured product of even higher value. This can continue until the final product in the production chain is consumed by a final customer. The process of consuming a product of low value and creating a more advanced one with a value-add can resemble the process in a food web. Consequently, this is another area of future research for us. 


\section{Appendix A: References and tables of result}

Table of country codes and names according to the ISO standard.

\begin{tabular}{|c|c|c|c|c|c|}
\hline Country Name & Country code & ISO 2-digit & ISO 3-digit & Start year & End Year \\
\hline Afghanistan & 4 & $\mathrm{AF}$ & AFG & 1962 & 2061 \\
\hline Africa CAMEU region, nes & 472 & NULL & NULL & 1962 & 2004 \\
\hline Albania & 8 & $\mathrm{AL}$ & ALB & 1962 & 2061 \\
\hline Algeria & 12 & $\mathrm{DZ}$ & DZA & 1962 & 2061 \\
\hline American Samoa & 16 & NULL & ASM & 1962 & 2061 \\
\hline Andorra & 20 & $\mathrm{AD}$ & AND & 1962 & 2061 \\
\hline Angola & 24 & $\mathrm{AO}$ & AGO & 1962 & 2061 \\
\hline Anguilla & 660 & AI & AIA & 1981 & 2061 \\
\hline Antarctica & 10 & NULL & ANT & 1962 & 2061 \\
\hline Antigua and Barbuda & 28 & AG & ATG & 1962 & 2061 \\
\hline Areas, nes & 899 & NULL & NULL & 1962 & 2061 \\
\hline Argentina & 32 & $\mathrm{AR}$ & ARG & 1962 & 2061 \\
\hline Armenia & 51 & $\mathrm{AM}$ & ARM & 1992 & 2061 \\
\hline Aruba & 533 & $\mathrm{AB}$ & ARB & 1988 & 2061 \\
\hline Australia & 36 & $\mathrm{AU}$ & AUS & 1962 & 2061 \\
\hline Austria & 40 & AT & AUT & 1962 & 2061 \\
\hline Azerbaijan & 31 & $\mathrm{AZ}$ & AZE & 1992 & 2061 \\
\hline Bahamas & 44 & BS & BHS & 1962 & 2061 \\
\hline Bahrain & 48 & $\mathrm{BH}$ & BHR & 1962 & 2061 \\
\hline Bangladesh & 50 & $\mathrm{BD}$ & BGD & 1972 & 2061 \\
\hline Barbados & 52 & BB & BRB & 1962 & 2061 \\
\hline Belarus & 112 & BY & BLR & 1992 & 2061 \\
\hline Belgium & 56 & $\mathrm{BE}$ & BEL & 1999 & 2061 \\
\hline Belgium-Luxembourg & 58 & $\mathrm{BE}$ & BEL & 1962 & 1998 \\
\hline Belize & 84 & $\mathrm{BZ}$ & BLZ & 1962 & 2061 \\
\hline Benin & 204 & BJ & BEN & 1962 & 2061 \\
\hline Bermuda & 60 & BM & BMU & 1962 & 2061 \\
\hline Bhutan & 64 & BT & BTN & 1962 & 2061 \\
\hline Bolivia & 68 & BO & BOL & 1962 & 2061 \\
\hline Bosnia Herzegovina & 70 & $\mathrm{BA}$ & $\mathrm{BIH}$ & 1992 & 2061 \\
\hline Botswana & 72 & BW & BWA & 2000 & 2061 \\
\hline Bouvet Island & 74 & NULL & BVT & 1962 & 2061 \\
\hline Br. Antarctic Terr. & 80 & NULL & NULL & 1962 & 2061 \\
\hline Br. Indian Ocean Terr. & 86 & IO & IOT & 1962 & 2061 \\
\hline Br. Virgin Isds & 92 & VG & VGB & 1962 & 2061 \\
\hline Brazil & 76 & BR & BRA & 1962 & 2061 \\
\hline Brunei Darussalam & 96 & $\mathrm{BN}$ & BRN & 1962 & 2061 \\
\hline Bulgaria & 100 & BG & BGR & 1962 & 2061 \\
\hline Bunkers & 837 & NULL & NULL & 1962 & 2061 \\
\hline Burkina Faso & 854 & $\mathrm{BF}$ & BFA & 1962 & 2061 \\
\hline Burundi & 108 & BI & BDI & 1962 & 2061 \\
\hline CACM, nes & 471 & NULL & NULL & 1962 & 2004 \\
\hline Cambodia & 116 & $\mathrm{KH}$ & KHM & 1962 & 2061 \\
\hline Cameroon & 120 & $\mathrm{CM}$ & CMR & 1962 & 2061 \\
\hline Canada & 124 & $\mathrm{CA}$ & CAN & 1962 & 2061 \\
\hline Cape Verde & 132 & $\mathrm{CV}$ & $\mathrm{CPV}$ & 1962 & 2061 \\
\hline Caribbean, nes & 129 & NULL & NULL & 1962 & 2004 \\
\hline Cayman Isds & 136 & $\mathrm{KY}$ & CYM & 1962 & 2061 \\
\hline Central African Rep. & 140 & $\mathrm{CF}$ & $\mathrm{CAF}$ & 1962 & 2061 \\
\hline
\end{tabular}


Table of country codes and names according to the ISO standard (cont.).

\begin{tabular}{|c|c|c|c|c|c|}
\hline Country Name & Country code & ISO 2-digit & ISO 3-digit & Start year & End Year \\
\hline Chad & 148 & TD & TCD & 1962 & 2061 \\
\hline Chile & 152 & CL & CHL & 1962 & 2061 \\
\hline China & 156 & $\mathrm{CN}$ & CHN & 1962 & 2061 \\
\hline China, Hong Kong SAR & 344 & $\mathrm{HK}$ & HKG & 1962 & 2061 \\
\hline China, Macao SAR & 446 & MO & MAC & 1962 & 2061 \\
\hline Christmas Isds & 162 & $\mathrm{CX}$ & CXR & 1962 & 2061 \\
\hline Cocos Isds & 166 & $\mathrm{CC}$ & CCK & 1962 & 2061 \\
\hline Colombia & 170 & $\mathrm{CO}$ & COL & 1962 & 2061 \\
\hline Comoros & 174 & KM & $\mathrm{COM}$ & 1962 & 2061 \\
\hline Congo & 178 & CG & COG & 1962 & 2061 \\
\hline Cook Isds & 184 & CK & $\mathrm{COK}$ & 1962 & 2061 \\
\hline Costa Rica & 188 & $\mathrm{CR}$ & CRI & 1962 & 2061 \\
\hline Côte d'Ivoire & 384 & $\mathrm{CI}$ & $\mathrm{CIV}$ & 1962 & 2061 \\
\hline Croatia & 191 & $\mathrm{HR}$ & HRV & 1992 & 2061 \\
\hline Cuba & 192 & CU & CUB & 1962 & 2061 \\
\hline Cyprus & 196 & $\mathrm{CY}$ & CYP & 1962 & 2061 \\
\hline Czech Rep. & 203 & $\mathrm{CZ}$ & CZE & 1993 & 2061 \\
\hline Czechoslovakia & 200 & CS & CSK & 1962 & 1992 \\
\hline Dem. People's Rep. of Korea & 408 & $\mathrm{KP}$ & PRK & 1962 & 2061 \\
\hline Dem. Rep. of the Congo & 180 & $\mathrm{CD}$ & COD & 1962 & 2061 \\
\hline Denmark & 208 & DK & DNK & 1962 & 2061 \\
\hline Djibouti & 262 & DJ & DJI & 1962 & 2061 \\
\hline Dominica & 212 & DM & DMA & 1962 & 2061 \\
\hline Dominican Rep. & 214 & DO & DOM & 1962 & 2061 \\
\hline East and West Pakistan & 588 & PK & PAK & 1962 & 1971 \\
\hline Eastern Europe, nes & 221 & NULL & NULL & 1962 & 2004 \\
\hline Ecuador & 218 & EC & ECU & 1962 & 2061 \\
\hline Egypt & 818 & EG & EGY & 1962 & 2061 \\
\hline El Salvador & 222 & SV & SLV & 1962 & 2061 \\
\hline Equatorial Guinea & 226 & GQ & GNQ & 1962 & 2061 \\
\hline Eritrea & 232 & ER & ERI & 1993 & 2061 \\
\hline Estonia & 233 & $\mathrm{EE}$ & EST & 1992 & 2061 \\
\hline Ethiopia & 231 & ET & ETH & 1993 & 2061 \\
\hline EU-27 & 97 & $\mathrm{EU}$ & EU2 & 1962 & 1962 \\
\hline Europe EFTA, nes & 697 & NULL & NULL & 1962 & 2004 \\
\hline Europe EU, nes & 492 & NULL & NULL & NULL & NULL \\
\hline Faeroe Isds & 234 & FO & FRO & 1962 & 2061 \\
\hline Falkland Isds (Malvinas) & 238 & FK & FLK & 1962 & 2061 \\
\hline Fiji & 242 & FJ & FJI & 1962 & 2061 \\
\hline Finland & 246 & FI & FIN & 1962 & 2061 \\
\hline Fmr Arab Rep. of Yemen & 886 & YE & YEM & 1962 & 1990 \\
\hline Fmr Dem. Rep. of Germany & 278 & $\mathrm{DD}$ & DDR & 1962 & 1990 \\
\hline Fmr Dem. Rep. of Vietnam & 866 & VD & VDR & 1962 & 1974 \\
\hline Fmr Dem. Yemen & 720 & YD & YMD & 1962 & 1990 \\
\hline Fmr Ethiopia & 230 & ET & ETH & 1962 & 1992 \\
\hline Fmr Fed. Rep. of Germany & 280 & $\mathrm{DE}$ & $\mathrm{DEU}$ & 1962 & 1990 \\
\hline Fmr Pacific Isds & 582 & PC & PCI & 1962 & 1991 \\
\hline Fmr Panama, excl.Canal Zone & 590 & $\mathrm{PA}$ & PAN & 1962 & 1977 \\
\hline Fmr Panama-Canal-Zone & 592 & $\mathrm{PZ}$ & PCZ & 1962 & 1977 \\
\hline Fmr Rep. of Vietnam & 868 & $\mathrm{VN}$ & VNM & 1962 & 1974 \\
\hline Fmr Rhodesia Nyas & 717 & NULL & NULL & 1962 & 1964 \\
\hline Fmr Tanganyika & 835 & NULL & NULL & 1962 & 1964 \\
\hline Fmr USSR & 810 & SU & SUN & 1962 & 1991 \\
\hline Fmr Yugoslavia & 890 & YU & YUG & 1962 & 1991 \\
\hline Fmr Zanzibar and Pemba Isd & 836 & NULL & NULL & 1962 & 1964 \\
\hline Fr. South Antarctic Terr. & 260 & $\mathrm{FQ}$ & ATF & 1962 & 2061 \\
\hline France & 251 & FR & FRA & 1962 & 2061 \\
\hline Free Zones & 838 & NULL & NULL & 1962 & 2061 \\
\hline French Guiana & 254 & GF & GUF & 1962 & 1995 \\
\hline French Polynesia & 258 & $\mathrm{PF}$ & PYF & 1962 & 2061 \\
\hline FS Micronesia & 583 & FM & FSM & 1992 & 2061 \\
\hline Gabon & 266 & GA & GAB & 1962 & 2061 \\
\hline Gambia & 270 & GM & GMB & 1962 & 2061 \\
\hline Georgia & 268 & GE & GEO & 1992 & 2061 \\
\hline Germany & 276 & $\mathrm{DE}$ & DEU & 1991 & 2061 \\
\hline Ghana & 288 & $\mathrm{GH}$ & GHA & 1962 & 2061 \\
\hline Gibraltar & 292 & GI & GIB & 1962 & 2061 \\
\hline Greece & 300 & GR & GRC & 1962 & 2061 \\
\hline Greenland & 304 & GL & GRL & 1962 & 2061 \\
\hline Grenada & 308 & GD & GRD & 1962 & 2061 \\
\hline
\end{tabular}


Table of country codes and names according to the ISO standard (cont.).

\begin{tabular}{|c|c|c|c|c|c|}
\hline Country Name & Country code & ISO 2-digit & ISO 3-digit & Start year & End Year \\
\hline Guadeloupe & 312 & GP & GLP & 1962 & 1995 \\
\hline Guam & 316 & NULL & GUM & 1962 & 2061 \\
\hline Guatemala & 320 & GT & GTM & 1962 & 2061 \\
\hline Guinea & 324 & GN & GIN & 1962 & 2061 \\
\hline Guinea-Bissau & 624 & GW & GNB & 1962 & 2061 \\
\hline Guyana & 328 & GY & GUY & 1962 & 2061 \\
\hline Haiti & 332 & HT & HTI & 1962 & 2061 \\
\hline Heard Island and McDonald Islands & 334 & NULL & HMD & 1962 & 2061 \\
\hline Holy See (Vatican City State) & 336 & $\mathrm{VA}$ & VAT & 2000 & 2061 \\
\hline Honduras & 340 & $\mathrm{HN}$ & HND & 1962 & 2061 \\
\hline Hungary & 348 & HU & HUN & 1962 & 2061 \\
\hline Iceland & 352 & IS & ISL & 1962 & 2061 \\
\hline India & 699 & IN & IND & 1975 & 2061 \\
\hline India, excl. Sikkim & 356 & IN & IND & 1962 & 1974 \\
\hline Indonesia & 360 & ID & IDN & 1962 & 2061 \\
\hline Iran & 364 & IR & IRN & 1962 & 2061 \\
\hline Iraq & 368 & IQ & IRQ & 1962 & 2061 \\
\hline Ireland & 372 & IE & IRL & 1962 & 2061 \\
\hline Israel & 376 & IL & ISR & 1962 & 2061 \\
\hline Italy & 381 & IT & ITA & 1962 & 2061 \\
\hline Jamaica & 388 & JM & JAM & 1962 & 2061 \\
\hline Japan & 392 & JP & JPN & 1962 & 2061 \\
\hline Jordan & 400 & JO & JOR & 1962 & 2061 \\
\hline Kazakhstan & 398 & $\mathrm{KZ}$ & $\mathrm{KAZ}$ & 1992 & 2061 \\
\hline Kenya & 404 & $\mathrm{KE}$ & KEN & 1962 & 2061 \\
\hline Kiribati & 296 & KI & KIR & 1962 & 2061 \\
\hline Kuwait & 414 & KW & KWT & 1962 & 2061 \\
\hline Kyrgyzstan & 417 & KG & KGZ & 1992 & 2061 \\
\hline LAIA, nes & 473 & NULL & NULL & 1962 & 2061 \\
\hline Lao People's Dem. Rep. & 418 & LA & LAO & 1962 & 2061 \\
\hline Latvia & 428 & LV & LVA & 1992 & 2061 \\
\hline Lebanon & 422 & LB & LBN & 1962 & 2061 \\
\hline Lesotho & 426 & LS & LSO & 2000 & 2061 \\
\hline Liberia & 430 & LR & LBR & 1962 & 2061 \\
\hline Libya & 434 & LY & LBY & 1962 & 2061 \\
\hline Lithuania & 440 & LT & LTU & 1992 & 2061 \\
\hline Luxembourg & 442 & $\mathrm{LU}$ & LUX & 1999 & 2061 \\
\hline Madagascar & 450 & MG & MDG & 1962 & 2061 \\
\hline Malawi & 454 & MW & MWI & 1965 & 2061 \\
\hline Malaysia & 458 & MY & MYS & 1964 & 2061 \\
\hline Maldives & 462 & MV & MDV & 1962 & 2061 \\
\hline Mali & 466 & ML & MLI & 1962 & 2061 \\
\hline Malta & 470 & MT & MLT & 1962 & 2061 \\
\hline Marshall Isds & 584 & MH & MHL & 1992 & 2061 \\
\hline Martinique & 474 & MQ & MTQ & 1962 & 1995 \\
\hline Mauritania & 478 & MR & MRT & 1962 & 2061 \\
\hline Mauritius & 480 & MU & MUS & 1962 & 2061 \\
\hline Mayotte & 175 & YT & MYT & 1962 & 2061 \\
\hline Mexico & 484 & MX & MEX & 1962 & 2061 \\
\hline Mongolia & 496 & MN & MNG & 1962 & 2061 \\
\hline Montenegro & 499 & $\mathrm{ME}$ & MNE & 2006 & 2061 \\
\hline Montserrat & 500 & MS & MSR & 1962 & 2061 \\
\hline Morocco & 504 & MA & MAR & 1962 & 2061 \\
\hline Mozambique & 508 & MZ & MOZ & 1962 & 2061 \\
\hline Myanmar & 104 & MM & MMR & 1962 & 2061 \\
\hline N. Mariana Isds & 580 & MP & MNP & 1992 & 2061 \\
\hline Namibia & 516 & NA & NAM & 2000 & 2061 \\
\hline Nauru & 520 & NR & NRU & 1962 & 2061 \\
\hline Nepal & 524 & NP & NPL & 1962 & 2061 \\
\hline Neth. Antilles & 530 & AN & ANT & 1988 & 2061 \\
\hline Neth. Antilles and Aruba & 532 & AN & ANT & 1962 & 1987 \\
\hline Netherlands & 528 & NL & NLD & 1962 & 2061 \\
\hline Neutral Zone & 536 & NULL & NULL & 1962 & 2061 \\
\hline New Caledonia & 540 & NC & NCL & 1962 & 2061 \\
\hline New Zealand & 554 & NZ & NZL & 1962 & 2061 \\
\hline Nicaragua & 558 & NI & NIC & 1962 & 2061 \\
\hline Niger & 562 & $\mathrm{NE}$ & NER & 1962 & 2061 \\
\hline Nigeria & 566 & NG & NGA & 1962 & 2061 \\
\hline Niue & 570 & NU & NIU & 1962 & 2061 \\
\hline Norfolk Isds & 574 & NF & NFK & 1962 & 2061 \\
\hline
\end{tabular}


Table of country codes and names according to the ISO standard (cont.).

\begin{tabular}{|c|c|c|c|c|c|}
\hline Country Name & Country code & ISO 2-digit & ISO 3-digit & Start year & End Year \\
\hline $\begin{array}{l}\text { North America and Central America, } \\
\text { nes }\end{array}$ & 637 & NULL & NULL & 1962 & 2061 \\
\hline Northern Africa, nes & 290 & NULL & NULL & 1962 & 2004 \\
\hline Norway & 579 & NO & NOR & 1962 & 2061 \\
\hline Occ. Palestinian Terr. & 275 & PS & PSE & 2000 & 2061 \\
\hline Oceania, nes & 527 & NULL & NULL & 1962 & 2061 \\
\hline Oman & 512 & $\mathrm{OM}$ & OMN & 1962 & 2061 \\
\hline Other Africa, nes & 577 & NULL & NULL & 1962 & 2061 \\
\hline Other Asia, nes & 490 & NULL & NULL & 1962 & 2061 \\
\hline Other Europe, nes & 568 & NULL & NULL & 1962 & 2061 \\
\hline Pakistan & 586 & PK & PAK & 1972 & 2061 \\
\hline Palau & 585 & PW & PLW & 1992 & 2061 \\
\hline Panama & 591 & PA & PAN & 1978 & 2061 \\
\hline Papua New Guinea & 598 & PG & PNG & 1962 & 2061 \\
\hline Paraguay & 600 & PY & PRY & 1962 & 2061 \\
\hline Peninsula Malaysia & 459 & NULL & NULL & 1962 & 1963 \\
\hline Peru & 604 & $\mathrm{PE}$ & PER & 1962 & 2061 \\
\hline Philippines & 608 & $\mathrm{PH}$ & PHL & 1962 & 2061 \\
\hline Pitcairn & 612 & $\mathrm{PN}$ & PCN & 1962 & 2061 \\
\hline Poland & 616 & PL & POL & 1962 & 2061 \\
\hline Portugal & 620 & PT & PRT & 1962 & 2061 \\
\hline Qatar & 634 & QA & QAT & 1962 & 2061 \\
\hline Rep. of Korea & 410 & $\mathrm{KR}$ & KOR & 1962 & 2061 \\
\hline Rep. of Moldova & 498 & MD & MDA & 1992 & 2061 \\
\hline Rest of America, nes & 636 & NULL & NULL & 1962 & 2004 \\
\hline Réunion & 638 & $\mathrm{RE}$ & REU & 1962 & 1995 \\
\hline Romania & 642 & RO & ROM & 1962 & 2061 \\
\hline Russian Federation & 643 & RU & RUS & 1992 & 2061 \\
\hline Rwanda & 646 & RW & RWA & 1962 & 2061 \\
\hline Ryukyu Isd & 647 & NULL & NULL & 1962 & 1972 \\
\hline Sabah & 461 & NULL & NULL & 1962 & 1963 \\
\hline Saint Helena & 654 & $\mathrm{SH}$ & SHN & 1962 & 2061 \\
\hline Saint Kitts and Nevis & 659 & $\mathrm{KN}$ & KNA & 1981 & 2061 \\
\hline Saint Kitts, Nevis and Anguilla & 658 & $\mathrm{KN}$ & KNA & 1962 & 1980 \\
\hline Saint Lucia & 662 & LC & LCA & 1962 & 2061 \\
\hline Saint Pierre and Miquelon & 666 & PM & SPM & 1962 & 2061 \\
\hline Saint Vincent and the Grenadines & 670 & VC & VCT & 1962 & 2061 \\
\hline Samoa & 882 & WS & WSM & 1962 & 2061 \\
\hline San Marino & 674 & SM & SMR & 2000 & 2061 \\
\hline Sao Tome and Principe & 678 & ST & STP & 1962 & 2061 \\
\hline Sarawak & 457 & NULL & NULL & 1962 & 1963 \\
\hline Saudi Arabia & 682 & SA & SAU & 1962 & 2061 \\
\hline Senegal & 686 & $\mathrm{SN}$ & SEN & 1962 & 2061 \\
\hline Serbia & 688 & RS & $\mathrm{SRB}$ & 2006 & 2061 \\
\hline Serbia and Montenegro & 891 & CS & SCG & 1992 & 2005 \\
\hline Seychelles & 690 & SC & SYC & 1962 & 2061 \\
\hline Sierra Leone & 694 & SL & SLE & 1962 & 2061 \\
\hline Sikkim & 698 & NULL & NULL & 1962 & 1974 \\
\hline Singapore & 702 & SG & SGP & 1962 & 2061 \\
\hline Slovakia & 703 & SK & SVK & 1993 & 2061 \\
\hline Slovenia & 705 & SI & SVN & 1992 & 2061 \\
\hline So. African Customs Union & 711 & $\mathrm{ZA}$ & $\mathrm{ZAF}$ & 1962 & 1999 \\
\hline Solomon Isds & 90 & SB & SLB & 1962 & 2061 \\
\hline Somalia & 706 & so & SOM & 1962 & 2061 \\
\hline South Africa & 710 & $\mathrm{ZA}$ & ZAF & 2000 & 2061 \\
\hline $\begin{array}{l}\text { South Georgia and the South Sand- } \\
\text { wich Islands }\end{array}$ & 239 & NULL & SGS & 1962 & 2061 \\
\hline Spain & 724 & ES & ESP & 1962 & 2061 \\
\hline Special Categories & 839 & NULL & NULL & 1962 & 2061 \\
\hline Sri Lanka & 144 & LK & LKA & 1962 & 2061 \\
\hline Sudan & 736 & SD & SDN & 1962 & 2061 \\
\hline Suriname & 740 & SR & SUR & 1962 & 2061 \\
\hline Swaziland & 748 & SZ & SWZ & 2000 & 2061 \\
\hline Sweden & 752 & $\mathrm{SE}$ & SWE & 1962 & 2061 \\
\hline Switzerland & 757 & $\mathrm{CH}$ & $\mathrm{CHE}$ & 1962 & 2061 \\
\hline Syria & 760 & SY & SYR & 1962 & 2061 \\
\hline Tajikistan & 762 & $\mathrm{TJ}$ & TJK & 1992 & 2061 \\
\hline TFYR of Macedonia & 807 & MK & MKD & 1993 & 2061 \\
\hline Thailand & 764 & $\mathrm{TH}$ & THA & 1962 & 2061 \\
\hline Timor-Leste & 626 & TP & TMP & 1962 & 2061 \\
\hline Togo & 768 & TG & TGO & 1962 & 2061 \\
\hline Tokelau & 772 & TK & TKL & 1962 & 2061 \\
\hline
\end{tabular}


Table of country codes and names according to the ISO standard (cont.).

\begin{tabular}{|c|c|c|c|c|c|}
\hline Country Name & Country code & ISO 2-digit & ISO 3-digit & Start year & End Year \\
\hline Tonga & 776 & TO & TON & 1962 & 2061 \\
\hline Trinidad and Tobago & 780 & TT & TTO & 1962 & 2061 \\
\hline Tunisia & 788 & $\mathrm{TN}$ & TUN & 1962 & 2061 \\
\hline Turkey & 792 & TR & TUR & 1962 & 2061 \\
\hline Turkmenistan & 795 & TM & TKM & 1992 & 2061 \\
\hline Turks and Caicos Isds & 796 & TC & TCA & 1962 & 2061 \\
\hline Tuvalu & 798 & TV & TUV & 1962 & 2061 \\
\hline Uganda & 800 & UG & UGA & 1962 & 2061 \\
\hline Ukraine & 804 & UA & UKR & 1992 & 2061 \\
\hline United Arab Emirates & 784 & $\mathrm{AE}$ & ARE & 1962 & 2061 \\
\hline United Kingdom & 826 & GB & GBR & 1962 & 2061 \\
\hline United Rep. of Tanzania & 834 & $\mathrm{TZ}$ & TZA & 1965 & 2061 \\
\hline United States Minor Outlying Islands & 581 & UM & UMI & 1962 & 2061 \\
\hline Uruguay & 858 & UY & URY & 1962 & 2061 \\
\hline US Misc. Pacific Isds & 849 & NULL & NULL & 1962 & 1962 \\
\hline US Virgin Isds & 850 & VI & VIR & 1962 & 1980 \\
\hline USA & 842 & US & USA & 1981 & 2061 \\
\hline USA (before 1981) & 841 & US & USA & 1962 & 1980 \\
\hline Uzbekistan & 860 & UZ & UZB & 1992 & 2061 \\
\hline Vanuatu & 548 & $\mathrm{vU}$ & VUT & 1962 & 2061 \\
\hline Venezuela & 862 & VE & VEN & 1962 & 2061 \\
\hline Viet Nam & 704 & VN & VNM & 1975 & 2061 \\
\hline Wallis and Futuna Isds & 876 & $\mathrm{WF}$ & WLF & 1962 & 2061 \\
\hline Western Asia, nes & 879 & NULL & NULL & 1962 & 2004 \\
\hline Western Sahara & 732 & $\mathrm{EH}$ & ESH & 1962 & 2061 \\
\hline World & 0 & WL & WLD & 1962 & 2061 \\
\hline Yemen & 887 & YE & YEM & 1991 & 2061 \\
\hline Zambia & 894 & ZM & $\mathrm{ZMB}$ & 1965 & 2061 \\
\hline Zimbabwe & 716 & $\mathrm{ZW}$ & ZWE & 1965 & 2061 \\
\hline
\end{tabular}


Table of results for the $\beta$ exponent for the exporters without RSP and RPP filters.

\begin{tabular}{|c|c|c|c|c|c|c|c|c|}
\hline & 1995 & & 1999 & & 2004 & & 2009 & \\
\hline HS2 & $\beta$ & $R^{2}$ & $\beta$ & $R^{2}$ & $\beta$ & $R^{2}$ & $\beta$ & $R^{2}$ \\
\hline 1 & $2.12 \pm 0.29$ & 0.825 & $1.92 \pm 0.3$ & 0.768 & $2.04 \pm 0.25$ & 0.835 & $1.96 \pm 0.29$ & 0.773 \\
\hline 2 & $2.28 \pm 0.33$ & 0.801 & $2.33 \pm 0.3$ & 0.833 & $2.37 \pm 0.3$ & 0.82 & $2.22 \pm 0.25$ & 0.861 \\
\hline 3 & $2.1 \pm 0.23$ & 0.836 & $2.17 \pm 0.23$ & 0.834 & $2.24 \pm 0.23$ & 0.829 & $2.19 \pm 0.22$ & 0.84 \\
\hline 4 & $2.19 \pm 0.27$ & 0.829 & $2.16 \pm 0.29$ & 0.787 & $2.18 \pm 0.27$ & 0.797 & $2.09 \pm 0.25$ & 0.821 \\
\hline 5 & $2.22 \pm 0.2$ & 0.905 & $2.2 \pm 0.2$ & 0.902 & $1.98 \pm 0.21$ & 0.867 & $2.11 \pm 0.21$ & 0.873 \\
\hline 6 & $2.45 \pm 0.24$ & 0.884 & $2.33 \pm 0.25$ & 0.86 & $2.45 \pm 0.27$ & 0.851 & $2.33 \pm 0.26$ & 0.845 \\
\hline 7 & $2.15 \pm 0.19$ & 0.893 & $1.95 \pm 0.23$ & 0.808 & $2.14 \pm 0.21$ & 0.86 & $2.11 \pm 0.17$ & 0.899 \\
\hline 8 & $1.84 \pm 0.26$ & 0.747 & $2.13 \pm 0.25$ & 0.791 & $2.09 \pm 0.27$ & 0.752 & $2.09 \pm 0.22$ & 0.825 \\
\hline 9 & $2.1 \pm 0.23$ & 0.825 & $1.79 \pm 0.22$ & 0.773 & $1.9 \pm 0.22$ & 0.785 & $1.98 \pm 0.21$ & 0.819 \\
\hline 10 & $1.98 \pm 0.32$ & 0.763 & $1.88 \pm 0.25$ & 0.818 & $1.86 \pm 0.34$ & 0.71 & $1.6 \pm 0.28$ & 0.712 \\
\hline 11 & $1.53 \pm 0.27$ & 0.751 & $1.59 \pm 0.22$ & 0.811 & $1.79 \pm 0.21$ & 0.848 & $1.86 \pm 0.21$ & 0.848 \\
\hline 12 & $2.15 \pm 0.22$ & 0.854 & $2.05 \pm 0.24$ & 0.804 & $2.05 \pm 0.29$ & 0.724 & $2.02 \pm 0.29$ & 0.712 \\
\hline 13 & $2.12 \pm 0.24$ & 0.869 & $2 \pm 0.28$ & 0.807 & $2.04 \pm 0.32$ & 0.737 & $1.75 \pm 0.27$ & 0.747 \\
\hline 14 & $1.66 \pm 0.36$ & 0.689 & $1.42 \pm 0.35$ & 0.62 & $1.91 \pm 0.26$ & 0.832 & $1.78 \pm 0.26$ & 0.826 \\
\hline 15 & $2.09 \pm 0.22$ & 0.861 & $2.13 \pm 0.26$ & 0.809 & $2.21 \pm 0.21$ & 0.868 & $2.18 \pm 0.24$ & 0.82 \\
\hline 16 & $2.27 \pm 0.25$ & 0.857 & $2.14 \pm 0.28$ & 0.79 & $2.23 \pm 0.23$ & 0.855 & $2.24 \pm 0.2$ & 0.889 \\
\hline 17 & $2.02 \pm 0.22$ & 0.848 & $1.9 \pm 0.24$ & 0.793 & $1.93 \pm 0.23$ & 0.804 & $2 \pm 0.22$ & 0.831 \\
\hline 18 & $2.51 \pm 0.25$ & 0.88 & $2.33 \pm 0.28$ & 0.834 & $2.43 \pm 0.24$ & 0.856 & $2.28 \pm 0.25$ & 0.831 \\
\hline 19 & $2.37 \pm 0.22$ & 0.897 & $2.08 \pm 0.27$ & 0.795 & $2.17 \pm 0.24$ & 0.833 & $2.19 \pm 0.22$ & 0.848 \\
\hline 20 & $2.23 \pm 0.22$ & 0.858 & $2.06 \pm 0.25$ & 0.784 & $2.28 \pm 0.2$ & 0.865 & $2.28 \pm 0.2$ & 0.867 \\
\hline 21 & $2.1 \pm 0.23$ & 0.842 & $1.94 \pm 0.24$ & 0.787 & $2.21 \pm 0.23$ & 0.832 & $1.88 \pm 0.23$ & 0.76 \\
\hline 22 & $2.17 \pm 0.23$ & 0.834 & $2.2 \pm 0.25$ & 0.799 & $2.14 \pm 0.23$ & 0.809 & $2.16 \pm 0.21$ & 0.839 \\
\hline 23 & $2.06 \pm 0.28$ & 0.807 & $2.21 \pm 0.24$ & 0.857 & $2.29 \pm 0.26$ & 0.845 & $2.25 \pm 0.24$ & 0.854 \\
\hline 24 & $1.7 \pm 0.3$ & 0.72 & $1.73 \pm 0.26$ & 0.741 & $1.94 \pm 0.2$ & 0.859 & $1.87 \pm 0.17$ & 0.877 \\
\hline 25 & $1.94 \pm 0.19$ & 0.858 & $1.73 \pm 0.22$ & 0.774 & $2.01 \pm 0.18$ & 0.861 & $1.64 \pm 0.18$ & 0.82 \\
\hline 26 & $2.14 \pm 0.28$ & 0.843 & $2.21 \pm 0.34$ & 0.774 & $2.31 \pm 0.32$ & 0.802 & $2.22 \pm 0.36$ & 0.762 \\
\hline 27 & $2 \pm 0.35$ & 0.659 & $1.59 \pm 0.36$ & 0.493 & $2.05 \pm 0.45$ & 0.508 & $1.69 \pm 0.37$ & 0.506 \\
\hline 28 & $2.22 \pm 0.4$ & 0.654 & $2.03 \pm 0.36$ & 0.625 & $2.12 \pm 0.35$ & 0.669 & $2.01 \pm 0.35$ & 0.632 \\
\hline 29 & $2.49 \pm 0.38$ & 0.695 & $2.47 \pm 0.41$ & 0.637 & $2.41 \pm 0.46$ & 0.573 & $2.42 \pm 0.4$ & 0.635 \\
\hline 30 & $2.6 \pm 0.34$ & 0.776 & $2.76 \pm 0.3$ & 0.817 & $2.52 \pm 0.37$ & 0.709 & $2.48 \pm 0.36$ & 0.698 \\
\hline 31 & $2.13 \pm 0.27$ & 0.848 & $1.88 \pm 0.31$ & 0.741 & $1.73 \pm 0.34$ & 0.683 & $1.92 \pm 0.28$ & 0.769 \\
\hline 32 & $2.63 \pm 0.29$ & 0.84 & $2.51 \pm 0.31$ & 0.785 & $2.34 \pm 0.31$ & 0.743 & $2.23 \pm 0.29$ & 0.757 \\
\hline 33 & $2.41 \pm 0.2$ & 0.899 & $2.31 \pm 0.22$ & 0.845 & $2.38 \pm 0.23$ & 0.84 & $2.43 \pm 0.17$ & 0.902 \\
\hline 34 & $2.21 \pm 0.25$ & 0.84 & $2.07 \pm 0.32$ & 0.721 & $2.34 \pm 0.25$ & 0.824 & $2.22 \pm 0.23$ & 0.83 \\
\hline 35 & $2.56 \pm 0.39$ & 0.762 & $2.39 \pm 0.41$ & 0.704 & $2.58 \pm 0.35$ & 0.773 & $2.41 \pm 0.34$ & 0.764 \\
\hline 36 & $1.63 \pm 0.32$ & 0.732 & $1.56 \pm 0.35$ & 0.668 & $1.7 \pm 0.33$ & 0.727 & $2.02 \pm 0.22$ & 0.887 \\
\hline 37 & $2.57 \pm 0.33$ & 0.826 & $2.44 \pm 0.41$ & 0.725 & $2.39 \pm 0.36$ & 0.755 & $2.55 \pm 0.25$ & 0.886 \\
\hline 38 & $2.3 \pm 0.3$ & 0.774 & $2.39 \pm 0.34$ & 0.725 & $2.44 \pm 0.34$ & 0.726 & $2.62 \pm 0.28$ & 0.8 \\
\hline 39 & $2.52 \pm 0.34$ & 0.727 & $2.35 \pm 0.35$ & 0.669 & $2.45 \pm 0.33$ & 0.684 & $2.41 \pm 0.28$ & 0.731 \\
\hline 40 & $2.45 \pm 0.27$ & 0.809 & $2.28 \pm 0.3$ & 0.734 & $2.6 \pm 0.27$ & 0.812 & $2.32 \pm 0.3$ & 0.729 \\
\hline 41 & $2.17 \pm 0.24$ & 0.837 & $2.04 \pm 0.26$ & 0.791 & $2.13 \pm 0.29$ & 0.754 & $2.13 \pm 0.22$ & 0.86 \\
\hline 42 & $2.57 \pm 0.26$ & 0.846 & $2.54 \pm 0.26$ & 0.837 & $2.44 \pm 0.26$ & 0.814 & $2.47 \pm 0.21$ & 0.865 \\
\hline 43 & $2.27 \pm 0.31$ & 0.825 & $1.96 \pm 0.3$ & 0.81 & $2.39 \pm 0.28$ & 0.846 & $2.24 \pm 0.33$ & 0.782 \\
\hline 44 & $2.42 \pm 0.21$ & 0.864 & $2.57 \pm 0.17$ & 0.91 & $2.39 \pm 0.22$ & 0.829 & $2.25 \pm 0.19$ & 0.847 \\
\hline 45 & $2.2 \pm 0.54$ & 0.713 & $2.1 \pm 0.46$ & 0.711 & $2.21 \pm 0.46$ & 0.733 & $2.56 \pm 0.44$ & 0.81 \\
\hline 46 & $2.16 \pm 0.26$ & 0.865 & $2.12 \pm 0.3$ & 0.822 & $2.2 \pm 0.27$ & 0.858 & $2.11 \pm 0.25$ & 0.86 \\
\hline 47 & $2.63 \pm 0.37$ & 0.823 & $2.6 \pm 0.36$ & 0.844 & $2.76 \pm 0.34$ & 0.849 & $2.88 \pm 0.25$ & 0.922 \\
\hline 48 & $2.63 \pm 0.31$ & 0.784 & $2.62 \pm 0.32$ & 0.759 & $2.67 \pm 0.28$ & 0.802 & $2.64 \pm 0.24$ & 0.845 \\
\hline 49 & $2.43 \pm 0.21$ & 0.883 & $2.36 \pm 0.23$ & 0.84 & $2.4 \pm 0.2$ & 0.872 & $2.56 \pm 0.18$ & 0.905 \\
\hline
\end{tabular}


Table of results for the $\beta$ exponent for the exporters without RSP and RPP filters (cont.)

\begin{tabular}{|c|c|c|c|c|c|c|c|c|}
\hline & 1995 & & 1999 & & 2004 & & 2009 & \\
\hline HS2 & $\beta$ & $R^{2}$ & $\beta$ & $R^{2}$ & $\beta$ & $R^{2}$ & $\beta$ & $R^{2}$ \\
\hline 50 & $2.44 \pm 0.42$ & 0.781 & $2.34 \pm 0.39$ & 0.791 & $2.39 \pm 0.35$ & 0.824 & $2.13 \pm 0.34$ & 0.804 \\
\hline 51 & $2.51 \pm 0.22$ & 0.906 & $2.33 \pm 0.2$ & 0.904 & $2.04 \pm 0.26$ & 0.816 & $2.05 \pm 0.26$ & 0.825 \\
\hline 52 & $2.17 \pm 0.19$ & 0.885 & $2.24 \pm 0.2$ & 0.855 & $2.15 \pm 0.19$ & 0.874 & $1.96 \pm 0.25$ & 0.779 \\
\hline 53 & $2.17 \pm 0.24$ & 0.883 & $2.23 \pm 0.24$ & 0.877 & $1.82 \pm 0.3$ & 0.744 & $1.89 \pm 0.27$ & 0.792 \\
\hline 54 & $2.1 \pm 0.34$ & 0.716 & $2.44 \pm 0.28$ & 0.811 & $2.39 \pm 0.25$ & 0.84 & $2.13 \pm 0.28$ & 0.783 \\
\hline 55 & $2.32 \pm 0.21$ & 0.874 & $2.25 \pm 0.25$ & 0.831 & $2.34 \pm 0.19$ & 0.9 & $2.02 \pm 0.23$ & 0.82 \\
\hline 56 & $2.32 \pm 0.27$ & 0.836 & $2.34 \pm 0.25$ & 0.856 & $2.22 \pm 0.28$ & 0.782 & $1.95 \pm 0.29$ & 0.715 \\
\hline 57 & $2.51 \pm 0.23$ & 0.89 & $2.13 \pm 0.29$ & 0.775 & $1.98 \pm 0.32$ & 0.7 & $2.21 \pm 0.25$ & 0.824 \\
\hline 58 & $2.32 \pm 0.19$ & 0.911 & $2.12 \pm 0.23$ & 0.854 & $2.25 \pm 0.23$ & 0.849 & $1.95 \pm 0.24$ & 0.812 \\
\hline 59 & $2.45 \pm 0.34$ & 0.802 & $2.38 \pm 0.33$ & 0.774 & $2.56 \pm 0.29$ & 0.84 & $2.55 \pm 0.23$ & 0.887 \\
\hline 60 & $2.17 \pm 0.29$ & 0.814 & $1.94 \pm 0.33$ & 0.72 & $2.11 \pm 0.29$ & 0.778 & $2.01 \pm 0.3$ & 0.76 \\
\hline 61 & $2.4 \pm 0.24$ & 0.838 & $2.32 \pm 0.26$ & 0.783 & $2.36 \pm 0.24$ & 0.794 & $2.46 \pm 0.25$ & 0.79 \\
\hline 62 & $2.51 \pm 0.22$ & 0.868 & $2.47 \pm 0.26$ & 0.788 & $2.48 \pm 0.23$ & 0.816 & $2.53 \pm 0.23$ & 0.82 \\
\hline 63 & $2.34 \pm 0.18$ & 0.902 & $2.12 \pm 0.22$ & 0.819 & $2.25 \pm 0.21$ & 0.832 & $2.44 \pm 0.18$ & 0.887 \\
\hline 64 & $2.49 \pm 0.25$ & 0.85 & $2.51 \pm 0.24$ & 0.853 & $2.56 \pm 0.2$ & 0.883 & $2.55 \pm 0.21$ & 0.878 \\
\hline 65 & $2.28 \pm 0.24$ & 0.863 & $2.21 \pm 0.25$ & 0.836 & $2.34 \pm 0.24$ & 0.843 & $2.1 \pm 0.22$ & 0.839 \\
\hline 66 & $2.08 \pm 0.42$ & 0.725 & $2.42 \pm 0.31$ & 0.855 & $2.28 \pm 0.31$ & 0.831 & $1.71 \pm 0.34$ & 0.728 \\
\hline 67 & $2.57 \pm 0.27$ & 0.902 & $2.53 \pm 0.28$ & 0.896 & $2.22 \pm 0.29$ & 0.843 & $1.88 \pm 0.29$ & 0.784 \\
\hline 68 & $2.29 \pm 0.28$ & 0.801 & $2.19 \pm 0.27$ & 0.783 & $2.01 \pm 0.3$ & 0.697 & $2.21 \pm 0.23$ & 0.821 \\
\hline 69 & $2.43 \pm 0.23$ & 0.861 & $2.38 \pm 0.2$ & 0.885 & $2.43 \pm 0.19$ & 0.895 & $2.12 \pm 0.22$ & 0.833 \\
\hline 70 & $2.53 \pm 0.31$ & 0.791 & $2.27 \pm 0.35$ & 0.686 & $2.54 \pm 0.31$ & 0.777 & $2.46 \pm 0.26$ & 0.812 \\
\hline 71 & $2.43 \pm 0.33$ & 0.761 & $2.44 \pm 0.29$ & 0.789 & $2.47 \pm 0.32$ & 0.749 & $2.36 \pm 0.35$ & 0.706 \\
\hline 72 & $2.63 \pm 0.22$ & 0.882 & $2.49 \pm 0.28$ & 0.808 & $2.45 \pm 0.25$ & 0.825 & $2.53 \pm 0.17$ & 0.911 \\
\hline 73 & $2.44 \pm 0.26$ & 0.822 & $2.3 \pm 0.25$ & 0.789 & $2.42 \pm 0.24$ & 0.807 & $2.6 \pm 0.21$ & 0.851 \\
\hline 74 & $2.9 \pm 0.2$ & 0.923 & $2.42 \pm 0.31$ & 0.782 & $2.87 \pm 0.25$ & 0.878 & $2.62 \pm 0.28$ & 0.821 \\
\hline 75 & $2.74 \pm 0.43$ & 0.813 & $2.39 \pm 0.55$ & 0.652 & $2.98 \pm 0.45$ & 0.796 & $2.75 \pm 0.48$ & 0.757 \\
\hline 76 & $2.67 \pm 0.26$ & 0.854 & $2.47 \pm 0.28$ & 0.796 & $2.35 \pm 0.27$ & 0.778 & $2.3 \pm 0.23$ & 0.814 \\
\hline 78 & $2.18 \pm 0.32$ & 0.836 & $2.13 \pm 0.28$ & 0.877 & $2.3 \pm 0.28$ & 0.86 & $2.2 \pm 0.23$ & 0.901 \\
\hline 79 & $2.28 \pm 0.27$ & 0.874 & $1.97 \pm 0.32$ & 0.767 & $2.1 \pm 0.28$ & 0.833 & $2.37 \pm 0.28$ & 0.861 \\
\hline 80 & $2.44 \pm 0.34$ & 0.858 & $2.25 \pm 0.32$ & 0.853 & $2.46 \pm 0.32$ & 0.859 & $2.47 \pm 0.31$ & 0.869 \\
\hline 81 & $2.37 \pm 0.38$ & 0.78 & $2.35 \pm 0.41$ & 0.748 & $2.34 \pm 0.38$ & 0.768 & $2.25 \pm 0.36$ & 0.752 \\
\hline 82 & $2.65 \pm 0.21$ & 0.9 & $2.8 \pm 0.23$ & 0.887 & $2.54 \pm 0.25$ & 0.837 & $2.24 \pm 0.27$ & 0.758 \\
\hline 83 & $2.46 \pm 0.28$ & 0.827 & $2.43 \pm 0.25$ & 0.837 & $2.4 \pm 0.29$ & 0.772 & $2.36 \pm 0.28$ & 0.783 \\
\hline 84 & $2.73 \pm 0.25$ & 0.826 & $2.48 \pm 0.29$ & 0.722 & $2.65 \pm 0.25$ & 0.784 & $2.75 \pm 0.26$ & 0.777 \\
\hline 85 & $2.46 \pm 0.34$ & 0.676 & $2.54 \pm 0.3$ & 0.72 & $2.76 \pm 0.27$ & 0.78 & $2.65 \pm 0.25$ & 0.786 \\
\hline 86 & $2.51 \pm 0.23$ & 0.915 & $2.22 \pm 0.34$ & 0.789 & $2.59 \pm 0.3$ & 0.836 & $2.34 \pm 0.3$ & 0.805 \\
\hline 87 & $2.78 \pm 0.23$ & 0.878 & $2.61 \pm 0.29$ & 0.775 & $2.65 \pm 0.27$ & 0.806 & $2.71 \pm 0.23$ & 0.843 \\
\hline 88 & $1.74 \pm 0.38$ & 0.615 & $1.81 \pm 0.37$ & 0.624 & $1.99 \pm 0.26$ & 0.772 & $1.87 \pm 0.29$ & 0.704 \\
\hline 89 & $1.87 \pm 0.36$ & 0.7 & $1.74 \pm 0.29$ & 0.726 & $1.98 \pm 0.31$ & 0.733 & $1.97 \pm 0.33$ & 0.694 \\
\hline 90 & $2.51 \pm 0.3$ & 0.756 & $2.57 \pm 0.28$ & 0.769 & $2.61 \pm 0.25$ & 0.807 & $2.64 \pm 0.24$ & 0.815 \\
\hline 91 & $2.56 \pm 0.33$ & 0.808 & $2.4 \pm 0.32$ & 0.792 & $2.37 \pm 0.27$ & 0.828 & $2.29 \pm 0.28$ & 0.814 \\
\hline 92 & $2.27 \pm 0.31$ & 0.799 & $2.15 \pm 0.29$ & 0.797 & $2.42 \pm 0.23$ & 0.882 & $2.45 \pm 0.2$ & 0.912 \\
\hline 93 & $1.61 \pm 0.38$ & 0.628 & $1.83 \pm 0.39$ & 0.651 & $2.01 \pm 0.26$ & 0.838 & $2.07 \pm 0.26$ & 0.848 \\
\hline 94 & $2.44 \pm 0.25$ & 0.834 & $2.47 \pm 0.25$ & 0.824 & $2.55 \pm 0.24$ & 0.815 & $2.47 \pm 0.21$ & 0.842 \\
\hline 95 & $2.49 \pm 0.31$ & 0.777 & $2.62 \pm 0.26$ & 0.834 & $2.7 \pm 0.27$ & 0.819 & $2.76 \pm 0.25$ & 0.853 \\
\hline 96 & $2.37 \pm 0.27$ & 0.813 & $2.48 \pm 0.24$ & 0.852 & $2.35 \pm 0.27$ & 0.788 & $2.16 \pm 0.26$ & 0.776 \\
\hline 97 & $2.26 \pm 0.25$ & 0.853 & $2.16 \pm 0.25$ & 0.837 & $2.1 \pm 0.26$ & 0.802 & $2.12 \pm 0.29$ & 0.776 \\
\hline
\end{tabular}


Table of results for the $\beta$ exponent for the importers without RSP and RPP filters.

\begin{tabular}{|c|c|c|c|c|c|c|c|c|}
\hline & 1995 & & 1999 & & 2004 & & 2009 & \\
\hline HS2 & $\beta$ & $R^{2}$ & $\beta$ & $R^{2}$ & $\beta$ & $R^{2}$ & $\beta$ & $R^{2}$ \\
\hline 1 & $1.66 \pm 0.41$ & 0.582 & $1.63 \pm 0.37$ & 0.606 & $1.8 \pm 0.35$ & 0.662 & $1.79 \pm 0.33$ & 0.684 \\
\hline 2 & $1.99 \pm 0.24$ & 0.841 & $2.12 \pm 0.23$ & 0.862 & $2.03 \pm 0.25$ & 0.819 & $2.11 \pm 0.27$ & 0.807 \\
\hline 3 & $2.04 \pm 0.2$ & 0.856 & $2.05 \pm 0.21$ & 0.836 & $1.76 \pm 0.22$ & 0.76 & $1.89 \pm 0.2$ & 0.825 \\
\hline 4 & $2.02 \pm 0.18$ & 0.885 & $2.06 \pm 0.18$ & 0.883 & $1.98 \pm 0.2$ & 0.842 & $1.92 \pm 0.17$ & 0.881 \\
\hline 5 & $1.96 \pm 0.22$ & 0.863 & $1.89 \pm 0.21$ & 0.855 & $1.89 \pm 0.2$ & 0.853 & $1.81 \pm 0.21$ & 0.837 \\
\hline 6 & $2.3 \pm 0.23$ & 0.88 & $2.19 \pm 0.23$ & 0.88 & $2.11 \pm 0.25$ & 0.827 & $2.19 \pm 0.23$ & 0.857 \\
\hline 7 & $1.92 \pm 0.18$ & 0.878 & $1.86 \pm 0.22$ & 0.802 & $1.88 \pm 0.22$ & 0.802 & $2.12 \pm 0.22$ & 0.83 \\
\hline 8 & $1.77 \pm 0.25$ & 0.729 & $2.07 \pm 0.24$ & 0.793 & $2.1 \pm 0.23$ & 0.808 & $1.98 \pm 0.22$ & 0.801 \\
\hline 9 & $1.83 \pm 0.25$ & 0.764 & $1.97 \pm 0.21$ & 0.817 & $1.98 \pm 0.19$ & 0.846 & $2.15 \pm 0.2$ & 0.84 \\
\hline 10 & $1.94 \pm 0.28$ & 0.787 & $1.99 \pm 0.27$ & 0.794 & $1.95 \pm 0.29$ & 0.758 & $1.93 \pm 0.33$ & 0.706 \\
\hline 11 & $1.73 \pm 0.26$ & 0.785 & $1.67 \pm 0.23$ & 0.78 & $1.73 \pm 0.21$ & 0.793 & $1.64 \pm 0.23$ & 0.764 \\
\hline 12 & $1.89 \pm 0.21$ & 0.833 & $1.78 \pm 0.22$ & 0.785 & $1.86 \pm 0.21$ & 0.791 & $1.86 \pm 0.23$ & 0.774 \\
\hline 13 & $1.65 \pm 0.24$ & 0.796 & $1.78 \pm 0.22$ & 0.817 & $1.69 \pm 0.24$ & 0.776 & $1.71 \pm 0.23$ & 0.792 \\
\hline 14 & $1.29 \pm 0.29$ & 0.661 & $1.36 \pm 0.24$ & 0.744 & $1.38 \pm 0.24$ & 0.743 & $1.26 \pm 0.25$ & 0.696 \\
\hline 15 & $1.76 \pm 0.2$ & 0.833 & $1.89 \pm 0.2$ & 0.837 & $1.79 \pm 0.17$ & 0.851 & $1.71 \pm 0.22$ & 0.758 \\
\hline 16 & $1.64 \pm 0.2$ & 0.808 & $1.86 \pm 0.21$ & 0.821 & $1.73 \pm 0.21$ & 0.802 & $1.78 \pm 0.18$ & 0.852 \\
\hline 17 & $1.37 \pm 0.26$ & 0.633 & $1.43 \pm 0.22$ & 0.672 & $1.3 \pm 0.21$ & 0.651 & $1.46 \pm 0.22$ & 0.696 \\
\hline 18 & $1.82 \pm 0.23$ & 0.813 & $1.87 \pm 0.23$ & 0.804 & $1.9 \pm 0.24$ & 0.787 & $1.76 \pm 0.26$ & 0.716 \\
\hline 19 & $1.79 \pm 0.21$ & 0.819 & $1.93 \pm 0.21$ & 0.819 & $1.99 \pm 0.18$ & 0.844 & $1.95 \pm 0.18$ & 0.85 \\
\hline 20 & $2.1 \pm 0.17$ & 0.89 & $2.07 \pm 0.18$ & 0.883 & $2.17 \pm 0.18$ & 0.877 & $1.93 \pm 0.17$ & 0.868 \\
\hline 21 & $2.07 \pm 0.16$ & 0.901 & $2.12 \pm 0.17$ & 0.891 & $1.98 \pm 0.19$ & 0.838 & $1.96 \pm 0.18$ & 0.855 \\
\hline 22 & $2.1 \pm 0.16$ & 0.903 & $2.04 \pm 0.16$ & 0.891 & $2.17 \pm 0.19$ & 0.852 & $2.04 \pm 0.17$ & 0.861 \\
\hline 23 & $1.93 \pm 0.2$ & 0.865 & $1.81 \pm 0.2$ & 0.832 & $1.78 \pm 0.22$ & 0.797 & $1.88 \pm 0.19$ & 0.853 \\
\hline 24 & $1.92 \pm 0.23$ & 0.837 & $1.57 \pm 0.2$ & 0.79 & $1.69 \pm 0.19$ & 0.822 & $1.86 \pm 0.17$ & 0.868 \\
\hline 25 & $1.82 \pm 0.15$ & 0.893 & $1.8 \pm 0.18$ & 0.846 & $1.85 \pm 0.12$ & 0.918 & $1.67 \pm 0.16$ & 0.846 \\
\hline 26 & $1.43 \pm 0.36$ & 0.592 & $1.44 \pm 0.35$ & 0.572 & $1.3 \pm 0.32$ & 0.563 & $1.24 \pm 0.38$ & 0.445 \\
\hline 27 & $1.51 \pm 0.32$ & 0.529 & $1.55 \pm 0.31$ & 0.544 & $1.44 \pm 0.29$ & 0.521 & $1.32 \pm 0.32$ & 0.44 \\
\hline 28 & $1.76 \pm 0.22$ & 0.779 & $1.88 \pm 0.24$ & 0.767 & $1.99 \pm 0.23$ & 0.788 & $1.83 \pm 0.24$ & 0.757 \\
\hline 29 & $2.07 \pm 0.27$ & 0.77 & $2.21 \pm 0.26$ & 0.779 & $2.45 \pm 0.27$ & 0.781 & $2.41 \pm 0.3$ & 0.757 \\
\hline 30 & $2.18 \pm 0.2$ & 0.867 & $2.33 \pm 0.21$ & 0.857 & $2.42 \pm 0.25$ & 0.81 & $2.35 \pm 0.25$ & 0.789 \\
\hline 31 & $1.46 \pm 0.29$ & 0.665 & $1.6 \pm 0.22$ & 0.781 & $1.64 \pm 0.25$ & 0.743 & $1.63 \pm 0.25$ & 0.702 \\
\hline 32 & $2.17 \pm 0.18$ & 0.895 & $2.19 \pm 0.22$ & 0.833 & $2.22 \pm 0.2$ & 0.851 & $2.07 \pm 0.21$ & 0.819 \\
\hline 33 & $2.04 \pm 0.18$ & 0.881 & $2.13 \pm 0.18$ & 0.875 & $2.1 \pm 0.21$ & 0.82 & $2.19 \pm 0.22$ & 0.803 \\
\hline 34 & $1.98 \pm 0.17$ & 0.895 & $1.99 \pm 0.21$ & 0.826 & $2 \pm 0.18$ & 0.852 & $1.87 \pm 0.18$ & 0.836 \\
\hline 35 & $1.88 \pm 0.19$ & 0.874 & $2.05 \pm 0.18$ & 0.885 & $2.12 \pm 0.2$ & 0.866 & $2.06 \pm 0.22$ & 0.824 \\
\hline 36 & $1.49 \pm 0.17$ & 0.867 & $1.53 \pm 0.21$ & 0.826 & $1.62 \pm 0.19$ & 0.859 & $1.54 \pm 0.18$ & 0.847 \\
\hline 37 & $2.21 \pm 0.28$ & 0.824 & $2.28 \pm 0.28$ & 0.81 & $2.26 \pm 0.27$ & 0.814 & $2.27 \pm 0.25$ & 0.849 \\
\hline 38 & $2.1 \pm 0.22$ & 0.845 & $2.15 \pm 0.2$ & 0.849 & $2.29 \pm 0.21$ & 0.844 & $2.22 \pm 0.21$ & 0.832 \\
\hline 39 & $2.47 \pm 0.22$ & 0.849 & $2.61 \pm 0.22$ & 0.845 & $2.62 \pm 0.24$ & 0.823 & $2.44 \pm 0.23$ & 0.81 \\
\hline 40 & $2.15 \pm 0.19$ & 0.86 & $2.23 \pm 0.2$ & 0.849 & $2.23 \pm 0.21$ & 0.828 & $2.2 \pm 0.22$ & 0.818 \\
\hline 41 & $2.02 \pm 0.17$ & 0.9 & $2.04 \pm 0.22$ & 0.851 & $1.93 \pm 0.2$ & 0.846 & $1.92 \pm 0.19$ & 0.863 \\
\hline 42 & $2.25 \pm 0.26$ & 0.805 & $2.17 \pm 0.24$ & 0.807 & $2.36 \pm 0.21$ & 0.866 & $2.26 \pm 0.23$ & 0.819 \\
\hline 43 & $2.07 \pm 0.31$ & 0.798 & $1.88 \pm 0.28$ & 0.797 & $1.99 \pm 0.32$ & 0.74 & $1.82 \pm 0.37$ & 0.65 \\
\hline 44 & $2.36 \pm 0.24$ & 0.828 & $2.37 \pm 0.24$ & 0.796 & $2.39 \pm 0.24$ & 0.795 & $2.3 \pm 0.22$ & 0.812 \\
\hline 45 & $1.84 \pm 0.31$ & 0.8 & $1.68 \pm 0.36$ & 0.697 & $1.65 \pm 0.36$ & 0.669 & $1.74 \pm 0.34$ & 0.728 \\
\hline 46 & $1.78 \pm 0.32$ & 0.741 & $1.74 \pm 0.24$ & 0.815 & $1.84 \pm 0.24$ & 0.83 & $1.72 \pm 0.26$ & 0.775 \\
\hline 47 & $2.12 \pm 0.33$ & 0.793 & $2.12 \pm 0.36$ & 0.748 & $2.17 \pm 0.29$ & 0.812 & $2.23 \pm 0.33$ & 0.793 \\
\hline 48 & $2.4 \pm 0.18$ & 0.892 & $2.44 \pm 0.22$ & 0.847 & $2.39 \pm 0.2$ & 0.864 & $2.13 \pm 0.25$ & 0.76 \\
\hline 49 & $2.43 \pm 0.21$ & 0.867 & $2.33 \pm 0.22$ & 0.841 & $2.12 \pm 0.24$ & 0.766 & $2.22 \pm 0.24$ & 0.782 \\
\hline
\end{tabular}


Table of results for the $\beta$ exponent for the importers without RSP and RPP filters (cont.)

\begin{tabular}{|c|c|c|c|c|c|c|c|c|}
\hline & 1995 & & 1999 & & 2004 & & 2009 & \\
\hline HS2 & $\beta$ & $R^{2}$ & $\beta$ & $R^{2}$ & $\beta$ & $R^{2}$ & $\beta$ & $R^{2}$ \\
\hline 50 & $1.74 \pm 0.4$ & 0.685 & $1.73 \pm 0.37$ & 0.704 & $1.83 \pm 0.36$ & 0.694 & $1.75 \pm 0.38$ & 0.68 \\
\hline 51 & $1.9 \pm 0.26$ & 0.793 & $1.98 \pm 0.23$ & 0.824 & $1.92 \pm 0.24$ & 0.807 & $1.81 \pm 0.27$ & 0.774 \\
\hline 52 & $1.77 \pm 0.21$ & 0.795 & $2.08 \pm 0.18$ & 0.862 & $2.08 \pm 0.21$ & 0.816 & $1.66 \pm 0.22$ & 0.757 \\
\hline 53 & $1.69 \pm 0.29$ & 0.762 & $1.78 \pm 0.26$ & 0.783 & $1.77 \pm 0.23$ & 0.805 & $1.87 \pm 0.26$ & 0.801 \\
\hline 54 & $2.1 \pm 0.24$ & 0.811 & $2.27 \pm 0.22$ & 0.859 & $2.24 \pm 0.22$ & 0.848 & $2.07 \pm 0.23$ & 0.821 \\
\hline 55 & $1.98 \pm 0.2$ & 0.858 & $2.15 \pm 0.21$ & 0.845 & $2.15 \pm 0.18$ & 0.873 & $1.95 \pm 0.21$ & 0.843 \\
\hline 56 & $1.88 \pm 0.16$ & 0.9 & $1.85 \pm 0.18$ & 0.86 & $1.98 \pm 0.18$ & 0.856 & $1.97 \pm 0.2$ & 0.843 \\
\hline 57 & $2.29 \pm 0.22$ & 0.876 & $2.24 \pm 0.2$ & 0.9 & $2.1 \pm 0.21$ & 0.846 & $2.17 \pm 0.22$ & 0.857 \\
\hline 58 & $2.07 \pm 0.2$ & 0.866 & $2.18 \pm 0.2$ & 0.878 & $2.13 \pm 0.19$ & 0.871 & $2.11 \pm 0.21$ & 0.863 \\
\hline 59 & $1.91 \pm 0.22$ & 0.837 & $2.2 \pm 0.23$ & 0.849 & $2.15 \pm 0.23$ & 0.839 & $2 \pm 0.22$ & 0.837 \\
\hline 60 & $1.95 \pm 0.21$ & 0.861 & $2.02 \pm 0.24$ & 0.832 & $1.95 \pm 0.21$ & 0.844 & $1.82 \pm 0.23$ & 0.787 \\
\hline 61 & $2.19 \pm 0.24$ & 0.797 & $2.28 \pm 0.23$ & 0.806 & $2.35 \pm 0.23$ & 0.809 & $2.4 \pm 0.28$ & 0.763 \\
\hline 62 & $2.23 \pm 0.21$ & 0.845 & $2.3 \pm 0.2$ & 0.845 & $2.43 \pm 0.22$ & 0.821 & $2.49 \pm 0.26$ & 0.787 \\
\hline 63 & $1.97 \pm 0.17$ & 0.871 & $2.05 \pm 0.19$ & 0.836 & $2.12 \pm 0.19$ & 0.834 & $2.05 \pm 0.22$ & 0.785 \\
\hline 64 & $2.07 \pm 0.21$ & 0.839 & $2.08 \pm 0.24$ & 0.793 & $2.27 \pm 0.21$ & 0.847 & $2.18 \pm 0.22$ & 0.816 \\
\hline 65 & $1.83 \pm 0.23$ & 0.819 & $1.98 \pm 0.23$ & 0.813 & $2.02 \pm 0.23$ & 0.82 & $1.88 \pm 0.24$ & 0.78 \\
\hline 66 & $1.83 \pm 0.25$ & 0.84 & $1.9 \pm 0.22$ & 0.867 & $1.67 \pm 0.22$ & 0.826 & $1.68 \pm 0.25$ & 0.792 \\
\hline 67 & $1.99 \pm 0.26$ & 0.844 & $1.95 \pm 0.23$ & 0.862 & $1.86 \pm 0.24$ & 0.825 & $1.85 \pm 0.24$ & 0.838 \\
\hline 68 & $2.15 \pm 0.2$ & 0.874 & $2.13 \pm 0.18$ & 0.88 & $2.15 \pm 0.17$ & 0.882 & $2.08 \pm 0.19$ & 0.839 \\
\hline 69 & $2.27 \pm 0.2$ & 0.877 & $2.23 \pm 0.23$ & 0.82 & $2.22 \pm 0.2$ & 0.847 & $2.06 \pm 0.2$ & 0.838 \\
\hline 70 & $2.07 \pm 0.18$ & 0.869 & $2.27 \pm 0.21$ & 0.846 & $2.3 \pm 0.19$ & 0.861 & $2.1 \pm 0.2$ & 0.83 \\
\hline 71 & $1.41 \pm 0.32$ & 0.545 & $1.8 \pm 0.29$ & 0.68 & $1.9 \pm 0.27$ & 0.712 & $1.71 \pm 0.28$ & 0.66 \\
\hline 72 & $2.22 \pm 0.18$ & 0.901 & $2.13 \pm 0.19$ & 0.86 & $2.09 \pm 0.19$ & 0.835 & $2.2 \pm 0.19$ & 0.864 \\
\hline 73 & $2.33 \pm 0.19$ & 0.873 & $2.23 \pm 0.21$ & 0.831 & $2.41 \pm 0.21$ & 0.821 & $2.35 \pm 0.22$ & 0.818 \\
\hline 74 & $2.02 \pm 0.24$ & 0.809 & $2.06 \pm 0.24$ & 0.799 & $1.93 \pm 0.26$ & 0.74 & $1.88 \pm 0.27$ & 0.72 \\
\hline 75 & $1.81 \pm 0.44$ & 0.622 & $1.59 \pm 0.5$ & 0.479 & $1.95 \pm 0.49$ & 0.572 & $1.77 \pm 0.44$ & 0.584 \\
\hline 76 & $2.11 \pm 0.21$ & 0.846 & $2.08 \pm 0.21$ & 0.816 & $2.15 \pm 0.22$ & 0.816 & $2.19 \pm 0.25$ & 0.781 \\
\hline 78 & $1.77 \pm 0.26$ & 0.839 & $1.66 \pm 0.26$ & 0.795 & $1.71 \pm 0.26$ & 0.778 & $1.59 \pm 0.21$ & 0.845 \\
\hline 79 & $1.72 \pm 0.32$ & 0.711 & $1.92 \pm 0.32$ & 0.728 & $1.83 \pm 0.32$ & 0.722 & $1.81 \pm 0.33$ & 0.697 \\
\hline 80 & $1.79 \pm 0.4$ & 0.659 & $1.81 \pm 0.39$ & 0.68 & $1.76 \pm 0.35$ & 0.707 & $1.93 \pm 0.39$ & 0.723 \\
\hline 81 & $1.86 \pm 0.32$ & 0.735 & $1.81 \pm 0.32$ & 0.739 & $1.87 \pm 0.32$ & 0.728 & $1.74 \pm 0.34$ & 0.683 \\
\hline 82 & $2.24 \pm 0.2$ & 0.861 & $2.26 \pm 0.21$ & 0.855 & $2.25 \pm 0.21$ & 0.838 & $2.2 \pm 0.22$ & 0.812 \\
\hline 83 & $2.21 \pm 0.21$ & 0.866 & $2.26 \pm 0.22$ & 0.845 & $2.32 \pm 0.21$ & 0.841 & $2.12 \pm 0.23$ & 0.799 \\
\hline 84 & $2.76 \pm 0.24$ & 0.828 & $2.78 \pm 0.27$ & 0.796 & $2.76 \pm 0.3$ & 0.722 & $2.76 \pm 0.29$ & 0.749 \\
\hline 85 & $2.56 \pm 0.3$ & 0.749 & $2.62 \pm 0.3$ & 0.728 & $2.85 \pm 0.3$ & 0.749 & $2.8 \pm 0.29$ & 0.753 \\
\hline 86 & $2.18 \pm 0.25$ & 0.866 & $2.15 \pm 0.26$ & 0.831 & $2.08 \pm 0.28$ & 0.783 & $1.94 \pm 0.32$ & 0.711 \\
\hline 87 & $2.57 \pm 0.26$ & 0.819 & $2.65 \pm 0.26$ & 0.804 & $2.69 \pm 0.29$ & 0.764 & $2.52 \pm 0.26$ & 0.785 \\
\hline 88 & $1.79 \pm 0.4$ & 0.597 & $1.84 \pm 0.34$ & 0.663 & $1.82 \pm 0.28$ & 0.711 & $2.21 \pm 0.26$ & 0.818 \\
\hline 89 & $1.74 \pm 0.33$ & 0.702 & $1.74 \pm 0.26$ & 0.773 & $1.68 \pm 0.27$ & 0.697 & $1.58 \pm 0.28$ & 0.669 \\
\hline 90 & $2.32 \pm 0.25$ & 0.798 & $2.4 \pm 0.24$ & 0.802 & $2.64 \pm 0.26$ & 0.799 & $2.52 \pm 0.26$ & 0.776 \\
\hline 91 & $2 \pm 0.31$ & 0.723 & $2.04 \pm 0.28$ & 0.764 & $1.96 \pm 0.27$ & 0.759 & $1.93 \pm 0.28$ & 0.724 \\
\hline 92 & $2.15 \pm 0.26$ & 0.847 & $2.3 \pm 0.21$ & 0.896 & $2.26 \pm 0.23$ & 0.86 & $2.21 \pm 0.26$ & 0.836 \\
\hline 93 & $1.33 \pm 0.3$ & 0.63 & $1.75 \pm 0.32$ & 0.712 & $1.76 \pm 0.28$ & 0.759 & $1.72 \pm 0.3$ & 0.714 \\
\hline 94 & $2.44 \pm 0.22$ & 0.849 & $2.58 \pm 0.22$ & 0.854 & $2.58 \pm 0.23$ & 0.838 & $2.42 \pm 0.24$ & 0.803 \\
\hline 95 & $2.34 \pm 0.24$ & 0.835 & $2.39 \pm 0.26$ & 0.821 & $2.41 \pm 0.24$ & 0.824 & $2.37 \pm 0.23$ & 0.824 \\
\hline 96 & $2.03 \pm 0.21$ & 0.838 & $2.08 \pm 0.2$ & 0.833 & $2.17 \pm 0.2$ & 0.84 & $2.09 \pm 0.21$ & 0.823 \\
\hline 97 & $1.94 \pm 0.33$ & 0.714 & $2.06 \pm 0.27$ & 0.787 & $1.9 \pm 0.32$ & 0.709 & $1.91 \pm 0.3$ & 0.724 \\
\hline
\end{tabular}


Table of results for the $\rho$ exponent for the exporters without RSP and RPP filters.

\begin{tabular}{|c|c|c|c|c|c|c|c|c|}
\hline & 1995 & & 1999 & & 2004 & & 2009 & \\
\hline HS2 & $\rho$ & $R^{2}$ & $\rho$ & $R^{2}$ & $\rho$ & $R^{2}$ & $\rho$ & $R^{2}$ \\
\hline 1 & $0.09 \pm 0.08$ & 0.108 & $0.09 \pm 0.08$ & 0.087 & $0.12 \pm 0.06$ & 0.206 & $0.07 \pm 0.09$ & 0.043 \\
\hline 2 & $-0.01 \pm 0.05$ & 0.002 & $0.01 \pm 0.04$ & 0.002 & $0.04 \pm 0.02$ & 0.187 & $-0.01 \pm 0.04$ & 0.007 \\
\hline 3 & $0.02 \pm 0.04$ & 0.014 & $0.03 \pm 0.03$ & 0.056 & $0.01 \pm 0.03$ & 0.01 & $-0.01 \pm 0.03$ & 0.009 \\
\hline 4 & $0.06 \pm 0.03$ & 0.18 & $0.04 \pm 0.03$ & 0.082 & $0.06 \pm 0.04$ & 0.144 & $0.05 \pm 0.05$ & 0.085 \\
\hline 5 & $0 \pm 0.04$ & 0 & $-0.01 \pm 0.04$ & 0.004 & $0.01 \pm 0.03$ & 0.002 & $-0.02 \pm 0.03$ & 0.021 \\
\hline 6 & $0.11 \pm 0.04$ & 0.286 & $0.11 \pm 0.04$ & 0.293 & $0.1 \pm 0.03$ & 0.39 & $0.09 \pm 0.04$ & 0.299 \\
\hline 7 & $0.02 \pm 0.05$ & 0.01 & $0.03 \pm 0.04$ & 0.021 & $0.01 \pm 0.04$ & 0.005 & $0 \pm 0.03$ & 0 \\
\hline 8 & $0.01 \pm 0.03$ & 0.002 & $0.02 \pm 0.03$ & 0.014 & $0 \pm 0.02$ & 0 & $-0.01 \pm 0.03$ & 0.009 \\
\hline 9 & $-0.03 \pm 0.03$ & 0.035 & $0.05 \pm 0.03$ & 0.108 & $0.06 \pm 0.02$ & 0.226 & $0.03 \pm 0.03$ & 0.041 \\
\hline 10 & $0 \pm 0.06$ & 0 & $0 \pm 0.05$ & 0.001 & $-0.02 \pm 0.06$ & 0.009 & $-0.07 \pm 0.06$ & 0.088 \\
\hline 11 & $0.05 \pm 0.06$ & 0.064 & $0.07 \pm 0.05$ & 0.157 & $0.14 \pm 0.06$ & 0.339 & $0.07 \pm 0.05$ & 0.117 \\
\hline 12 & $0.05 \pm 0.04$ & 0.122 & $0.04 \pm 0.03$ & 0.067 & $0.06 \pm 0.04$ & 0.094 & $0.02 \pm 0.03$ & 0.022 \\
\hline 13 & $-0.06 \pm 0.04$ & 0.127 & $-0.02 \pm 0.04$ & 0.013 & $0.01 \pm 0.04$ & 0.002 & $0 \pm 0.04$ & 0 \\
\hline 14 & $0.01 \pm 0.06$ & 0.002 & $0.02 \pm 0.07$ & 0.008 & $0 \pm 0.06$ & 0 & $0.03 \pm 0.06$ & 0.021 \\
\hline 15 & $0.03 \pm 0.03$ & 0.078 & $0.07 \pm 0.03$ & 0.231 & $0.06 \pm 0.04$ & 0.112 & $0.05 \pm 0.05$ & 0.048 \\
\hline 16 & $0.05 \pm 0.04$ & 0.082 & $0.06 \pm 0.04$ & 0.111 & $0.07 \pm 0.05$ & 0.136 & $0.07 \pm 0.05$ & 0.121 \\
\hline 17 & $0.03 \pm 0.06$ & 0.021 & $0.07 \pm 0.05$ & 0.118 & $0.08 \pm 0.04$ & 0.196 & $0.09 \pm 0.06$ & 0.118 \\
\hline 18 & $0.03 \pm 0.05$ & 0.029 & $0.04 \pm 0.04$ & 0.055 & $0.08 \pm 0.03$ & 0.236 & $0.02 \pm 0.05$ & 0.011 \\
\hline 19 & $0.1 \pm 0.05$ & 0.228 & $0.13 \pm 0.05$ & 0.307 & $0.1 \pm 0.05$ & 0.2 & $0.07 \pm 0.05$ & 0.099 \\
\hline 20 & $0.04 \pm 0.04$ & 0.053 & $0.05 \pm 0.03$ & 0.103 & $0.07 \pm 0.03$ & 0.236 & $0.06 \pm 0.03$ & 0.151 \\
\hline 21 & $0.1 \pm 0.03$ & 0.345 & $0.1 \pm 0.05$ & 0.212 & $0.06 \pm 0.03$ & 0.182 & $0.01 \pm 0.04$ & 0.004 \\
\hline 22 & $0.13 \pm 0.04$ & 0.428 & $0.11 \pm 0.04$ & 0.316 & $0.1 \pm 0.03$ & 0.322 & $0.06 \pm 0.04$ & 0.128 \\
\hline 23 & $0.05 \pm 0.05$ & 0.071 & $0.1 \pm 0.05$ & 0.19 & $0.08 \pm 0.04$ & 0.227 & $0.09 \pm 0.06$ & 0.134 \\
\hline 24 & $0.08 \pm 0.04$ & 0.27 & $0.12 \pm 0.05$ & 0.249 & $0.11 \pm 0.04$ & 0.281 & $0.15 \pm 0.05$ & 0.337 \\
\hline 25 & $0.11 \pm 0.04$ & 0.26 & $0.09 \pm 0.04$ & 0.191 & $0.13 \pm 0.06$ & 0.2 & $0.07 \pm 0.06$ & 0.08 \\
\hline 26 & $-0.02 \pm 0.05$ & 0.015 & $-0.07 \pm 0.06$ & 0.087 & $-0.06 \pm 0.05$ & 0.084 & $-0.06 \pm 0.05$ & 0.104 \\
\hline 27 & $-0.07 \pm 0.05$ & 0.111 & $-0.06 \pm 0.04$ & 0.085 & $-0.04 \pm 0.05$ & 0.028 & $-0.02 \pm 0.05$ & 0.009 \\
\hline 28 & $0 \pm 0.04$ & 0 & $0.02 \pm 0.04$ & 0.011 & $0.02 \pm 0.04$ & 0.011 & $0.02 \pm 0.03$ & 0.026 \\
\hline 29 & $0.03 \pm 0.03$ & 0.075 & $0.06 \pm 0.02$ & 0.226 & $0.04 \pm 0.03$ & 0.078 & $0.03 \pm 0.02$ & 0.168 \\
\hline 30 & $0.06 \pm 0.03$ & 0.164 & $0.07 \pm 0.03$ & 0.26 & $0.06 \pm 0.02$ & 0.265 & $0.04 \pm 0.03$ & 0.085 \\
\hline 31 & $0.08 \pm 0.05$ & 0.206 & $0.08 \pm 0.06$ & 0.131 & $0.09 \pm 0.04$ & 0.271 & $0.03 \pm 0.05$ & 0.034 \\
\hline 32 & $0.08 \pm 0.03$ & 0.381 & $0.09 \pm 0.04$ & 0.253 & $0.11 \pm 0.04$ & 0.289 & $0.09 \pm 0.03$ & 0.278 \\
\hline 33 & $0.09 \pm 0.03$ & 0.364 & $0.11 \pm 0.02$ & 0.505 & $0.08 \pm 0.02$ & 0.321 & $0.03 \pm 0.03$ & 0.064 \\
\hline 34 & $0.1 \pm 0.05$ & 0.216 & $0.09 \pm 0.05$ & 0.188 & $0.08 \pm 0.05$ & 0.148 & $0.05 \pm 0.05$ & 0.053 \\
\hline 35 & $0.06 \pm 0.03$ & 0.208 & $0.09 \pm 0.04$ & 0.314 & $0.08 \pm 0.04$ & 0.169 & $0.03 \pm 0.03$ & 0.087 \\
\hline 36 & $0.13 \pm 0.07$ & 0.273 & $0.16 \pm 0.08$ & 0.323 & $0.18 \pm 0.05$ & 0.56 & $0.11 \pm 0.07$ & 0.194 \\
\hline 37 & $0.09 \pm 0.02$ & 0.674 & $0.1 \pm 0.02$ & 0.564 & $0.12 \pm 0.03$ & 0.496 & $0.08 \pm 0.03$ & 0.378 \\
\hline 38 & $0.05 \pm 0.02$ & 0.247 & $0.07 \pm 0.03$ & 0.284 & $0.06 \pm 0.02$ & 0.321 & $0.03 \pm 0.03$ & 0.056 \\
\hline 39 & $0.05 \pm 0.02$ & 0.24 & $0.05 \pm 0.03$ & 0.14 & $0.05 \pm 0.03$ & 0.115 & $0.04 \pm 0.02$ & 0.106 \\
\hline 40 & $0.01 \pm 0.03$ & 0.01 & $0.05 \pm 0.02$ & 0.234 & $0.03 \pm 0.02$ & 0.163 & $0.03 \pm 0.02$ & 0.07 \\
\hline 41 & $0.03 \pm 0.04$ & 0.049 & $0.08 \pm 0.05$ & 0.121 & $0.07 \pm 0.05$ & 0.1 & $0.05 \pm 0.05$ & 0.064 \\
\hline 42 & $0.04 \pm 0.02$ & 0.159 & $0.07 \pm 0.03$ & 0.255 & $0.07 \pm 0.02$ & 0.358 & $0.03 \pm 0.02$ & 0.08 \\
\hline 43 & $-0.05 \pm 0.04$ & 0.105 & $-0.06 \pm 0.05$ & 0.132 & $-0.01 \pm 0.05$ & 0.004 & $-0.01 \pm 0.05$ & 0.003 \\
\hline 44 & $0.01 \pm 0.03$ & 0.006 & $0.03 \pm 0.03$ & 0.043 & $0.02 \pm 0.02$ & 0.043 & $0.02 \pm 0.03$ & 0.023 \\
\hline 45 & $0.11 \pm 0.11$ & 0.123 & $0.13 \pm 0.05$ & 0.415 & $0.08 \pm 0.05$ & 0.236 & $0.13 \pm 0.05$ & 0.478 \\
\hline 46 & $0.1 \pm 0.06$ & 0.176 & $0.18 \pm 0.04$ & 0.645 & $0.15 \pm 0.03$ & 0.636 & $0.11 \pm 0.04$ & 0.37 \\
\hline 47 & $0.02 \pm 0.06$ & 0.009 & $0.02 \pm 0.06$ & 0.014 & $0.02 \pm 0.04$ & 0.029 & $0 \pm 0.04$ & 0 \\
\hline 48 & $0.05 \pm 0.03$ & 0.125 & $0.06 \pm 0.03$ & 0.162 & $0.07 \pm 0.03$ & 0.211 & $0.04 \pm 0.03$ & 0.087 \\
\hline 49 & $0.07 \pm 0.02$ & 0.327 & $0.08 \pm 0.03$ & 0.293 & $0.09 \pm 0.03$ & 0.275 & $0.04 \pm 0.03$ & 0.063 \\
\hline
\end{tabular}


Table of results for the $\rho$ exponent for the exporters without RSP and RPP filters (cont.)

\begin{tabular}{|c|c|c|c|c|c|c|c|c|}
\hline & 1995 & & 1999 & & 2004 & & 2009 & \\
\hline HS2 & $\rho$ & $R^{2}$ & $\rho$ & $R^{2}$ & $\rho$ & $R^{2}$ & $\rho$ & $R^{2}$ \\
\hline 50 & $0.09 \pm 0.05$ & 0.297 & $0.05 \pm 0.06$ & 0.077 & $0.1 \pm 0.04$ & 0.403 & $0.08 \pm 0.03$ & 0.357 \\
\hline 51 & $0.02 \pm 0.05$ & 0.014 & $0.03 \pm 0.04$ & 0.042 & $0.05 \pm 0.04$ & 0.083 & $0.02 \pm 0.04$ & 0.017 \\
\hline 52 & $0 \pm 0.04$ & 0.001 & $0 \pm 0.05$ & 0 & $0.02 \pm 0.03$ & 0.017 & $0.03 \pm 0.03$ & 0.051 \\
\hline 53 & $0.04 \pm 0.02$ & 0.191 & $0.04 \pm 0.04$ & 0.074 & $0.06 \pm 0.03$ & 0.189 & $0.02 \pm 0.04$ & 0.031 \\
\hline 54 & $0.02 \pm 0.03$ & 0.023 & $-0.01 \pm 0.02$ & 0.018 & $0.02 \pm 0.02$ & 0.041 & $0.01 \pm 0.03$ & 0.01 \\
\hline 55 & $0.04 \pm 0.02$ & 0.155 & $0.04 \pm 0.03$ & 0.068 & $0.03 \pm 0.04$ & 0.038 & $0.01 \pm 0.03$ & 0.003 \\
\hline 56 & $0.04 \pm 0.03$ & 0.124 & $0.05 \pm 0.03$ & 0.115 & $0.06 \pm 0.03$ & 0.233 & $0.04 \pm 0.03$ & 0.095 \\
\hline 57 & $0.04 \pm 0.03$ & 0.139 & $0.03 \pm 0.03$ & 0.058 & $0.05 \pm 0.03$ & 0.171 & $0.04 \pm 0.03$ & 0.121 \\
\hline 58 & $0.04 \pm 0.03$ & 0.1 & $0.04 \pm 0.03$ & 0.135 & $0.05 \pm 0.03$ & 0.168 & $0.02 \pm 0.03$ & 0.03 \\
\hline 59 & $0.07 \pm 0.03$ & 0.281 & $0.06 \pm 0.04$ & 0.166 & $0.05 \pm 0.03$ & 0.146 & $0.02 \pm 0.03$ & 0.016 \\
\hline 60 & $0.05 \pm 0.05$ & 0.078 & $0.05 \pm 0.05$ & 0.083 & $0.03 \pm 0.04$ & 0.053 & $0 \pm 0.04$ & 0 \\
\hline 61 & $0.01 \pm 0.03$ & 0.004 & $0.01 \pm 0.03$ & 0.004 & $0.01 \pm 0.02$ & 0.011 & $0.03 \pm 0.02$ & 0.052 \\
\hline 62 & $-0.01 \pm 0.03$ & 0.005 & $0.01 \pm 0.03$ & 0.01 & $0.03 \pm 0.02$ & 0.083 & $0.04 \pm 0.02$ & 0.088 \\
\hline 63 & $0.03 \pm 0.02$ & 0.094 & $0.02 \pm 0.03$ & 0.018 & $0.05 \pm 0.02$ & 0.23 & $0.03 \pm 0.02$ & 0.063 \\
\hline 64 & $0.02 \pm 0.03$ & 0.025 & $0.04 \pm 0.03$ & 0.086 & $0.06 \pm 0.03$ & 0.123 & $0.05 \pm 0.02$ & 0.182 \\
\hline 65 & $0.04 \pm 0.03$ & 0.124 & $0.07 \pm 0.03$ & 0.226 & $0.1 \pm 0.02$ & 0.518 & $0.04 \pm 0.03$ & 0.122 \\
\hline 66 & $0.09 \pm 0.03$ & 0.441 & $0.1 \pm 0.04$ & 0.442 & $0.15 \pm 0.03$ & 0.67 & $0.09 \pm 0.03$ & 0.502 \\
\hline 67 & $0.17 \pm 0.04$ & 0.677 & $0.2 \pm 0.04$ & 0.735 & $0.1 \pm 0.05$ & 0.272 & $0.03 \pm 0.04$ & 0.047 \\
\hline 68 & $0.09 \pm 0.03$ & 0.439 & $0.09 \pm 0.03$ & 0.344 & $0.07 \pm 0.03$ & 0.204 & $0.03 \pm 0.03$ & 0.073 \\
\hline 69 & $0.08 \pm 0.02$ & 0.393 & $0.08 \pm 0.02$ & 0.385 & $0.1 \pm 0.03$ & 0.399 & $0.03 \pm 0.03$ & 0.077 \\
\hline 70 & $0.07 \pm 0.05$ & 0.098 & $0.05 \pm 0.03$ & 0.11 & $0.05 \pm 0.03$ & 0.164 & $0.04 \pm 0.02$ & 0.116 \\
\hline 71 & $-0.04 \pm 0.04$ & 0.061 & $-0.01 \pm 0.03$ & 0.003 & $-0.02 \pm 0.02$ & 0.023 & $-0.03 \pm 0.03$ & 0.045 \\
\hline 72 & $0.07 \pm 0.03$ & 0.228 & $0.08 \pm 0.03$ & 0.279 & $0.07 \pm 0.02$ & 0.318 & $0.03 \pm 0.03$ & 0.059 \\
\hline 73 & $0.05 \pm 0.02$ & 0.297 & $0.07 \pm 0.03$ & 0.219 & $0.07 \pm 0.02$ & 0.303 & $0.02 \pm 0.02$ & 0.048 \\
\hline 74 & $-0.02 \pm 0.05$ & 0.006 & $-0.01 \pm 0.04$ & 0.001 & $-0.02 \pm 0.04$ & 0.009 & $-0.06 \pm 0.04$ & 0.099 \\
\hline 75 & $-0.07 \pm 0.06$ & 0.105 & $-0.06 \pm 0.05$ & 0.093 & $-0.09 \pm 0.06$ & 0.168 & $-0.08 \pm 0.06$ & 0.163 \\
\hline 76 & $0.03 \pm 0.03$ & 0.038 & $0.05 \pm 0.03$ & 0.132 & $0.04 \pm 0.03$ & 0.106 & $0.02 \pm 0.03$ & 0.014 \\
\hline 78 & $-0.04 \pm 0.1$ & 0.017 & $0.03 \pm 0.09$ & 0.01 & $0.02 \pm 0.08$ & 0.009 & $-0.05 \pm 0.06$ & 0.064 \\
\hline 79 & $0.03 \pm 0.06$ & 0.019 & $0.03 \pm 0.05$ & 0.027 & $0.01 \pm 0.06$ & 0.005 & $-0.04 \pm 0.05$ & 0.04 \\
\hline 80 & $-0.03 \pm 0.06$ & 0.032 & $0.01 \pm 0.06$ & 0.001 & $0 \pm 0.05$ & 0.001 & $-0.04 \pm 0.06$ & 0.037 \\
\hline 81 & $-0.04 \pm 0.04$ & 0.107 & $0 \pm 0.04$ & 0.001 & $-0.03 \pm 0.06$ & 0.02 & $-0.01 \pm 0.05$ & 0.006 \\
\hline 82 & $0.06 \pm 0.03$ & 0.169 & $0.06 \pm 0.02$ & 0.305 & $0.06 \pm 0.02$ & 0.407 & $0.02 \pm 0.01$ & 0.083 \\
\hline 83 & $0.06 \pm 0.03$ & 0.194 & $0.06 \pm 0.03$ & 0.189 & $0.05 \pm 0.02$ & 0.205 & $0.05 \pm 0.02$ & 0.175 \\
\hline 84 & $0.03 \pm 0.01$ & 0.303 & $0.03 \pm 0.01$ & 0.258 & $0.04 \pm 0.01$ & 0.226 & $0.03 \pm 0.01$ & 0.169 \\
\hline 85 & $0.02 \pm 0.01$ & 0.073 & $0.04 \pm 0.01$ & 0.235 & $0.01 \pm 0.01$ & 0.03 & $0 \pm 0.01$ & 0 \\
\hline 86 & $0 \pm 0.07$ & 0 & $0.02 \pm 0.05$ & 0.02 & $-0.01 \pm 0.05$ & 0.005 & $0 \pm 0.05$ & 0 \\
\hline 87 & $0.06 \pm 0.02$ & 0.354 & $0.06 \pm 0.02$ & 0.282 & $0.04 \pm 0.01$ & 0.294 & $0 \pm 0.02$ & 0 \\
\hline 88 & $0.03 \pm 0.04$ & 0.055 & $0.07 \pm 0.05$ & 0.146 & $0.06 \pm 0.04$ & 0.155 & $0.07 \pm 0.03$ & 0.317 \\
\hline 89 & $-0.07 \pm 0.06$ & 0.118 & $-0.05 \pm 0.05$ & 0.063 & $-0.02 \pm 0.05$ & 0.009 & $-0.02 \pm 0.04$ & 0.017 \\
\hline 90 & $0.02 \pm 0.01$ & 0.166 & $0.03 \pm 0.01$ & 0.196 & $0.04 \pm 0.01$ & 0.192 & $0.01 \pm 0.01$ & 0.036 \\
\hline 91 & $0.06 \pm 0.02$ & 0.313 & $0.05 \pm 0.03$ & 0.21 & $0.08 \pm 0.02$ & 0.467 & $0.04 \pm 0.02$ & 0.246 \\
\hline 92 & $0.03 \pm 0.02$ & 0.15 & $0.06 \pm 0.02$ & 0.363 & $0.06 \pm 0.02$ & 0.398 & $0.06 \pm 0.03$ & 0.259 \\
\hline 93 & $-0.04 \pm 0.08$ & 0.019 & $0 \pm 0.07$ & 0 & $0.01 \pm 0.1$ & 0.001 & $0.02 \pm 0.04$ & 0.015 \\
\hline 94 & $0.06 \pm 0.02$ & 0.282 & $0.06 \pm 0.02$ & 0.244 & $0.06 \pm 0.02$ & 0.265 & $0.03 \pm 0.02$ & 0.065 \\
\hline 95 & $0.03 \pm 0.02$ & 0.102 & $0.06 \pm 0.02$ & 0.252 & $0.06 \pm 0.02$ & 0.405 & $0.05 \pm 0.02$ & 0.315 \\
\hline 96 & $0.04 \pm 0.02$ & 0.191 & $0.06 \pm 0.02$ & 0.351 & $0.07 \pm 0.02$ & 0.352 & $0.06 \pm 0.02$ & 0.245 \\
\hline 97 & $0.02 \pm 0.03$ & 0.037 & $0.02 \pm 0.05$ & 0.013 & $0.02 \pm 0.02$ & 0.041 & $0.05 \pm 0.03$ & 0.156 \\
\hline
\end{tabular}


Table of results for the $\rho$ exponent for the importers without RSP and RPP filters.

\begin{tabular}{|c|c|c|c|c|c|c|c|c|}
\hline & 1995 & & 1999 & & 2004 & & 2009 & \\
\hline HS2 & $\rho$ & $R^{2}$ & $\rho$ & $R^{2}$ & $\rho$ & $R^{2}$ & $\rho$ & $R^{2}$ \\
\hline 1 & $0.05 \pm 0.07$ & 0.04 & $0.05 \pm 0.07$ & 0.039 & $0.1 \pm 0.08$ & 0.093 & $0.04 \pm 0.09$ & 0.015 \\
\hline 2 & $0.09 \pm 0.05$ & 0.178 & $0.12 \pm 0.05$ & 0.326 & $0.11 \pm 0.04$ & 0.299 & $0.07 \pm 0.06$ & 0.079 \\
\hline 3 & $0.03 \pm 0.03$ & 0.05 & $0.05 \pm 0.03$ & 0.1 & $0.04 \pm 0.03$ & 0.093 & $0.02 \pm 0.03$ & 0.039 \\
\hline 4 & $0.09 \pm 0.05$ & 0.207 & $0.1 \pm 0.04$ & 0.232 & $0.09 \pm 0.04$ & 0.211 & $0.07 \pm 0.04$ & 0.132 \\
\hline 5 & $0 \pm 0.04$ & 0 & $0 \pm 0.03$ & 0 & $0.01 \pm 0.08$ & 0.001 & $0.02 \pm 0.06$ & 0.014 \\
\hline 6 & $0.06 \pm 0.05$ & 0.095 & $0.07 \pm 0.04$ & 0.202 & $0.07 \pm 0.05$ & 0.093 & $0.05 \pm 0.05$ & 0.055 \\
\hline 7 & $0.04 \pm 0.05$ & 0.034 & $0.05 \pm 0.06$ & 0.035 & $0.02 \pm 0.05$ & 0.009 & $0 \pm 0.06$ & 0 \\
\hline 8 & $0.02 \pm 0.06$ & 0.004 & $0.01 \pm 0.05$ & 0.003 & $0.04 \pm 0.05$ & 0.033 & $0.01 \pm 0.04$ & 0.005 \\
\hline 9 & $0.05 \pm 0.04$ & 0.063 & $0.05 \pm 0.04$ & 0.078 & $0.05 \pm 0.04$ & 0.075 & $0.05 \pm 0.04$ & 0.086 \\
\hline 10 & $0.05 \pm 0.05$ & 0.063 & $0.07 \pm 0.05$ & 0.11 & $0.07 \pm 0.07$ & 0.063 & $0.01 \pm 0.07$ & 0 \\
\hline 11 & $0.08 \pm 0.07$ & 0.093 & $0.09 \pm 0.06$ & 0.123 & $0.09 \pm 0.07$ & 0.085 & $0.11 \pm 0.07$ & 0.15 \\
\hline 12 & $0.04 \pm 0.04$ & 0.083 & $0.04 \pm 0.04$ & 0.053 & $0.04 \pm 0.03$ & 0.079 & $0.07 \pm 0.05$ & 0.077 \\
\hline 13 & $-0.01 \pm 0.06$ & 0.001 & $0 \pm 0.05$ & 0 & $0 \pm 0.04$ & 0 & $0.03 \pm 0.05$ & 0.015 \\
\hline 14 & $0.03 \pm 0.05$ & 0.025 & $0.02 \pm 0.06$ & 0.009 & $0.04 \pm 0.07$ & 0.038 & $0.02 \pm 0.06$ & 0.007 \\
\hline 15 & $0.05 \pm 0.08$ & 0.019 & $0.06 \pm 0.06$ & 0.059 & $0.09 \pm 0.05$ & 0.131 & $0.05 \pm 0.04$ & 0.055 \\
\hline 16 & $0.07 \pm 0.06$ & 0.092 & $0.09 \pm 0.04$ & 0.284 & $0.09 \pm 0.05$ & 0.137 & $0.07 \pm 0.05$ & 0.118 \\
\hline 17 & $0.02 \pm 0.06$ & 0.004 & $0.05 \pm 0.06$ & 0.034 & $0.03 \pm 0.04$ & 0.022 & $0.06 \pm 0.05$ & 0.06 \\
\hline 18 & $0.04 \pm 0.05$ & 0.039 & $0.05 \pm 0.05$ & 0.045 & $0.05 \pm 0.05$ & 0.055 & $0.04 \pm 0.06$ & 0.026 \\
\hline 19 & $0.07 \pm 0.05$ & 0.131 & $0.09 \pm 0.05$ & 0.141 & $0.08 \pm 0.04$ & 0.134 & $0.09 \pm 0.04$ & 0.159 \\
\hline 20 & $0.07 \pm 0.03$ & 0.169 & $0.09 \pm 0.07$ & 0.084 & $0.07 \pm 0.04$ & 0.121 & $0.08 \pm 0.04$ & 0.143 \\
\hline 21 & $0.09 \pm 0.04$ & 0.204 & $0.06 \pm 0.04$ & 0.128 & $0.07 \pm 0.04$ & 0.129 & $0.06 \pm 0.03$ & 0.127 \\
\hline 22 & $0.09 \pm 0.04$ & 0.164 & $0.1 \pm 0.05$ & 0.208 & $0.09 \pm 0.04$ & 0.149 & $0.08 \pm 0.04$ & 0.144 \\
\hline 23 & $0.09 \pm 0.04$ & 0.274 & $0.08 \pm 0.04$ & 0.243 & $0.09 \pm 0.03$ & 0.302 & $0.09 \pm 0.04$ & 0.231 \\
\hline 24 & $0.06 \pm 0.06$ & 0.075 & $0.08 \pm 0.06$ & 0.093 & $0.08 \pm 0.05$ & 0.117 & $0.08 \pm 0.08$ & 0.061 \\
\hline 25 & $0.09 \pm 0.05$ & 0.184 & $0.1 \pm 0.05$ & 0.165 & $0.1 \pm 0.05$ & 0.189 & $0.09 \pm 0.08$ & 0.06 \\
\hline 26 & $0 \pm 0.03$ & 0 & $0.03 \pm 0.04$ & 0.052 & $0.01 \pm 0.04$ & 0.01 & $0.03 \pm 0.05$ & 0.025 \\
\hline 27 & $0.07 \pm 0.04$ & 0.105 & $0.06 \pm 0.05$ & 0.071 & $0.06 \pm 0.04$ & 0.073 & $0.03 \pm 0.04$ & 0.023 \\
\hline 28 & $0.03 \pm 0.03$ & 0.06 & $0.05 \pm 0.02$ & 0.165 & $0.06 \pm 0.03$ & 0.167 & $0.04 \pm 0.03$ & 0.068 \\
\hline 29 & $0.03 \pm 0.03$ & 0.064 & $0.04 \pm 0.02$ & 0.169 & $0.03 \pm 0.02$ & 0.093 & $0.05 \pm 0.02$ & 0.177 \\
\hline 30 & $0.04 \pm 0.03$ & 0.142 & $0.05 \pm 0.03$ & 0.13 & $0.06 \pm 0.03$ & 0.164 & $0.05 \pm 0.03$ & 0.09 \\
\hline 31 & $0.1 \pm 0.06$ & 0.166 & $0.1 \pm 0.05$ & 0.208 & $0.09 \pm 0.05$ & 0.188 & $0.08 \pm 0.04$ & 0.181 \\
\hline 32 & $0.08 \pm 0.04$ & 0.222 & $0.07 \pm 0.04$ & 0.145 & $0.07 \pm 0.04$ & 0.131 & $0.04 \pm 0.03$ & 0.066 \\
\hline 33 & $0.03 \pm 0.05$ & 0.02 & $0.01 \pm 0.05$ & 0.001 & $0.01 \pm 0.04$ & 0.003 & $0.01 \pm 0.04$ & 0.004 \\
\hline 34 & $0.09 \pm 0.05$ & 0.179 & $0.09 \pm 0.07$ & 0.077 & $0.09 \pm 0.05$ & 0.139 & $0.06 \pm 0.04$ & 0.112 \\
\hline 35 & $0.1 \pm 0.04$ & 0.271 & $0.09 \pm 0.06$ & 0.142 & $0.11 \pm 0.04$ & 0.318 & $0.1 \pm 0.04$ & 0.22 \\
\hline 36 & $0.13 \pm 0.08$ & 0.219 & $0.13 \pm 0.07$ & 0.223 & $0.12 \pm 0.06$ & 0.247 & $0.11 \pm 0.07$ & 0.191 \\
\hline 37 & $0.03 \pm 0.04$ & 0.032 & $0.05 \pm 0.04$ & 0.111 & $0.05 \pm 0.04$ & 0.08 & $0.02 \pm 0.05$ & 0.018 \\
\hline 38 & $0.05 \pm 0.03$ & 0.174 & $0.06 \pm 0.04$ & 0.098 & $0.05 \pm 0.03$ & 0.114 & $0.04 \pm 0.04$ & 0.059 \\
\hline 39 & $0.07 \pm 0.03$ & 0.213 & $0.06 \pm 0.03$ & 0.125 & $0.04 \pm 0.03$ & 0.055 & $0.02 \pm 0.03$ & 0.018 \\
\hline 40 & $0.07 \pm 0.03$ & 0.215 & $0.06 \pm 0.03$ & 0.188 & $0.05 \pm 0.03$ & 0.147 & $0.04 \pm 0.03$ & 0.107 \\
\hline 41 & $0 \pm 0.03$ & 0 & $0.01 \pm 0.04$ & 0.004 & $0 \pm 0.03$ & 0.001 & $0.01 \pm 0.04$ & 0.006 \\
\hline 42 & $0.07 \pm 0.05$ & 0.093 & $0.05 \pm 0.04$ & 0.071 & $0.06 \pm 0.05$ & 0.079 & $0.04 \pm 0.04$ & 0.034 \\
\hline 43 & $0.05 \pm 0.04$ & 0.127 & $0.02 \pm 0.03$ & 0.027 & $0.05 \pm 0.04$ & 0.124 & $0.03 \pm 0.04$ & 0.038 \\
\hline 44 & $0.06 \pm 0.04$ & 0.109 & $0.04 \pm 0.04$ & 0.046 & $0.04 \pm 0.03$ & 0.048 & $0.03 \pm 0.04$ & 0.022 \\
\hline 45 & $0.09 \pm 0.05$ & 0.303 & $0.06 \pm 0.06$ & 0.091 & $0.08 \pm 0.05$ & 0.186 & $0.1 \pm 0.07$ & 0.17 \\
\hline 46 & $0.05 \pm 0.06$ & 0.06 & $0.06 \pm 0.11$ & 0.026 & $0.03 \pm 0.06$ & 0.025 & $0.03 \pm 0.07$ & 0.011 \\
\hline 47 & $0.09 \pm 0.04$ & 0.343 & $0.05 \pm 0.04$ & 0.133 & $0.05 \pm 0.03$ & 0.152 & $0.1 \pm 0.06$ & 0.19 \\
\hline 48 & $0.07 \pm 0.03$ & 0.182 & $0.06 \pm 0.03$ & 0.138 & $0.07 \pm 0.04$ & 0.118 & $0.05 \pm 0.03$ & 0.084 \\
\hline 49 & $0.05 \pm 0.04$ & 0.058 & $0.02 \pm 0.04$ & 0.016 & $0.02 \pm 0.04$ & 0.009 & $0.03 \pm 0.04$ & 0.019 \\
\hline
\end{tabular}


Table of results for the $\rho$ exponent for the importers without RSP and RPP filters (cont.)

\begin{tabular}{|c|c|c|c|c|c|c|c|c|}
\hline & 1995 & & 1999 & & 2004 & & 2009 & \\
\hline HS2 & $\rho$ & $R^{2}$ & $\rho$ & $R^{2}$ & $\rho$ & $R^{2}$ & $\rho$ & $R^{2}$ \\
\hline 50 & $0.07 \pm 0.04$ & 0.291 & $0.03 \pm 0.05$ & 0.046 & $0.06 \pm 0.08$ & 0.052 & $0 \pm 0.06$ & 0 \\
\hline 51 & $0.04 \pm 0.03$ & 0.112 & $0.07 \pm 0.04$ & 0.143 & $0.04 \pm 0.03$ & 0.065 & $0.03 \pm 0.05$ & 0.042 \\
\hline 52 & $0.04 \pm 0.02$ & 0.11 & $0.04 \pm 0.03$ & 0.084 & $0.04 \pm 0.03$ & 0.063 & $0.02 \pm 0.04$ & 0.02 \\
\hline 53 & $0.06 \pm 0.05$ & 0.141 & $0.08 \pm 0.06$ & 0.123 & $0.08 \pm 0.06$ & 0.101 & $0.1 \pm 0.06$ & 0.204 \\
\hline 54 & $0.06 \pm 0.04$ & 0.112 & $0.08 \pm 0.03$ & 0.287 & $0.07 \pm 0.04$ & 0.155 & $0.05 \pm 0.04$ & 0.1 \\
\hline 55 & $0.05 \pm 0.04$ & 0.093 & $0.07 \pm 0.04$ & 0.135 & $0.07 \pm 0.04$ & 0.12 & $0.06 \pm 0.05$ & 0.093 \\
\hline 56 & $0.07 \pm 0.04$ & 0.163 & $0.07 \pm 0.04$ & 0.118 & $0.07 \pm 0.04$ & 0.135 & $0.05 \pm 0.03$ & 0.118 \\
\hline 57 & $0.04 \pm 0.05$ & 0.031 & $0.1 \pm 0.06$ & 0.154 & $0.05 \pm 0.05$ & 0.055 & $0.08 \pm 0.05$ & 0.139 \\
\hline 58 & $0.06 \pm 0.04$ & 0.119 & $0.05 \pm 0.06$ & 0.043 & $0.04 \pm 0.05$ & 0.043 & $0.02 \pm 0.04$ & 0.015 \\
\hline 59 & $0.07 \pm 0.04$ & 0.192 & $0.06 \pm 0.04$ & 0.151 & $0.06 \pm 0.05$ & 0.096 & $0.05 \pm 0.03$ & 0.161 \\
\hline 60 & $0.03 \pm 0.05$ & 0.03 & $0.01 \pm 0.06$ & 0.005 & $0.02 \pm 0.04$ & 0.01 & $0.02 \pm 0.05$ & 0.008 \\
\hline 61 & $0.04 \pm 0.04$ & 0.061 & $0.05 \pm 0.04$ & 0.071 & $0.04 \pm 0.03$ & 0.059 & $0.05 \pm 0.03$ & 0.079 \\
\hline 62 & $0.02 \pm 0.03$ & 0.014 & $0.03 \pm 0.03$ & 0.041 & $0.04 \pm 0.03$ & 0.052 & $0.04 \pm 0.03$ & 0.052 \\
\hline 63 & $0.06 \pm 0.04$ & 0.103 & $0.07 \pm 0.04$ & 0.147 & $0.07 \pm 0.04$ & 0.104 & $0.04 \pm 0.04$ & 0.048 \\
\hline 64 & $0.07 \pm 0.04$ & 0.138 & $0.09 \pm 0.05$ & 0.151 & $0.09 \pm 0.06$ & 0.111 & $0.1 \pm 0.05$ & 0.172 \\
\hline 65 & $0.07 \pm 0.04$ & 0.158 & $0.11 \pm 0.05$ & 0.207 & $0.09 \pm 0.06$ & 0.12 & $0.1 \pm 0.06$ & 0.131 \\
\hline 66 & $0.07 \pm 0.06$ & 0.135 & $0.08 \pm 0.07$ & 0.106 & $0.09 \pm 0.08$ & 0.106 & $0.12 \pm 0.08$ & 0.155 \\
\hline 67 & $0.08 \pm 0.06$ & 0.143 & $0.11 \pm 0.06$ & 0.239 & $0.08 \pm 0.07$ & 0.084 & $0.08 \pm 0.06$ & 0.144 \\
\hline 68 & $0.08 \pm 0.04$ & 0.237 & $0.09 \pm 0.04$ & 0.191 & $0.08 \pm 0.05$ & 0.127 & $0.05 \pm 0.04$ & 0.076 \\
\hline 69 & $0.05 \pm 0.04$ & 0.074 & $0.07 \pm 0.04$ & 0.134 & $0.07 \pm 0.05$ & 0.09 & $0.06 \pm 0.03$ & 0.148 \\
\hline 70 & $0.1 \pm 0.03$ & 0.312 & $0.07 \pm 0.05$ & 0.087 & $0.07 \pm 0.03$ & 0.169 & $0.06 \pm 0.04$ & 0.123 \\
\hline 71 & $-0.03 \pm 0.02$ & 0.1 & $-0.01 \pm 0.03$ & 0.011 & $-0.01 \pm 0.03$ & 0.006 & $0.01 \pm 0.02$ & 0.002 \\
\hline 72 & $0.06 \pm 0.03$ & 0.195 & $0.06 \pm 0.04$ & 0.126 & $0 \pm 0.03$ & 0 & $-0.04 \pm 0.04$ & 0.043 \\
\hline 73 & $0.06 \pm 0.03$ & 0.138 & $0.05 \pm 0.03$ & 0.079 & $0.04 \pm 0.03$ & 0.038 & $0.03 \pm 0.03$ & 0.039 \\
\hline 74 & $0.01 \pm 0.03$ & 0.005 & $0 \pm 0.02$ & 0 & $0 \pm 0.04$ & 0 & $0.01 \pm 0.03$ & 0.008 \\
\hline 75 & $-0.01 \pm 0.03$ & 0.006 & $0.01 \pm 0.03$ & 0.013 & $0 \pm 0.04$ & 0 & $0.01 \pm 0.05$ & 0.001 \\
\hline 76 & $0.04 \pm 0.02$ & 0.127 & $0.06 \pm 0.04$ & 0.108 & $0.03 \pm 0.03$ & 0.068 & $0.02 \pm 0.03$ & 0.014 \\
\hline 78 & $0.05 \pm 0.06$ & 0.066 & $0.04 \pm 0.06$ & 0.043 & $0.01 \pm 0.06$ & 0.005 & $-0.03 \pm 0.05$ & 0.035 \\
\hline 79 & $0.03 \pm 0.06$ & 0.018 & $0.05 \pm 0.06$ & 0.051 & $0.03 \pm 0.04$ & 0.055 & $0.01 \pm 0.04$ & 0.003 \\
\hline 80 & $0.01 \pm 0.05$ & 0.003 & $0.06 \pm 0.08$ & 0.058 & $0.04 \pm 0.06$ & 0.049 & $0.08 \pm 0.06$ & 0.15 \\
\hline 81 & $-0.01 \pm 0.04$ & 0.002 & $0.03 \pm 0.04$ & 0.058 & $0 \pm 0.04$ & 0 & $0.03 \pm 0.03$ & 0.06 \\
\hline 82 & $0.05 \pm 0.03$ & 0.096 & $0.04 \pm 0.03$ & 0.097 & $0.03 \pm 0.04$ & 0.032 & $0.03 \pm 0.03$ & 0.057 \\
\hline 83 & $0.08 \pm 0.03$ & 0.235 & $0.07 \pm 0.05$ & 0.101 & $0.06 \pm 0.04$ & 0.124 & $0.03 \pm 0.03$ & 0.049 \\
\hline 84 & $0.03 \pm 0.03$ & 0.05 & $0.05 \pm 0.02$ & 0.152 & $0.01 \pm 0.02$ & 0.012 & $-0.01 \pm 0.02$ & 0.009 \\
\hline 85 & $0.03 \pm 0.03$ & 0.046 & $0.03 \pm 0.03$ & 0.043 & $0 \pm 0.03$ & 0 & $-0.02 \pm 0.03$ & 0.019 \\
\hline 86 & $0.05 \pm 0.05$ & 0.074 & $0.05 \pm 0.06$ & 0.06 & $0.05 \pm 0.04$ & 0.106 & $0.02 \pm 0.04$ & 0.019 \\
\hline 87 & $0.05 \pm 0.04$ & 0.079 & $0.05 \pm 0.04$ & 0.067 & $0.03 \pm 0.03$ & 0.027 & $0.01 \pm 0.03$ & 0.003 \\
\hline 88 & $0 \pm 0.06$ & 0 & $0 \pm 0.05$ & 0 & $0.02 \pm 0.08$ & 0.004 & $0 \pm 0.04$ & 0.001 \\
\hline 89 & $-0.01 \pm 0.04$ & 0.003 & $-0.02 \pm 0.05$ & 0.011 & $0.01 \pm 0.04$ & 0.005 & $-0.01 \pm 0.04$ & 0.004 \\
\hline 90 & $-0.02 \pm 0.03$ & 0.012 & $0.01 \pm 0.03$ & 0.002 & $0 \pm 0.02$ & 0.001 & $0 \pm 0.02$ & 0.001 \\
\hline 91 & $0.03 \pm 0.04$ & 0.032 & $0.05 \pm 0.04$ & 0.067 & $0.02 \pm 0.04$ & 0.01 & $0.03 \pm 0.04$ & 0.018 \\
\hline 92 & $0.02 \pm 0.04$ & 0.031 & $0.05 \pm 0.04$ & 0.076 & $0.06 \pm 0.06$ & 0.071 & $0.06 \pm 0.04$ & 0.129 \\
\hline 93 & $0.02 \pm 0.08$ & 0.005 & $0 \pm 0.07$ & 0 & $0.08 \pm 0.07$ & 0.103 & $0.05 \pm 0.06$ & 0.054 \\
\hline 94 & $0.07 \pm 0.03$ & 0.17 & $0.06 \pm 0.03$ & 0.132 & $0.05 \pm 0.04$ & 0.07 & $0.05 \pm 0.03$ & 0.068 \\
\hline 95 & $0.06 \pm 0.04$ & 0.116 & $0.06 \pm 0.04$ & 0.118 & $0.06 \pm 0.05$ & 0.073 & $0.03 \pm 0.04$ & 0.03 \\
\hline 96 & $0.05 \pm 0.04$ & 0.089 & $0.07 \pm 0.04$ & 0.117 & $0.05 \pm 0.04$ & 0.063 & $0.05 \pm 0.03$ & 0.088 \\
\hline 97 & $0.02 \pm 0.04$ & 0.014 & $0.04 \pm 0.04$ & 0.064 & $0 \pm 0.05$ & 0 & $-0.02 \pm 0.04$ & 0.019 \\
\hline
\end{tabular}


Table of results for the $\sigma$ exponent for the exporters without RSP and RPP filters.

\begin{tabular}{|c|c|c|c|c|c|c|c|c|}
\hline & 1995 & & 1999 & & 2004 & & 2009 & \\
\hline HS2 & $\sigma$ & $R^{2}$ & $\sigma$ & $R^{2}$ & $\sigma$ & $R^{2}$ & $\sigma$ & $R^{2}$ \\
\hline 1 & $-0.52 \pm 0.06$ & 0.877 & $-0.46 \pm 0.05$ & 0.852 & $-0.44 \pm 0.05$ & 0.861 & $-0.49 \pm 0.05$ & 0.863 \\
\hline 2 & $-0.55 \pm 0.05$ & 0.906 & $-0.48 \pm 0.07$ & 0.822 & $-0.44 \pm 0.07$ & 0.735 & $-0.52 \pm 0.06$ & 0.858 \\
\hline 3 & $-0.44 \pm 0.04$ & 0.891 & $-0.4 \pm 0.04$ & 0.841 & $-0.37 \pm 0.04$ & 0.792 & $-0.39 \pm 0.05$ & 0.783 \\
\hline 4 & $-0.5 \pm 0.06$ & 0.822 & $-0.37 \pm 0.07$ & 0.675 & $-0.31 \pm 0.07$ & 0.569 & $-0.36 \pm 0.07$ & 0.677 \\
\hline 5 & $-0.52 \pm 0.05$ & 0.896 & $-0.51 \pm 0.05$ & 0.898 & $-0.49 \pm 0.05$ & 0.862 & $-0.52 \pm 0.05$ & 0.878 \\
\hline 6 & $-0.52 \pm 0.05$ & 0.888 & $-0.46 \pm 0.06$ & 0.807 & $-0.46 \pm 0.05$ & 0.83 & $-0.47 \pm 0.05$ & 0.849 \\
\hline 7 & $-0.45 \pm 0.05$ & 0.838 & $-0.45 \pm 0.05$ & 0.82 & $-0.38 \pm 0.06$ & 0.74 & $-0.4 \pm 0.05$ & 0.793 \\
\hline 8 & $-0.42 \pm 0.04$ & 0.866 & $-0.36 \pm 0.04$ & 0.815 & $-0.33 \pm 0.04$ & 0.746 & $-0.35 \pm 0.04$ & 0.765 \\
\hline 9 & $-0.38 \pm 0.04$ & 0.822 & $-0.32 \pm 0.04$ & 0.739 & $-0.32 \pm 0.04$ & 0.719 & $-0.31 \pm 0.04$ & 0.72 \\
\hline 10 & $-0.49 \pm 0.05$ & 0.874 & $-0.38 \pm 0.06$ & 0.772 & $-0.38 \pm 0.06$ & 0.761 & $-0.35 \pm 0.06$ & 0.692 \\
\hline 11 & $-0.54 \pm 0.07$ & 0.858 & $-0.51 \pm 0.06$ & 0.868 & $-0.38 \pm 0.07$ & 0.708 & $-0.38 \pm 0.07$ & 0.714 \\
\hline 12 & $-0.46 \pm 0.04$ & 0.868 & $-0.39 \pm 0.05$ & 0.762 & $-0.32 \pm 0.05$ & 0.719 & $-0.35 \pm 0.05$ & 0.752 \\
\hline 13 & $-0.52 \pm 0.08$ & 0.799 & $-0.48 \pm 0.07$ & 0.804 & $-0.41 \pm 0.06$ & 0.742 & $-0.4 \pm 0.06$ & 0.747 \\
\hline 14 & $-0.54 \pm 0.08$ & 0.837 & $-0.52 \pm 0.08$ & 0.798 & $-0.48 \pm 0.07$ & 0.821 & $-0.51 \pm 0.07$ & 0.851 \\
\hline 15 & $-0.46 \pm 0.06$ & 0.806 & $-0.41 \pm 0.08$ & 0.651 & $-0.39 \pm 0.07$ & 0.672 & $-0.39 \pm 0.05$ & 0.765 \\
\hline 16 & $-0.48 \pm 0.04$ & 0.89 & $-0.36 \pm 0.04$ & 0.821 & $-0.33 \pm 0.05$ & 0.755 & $-0.37 \pm 0.05$ & 0.783 \\
\hline 17 & $-0.39 \pm 0.06$ & 0.758 & $-0.31 \pm 0.06$ & 0.635 & $-0.29 \pm 0.05$ & 0.65 & $-0.31 \pm 0.05$ & 0.708 \\
\hline 18 & $-0.5 \pm 0.08$ & 0.763 & $-0.39 \pm 0.08$ & 0.665 & $-0.37 \pm 0.08$ & 0.596 & $-0.41 \pm 0.06$ & 0.759 \\
\hline 19 & $-0.42 \pm 0.06$ & 0.799 & $-0.37 \pm 0.06$ & 0.705 & $-0.3 \pm 0.06$ & 0.6 & $-0.34 \pm 0.06$ & 0.677 \\
\hline 20 & $-0.42 \pm 0.05$ & 0.795 & $-0.37 \pm 0.05$ & 0.761 & $-0.33 \pm 0.05$ & 0.648 & $-0.34 \pm 0.05$ & 0.712 \\
\hline 21 & $-0.41 \pm 0.06$ & 0.722 & $-0.34 \pm 0.06$ & 0.68 & $-0.3 \pm 0.05$ & 0.674 & $-0.34 \pm 0.05$ & 0.723 \\
\hline 22 & $-0.47 \pm 0.06$ & 0.798 & $-0.39 \pm 0.05$ & 0.737 & $-0.33 \pm 0.06$ & 0.63 & $-0.38 \pm 0.05$ & 0.73 \\
\hline 23 & $-0.38 \pm 0.07$ & 0.707 & $-0.35 \pm 0.06$ & 0.688 & $-0.29 \pm 0.06$ & 0.589 & $-0.28 \pm 0.08$ & 0.487 \\
\hline 24 & $-0.43 \pm 0.08$ & 0.73 & $-0.33 \pm 0.07$ & 0.627 & $-0.3 \pm 0.06$ & 0.638 & $-0.31 \pm 0.05$ & 0.674 \\
\hline 25 & $-0.37 \pm 0.05$ & 0.746 & $-0.36 \pm 0.05$ & 0.752 & $-0.3 \pm 0.05$ & 0.672 & $-0.34 \pm 0.05$ & 0.69 \\
\hline 26 & $-0.54 \pm 0.07$ & 0.861 & $-0.56 \pm 0.05$ & 0.908 & $-0.53 \pm 0.06$ & 0.862 & $-0.52 \pm 0.05$ & 0.893 \\
\hline 27 & $-0.42 \pm 0.06$ & 0.723 & $-0.34 \pm 0.04$ & 0.753 & $-0.27 \pm 0.05$ & 0.613 & $-0.31 \pm 0.05$ & 0.676 \\
\hline 28 & $-0.38 \pm 0.05$ & 0.75 & $-0.34 \pm 0.05$ & 0.748 & $-0.27 \pm 0.05$ & 0.642 & $-0.31 \pm 0.05$ & 0.694 \\
\hline 29 & $-0.41 \pm 0.05$ & 0.797 & $-0.4 \pm 0.05$ & 0.722 & $-0.32 \pm 0.05$ & 0.669 & $-0.36 \pm 0.05$ & 0.735 \\
\hline 30 & $-0.42 \pm 0.07$ & 0.701 & $-0.35 \pm 0.06$ & 0.619 & $-0.3 \pm 0.06$ & 0.608 & $-0.34 \pm 0.05$ & 0.677 \\
\hline 31 & $-0.41 \pm 0.06$ & 0.809 & $-0.36 \pm 0.06$ & 0.743 & $-0.3 \pm 0.07$ & 0.631 & $-0.38 \pm 0.05$ & 0.786 \\
\hline 32 & $-0.36 \pm 0.06$ & 0.719 & $-0.35 \pm 0.06$ & 0.659 & $-0.27 \pm 0.06$ & 0.517 & $-0.31 \pm 0.05$ & 0.649 \\
\hline 33 & $-0.5 \pm 0.06$ & 0.787 & $-0.39 \pm 0.06$ & 0.684 & $-0.36 \pm 0.05$ & 0.694 & $-0.36 \pm 0.05$ & 0.723 \\
\hline 34 & $-0.39 \pm 0.06$ & 0.708 & $-0.29 \pm 0.06$ & 0.593 & $-0.29 \pm 0.06$ & 0.586 & $-0.32 \pm 0.06$ & 0.634 \\
\hline 35 & $-0.39 \pm 0.06$ & 0.786 & $-0.3 \pm 0.06$ & 0.657 & $-0.27 \pm 0.05$ & 0.622 & $-0.31 \pm 0.05$ & 0.721 \\
\hline 36 & $-0.35 \pm 0.07$ & 0.749 & $-0.26 \pm 0.08$ & 0.533 & $-0.26 \pm 0.06$ & 0.632 & $-0.3 \pm 0.06$ & 0.686 \\
\hline 37 & $-0.54 \pm 0.08$ & 0.778 & $-0.44 \pm 0.08$ & 0.695 & $-0.38 \pm 0.07$ & 0.643 & $-0.46 \pm 0.08$ & 0.729 \\
\hline 38 & $-0.4 \pm 0.05$ & 0.795 & $-0.34 \pm 0.07$ & 0.581 & $-0.31 \pm 0.06$ & 0.61 & $-0.35 \pm 0.05$ & 0.678 \\
\hline 39 & $-0.41 \pm 0.06$ & 0.685 & $-0.35 \pm 0.05$ & 0.674 & $-0.35 \pm 0.04$ & 0.729 & $-0.34 \pm 0.05$ & 0.665 \\
\hline 40 & $-0.41 \pm 0.06$ & 0.685 & $-0.36 \pm 0.06$ & 0.662 & $-0.34 \pm 0.05$ & 0.649 & $-0.37 \pm 0.05$ & 0.677 \\
\hline 41 & $-0.41 \pm 0.05$ & 0.841 & $-0.36 \pm 0.06$ & 0.698 & $-0.38 \pm 0.05$ & 0.742 & $-0.42 \pm 0.06$ & 0.787 \\
\hline 42 & $-0.46 \pm 0.05$ & 0.818 & $-0.41 \pm 0.05$ & 0.771 & $-0.37 \pm 0.06$ & 0.676 & $-0.41 \pm 0.05$ & 0.775 \\
\hline 43 & $-0.4 \pm 0.06$ & 0.794 & $-0.41 \pm 0.06$ & 0.835 & $-0.41 \pm 0.05$ & 0.831 & $-0.47 \pm 0.05$ & 0.874 \\
\hline 44 & $-0.38 \pm 0.05$ & 0.772 & $-0.33 \pm 0.04$ & 0.722 & $-0.31 \pm 0.04$ & 0.736 & $-0.33 \pm 0.04$ & 0.769 \\
\hline 45 & $-0.51 \pm 0.1$ & 0.793 & $-0.5 \pm 0.09$ & 0.776 & $-0.51 \pm 0.08$ & 0.839 & $-0.49 \pm 0.09$ & 0.781 \\
\hline 46 & $-0.52 \pm 0.07$ & 0.85 & $-0.45 \pm 0.08$ & 0.728 & $-0.46 \pm 0.09$ & 0.706 & $-0.5 \pm 0.08$ & 0.796 \\
\hline 47 & $-0.47 \pm 0.06$ & 0.839 & $-0.48 \pm 0.08$ & 0.786 & $-0.45 \pm 0.07$ & 0.804 & $-0.46 \pm 0.07$ & 0.817 \\
\hline 48 & $-0.41 \pm 0.06$ & 0.711 & $-0.32 \pm 0.05$ & 0.644 & $-0.29 \pm 0.05$ & 0.577 & $-0.32 \pm 0.05$ & 0.663 \\
\hline 49 & $-0.48 \pm 0.07$ & 0.741 & $-0.41 \pm 0.06$ & 0.713 & $-0.34 \pm 0.06$ & 0.586 & $-0.36 \pm 0.06$ & 0.616 \\
\hline
\end{tabular}


Table of results for the $\sigma$ exponent for the exporters without RSP and RPP filters (cont.)

\begin{tabular}{|c|c|c|c|c|c|c|c|c|}
\hline & 1995 & & 1999 & & 2004 & & 2009 & \\
\hline HS2 & $\sigma$ & $R^{2}$ & $\sigma$ & $R^{2}$ & $\sigma$ & $R^{2}$ & $\sigma$ & $R^{2}$ \\
\hline 50 & $-0.6 \pm 0.12$ & 0.748 & $-0.56 \pm 0.13$ & 0.671 & $-0.57 \pm 0.09$ & 0.794 & $-0.59 \pm 0.1$ & 0.789 \\
\hline 51 & $-0.42 \pm 0.05$ & 0.82 & $-0.43 \pm 0.05$ & 0.838 & $-0.38 \pm 0.06$ & 0.777 & $-0.41 \pm 0.06$ & 0.79 \\
\hline 52 & $-0.41 \pm 0.05$ & 0.81 & $-0.39 \pm 0.04$ & 0.811 & $-0.38 \pm 0.04$ & 0.814 & $-0.41 \pm 0.05$ & 0.781 \\
\hline 53 & $-0.45 \pm 0.05$ & 0.872 & $-0.38 \pm 0.06$ & 0.741 & $-0.34 \pm 0.06$ & 0.754 & $-0.36 \pm 0.06$ & 0.749 \\
\hline 54 & $-0.41 \pm 0.05$ & 0.813 & $-0.38 \pm 0.04$ & 0.824 & $-0.38 \pm 0.05$ & 0.75 & $-0.34 \pm 0.05$ & 0.746 \\
\hline 55 & $-0.41 \pm 0.05$ & 0.817 & $-0.36 \pm 0.05$ & 0.786 & $-0.36 \pm 0.06$ & 0.698 & $-0.36 \pm 0.05$ & 0.726 \\
\hline 56 & $-0.39 \pm 0.06$ & 0.781 & $-0.36 \pm 0.05$ & 0.752 & $-0.31 \pm 0.05$ & 0.707 & $-0.34 \pm 0.05$ & 0.765 \\
\hline 57 & $-0.45 \pm 0.05$ & 0.827 & $-0.41 \pm 0.06$ & 0.747 & $-0.39 \pm 0.05$ & 0.772 & $-0.4 \pm 0.05$ & 0.805 \\
\hline 58 & $-0.41 \pm 0.06$ & 0.749 & $-0.38 \pm 0.05$ & 0.787 & $-0.38 \pm 0.05$ & 0.758 & $-0.41 \pm 0.05$ & 0.786 \\
\hline 59 & $-0.36 \pm 0.07$ & 0.707 & $-0.35 \pm 0.06$ & 0.71 & $-0.32 \pm 0.06$ & 0.641 & $-0.39 \pm 0.06$ & 0.76 \\
\hline 60 & $-0.44 \pm 0.06$ & 0.801 & $-0.37 \pm 0.07$ & 0.682 & $-0.39 \pm 0.06$ & 0.768 & $-0.41 \pm 0.06$ & 0.756 \\
\hline 61 & $-0.42 \pm 0.05$ & 0.801 & $-0.38 \pm 0.04$ & 0.771 & $-0.33 \pm 0.04$ & 0.728 & $-0.33 \pm 0.04$ & 0.727 \\
\hline 62 & $-0.44 \pm 0.05$ & 0.802 & $-0.38 \pm 0.04$ & 0.77 & $-0.32 \pm 0.04$ & 0.693 & $-0.33 \pm 0.04$ & 0.724 \\
\hline 63 & $-0.44 \pm 0.06$ & 0.764 & $-0.38 \pm 0.05$ & 0.71 & $-0.33 \pm 0.05$ & 0.692 & $-0.38 \pm 0.05$ & 0.741 \\
\hline 64 & $-0.39 \pm 0.05$ & 0.747 & $-0.38 \pm 0.05$ & 0.748 & $-0.32 \pm 0.05$ & 0.676 & $-0.36 \pm 0.04$ & 0.761 \\
\hline 65 & $-0.46 \pm 0.06$ & 0.799 & $-0.36 \pm 0.05$ & 0.745 & $-0.34 \pm 0.05$ & 0.737 & $-0.38 \pm 0.05$ & 0.794 \\
\hline 66 & $-0.55 \pm 0.07$ & 0.887 & $-0.48 \pm 0.07$ & 0.814 & $-0.37 \pm 0.07$ & 0.735 & $-0.36 \pm 0.06$ & 0.824 \\
\hline 67 & $-0.53 \pm 0.08$ & 0.823 & $-0.38 \pm 0.1$ & 0.616 & $-0.43 \pm 0.08$ & 0.717 & $-0.51 \pm 0.07$ & 0.808 \\
\hline 68 & $-0.42 \pm 0.06$ & 0.739 & $-0.34 \pm 0.06$ & 0.642 & $-0.32 \pm 0.05$ & 0.649 & $-0.35 \pm 0.05$ & 0.691 \\
\hline 69 & $-0.44 \pm 0.06$ & 0.766 & $-0.37 \pm 0.06$ & 0.688 & $-0.34 \pm 0.05$ & 0.67 & $-0.37 \pm 0.06$ & 0.704 \\
\hline 70 & $-0.36 \pm 0.06$ & 0.695 & $-0.36 \pm 0.06$ & 0.618 & $-0.3 \pm 0.05$ & 0.628 & $-0.35 \pm 0.05$ & 0.684 \\
\hline 71 & $-0.53 \pm 0.06$ & 0.805 & $-0.51 \pm 0.06$ & 0.809 & $-0.49 \pm 0.06$ & 0.779 & $-0.51 \pm 0.05$ & 0.831 \\
\hline 72 & $-0.43 \pm 0.06$ & 0.752 & $-0.31 \pm 0.06$ & 0.598 & $-0.33 \pm 0.06$ & 0.625 & $-0.4 \pm 0.05$ & 0.732 \\
\hline 73 & $-0.41 \pm 0.07$ & 0.663 & $-0.34 \pm 0.06$ & 0.581 & $-0.33 \pm 0.05$ & 0.623 & $-0.38 \pm 0.05$ & 0.718 \\
\hline 74 & $-0.51 \pm 0.06$ & 0.825 & $-0.44 \pm 0.05$ & 0.795 & $-0.39 \pm 0.06$ & 0.684 & $-0.44 \pm 0.06$ & 0.747 \\
\hline 75 & $-0.5 \pm 0.1$ & 0.732 & $-0.53 \pm 0.09$ & 0.774 & $-0.47 \pm 0.09$ & 0.738 & $-0.48 \pm 0.08$ & 0.784 \\
\hline 76 & $-0.43 \pm 0.05$ & 0.778 & $-0.35 \pm 0.06$ & 0.662 & $-0.34 \pm 0.05$ & 0.669 & $-0.35 \pm 0.05$ & 0.657 \\
\hline 78 & $-0.6 \pm 0.07$ & 0.897 & $-0.56 \pm 0.08$ & 0.87 & $-0.5 \pm 0.08$ & 0.795 & $-0.61 \pm 0.06$ & 0.912 \\
\hline 79 & $-0.47 \pm 0.09$ & 0.727 & $-0.42 \pm 0.08$ & 0.708 & $-0.36 \pm 0.08$ & 0.665 & $-0.42 \pm 0.06$ & 0.787 \\
\hline 80 & $-0.51 \pm 0.1$ & 0.757 & $-0.43 \pm 0.09$ & 0.719 & $-0.42 \pm 0.1$ & 0.668 & $-0.45 \pm 0.07$ & 0.834 \\
\hline 81 & $-0.55 \pm 0.08$ & 0.832 & $-0.51 \pm 0.07$ & 0.814 & $-0.52 \pm 0.07$ & 0.82 & $-0.49 \pm 0.06$ & 0.832 \\
\hline 82 & $-0.48 \pm 0.06$ & 0.756 & $-0.35 \pm 0.07$ & 0.575 & $-0.4 \pm 0.06$ & 0.695 & $-0.41 \pm 0.06$ & 0.682 \\
\hline 83 & $-0.44 \pm 0.06$ & 0.773 & $-0.35 \pm 0.06$ & 0.636 & $-0.35 \pm 0.07$ & 0.583 & $-0.35 \pm 0.06$ & 0.654 \\
\hline 84 & $-0.48 \pm 0.06$ & 0.722 & $-0.43 \pm 0.05$ & 0.71 & $-0.38 \pm 0.04$ & 0.712 & $-0.4 \pm 0.05$ & 0.711 \\
\hline 85 & $-0.49 \pm 0.05$ & 0.774 & $-0.41 \pm 0.06$ & 0.659 & $-0.41 \pm 0.05$ & 0.73 & $-0.44 \pm 0.05$ & 0.743 \\
\hline 86 & $-0.52 \pm 0.06$ & 0.864 & $-0.5 \pm 0.06$ & 0.856 & $-0.45 \pm 0.06$ & 0.819 & $-0.5 \pm 0.06$ & 0.838 \\
\hline 87 & $-0.49 \pm 0.06$ & 0.757 & $-0.37 \pm 0.07$ & 0.569 & $-0.42 \pm 0.05$ & 0.762 & $-0.44 \pm 0.05$ & 0.769 \\
\hline 88 & $-0.64 \pm 0.06$ & 0.884 & $-0.52 \pm 0.06$ & 0.832 & $-0.51 \pm 0.06$ & 0.822 & $-0.53 \pm 0.06$ & 0.818 \\
\hline 89 & $-0.54 \pm 0.07$ & 0.854 & $-0.5 \pm 0.07$ & 0.81 & $-0.45 \pm 0.06$ & 0.775 & $-0.47 \pm 0.06$ & 0.82 \\
\hline 90 & $-0.53 \pm 0.06$ & 0.775 & $-0.43 \pm 0.07$ & 0.611 & $-0.42 \pm 0.05$ & 0.709 & $-0.45 \pm 0.05$ & 0.742 \\
\hline 91 & $-0.55 \pm 0.07$ & 0.819 & $-0.46 \pm 0.09$ & 0.663 & $-0.5 \pm 0.07$ & 0.786 & $-0.56 \pm 0.06$ & 0.859 \\
\hline 92 & $-0.54 \pm 0.07$ & 0.812 & $-0.51 \pm 0.08$ & 0.76 & $-0.51 \pm 0.07$ & 0.801 & $-0.49 \pm 0.06$ & 0.804 \\
\hline 93 & $-0.56 \pm 0.08$ & 0.834 & $-0.53 \pm 0.08$ & 0.804 & $-0.45 \pm 0.08$ & 0.759 & $-0.47 \pm 0.09$ & 0.712 \\
\hline 94 & $-0.45 \pm 0.06$ & 0.748 & $-0.38 \pm 0.06$ & 0.62 & $-0.34 \pm 0.06$ & 0.585 & $-0.38 \pm 0.05$ & 0.715 \\
\hline 95 & $-0.48 \pm 0.06$ & 0.791 & $-0.42 \pm 0.06$ & 0.733 & $-0.38 \pm 0.05$ & 0.711 & $-0.39 \pm 0.06$ & 0.701 \\
\hline 96 & $-0.46 \pm 0.06$ & 0.767 & $-0.34 \pm 0.07$ & 0.572 & $-0.35 \pm 0.05$ & 0.718 & $-0.37 \pm 0.05$ & 0.723 \\
\hline 97 & $-0.57 \pm 0.06$ & 0.876 & $-0.51 \pm 0.05$ & 0.865 & $-0.49 \pm 0.05$ & 0.848 & $-0.54 \pm 0.05$ & 0.867 \\
\hline
\end{tabular}


Table of results for the $\sigma$ exponent for the importers without RSP and RPP filters.

\begin{tabular}{|c|c|c|c|c|c|c|c|c|}
\hline & 1995 & & 1999 & & 2004 & & 2009 & \\
\hline HS2 & $\sigma$ & $R^{2}$ & $\sigma$ & $R^{2}$ & $\sigma$ & $R^{2}$ & $\sigma$ & $R^{2}$ \\
\hline 1 & $-0.46 \pm 0.07$ & 0.763 & $-0.5 \pm 0.06$ & 0.831 & $-0.45 \pm 0.05$ & 0.833 & $-0.44 \pm 0.06$ & 0.811 \\
\hline 2 & $-0.44 \pm 0.05$ & 0.847 & $-0.38 \pm 0.05$ & 0.828 & $-0.38 \pm 0.04$ & 0.833 & $-0.39 \pm 0.05$ & 0.822 \\
\hline 3 & $-0.52 \pm 0.05$ & 0.845 & $-0.43 \pm 0.04$ & 0.867 & $-0.4 \pm 0.04$ & 0.868 & $-0.37 \pm 0.05$ & 0.783 \\
\hline 4 & $-0.37 \pm 0.04$ & 0.834 & $-0.33 \pm 0.03$ & 0.84 & $-0.32 \pm 0.03$ & 0.871 & $-0.31 \pm 0.04$ & 0.8 \\
\hline 5 & $-0.53 \pm 0.05$ & 0.886 & $-0.49 \pm 0.05$ & 0.889 & $-0.44 \pm 0.05$ & 0.838 & $-0.44 \pm 0.05$ & 0.866 \\
\hline 6 & $-0.55 \pm 0.05$ & 0.905 & $-0.46 \pm 0.05$ & 0.881 & $-0.45 \pm 0.04$ & 0.872 & $-0.41 \pm 0.05$ & 0.838 \\
\hline 7 & $-0.45 \pm 0.04$ & 0.869 & $-0.4 \pm 0.04$ & 0.858 & $-0.36 \pm 0.05$ & 0.777 & $-0.39 \pm 0.05$ & 0.79 \\
\hline 8 & $-0.44 \pm 0.05$ & 0.831 & $-0.38 \pm 0.05$ & 0.779 & $-0.32 \pm 0.06$ & 0.606 & $-0.35 \pm 0.04$ & 0.773 \\
\hline 9 & $-0.43 \pm 0.05$ & 0.793 & $-0.37 \pm 0.04$ & 0.815 & $-0.33 \pm 0.05$ & 0.686 & $-0.35 \pm 0.04$ & 0.795 \\
\hline 10 & $-0.46 \pm 0.06$ & 0.839 & $-0.37 \pm 0.05$ & 0.802 & $-0.32 \pm 0.07$ & 0.571 & $-0.34 \pm 0.06$ & 0.716 \\
\hline 11 & $-0.42 \pm 0.06$ & 0.808 & $-0.37 \pm 0.04$ & 0.823 & $-0.37 \pm 0.04$ & 0.823 & $-0.35 \pm 0.06$ & 0.684 \\
\hline 12 & $-0.43 \pm 0.05$ & 0.833 & $-0.36 \pm 0.04$ & 0.804 & $-0.34 \pm 0.04$ & 0.792 & $-0.31 \pm 0.07$ & 0.529 \\
\hline 13 & $-0.43 \pm 0.06$ & 0.787 & $-0.39 \pm 0.04$ & 0.857 & $-0.38 \pm 0.04$ & 0.864 & $-0.37 \pm 0.07$ & 0.691 \\
\hline 14 & $-0.46 \pm 0.06$ & 0.843 & $-0.47 \pm 0.06$ & 0.846 & $-0.44 \pm 0.06$ & 0.833 & $-0.5 \pm 0.06$ & 0.863 \\
\hline 15 & $-0.39 \pm 0.04$ & 0.888 & $-0.32 \pm 0.04$ & 0.82 & $-0.33 \pm 0.03$ & 0.882 & $-0.32 \pm 0.04$ & 0.745 \\
\hline 16 & $-0.42 \pm 0.06$ & 0.786 & $-0.36 \pm 0.05$ & 0.778 & $-0.34 \pm 0.05$ & 0.723 & $-0.36 \pm 0.05$ & 0.743 \\
\hline 17 & $-0.33 \pm 0.05$ & 0.705 & $-0.3 \pm 0.03$ & 0.805 & $-0.27 \pm 0.04$ & 0.651 & $-0.28 \pm 0.04$ & 0.684 \\
\hline 18 & $-0.42 \pm 0.04$ & 0.906 & $-0.39 \pm 0.04$ & 0.886 & $-0.35 \pm 0.04$ & 0.85 & $-0.36 \pm 0.04$ & 0.786 \\
\hline 19 & $-0.34 \pm 0.04$ & 0.809 & $-0.31 \pm 0.03$ & 0.829 & $-0.3 \pm 0.03$ & 0.831 & $-0.3 \pm 0.03$ & 0.814 \\
\hline 20 & $-0.38 \pm 0.04$ & 0.795 & $-0.32 \pm 0.04$ & 0.753 & $-0.29 \pm 0.04$ & 0.715 & $-0.31 \pm 0.04$ & 0.792 \\
\hline 21 & $-0.36 \pm 0.04$ & 0.82 & $-0.3 \pm 0.03$ & 0.82 & $-0.27 \pm 0.03$ & 0.765 & $-0.28 \pm 0.03$ & 0.775 \\
\hline 22 & $-0.35 \pm 0.03$ & 0.853 & $-0.29 \pm 0.03$ & 0.798 & $-0.28 \pm 0.03$ & 0.813 & $-0.32 \pm 0.03$ & 0.867 \\
\hline 23 & $-0.39 \pm 0.05$ & 0.801 & $-0.33 \pm 0.05$ & 0.756 & $-0.31 \pm 0.04$ & 0.755 & $-0.29 \pm 0.06$ & 0.588 \\
\hline 24 & $-0.41 \pm 0.05$ & 0.82 & $-0.34 \pm 0.05$ & 0.748 & $-0.33 \pm 0.04$ & 0.798 & $-0.29 \pm 0.06$ & 0.564 \\
\hline 25 & $-0.35 \pm 0.04$ & 0.801 & $-0.31 \pm 0.05$ & 0.701 & $-0.3 \pm 0.04$ & 0.738 & $-0.31 \pm 0.05$ & 0.689 \\
\hline 26 & $-0.51 \pm 0.06$ & 0.879 & $-0.49 \pm 0.05$ & 0.865 & $-0.45 \pm 0.04$ & 0.895 & $-0.48 \pm 0.05$ & 0.875 \\
\hline 27 & $-0.36 \pm 0.04$ & 0.816 & $-0.29 \pm 0.04$ & 0.721 & $-0.23 \pm 0.03$ & 0.689 & $-0.29 \pm 0.03$ & 0.783 \\
\hline 28 & $-0.34 \pm 0.04$ & 0.77 & $-0.28 \pm 0.03$ & 0.807 & $-0.26 \pm 0.04$ & 0.721 & $-0.27 \pm 0.04$ & 0.706 \\
\hline 29 & $-0.37 \pm 0.04$ & 0.848 & $-0.3 \pm 0.04$ & 0.779 & $-0.27 \pm 0.03$ & 0.75 & $-0.3 \pm 0.04$ & 0.765 \\
\hline 30 & $-0.3 \pm 0.02$ & 0.935 & $-0.27 \pm 0.02$ & 0.881 & $-0.24 \pm 0.03$ & 0.774 & $-0.25 \pm 0.03$ & 0.715 \\
\hline 31 & $-0.39 \pm 0.05$ & 0.843 & $-0.33 \pm 0.04$ & 0.828 & $-0.3 \pm 0.04$ & 0.779 & $-0.33 \pm 0.05$ & 0.751 \\
\hline 32 & $-0.33 \pm 0.03$ & 0.845 & $-0.28 \pm 0.04$ & 0.764 & $-0.24 \pm 0.04$ & 0.638 & $-0.28 \pm 0.03$ & 0.809 \\
\hline 33 & $-0.35 \pm 0.03$ & 0.855 & $-0.3 \pm 0.04$ & 0.776 & $-0.28 \pm 0.03$ & 0.778 & $-0.3 \pm 0.04$ & 0.739 \\
\hline 34 & $-0.35 \pm 0.04$ & 0.809 & $-0.28 \pm 0.04$ & 0.743 & $-0.26 \pm 0.03$ & 0.791 & $-0.26 \pm 0.04$ & 0.71 \\
\hline 35 & $-0.39 \pm 0.04$ & 0.863 & $-0.31 \pm 0.04$ & 0.791 & $-0.29 \pm 0.04$ & 0.736 & $-0.28 \pm 0.05$ & 0.613 \\
\hline 36 & $-0.34 \pm 0.08$ & 0.631 & $-0.32 \pm 0.08$ & 0.575 & $-0.38 \pm 0.07$ & 0.705 & $-0.37 \pm 0.08$ & 0.631 \\
\hline 37 & $-0.4 \pm 0.03$ & 0.932 & $-0.33 \pm 0.04$ & 0.828 & $-0.29 \pm 0.04$ & 0.775 & $-0.35 \pm 0.04$ & 0.853 \\
\hline 38 & $-0.33 \pm 0.03$ & 0.886 & $-0.3 \pm 0.03$ & 0.817 & $-0.26 \pm 0.02$ & 0.832 & $-0.28 \pm 0.04$ & 0.735 \\
\hline 39 & $-0.3 \pm 0.02$ & 0.883 & $-0.26 \pm 0.02$ & 0.844 & $-0.23 \pm 0.02$ & 0.829 & $-0.26 \pm 0.02$ & 0.817 \\
\hline 40 & $-0.32 \pm 0.02$ & 0.896 & $-0.27 \pm 0.02$ & 0.87 & $-0.24 \pm 0.02$ & 0.876 & $-0.27 \pm 0.02$ & 0.866 \\
\hline 41 & $-0.44 \pm 0.05$ & 0.833 & $-0.36 \pm 0.06$ & 0.729 & $-0.38 \pm 0.05$ & 0.816 & $-0.4 \pm 0.05$ & 0.792 \\
\hline 42 & $-0.38 \pm 0.06$ & 0.686 & $-0.33 \pm 0.04$ & 0.747 & $-0.31 \pm 0.04$ & 0.734 & $-0.32 \pm 0.04$ & 0.783 \\
\hline 43 & $-0.37 \pm 0.08$ & 0.69 & $-0.43 \pm 0.08$ & 0.74 & $-0.36 \pm 0.07$ & 0.676 & $-0.44 \pm 0.07$ & 0.757 \\
\hline 44 & $-0.4 \pm 0.04$ & 0.836 & $-0.35 \pm 0.03$ & 0.828 & $-0.31 \pm 0.03$ & 0.784 & $-0.33 \pm 0.04$ & 0.748 \\
\hline 45 & $-0.43 \pm 0.07$ & 0.805 & $-0.35 \pm 0.08$ & 0.656 & $-0.34 \pm 0.07$ & 0.691 & $-0.4 \pm 0.07$ & 0.756 \\
\hline 46 & $-0.46 \pm 0.07$ & 0.829 & $-0.41 \pm 0.1$ & 0.593 & $-0.42 \pm 0.05$ & 0.841 & $-0.4 \pm 0.07$ & 0.709 \\
\hline 47 & $-0.48 \pm 0.06$ & 0.849 & $-0.44 \pm 0.05$ & 0.851 & $-0.42 \pm 0.05$ & 0.842 & $-0.38 \pm 0.07$ & 0.739 \\
\hline 48 & $-0.32 \pm 0.03$ & 0.871 & $-0.26 \pm 0.03$ & 0.817 & $-0.24 \pm 0.03$ & 0.727 & $-0.26 \pm 0.03$ & 0.796 \\
\hline 49 & $-0.33 \pm 0.03$ & 0.859 & $-0.3 \pm 0.03$ & 0.866 & $-0.27 \pm 0.03$ & 0.828 & $-0.3 \pm 0.03$ & 0.823 \\
\hline
\end{tabular}


Table of results for the $\sigma$ exponent for the importers without RSP and RPP filters (cont.)

\begin{tabular}{|c|c|c|c|c|c|c|c|c|}
\hline & 1995 & & 1999 & & 2004 & & 2009 & \\
\hline HS2 & $\sigma$ & $R^{2}$ & $\sigma$ & $R^{2}$ & $\sigma$ & $R^{2}$ & $\sigma$ & $R^{2}$ \\
\hline 50 & $-0.39 \pm 0.06$ & 0.844 & $-0.36 \pm 0.05$ & 0.843 & $-0.39 \pm 0.06$ & 0.82 & $-0.41 \pm 0.05$ & 0.861 \\
\hline 51 & $-0.41 \pm 0.05$ & 0.816 & $-0.38 \pm 0.06$ & 0.753 & $-0.32 \pm 0.06$ & 0.662 & $-0.35 \pm 0.06$ & 0.735 \\
\hline 52 & $-0.39 \pm 0.04$ & 0.85 & $-0.34 \pm 0.04$ & 0.785 & $-0.35 \pm 0.04$ & 0.777 & $-0.35 \pm 0.04$ & 0.8 \\
\hline 53 & $-0.37 \pm 0.07$ & 0.755 & $-0.35 \pm 0.06$ & 0.689 & $-0.31 \pm 0.07$ & 0.575 & $-0.28 \pm 0.08$ & 0.474 \\
\hline 54 & $-0.35 \pm 0.04$ & 0.808 & $-0.31 \pm 0.04$ & 0.81 & $-0.29 \pm 0.05$ & 0.65 & $-0.32 \pm 0.06$ & 0.668 \\
\hline 55 & $-0.35 \pm 0.04$ & 0.806 & $-0.33 \pm 0.05$ & 0.72 & $-0.29 \pm 0.05$ & 0.652 & $-0.31 \pm 0.06$ & 0.626 \\
\hline 56 & $-0.35 \pm 0.04$ & 0.824 & $-0.3 \pm 0.04$ & 0.763 & $-0.28 \pm 0.05$ & 0.655 & $-0.32 \pm 0.04$ & 0.778 \\
\hline 57 & $-0.44 \pm 0.05$ & 0.853 & $-0.32 \pm 0.06$ & 0.706 & $-0.34 \pm 0.04$ & 0.771 & $-0.36 \pm 0.05$ & 0.776 \\
\hline 58 & $-0.36 \pm 0.04$ & 0.831 & $-0.34 \pm 0.05$ & 0.756 & $-0.32 \pm 0.04$ & 0.784 & $-0.35 \pm 0.04$ & 0.792 \\
\hline 59 & $-0.32 \pm 0.04$ & 0.834 & $-0.35 \pm 0.03$ & 0.871 & $-0.27 \pm 0.05$ & 0.64 & $-0.31 \pm 0.04$ & 0.789 \\
\hline 60 & $-0.4 \pm 0.05$ & 0.815 & $-0.36 \pm 0.05$ & 0.805 & $-0.37 \pm 0.04$ & 0.831 & $-0.36 \pm 0.05$ & 0.781 \\
\hline 61 & $-0.38 \pm 0.05$ & 0.779 & $-0.32 \pm 0.04$ & 0.716 & $-0.31 \pm 0.04$ & 0.693 & $-0.31 \pm 0.05$ & 0.661 \\
\hline 62 & $-0.38 \pm 0.04$ & 0.778 & $-0.31 \pm 0.04$ & 0.714 & $-0.3 \pm 0.04$ & 0.721 & $-0.29 \pm 0.04$ & 0.67 \\
\hline 63 & $-0.33 \pm 0.03$ & 0.839 & $-0.28 \pm 0.03$ & 0.774 & $-0.26 \pm 0.03$ & 0.757 & $-0.28 \pm 0.03$ & 0.797 \\
\hline 64 & $-0.34 \pm 0.04$ & 0.799 & $-0.3 \pm 0.04$ & 0.707 & $-0.28 \pm 0.04$ & 0.713 & $-0.29 \pm 0.04$ & 0.732 \\
\hline 65 & $-0.41 \pm 0.06$ & 0.777 & $-0.33 \pm 0.07$ & 0.573 & $-0.3 \pm 0.07$ & 0.529 & $-0.31 \pm 0.06$ & 0.644 \\
\hline 66 & $-0.44 \pm 0.06$ & 0.845 & $-0.37 \pm 0.06$ & 0.745 & $-0.37 \pm 0.08$ & 0.658 & $-0.39 \pm 0.08$ & 0.685 \\
\hline 67 & $-0.42 \pm 0.07$ & 0.778 & $-0.37 \pm 0.07$ & 0.734 & $-0.41 \pm 0.07$ & 0.75 & $-0.44 \pm 0.06$ & 0.818 \\
\hline 68 & $-0.37 \pm 0.03$ & 0.871 & $-0.31 \pm 0.04$ & 0.776 & $-0.26 \pm 0.04$ & 0.665 & $-0.32 \pm 0.03$ & 0.808 \\
\hline 69 & $-0.35 \pm 0.03$ & 0.85 & $-0.32 \pm 0.03$ & 0.86 & $-0.28 \pm 0.03$ & 0.798 & $-0.31 \pm 0.03$ & 0.815 \\
\hline 70 & $-0.33 \pm 0.03$ & 0.856 & $-0.27 \pm 0.03$ & 0.78 & $-0.27 \pm 0.03$ & 0.783 & $-0.29 \pm 0.03$ & 0.786 \\
\hline 71 & $-0.49 \pm 0.04$ & 0.919 & $-0.47 \pm 0.03$ & 0.926 & $-0.42 \pm 0.03$ & 0.882 & $-0.44 \pm 0.03$ & 0.899 \\
\hline 72 & $-0.37 \pm 0.03$ & 0.877 & $-0.3 \pm 0.03$ & 0.817 & $-0.3 \pm 0.02$ & 0.866 & $-0.34 \pm 0.03$ & 0.827 \\
\hline 73 & $-0.3 \pm 0.02$ & 0.88 & $-0.26 \pm 0.02$ & 0.87 & $-0.24 \pm 0.02$ & 0.849 & $-0.27 \pm 0.02$ & 0.829 \\
\hline 74 & $-0.45 \pm 0.03$ & 0.924 & $-0.39 \pm 0.04$ & 0.855 & $-0.35 \pm 0.03$ & 0.869 & $-0.38 \pm 0.03$ & 0.884 \\
\hline 75 & $-0.46 \pm 0.06$ & 0.852 & $-0.43 \pm 0.06$ & 0.846 & $-0.39 \pm 0.05$ & 0.816 & $-0.39 \pm 0.05$ & 0.829 \\
\hline 76 & $-0.36 \pm 0.03$ & 0.878 & $-0.3 \pm 0.04$ & 0.775 & $-0.32 \pm 0.03$ & 0.845 & $-0.33 \pm 0.04$ & 0.788 \\
\hline 78 & $-0.51 \pm 0.06$ & 0.897 & $-0.5 \pm 0.06$ & 0.884 & $-0.49 \pm 0.07$ & 0.81 & $-0.54 \pm 0.05$ & 0.904 \\
\hline 79 & $-0.41 \pm 0.07$ & 0.74 & $-0.4 \pm 0.06$ & 0.754 & $-0.36 \pm 0.05$ & 0.785 & $-0.33 \pm 0.08$ & 0.598 \\
\hline 80 & $-0.38 \pm 0.08$ & 0.689 & $-0.36 \pm 0.08$ & 0.651 & $-0.39 \pm 0.07$ & 0.737 & $-0.39 \pm 0.09$ & 0.661 \\
\hline 81 & $-0.47 \pm 0.05$ & 0.887 & $-0.4 \pm 0.08$ & 0.683 & $-0.4 \pm 0.05$ & 0.852 & $-0.46 \pm 0.06$ & 0.845 \\
\hline 82 & $-0.35 \pm 0.02$ & 0.915 & $-0.29 \pm 0.03$ & 0.841 & $-0.28 \pm 0.02$ & 0.86 & $-0.3 \pm 0.02$ & 0.887 \\
\hline 83 & $-0.35 \pm 0.03$ & 0.906 & $-0.28 \pm 0.03$ & 0.806 & $-0.28 \pm 0.03$ & 0.841 & $-0.27 \pm 0.03$ & 0.745 \\
\hline 84 & $-0.3 \pm 0.02$ & 0.894 & $-0.25 \pm 0.02$ & 0.873 & $-0.25 \pm 0.02$ & 0.85 & $-0.27 \pm 0.02$ & 0.828 \\
\hline 85 & $-0.3 \pm 0.02$ & 0.882 & $-0.28 \pm 0.02$ & 0.886 & $-0.28 \pm 0.02$ & 0.884 & $-0.3 \pm 0.02$ & 0.837 \\
\hline 86 & $-0.41 \pm 0.05$ & 0.831 & $-0.38 \pm 0.04$ & 0.842 & $-0.37 \pm 0.04$ & 0.837 & $-0.4 \pm 0.04$ & 0.889 \\
\hline 87 & $-0.3 \pm 0.02$ & 0.88 & $-0.24 \pm 0.02$ & 0.849 & $-0.24 \pm 0.02$ & 0.834 & $-0.25 \pm 0.02$ & 0.831 \\
\hline 88 & $-0.51 \pm 0.04$ & 0.911 & $-0.45 \pm 0.04$ & 0.897 & $-0.43 \pm 0.04$ & 0.886 & $-0.43 \pm 0.03$ & 0.904 \\
\hline 89 & $-0.45 \pm 0.05$ & 0.882 & $-0.42 \pm 0.05$ & 0.851 & $-0.37 \pm 0.05$ & 0.808 & $-0.39 \pm 0.04$ & 0.883 \\
\hline 90 & $-0.34 \pm 0.03$ & 0.892 & $-0.29 \pm 0.02$ & 0.881 & $-0.28 \pm 0.02$ & 0.879 & $-0.31 \pm 0.02$ & 0.882 \\
\hline 91 & $-0.42 \pm 0.04$ & 0.892 & $-0.33 \pm 0.04$ & 0.826 & $-0.37 \pm 0.03$ & 0.883 & $-0.37 \pm 0.04$ & 0.836 \\
\hline 92 & $-0.46 \pm 0.05$ & 0.867 & $-0.39 \pm 0.05$ & 0.8 & $-0.39 \pm 0.05$ & 0.797 & $-0.39 \pm 0.05$ & 0.791 \\
\hline 93 & $-0.45 \pm 0.05$ & 0.857 & $-0.35 \pm 0.05$ & 0.795 & $-0.34 \pm 0.05$ & 0.78 & $-0.36 \pm 0.09$ & 0.571 \\
\hline 94 & $-0.32 \pm 0.04$ & 0.798 & $-0.28 \pm 0.02$ & 0.859 & $-0.25 \pm 0.02$ & 0.811 & $-0.28 \pm 0.02$ & 0.842 \\
\hline 95 & $-0.38 \pm 0.04$ & 0.852 & $-0.32 \pm 0.04$ & 0.818 & $-0.28 \pm 0.04$ & 0.713 & $-0.31 \pm 0.04$ & 0.763 \\
\hline 96 & $-0.36 \pm 0.03$ & 0.89 & $-0.29 \pm 0.03$ & 0.774 & $-0.27 \pm 0.04$ & 0.669 & $-0.29 \pm 0.04$ & 0.7 \\
\hline 97 & $-0.61 \pm 0.05$ & 0.919 & $-0.55 \pm 0.05$ & 0.896 & $-0.58 \pm 0.04$ & 0.935 & $-0.54 \pm 0.04$ & 0.925 \\
\hline
\end{tabular}


Table of results for the $\nu$ exponent for the exporters without RSP and RPP filters.

\begin{tabular}{|c|c|c|c|c|c|c|c|c|}
\hline & 1995 & & 1999 & & 2004 & & 2009 & \\
\hline HS2 & $\nu$ & $R^{2}$ & $\nu$ & $R^{2}$ & $\nu$ & $R^{2}$ & $\nu$ & $R^{2}$ \\
\hline 1 & $-0.28 \pm 0.08$ & 0.509 & $-0.25 \pm 0.08$ & 0.436 & $-0.2 \pm 0.06$ & 0.44 & $-0.26 \pm 0.09$ & 0.369 \\
\hline 2 & $-0.33 \pm 0.06$ & 0.745 & $-0.26 \pm 0.05$ & 0.736 & $-0.22 \pm 0.05$ & 0.647 & $-0.31 \pm 0.05$ & 0.759 \\
\hline 3 & $-0.28 \pm 0.04$ & 0.78 & $-0.25 \pm 0.03$ & 0.752 & $-0.23 \pm 0.03$ & 0.718 & $-0.24 \pm 0.03$ & 0.716 \\
\hline 4 & $-0.27 \pm 0.04$ & 0.765 & $-0.17 \pm 0.05$ & 0.443 & $-0.13 \pm 0.05$ & 0.316 & $-0.16 \pm 0.05$ & 0.367 \\
\hline 5 & $-0.35 \pm 0.04$ & 0.835 & $-0.33 \pm 0.04$ & 0.811 & $-0.31 \pm 0.04$ & 0.814 & $-0.33 \pm 0.04$ & 0.815 \\
\hline 6 & $-0.27 \pm 0.04$ & 0.751 & $-0.24 \pm 0.04$ & 0.691 & $-0.24 \pm 0.04$ & 0.705 & $-0.24 \pm 0.04$ & 0.707 \\
\hline 7 & $-0.26 \pm 0.04$ & 0.717 & $-0.26 \pm 0.04$ & 0.703 & $-0.21 \pm 0.05$ & 0.543 & $-0.24 \pm 0.04$ & 0.712 \\
\hline 8 & $-0.27 \pm 0.03$ & 0.811 & $-0.22 \pm 0.04$ & 0.654 & $-0.21 \pm 0.03$ & 0.707 & $-0.24 \pm 0.04$ & 0.692 \\
\hline 9 & $-0.25 \pm 0.04$ & 0.728 & $-0.17 \pm 0.04$ & 0.521 & $-0.17 \pm 0.03$ & 0.58 & $-0.18 \pm 0.03$ & 0.594 \\
\hline 10 & $-0.29 \pm 0.05$ & 0.732 & $-0.19 \pm 0.05$ & 0.564 & $-0.23 \pm 0.05$ & 0.62 & $-0.24 \pm 0.06$ & 0.558 \\
\hline 11 & $-0.29 \pm 0.06$ & 0.674 & $-0.27 \pm 0.05$ & 0.693 & $-0.13 \pm 0.07$ & 0.232 & $-0.16 \pm 0.06$ & 0.374 \\
\hline 12 & $-0.26 \pm 0.03$ & 0.801 & $-0.21 \pm 0.04$ & 0.648 & $-0.16 \pm 0.04$ & 0.412 & $-0.2 \pm 0.04$ & 0.579 \\
\hline 13 & $-0.34 \pm 0.07$ & 0.699 & $-0.3 \pm 0.06$ & 0.701 & $-0.23 \pm 0.06$ & 0.525 & $-0.23 \pm 0.05$ & 0.586 \\
\hline 14 & $-0.39 \pm 0.08$ & 0.704 & $-0.34 \pm 0.08$ & 0.651 & $-0.32 \pm 0.08$ & 0.591 & $-0.31 \pm 0.08$ & 0.631 \\
\hline 15 & $-0.25 \pm 0.04$ & 0.753 & $-0.19 \pm 0.06$ & 0.396 & $-0.17 \pm 0.05$ & 0.396 & $-0.18 \pm 0.06$ & 0.364 \\
\hline 16 & $-0.28 \pm 0.04$ & 0.783 & $-0.2 \pm 0.04$ & 0.622 & $-0.17 \pm 0.05$ & 0.419 & $-0.21 \pm 0.04$ & 0.614 \\
\hline 17 & $-0.25 \pm 0.07$ & 0.491 & $-0.16 \pm 0.06$ & 0.317 & $-0.14 \pm 0.05$ & 0.342 & $-0.17 \pm 0.06$ & 0.29 \\
\hline 18 & $-0.28 \pm 0.07$ & 0.564 & $-0.2 \pm 0.05$ & 0.529 & $-0.17 \pm 0.05$ & 0.414 & $-0.23 \pm 0.06$ & 0.481 \\
\hline 19 & $-0.21 \pm 0.06$ & 0.493 & $-0.15 \pm 0.06$ & 0.335 & $-0.09 \pm 0.05$ & 0.146 & $-0.15 \pm 0.05$ & 0.33 \\
\hline 20 & $-0.23 \pm 0.05$ & 0.618 & $-0.21 \pm 0.03$ & 0.66 & $-0.15 \pm 0.04$ & 0.468 & $-0.16 \pm 0.04$ & 0.48 \\
\hline 21 & $-0.17 \pm 0.04$ & 0.479 & $-0.12 \pm 0.05$ & 0.235 & $-0.13 \pm 0.04$ & 0.425 & $-0.18 \pm 0.04$ & 0.553 \\
\hline 22 & $-0.2 \pm 0.04$ & 0.541 & $-0.17 \pm 0.04$ & 0.53 & $-0.12 \pm 0.04$ & 0.297 & $-0.18 \pm 0.04$ & 0.508 \\
\hline 23 & $-0.19 \pm 0.06$ & 0.434 & $-0.12 \pm 0.06$ & 0.218 & $-0.11 \pm 0.05$ & 0.26 & $-0.07 \pm 0.07$ & 0.059 \\
\hline 24 & $-0.2 \pm 0.06$ & 0.513 & $-0.09 \pm 0.06$ & 0.115 & $-0.08 \pm 0.05$ & 0.148 & $-0.09 \pm 0.06$ & 0.128 \\
\hline 25 & $-0.15 \pm 0.05$ & 0.332 & $-0.16 \pm 0.04$ & 0.448 & $-0.07 \pm 0.06$ & 0.059 & $-0.14 \pm 0.06$ & 0.209 \\
\hline 26 & $-0.36 \pm 0.05$ & 0.813 & $-0.41 \pm 0.05$ & 0.827 & $-0.37 \pm 0.06$ & 0.769 & $-0.35 \pm 0.05$ & 0.802 \\
\hline 27 & $-0.3 \pm 0.05$ & 0.64 & $-0.26 \pm 0.05$ & 0.621 & $-0.19 \pm 0.06$ & 0.351 & $-0.18 \pm 0.06$ & 0.32 \\
\hline 28 & $-0.23 \pm 0.05$ & 0.619 & $-0.21 \pm 0.05$ & 0.534 & $-0.14 \pm 0.04$ & 0.405 & $-0.16 \pm 0.04$ & 0.49 \\
\hline 29 & $-0.22 \pm 0.03$ & 0.723 & $-0.2 \pm 0.03$ & 0.621 & $-0.16 \pm 0.04$ & 0.457 & $-0.19 \pm 0.03$ & 0.656 \\
\hline 30 & $-0.2 \pm 0.05$ & 0.521 & $-0.14 \pm 0.04$ & 0.334 & $-0.11 \pm 0.04$ & 0.322 & $-0.16 \pm 0.04$ & 0.437 \\
\hline 31 & $-0.21 \pm 0.05$ & 0.625 & $-0.18 \pm 0.07$ & 0.388 & $-0.15 \pm 0.06$ & 0.364 & $-0.23 \pm 0.06$ & 0.549 \\
\hline 32 & $-0.14 \pm 0.04$ & 0.512 & $-0.13 \pm 0.05$ & 0.284 & $-0.06 \pm 0.05$ & 0.066 & $-0.12 \pm 0.04$ & 0.365 \\
\hline 33 & $-0.22 \pm 0.04$ & 0.648 & $-0.15 \pm 0.04$ & 0.456 & $-0.15 \pm 0.03$ & 0.608 & $-0.18 \pm 0.03$ & 0.584 \\
\hline 34 & $-0.15 \pm 0.05$ & 0.357 & $-0.09 \pm 0.05$ & 0.178 & $-0.09 \pm 0.05$ & 0.128 & $-0.13 \pm 0.05$ & 0.243 \\
\hline 35 & $-0.2 \pm 0.04$ & 0.591 & $-0.13 \pm 0.04$ & 0.38 & $-0.11 \pm 0.05$ & 0.229 & $-0.14 \pm 0.04$ & 0.48 \\
\hline 36 & $-0.16 \pm 0.08$ & 0.296 & $-0.06 \pm 0.1$ & 0.036 & $-0.05 \pm 0.05$ & 0.091 & $-0.1 \pm 0.09$ & 0.126 \\
\hline 37 & $-0.26 \pm 0.05$ & 0.707 & $-0.19 \pm 0.05$ & 0.544 & $-0.14 \pm 0.05$ & 0.372 & $-0.2 \pm 0.05$ & 0.558 \\
\hline 38 & $-0.19 \pm 0.03$ & 0.714 & $-0.15 \pm 0.05$ & 0.342 & $-0.12 \pm 0.04$ & 0.365 & $-0.17 \pm 0.04$ & 0.524 \\
\hline 39 & $-0.19 \pm 0.04$ & 0.546 & $-0.16 \pm 0.03$ & 0.536 & $-0.16 \pm 0.03$ & 0.475 & $-0.17 \pm 0.03$ & 0.507 \\
\hline 40 & $-0.23 \pm 0.04$ & 0.641 & $-0.19 \pm 0.03$ & 0.633 & $-0.17 \pm 0.03$ & 0.609 & $-0.21 \pm 0.03$ & 0.616 \\
\hline 41 & $-0.24 \pm 0.04$ & 0.741 & $-0.18 \pm 0.06$ & 0.368 & $-0.2 \pm 0.05$ & 0.471 & $-0.24 \pm 0.06$ & 0.536 \\
\hline 42 & $-0.26 \pm 0.04$ & 0.749 & $-0.2 \pm 0.04$ & 0.612 & $-0.17 \pm 0.04$ & 0.484 & $-0.22 \pm 0.03$ & 0.707 \\
\hline 43 & $-0.29 \pm 0.05$ & 0.768 & $-0.29 \pm 0.04$ & 0.852 & $-0.28 \pm 0.04$ & 0.77 & $-0.31 \pm 0.04$ & 0.808 \\
\hline 44 & $-0.21 \pm 0.03$ & 0.763 & $-0.16 \pm 0.03$ & 0.621 & $-0.16 \pm 0.02$ & 0.65 & $-0.18 \pm 0.03$ & 0.64 \\
\hline 45 & $-0.23 \pm 0.15$ & 0.271 & $-0.24 \pm 0.07$ & 0.579 & $-0.26 \pm 0.07$ & 0.653 & $-0.19 \pm 0.08$ & 0.434 \\
\hline 46 & $-0.29 \pm 0.07$ & 0.611 & $-0.19 \pm 0.06$ & 0.45 & $-0.18 \pm 0.06$ & 0.43 & $-0.21 \pm 0.05$ & 0.583 \\
\hline 47 & $-0.28 \pm 0.06$ & 0.662 & $-0.29 \pm 0.08$ & 0.592 & $-0.26 \pm 0.04$ & 0.745 & $-0.28 \pm 0.04$ & 0.788 \\
\hline 48 & $-0.19 \pm 0.04$ & 0.538 & $-0.12 \pm 0.04$ & 0.366 & $-0.1 \pm 0.03$ & 0.276 & $-0.15 \pm 0.03$ & 0.475 \\
\hline 49 & $-0.24 \pm 0.04$ & 0.687 & $-0.17 \pm 0.04$ & 0.532 & $-0.14 \pm 0.04$ & 0.341 & $-0.17 \pm 0.04$ & 0.417 \\
\hline
\end{tabular}


Table of results for the $\nu$ exponent for the exporters without RSP and RPP filters (cont.)

\begin{tabular}{|c|c|c|c|c|c|c|c|c|}
\hline & 1995 & & 1999 & & 2004 & & 2009 & \\
\hline HS2 & $\nu$ & $R^{2}$ & $\nu$ & $R^{2}$ & $\nu$ & $R^{2}$ & $\nu$ & $R^{2}$ \\
\hline 50 & $-0.31 \pm 0.08$ & 0.61 & $-0.29 \pm 0.1$ & 0.461 & $-0.27 \pm 0.06$ & 0.654 & $-0.29 \pm 0.06$ & 0.711 \\
\hline 51 & $-0.23 \pm 0.05$ & 0.607 & $-0.24 \pm 0.05$ & 0.649 & $-0.22 \pm 0.05$ & 0.576 & $-0.24 \pm 0.06$ & 0.548 \\
\hline 52 & $-0.27 \pm 0.04$ & 0.692 & $-0.23 \pm 0.05$ & 0.568 & $-0.23 \pm 0.04$ & 0.681 & $-0.23 \pm 0.04$ & 0.6 \\
\hline 53 & $-0.27 \pm 0.03$ & 0.855 & $-0.23 \pm 0.06$ & 0.585 & $-0.22 \pm 0.04$ & 0.654 & $-0.21 \pm 0.04$ & 0.65 \\
\hline 54 & $-0.24 \pm 0.04$ & 0.704 & $-0.23 \pm 0.03$ & 0.752 & $-0.21 \pm 0.04$ & 0.675 & $-0.2 \pm 0.04$ & 0.664 \\
\hline 55 & $-0.22 \pm 0.04$ & 0.701 & $-0.18 \pm 0.04$ & 0.495 & $-0.17 \pm 0.05$ & 0.393 & $-0.2 \pm 0.04$ & 0.594 \\
\hline 56 & $-0.2 \pm 0.04$ & 0.634 & $-0.18 \pm 0.04$ & 0.593 & $-0.14 \pm 0.04$ & 0.457 & $-0.2 \pm 0.03$ & 0.641 \\
\hline 57 & $-0.25 \pm 0.04$ & 0.776 & $-0.23 \pm 0.04$ & 0.65 & $-0.21 \pm 0.04$ & 0.683 & $-0.21 \pm 0.04$ & 0.651 \\
\hline 58 & $-0.22 \pm 0.04$ & 0.637 & $-0.2 \pm 0.04$ & 0.639 & $-0.18 \pm 0.04$ & 0.559 & $-0.22 \pm 0.04$ & 0.633 \\
\hline 59 & $-0.16 \pm 0.05$ & 0.49 & $-0.16 \pm 0.04$ & 0.466 & $-0.14 \pm 0.04$ & 0.483 & $-0.22 \pm 0.04$ & 0.626 \\
\hline 60 & $-0.24 \pm 0.06$ & 0.566 & $-0.18 \pm 0.05$ & 0.472 & $-0.21 \pm 0.04$ & 0.647 & $-0.24 \pm 0.05$ & 0.592 \\
\hline 61 & $-0.25 \pm 0.04$ & 0.7 & $-0.23 \pm 0.04$ & 0.627 & $-0.18 \pm 0.03$ & 0.584 & $-0.19 \pm 0.03$ & 0.584 \\
\hline 62 & $-0.27 \pm 0.04$ & 0.705 & $-0.22 \pm 0.04$ & 0.609 & $-0.18 \pm 0.03$ & 0.565 & $-0.17 \pm 0.03$ & 0.482 \\
\hline 63 & $-0.24 \pm 0.04$ & 0.683 & $-0.21 \pm 0.04$ & 0.593 & $-0.16 \pm 0.03$ & 0.569 & $-0.2 \pm 0.03$ & 0.617 \\
\hline 64 & $-0.21 \pm 0.04$ & 0.565 & $-0.19 \pm 0.04$ & 0.514 & $-0.13 \pm 0.04$ & 0.291 & $-0.17 \pm 0.03$ & 0.585 \\
\hline 65 & $-0.26 \pm 0.05$ & 0.709 & $-0.17 \pm 0.04$ & 0.515 & $-0.15 \pm 0.03$ & 0.535 & $-0.21 \pm 0.03$ & 0.719 \\
\hline 66 & $-0.3 \pm 0.04$ & 0.873 & $-0.21 \pm 0.05$ & 0.661 & $-0.13 \pm 0.05$ & 0.421 & $-0.14 \pm 0.04$ & 0.562 \\
\hline 67 & $-0.22 \pm 0.05$ & 0.626 & $-0.09 \pm 0.07$ & 0.175 & $-0.16 \pm 0.07$ & 0.353 & $-0.26 \pm 0.05$ & 0.686 \\
\hline 68 & $-0.2 \pm 0.04$ & 0.606 & $-0.16 \pm 0.04$ & 0.478 & $-0.15 \pm 0.03$ & 0.49 & $-0.19 \pm 0.03$ & 0.606 \\
\hline 69 & $-0.21 \pm 0.04$ & 0.625 & $-0.16 \pm 0.04$ & 0.492 & $-0.13 \pm 0.04$ & 0.325 & $-0.19 \pm 0.04$ & 0.554 \\
\hline 70 & $-0.15 \pm 0.05$ & 0.304 & $-0.18 \pm 0.04$ & 0.47 & $-0.12 \pm 0.03$ & 0.422 & $-0.18 \pm 0.04$ & 0.558 \\
\hline 71 & $-0.35 \pm 0.05$ & 0.72 & $-0.32 \pm 0.04$ & 0.738 & $-0.3 \pm 0.04$ & 0.734 & $-0.32 \pm 0.04$ & 0.756 \\
\hline 72 & $-0.2 \pm 0.04$ & 0.585 & $-0.12 \pm 0.04$ & 0.318 & $-0.15 \pm 0.03$ & 0.47 & $-0.2 \pm 0.04$ & 0.609 \\
\hline 73 & $-0.2 \pm 0.04$ & 0.572 & $-0.13 \pm 0.05$ & 0.233 & $-0.14 \pm 0.03$ & 0.419 & $-0.21 \pm 0.03$ & 0.692 \\
\hline 74 & $-0.32 \pm 0.05$ & 0.744 & $-0.27 \pm 0.04$ & 0.686 & $-0.25 \pm 0.05$ & 0.601 & $-0.29 \pm 0.05$ & 0.684 \\
\hline 75 & $-0.35 \pm 0.09$ & 0.616 & $-0.38 \pm 0.07$ & 0.721 & $-0.35 \pm 0.09$ & 0.611 & $-0.35 \pm 0.06$ & 0.747 \\
\hline 76 & $-0.25 \pm 0.04$ & 0.717 & $-0.15 \pm 0.05$ & 0.33 & $-0.17 \pm 0.03$ & 0.543 & $-0.19 \pm 0.04$ & 0.52 \\
\hline 78 & $-0.4 \pm 0.1$ & 0.625 & $-0.36 \pm 0.09$ & 0.646 & $-0.3 \pm 0.08$ & 0.552 & $-0.39 \pm 0.06$ & 0.826 \\
\hline 79 & $-0.3 \pm 0.08$ & 0.612 & $-0.26 \pm 0.07$ & 0.57 & $-0.22 \pm 0.07$ & 0.468 & $-0.28 \pm 0.06$ & 0.651 \\
\hline 80 & $-0.35 \pm 0.08$ & 0.689 & $-0.26 \pm 0.07$ & 0.588 & $-0.27 \pm 0.09$ & 0.525 & $-0.29 \pm 0.06$ & 0.702 \\
\hline 81 & $-0.38 \pm 0.05$ & 0.821 & $-0.32 \pm 0.05$ & 0.77 & $-0.35 \pm 0.06$ & 0.719 & $-0.31 \pm 0.05$ & 0.719 \\
\hline 82 & $-0.23 \pm 0.05$ & 0.56 & $-0.14 \pm 0.05$ & 0.334 & $-0.19 \pm 0.04$ & 0.571 & $-0.22 \pm 0.04$ & 0.624 \\
\hline 83 & $-0.21 \pm 0.04$ & 0.638 & $-0.15 \pm 0.04$ & 0.407 & $-0.17 \pm 0.04$ & 0.48 & $-0.16 \pm 0.04$ & 0.487 \\
\hline 84 & $-0.26 \pm 0.03$ & 0.682 & $-0.23 \pm 0.03$ & 0.643 & $-0.19 \pm 0.03$ & 0.615 & $-0.21 \pm 0.03$ & 0.603 \\
\hline 85 & $-0.27 \pm 0.03$ & 0.761 & $-0.21 \pm 0.04$ & 0.57 & $-0.23 \pm 0.03$ & 0.71 & $-0.25 \pm 0.03$ & 0.69 \\
\hline 86 & $-0.29 \pm 0.06$ & 0.663 & $-0.28 \pm 0.06$ & 0.693 & $-0.28 \pm 0.05$ & 0.667 & $-0.29 \pm 0.05$ & 0.699 \\
\hline 87 & $-0.25 \pm 0.04$ & 0.704 & $-0.17 \pm 0.05$ & 0.375 & $-0.22 \pm 0.03$ & 0.7 & $-0.25 \pm 0.03$ & 0.689 \\
\hline 88 & $-0.36 \pm 0.05$ & 0.821 & $-0.26 \pm 0.05$ & 0.645 & $-0.25 \pm 0.04$ & 0.689 & $-0.28 \pm 0.04$ & 0.735 \\
\hline 89 & $-0.39 \pm 0.07$ & 0.703 & $-0.33 \pm 0.06$ & 0.717 & $-0.31 \pm 0.06$ & 0.672 & $-0.3 \pm 0.04$ & 0.768 \\
\hline 90 & $-0.29 \pm 0.04$ & 0.745 & $-0.22 \pm 0.04$ & 0.514 & $-0.22 \pm 0.03$ & 0.654 & $-0.26 \pm 0.03$ & 0.737 \\
\hline 91 & $-0.3 \pm 0.05$ & 0.752 & $-0.22 \pm 0.06$ & 0.482 & $-0.24 \pm 0.04$ & 0.693 & $-0.31 \pm 0.04$ & 0.828 \\
\hline 92 & $-0.3 \pm 0.04$ & 0.781 & $-0.26 \pm 0.05$ & 0.675 & $-0.27 \pm 0.04$ & 0.728 & $-0.26 \pm 0.04$ & 0.714 \\
\hline 93 & $-0.39 \pm 0.1$ & 0.584 & $-0.36 \pm 0.1$ & 0.552 & $-0.27 \pm 0.11$ & 0.362 & $-0.27 \pm 0.07$ & 0.578 \\
\hline 94 & $-0.23 \pm 0.04$ & 0.667 & $-0.16 \pm 0.05$ & 0.371 & $-0.15 \pm 0.04$ & 0.369 & $-0.2 \pm 0.03$ & 0.585 \\
\hline 95 & $-0.25 \pm 0.04$ & 0.734 & $-0.2 \pm 0.04$ & 0.592 & $-0.18 \pm 0.03$ & 0.612 & $-0.2 \pm 0.03$ & 0.646 \\
\hline 96 & $-0.24 \pm 0.04$ & 0.68 & $-0.14 \pm 0.05$ & 0.346 & $-0.15 \pm 0.03$ & 0.5 & $-0.18 \pm 0.04$ & 0.569 \\
\hline 97 & $-0.36 \pm 0.04$ & 0.859 & $-0.32 \pm 0.05$ & 0.701 & $-0.29 \pm 0.04$ & 0.817 & $-0.32 \pm 0.04$ & 0.839 \\
\hline
\end{tabular}


Table of results for the $\nu$ exponent for the importers without RSP and RPP filters.

\begin{tabular}{|c|c|c|c|c|c|c|c|c|}
\hline & 1995 & & 1999 & & 2004 & & 2009 & \\
\hline HS2 & $\nu$ & $R^{2}$ & $\nu$ & $R^{2}$ & $\nu$ & $R^{2}$ & $\nu$ & $R^{2}$ \\
\hline 1 & $-0.28 \pm 0.07$ & 0.561 & $-0.3 \pm 0.08$ & 0.534 & $-0.25 \pm 0.08$ & 0.421 & $-0.26 \pm 0.08$ & 0.418 \\
\hline 2 & $-0.28 \pm 0.05$ & 0.712 & $-0.23 \pm 0.05$ & 0.609 & $-0.25 \pm 0.05$ & 0.674 & $-0.26 \pm 0.06$ & 0.522 \\
\hline 3 & $-0.3 \pm 0.04$ & 0.809 & $-0.25 \pm 0.03$ & 0.757 & $-0.23 \pm 0.03$ & 0.735 & $-0.22 \pm 0.03$ & 0.688 \\
\hline 4 & $-0.24 \pm 0.04$ & 0.694 & $-0.21 \pm 0.04$ & 0.591 & $-0.2 \pm 0.04$ & 0.649 & $-0.21 \pm 0.04$ & 0.618 \\
\hline 5 & $-0.36 \pm 0.04$ & 0.871 & $-0.32 \pm 0.04$ & 0.86 & $-0.29 \pm 0.09$ & 0.433 & $-0.28 \pm 0.07$ & 0.554 \\
\hline 6 & $-0.32 \pm 0.05$ & 0.773 & $-0.28 \pm 0.04$ & 0.764 & $-0.26 \pm 0.05$ & 0.664 & $-0.26 \pm 0.05$ & 0.668 \\
\hline 7 & $-0.27 \pm 0.05$ & 0.643 & $-0.25 \pm 0.05$ & 0.568 & $-0.22 \pm 0.05$ & 0.504 & $-0.26 \pm 0.05$ & 0.533 \\
\hline 8 & $-0.27 \pm 0.06$ & 0.53 & $-0.24 \pm 0.05$ & 0.526 & $-0.19 \pm 0.06$ & 0.324 & $-0.22 \pm 0.04$ & 0.554 \\
\hline 9 & $-0.26 \pm 0.05$ & 0.631 & $-0.22 \pm 0.04$ & 0.606 & $-0.2 \pm 0.04$ & 0.532 & $-0.21 \pm 0.04$ & 0.601 \\
\hline 10 & $-0.37 \pm 0.06$ & 0.758 & $-0.29 \pm 0.05$ & 0.685 & $-0.24 \pm 0.08$ & 0.38 & $-0.28 \pm 0.07$ & 0.51 \\
\hline 11 & $-0.31 \pm 0.07$ & 0.606 & $-0.26 \pm 0.06$ & 0.536 & $-0.26 \pm 0.07$ & 0.464 & $-0.21 \pm 0.07$ & 0.344 \\
\hline 12 & $-0.27 \pm 0.04$ & 0.724 & $-0.21 \pm 0.04$ & 0.664 & $-0.21 \pm 0.03$ & 0.655 & $-0.17 \pm 0.07$ & 0.246 \\
\hline 13 & $-0.31 \pm 0.06$ & 0.68 & $-0.27 \pm 0.04$ & 0.735 & $-0.27 \pm 0.04$ & 0.74 & $-0.25 \pm 0.06$ & 0.523 \\
\hline 14 & $-0.31 \pm 0.06$ & 0.711 & $-0.3 \pm 0.06$ & 0.682 & $-0.3 \pm 0.07$ & 0.637 & $-0.36 \pm 0.07$ & 0.726 \\
\hline 15 & $-0.28 \pm 0.08$ & 0.433 & $-0.22 \pm 0.05$ & 0.499 & $-0.2 \pm 0.04$ & 0.525 & $-0.21 \pm 0.04$ & 0.574 \\
\hline 16 & $-0.27 \pm 0.06$ & 0.58 & $-0.21 \pm 0.04$ & 0.643 & $-0.2 \pm 0.05$ & 0.463 & $-0.22 \pm 0.05$ & 0.538 \\
\hline 17 & $-0.24 \pm 0.05$ & 0.569 & $-0.22 \pm 0.05$ & 0.524 & $-0.2 \pm 0.04$ & 0.58 & $-0.18 \pm 0.05$ & 0.44 \\
\hline 18 & $-0.29 \pm 0.05$ & 0.724 & $-0.27 \pm 0.05$ & 0.661 & $-0.23 \pm 0.04$ & 0.629 & $-0.24 \pm 0.05$ & 0.59 \\
\hline 19 & $-0.23 \pm 0.04$ & 0.636 & $-0.2 \pm 0.05$ & 0.465 & $-0.19 \pm 0.03$ & 0.603 & $-0.18 \pm 0.04$ & 0.554 \\
\hline 20 & $-0.23 \pm 0.04$ & 0.69 & $-0.17 \pm 0.08$ & 0.211 & $-0.17 \pm 0.04$ & 0.435 & $-0.18 \pm 0.04$ & 0.488 \\
\hline 21 & $-0.24 \pm 0.04$ & 0.651 & $-0.2 \pm 0.04$ & 0.6 & $-0.17 \pm 0.04$ & 0.538 & $-0.18 \pm 0.04$ & 0.555 \\
\hline 22 & $-0.2 \pm 0.03$ & 0.654 & $-0.15 \pm 0.04$ & 0.368 & $-0.15 \pm 0.04$ & 0.444 & $-0.18 \pm 0.03$ & 0.584 \\
\hline 23 & $-0.25 \pm 0.04$ & 0.735 & $-0.22 \pm 0.04$ & 0.631 & $-0.2 \pm 0.04$ & 0.612 & $-0.19 \pm 0.05$ & 0.453 \\
\hline 24 & $-0.27 \pm 0.05$ & 0.648 & $-0.2 \pm 0.06$ & 0.382 & $-0.19 \pm 0.05$ & 0.424 & $-0.15 \pm 0.08$ & 0.17 \\
\hline 25 & $-0.2 \pm 0.05$ & 0.509 & $-0.16 \pm 0.05$ & 0.348 & $-0.16 \pm 0.05$ & 0.369 & $-0.15 \pm 0.09$ & 0.129 \\
\hline 26 & $-0.34 \pm 0.04$ & 0.865 & $-0.31 \pm 0.04$ & 0.806 & $-0.28 \pm 0.04$ & 0.839 & $-0.29 \pm 0.04$ & 0.795 \\
\hline 27 & $-0.23 \pm 0.04$ & 0.637 & $-0.19 \pm 0.05$ & 0.43 & $-0.14 \pm 0.04$ & 0.316 & $-0.2 \pm 0.04$ & 0.532 \\
\hline 28 & $-0.25 \pm 0.03$ & 0.742 & $-0.19 \pm 0.03$ & 0.711 & $-0.16 \pm 0.03$ & 0.569 & $-0.18 \pm 0.04$ & 0.539 \\
\hline 29 & $-0.26 \pm 0.03$ & 0.838 & $-0.2 \pm 0.02$ & 0.816 & $-0.18 \pm 0.02$ & 0.744 & $-0.2 \pm 0.03$ & 0.671 \\
\hline 30 & $-0.22 \pm 0.02$ & 0.847 & $-0.19 \pm 0.02$ & 0.789 & $-0.18 \pm 0.03$ & 0.683 & $-0.17 \pm 0.03$ & 0.526 \\
\hline 31 & $-0.26 \pm 0.06$ & 0.59 & $-0.22 \pm 0.05$ & 0.537 & $-0.2 \pm 0.05$ & 0.539 & $-0.23 \pm 0.05$ & 0.578 \\
\hline 32 & $-0.23 \pm 0.04$ & 0.671 & $-0.19 \pm 0.04$ & 0.534 & $-0.14 \pm 0.05$ & 0.31 & $-0.18 \pm 0.04$ & 0.565 \\
\hline 33 & $-0.24 \pm 0.04$ & 0.687 & $-0.22 \pm 0.04$ & 0.564 & $-0.19 \pm 0.04$ & 0.565 & $-0.2 \pm 0.04$ & 0.522 \\
\hline 34 & $-0.24 \pm 0.05$ & 0.593 & $-0.18 \pm 0.08$ & 0.208 & $-0.15 \pm 0.04$ & 0.41 & $-0.18 \pm 0.04$ & 0.506 \\
\hline 35 & $-0.28 \pm 0.04$ & 0.786 & $-0.21 \pm 0.06$ & 0.402 & $-0.18 \pm 0.04$ & 0.522 & $-0.17 \pm 0.05$ & 0.433 \\
\hline 36 & $-0.22 \pm 0.09$ & 0.34 & $-0.2 \pm 0.1$ & 0.249 & $-0.27 \pm 0.08$ & 0.508 & $-0.28 \pm 0.08$ & 0.484 \\
\hline 37 & $-0.29 \pm 0.03$ & 0.872 & $-0.22 \pm 0.03$ & 0.75 & $-0.19 \pm 0.04$ & 0.586 & $-0.25 \pm 0.04$ & 0.696 \\
\hline 38 & $-0.23 \pm 0.02$ & 0.842 & $-0.21 \pm 0.04$ & 0.572 & $-0.17 \pm 0.03$ & 0.623 & $-0.19 \pm 0.04$ & 0.53 \\
\hline 39 & $-0.18 \pm 0.02$ & 0.707 & $-0.15 \pm 0.03$ & 0.581 & $-0.14 \pm 0.02$ & 0.575 & $-0.16 \pm 0.02$ & 0.595 \\
\hline 40 & $-0.21 \pm 0.03$ & 0.781 & $-0.19 \pm 0.02$ & 0.772 & $-0.15 \pm 0.02$ & 0.669 & $-0.18 \pm 0.02$ & 0.752 \\
\hline 41 & $-0.29 \pm 0.04$ & 0.823 & $-0.24 \pm 0.04$ & 0.697 & $-0.26 \pm 0.04$ & 0.767 & $-0.26 \pm 0.05$ & 0.607 \\
\hline 42 & $-0.24 \pm 0.06$ & 0.445 & $-0.21 \pm 0.04$ & 0.579 & $-0.17 \pm 0.05$ & 0.394 & $-0.19 \pm 0.04$ & 0.497 \\
\hline 43 & $-0.23 \pm 0.05$ & 0.633 & $-0.29 \pm 0.06$ & 0.702 & $-0.21 \pm 0.05$ & 0.534 & $-0.28 \pm 0.06$ & 0.628 \\
\hline 44 & $-0.23 \pm 0.04$ & 0.627 & $-0.19 \pm 0.04$ & 0.531 & $-0.17 \pm 0.03$ & 0.52 & $-0.19 \pm 0.04$ & 0.488 \\
\hline 45 & $-0.31 \pm 0.06$ & 0.732 & $-0.26 \pm 0.07$ & 0.558 & $-0.24 \pm 0.06$ & 0.639 & $-0.29 \pm 0.08$ & 0.599 \\
\hline 46 & $-0.29 \pm 0.06$ & 0.667 & $-0.25 \pm 0.13$ & 0.249 & $-0.25 \pm 0.05$ & 0.672 & $-0.23 \pm 0.08$ & 0.438 \\
\hline 47 & $-0.32 \pm 0.05$ & 0.828 & $-0.3 \pm 0.04$ & 0.807 & $-0.27 \pm 0.04$ & 0.752 & $-0.21 \pm 0.07$ & 0.428 \\
\hline 48 & $-0.21 \pm 0.03$ & 0.737 & $-0.17 \pm 0.03$ & 0.572 & $-0.15 \pm 0.04$ & 0.415 & $-0.16 \pm 0.03$ & 0.599 \\
\hline 49 & $-0.2 \pm 0.03$ & 0.649 & $-0.19 \pm 0.03$ & 0.653 & $-0.16 \pm 0.03$ & 0.496 & $-0.18 \pm 0.03$ & 0.56 \\
\hline
\end{tabular}


Table of results for the $\nu$ exponent for the importers without RSP and RPP filters (cont.)

\begin{tabular}{|c|c|c|c|c|c|c|c|c|}
\hline & 1995 & & 1999 & & 2004 & & 2009 & \\
\hline HS2 & $\nu$ & $R^{2}$ & $\nu$ & $R^{2}$ & $\nu$ & $R^{2}$ & $\nu$ & $R^{2}$ \\
\hline 50 & $-0.28 \pm 0.05$ & 0.803 & $-0.25 \pm 0.05$ & 0.723 & $-0.27 \pm 0.08$ & 0.5 & $-0.32 \pm 0.05$ & 0.802 \\
\hline 51 & $-0.27 \pm 0.04$ & 0.788 & $-0.23 \pm 0.05$ & 0.56 & $-0.2 \pm 0.05$ & 0.561 & $-0.24 \pm 0.05$ & 0.639 \\
\hline 52 & $-0.26 \pm 0.03$ & 0.822 & $-0.21 \pm 0.03$ & 0.686 & $-0.23 \pm 0.04$ & 0.65 & $-0.24 \pm 0.04$ & 0.683 \\
\hline 53 & $-0.26 \pm 0.05$ & 0.693 & $-0.22 \pm 0.07$ & 0.442 & $-0.2 \pm 0.08$ & 0.321 & $-0.14 \pm 0.09$ & 0.169 \\
\hline 54 & $-0.23 \pm 0.05$ & 0.562 & $-0.2 \pm 0.04$ & 0.661 & $-0.18 \pm 0.05$ & 0.424 & $-0.24 \pm 0.05$ & 0.555 \\
\hline 55 & $-0.24 \pm 0.04$ & 0.669 & $-0.22 \pm 0.05$ & 0.534 & $-0.19 \pm 0.05$ & 0.431 & $-0.21 \pm 0.07$ & 0.378 \\
\hline 56 & $-0.24 \pm 0.04$ & 0.731 & $-0.2 \pm 0.04$ & 0.578 & $-0.18 \pm 0.05$ & 0.418 & $-0.22 \pm 0.04$ & 0.648 \\
\hline 57 & $-0.28 \pm 0.05$ & 0.669 & $-0.17 \pm 0.06$ & 0.403 & $-0.21 \pm 0.04$ & 0.563 & $-0.23 \pm 0.05$ & 0.572 \\
\hline 58 & $-0.25 \pm 0.04$ & 0.736 & $-0.23 \pm 0.07$ & 0.4 & $-0.22 \pm 0.04$ & 0.594 & $-0.25 \pm 0.04$ & 0.665 \\
\hline 59 & $-0.22 \pm 0.03$ & 0.769 & $-0.24 \pm 0.04$ & 0.747 & $-0.17 \pm 0.06$ & 0.347 & $-0.2 \pm 0.04$ & 0.64 \\
\hline 60 & $-0.28 \pm 0.06$ & 0.657 & $-0.26 \pm 0.06$ & 0.605 & $-0.28 \pm 0.04$ & 0.754 & $-0.27 \pm 0.05$ & 0.653 \\
\hline 61 & $-0.22 \pm 0.04$ & 0.556 & $-0.18 \pm 0.04$ & 0.422 & $-0.17 \pm 0.04$ & 0.462 & $-0.17 \pm 0.04$ & 0.431 \\
\hline 62 & $-0.24 \pm 0.04$ & 0.655 & $-0.18 \pm 0.03$ & 0.542 & $-0.17 \pm 0.03$ & 0.485 & $-0.16 \pm 0.04$ & 0.459 \\
\hline 63 & $-0.21 \pm 0.04$ & 0.584 & $-0.17 \pm 0.04$ & 0.476 & $-0.15 \pm 0.04$ & 0.44 & $-0.16 \pm 0.04$ & 0.471 \\
\hline 64 & $-0.21 \pm 0.04$ & 0.539 & $-0.17 \pm 0.05$ & 0.374 & $-0.14 \pm 0.06$ & 0.237 & $-0.14 \pm 0.04$ & 0.325 \\
\hline 65 & $-0.27 \pm 0.05$ & 0.679 & $-0.16 \pm 0.07$ & 0.25 & $-0.15 \pm 0.07$ & 0.194 & $-0.15 \pm 0.06$ & 0.239 \\
\hline 66 & $-0.32 \pm 0.06$ & 0.726 & $-0.23 \pm 0.07$ & 0.471 & $-0.24 \pm 0.08$ & 0.404 & $-0.26 \pm 0.09$ & 0.451 \\
\hline 67 & $-0.28 \pm 0.06$ & 0.673 & $-0.23 \pm 0.07$ & 0.524 & $-0.27 \pm 0.08$ & 0.467 & $-0.29 \pm 0.06$ & 0.708 \\
\hline 68 & $-0.24 \pm 0.03$ & 0.774 & $-0.18 \pm 0.04$ & 0.568 & $-0.13 \pm 0.04$ & 0.302 & $-0.21 \pm 0.04$ & 0.602 \\
\hline 69 & $-0.22 \pm 0.04$ & 0.596 & $-0.19 \pm 0.03$ & 0.656 & $-0.16 \pm 0.04$ & 0.462 & $-0.19 \pm 0.03$ & 0.647 \\
\hline 70 & $-0.21 \pm 0.03$ & 0.712 & $-0.17 \pm 0.04$ & 0.442 & $-0.16 \pm 0.03$ & 0.541 & $-0.18 \pm 0.03$ & 0.56 \\
\hline 71 & $-0.34 \pm 0.03$ & 0.901 & $-0.31 \pm 0.02$ & 0.898 & $-0.27 \pm 0.03$ & 0.831 & $-0.29 \pm 0.03$ & 0.865 \\
\hline 72 & $-0.24 \pm 0.03$ & 0.789 & $-0.19 \pm 0.04$ & 0.537 & $-0.19 \pm 0.03$ & 0.667 & $-0.23 \pm 0.03$ & 0.673 \\
\hline 73 & $-0.2 \pm 0.03$ & 0.704 & $-0.17 \pm 0.03$ & 0.581 & $-0.15 \pm 0.03$ & 0.512 & $-0.18 \pm 0.03$ & 0.663 \\
\hline 74 & $-0.32 \pm 0.02$ & 0.927 & $-0.28 \pm 0.02$ & 0.883 & $-0.25 \pm 0.03$ & 0.788 & $-0.26 \pm 0.02$ & 0.851 \\
\hline 75 & $-0.34 \pm 0.04$ & 0.889 & $-0.3 \pm 0.04$ & 0.853 & $-0.28 \pm 0.04$ & 0.818 & $-0.27 \pm 0.04$ & 0.802 \\
\hline 76 & $-0.24 \pm 0.03$ & 0.828 & $-0.19 \pm 0.04$ & 0.493 & $-0.21 \pm 0.03$ & 0.74 & $-0.22 \pm 0.04$ & 0.635 \\
\hline 78 & $-0.33 \pm 0.06$ & 0.791 & $-0.33 \pm 0.06$ & 0.781 & $-0.34 \pm 0.08$ & 0.59 & $-0.39 \pm 0.05$ & 0.853 \\
\hline 79 & $-0.3 \pm 0.07$ & 0.617 & $-0.26 \pm 0.07$ & 0.503 & $-0.24 \pm 0.04$ & 0.7 & $-0.21 \pm 0.06$ & 0.508 \\
\hline 80 & $-0.29 \pm 0.06$ & 0.667 & $-0.22 \pm 0.1$ & 0.318 & $-0.25 \pm 0.08$ & 0.5 & $-0.24 \pm 0.07$ & 0.566 \\
\hline 81 & $-0.33 \pm 0.04$ & 0.854 & $-0.27 \pm 0.07$ & 0.58 & $-0.28 \pm 0.04$ & 0.834 & $-0.31 \pm 0.04$ & 0.842 \\
\hline 82 & $-0.23 \pm 0.03$ & 0.77 & $-0.19 \pm 0.02$ & 0.75 & $-0.19 \pm 0.04$ & 0.577 & $-0.2 \pm 0.02$ & 0.763 \\
\hline 83 & $-0.24 \pm 0.03$ & 0.801 & $-0.17 \pm 0.04$ & 0.492 & $-0.17 \pm 0.03$ & 0.619 & $-0.18 \pm 0.03$ & 0.597 \\
\hline 84 & $-0.19 \pm 0.02$ & 0.772 & $-0.15 \pm 0.02$ & 0.787 & $-0.16 \pm 0.01$ & 0.785 & $-0.18 \pm 0.02$ & 0.712 \\
\hline 85 & $-0.2 \pm 0.02$ & 0.8 & $-0.17 \pm 0.02$ & 0.743 & $-0.19 \pm 0.02$ & 0.755 & $-0.19 \pm 0.02$ & 0.726 \\
\hline 86 & $-0.28 \pm 0.05$ & 0.718 & $-0.25 \pm 0.05$ & 0.624 & $-0.24 \pm 0.03$ & 0.765 & $-0.3 \pm 0.05$ & 0.711 \\
\hline 87 & $-0.19 \pm 0.03$ & 0.633 & $-0.15 \pm 0.03$ & 0.509 & $-0.14 \pm 0.02$ & 0.617 & $-0.17 \pm 0.02$ & 0.676 \\
\hline 88 & $-0.36 \pm 0.05$ & 0.8 & $-0.31 \pm 0.05$ & 0.741 & $-0.26 \pm 0.09$ & 0.347 & $-0.28 \pm 0.04$ & 0.799 \\
\hline 89 & $-0.36 \pm 0.04$ & 0.85 & $-0.33 \pm 0.05$ & 0.765 & $-0.28 \pm 0.04$ & 0.739 & $-0.31 \pm 0.04$ & 0.785 \\
\hline 90 & $-0.24 \pm 0.02$ & 0.839 & $-0.2 \pm 0.02$ & 0.816 & $-0.19 \pm 0.02$ & 0.818 & $-0.2 \pm 0.02$ & 0.84 \\
\hline 91 & $-0.3 \pm 0.04$ & 0.795 & $-0.22 \pm 0.04$ & 0.66 & $-0.26 \pm 0.03$ & 0.78 & $-0.23 \pm 0.04$ & 0.643 \\
\hline 92 & $-0.31 \pm 0.04$ & 0.798 & $-0.24 \pm 0.05$ & 0.664 & $-0.23 \pm 0.05$ & 0.569 & $-0.24 \pm 0.04$ & 0.67 \\
\hline 93 & $-0.32 \pm 0.09$ & 0.522 & $-0.25 \pm 0.06$ & 0.595 & $-0.2 \pm 0.06$ & 0.492 & $-0.24 \pm 0.09$ & 0.379 \\
\hline 94 & $-0.19 \pm 0.04$ & 0.563 & $-0.16 \pm 0.03$ & 0.603 & $-0.14 \pm 0.03$ & 0.467 & $-0.16 \pm 0.03$ & 0.569 \\
\hline 95 & $-0.24 \pm 0.03$ & 0.716 & $-0.18 \pm 0.04$ & 0.544 & $-0.16 \pm 0.05$ & 0.346 & $-0.19 \pm 0.04$ & 0.54 \\
\hline 96 & $-0.25 \pm 0.04$ & 0.74 & $-0.18 \pm 0.04$ & 0.487 & $-0.18 \pm 0.04$ & 0.442 & $-0.19 \pm 0.04$ & 0.551 \\
\hline 97 & $-0.38 \pm 0.04$ & 0.864 & $-0.32 \pm 0.04$ & 0.838 & $-0.36 \pm 0.04$ & 0.84 & $-0.35 \pm 0.03$ & 0.891 \\
\hline
\end{tabular}


Table of results for the $\theta$ exponent without RSP and RPP filters.

\begin{tabular}{|c|c|c|c|c|c|c|c|c|}
\hline & 1995 & & 1999 & & 2004 & & 2009 & \\
\hline HS2 & $\theta$ & $R^{2}$ & $\theta$ & $R^{2}$ & $\theta$ & $R^{2}$ & $\theta$ & $R^{2}$ \\
\hline 1 & $0.82 \pm 0.11$ & 0.188 & $0.81 \pm 0.1$ & 0.18 & $0.86 \pm 0.1$ & 0.215 & $0.84 \pm 0.11$ & 0.15 \\
\hline 2 & $1.28 \pm 0.1$ & 0.368 & $1.27 \pm 0.1$ & 0.358 & $1.28 \pm 0.1$ & 0.32 & $1.28 \pm 0.09$ & 0.351 \\
\hline 3 & $1.37 \pm 0.08$ & 0.386 & $1.36 \pm 0.08$ & 0.36 & $1.39 \pm 0.07$ & 0.383 & $1.27 \pm 0.08$ & 0.308 \\
\hline 4 & $1.22 \pm 0.09$ & 0.349 & $1.23 \pm 0.08$ & 0.369 & $1.27 \pm 0.08$ & 0.354 & $1.12 \pm 0.08$ & 0.291 \\
\hline 5 & $1.2 \pm 0.08$ & 0.424 & $1.19 \pm 0.08$ & 0.396 & $1.13 \pm 0.08$ & 0.369 & $1.1 \pm 0.08$ & 0.317 \\
\hline 6 & $1.3 \pm 0.09$ & 0.412 & $1.25 \pm 0.09$ & 0.397 & $1.25 \pm 0.08$ & 0.364 & $1.24 \pm 0.09$ & 0.352 \\
\hline 7 & $1.19 \pm 0.08$ & 0.336 & $1.09 \pm 0.08$ & 0.282 & $1.13 \pm 0.08$ & 0.264 & $1.21 \pm 0.08$ & 0.29 \\
\hline 8 & $1.25 \pm 0.08$ & 0.306 & $1.4 \pm 0.08$ & 0.351 & $1.45 \pm 0.07$ & 0.362 & $1.36 \pm 0.08$ & 0.32 \\
\hline 9 & $1.19 \pm 0.08$ & 0.325 & $1.12 \pm 0.07$ & 0.279 & $1.16 \pm 0.07$ & 0.31 & $1.21 \pm 0.07$ & 0.311 \\
\hline 10 & $0.98 \pm 0.12$ & 0.213 & $1.12 \pm 0.11$ & 0.273 & $0.96 \pm 0.1$ & 0.21 & $1.09 \pm 0.11$ & 0.228 \\
\hline 11 & $0.82 \pm 0.1$ & 0.21 & $0.81 \pm 0.09$ & 0.212 & $0.75 \pm 0.09$ & 0.159 & $0.82 \pm 0.09$ & 0.187 \\
\hline 12 & $1.15 \pm 0.08$ & 0.32 & $1.16 \pm 0.07$ & 0.309 & $1.19 \pm 0.07$ & 0.291 & $1.23 \pm 0.08$ & 0.307 \\
\hline 13 & $1.08 \pm 0.08$ & 0.411 & $1.08 \pm 0.08$ & 0.364 & $1.21 \pm 0.08$ & 0.405 & $1.13 \pm 0.08$ & 0.386 \\
\hline 14 & $0.7 \pm 0.1$ & 0.213 & $0.72 \pm 0.1$ & 0.238 & $0.62 \pm 0.09$ & 0.174 & $0.62 \pm 0.09$ & 0.185 \\
\hline 15 & $1.04 \pm 0.09$ & 0.268 & $1.13 \pm 0.08$ & 0.301 & $1.14 \pm 0.08$ & 0.279 & $1.16 \pm 0.09$ & 0.241 \\
\hline 16 & $1.1 \pm 0.08$ & 0.322 & $1.12 \pm 0.08$ & 0.303 & $1.1 \pm 0.08$ & 0.296 & $1.01 \pm 0.08$ & 0.268 \\
\hline 17 & $0.9 \pm 0.09$ & 0.212 & $0.89 \pm 0.08$ & 0.194 & $0.9 \pm 0.08$ & 0.181 & $0.89 \pm 0.08$ & 0.176 \\
\hline 18 & $1.15 \pm 0.09$ & 0.345 & $1.04 \pm 0.09$ & 0.294 & $1.16 \pm 0.08$ & 0.306 & $1.12 \pm 0.08$ & 0.265 \\
\hline 19 & $1.03 \pm 0.08$ & 0.282 & $1.11 \pm 0.07$ & 0.309 & $1.11 \pm 0.08$ & 0.274 & $1.14 \pm 0.07$ & 0.271 \\
\hline 20 & $1.26 \pm 0.07$ & 0.375 & $1.28 \pm 0.07$ & 0.372 & $1.34 \pm 0.07$ & 0.391 & $1.29 \pm 0.07$ & 0.341 \\
\hline 21 & $1.22 \pm 0.08$ & 0.369 & $1.26 \pm 0.07$ & 0.36 & $1.26 \pm 0.07$ & 0.321 & $1.26 \pm 0.07$ & 0.307 \\
\hline 22 & $1.24 \pm 0.08$ & 0.327 & $1.3 \pm 0.07$ & 0.337 & $1.34 \pm 0.07$ & 0.343 & $1.3 \pm 0.07$ & 0.298 \\
\hline 23 & $1.03 \pm 0.09$ & 0.302 & $0.96 \pm 0.08$ & 0.264 & $0.98 \pm 0.09$ & 0.232 & $1.05 \pm 0.09$ & 0.271 \\
\hline 24 & $0.95 \pm 0.09$ & 0.253 & $0.9 \pm 0.08$ & 0.202 & $0.89 \pm 0.08$ & 0.223 & $0.92 \pm 0.08$ & 0.221 \\
\hline 25 & $1.04 \pm 0.08$ & 0.265 & $1.04 \pm 0.08$ & 0.25 & $1.07 \pm 0.07$ & 0.242 & $0.92 \pm 0.08$ & 0.17 \\
\hline 26 & $1.1 \pm 0.11$ & 0.279 & $1.1 \pm 0.12$ & 0.253 & $1.02 \pm 0.12$ & 0.208 & $1.13 \pm 0.13$ & 0.228 \\
\hline 27 & $0.99 \pm 0.1$ & 0.148 & $1.02 \pm 0.1$ & 0.137 & $1.17 \pm 0.11$ & 0.149 & $1.06 \pm 0.11$ & 0.128 \\
\hline 28 & $1.34 \pm 0.08$ & 0.364 & $1.36 \pm 0.08$ & 0.359 & $1.39 \pm 0.08$ & 0.328 & $1.38 \pm 0.08$ & 0.307 \\
\hline 29 & $1.75 \pm 0.08$ & 0.454 & $1.81 \pm 0.08$ & 0.455 & $1.89 \pm 0.08$ & 0.446 & $1.94 \pm 0.08$ & 0.454 \\
\hline 30 & $1.48 \pm 0.08$ & 0.402 & $1.61 \pm 0.07$ & 0.43 & $1.64 \pm 0.08$ & 0.393 & $1.82 \pm 0.08$ & 0.406 \\
\hline 31 & $0.83 \pm 0.1$ & 0.205 & $0.78 \pm 0.1$ & 0.17 & $0.91 \pm 0.1$ & 0.214 & $0.88 \pm 0.1$ & 0.168 \\
\hline 32 & $1.4 \pm 0.07$ & 0.414 & $1.47 \pm 0.07$ & 0.386 & $1.47 \pm 0.08$ & 0.359 & $1.54 \pm 0.07$ & 0.391 \\
\hline 33 & $1.3 \pm 0.08$ & 0.379 & $1.34 \pm 0.07$ & 0.366 & $1.51 \pm 0.07$ & 0.381 & $1.53 \pm 0.07$ & 0.37 \\
\hline 34 & $1.08 \pm 0.08$ & 0.305 & $1.05 \pm 0.08$ & 0.258 & $1.2 \pm 0.07$ & 0.291 & $1.22 \pm 0.08$ & 0.279 \\
\hline 35 & $1.26 \pm 0.09$ & 0.38 & $1.23 \pm 0.08$ & 0.362 & $1.39 \pm 0.08$ & 0.394 & $1.38 \pm 0.08$ & 0.394 \\
\hline 36 & $0.69 \pm 0.09$ & 0.214 & $0.67 \pm 0.09$ & 0.204 & $0.72 \pm 0.09$ & 0.213 & $0.66 \pm 0.1$ & 0.165 \\
\hline 37 & $1.53 \pm 0.1$ & 0.438 & $1.44 \pm 0.09$ & 0.416 & $1.45 \pm 0.08$ & 0.409 & $1.43 \pm 0.08$ & 0.43 \\
\hline 38 & $1.45 \pm 0.07$ & 0.42 & $1.56 \pm 0.07$ & 0.415 & $1.65 \pm 0.07$ & 0.407 & $1.73 \pm 0.07$ & 0.429 \\
\hline 39 & $1.86 \pm 0.07$ & 0.445 & $1.9 \pm 0.07$ & 0.463 & $1.97 \pm 0.07$ & 0.435 & $2.06 \pm 0.07$ & 0.441 \\
\hline 40 & $1.6 \pm 0.07$ & 0.43 & $1.61 \pm 0.07$ & 0.446 & $1.8 \pm 0.07$ & 0.46 & $1.82 \pm 0.07$ & 0.452 \\
\hline 41 & $1.26 \pm 0.08$ & 0.386 & $1.22 \pm 0.08$ & 0.371 & $1.32 \pm 0.08$ & 0.375 & $1.19 \pm 0.08$ & 0.325 \\
\hline 42 & $1.55 \pm 0.07$ & 0.457 & $1.52 \pm 0.07$ & 0.443 & $1.66 \pm 0.07$ & 0.451 & $1.63 \pm 0.07$ & 0.413 \\
\hline 43 & $1.23 \pm 0.1$ & 0.409 & $1.14 \pm 0.1$ & 0.402 & $1.29 \pm 0.1$ & 0.379 & $1.14 \pm 0.1$ & 0.315 \\
\hline 44 & $1.51 \pm 0.07$ & 0.392 & $1.6 \pm 0.07$ & 0.382 & $1.69 \pm 0.07$ & 0.38 & $1.59 \pm 0.07$ & 0.376 \\
\hline 45 & $0.89 \pm 0.12$ & 0.3 & $0.93 \pm 0.11$ & 0.297 & $0.87 \pm 0.12$ & 0.26 & $0.91 \pm 0.11$ & 0.285 \\
\hline 46 & $1 \pm 0.09$ & 0.359 & $0.9 \pm 0.08$ & 0.333 & $0.98 \pm 0.08$ & 0.367 & $0.93 \pm 0.08$ & 0.312 \\
\hline 47 & $1.35 \pm 0.12$ & 0.379 & $1.24 \pm 0.12$ & 0.369 & $1.34 \pm 0.11$ & 0.362 & $1.35 \pm 0.11$ & 0.376 \\
\hline 48 & $1.64 \pm 0.08$ & 0.403 & $1.67 \pm 0.07$ & 0.407 & $1.68 \pm 0.08$ & 0.379 & $1.71 \pm 0.07$ & 0.402 \\
\hline 49 & $1.56 \pm 0.07$ & 0.408 & $1.47 \pm 0.07$ & 0.374 & $1.5 \pm 0.07$ & 0.347 & $1.51 \pm 0.07$ & 0.345 \\
\hline
\end{tabular}


Table of results for the $\theta$ exponent without RSP and RPP filters (cont.)

\begin{tabular}{|c|c|c|c|c|c|c|c|c|}
\hline & 1995 & & 1999 & & 2004 & & 2009 & \\
\hline HS2 & $\theta$ & $R^{2}$ & $\theta$ & $R^{2}$ & $\theta$ & $R^{2}$ & $\theta$ & $R^{2}$ \\
\hline 50 & $1.07 \pm 0.11$ & 0.333 & $0.95 \pm 0.11$ & 0.317 & $1.08 \pm 0.1$ & 0.36 & $1.09 \pm 0.11$ & 0.34 \\
\hline 51 & $1.31 \pm 0.09$ & 0.417 & $1.27 \pm 0.09$ & 0.378 & $1.3 \pm 0.09$ & 0.383 & $1.18 \pm 0.09$ & 0.365 \\
\hline 52 & $1.27 \pm 0.07$ & 0.367 & $1.36 \pm 0.07$ & 0.375 & $1.39 \pm 0.07$ & 0.367 & $1.27 \pm 0.08$ & 0.325 \\
\hline 53 & $1.04 \pm 0.09$ & 0.365 & $1.09 \pm 0.09$ & 0.356 & $0.99 \pm 0.08$ & 0.329 & $0.97 \pm 0.09$ & 0.297 \\
\hline 54 & $1.48 \pm 0.08$ & 0.426 & $1.51 \pm 0.07$ & 0.453 & $1.52 \pm 0.07$ & 0.453 & $1.32 \pm 0.08$ & 0.375 \\
\hline 55 & $1.34 \pm 0.08$ & 0.389 & $1.39 \pm 0.07$ & 0.411 & $1.44 \pm 0.07$ & 0.412 & $1.33 \pm 0.08$ & 0.372 \\
\hline 56 & $1.17 \pm 0.08$ & 0.374 & $1.28 \pm 0.07$ & 0.426 & $1.25 \pm 0.07$ & 0.357 & $1.26 \pm 0.07$ & 0.348 \\
\hline 57 & $1.39 \pm 0.08$ & 0.443 & $1.35 \pm 0.07$ & 0.466 & $1.36 \pm 0.08$ & 0.383 & $1.37 \pm 0.08$ & 0.412 \\
\hline 58 & $1.28 \pm 0.07$ & 0.42 & $1.28 \pm 0.08$ & 0.412 & $1.37 \pm 0.07$ & 0.395 & $1.26 \pm 0.07$ & 0.383 \\
\hline 59 & $1.2 \pm 0.09$ & 0.362 & $1.43 \pm 0.08$ & 0.441 & $1.43 \pm 0.08$ & 0.403 & $1.4 \pm 0.08$ & 0.415 \\
\hline 60 & $1.15 \pm 0.09$ & 0.328 & $1.17 \pm 0.09$ & 0.327 & $1.16 \pm 0.09$ & 0.302 & $1.15 \pm 0.09$ & 0.286 \\
\hline 61 & $1.63 \pm 0.08$ & 0.417 & $1.72 \pm 0.08$ & 0.413 & $1.91 \pm 0.07$ & 0.438 & $1.88 \pm 0.07$ & 0.445 \\
\hline 62 & $1.88 \pm 0.07$ & 0.479 & $1.83 \pm 0.07$ & 0.446 & $1.88 \pm 0.07$ & 0.441 & $1.94 \pm 0.07$ & 0.457 \\
\hline 63 & $1.36 \pm 0.07$ & 0.422 & $1.43 \pm 0.06$ & 0.39 & $1.48 \pm 0.06$ & 0.383 & $1.49 \pm 0.07$ & 0.364 \\
\hline 64 & $1.53 \pm 0.08$ & 0.427 & $1.47 \pm 0.07$ & 0.386 & $1.56 \pm 0.07$ & 0.404 & $1.55 \pm 0.08$ & 0.372 \\
\hline 65 & $1.18 \pm 0.08$ & 0.397 & $1.22 \pm 0.07$ & 0.408 & $1.27 \pm 0.07$ & 0.39 & $1.19 \pm 0.07$ & 0.37 \\
\hline 66 & $1.03 \pm 0.1$ & 0.363 & $1.01 \pm 0.09$ & 0.355 & $0.96 \pm 0.09$ & 0.307 & $0.84 \pm 0.1$ & 0.263 \\
\hline 67 & $1 \pm 0.09$ & 0.373 & $1.02 \pm 0.09$ & 0.371 & $0.98 \pm 0.08$ & 0.335 & $0.99 \pm 0.09$ & 0.332 \\
\hline 68 & $1.33 \pm 0.08$ & 0.404 & $1.35 \pm 0.07$ & 0.382 & $1.43 \pm 0.07$ & 0.39 & $1.39 \pm 0.07$ & 0.377 \\
\hline 69 & $1.5 \pm 0.07$ & 0.475 & $1.53 \pm 0.06$ & 0.456 & $1.53 \pm 0.07$ & 0.417 & $1.42 \pm 0.07$ & 0.396 \\
\hline 70 & $1.37 \pm 0.07$ & 0.371 & $1.45 \pm 0.07$ & 0.379 & $1.55 \pm 0.07$ & 0.395 & $1.56 \pm 0.07$ & 0.388 \\
\hline 71 & $1.69 \pm 0.1$ & 0.395 & $1.83 \pm 0.09$ & 0.408 & $1.85 \pm 0.09$ & 0.402 & $1.91 \pm 0.1$ & 0.389 \\
\hline 72 & $1.57 \pm 0.08$ & 0.417 & $1.48 \pm 0.08$ & 0.383 & $1.61 \pm 0.08$ & 0.368 & $1.67 \pm 0.07$ & 0.406 \\
\hline 73 & $1.73 \pm 0.07$ & 0.468 & $1.68 \pm 0.07$ & 0.428 & $1.87 \pm 0.07$ & 0.425 & $2 \pm 0.07$ & 0.453 \\
\hline 74 & $1.49 \pm 0.09$ & 0.381 & $1.42 \pm 0.08$ & 0.353 & $1.47 \pm 0.08$ & 0.336 & $1.55 \pm 0.09$ & 0.348 \\
\hline 75 & $1.31 \pm 0.12$ & 0.381 & $1.22 \pm 0.12$ & 0.316 & $1.46 \pm 0.12$ & 0.373 & $1.42 \pm 0.12$ & 0.392 \\
\hline 76 & $1.43 \pm 0.08$ & 0.347 & $1.42 \pm 0.07$ & 0.342 & $1.53 \pm 0.08$ & 0.359 & $1.5 \pm 0.08$ & 0.358 \\
\hline 78 & $0.76 \pm 0.12$ & 0.196 & $0.71 \pm 0.12$ & 0.173 & $0.78 \pm 0.12$ & 0.176 & $0.84 \pm 0.11$ & 0.211 \\
\hline 79 & $0.89 \pm 0.11$ & 0.23 & $1 \pm 0.1$ & 0.257 & $0.94 \pm 0.11$ & 0.221 & $1.02 \pm 0.1$ & 0.256 \\
\hline 80 & $0.98 \pm 0.12$ & 0.306 & $0.88 \pm 0.12$ & 0.243 & $0.96 \pm 0.12$ & 0.27 & $1 \pm 0.13$ & 0.257 \\
\hline 81 & $1.35 \pm 0.1$ & 0.42 & $1.36 \pm 0.1$ & 0.455 & $1.48 \pm 0.1$ & 0.453 & $1.4 \pm 0.1$ & 0.42 \\
\hline 82 & $1.61 \pm 0.07$ & 0.482 & $1.61 \pm 0.07$ & 0.461 & $1.75 \pm 0.07$ & 0.477 & $1.81 \pm 0.06$ & 0.475 \\
\hline 83 & $1.48 \pm 0.07$ & 0.436 & $1.5 \pm 0.07$ & 0.425 & $1.62 \pm 0.07$ & 0.442 & $1.6 \pm 0.07$ & 0.408 \\
\hline 84 & $2.46 \pm 0.07$ & 0.547 & $2.55 \pm 0.07$ & 0.531 & $2.75 \pm 0.07$ & 0.524 & $2.66 \pm 0.07$ & 0.493 \\
\hline 85 & $2.4 \pm 0.07$ & 0.533 & $2.58 \pm 0.07$ & 0.53 & $2.69 \pm 0.07$ & 0.515 & $2.78 \pm 0.07$ & 0.53 \\
\hline 86 & $1.19 \pm 0.11$ & 0.335 & $1.2 \pm 0.1$ & 0.324 & $1.18 \pm 0.1$ & 0.29 & $1.27 \pm 0.09$ & 0.316 \\
\hline 87 & $1.99 \pm 0.07$ & 0.494 & $1.99 \pm 0.07$ & 0.447 & $2.18 \pm 0.08$ & 0.456 & $2.13 \pm 0.07$ & 0.439 \\
\hline 88 & $1.48 \pm 0.12$ & 0.35 & $1.43 \pm 0.11$ & 0.311 & $1.44 \pm 0.1$ & 0.299 & $1.6 \pm 0.1$ & 0.353 \\
\hline 89 & $1.05 \pm 0.11$ & 0.244 & $1.1 \pm 0.1$ & 0.248 & $1.19 \pm 0.1$ & 0.268 & $1.14 \pm 0.1$ & 0.224 \\
\hline 90 & $2.08 \pm 0.07$ & 0.555 & $2.15 \pm 0.07$ & 0.528 & $2.25 \pm 0.07$ & 0.532 & $2.36 \pm 0.06$ & 0.541 \\
\hline 91 & $1.55 \pm 0.09$ & 0.415 & $1.47 \pm 0.08$ & 0.425 & $1.47 \pm 0.08$ & 0.415 & $1.53 \pm 0.08$ & 0.43 \\
\hline 92 & $1.47 \pm 0.08$ & 0.525 & $1.41 \pm 0.07$ & 0.506 & $1.47 \pm 0.07$ & 0.535 & $1.41 \pm 0.08$ & 0.475 \\
\hline 93 & $1.03 \pm 0.11$ & 0.293 & $1.09 \pm 0.1$ & 0.312 & $0.98 \pm 0.09$ & 0.301 & $1.15 \pm 0.1$ & 0.323 \\
\hline 94 & $1.62 \pm 0.07$ & 0.442 & $1.74 \pm 0.07$ & 0.467 & $1.91 \pm 0.07$ & 0.465 & $1.85 \pm 0.07$ & 0.424 \\
\hline 95 & $1.77 \pm 0.07$ & 0.524 & $1.73 \pm 0.07$ & 0.511 & $1.77 \pm 0.07$ & 0.471 & $1.78 \pm 0.07$ & 0.491 \\
\hline 96 & $1.48 \pm 0.07$ & 0.465 & $1.46 \pm 0.07$ & 0.431 & $1.49 \pm 0.07$ & 0.426 & $1.42 \pm 0.07$ & 0.385 \\
\hline 97 & $1.38 \pm 0.09$ & 0.444 & $1.45 \pm 0.08$ & 0.465 & $1.41 \pm 0.08$ & 0.444 & $1.45 \pm 0.08$ & 0.45 \\
\hline
\end{tabular}





\title{
Appendix B: Data visualization
}

\author{
By visualizing information, we turn it into a \\ landscape that you can explore with your eyes, a \\ sort of information map. And when you're lost in \\ information, an information map is kind of \\ useful.
}

\section{- David McCandless}

(World leading expert in data visualization )

When we deal with complex systems, we also deal with the problem of the consumption and assimilation of a large quantity of data. For example, financial markets generate huge volumes of data that come from the trading activities hourly, daily, monthly, locally, regionally, worldwide and so on. Common sense suggests that looking at interactive charts to visualize the time series derived from the prices and trade volumes of a given stock is a reasonable action. The human brain seems to be highly efficient in processing and understanding visual patterns as a consequent of our evolution process. Furthermore, popular culture commonly supports the concept in which "a picture says a thousand words". Based on this premise, it can be argued that we might formally take advantage of the physical attributes of human vision in order to efficiently consume and understand large datasets. This, in addition to exponentially growing data availablity early in the XXI century, has led to the introduction of a new discipline, which is often referred to as Data Visualization.

Yet, data visualization is not new to humanity. Let us consider the work of Charles Minard (1781-1870), a civil engineer who was highly recognized for his information graphics. In 1869, Minard created a map of Napoleon's Russian campaign of 1812 (in French, Carte figurative des pertes successives en hommes de l'Armée Française dans la campagne de Russie 1812-1813). This figure portraits the loss of men over time and different locations, where the thickness of the line depicts the number of soldiers of his army in addition to other variables such as traveled distance and temperature, for instance. Now, Minard's design is highly innovative (even by today's standards) since he was able to collate, mix and simplify that is heterogeneous in nature while portraiting the variables in a very simple way. The delivery is not without a sense of elegance, because mixing geo-spatial and quantitative data continues to be a very 


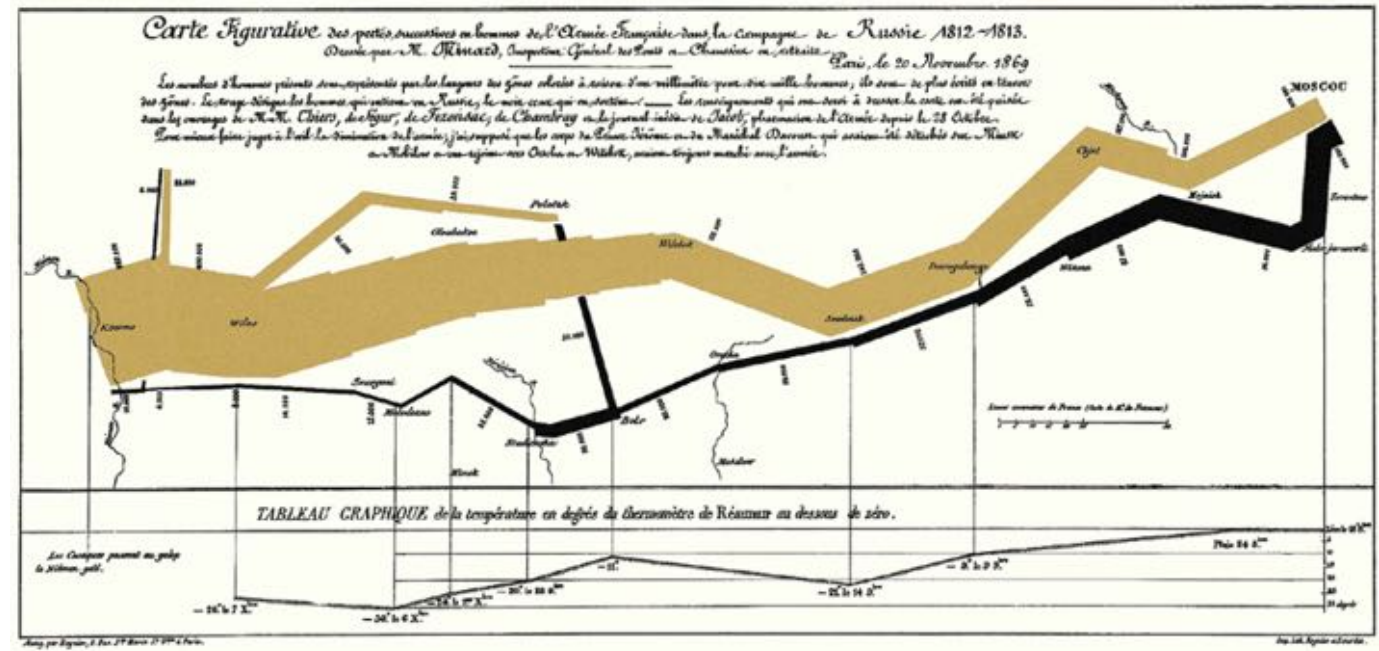

Fig. B.1.: Map of Napoleon's Russian Campaign of 1812 by Charles Minard. This figure shows one of the first visualizations of complex data. This works describe the campaign of Napoleon Bonaparte to Russia with a great deal of detail, an outstanding achievement if we consider the period in which was performed.

complex task. The resulting visualization (Figure B.1) is not only a master piece, but also an inspiration to the data visualization community nowadays.

Even though this is not the only information graphic from Minard, it is highly representative of the essence of data visualization: a great design that tells a story in a simple way by using visual tools. Of course, the biggest constraint for Minard to expand this discipline in his times was not a lack of inspiration or design skills, but the limited access to data. This constraint is no longer a concern in our century, and we can benefit from the massive datasets in almost any field or discipline.

\section{B.1 Principles of Design}

The main objective of any visualization is to effectively communicate an idea or fact in a visual way. Confusing and ambiguious graphics are among the main ways of destroying an effective visual communication tool. For this reason, we follow simple 10 rules to maximize comprehensiveness of visualizations. These are not the only rules that could be applied to the design of visual tool, but they are certainly the ones that can drastically improve a design at a minimum cost of implementation.

Rule 1: Confusing vs merely informative. Including too much information in a visualization can make it confusing rather than an effective communication tool. In contrast, excluding critical information can turn the visualization into a merely informative figure. Finding the correct spot between information excess and just 
informative data is critical for a balanced visualization that delivers a clear message while making complexity accessible to the reader.

Rule 2: Beautiful design. Attractiveness is within the main reasons why a human being would pay attention to graphics. A sense of proportion and symmetry while using the appropriate colors will increase the attractiness of a visualization.

Rule 3: Normalized spacing and sizing. Maintaining similar spacing and size of supporting element can enhance the focus and attention towards the critical parts of the visualization while simplifying the overall visual complexity.

Rule 4: No redundancy. By removing elements that are redundant, we can increase simplicity and drive attention to aspects that contribute to the message delivery.

Rule 5: Manage opacity. Whenever a reference is required, low opacity should be considered in order to maintain graphical density to avoid unnecessary confusion. In this way, the different layers of the visualization can be easily differentiated.

Rule 6: Consistency is the key. References, axis, fonts, sizes, colors should always follow the same rules. Whenever these rules are missed, a mistaken interpretation will occur since the reader may assume this is because an incorrect reason, causing noise in the message delivery.

Rule 7: Color perception is tricky. We need to manage our colors accordingly. In some cases, perceived brightness can create a sense of importance in a erroneous way. For example, yellow appears brighter than blue even when their theoretical brightness and color saturation may be the same.

Rule 8: Uniformity and grid layout. Setting a standard for the whole visualization helps us accomplish uniformity, which generates a sense of visual simplicity. A hidden grip layout is frequently a smart trick to secure uniformity in terms of proportion and spacing.

Rule 9: Patterns are usually hidden. The visualization should help the reader unveil any hidden pattern in the data. A proper selection of the visualization type will be critical to accomplish this objective. We will review more details of this concept in the next section.

Rule 10: Focus on the data. Lack of alignment, changing scales and thick boxes certainly reduce the attention on the data. Although this is a common mistake, it is only logical to use the previous rules to avoid this. 


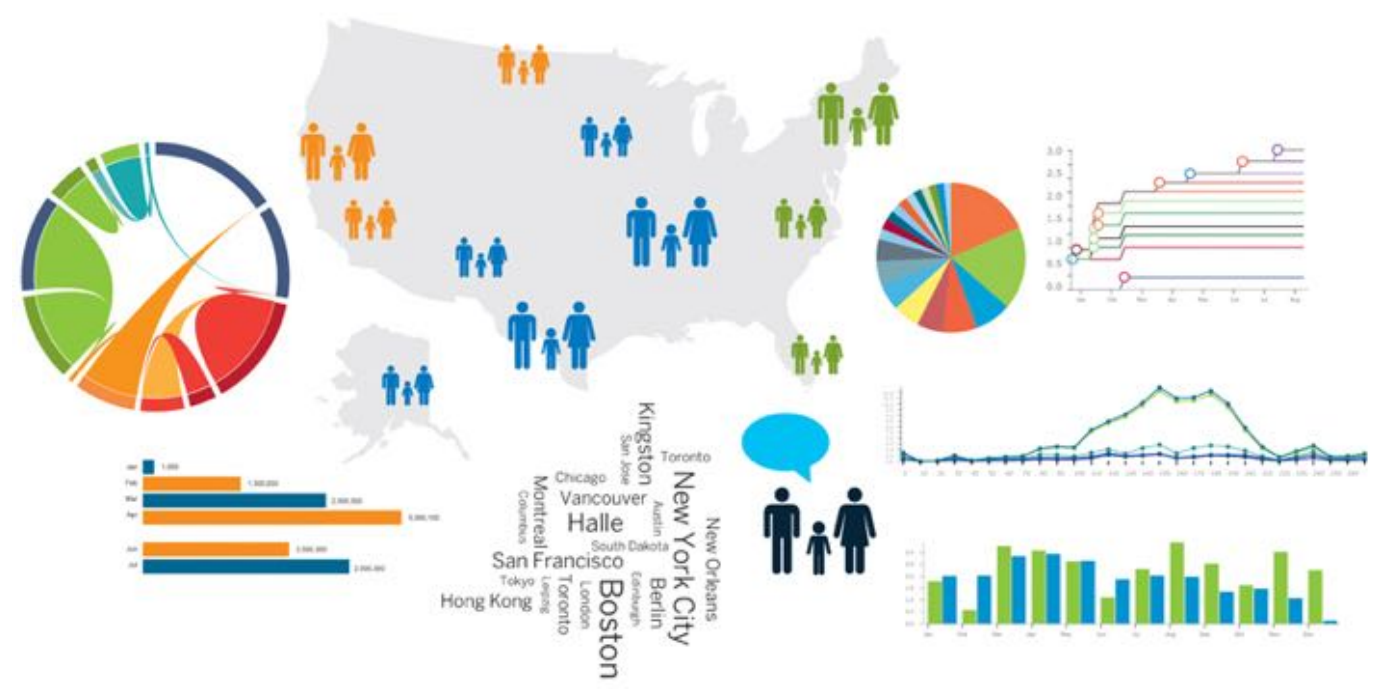

Fig. B.2.: Examples of multiple forms of visualizations. We show a variety of data visualizations. From left to right, a circular layout network, a bar chart, a geolocated data map, a word cloud a pie chart, a time series graph and a tree diagram. All of these graphs may be used for a suitable purpose. The goal of data visualization is to provide a framework to optimize the visual design.

These rules work independently of the type of visualization that are used to convey different messages. For example, time series of a continuous variable is not a perfect fit for bar charts. Rather, they are more suitable for a line chart, where trends and seasonality effects can easily be displayed. But there are more types of visualizations such as circular graphs (highly efficient for networks), geo-spatial graphs (useful for geo-located data) or word clouds (special for visualizing word frequency) as seen in figure B.2.

For this study, we decided to develop our own visualization tools, which we review in the following sections along with an overview of our code to implement them.

\section{B.2 Bipartite graph visualization}

There are several possible layouts to visualize complex networks. Unfortunately, most of them are not strictly suitable for weighted bipartite networks. But this is not the case of the circular layout graphs. This particular layout presents several benefits to accomodate the special features of our WBTNs. First, the exporter and importer subsets of node can easly be separated, allowing for node segmentation based on a combination of node position and color coding. Secondly, the weighted nature of the networks can be portraited through the thickness of each link in addition to further color coding. Finally, the circular configuration creates equal focus across 


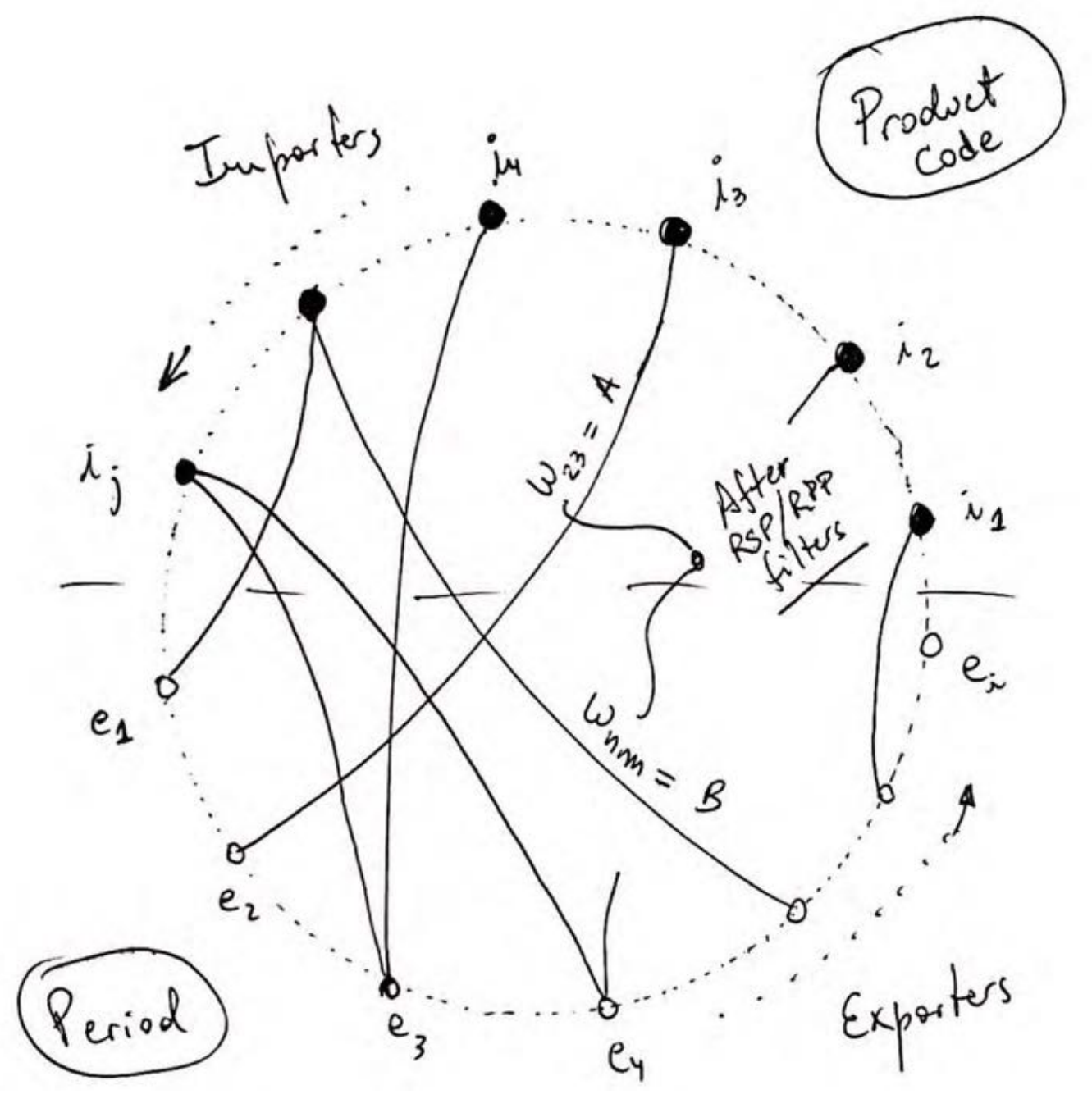

Fig. B.3.: Design of circular graph visualizations. This figure shows shows two examples of complex networks in a circular graph configuration. Both networks have been created randomly and we have assigned a color code to distinguish connections between nodes.

the nodes, leaving room for the display of link concentration in the center. We show the basic design concept in figure B.3.

Based on the proposed design, we apply it to a couple of random networks to test its validity. Figure B.4 shows the results of this test.

In the examples, we use binary random networks due to the simplicity to create them. Nonetheless, we can observe the patterns that arise from the proposed combination of node positioning and color coding. Consequently, we will adopt this layout configuration for the visualization of our results. But this needs to incorporate further definitions: 

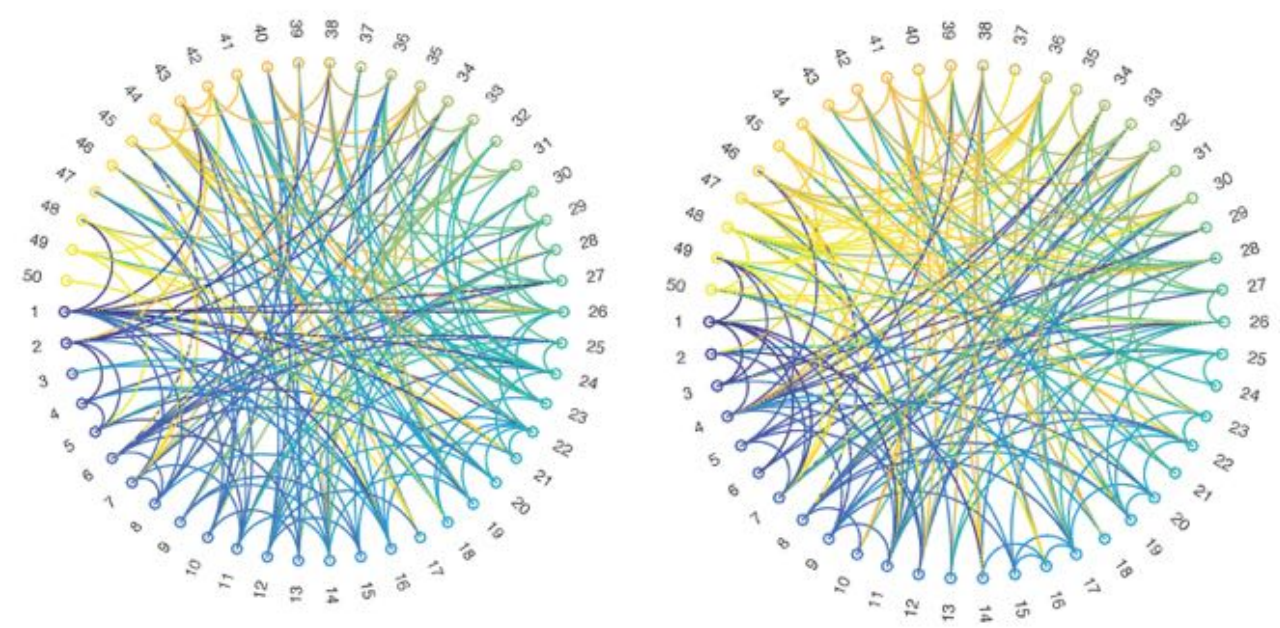

Fig. B.4.: Examples of circular graph visualizations (random networks). This figure shows shows two examples of complex networks in a circular graph configuration. Both networks have been created randomly and we have assigned a color code to distinguish connections between nodes.

Node position: we adopt positions along a circle where nodes are equally separated in distance among the $2 \pi R$ circumference. The radius $R$ is selected conveniently to fit both the nodes and the node names. In terms of ordering, we position the exporters on the bottom side of the circle, sorting them based on the ISO-3 country code in ascending order. The same is true for the importers on the upper side of the circle.

Node size: we adopt a constant size for both the exporter and importer nodes. Despite the fact that node size could be proportional to the node degree, this is not the main purpose of our design. Additionally, the node degree property can anyway be appreciated by the number of links that arrive to (or depart from) it. This avoids some redundancy.

Link thickness: we adopt a scaled definition for the thickness of each link. The maximum thickness will be of 4 points, whereas the minimum will be 0.5 points. We assign the maximum thickness to those links that are at least 0.25 times the maximum trade volume.

Node labels: we assign the standard ISO country code to each node. It can be observed that they start from the left-middle position (9 o'clock position), are ordered by ISO country code and continue to be positioned with an anti-clockwise direction. We start with the exporters first and continue with the importers (also in anti-clockwise direction). 


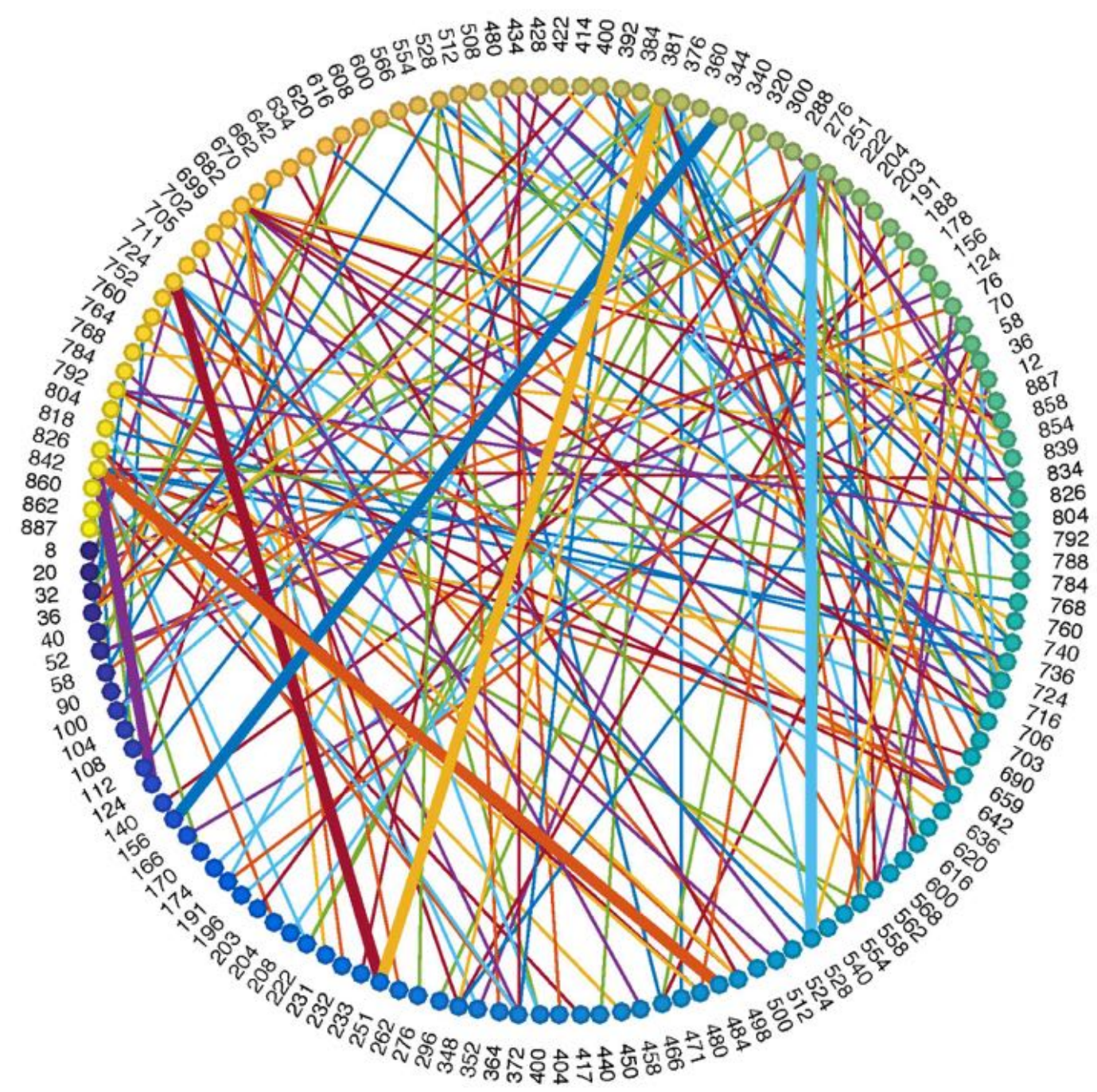

Fig. B.5.: Design application to an actual WBTN (HS2 $=01$ in 1995). This figure shows shows two examples of bipartite binary matrices. This means that each white spot represents an link active link that connects the $i$ element of the first of nodes with the $j$ element of the other set.

Visible links and nodes: for vital link and node visualization, we restrict the RSP and RPP values to $R S P>10^{-1}$ and $R P P>10^{-1}$. These filter settings ensure that only the most important links and nodes are visualized.

As a result, we show an example of an actual WBTN for the industry HS2 $=01$ (Live Animals) in the year 1995 (figure B.5).

In order to build our own visualizations, an adaptation of the code is required. Therefore, we include our Matlab code for a simple circular layout visualization, which shows the simplicity of creating special layouts (no color management or sizing in this case though): 


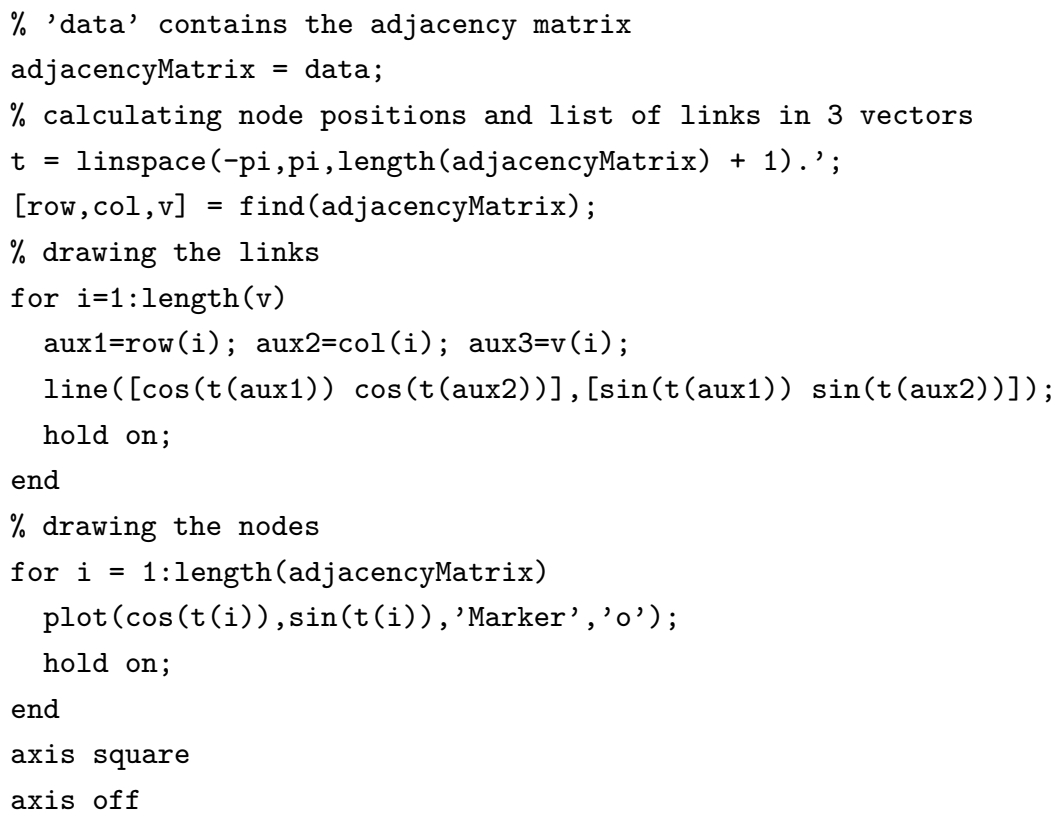

This basic code can be improved so that it reflects all visual features as shown in figure B.4. But even though we adopt this layout as our main visualization, we believe that increased and integrated visual tools are a must. In the next sections, we discuss alternative layouts and dashboards.

\section{B.3 Geo-spatial graph visualization}

Bipartite graphs are useful to understand the topological features of markets, as concluded in the previous section. However, we are dealing with market agents that represent different countries and consequently, the geographical distance is a very important variable to account for. For example, gravity models of international trade propose that countries will be more likely to exchange goods and services when the size of their economies are higher (big economies attract other economies in the same way a big physical mass works for gravity) and, at the same time, they will be less likely to trade when the geographical distance is higher. Furthermore, geographical constraints may also condition the clustering and assortative features of trade networks by increasing the probability of cluster formation. When a set of countries are mutually closer to each other, they will be more likely to form regional communities between them than with other countries. We can find several examples of this effect, such as the European cluster, the South American cluster or the Middle East cluster, where countries are very close to each other locally, but they are somewhat distant from other geographies. We propose the design of figure B.6. 


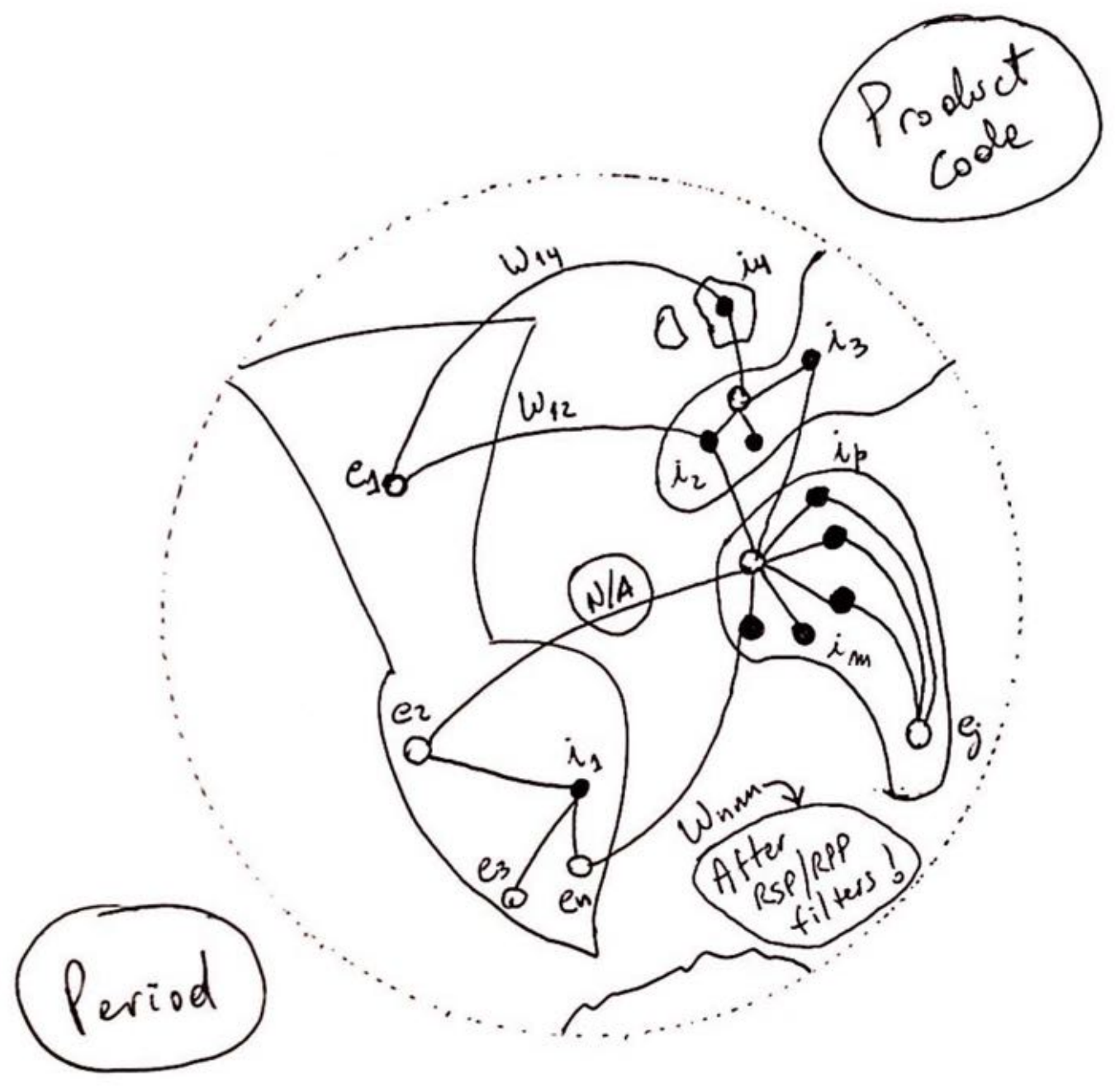

Fig. B.6.: Design of Geo-spatial graph visualizations. This figure shows shows two examples of complex networks in a circular graph configuration. Both networks have been created randomly and we have assigned a color code to distinguish connections between nodes. 
The design concept of a geo-spatial network is not difficult to implement from a coding perspective. The main difference in comparison to the previous circular layout is that node positions will now be determined by the geographical coordinates of the country instead of the sequential position around the circular space. This is a simplified version of our matlab code for descriptive purposes:

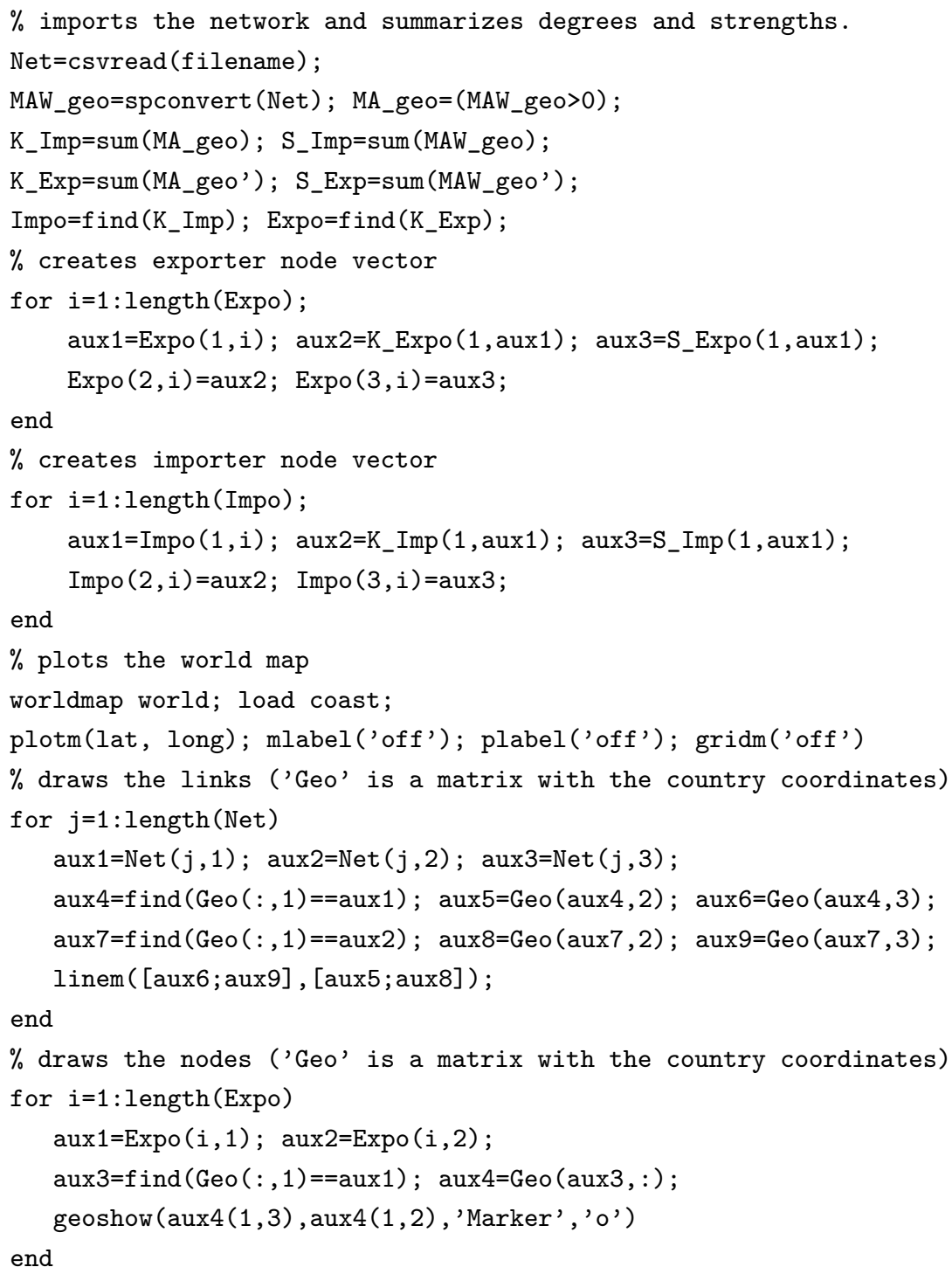

As with the previous visualization, we define a set of rules for our geo-located network layout. They are fundamental to the standardization of the visual features for all products and level of aggregation, as well as the different periods. The main goal is again to secure visual consistency.

Node position: we adopt the coordinates of the capital city for as a convention. Some exceptions are made when capital cities of two countries are located too close to 
each other, creating some confusion in the visualization. In these cases, we change the coordinates and use an approximate country central point.

Node size: we define 4 node categories based on the node degree. A "high-degree node" is represented if the node degree is higher than 0.7 times the maximum degree in the network. An "average degree node" is represented if the node degree is between 0.4 and 0.7 times the maximum degree in the network. A "low-degree node" will be between 0.15 and 0.4 times the maximum degree and the rest will be considered a "perispheric node".

Node color: based on the 4 categories defined by the node degree ranges, we also assign a different color to each category so that it helps the reader better differentiate them.

Link thickness: we also adopt 4 categories to depict the weight of each link. These categories are: a) High-volume link (higher than 0.25 times the maximum weight), b) Average-volume link (between 0.10 and 0.25 times the maximum weight), c) Low-volume link (between 0.005 and 0.10 times the maximum weight) and d) Marginal Link (lower than 0.005 times the maximum weight).

Link color: we align the color to each category. In this way, both links and node have a consistent definition of size-color combined coding.

Finally, we take care of some irregular pieces of the data (old countries, lack of country reporting or free trade zones) by assigning alternative coordinates upon the Greenwich meridian. In this way, the visualization does not incorporate noise upon the core countries while still accounting for the activity records. Additionally, we run our code for the first HS2 industry (01) and the first period (1995) and build the geo-located graph for this scenario. For the example, we set a filter of $R S P>10^{-2}$ for a balanced graph density. Thus, we show the outcome on the figure B.7. It can be appreciated that our design premises have been materialized in the visualization, where the core of the trade network is located in Western Eupore with some more peripheric, still significant nodes in America, the south of Africa and Australia. We also observe that some of these peripheric nodes have high trade volumes despite the fact that their node degree is not specially high on the other hand. For other nodes, the opposite is true. 


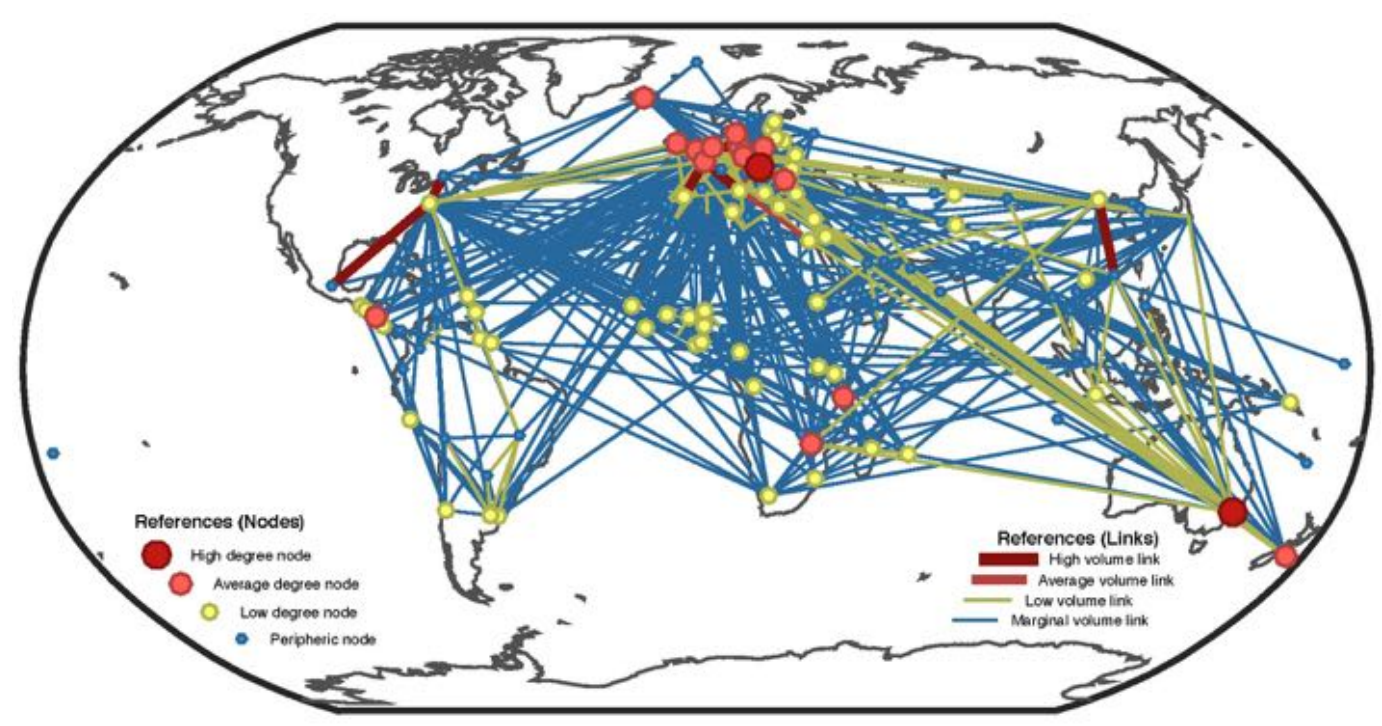

Fig. B.7.: Example of Geo-spatial graph visualization (HS2=01 in 1995). This figure shows an international trade network where nodes have been geolocated as described in section B.4. This layout provides an alternative view of the roles that countries play in the system, displaying areas of concentration of high degree nodes and relationaship between the different continents. Geographic constraints and trade preferences among countries can easily be visualized with this format.

\section{B.4 Other network layouts}

Despite the fact that we choose to design and implement our own vizualizations and their respective code, other highly professional and valuable tools and layouts are freely available for the scientific community to use. We acknowledge this fact by providing an overview of a few relevant tools and the underlying concepts behind them. In figure B.8 we show a few examples of these tools.

Force-Directed Layout. This layout is commonly available in most of the network visualization tools, like Cytoscape and Gephi. The importance of this layout is related to the concept of centrality, among others. In this layout, nodes that are located in the center of the graph tend to be more important than are those in the periphery based on some network parameter (degree, strength, and eigenvector centrality, among others). It is also the most common layout for general networks. Several algorithms are also available to set up the distances and positions of nodes. This, coupled with additional parameters like color coding and size, helps any data scientist create network visualizations that are both insightful and beautiful. The upper left layout in figure B.8 represent an example of this layout. On the other hand, Figure B.9 shows 8 different layouts, based on 8 different algorithms, of the same network. 

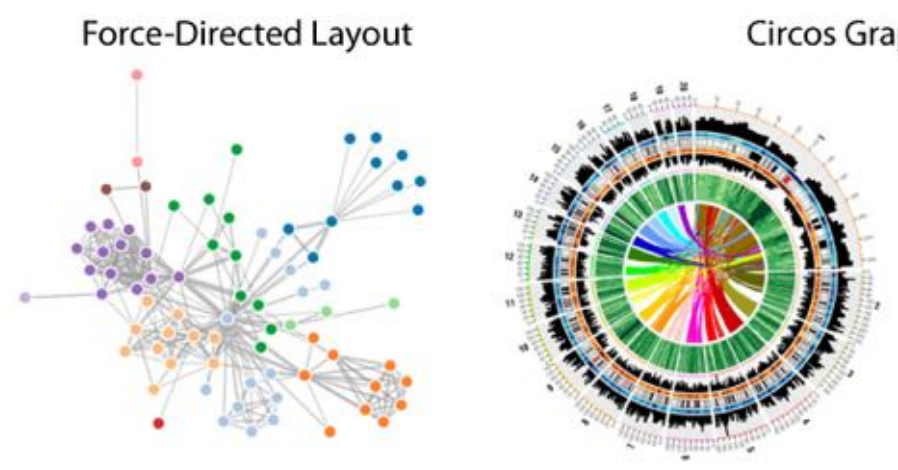

Circos Graph Layouts
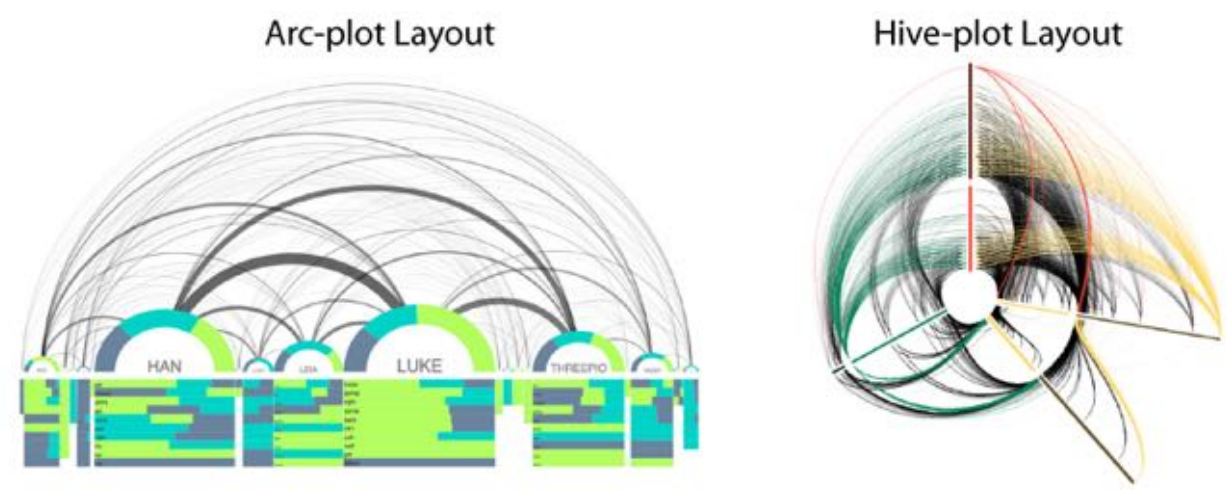

Fig. B.8.: Examples of other graph layouts. This figure shows different type of network layouts. First (upper-left layout), we show a force-directed network, which is the most commonly used layout for graph representation. Secondly (upper-right layouts), we find the Circos layout, which incorporates enriched data on top of a circular layout network, a highly professional tool. Thirdly (bottom-left layout), we observe the arc-plot layout, which accomodates nodes along a single axes, allowing for extended data beneath it. Finally (bottom-right layout), we find an example of a Hive Plot, a graph based on multiple radial axis where nodes can conveniently be distributed. 
Circos tool. This tool uses a circular layout to depict networks, but it does so with outstanding color management and enriched data that extends to other variables, which are located in different layers of the graph. This layout is commonly used to portrait complex findings from genomic and social science data. Based on information provided by the authors: "Circos uses a circular composition to show connections between objects or between positions, which are difficult to visually organize when the underlying layout is linear (or a graph, which can quickly become a hairball). In many cases, a linear layout makes impossible keeping the relationship lines from crossing other structures, deteriorates the effectiveness of the graphic". In figure B.8, the upper right visualizations are examples of the circos layout, which do not only contain the network graph in the center, but also incorporates quantitative data on the topic in the outside layers. This shows the level of complexity that can be depicted by such a tool.

Hive plots. This tool, similarly to Circos, is a professional network visualization one. The main feature is related to the usage of axis to locate nodes in a geometrically convenient form. It is particularly useful to visualize multi-layer networks. According to the tool authors: "The hive plot is a rational visualization method for drawing networks. Nodes are mapped to and positioned on radially distributed linear axes this mapping is based on network structural properties. Edges are drawn as curved links. Simple and interpretable. They also define that: "The purpose of the hive plot is to establish a new baseline for visualization of large networks - a method that is both general and tunable and useful as a starting point in visually exploring network structure. In figure B.8, the lower right layout is an example of the hive plots.

Arc Layout. The arc layout is quite close to the previous case of hive plots. We can conceptualize this layout as a hive plot of one axes only where links are depicted by using arcs that connect nodes accordingly. In this way, and similarly to Circos, Arc layout allows for additional quantitative data beneath the axes. In figure B.8, we show an example of this layout in the lower left graph. 


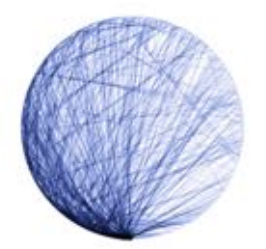

CIRCULAR BY DEGREE

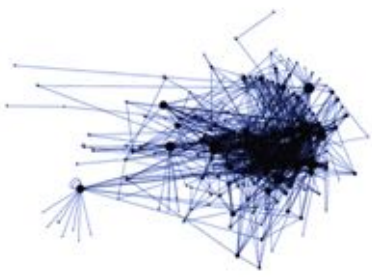

RADIAL TREE

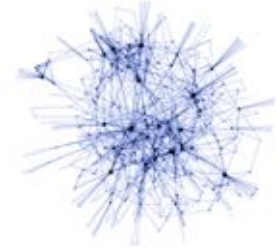

EDGE-WEIGHTED SPRING EMBEDDED

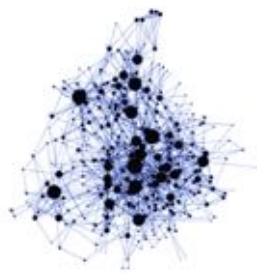

ORTHOGONAL

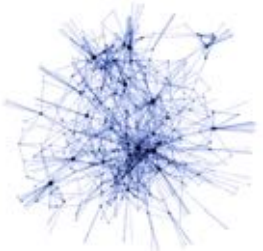

SPRING EMBEDDED

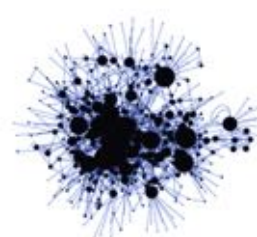

FORCE DIRECTED
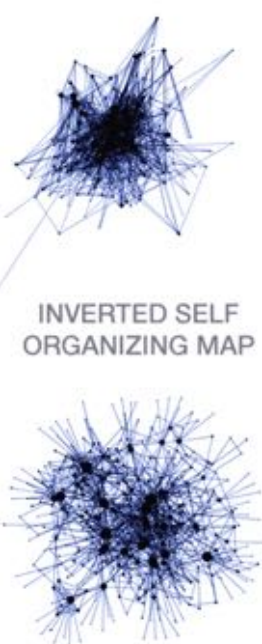

FORCE DIRECTED

Fig. B.9.: Examples of other graph layouts. This figure shows 8 alternative layouts of the same network, each of them depicting a different way of visualizing the network and its topological features from different perspectives. Source: authors of Circos (www.circos.ca) 

Appendix C: Selected HS2 visualizations 

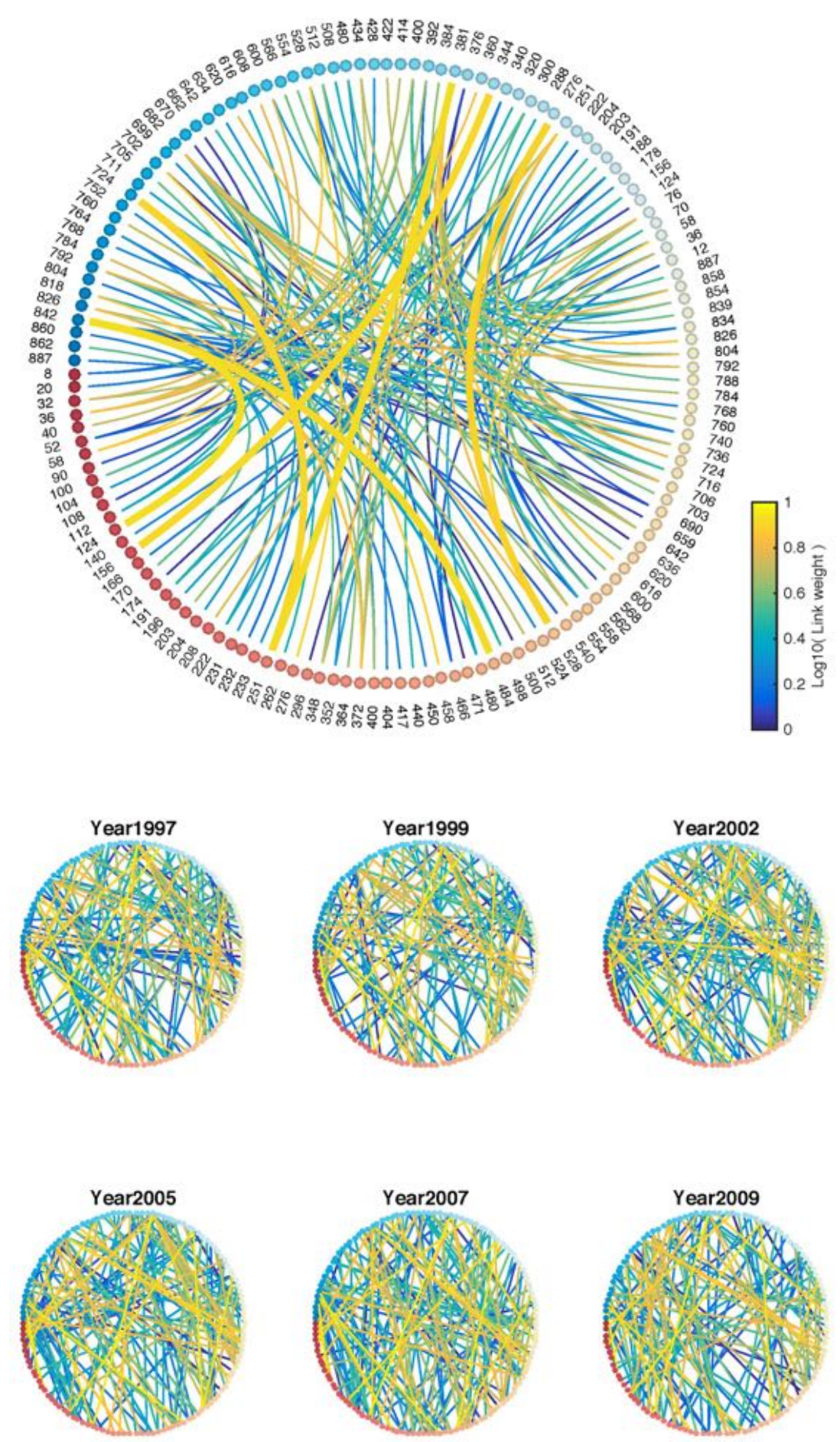

Fig. C.1.: Selected HS2 $=01$ trade network visualization. This figure shows the circular layout visualization for the 'Live Animals' industry (HS2 $=01$ ). We set a threshold of $R S P>0.1$ and $R P P>0.1$ for visible links. Exporters are located at the bottom of the graph with a declining red color gradient. Importers are located on the top of the graph with a declining blue color gradient. The thickness of the line is proportional to the weight of the link. Note: the importers start at approximate 2 o'clock between the last exporter (887) and the first importer (12). The label of the node is the ISO country code (see Appendix A for references). 

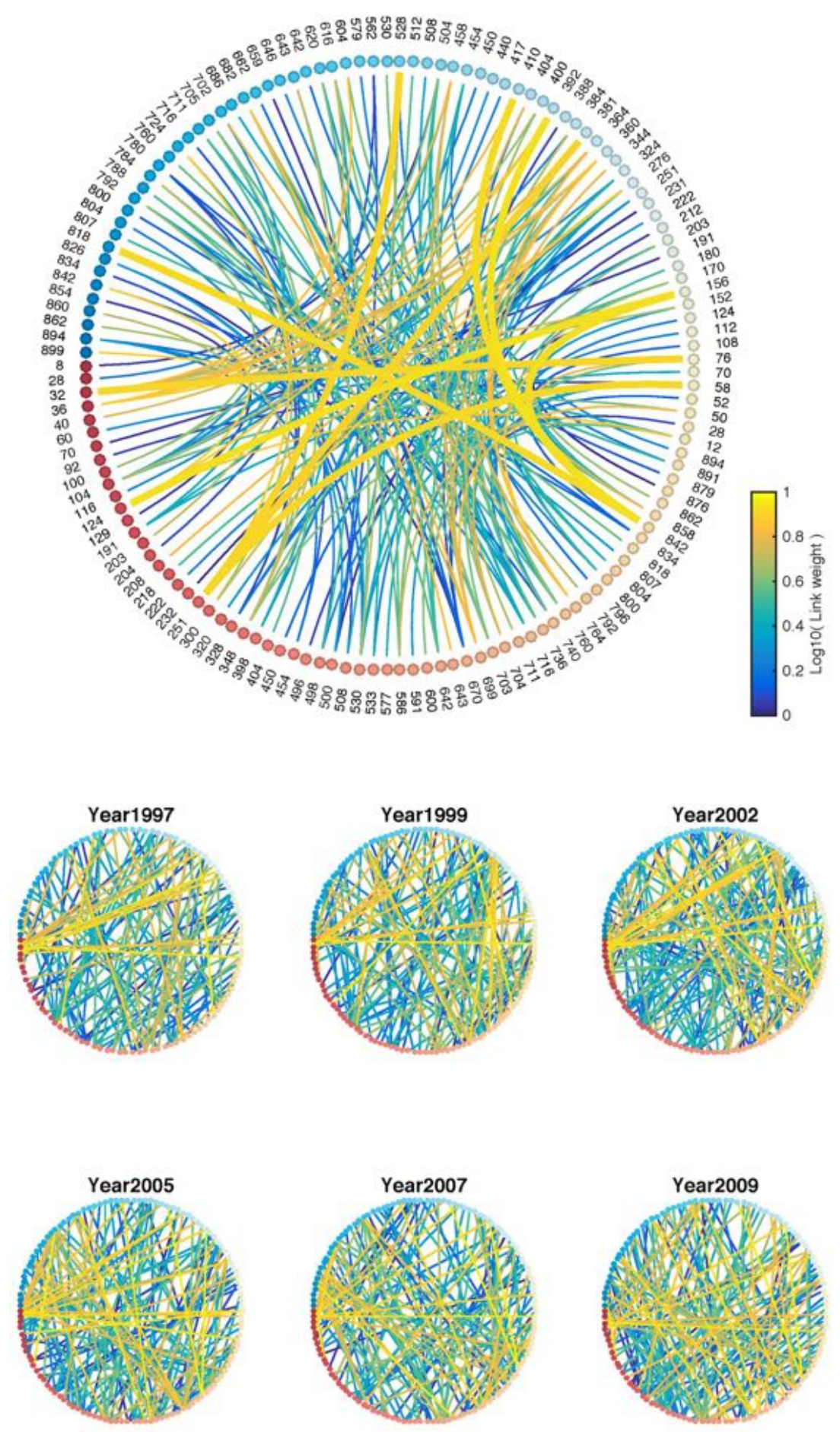

Fig. C.2.: Selected HS2 $=10$ trade network visualization. This figure shows the circular layout visualization for the 'Cereals' industry (HS2 $=10$ ). We set a threshold of $R S P>0.1$ and $R P P>0.1$ for visible links. Exporters are located at the bottom of the graph with a declining red color gradient. Importers are located on the top of the graph with a declining blue color gradient. The thickness of the line is proportional to the weight of the link. Note: the importers start at approximate 4 o'clock between the last exporter (894) and the first importer (12). The label of the node is the ISO country code (see Appendix A for references). 

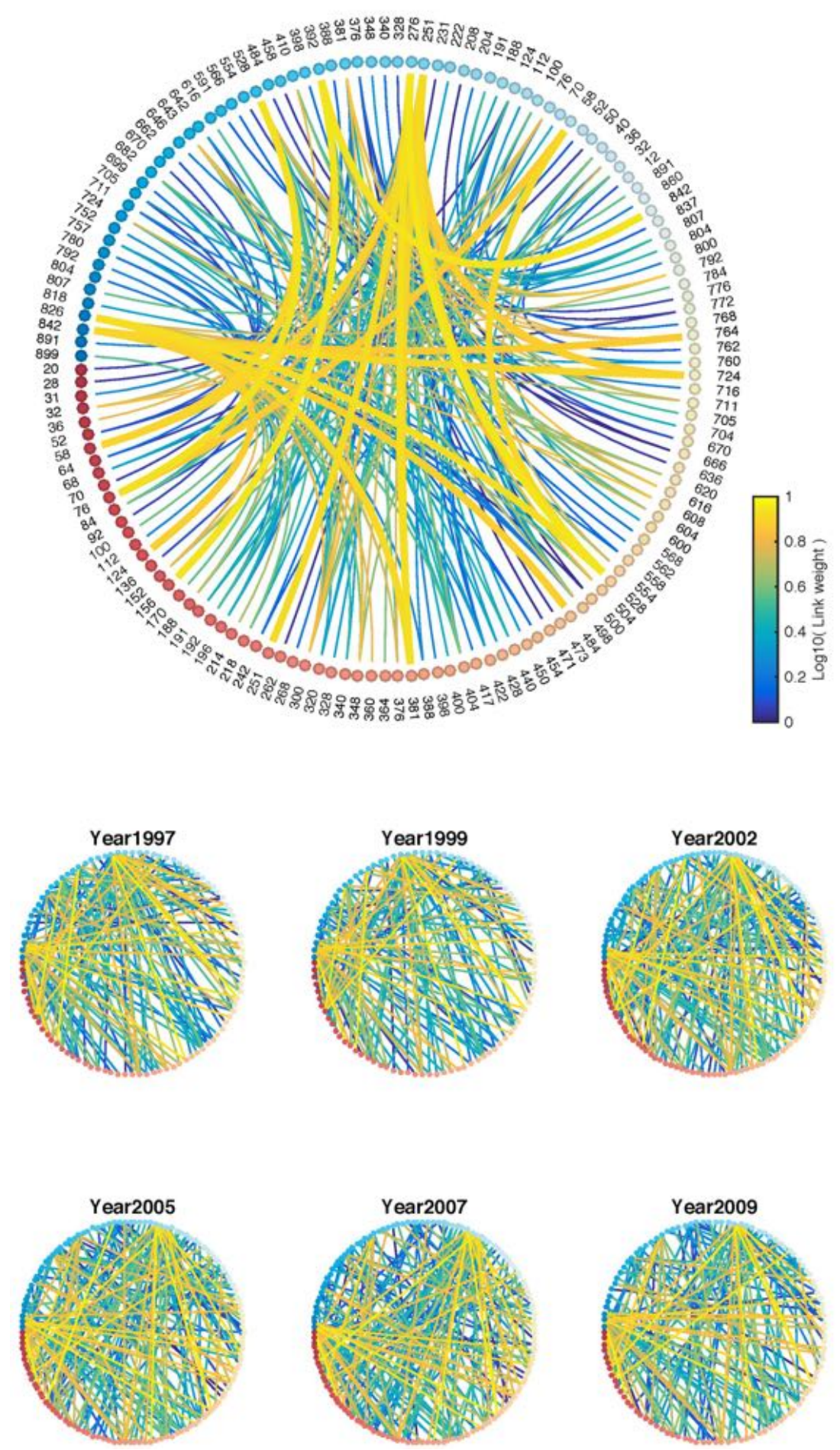

Fig. C.3.: Selected HS2 $=20$ trade network visualization. This figure shows the circular layout visualization for the 'Vegetables and Fruits' industry (HS2 $=20$ ). We set a threshold of $R S P>0.1$ and $R P P>0.1$ for visible links. Exporters are located at the bottom of the graph with a declining red color gradient. Importers are located on the top of the graph with a declining blue color gradient. The thickness of the line is proportional to the weight of the link. Note: the importers start at approximate 2 o'clock between the last exporter (891) and the first importer (12). The label of the node is the ISO country code (see Appendix A for references). 

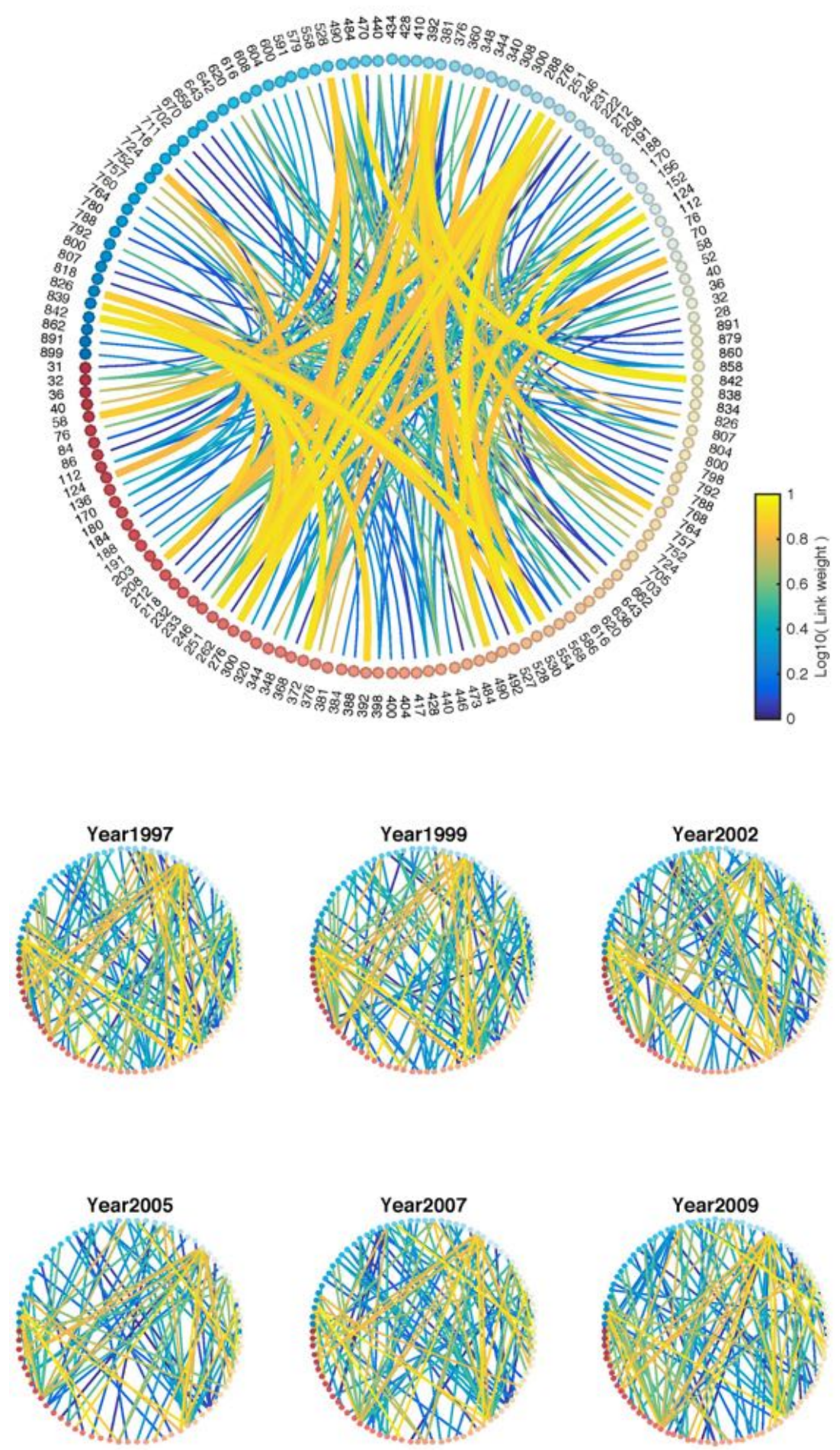

Fig. C.4.: Selected HS2 $=35$ trade network visualization. This figure shows the circular layout visualization for the 'Glues and Enzymes' industry (HS2=35). We set a threshold of $R S P>0.1$ and $R P P>0.1$ for visible links. Exporters are located at the bottom of the graph with a declining red color gradient. Importers are located on the top of the graph with a declining blue color gradient. The thickness of the line is proportional to the weight of the link. Note: the importers start at approximate 3 o'clock between the last exporter (891) and the first importer (32). The label of the node is the ISO country code (see Appendix A for references). 

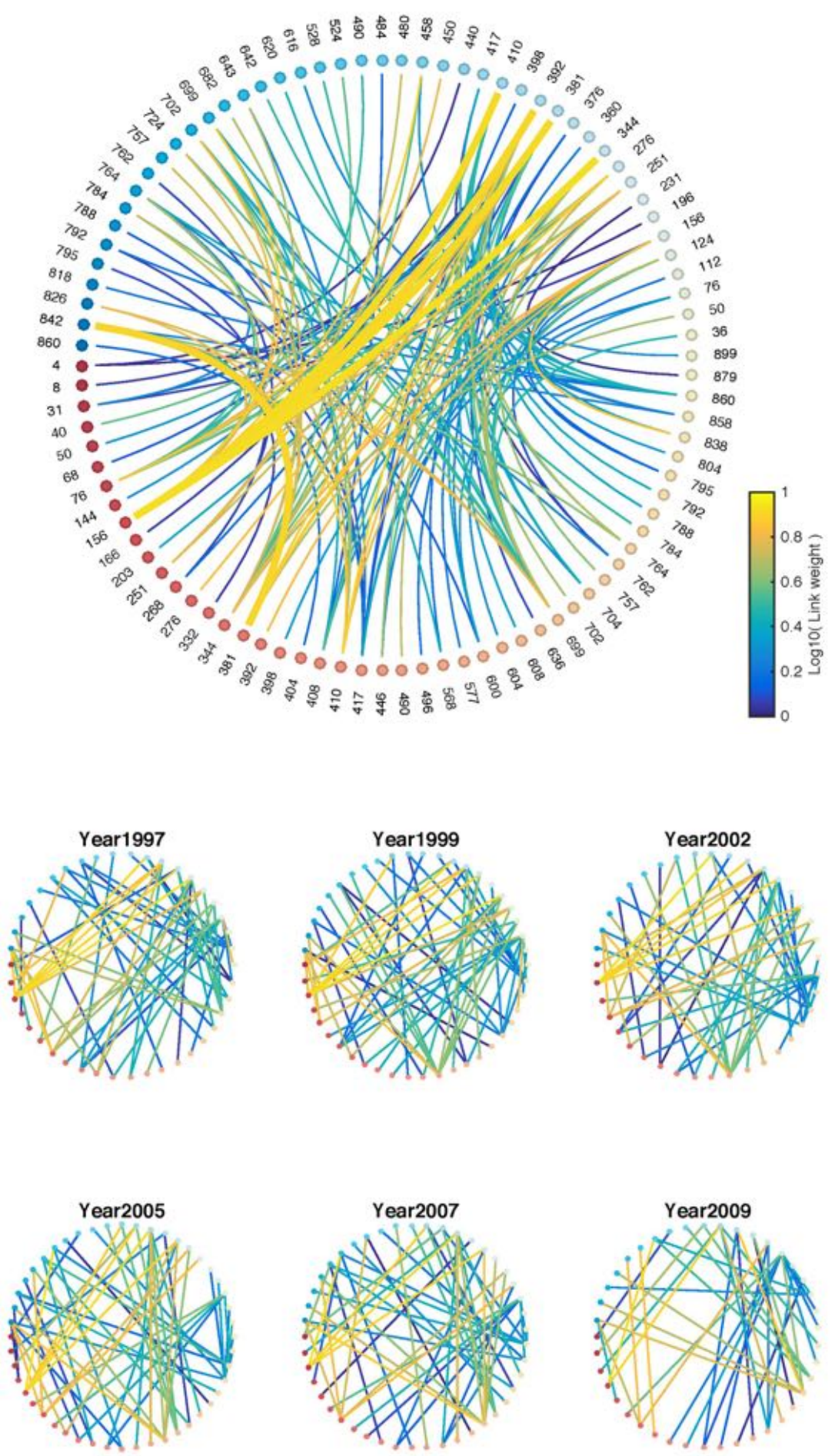

Fig. C.5.: Selected HS2 $=50$ trade network visualization. This figure shows the circular layout visualization for the 'Silk' industry $(\mathrm{HS} 2=50)$. We set a threshold of $R S P>0.1$ and $R P P>0.1$ for visible links. Exporters are located at the bottom of the graph with a declining red color gradient. Importers are located on the top of the graph with a declining blue color gradient. The thickness of the line is proportional to the weight of the link. Note: the importers start at approximate 3 o'clock between the last exporter (860) and the first importer (50). The label of the node is the ISO country code (see Appendix A for references). 

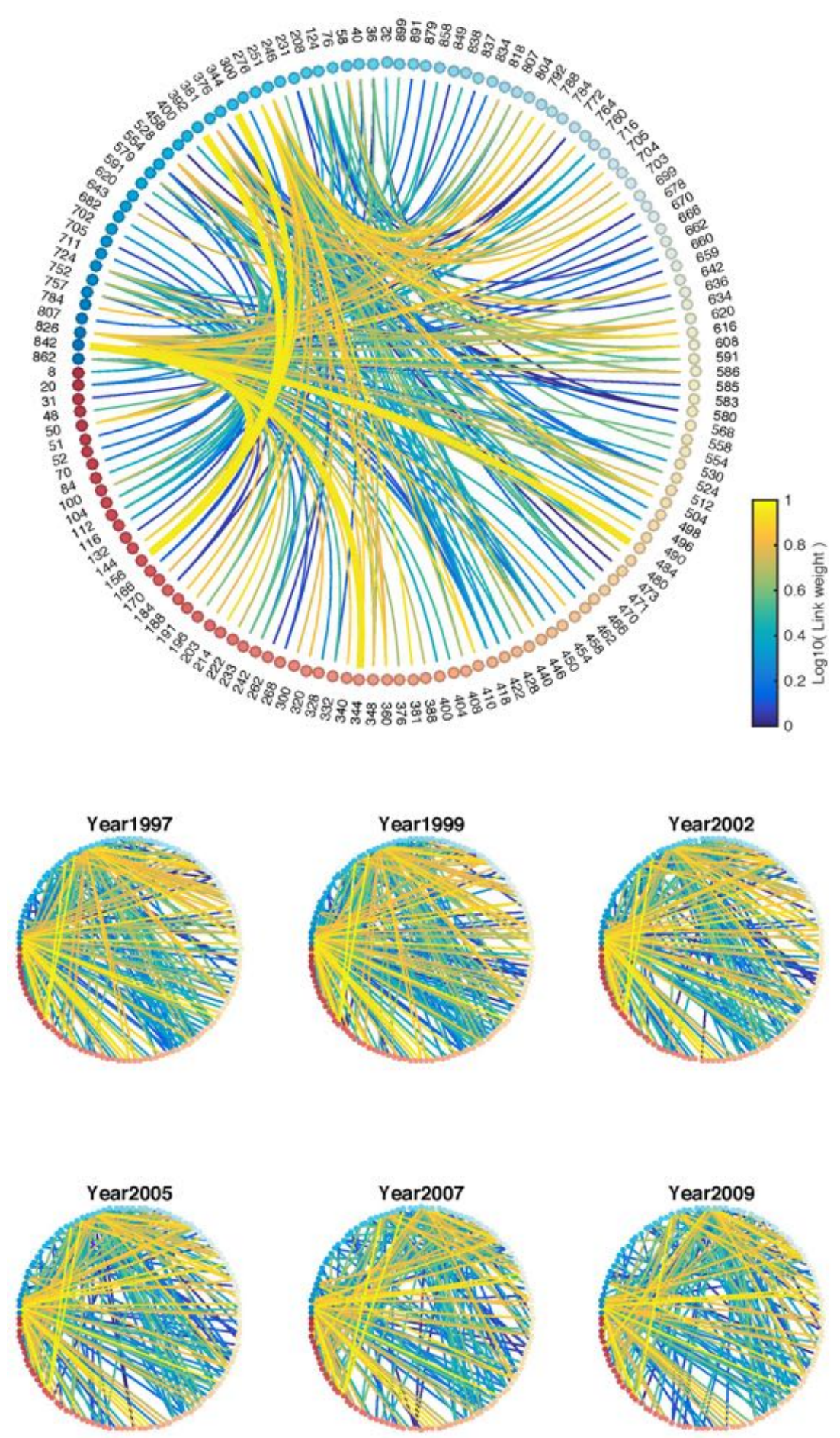

Fig. C.6.: Selected HS2 $=62$ trade network visualization. This figure shows the circular layout visualization for the 'Apparel' industry (HS2 $=62$ ). We set a threshold of $R S P>0.1$ and $R P P>0.1$ for visible links. Exporters are located at the bottom of the graph with a declining red color gradient. Importers are located on the top of the graph with a declining blue color gradient. The thickness of the line is proportional to the weight of the link. Note: the importers start at approximate 12 o'clock between the last exporter (899) and the first importer (32). The label of the node is the ISO country code (see Appendix A for references). 

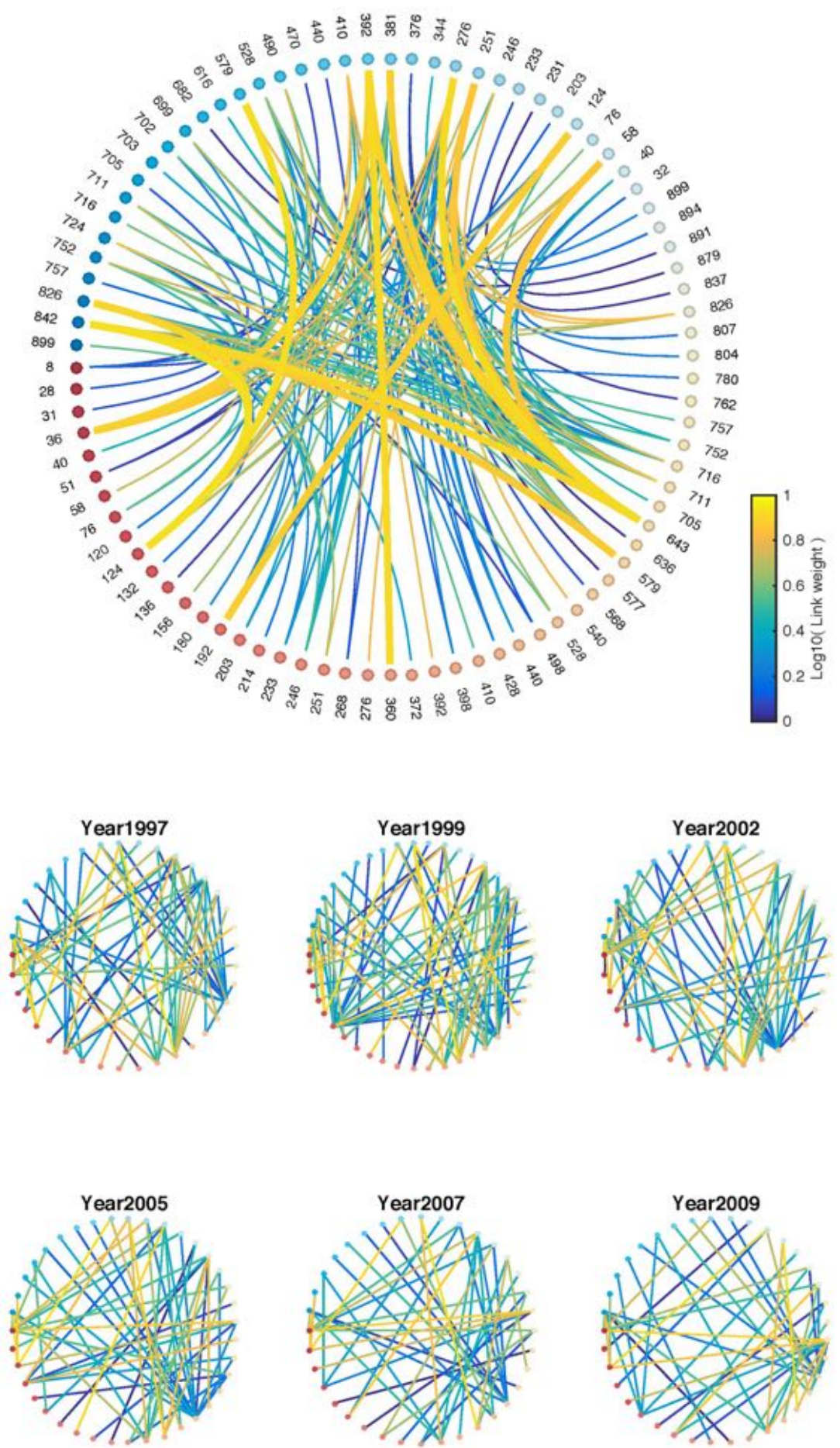

Fig. C.7.: Selected HS2 $=75$ trade network visualization. This figure shows the circular layout visualization for the 'Nickel' industry (HS2 $=75$ ). We set a threshold of $R S P>0.1$ and $R P P>0.1$ for visible links. Exporters are located at the bottom of the graph with a declining red color gradient. Importers are located on the top of the graph with a declining blue color gradient. The thickness of the line is proportional to the weight of the link. Note: the importers start at approximate 2 o'clock between the last exporter (894) and the first importer (40). The label of the node is the ISO country code (see Appendix A for references). 

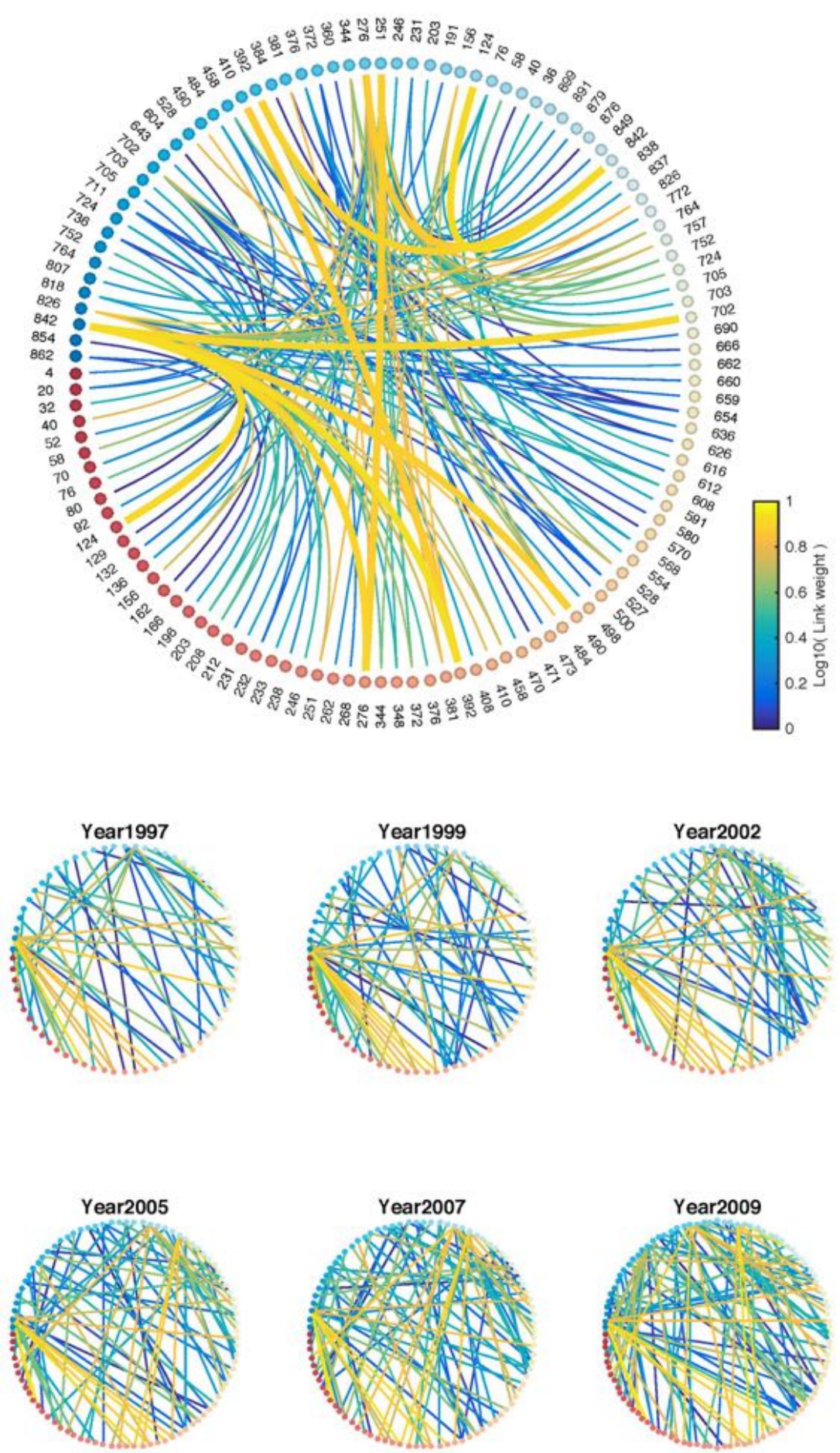

Fig. C.8.: Selected HS2 $=84$ trade network visualization. This figure shows the circular layout visualization for the 'Machinery' industry (HS2 $=84$ ). We set a threshold of $R S P>0.1$ and $R P P>0.1$ for visible links. Exporters are located at the bottom of the graph with a declining red color gradient. Importers are located on the top of the graph with a declining blue color gradient. The thickness of the line is proportional to the weight of the link. Note: the importers start at approximate 1 o'clock between the last exporter (899) and the first importer (58). The label of the node is the ISO country code (see Appendix A for references). 

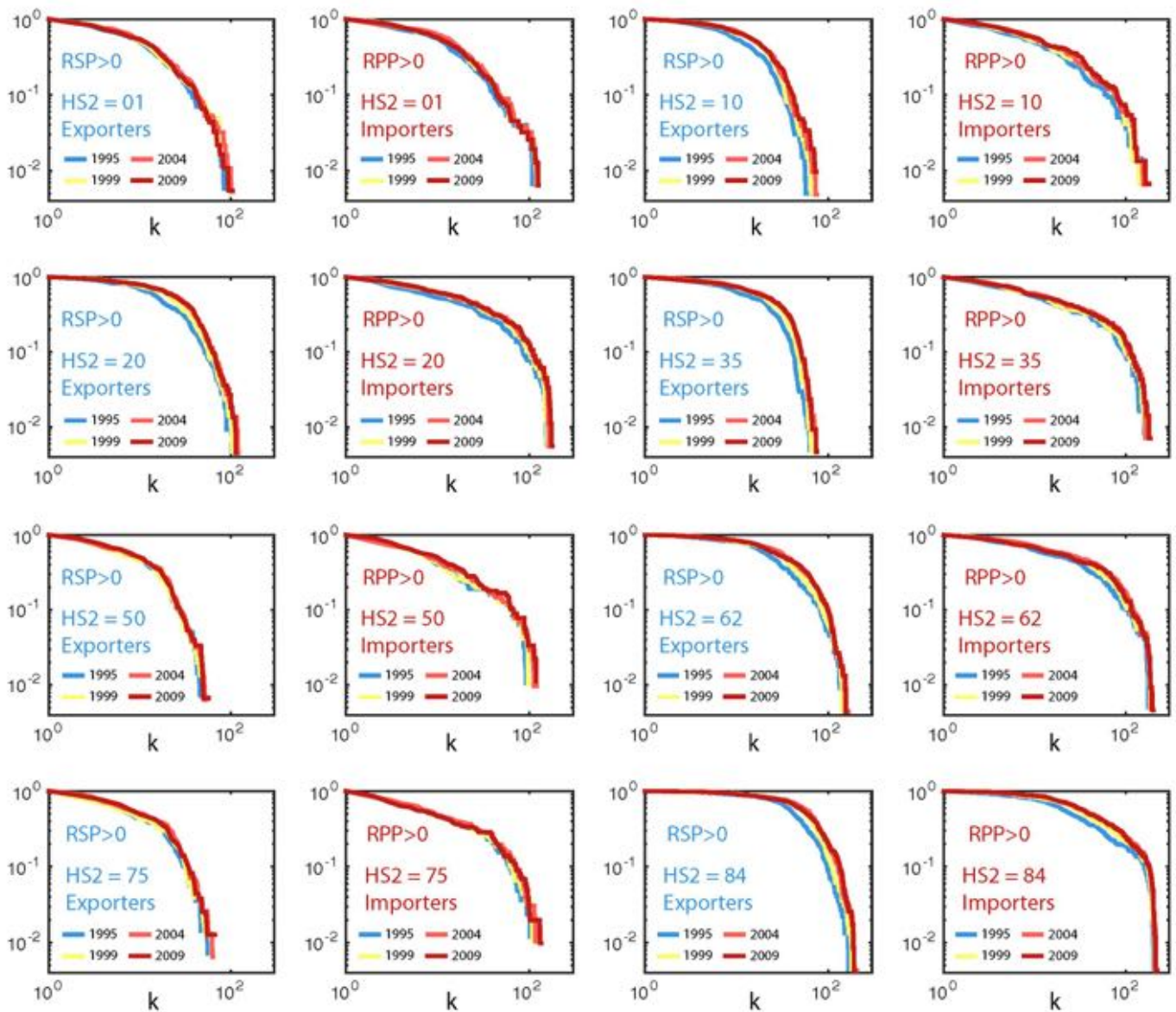

Fig. C.9.: Cummulative Degree Ditribution without RSP and RPP filters. This figure shows the $\mathrm{P}(\mathrm{K})$ degree distribution for the selected HS2 industries. Columns 1 and 3 show the exporter subsets results, whereas columns 2 and 4 show the importers performance. No RSP or RPP filters here. The $\mathrm{P}(\mathrm{K})$ performances include the results from 1995, 1999, 2004 and 2009. In general, no major differences can be found across periods. 

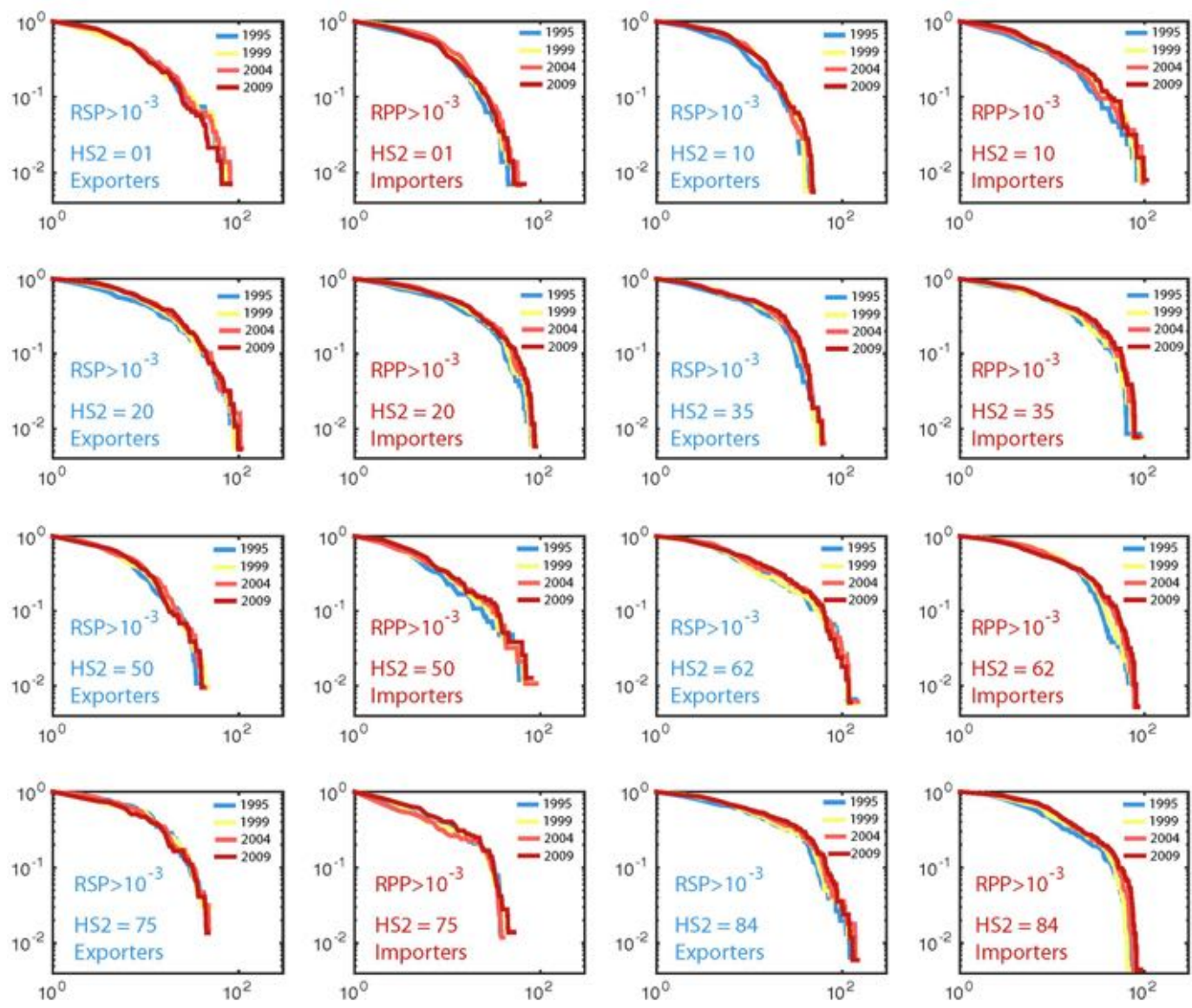

Fig. C.10.: Cummulative Degree Ditribution with RSP and RPP filters. This figure shows the $\mathrm{P}(\mathrm{K})$ degree distribution for the selected HS2 industries. Columns 1 and 3 show the exporter subsets results, whereas columns 2 and 4 show the importers performance. RSP or RPP filters have been applied here. The $\mathrm{P}(\mathrm{K})$ performances include the results from 1995, 1999, 2004 and 2009. In general, no major differences can be found across periods. 

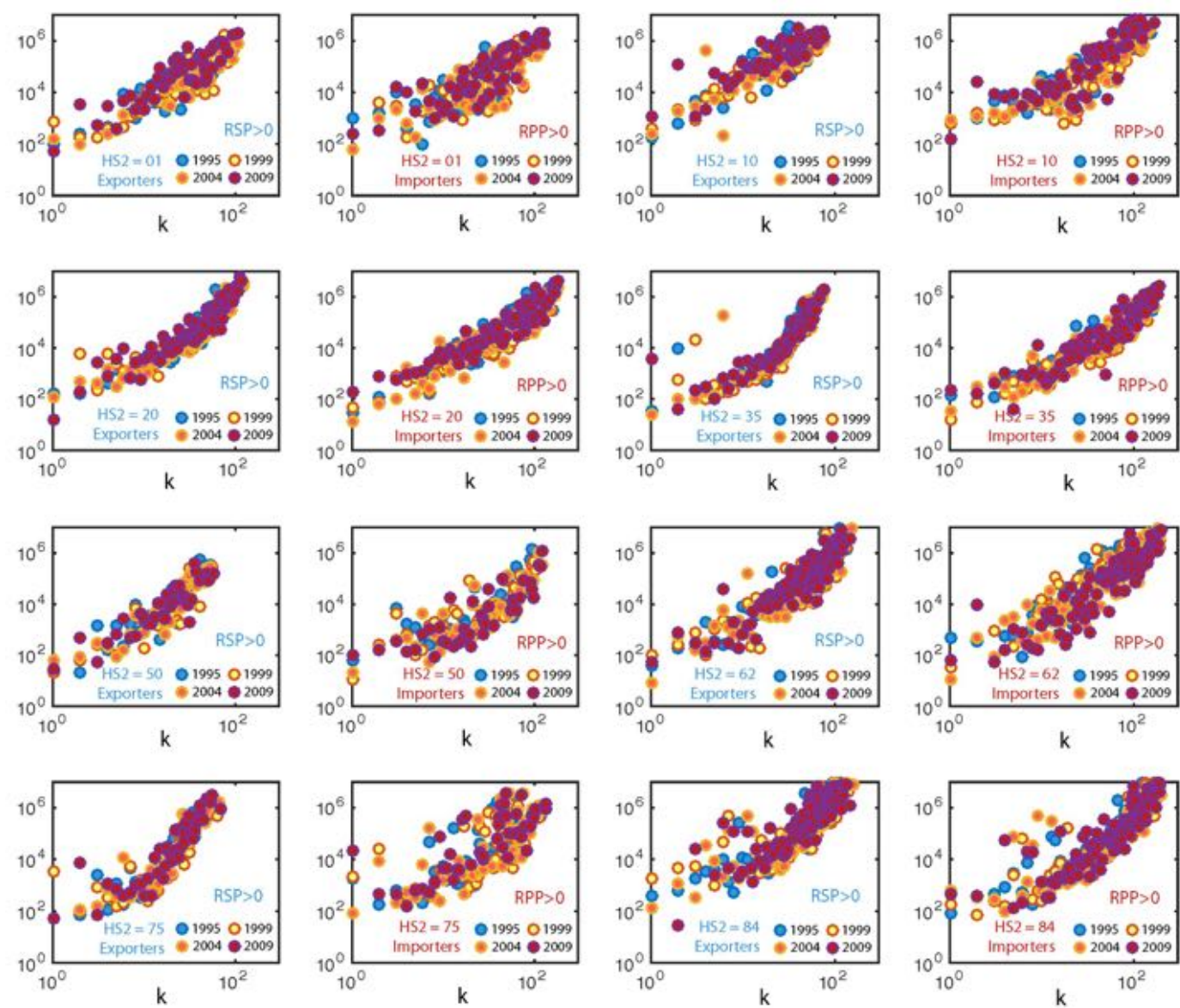

Fig. C.11.: Strength-Degree correlation, unfiltered scenario. This figure shows the strengthdegree correlation for the selected HS2 industries over the selected periods. 

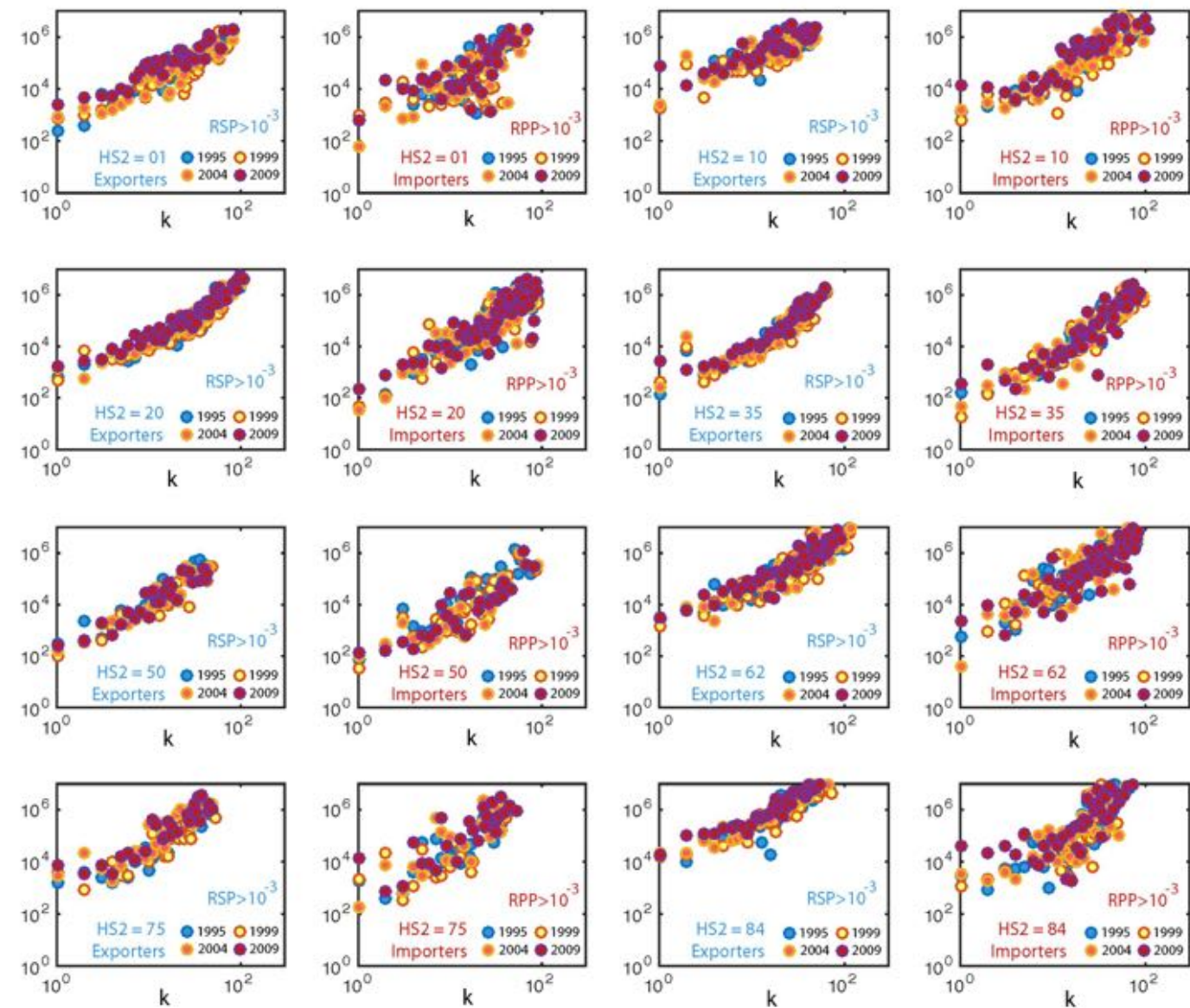

Fig. C.12.: Strength-Degree correlation with RSP and RPP filters. This figure shows the strength-degree correlation for the selected HS2 industries over the selected periods in its filtered scenario. 

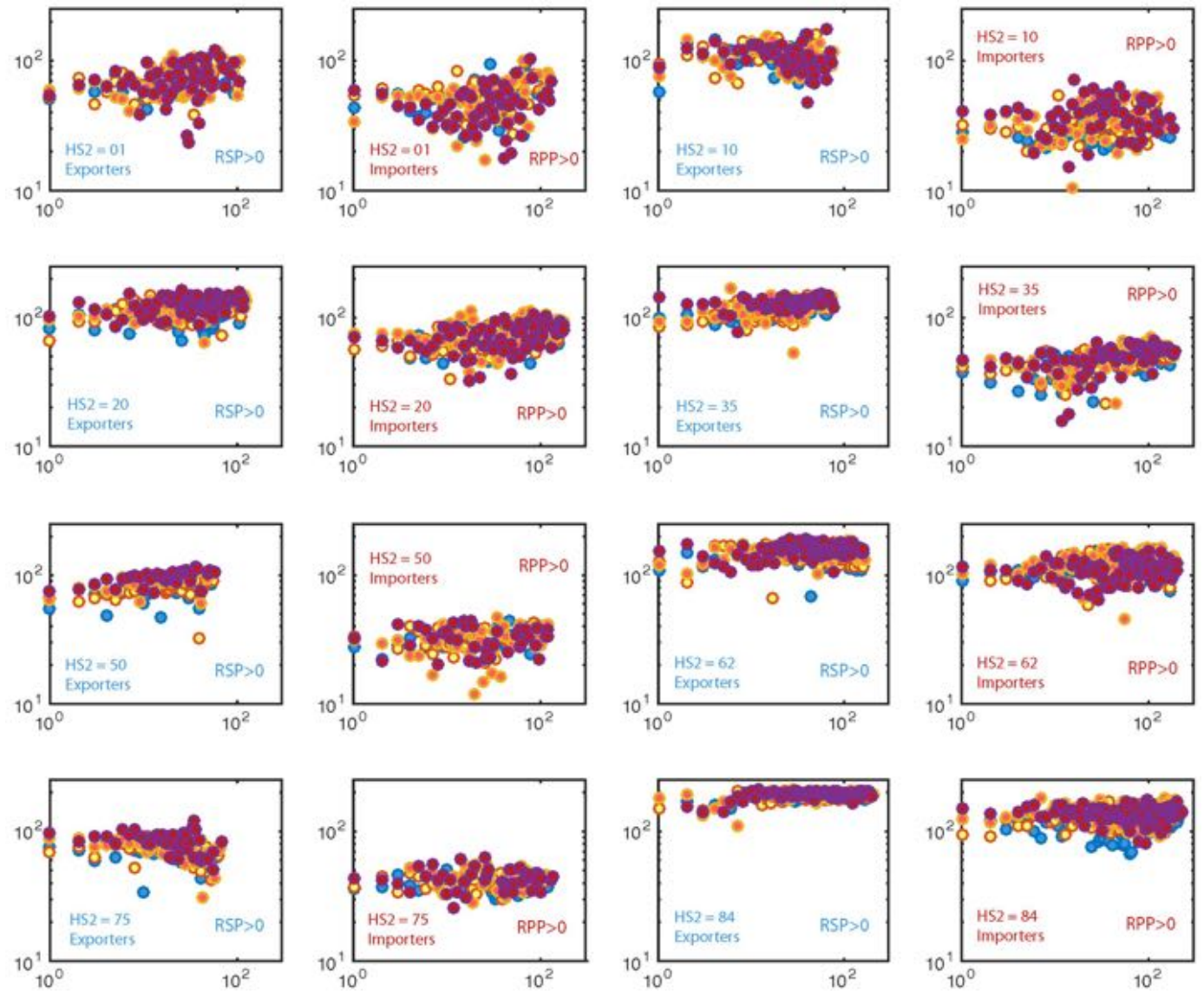

Fig. C.13.: Nearest neighbor degree (weighted) without RSP and RPP filters. This figure shows the performance of $\mathrm{Knn}(\mathrm{k})$ for the exporters (colums 1 and 3 ) and the importers (columns 2 and 4). We observe an uncorrelated pattern in all cases of the unfiltered scenario. 

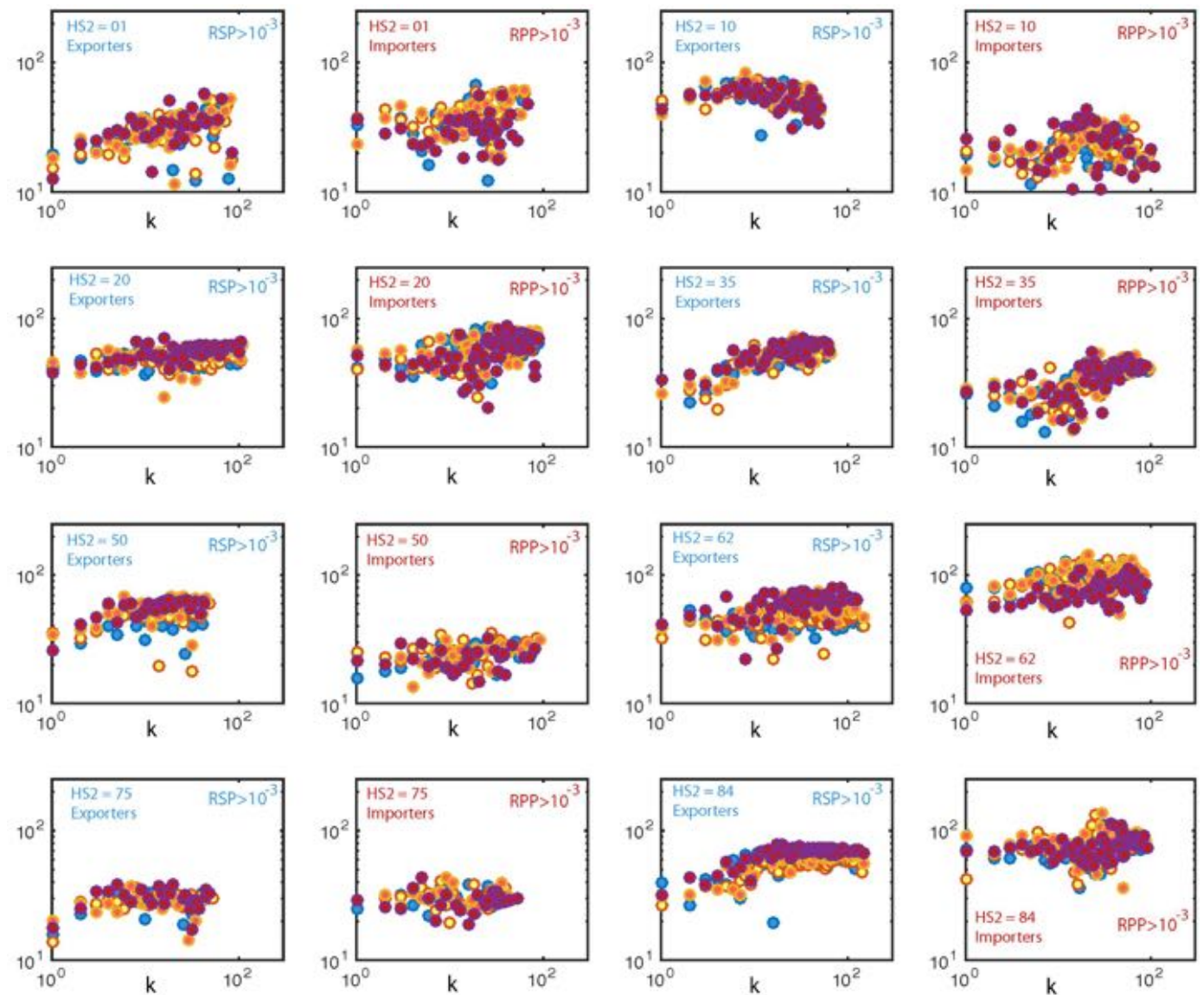

Fig. C.14.: Nearest neighbor degree (weighted) with RSP and RPP filters. This figure shows the performance of $\mathrm{Knn}(\mathrm{k})$ for the exporters (colums 1 and 3) and the importers (columns 2 and 4). We observe an uncorrelated pattern in most cases of the filtered scenario. 

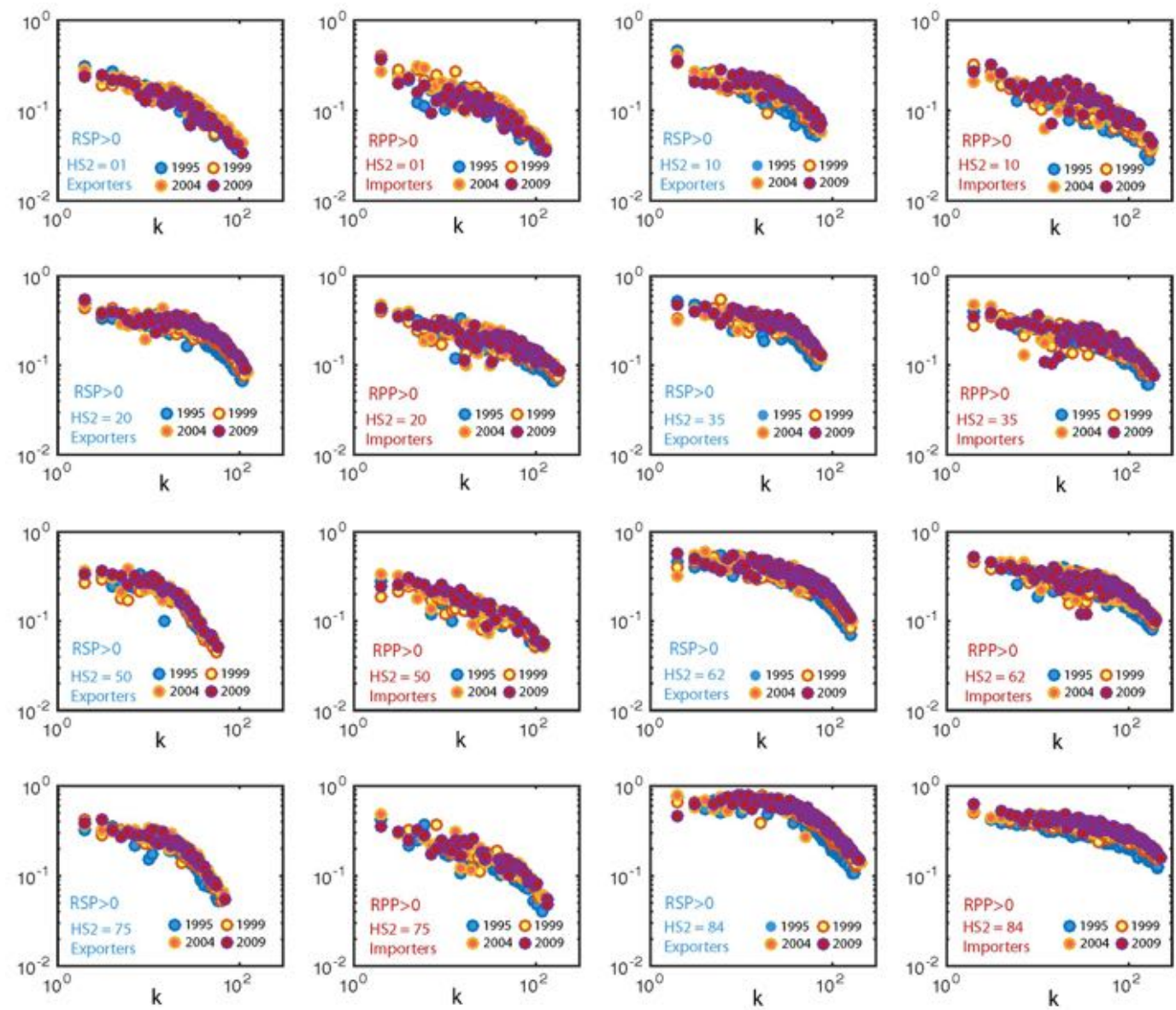

Fig. C.15.: Bipartite clustering (unweighted) without RSP and RPP filters. This figure shows the performance of $\mathrm{C} 4 \mathrm{~b}(\mathrm{k})$ for the exporters (colums 1 and 3) and the importers (columns 2 and 4). We observe a declining clustering performance in all cases of the unfiltered scenario. 

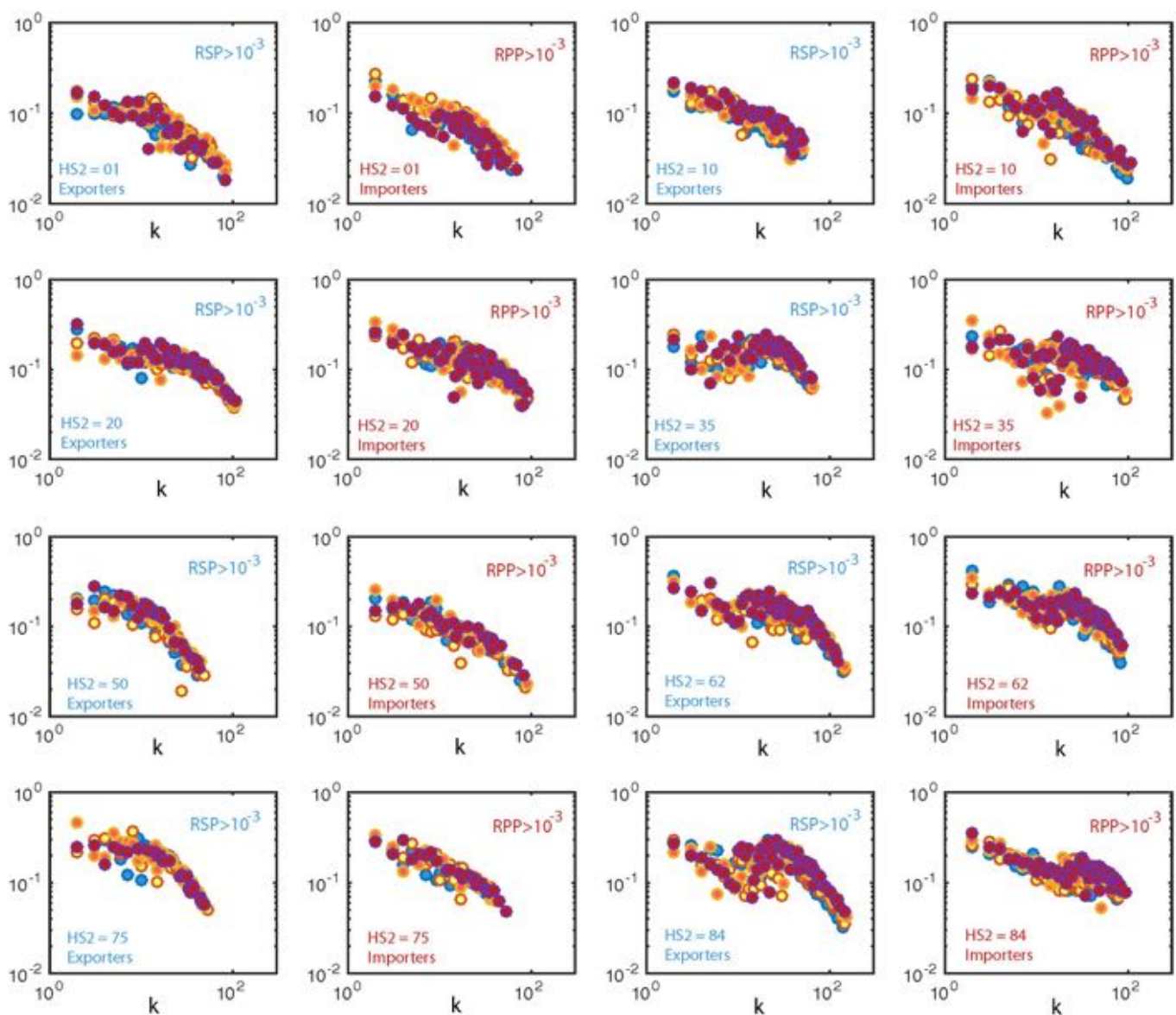

Fig. C.16.: Bipartite clustering (unweighted) with RSP and RPP filters. This figure shows the performance of $\mathrm{C} 4 \mathrm{~b}(\mathrm{k})$ for the exporters (colums 1 and 3) and the importers (columns 2 and 4). We observe a declining clustering performance in all cases of the filtered scenario. 

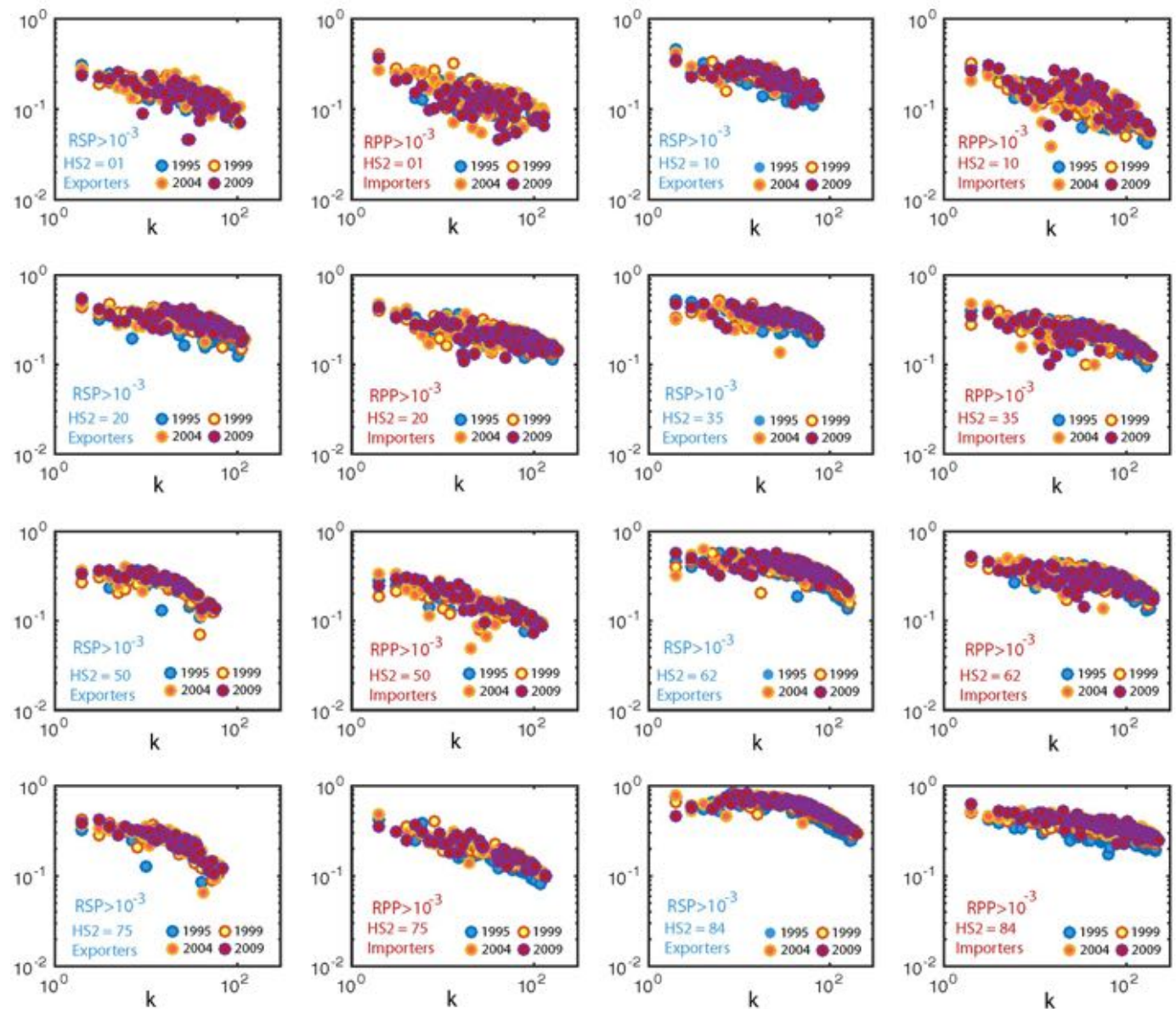

Fig. C.17.: Bipartite clustering (weighted) without RSP and RPP filters. This figure shows the performance of $\mathrm{C} 4 \mathrm{w}(\mathrm{k})$ for the exporters (colums 1 and 3 ) and the importers (columns 2 and 4). We observe a declining clustering performance in all cases of the unfiltered scenario. 

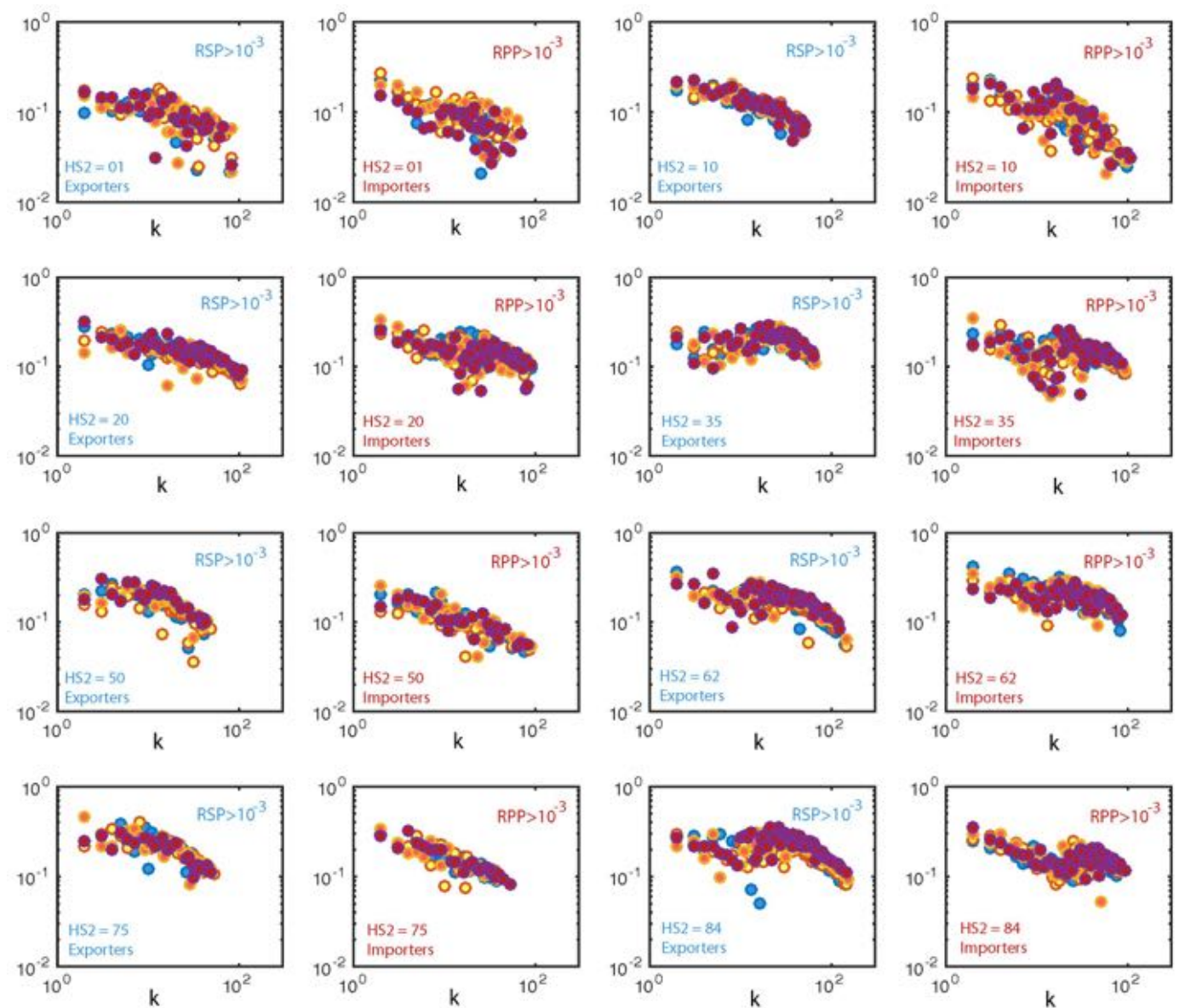

Fig. C.18.: Bipartite clustering (weighted) without RSP and RPP filters. This figure shows the performance of $\mathrm{C} 4 \mathrm{w}(\mathrm{k})$ for the exporters (colums 1 and 3 ) and the importers (columns 2 and 4). We observe a declining clustering performance in all cases of the filtered scenario. 

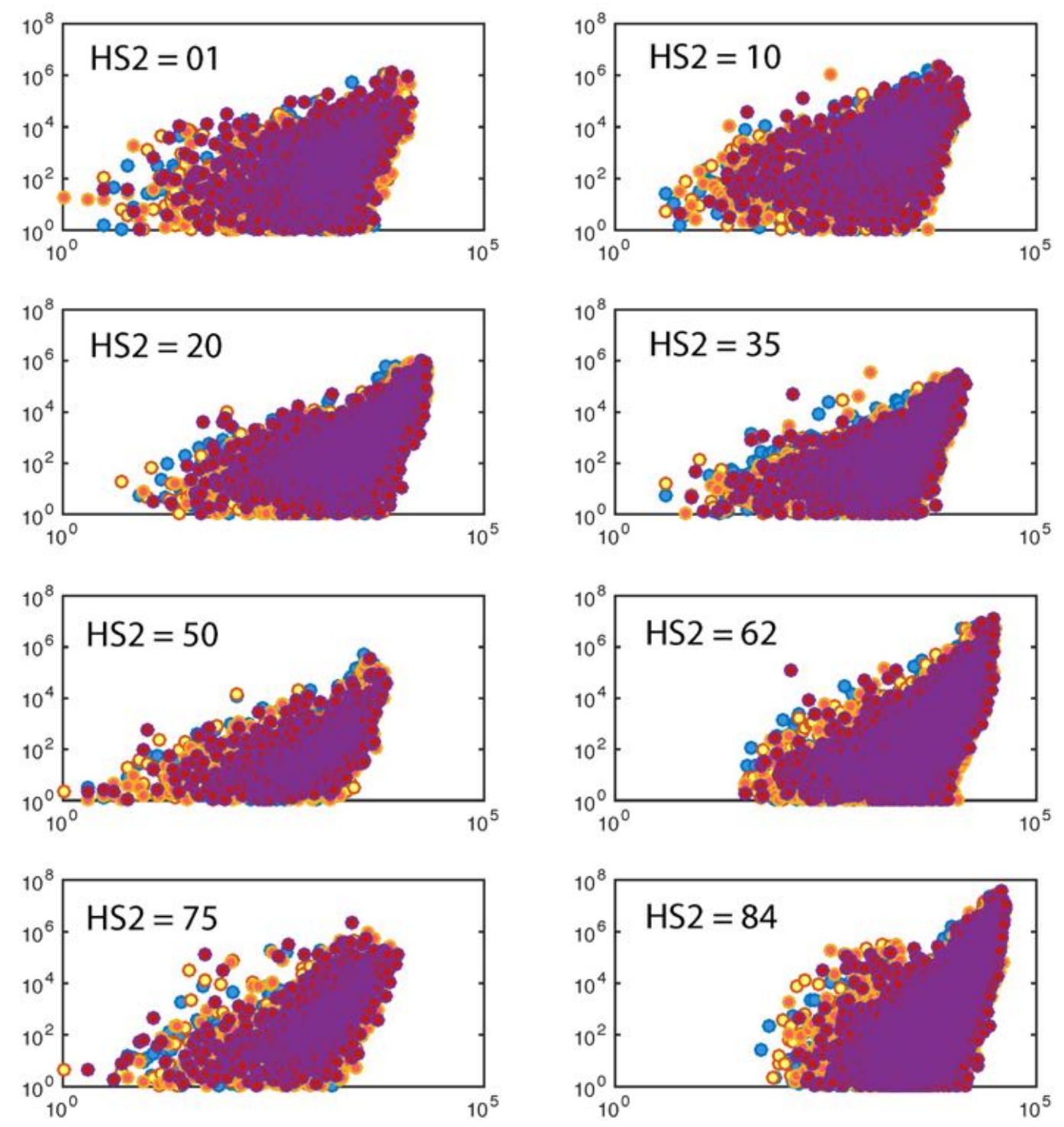

Fig. C.19.: Average weight as a function of the end-point degree without RSP and RPP filters. This figure shows the performance of $<\mathrm{w}>(\mathrm{Ki} . \mathrm{Kj})$ for HS2 selected industries. 

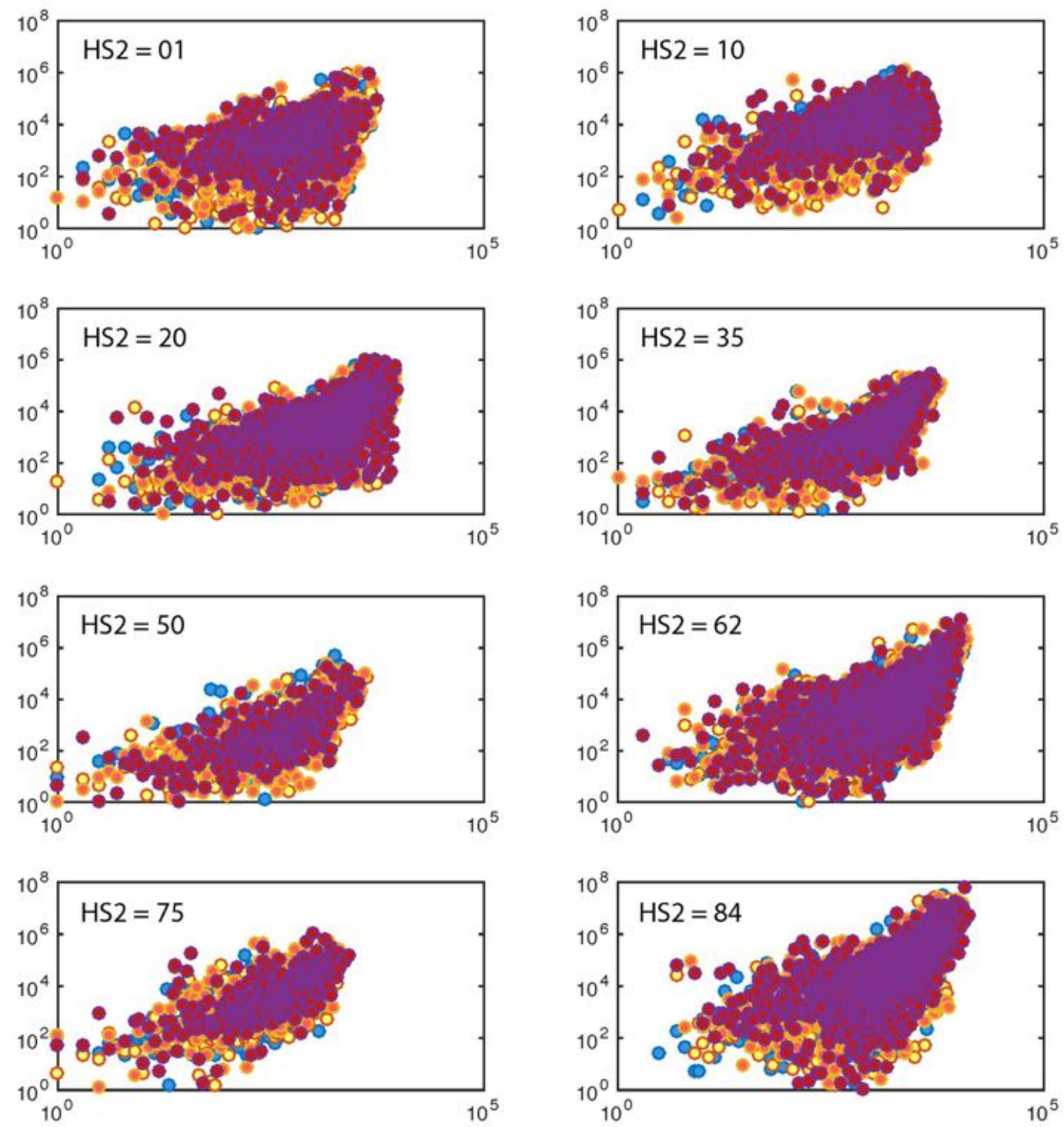

Fig. C.20.: Average weight as a function of the end-point degree with RSP and RPP filters. This figure shows the performance of $<\mathrm{w}>$ (Ki.Kj) for HS2 selected industries. 

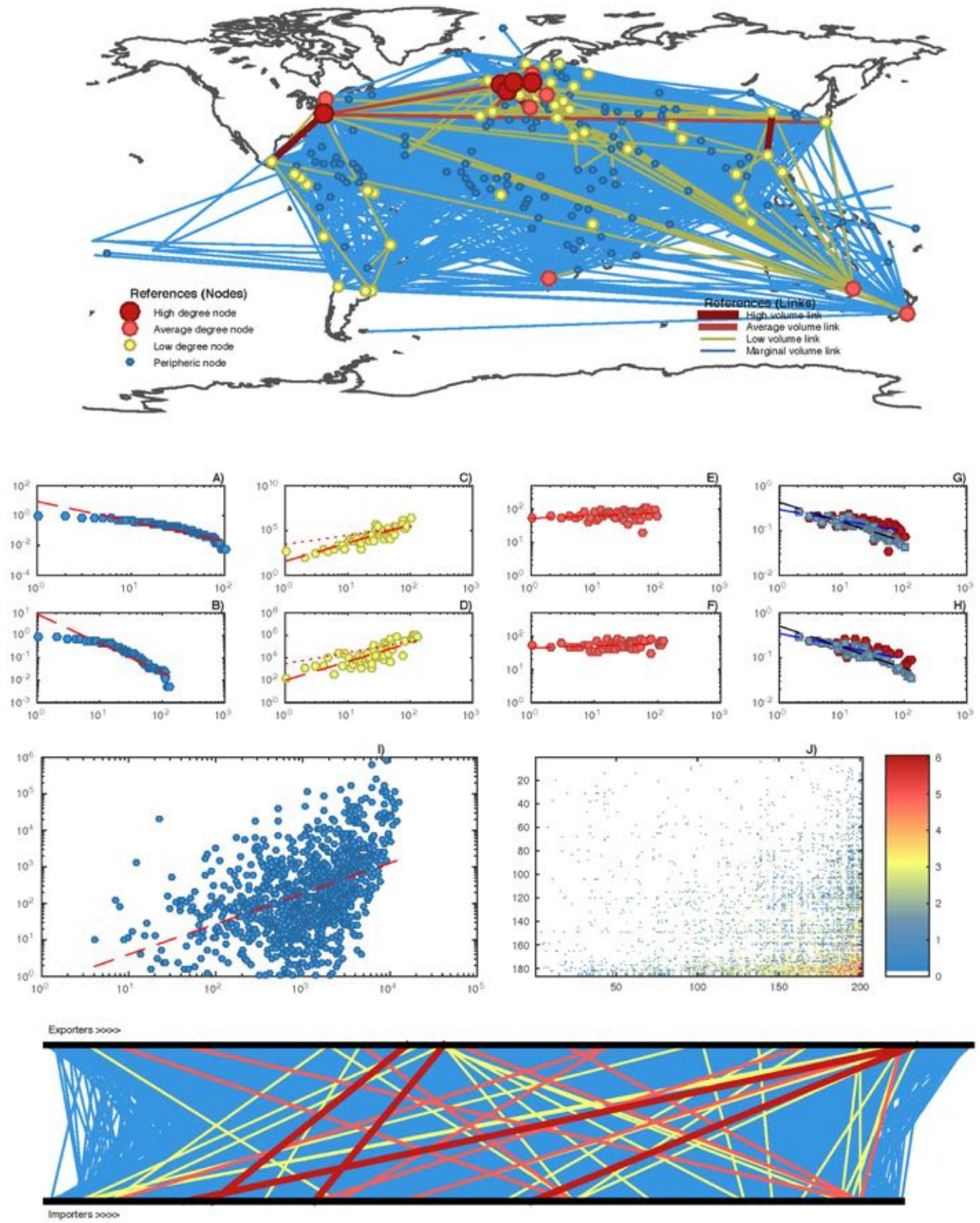

Fig. C.21.: Market dashboard HS2=01, $\mathrm{Yr}=2000$. A) $\mathrm{P}(\mathrm{k})$ Exporters, B) $\mathrm{P}(\mathrm{k})$ Importers, $\mathrm{C}) \mathrm{S}(\mathrm{k})$ Exporters, D) S(k) Importers, E) Knn(k) Exporters, F) Knn(k) Importers, G) C4b/w(k) Exporters, H) C4b/w(k) Importers, I) $<\mathrm{w}>$ (ki.kj) and J) Bipartite Matrix. 


\section{Geo-Network Chart - Product10 (Year2000)}
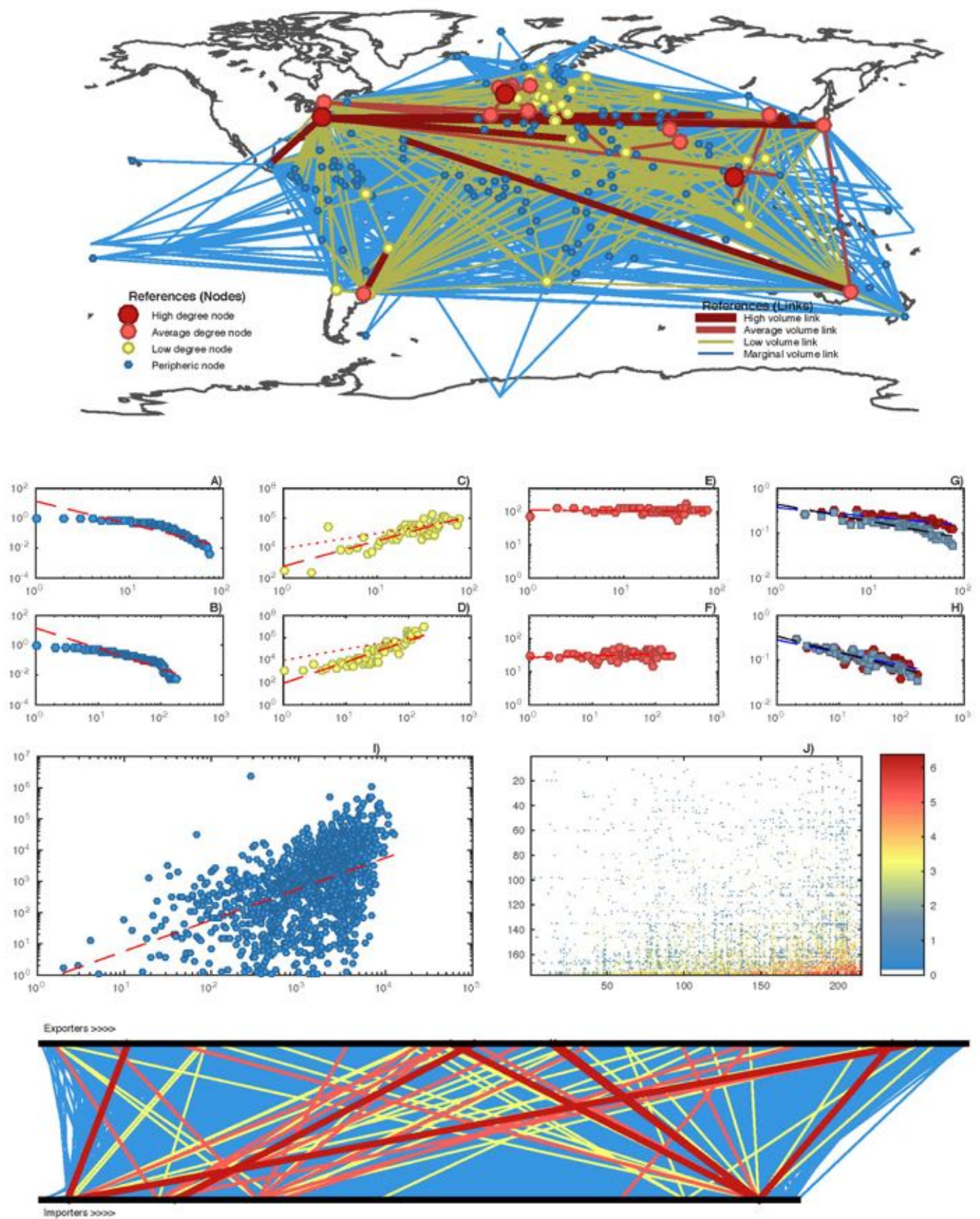

Fig. C.22.: Market dashboard HS2=10, Yr=2000. A) P(k) Exporters, B) P(k) Importers, C) S(k) Exporters, D) S(k) Importers, E) Knn(k) Exporters, F) Knn(k) Importers, G) C4b/w(k) Exporters, H) C4b/w(k) Importers, I) $<\mathrm{w}>($ ki.kj) and J) Bipartite Matrix. 


\section{Geo-Network Chart - Product20 (Year2000)}
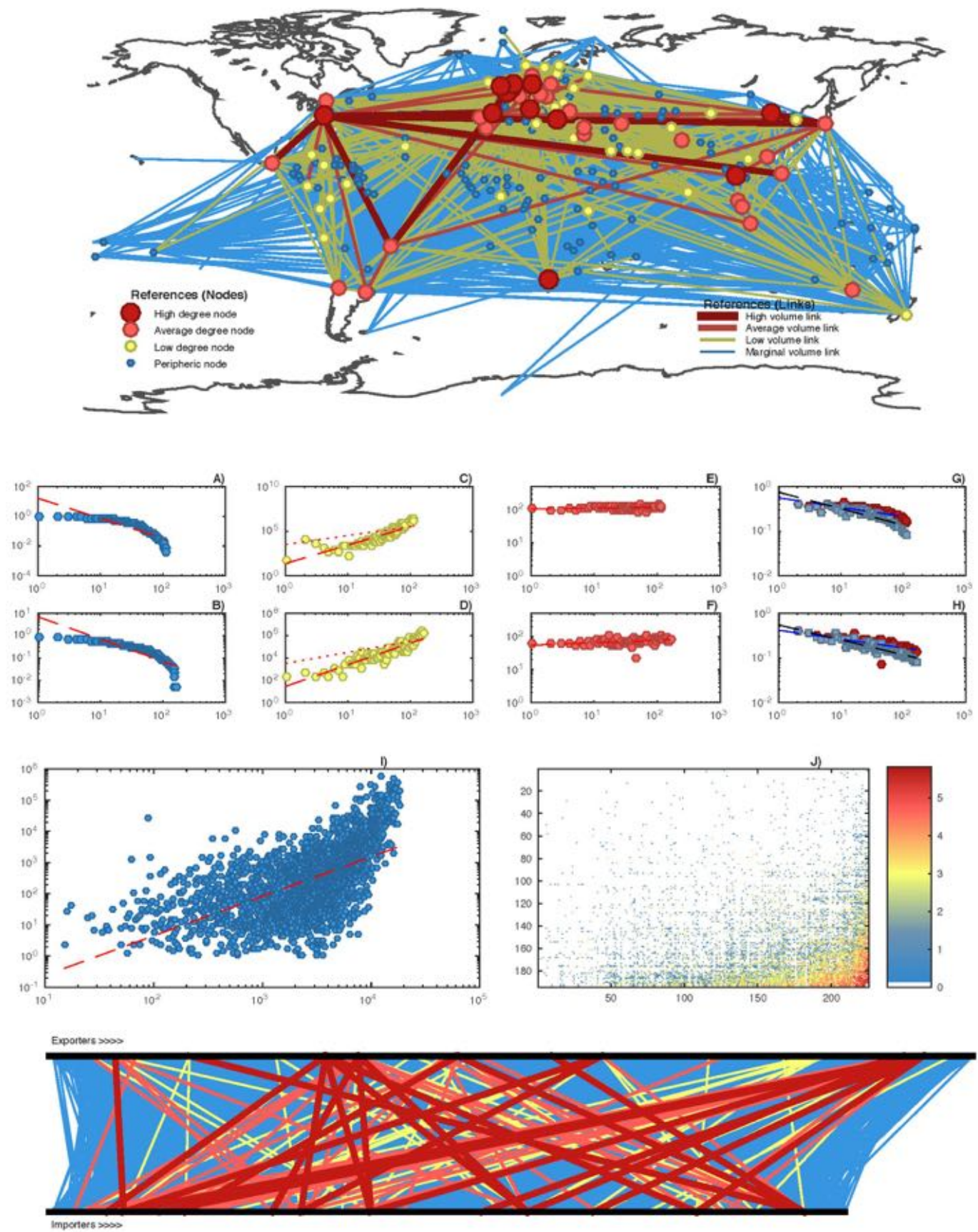

Fig. C.23.: Market dashboard HS2=20, Yr=2000. A) P(k) Exporters, B) P(k) Importers, C) $\mathrm{S}(\mathrm{k})$ Exporters, D) S(k) Importers, E) Knn(k) Exporters, F) Knn(k) Importers, G) C4b/w(k) Exporters, H) C4b/w(k) Importers, I) $<\mathrm{w}>(\mathrm{ki} . \mathrm{kj})$ and J) Bipartite Matrix. 

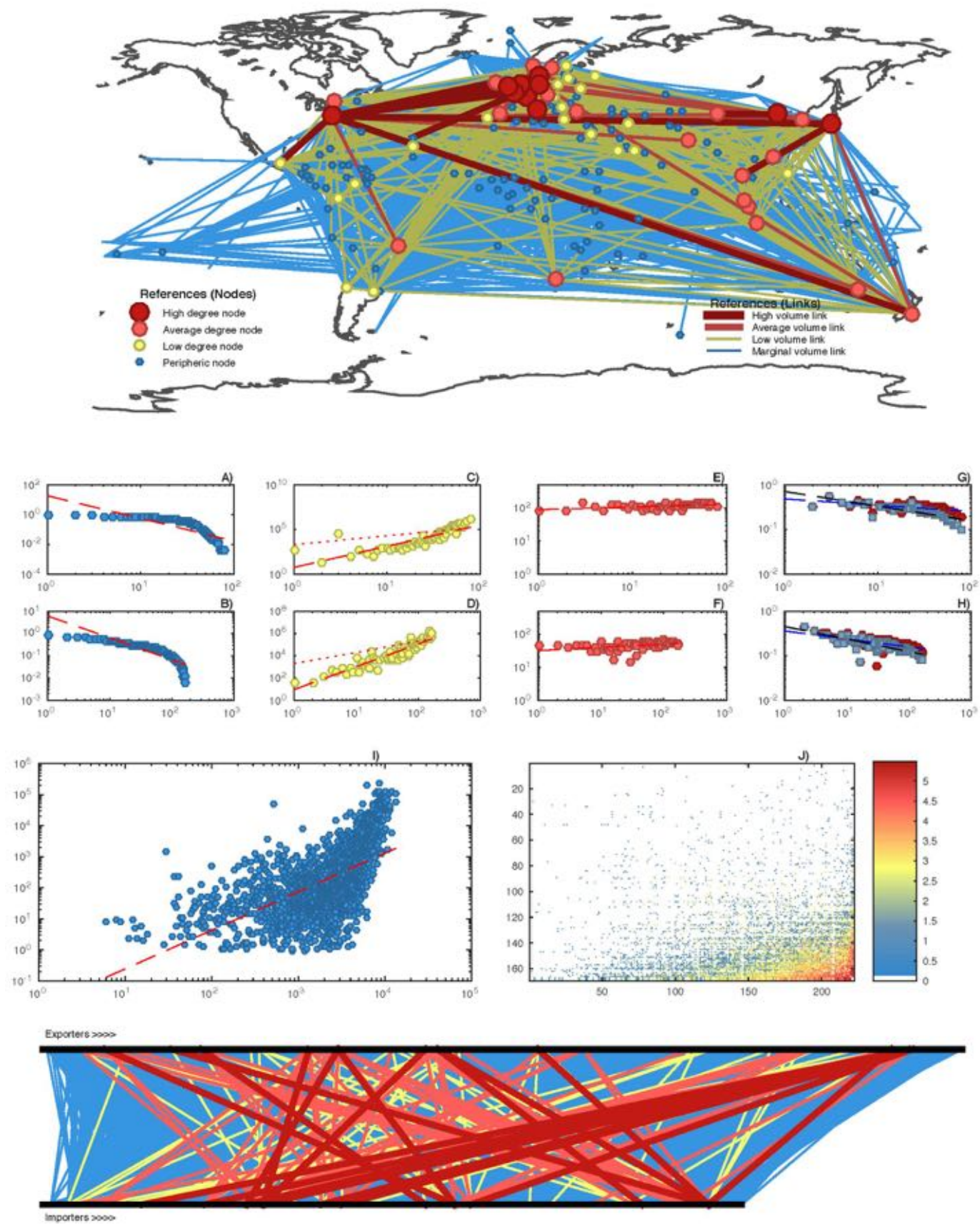

Fig. C.24.: Market dashboard HS2=35, Yr=2000. A) P(k) Exporters, B) P(k) Importers, C) S(k) Exporters, D) S(k) Importers, E) Knn(k) Exporters, F) Knn(k) Importers, G) C4b/w(k) Exporters, H) C4b/w(k) Importers, I) $<\mathrm{w}>$ (ki.kj) and J) Bipartite Matrix. 


\section{Geo-Network Chart - Product50 (Year2000)}
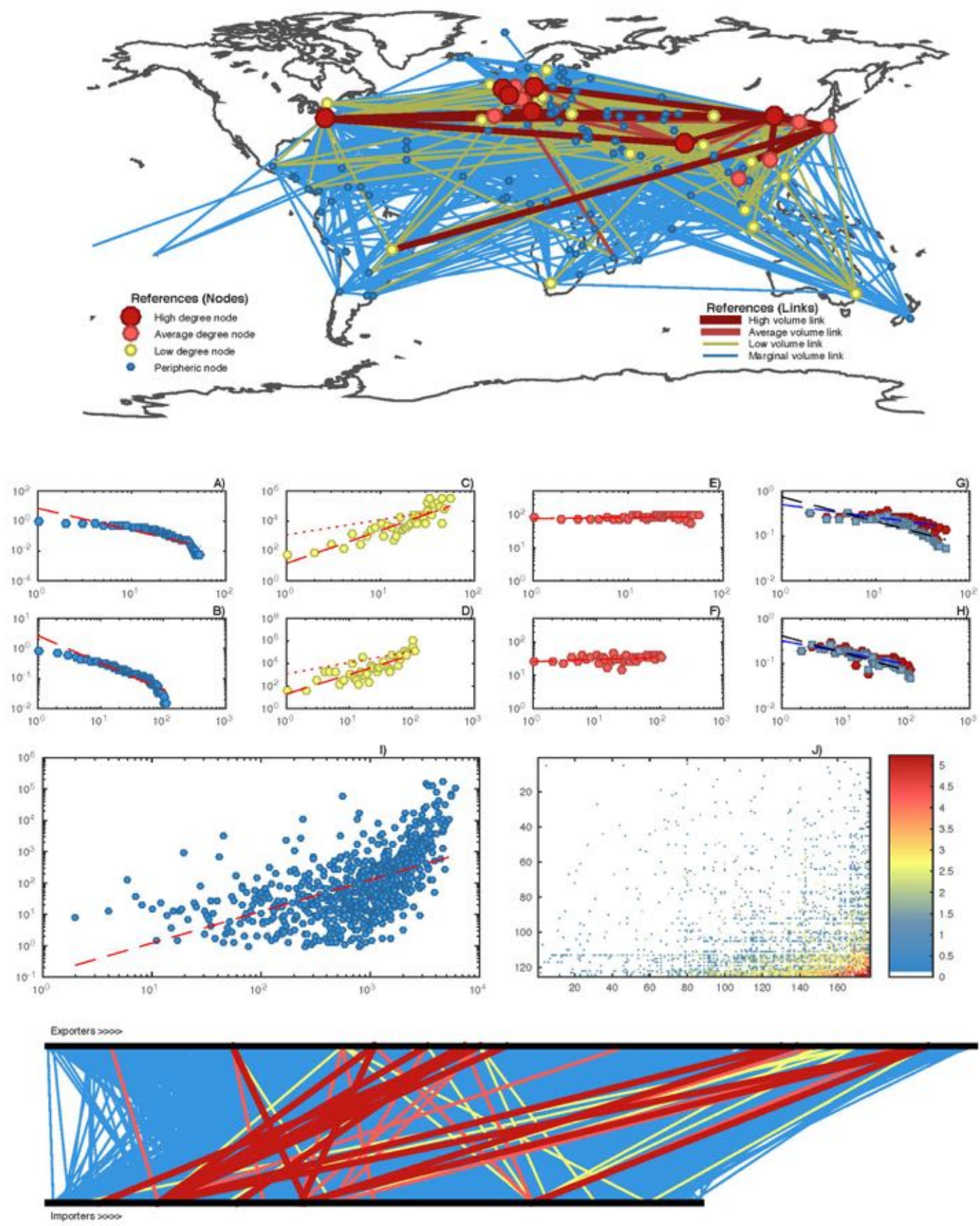

Fig. C.25.: Market dashboard HS2=50, Yr=2000. A) P(k) Exporters, B) P(k) Importers, C) $S(k)$ Exporters, D) S(k) Importers, E) Knn(k) Exporters, F) Knn(k) Importers, G) C4b/w(k) Exporters, H) C4b/w(k) Importers, I) $<w>(k i . k j)$ and J) Bipartite Matrix. 

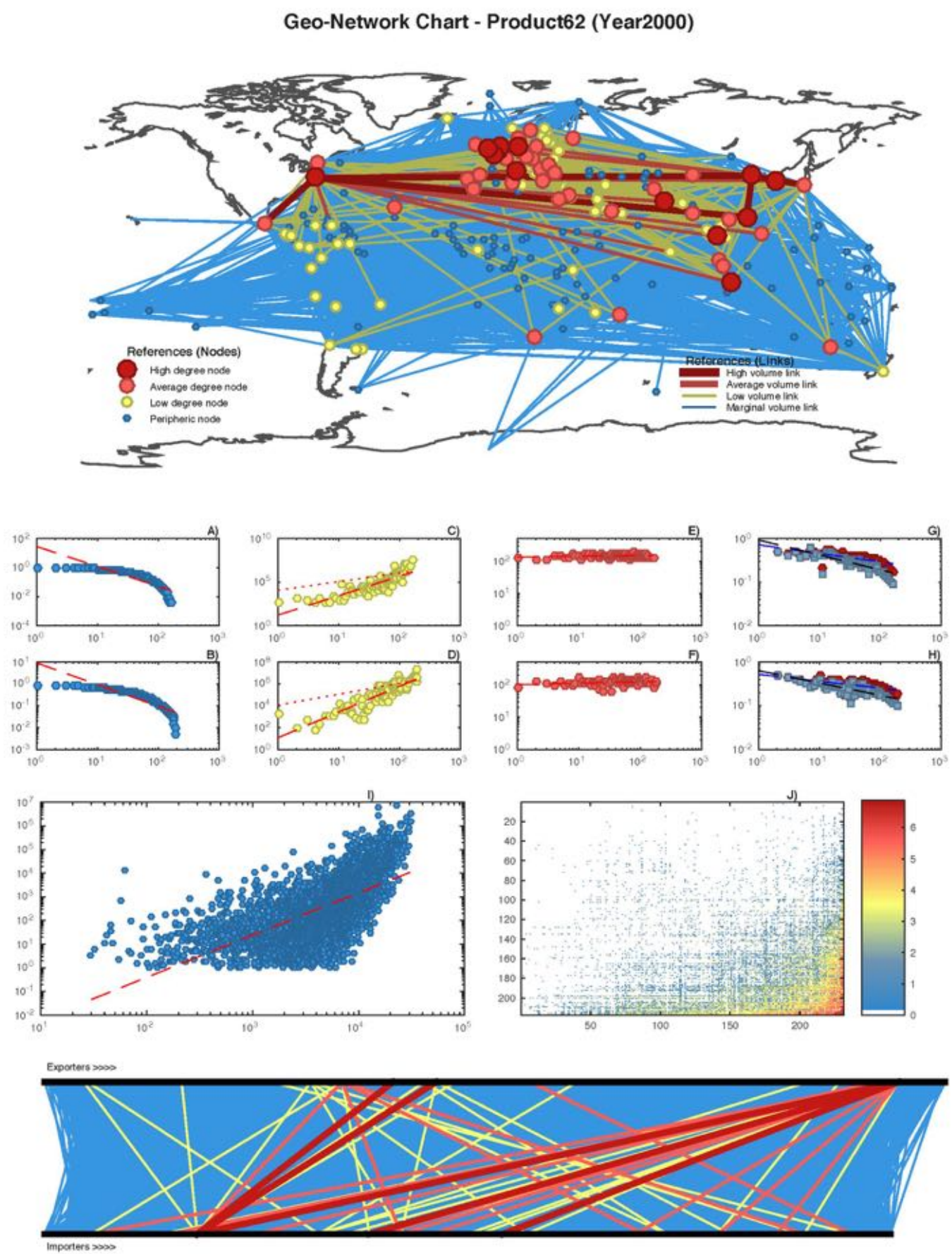

Fig. C.26.: Market dashboard HS2=62, $Y r=2000$. A) $P(k)$ Exporters, B) $P(k)$ Importers, C) $S(k)$ Exporters, D) S(k) Importers, E) Knn(k) Exporters, F) Knn(k) Importers, G) C4b/w(k) Exporters, H) C4b/w(k) Importers, I) $<\mathrm{w}>$ (ki.kj) and J) Bipartite Matrix. 

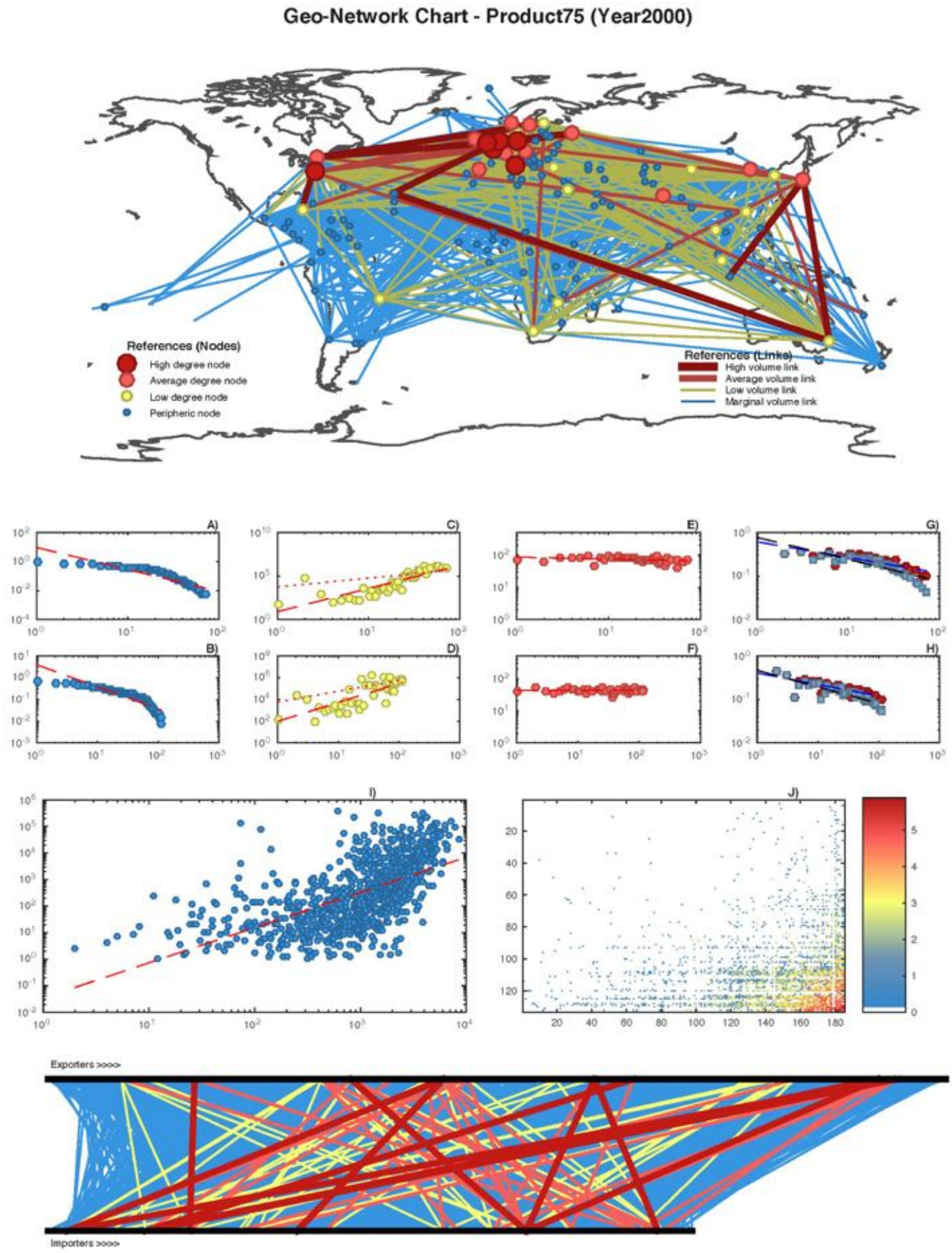

Fig. C.27.: Market dashboard HS2=75, Yr=2000. A) P(k) Exporters, B) P(k) Importers, C) $S(k)$ Exporters, D) S(k) Importers, E) Knn(k) Exporters, F) Knn(k) Importers, G) C4b/w(k) Exporters, H) C4b/w(k) Importers, I) $<\mathrm{w}>(\mathrm{ki.kj})$ and J) Bipartite Matrix. 
Geo-Network Chart - Product84 (Year2000)
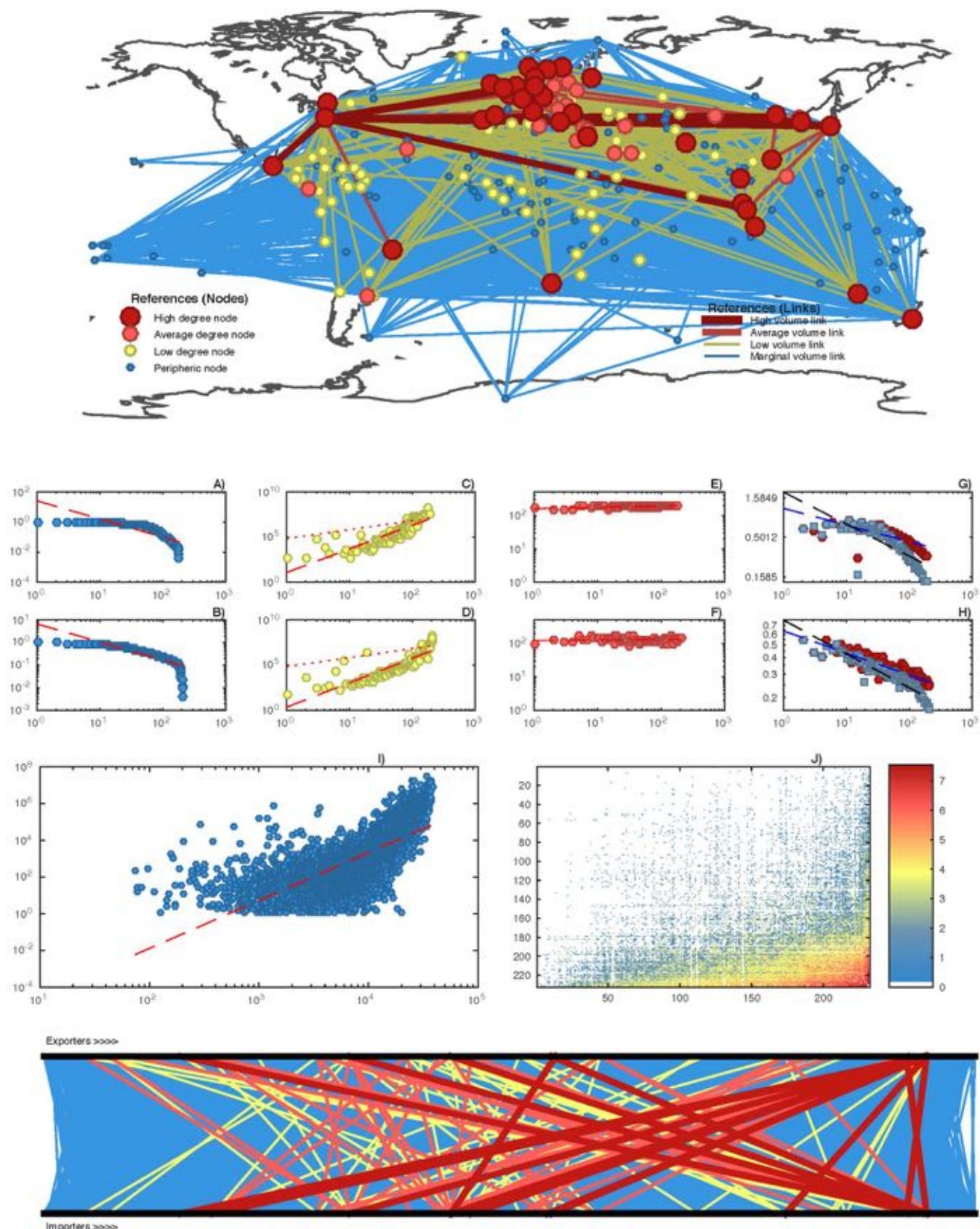

Fig. C.28.: Market dashboard HS2=84, Yr=2000. A) P(k) Exporters, B) P(k) Importers, C) S(k) Exporters, D) S(k) Importers, E) Knn(k) Exporters, F) Knn(k) Importers, G) C4b/w(k) Exporters, H) C4b/w(k) Importers, I) $<\mathrm{w}>(\mathrm{ki} . \mathrm{kj})$ and J) Bipartite Matrix. 

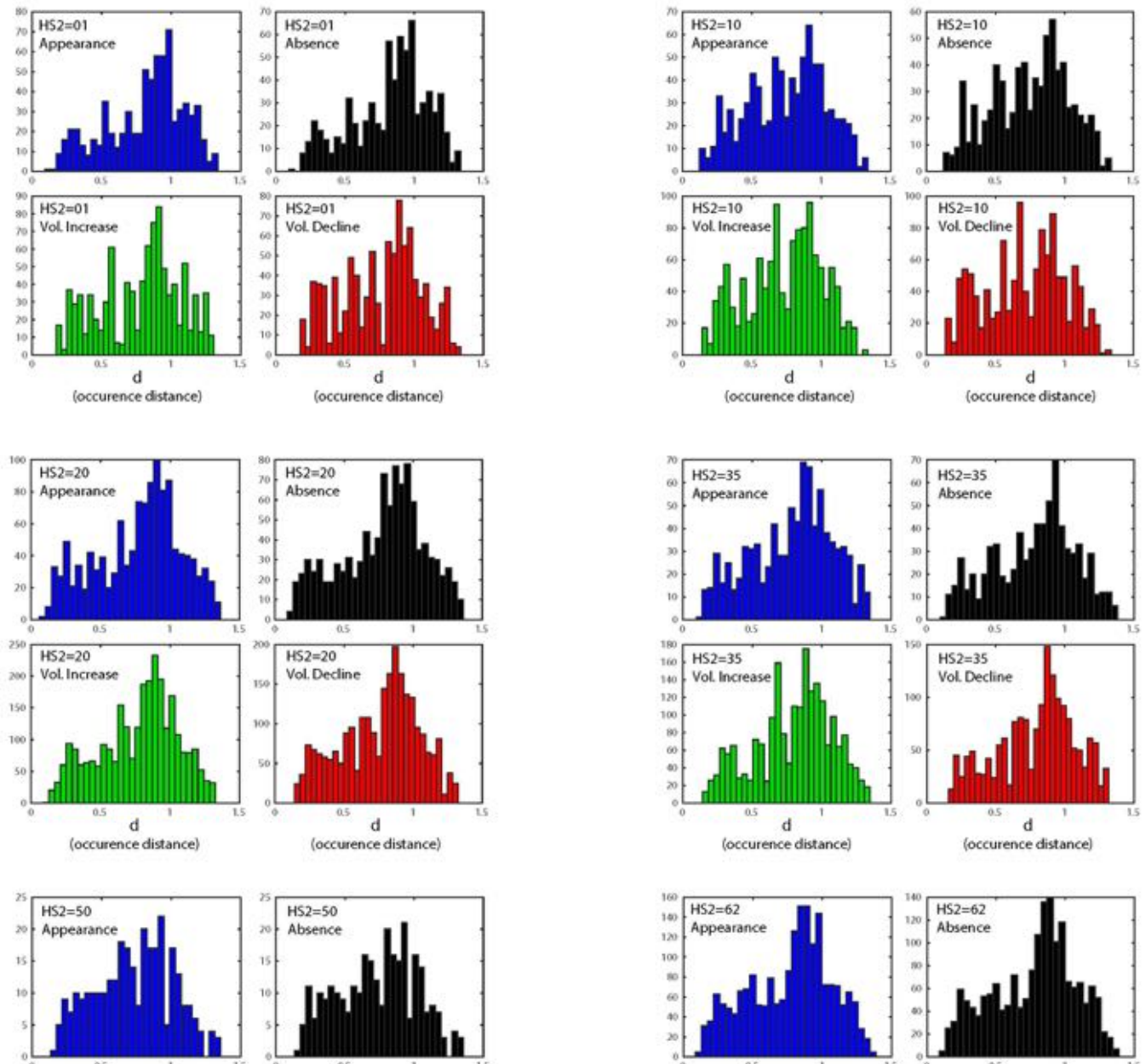

d
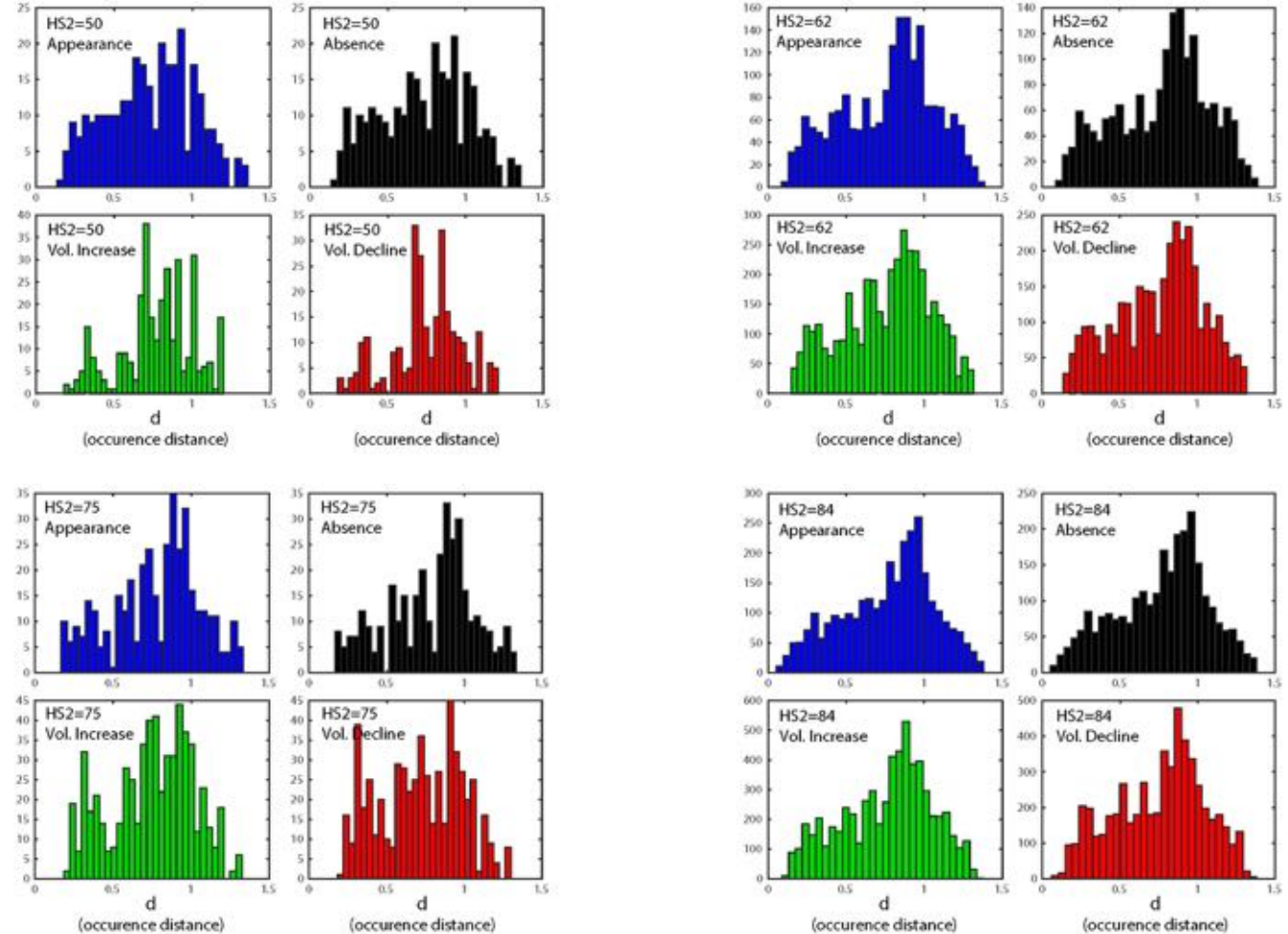

Fig. C.29.: Dynamical distributions for selected industries HS2. This figure shows, each of the selected industries HS2, the frequency of each type of dynamical variance as a function of the occurrense distance: link appearance (blue), link absence (black), volume increase (green) and volume decline (red). 


\section{Appendix D: Market visualizations}
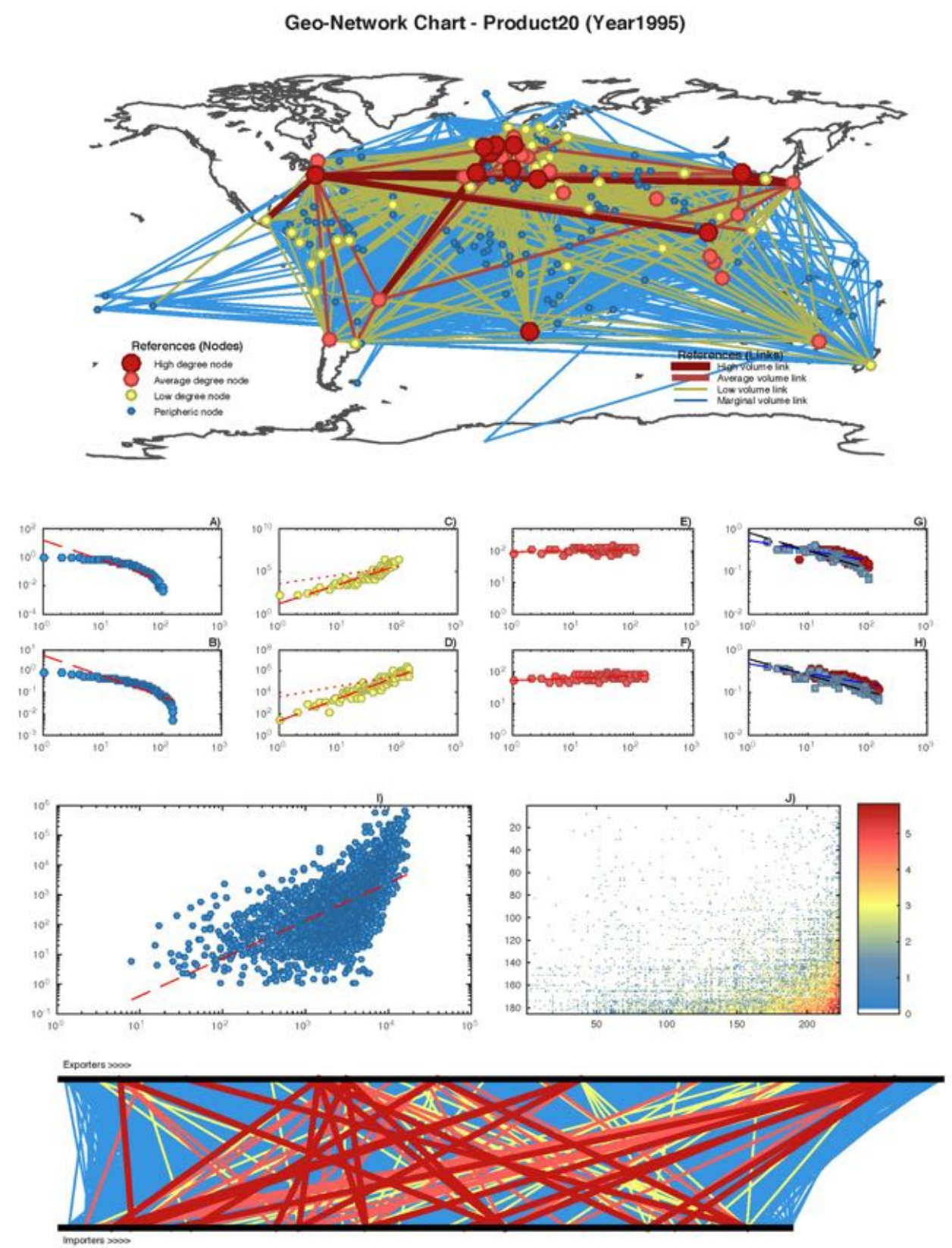

Fig. D.1.: Market dashboard HS2=20, $Y r=1995$. A) $P(k)$ Exporters, B) $P(k)$ Importers, C) $S(k)$ Exporters, D) S(k) Importers, E) Knn(k) Exporters, F) Knn(k) Importers, G) C4b/w(k) Exporters, H) C4b/w(k) Importers, I) $<\mathrm{w}>$ (ki.kj) and J) Bipartite Matrix. 

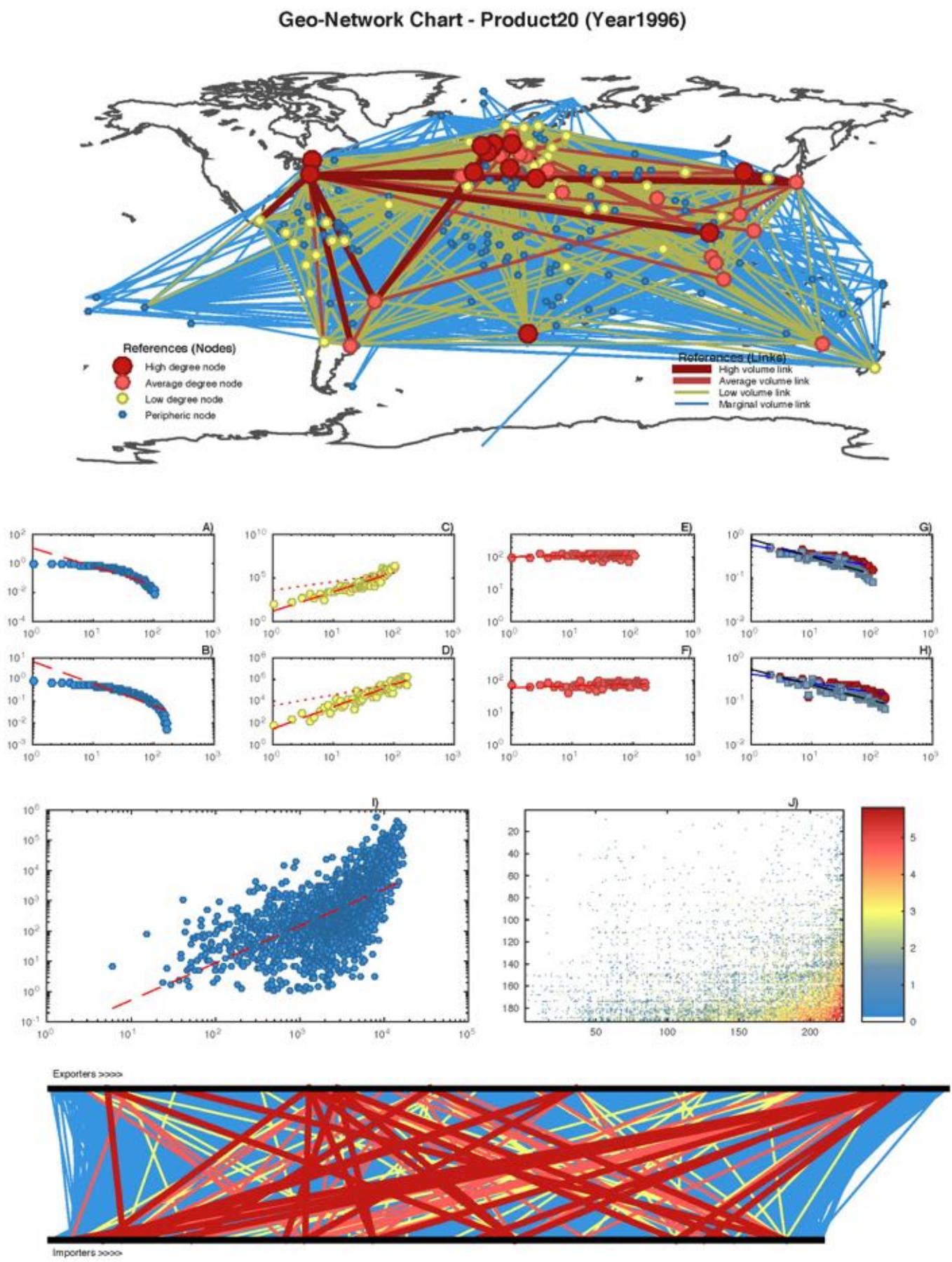

Fig. D.2.: Market dashboard HS2=20, $\mathrm{Yr}=1996$. A) $\mathrm{P}(\mathrm{k})$ Exporters, B) $\mathrm{P}(\mathrm{k})$ Importers, C) $\mathrm{S}(\mathrm{k})$ Exporters, D) S(k) Importers, E) Knn(k) Exporters, F) Knn(k) Importers, G) C4b/w(k) Exporters, H) C4b/w(k) Importers, I) $<\mathrm{w}>$ (ki.kj) and J) Bipartite Matrix. 


\section{Geo-Network Chart - Product20 (Year1997)}
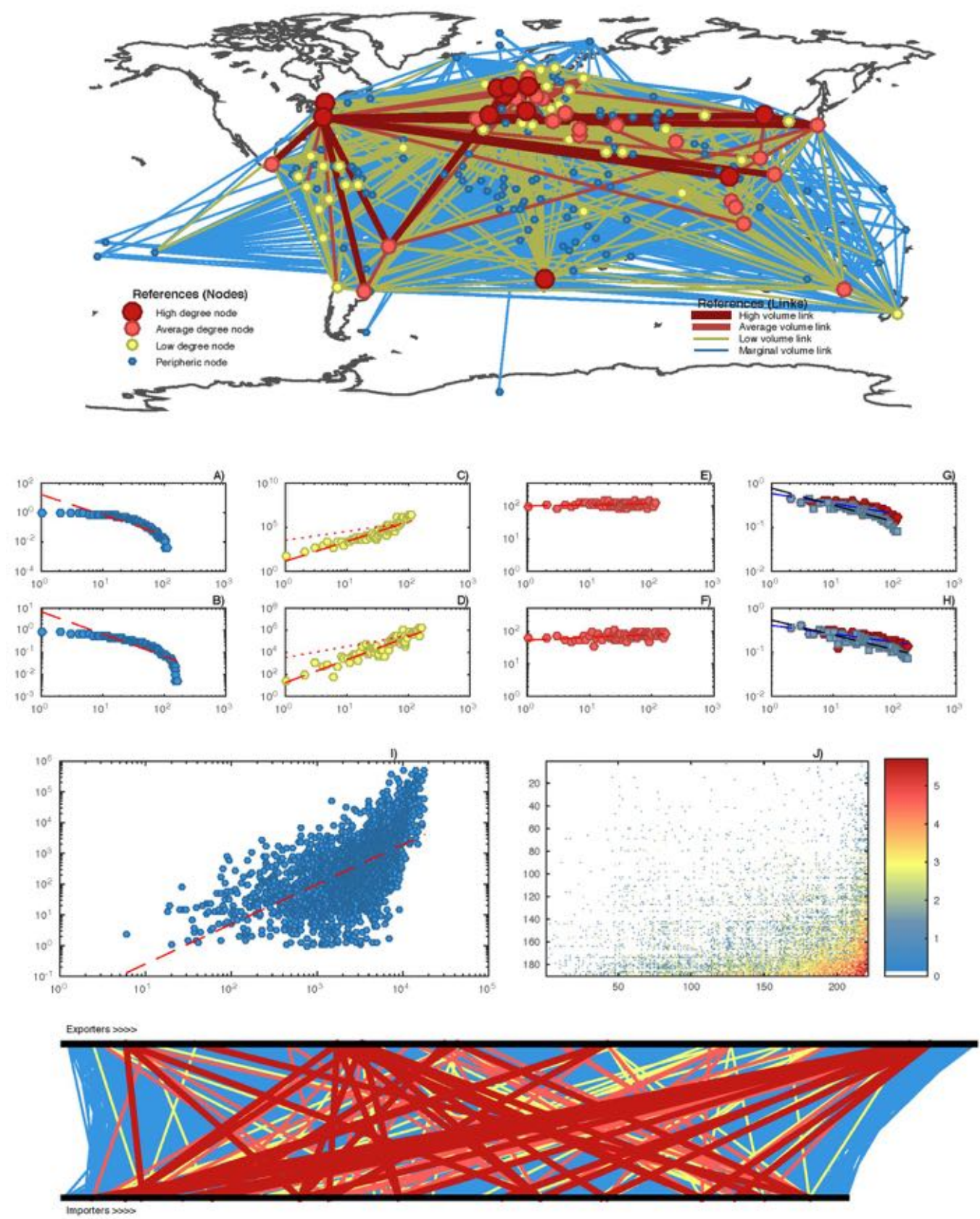

Fig. D.3.: Market dashboard HS2=20, $\mathrm{Yr}=1997$. A) $\mathrm{P}(\mathrm{k})$ Exporters, B) $\mathrm{P}(\mathrm{k})$ Importers, C) $\mathrm{S}(\mathrm{k})$ Exporters, D) S(k) Importers, E) Knn(k) Exporters, F) Knn(k) Importers, G) C4b/w(k) Exporters, H) C4b/w(k) Importers, I) $<\mathrm{w}>$ (ki.kj) and J) Bipartite Matrix. 


\section{Geo-Network Chart - Product20 (Year1998)}
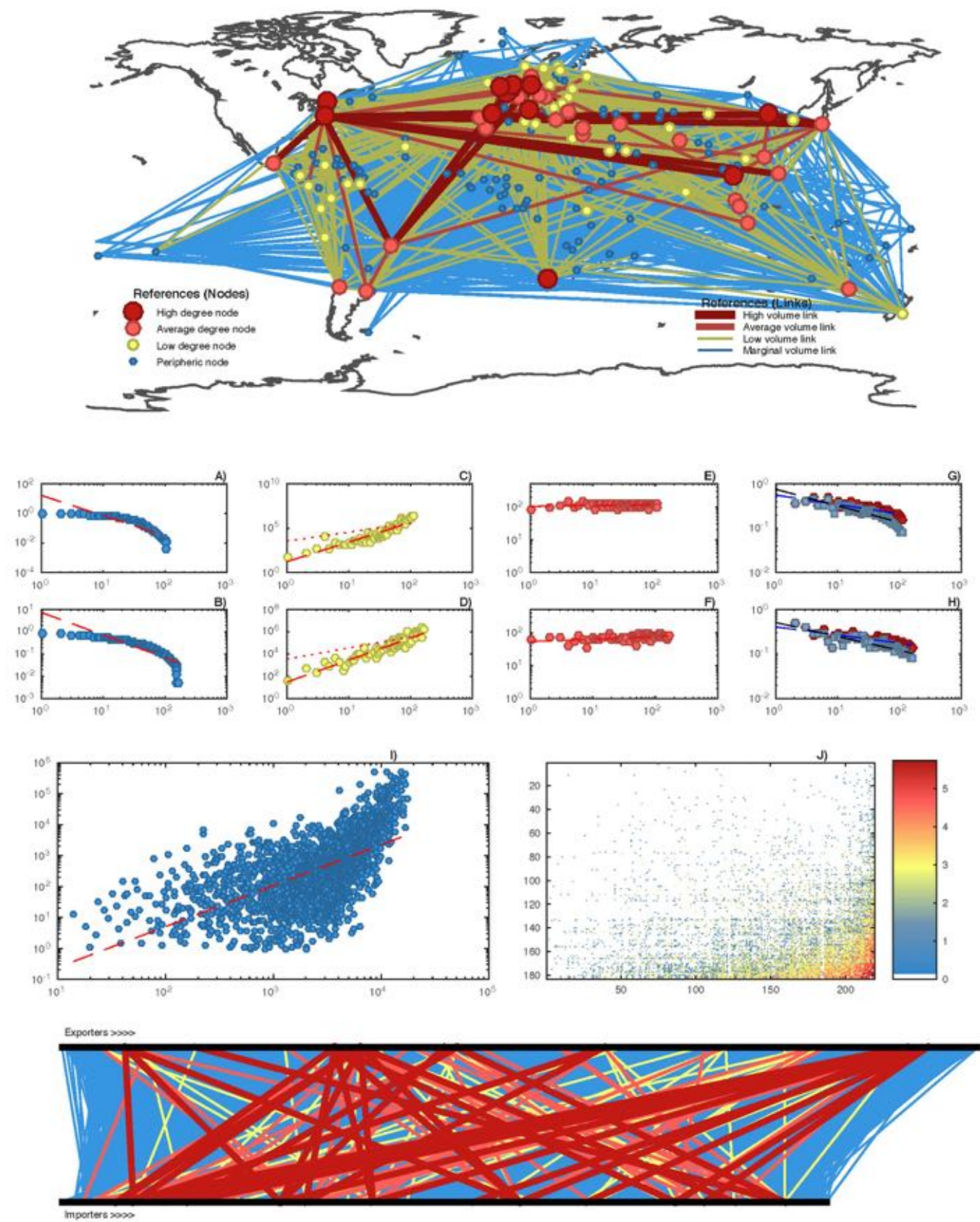

Fig. D.4.: Market dashboard HS2=20, $\mathrm{Yr}=1998$. A) $\mathrm{P}(\mathrm{k})$ Exporters, B) $\mathrm{P}(\mathrm{k})$ Importers, C) $\mathrm{S}(\mathrm{k})$ Exporters, D) S(k) Importers, E) Knn(k) Exporters, F) Knn(k) Importers, G) C4b/w(k) Exporters, H) C4b/w(k) Importers, I) $<w>$ (ki.kj) and J) Bipartite Matrix. 


\section{Geo-Network Chart - Product20 (Year1999)}
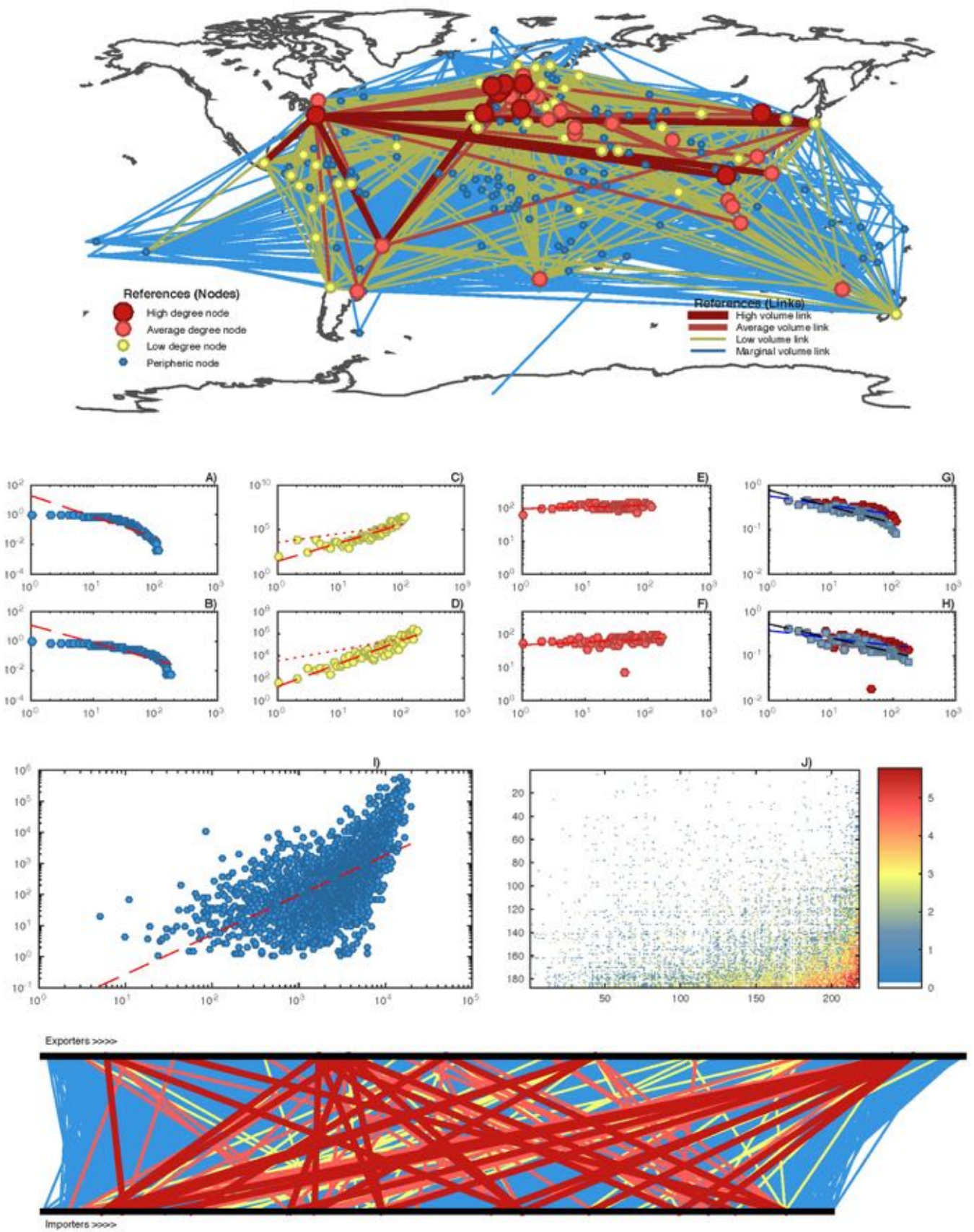

Fig. D.5.: Market dashboard HS2=20, Yr=1999. A) P(k) Exporters, B) P(k) Importers, C) S(k) Exporters, D) S(k) Importers, E) Knn(k) Exporters, F) Knn(k) Importers, G) C4b/w(k) Exporters, H) C4b/w(k) Importers, I) $<\mathrm{w}>($ ki.kj) and J) Bipartite Matrix. 


\section{Geo-Network Chart - Product20 (Year2000)}
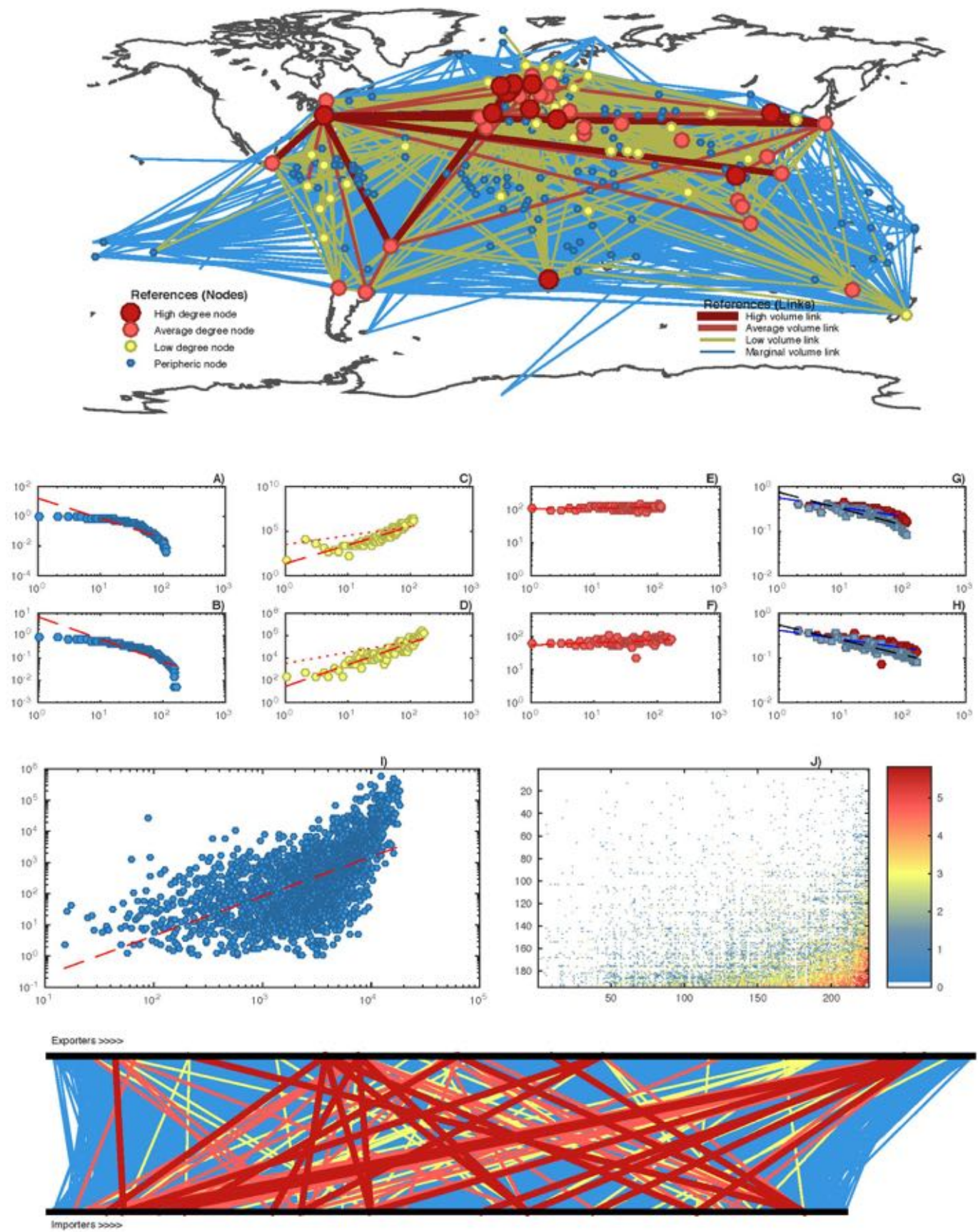

Fig. D.6.: Market dashboard HS2=20, $\mathrm{Yr}=2000$. A) $\mathrm{P}(\mathrm{k})$ Exporters, $\mathrm{B}) \mathrm{P}(\mathrm{k})$ Importers, C) $\mathrm{S}(\mathrm{k})$ Exporters, D) S(k) Importers, E) Knn(k) Exporters, F) Knn(k) Importers, G) C4b/w(k) Exporters, H) C4b/w(k) Importers, I) $<w>$ (ki.kj) and J) Bipartite Matrix. 
Geo-Network Chart - Product20 (Year2001)
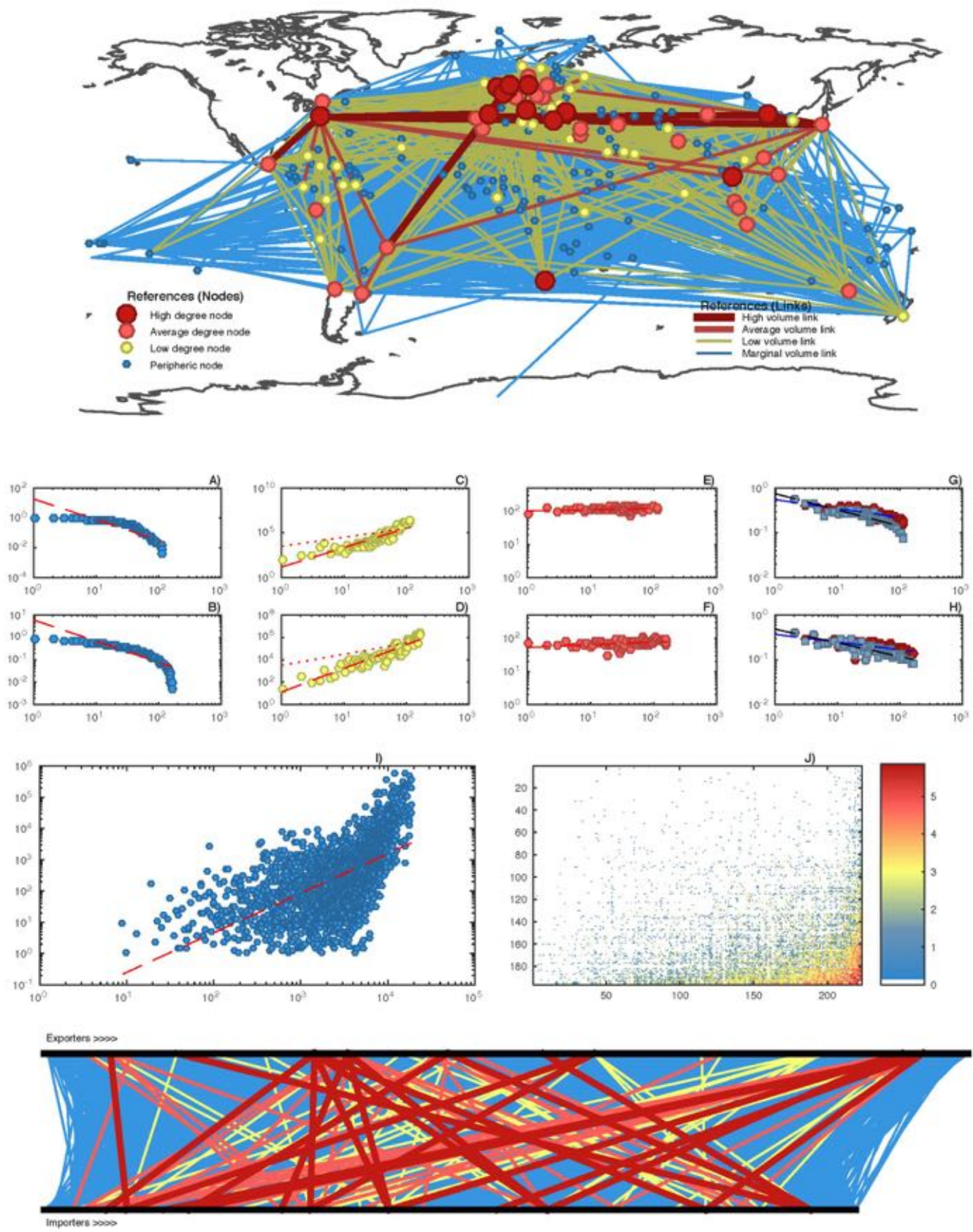

Fig. D.7.: Market dashboard HS2=20, Yr=2001. A) P(k) Exporters, B) P(k) Importers, C) S(k) Exporters, D) S(k) Importers, E) Knn(k) Exporters, F) Knn(k) Importers, G) C4b/w(k) Exporters, H) C4b/w(k) Importers, I) $<\mathrm{w}>($ ki.kj) and J) Bipartite Matrix. 


\section{Geo-Network Chart - Product20 (Year2002)}
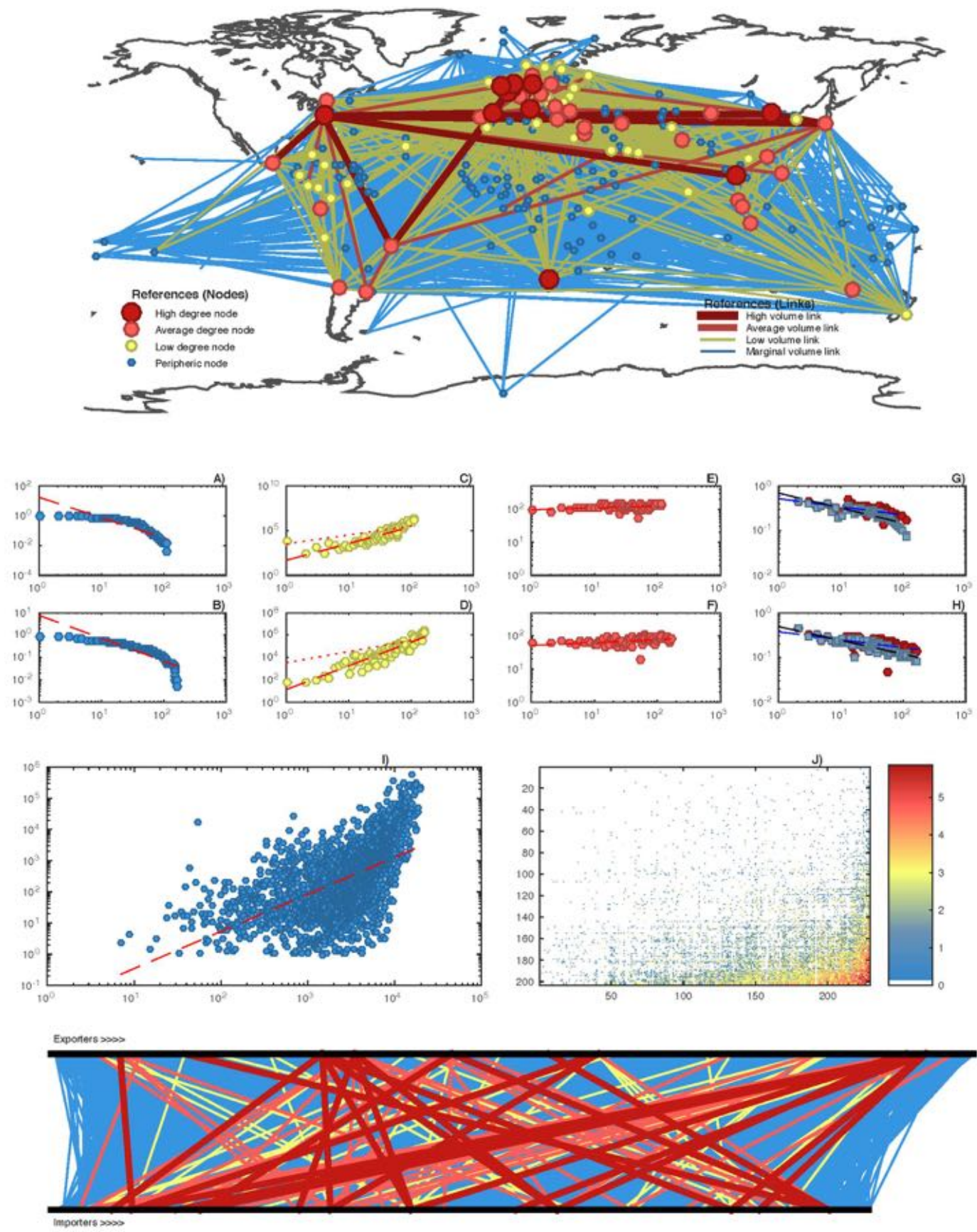

Fig. D.8.: Market dashboard HS2=20, $Y r=2002$. A) $P(k)$ Exporters, B) $P(k)$ Importers, C) $S(k)$ Exporters, D) S(k) Importers, E) Knn(k) Exporters, F) Knn(k) Importers, G) C4b/w(k) Exporters, H) C4b/w(k) Importers, I) $<w>(k i . k j)$ and J) Bipartite Matrix. 


\section{Geo-Network Chart - Product20 (Year2003)}
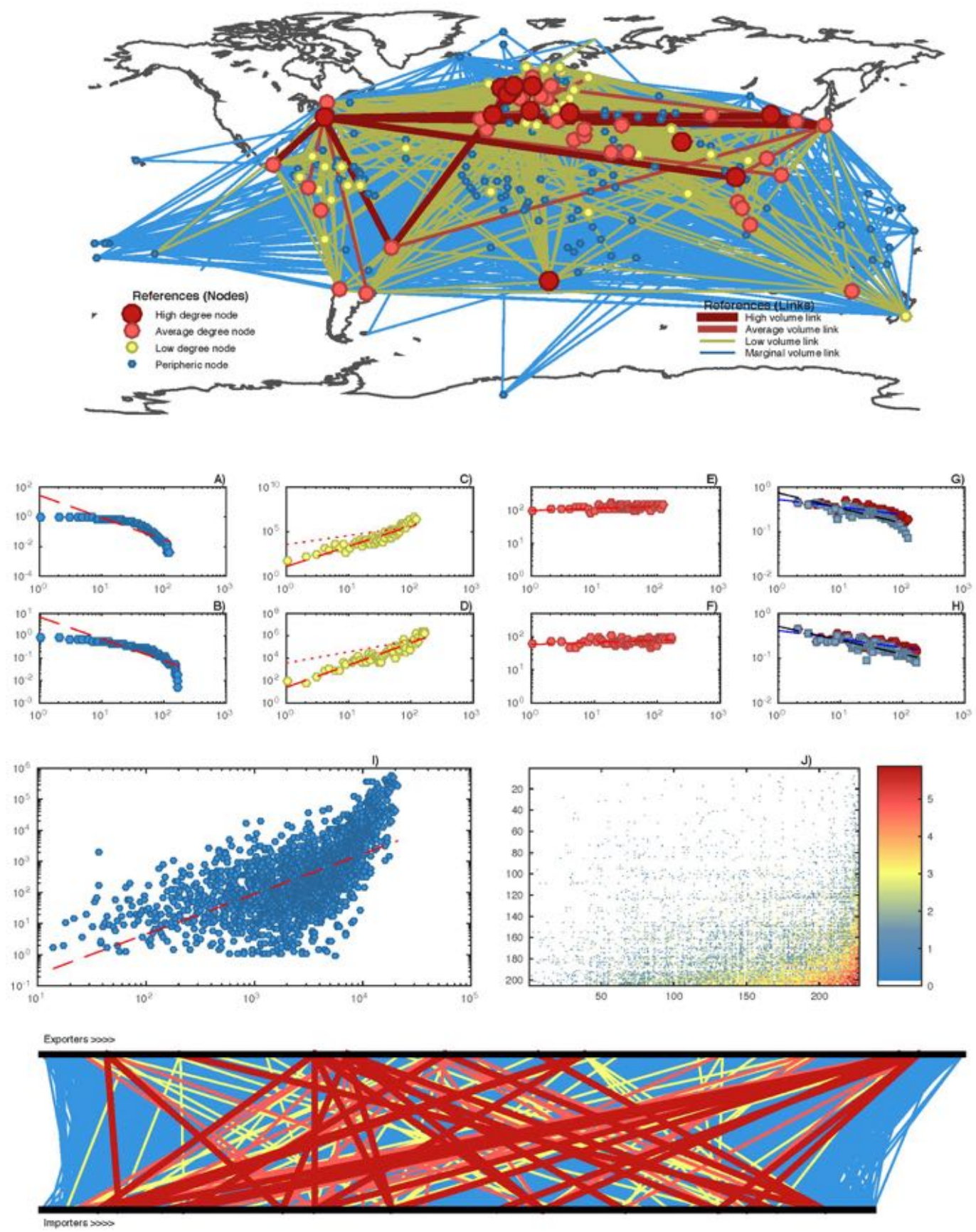

Fig. D.9.: Market dashboard HS2=20, Yr=2003. A) P(k) Exporters, B) P(k) Importers, C) S(k) Exporters, D) S(k) Importers, E) Knn(k) Exporters, F) Knn(k) Importers, G) C4b/w(k) Exporters, H) C4b/w(k) Importers, I) $<\mathrm{w}>$ (ki.kj) and J) Bipartite Matrix. 


\section{Geo-Network Chart - Product20 (Year2004)}
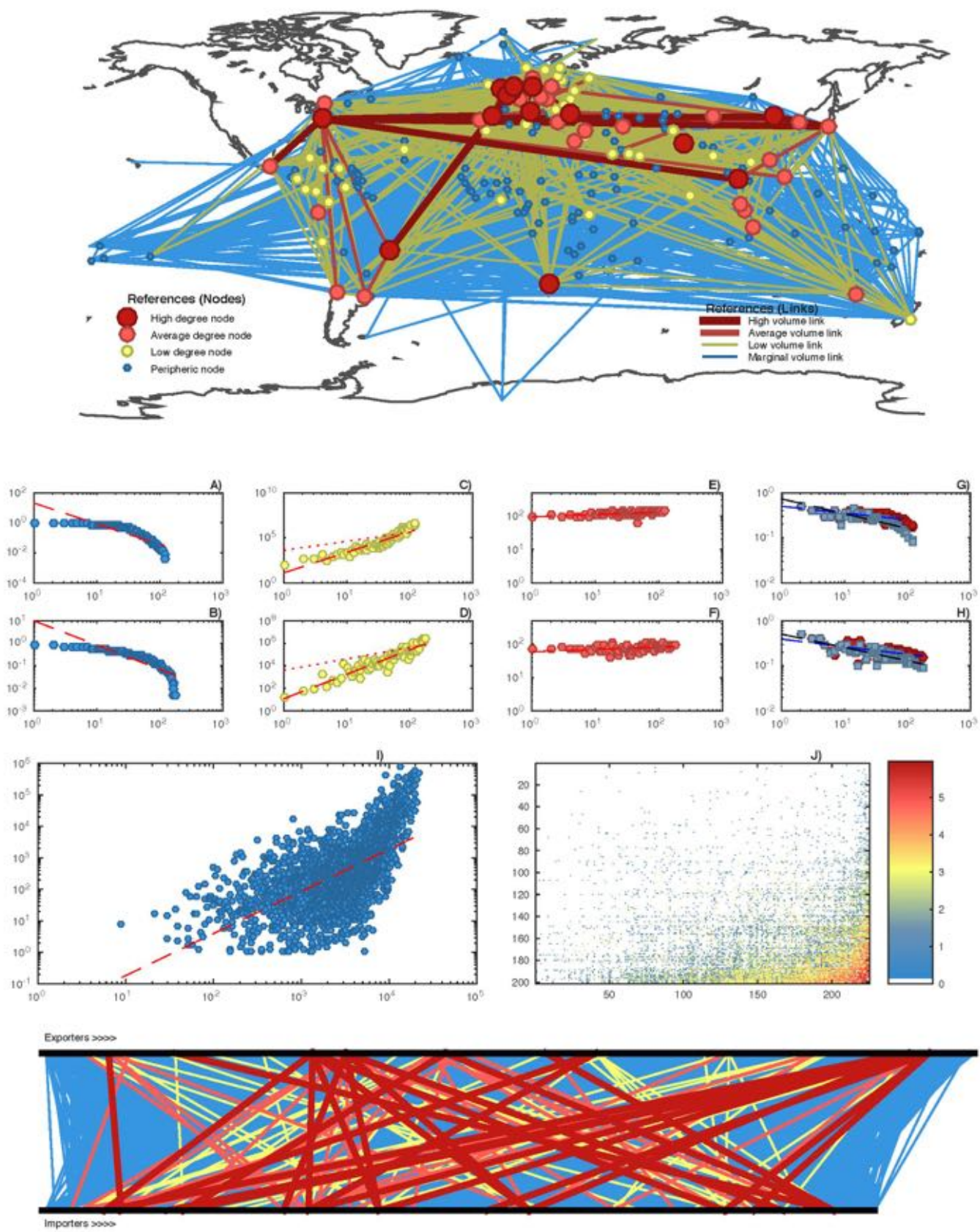

Fig. D.10.: Market dashboard HS2=20, $Y r=2004$. A) $P(k)$ Exporters, B) $P(k)$ Importers, C) $S(k)$ Exporters, D) S(k) Importers, E) Knn(k) Exporters, F) Knn(k) Importers, G) C4b/w(k) Exporters, H) C4b/w(k) Importers, I) $<\mathrm{w}>$ (ki.kj) and J) Bipartite Matrix. 
Geo-Network Chart - Product20 (Year2005)
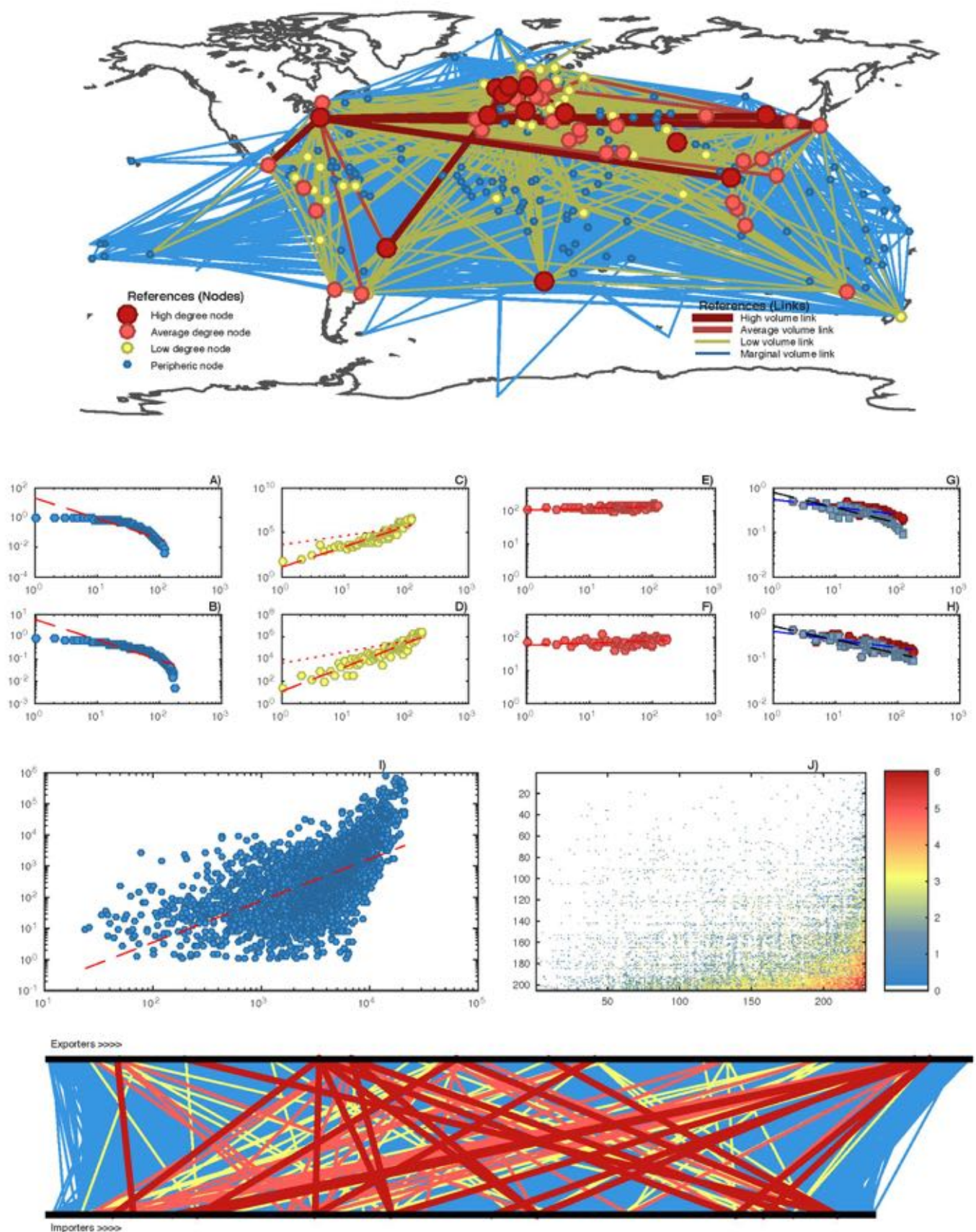

Fig. D.11.: Market dashboard HS2=20, $\mathrm{Yr}=2005$. A) $\mathrm{P}(\mathrm{k})$ Exporters, B) $\mathrm{P}(\mathrm{k})$ Importers, C) $\mathrm{S}(\mathrm{k})$ Exporters, D) S(k) Importers, E) Knn(k) Exporters, F) Knn(k) Importers, G) C4b/w(k) Exporters, H) C4b/w(k) Importers, I) $<\mathrm{w}>(\mathrm{ki} . \mathrm{kj})$ and J) Bipartite Matrix. 


\section{Geo-Network Chart - Product20 (Year2006)}
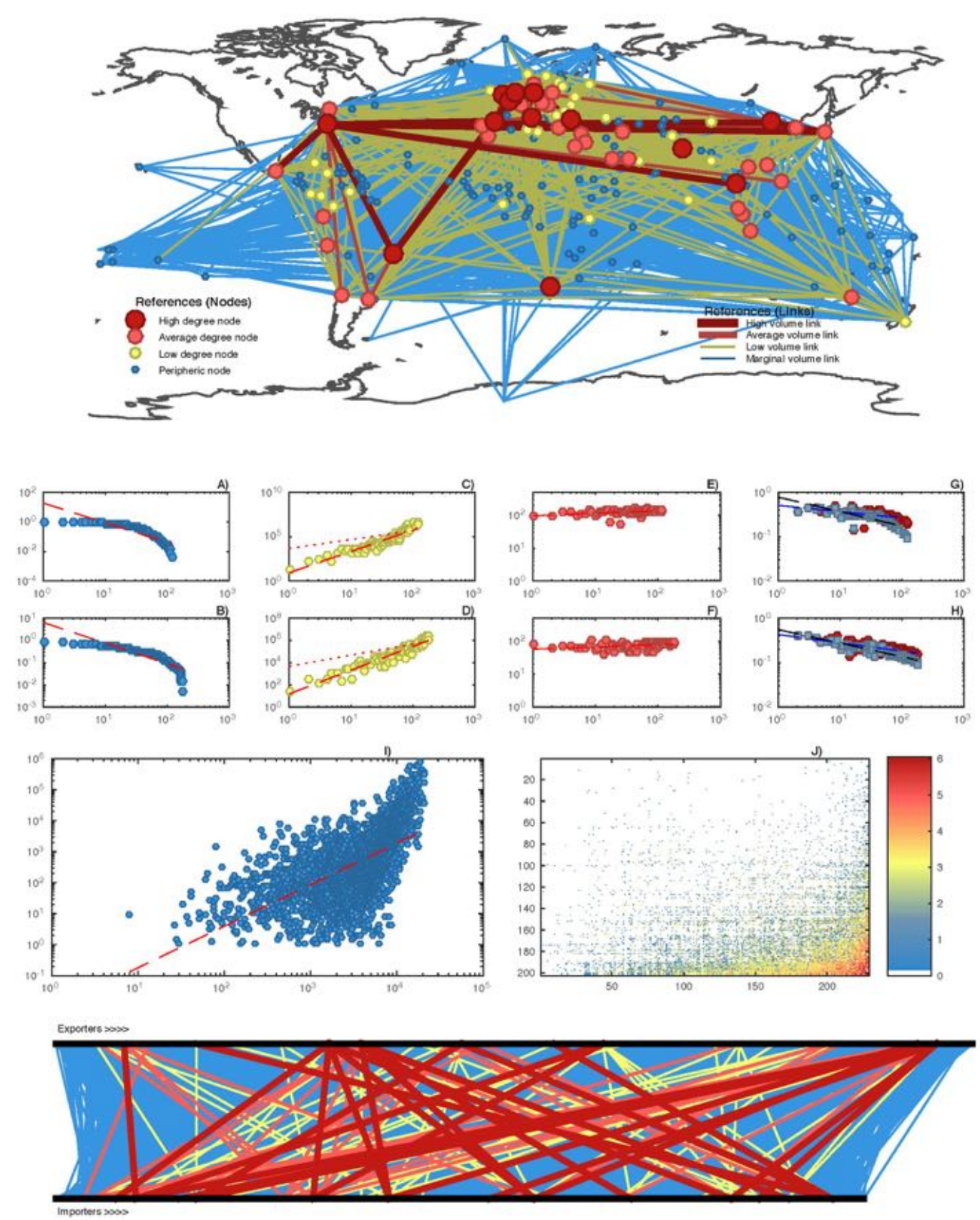

Fig. D.12.: Market dashboard HS2=20, $Y r=2006$. A) $P(k)$ Exporters, B) $P(k)$ Importers, C) $S(k)$ Exporters, D) S(k) Importers, E) Knn(k) Exporters, F) Knn(k) Importers, G) C4b/w(k) Exporters, H) C4b/w(k) Importers, I) <w>(ki.kj) and J) Bipartite Matrix. 


\section{Geo-Network Chart - Product20 (Year2007)}
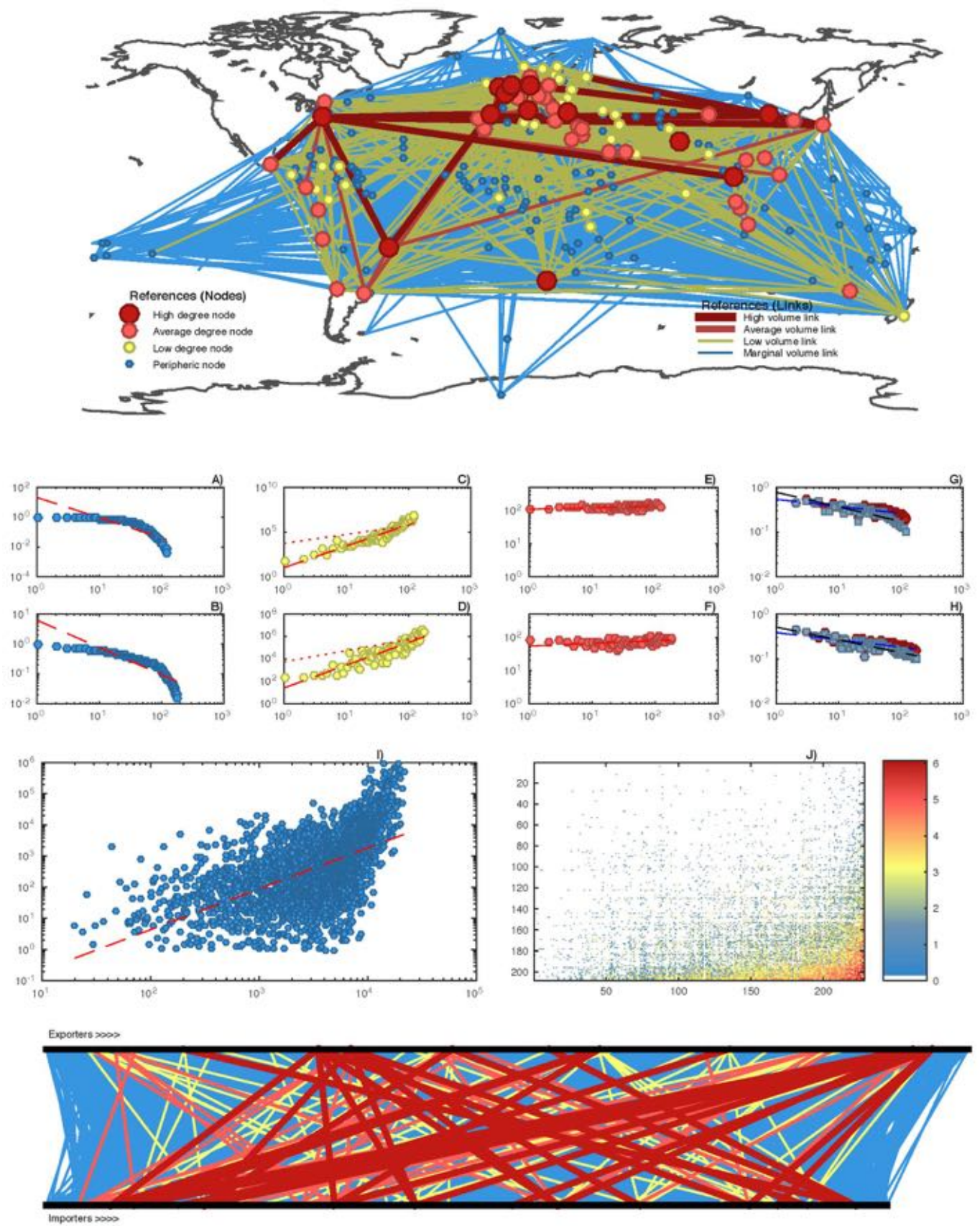

Fig. D.13.: Market dashboard HS2=20, $Y r=2007$. A) $P(k)$ Exporters, B) $P(k)$ Importers, C) $S(k)$ Exporters, D) S(k) Importers, E) Knn(k) Exporters, F) Knn(k) Importers, G) C4b/w(k) Exporters, H) C4b/w(k) Importers, I) $<w>(k i . k j)$ and J) Bipartite Matrix. 

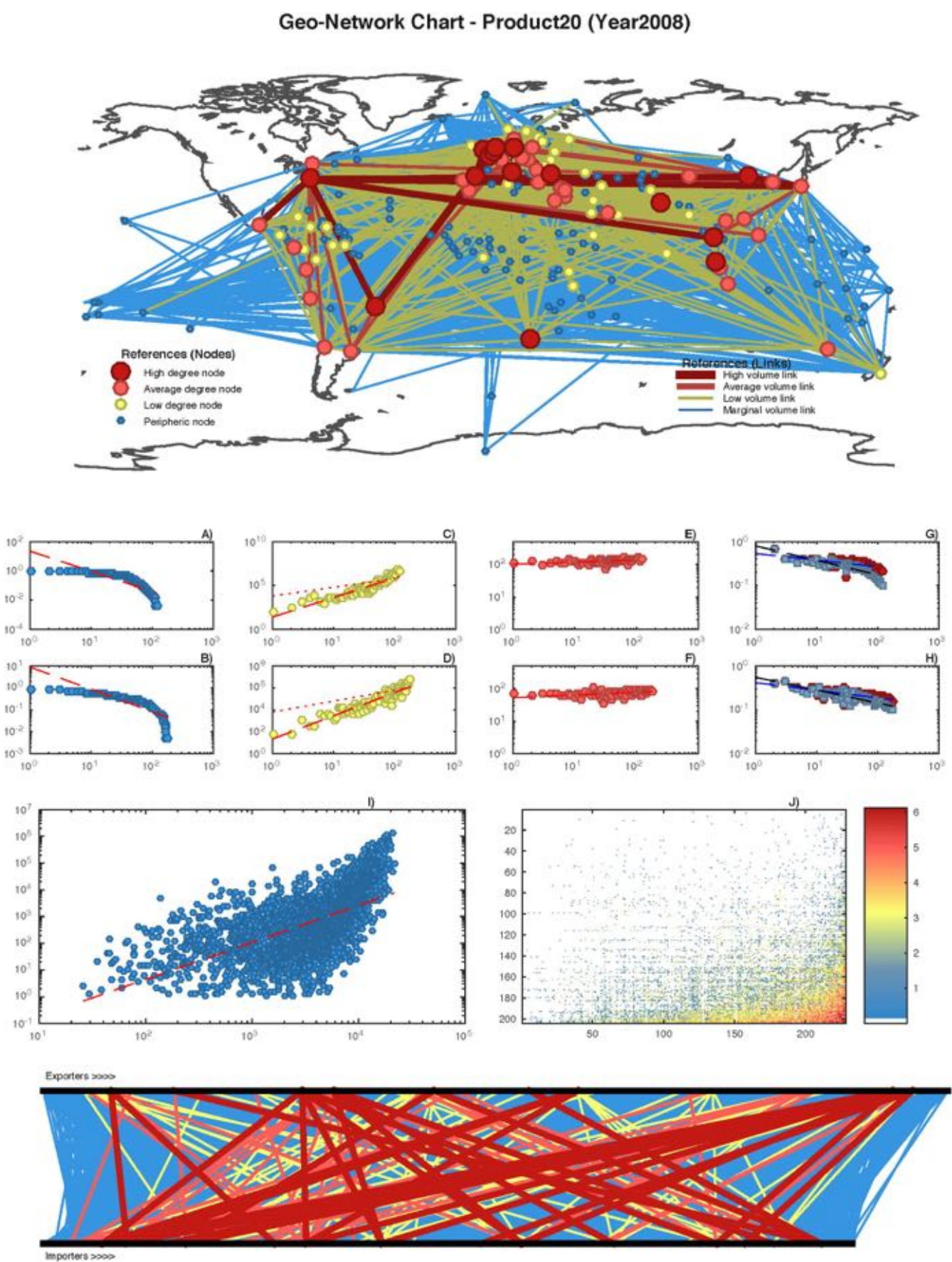

Fig. D.14.: Market dashboard HS2=20, $\mathrm{Yr}=2008$. A) $\mathrm{P}(\mathrm{k})$ Exporters, B) $\mathrm{P}(\mathrm{k})$ Importers, C) $\mathrm{S}(\mathrm{k})$ Exporters, D) S(k) Importers, E) Knn(k) Exporters, F) Knn(k) Importers, G) C4b/w(k) Exporters, H) C4b/w(k) Importers, I) $<\mathrm{w}>(\mathrm{ki.kj})$ and J) Bipartite Matrix. 

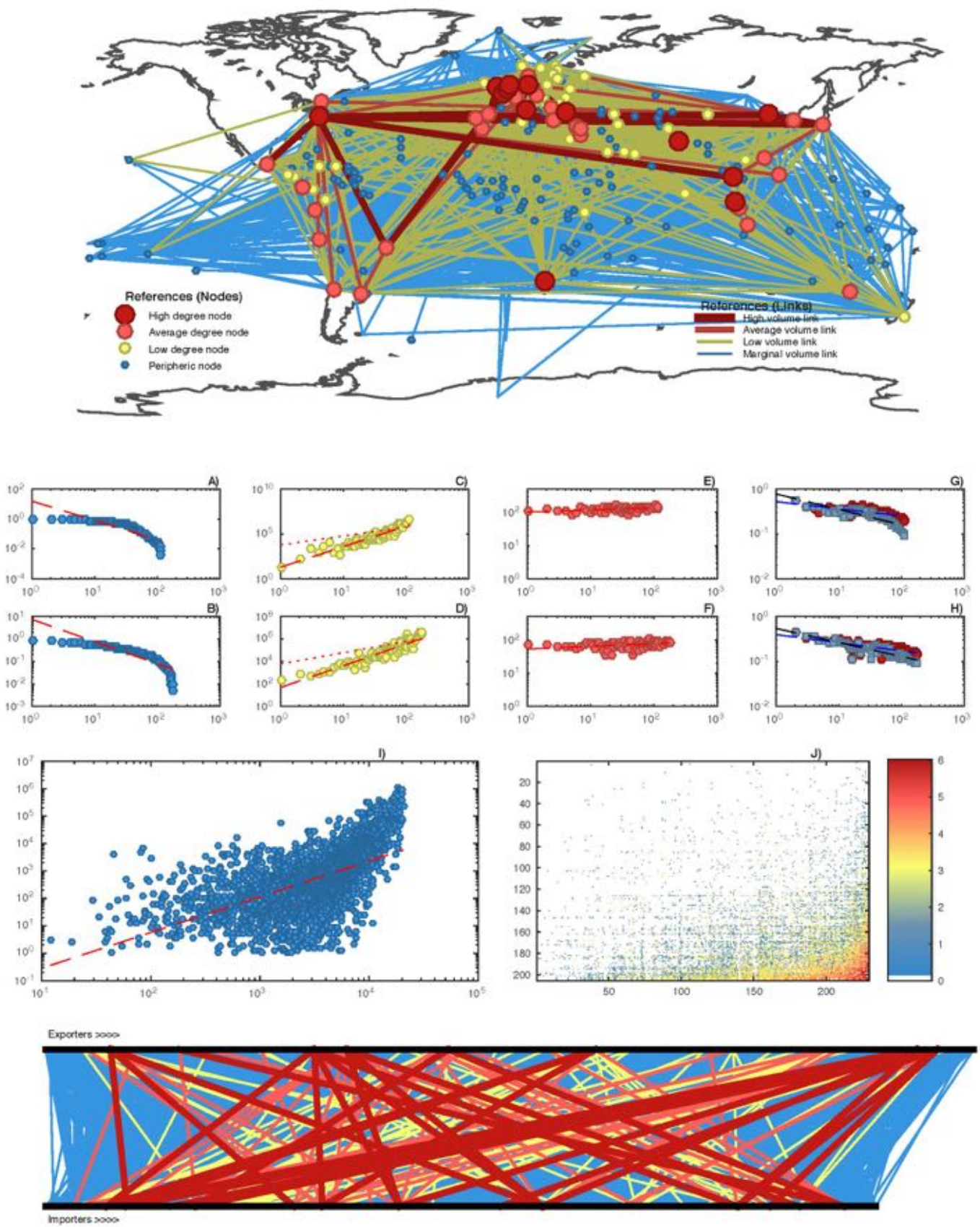

Fig. D.15.: Market dashboard HS2=20, $Y r=2009$. A) P(k) Exporters, B) P(k) Importers, C) S(k) Exporters, D) S(k) Importers, E) Knn(k) Exporters, F) Knn(k) Importers, G) C4b/w(k) Exporters, H) C4b/w(k) Importers, I) $<\mathrm{w}>$ (ki.kj) and J) Bipartite Matrix. 



\section{Bibliography}

[1] D. Acemoglu, A. Ozdaglar, A. Tahbaz-Salehi , Systemic Risk and Stability in Networks. American Economic Review 105(2), p. 564-608. (2013).

[2] L.A. Adamic, B.A. Huberman, Power-law distribution of the world wide web. Science 287, p. 2115 (2000).

[3] L.A. Adamic, N. Glance, The Political Blogosphere and the 2004 U.S. Election: Divided They Blog. LinkKDD '05 Proceedings of the 3rd international workshop on Link discovery, p. 36-43 (2005).

[4] D.P. Vázquez, Diameter of the world wide web. Nature 401, p. 130-131 (1999).

[5] R. Albert and A.L. Barabási, Statistical mechanics of complex networks. Reviews of Modern Physics, Vol. 74, p. 47-97, (2002).

[6] F. Allen, D. Gale, Financial contagion. Journal of Political Economy, Vol. 108, Issue 1, p. 1-33 (2000).

[7] F. Allen, A. Babus, E. Carletti, Asset commonality, debt maturity and systemic risk. Journal of Financial Economics, Vol. 104, Issue 3, p. 519-534 (2012).

[8] A. Allen-Perkins, J. Galeano, J.M. Pastor, Inducing self-organized criticality in a network toy model by neighborhood assortativity. Physical Review E 94 (5), 052304 (2016).

[9] M. Almeida-Neto, P. Guimarães Jr, T.M. Lewinsohn, On nestedness analyses: Rethinking matrix temperature and anti-nestedness. Oikos 116 (4), p. 716-722 (2007).

[10] M. Almeida-Neto, P. Guimarães, P. Guimarães, R.D. Loyola, W. Ulrich, A consistent metric for nestedness analysis in ecological systems: reconciling concept 
and measurement. Oikos 117 (8), p. 1227-1239 (2008).

[11] M. Almeida-Neto, W. Ulrich, A straightforward computational approach for measuring nestedness using quantitative matrices. Environmental Modelling \& Software, Vol. 26, Issue 2, p. 173-178 (2011).

[12] J.E. Anderson, The gravity model. Working paper 16576 of the NATIONAL BUREAU OF ECONOMIC RESEARCH (2010).

[13] W. Atmar, B.D. Patterson, The measure of order and disorder in the distribution of species in fragmented habitat. Oecologia, 96, p. 373-382 (1993).

[14] B. Balassa, Comparative Advantage in Manufactured Goods: A Reappraisal. The Review of Economics and Statistics, vol. 68, issue 2, p. 315-19 (1986).

[15] G. Ballantyne, K.C.R. Baldock, P.G. Willmer, Constructing more informative plant-pollinator networks: visitation and pollen deposition networks in a heathland plant community. Proc. R. Soc. B., Vol. 282. 1814. The Royal Society (2015).

[16] A.L. Barabási, R. Albert, Emergence of scaling in random networks. Science, Vol. 286, p. 509-512. (1999).

[17] A.L. Barabási, R. Albert, H. Jeong, Scale-free characteristics of random networks: the topology of the world wide web. Physica A 281, p. 69-77 (2000).

[18] A.L. Barabási, The physics of the web. Physics World 14, p. 33-38 (2001).

[19] M.J. Barber, Modularity and community detection in bipartite networks. Physical Review E, Vol. 76, Issue 6, p. 066102 (2007).

[20] A. Barrat, M. Barthelemy, R. Pastor-Satorras, A. Vespignani, The Architecture of Complex Weighted Networks. PNAS, Vol. 101 (2004).

[21] J. Bascompte, P. Jordano, C.J. Melián, J.M. Olesen, The Nested Assembly of Plant-Animal Mutualistic Networks. PNAS, Vol. 100, no. 16 (2004).

[22] J. Bascompte, P. Jordano, Plant-animal mutualistic networks: the architecture of biodiversity. Annual Review of Ecology, Evolution, and Systematic, p. 567-593 (2007).

[23] J. Bascompte, Disentangling the web of life. Science 325, p. 416-419 (2009). 
[24] U. Bastolla, M. Lässig, S.C. Manrubia, A. Valleriani, Biodiversity in model ecosystems, II: species assembly and food web structure. J Theor Biol, Vol. 235, p. 531-539 (2005).

[25] U. Bastolla, M.A. Fortuna, A. Pascual-García, A. Ferrera, B. Luque, The architecture of mutualistic networks minimizes competition and increases biodiversity. Nature 458, p. 1018-1020 (2009).

[26] E.B.E. Santos, R. Cont, The brazilian interbank network structure and systemic risk. Tech. Rep. 219, Banco Central do Brasil, Working Paper Series (2010).

[27] L. Bettencourt, G. West, A unified theory of urban living. Nature 467, p. 912-913 (2010).

[28] J. Borondo, AJ Morales, R.M. Benito, J.C. Losada, Multiple leaders on a multilayer social media. Chaos, Solitons \& Fractals 72, p. 90-98 (2013).

[29] J. Borondo, F. Borondo, C. Rodriguez-Sickert, C.A. Hidalgo, To each according to its degree: The meritocracy and topocracy of embedded markets. Scientific reports 4, 3784 (2014).

[30] J. Borondo, AJ Morales, R.M. Benito, J.C. Losada, Mapping the online communication patterns of political conversations. Physica A: Statistical Mechanics and its Applications 414, p. 403-413 (2014).

[31] M. Boss, H. Elsinger, M. Summer, S. Thurner, Network topology of the interbank market. Quantitative Finance 4, p. 677-684 (2004).

[32] G.E.P. Box, Robustness in the strategy of scientific model building. Robustness in statistics, Vol. 1, p. 201-236 (1979).

[33] Bureau van Dijk, Bankscope database. URL http://bankscope.bvdinfo.com.

[34] M. Burger, F. Van Oort, G.J. Linders, On the Specification of the Gravity Model of Trade: Zeros, Excess Zeros and Zero-inflated Estimation. Spatial Economic Analysis, vol. 4, issue 2, p. 167-190 (2009).

[35] S. Bustos, C. Gomez, R. Hausmann, C.A. Hidalgo, The Dynamics of Nestedness Predicts the Evolution of Industrial Ecosystems. PloS one, Vol. 7, Issue 11, e49393 (2012). 
[36] T.C. Silva, L. Zhao, Machine Learning in Complex Networks. Springer International Publishing Switzerland (2016).

[37] G. Caldarelli, M. Cristelli, A. Gabrielli, L. Pietronero, A network analysis of countries' export flows: firm grounds for the building blocks of the economy. PLoS ONE 7(10): e47278. doi:10.1371/journal.pone.0047278 (2012).

[38] J. Camacho, R. Guimera, L.A.N. Amaral, Analytical solution of a model for complex food webs. Phys. Rev. E 65, 030901(R) (2002).

[39] C. Campbell, S. Yang, R. Albert, A Network Model for Plant-Pollinator Community Assembly. PNAS, Vol. 108, Issue 1, p. 197-202 (2011).

[40] J.A. Capitán, A. Arenas, R. Guimerà, Degree of intervality of food webs: From body-size data to models. J. Theor. Biol., Vol. 334, p. 35-44 (2013).

[41] J.A. Capitán, S. Manrubia, Demography-based adaptive network model reproduces the spatial organization of human linguistic groups. Physical Review E 92, 062811 (2015).

[42] J.A. Capitán, J.B. Axelsen, New patterns in human biogeography revealed by networks of contacts between linguistic groups. Proc. R. Soc. B 282, 20142947 (2015).

[43] C. Carrere, Revisiting the effects of regional trade agreements on trade flows with proper specification of the gravity model. European Economic Review, Volume 50, Issue 2, p. 223-247 (2006).

[44] P.L. Chagnon, Characterizing topology of ecological networks along gradients: The limits of metrics standardization. Ecological Complexity, Vol. 22, p. 36-39. (2015).

[45] A. Chakraborty, S.S. Manna, Weighted Trade Network in a Model of Preferential Bipartite Transactions. Phys. Rev. E 81, 016111 (2010).

[46] A. Clauset, CR Shalizi, M.E.J. Newman, Power-law distributions in empirical data. Siam Rev., Vol. 51, Issue 4, p. 661-703 (2009).

[47] V. Colizza, A. Flammini, M.A. Serrano, A. Vespignani, Detecting rich-club ordering in complex networks. Nat. Phys. 2(2), p. 110-115 (2006). 
[48] M. Cristelli, A. Gabrielli, A. Tacchella, G. Caldarelli, Measuring the Intangibles: A Metrics for the Economic Complexity of Countries and Products. PLoS ONE, Volume 8, Issue 8, e70726 (2013).

[49] S. Cuenda, M. Fernández, J. Galeano, J.A. Capitán, A Minimal Agent-Based Model Reproduces the Overall Topology of Interbank Networks. Social Science Research Network, http://ssrn.com/abstract=2841753 (2016).

[50] G. De Masi, G. Iori, G. Caldarelli, Fitness model for the Italian interbank money market. Physical Review E 74(6), p. 1-5 (2006).

[51] M.A. De Menezes, A.L. Barabási, Fluctuations in network dynamics. Physical Review Letters 92, 28701 (2004).

[52] Z. Eisler, J. Kertesz, S.H. Yook, Multiscaling and non-universality in fluctuations of driven complex systems. Europhysics Letters 69, p. 664-670 (2005).

[53] P. Erdos, A. Rényi, On random graphs. Publicationes Mathematicae, Vol. 6, p. 290-297 (1959).

[54] P. Erdos, A. Rényi, On the evolution of random graphs. Publications of the Mathematical Institute of the Hungarian Academy of Sciences, Vol. 5, p. 17-61 (1960).

[55] L. Ermann, D.L. Shepelyansky, Ecological analysis of world trade. Physics Letters A, Vol. 377, Issue 3-4, p. 250-256 (2013).

[56] European Central Bank, Recent advances in modelling systemic risk using network analysis. Working paper (2010).

[57] European Central Bank, Target Annual Report 2014. Tech. Rep. June.. URL https://www.ecb.europa.eu/pub/pdf/other/targetar2014.en.pdf (2015).

[58] G. Fagiolo, J. Reyes, S. Schiavo, On the Topological Properties of the World Trade Web: A Weighted Network Analysis. Physica A, Vol. 387, p. 3868-3873 (2008).

[59] G. Fagiolo, J. Reyes, S. Schiavo, The World Trade Web: Topological Properties, Dynamics, and Evolution. Phys. Rev. E 79, 036115 (2009).

[60] G. Fagiolo, J. Reyes, S. Schiavo, The Evolution of the World Trade Web. Journal of Evolutionary Economics, Vol. 20, p. 479-514 (2010). 
[61] M. Fernandez, J. Galeano, C. Hidalgo, Bipartite networks provide new insights on international trade markets. Networks and Heterogeneous Media, Vol. 7, No. 3 (2012).

[62] M.A. Fortuna, DB Stouffer, J.M. Olesen, Nestedness versus modularity in ecological networks: two sides of the same coin?. Journal of Animal Ecology, Vol. 79, Issue 4, p. 811-817 (2010).

[63] S. Fortunato, Community detection in graphs. Phys. Rep. 486, p. 75-174 (2010).

[64] X. Freixas, BM Parigi, J.C. Rochet, Systemic risk, interbank relations, and liquidity provision by the central bank. Journal of Money, Credit, and Banking, Vol. 32, Issue 3, p. 611-38. (2000).

[65] D. Fricke, T. Lux, On the distribution of links in the interbank network: evidence from the e-mid overnight money market. Empirical Economics 49, p. 1467-1495 (2015).

[66] P. Gai, S. Kapadia, Contagion in financial networks. Proceedings of the Royal Society of London, 0410 (2010).

[67] J. Galeano, J. Buceta, K. Juarez, Dynamical scaling analysis of plant callus growth. EPL (Europhysics Letters) 63 (1), p. 83 (2003).

[68] J. Galeano, J.M. Pastor, J.M. Iriondo, Weighted-Interaction Nestedness Estimator (WINE): A new estimator to calculate over frequency matrices. Environmental Modelling \& Software 24, Issue 11, p. 1342-1346 (2009).

[69] J. Gao, B. Barzel, A.L. Barabási, Universal resilience patterns in complex networks. Nature 530.7590, p. 307-312 (2016).

[70] J. García-Algarra, J. Galeano, J.M. Pastor, Rethinking the logistic approach for population dynamics of mutualistic interactions. Journal of theoretical biology, Vol. 363, p. 332-343 (2014).

[71] J. García-Algarra, J.M. Pastor, J.M. Iriondo, J. Galeano, Ranking of critical species to preserve the functionality of mutualistic networks using the $k$-core decomposition. PeerJ 5, e3321 (2017).

[72] G. Gaulier, S. Zignago, BACI: International Trade Database at the Productlevel. Working paper 2010-23 of the CENTRE D'ÉTUDES PROSPECTIVES ET D' INFORMATIONS INTERNATIONALES (2010). 
[73] C.P. Georg, The effect of the interbank network structure on contagion and common shocks. Journal of Banking \& Finance 37 (7), p. 2216-2228 (2013).

[74] L.J. Gilarranz, J.M. Pastor, J. Galeano, The Architecture of Weighted Mutualistic Networks. Currently under revision for publication.

[75] M. Girvan, M.E.J. Newman, Community structure in social and biological networks. Proc. Natl. Acad. Sci. USA 99(12), p. 7821-7826 (2002).

[76] K.I. Goh, M.E. Cusick, D. Valle, B. Childs, M. Vidal, A.L. Barabasi, The human disease network. PNAS, Vol. 104, No. 21 (2007).

[77] M.C. Gonzalez, C.A. Hidalgo, A.L. Barabasi, Understanding individual human mobility patterns. Nature 453, p. 779-782 (2008).

[78] P.A. Grabowicz, J.J. Ramasco, E. Moro, J.M. Pujol, Social features of online networks: The strength of intermediary ties in online social media. PloS one 7 (1), e29358 (2012).

[79] A.G. Haldane, R.M. May, Systemic risk in banking ecosystems. Nature 469(7330), p. 351-355 (2011).

[80] R. Hausmann, C.A. Hidalgo, The network structure of economic output. J Econ Growth 16: 309342 (2011).

[81] R. Hausmann, C.A. Hidalgo, S. Bustos, M. Coscia, The atlas of economic complexity: Mapping paths to prosperity. Mit Press (2014).

[82] C.A. Hidalgo, B. Klinger, A.L. Barabási, The Product Space Conditions the Development of Nations. Science 317, 482 (2007).

[83] C.A. Hidalgo, R. Hausmann, The building blocks of economic complexity. PNAS, Vol. 106, Issue 26, p. 10570-10575 (2009).

[84] G. Iori, S. Jafarey, F.G. Padilla, Systemic risk on the interbank market. Journal of Economic Behavior \& Organization 61(4), p. 525-542 (2006).

[85] G. Iori, G. De Masi, O.V. Precup, G. Gabbi, A network analysis of the italian overnight money market. Journal of Economic Dynamics and Control 32, p. 259-278 (2008). 
[86] J.L. Iribarren, E. Moro, Impact of human activity patterns on the dynamics of information diffusion. Physical review letters 103 (3), 038702 (2009).

[87] J.L. Iribarren, E. Moro, Branching dynamics of viral information spreading. Physical Review E 84 (4), 046116 (2011).

[88] A. James, J.W. Pitchford, M.J. Plank, Disentangling nestedness from models of ecological complexity. Nature 487.7406, p. 227-230 (2012).

[89] M. Jiménez-Martín, J.M. Pastor, J.C. Losada, J. Galeano, Link aggregation process for modelling weighted mutualistic networks. arXiv:1403.5519 (2014).

[90] M. Kitsak, L.K. Gallos, S. Havlin, F. Liljeros, L. Muchnik, Identification of influential spreaders in complex networks. Nature Physics 6.11, p. 888-893 (2010).

[91] P. Krugman, The Self-Organizing Economy. Oxford University Press, Oxford (1996).

[92] C. Krumme, A. Llorente, A. Manuel Cebrian, The predictability of consumer visitation patterns. SCIENTIFIC REPORTS, Vol. 3 : 1645 (2013).

[93] M. Kuperman, G. Abramson, Small world effect in an epidemiological model. Phys. Rev. Lett. 86(13), p. 2909-2912 (2001).

[94] J.J. Lewer, H. Van den Berg, A Gravity Model of Immigration. Economics Letters 99:1, p. 164-167 (2008).

[95] P.G. Lind, M.C. Gonzalez, H.J. Herrmann, Cycles and clustering in bipartite networks. PHYSICAL REVIEW E 72, 056127 (2005).

[96] S. Martinez-Jaramillo, B. Alexandrova-Kabadjova, An empirical study of the mexican banking system's network and its implications for systemic risk. Banco de México Working Papers (2012-07), p. 1-47 (2012).

[97] L. Mátyás, Proper Econometric Specification of the Gravity Model. The World Economy, vol. 20, issue 3, p. 363-368 (1997).

[98] R.M. May, S.A. Levin, G. Sugihara, Ecology for bankers. Nature 451, p. 893-895 (2008).

[99] J.J. McAuley, L. da Fontoura Costa, Rich-club phenomenon across complex network hierarchies. Appl. Phys. Lett. 91, 084103 (2007). 
[100] K. McCann, Protecting biostructure. Nature 446.7131, p. 29 (2007).

[101] S. Millard, M. Polenghi, The relationship between the overnight interbank unsecured loan market and the CHAPS Sterling system. Bank of England Quarterly Bulletin, Spring 2004 (2), p. 42-47. URL http://papers.ssrn.com/sol3/papers.cfm?abstract_id=701274 (2004).

[102] G. Miritello, E. Moro, R. Lara, Dynamical strength of social ties in information spreading. Physical Review E 83 (4), 045102 (2011).

[103] G. Miritello, E. Moro, R. Lara, R. Martínez-López, Time as a limited resource: Communication strategy in mobile phone networks. Social Networks 35 (1), p. 89-95 (2013).

[104] F.S. Mishkin, The economics of money, banking, and financial markets. Pearson education (2007).

[105] F.S. Mishkin, Central Banking After The Crisis. 16 th Annual Conference of the Central Bank of Chile (November), p. 0-45. URL http://www0.gsb.columbia.edu/faculty/fmishkin/papers/12chile.pdf. (2012).

[106] J.M. Montoya, S.L. Pimm, R.V. Solé, Ecological networks and their fragility. Nature 442.7100, p. 259-264 (2006).

[107] A.J. Morales, W. Creixell, J. Borondo, J. Losada, R.M. Benito, Understanding ethnical interactions on ivory coast. Proceedings of the Third Conference on the Analysis of Mobile Phone Datasets, p. 116-122 (2013).

[108] A.J. Morales, J. Borondo, J.C. Losada, R.M. Benito, Efficiency of human activity on information spreading on Twitter. Social Networks 39, p. 1-11 (2014).

[109] A.J. Morales, J. Borondo, J.C. Losada, R.M. Benito, Measuring political polarization: Twitter shows the two sides of Venezuela. Chaos: An Interdisciplinary Journal of Nonlinear Science 25 (3), 033114 (2015).

[110] E. Moro, J. Vicente, L.G. Moyano, A. Gerig, J.D. Farmer, Market impact and trading profile of hidden orders in stock markets. Physical Review E 80 (6), 066102 (2009).

[111] M.E.J. Newman, S.H. Strogatz, D.J. Watts, Random graphs with arbitrary degree distributions and their applications. Physical Review E, Vol. 64, Issue 2 (2001). 
[112] M.E.J. Newman, Assortative Mixing in Networks. Physical Review Letters 89(20):208701 (2002).

[113] M.E.J. Newman, Mixing patterns in networks. Phys. Rev. E 67(2), 026126 (2003).

[114] M.E.J. Newman, Power laws, Pareto distributions and Zipf's law. Contemporary Physics 46, 5 (2004).

[115] M.E.J. Newman, Analysis of weighted networks. Phys. Rev. E 70, 056131 (2004).

[116] M.E.J. Newman, Modularity and community structure in networks. Proc. Natl. Acad. Sci. 103(23), p. 8577-8582 (2006).

[117] J.P. Onnela, J. Saramäki, J. Hyvönen, Structure and tie strengths in mobile communication networks. Proceedings of the National Academy of Sciences 104 (18), p. 7332-7336 (2007).

[118] L. Page, S. Brin, R. Motwani, T. Winograd, The pagerank citation ranking: Bringing order to the web. Technical Report. Stanford InfoLab (1999).

[119] J.M. Pastor Ruiz, J. Galeano, New dynamic scaling in increasing systems. Central European Journal of Physics 5 (4), p. 539-548 (2007).

[120] J.M. Pastor Ruiz, S. Santamaria, M. Méndez, J. Galeano, Effects of topology on robustness in ecological bipartite networks. Network and Heterogeneous Media 7 (3), p. 429 - 440 (2012).

[121] J.M. Pastor Ruiz, F.J. García Algarra, J. Galeano, J.M. Irondo, J.J. Ramasco, A simple and bounded model of population dynamics for mutualistic networks. Networks \& Heterogeneous Media 10.1 (2015).

[122] J.M. Pastor Ruiz, F.J. García Algarra, Dragging in Mutualistic Networks. Networks \& Heterogeneous Media 10.1 (2015).

[123] R. Pastor-Satorras, C. Castellano, P. Van Mieghem, Epidemic processes in complex networks. Rev. Mod. Phys. 87, 925 (2015).

[124] N. Perra, S. Fortunato, Spectral centrality measures in complex networks. Phys. Rev. E 78, 036107 (2008). 
[125] M. Raddant, Structure in the Italian overnight loan market. Journal of International Money and Finance 41, p. 197-213. URL http://dx.doi.org/10.1016/j.jimonfin.2013.11.005 (2014).

[126] E. Ravasz, A.L. Barabási, Hierarchical organization in complex networks. Physical Review E 67, 026112 (2003).

[127] E.L. Rezende, J.E. Lavabre, P. Guimarães, P. Jordano, Non-random coextinctions in phylogenetically structured mutualistic networks. Nature 448, p. 925-928 (2007).

[128] P. Sraffa, On the Principles of Political Economy and Taxation. John Murray (1817).

[129] G.J. Rodgers, K. Austin, B. Kahng, Eigenvalue spectra of complex networks. JOURNAL OF PHYSICS A, Math. Gen. 38, p. 9431-9437 (2005).

[130] A. Rohatgi, Webplotdigitizer. URL http://arohatgi.info/WebPlotDigitizer (2015).

[131] M.P. Rombach, M.A. Porter, J.H. Fowler, P.J. Mucha, Core-periphery structure in networks. SIAM J. Appl. Math. 74(1), p. 167-190 (2014).

[132] S. Saavedra, F. Reed-Tsochas, B. Uzzi, Asymmetric disassembly and robustness in declining networks. PNAS Vol. 105, no. 43, p. 16466-16471 (2008).

[133] S. Saavedra, F. Reed-Tsochas, B. Uzzi, A simple model of bipartite cooperation for ecological and organizational networks. Nature 457, p. 463-466 (2009).

[134] S. Saavedra, DB Stouffer, B. Uzzi, J. Bascompte, Strong contributors to network persistence are the most vulnerable to extinction. Nature 478, p. 233-235 (2011).

[135] P.A. Samuelson, Economics. Irwin/McGraw-Hill (1948).

[136] S. Santamaría, J. Galeano, J.M. Pastor, M. Méndez, Robustness of alpine pollination networks: effects of network structure and consequences for endemic plants. Arctic, Antarctic, and Alpine Research 46 (3), p. 568-580 (2014).

[137] S. Santamaría, J. Galeano, J.M. Pastor, M. Méndez, Removing interactions, rather than species, casts doubt on the high robustness of pollination networks. Oikos 125 (4), p. 526-534 (2016). 
[138] A. Santiago, R.M. Benito, An extended formalism for preferential attachment in heterogeneous complex networks. EPL (Europhysics Letters) 82 (5), 58004 (2008).

[139] M.A. Serrano, M. Boguná, Topology of the World Trade Web. Phys. Rev. E 68, 015101(R) (2003).

[140] M.A. Serrano, M. Boguñá, A. Vespignani, Patterns of Dominant Flows in the World Trade Web. J Econ Interac Coord. (2007).

[141] F. Simini, M.C. González, A. Maritan, A.L. Barabási, A universal model for mobility and migration patterns. Nature 484, p. 96-100 (2012).

[142] A. Smith, An Inquiry into the Nature and Causes of the Wealth of Nations. W. Strahan and T. Cadell, London (1776).

[143] D.A. Smith, D.R. White, Structure and dynamics of the global economy: network analysis of international trade. Soc. Forces 70(4), p. 857-893 (1992).

[144] C. Song, Z. Qu, N. Blumm, A.L. Barabási, Limits of Predictability in Human Mobility. Science 327, p. 1018-1021 (2010).

[145] K. Soramäki, ML Bech, J. Arnold, RJ Glass, The topology of interbank payment flows. Phys. A. Vol. 379, p. 317-333 (2007).

[146] D. Sornette, P. Cauwels, Financial bubbles: mechanisms and diagnostics. arXiv:1404.2140 (2014).

[147] T. Squartini, G. Fagiolo, D. Garlaschelli, Rewiring World Trade. Part I: A Binary Network Analysis. arXiv:1103.1243v2 (2011).

[148] T. Squartini, G. Fagiolo, D. Garlaschelli, Rewiring World Trade. Part II: A Weighted Network Analysis. arXiv:1103.1249v2 (2011).

[149] T. Squartini, G. Fagiolo, D. Garlaschelli, Randomizing world trade. i. a binary network analysis. Physical Review E 84: 046117 (2011).

[150] T. Squartini, G. Fagiolo, D. Garlaschelli, Randomizing world trade. ii. a weighted network analysis. Physical Review E 84: 046118 (2011).

[151] T. Squartini, I. Van Lelyveld, D. Garlaschelli, Early-warning signals of topological collapse in interbank networks. Scientific reports 3, 3357 (2013). 
[152] P.P.A. Staniczenko, J.C. Kopp, S. Allesina, The ghost of nestedness in ecological networks. Nature Communications, 4, 1391 (2012).

[153] D.B. Stouffer, J. Camacho, A robust measure of food web intervality. Proc. Natl. Acad. Sci. USA, Vol. 103, p. 19015-19020 (2006).

[154] A. Tacchella, M. Cristelli, G. Caldarelli, A. Gabrielli, A new metrics for countries' fitness and products' complexity. Scientific reports 2 (2012).

[155] A. Tacchella, M. Cristelli, G. Caldarelli, A. Gabrielli, Economic complexity: conceptual grounding of a new metrics for global competitiveness. Journal of Economic Dynamics and Control 37, p. 1683-1691 (2013).

[156] W. Ulrich, M. Almeida-Neto, N.J. Gotelli, A consumer's guide to nestedness analysis. Oikos 118, p. 3-17, (2009).

[157] W. Ulrich, M. Almeida-Neto, On the meanings of nestedness: back to the basics. Ecography 35 (10), p. 865-871 (2012).

[158] United Nations Organization, COMTRADE Database. URL = https://comtrade.un.org

[159] United States of America Department of Justice, Freedom of Information Act (FOIA). https://www.foia.gov

[160] A. Vázquez, R. Pastor-Satorras, A. Vespignani, Large-scale topological and dynamical properties of the Internet. Phys Rev E Stat Nonlin Soft Matter Phys 65:066130 (2002).

[161] D.P. Vázquez, W.F. Morris, P. Jordano, Interaction frequency as a surrogate for the total effect of animal mutualists on plants. Ecology Letters, Vol. 8, Issue 10, p. 1088-1094 (2005).

[162] D.P. Vázquez, Degree distribution in plant-animal mutualistic networks: forbidden links or random interactions?. Oikos 108 (2), p. 421-426 (2005).

[163] D.P. Vázquez, R. Poulin, BR Krasnov, Species abundance and the distribution of specialization in host-parasite interaction networks. Journal of Animal Ecology 74 (5), p. 946-955 (2005). 
[164] R. Vida, J. Galeano, S. Cuenda, Vulnerability of state-interdependent networks under malware spreading. Physica A: Statistical Mechanics and its Applications 421, p. 134-140 (2015).

[165] D.J. Watts, S.H. Strogatz, Collective dynamics of 'small-world' networks. Nature, Vol. 393 (6684), p. 440-442 (1998).

[166] R.J. Williams, N.D. Martinez, Simple rules yield complex food webs. Nature, Vol. 404, p. 180-183 (2000).

[167] L. Wittgenstein, Tractatus Logico-Philosophicus. Wilhelm Ostwald (ed.), Annalen der Naturphilosophie, 14 (1921).

[168] J. Yang, J. Leskovec, Overlapping communities explain core-periphery organization of networks. Proc. IEEE 102(12), p. 1892-1902 (2014).

[169] S.H. Yook, H. Jeong, A.L. Barabási, Weighted evolving networks. Physical Review Letters 86, p. 5835-5838 (2001).

[170] S.H. Yook, H. Jeong, A.L. Barabási, Modeling the internet's large-scale topology. Proceedings of the National Academy of Sciences 99, p. 13382-13386 (2002). 


\section{List of Figures}

2.1 Types of market described by microeconomic theory . . . . . . . . . . 9

2.2 The law of Supply and Demand . . . . . . . . . . . . . . 10

3.1 Example of a undirected, directed and weighted networks . . . . . 16

3.2 Bipartite network graph . . . . . . . . . . . . . 17

3.3 Bipartite network of phenome-genome (Diseasome) . . . . . . . . . 19

3.4 Bipartite (mutualistic) network of plants and pollinators. Rezende et al. (2007) . . . . . . . . . . . . . . . . . 20

3.5 Number of coexisting species determined by the structure of the network. Bastolla et al.[25]. Each panel represents a plant-animal network with different structures. Subfigure A depicts a fully connected structure. Subfigure B depicts a nested structure. Subfigure C depicts a compartmentalized structure. . . . . . . . . . . . . . . 21

3.6 Correlations between nestedness, randomizations and biodiversity. Bastolla et al.[25] . . . . . . . . . . . . . . . . . . . . 23

3.7 Process to determine individual contribution to nestedness and results after the randomizations. Saavedra et al.[134] . . . . . . . . . . . . . 24

3.8 Extinction exposure as a function of individual contribution to nestedness. Saavedra et al.[134] . . . . . . . . . . . . . . . . . . 24

3.9 Results on the topology of the World Trade Web. Serrano et al. (2003) 26

3.10 Topology weighted results on dominant flows in the WTW. Serrano et al. (2007) . . . . . . . . . . . . . . . . . . . 29

4.1 Example of data retrieval from COMTRADE . . . . . . . . . . . 37

4.2 Example of non-aggregated trade data . . . . . . . . . . . . 38

4.3 Example of the influence of distance on the estimated CIF rates . . . . 41

4.4 Definition of weighted bipartite trade networks . . . . . . . . . . . 42

4.5 Example of a weighted bipartite trade network . . . . . . . . . . 45

4.6 Probability distributions of the RSP and RPP (HS2 in 2009) . . . . . . 47

4.7 Probability distributions of the RSP and RPP (HS4 in 2009) . . . . . 47

4.8 Correlation between RSP and RPP (HS2 in 2009) . . . . . . . . . . . 48

4.9 Correlation between RSP and RPP from 1995 to 2009 . . . . . . . . . . 49

4.10 Effects of threshold values of RSP or RPP in our networks _ . . . . . 50

4.11 Using the RSP and RPP as efficient network filters . . . . . . . . . . . 52

4.12 Big data process for this study . . . . . . . . . . . . . . . 54 
5.1 Degree distributions of random and scale-free networks . . . . . . . . 60

5.2 Strength-degree correlation and the $\beta$ exponents . . . . . . . . . 62

5.3 End-point correlation and the $\theta$ exponents . . . . . . . . . . . . 63

5.4 Example of bipartite network for clustering analysis . . . . . . . . . . 64

5.5 Nearest Neighbor Degree Distribution Knn(k) . . . . . . . . . . . . . 66

6.1 Basic nestedness concept . . . . . . . . . . . . . . . 70

6.2 Example of a perfectly nested bipartite network . . . . . . . . . 71

6.3 Bipartite matrix temperature and measure of expectedness . . . . . . 72

6.4 NODF calculation process, Almeida-Neto et al. (2008) . . . . . . . 74

6.5 Weighted distances and packed matrices . . . . . . . . . . . 78

6.6 Binary matrices and spectral nestedness. Staniczenko et al. (2012) . . 80

6.7 Quantitative matrices and spectral nestedness. Staniczenko et al. (2012) 81

6.8 Weighted bipartite matrix visualization . . . . . . . . . . 83

6.9 Correlation between WNODF and WINE for a set of bipartite networks 84

7.1 Market links and volume over time . . . . . . . . . . . . . . . . 90

7.2 Trade networks at HS2, HS4 and HS6 levels of aggregation . . . . . . . 92

7.3 Selected HS2 $=01$ trade network visualization . . . . . . . . . . . 96

7.4 Cummulative Degree Distribution without RSP and RPP filters . . . . . 97

7.5 Degree distribution for all industries and periods (unfiltered) . . . . . 99

7.6 Cummulative Degree Ditribution with RSP and RPP filters . . . . . . . 101

7.7 Strength-Degree correlation, unfiltered scenario . . . . . . . . . . 103

7.8 Strength-Degree correlation with RSP and RPP filters . . . . . . . . . 105

7.9 Beta exponent results for all HS2 industries . . . . . . . . . . . . 106

7.10 Nearest neighbor degree (weighted) without RSP and RPP filters . . . 108

7.11 Nearest neighbor degree (weighted) with RSP and RPP filters . . . . 109

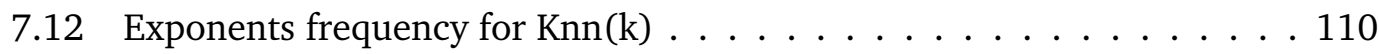

7.13 Bipartite clustering (unweighted) without RSP and RPP filters . . . . 112

7.14 Bipartite clustering (unweighted) with RSP and RPP filters . . . . . . . 113

7.15 Exponents frequency for $\mathrm{C} 4 \mathrm{~b}(\mathrm{k}) \ldots \ldots \ldots . \ldots . \ldots 114$

7.16 Bipartite clustering (weighted) with RSP and RPP filters . . . . . . . 115

7.17 Bipartite clustering (weighted) with RSP and RPP filters . . . . . . 116

7.18 Exponents frequency for $\mathrm{C} 4 \mathrm{w}(\mathrm{k}) \ldots \ldots$. . . . . . . . . 117

7.19 Average weight as a function of the end-point degree without RSP and RPP filters . . . . . . . . . . . . . . . . . . . 118

7.20 Average weight as a function of the end-point degree with RSP and RPP filters . . . . . . . . . . . . . . . . . . . . 119

7.21 Exponents frequency for end-point degree . . . . . . . . . . . . 120

7.22 Nestednes results for the selected HS2 industries . . . . . . . . . . 121

7.23 Nestednes results for selected HS2 industries (filtered) . . . . . . . . . 122

7.24 Nestednes results for all HS2 industries . . . . . . . . . . . . . . . . . 124 
7.25 Common topological features of WBTN . . . . . . . . . . . . 126

8.1 Nestednes results for different levels of aggregation . . . . . . . . . 130

8.2 Nestednes results for different periods . . . . . . . . . . . . . . . 132

8.3 Concept of dynamical phenomena tracking method . . . . . . . . . 134

8.4 Bipartite Matrices for all periods from 1995 to 2009 . . . . . . . . . . . 135

8.5 Dynamical variances for all periods from $1995 / 6$ to $2008 / 9$. . . . . 136

8.6 Dynamical distances for all type of variances . . . . . . . . . . . . 137

8.7 Dynamical distributions as a function of the occurrence distance . . . 138

9.1 Two approaches to market models . . . . . . . . . . . . . . . . 148

9.2 Example of the random bipartite weighted model . . . . . . . . . 150

9.3 Results of the random bipartite model M(200,200,12000) over 300 realizations . . . . . . . . . . . . . . . . . . 151

9.4 Nestedness results of the random bipartite model over 300 realizations 152

9.5 Gravity model fitting for $\mathrm{HS} 2=01 \ldots \ldots$. . . . . . . . . 158

9.6 Gravity model fitting for $\mathrm{HS} 2=01$ with $R S P>10^{-1} \ldots \ldots$

9.7 Results of gravity models for selected HS2 industries . . . . . . . . . 162

9.8 Results of gravity models for selected HS2 industries . . . . . . . . . 163

10.1 Step-by-step process of the mutualistic model ～. . . . . . . . . . . . 170

10.2 Example of a market simulation with our mutualistic model . . . . . . 172

10.3 Topological results of all 100 realizations . . . . . . . . . . . . . . 174

10.4 Degree distributions $\mathrm{p}(\mathrm{k})$ and $\mathrm{P}(\mathrm{K})$ for various $\mathrm{p}(\mathrm{FL}) \ldots \ldots$. . . . . . 175

10.5 Exponent $\gamma_{e x p}$ of $\mathrm{P}(\mathrm{K})$ for each $\mathrm{p}(\mathrm{FL}) \ldots \ldots \ldots \ldots$

10.6 Exponents of selected mutualistic indicators for various $\mathrm{p}(\mathrm{FL}) \ldots$

10.7 Nestedness performance for various $\mathrm{p}(\mathrm{fl}) . \quad \ldots \ldots$. . . . . . . . . 179

10.8 Actual market simulation with our mutualistic model for HS2 $=01$. . . 181

10.9 Actual market simulations with our mutualistic model (HS2 $=01$;

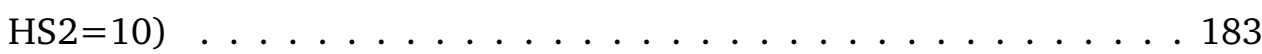

10.10 Actual market simulations with our mutualistic model (HS2 $=20$; $\mathrm{HS} 2=35) \ldots \ldots \ldots \ldots \ldots \ldots \ldots$. . . . . . . . . . . . . . . . . . . . . . . . . .

10.11 Actual market simulations with our mutualistic model (HS2 $=50$; $\mathrm{HS} 2=62) \ldots \ldots \ldots \ldots \ldots \ldots \ldots \ldots \ldots$

10.12 Actual market simulations with our mutualistic model (HS2 $=75$; $\mathrm{HS} 2=84) \ldots \ldots \ldots \ldots \ldots \ldots \ldots \ldots$

10.13 Actual market simulation with a modified mutualistic model for HS2 $=01.188$

10.14 Simulations of the special cases of markets. . . . . . . . . . . 190

11.1 Analytics based on global bank positions . . . . . . . . . . . . . . . 194

11.2 Analytics based on relative variables $x$ and $y \ldots \ldots$. . . . . 195

11.3 Bivariate distribution of relative variables $x$ and $y \ldots \ldots$. . . . 196

11.4 Circular layout graphs of interbank network examples . . . . . . . 200 
11.5 Network properties of our model . . . . . . . . . . . . . . . . 201

11.6 Network properties of our model . . . . . . . . . . . . . . 202

11.7 Network properties of our model . . . . . . . . . . . . . . 203

11.8 Network properties of our model . . . . . . . . . . . . . . . . 204

11.9 Network properties of our model _ . . . . . . . . . . . . 205

11.10 Network properties of our model . . . . . . . . . . . . 205

11.11 Network properties of our model . . . . . . . . . . . . 206

11.12 Network properties of our model . . . . . . . . . . . . . . . 206

11.13 Network properties of our model . . . . . . . . . . . . . . 207

11.14 Network properties of our model . . . . . . . . . . . . . . . . 209

12.1 Visual timeline of the thesis $\ldots \ldots \ldots . \ldots \ldots$

B.1 Map of Napoleon's Russian Campaign of 1812 by Charles Minard . . . 248

B.2 Examples of multiple forms of visualizations . . . . . . . . . . . 250

B.3 Design of circular graph visualizations . . . . . . . . . . . . . 251

B.4 Examples of circular graph visualizations (random networks) . . . . 252

B.5 Design application to an actual WBTN (HS2=01 in 1995) . . . . . . 253

B.6 Design of Geo-spatial graph visualizations . . . . . . . . . . . . 255

B.7 Example of Geo-spatial graph visualization (HS2=01 in 1995) . . . . . 258

B.8 Examples of other graph layouts . . . . . . . . . . . . . . . . 259

B.9 Examples of other graph layouts . . . . . . . . . . . . . . . . . 261

C.1 Selected HS2 $=01$ trade network visualization . . . . . . . . . . . . . 264

C.2 Selected HS2 $=10$ trade network visualization . . . . . . . . . . 265

C.3 Selected HS2 $=20$ trade network visualization . . . . . . . . . . . 266

C.4 Selected HS2 $=35$ trade network visualization . . . . . . . . . . . . 267

C.5 Selected HS2 $=50$ trade network visualization . . . . . . . . . . . 268

C.6 Selected HS2 $=62$ trade network visualization . . . . . . . . . . . . . . 269

C.7 Selected HS2 $=75$ trade network visualization . . . . . . . . . . . 270

C.8 Selected HS2 $=84$ trade network visualization $\ldots \ldots \ldots$. . . . . . 271

C.9 Cummulative Degree Ditribution without RSP and RPP filters . . . . 272

C.10 Cummulative Degree Ditribution with RSP and RPP filters . . . . . 273

C.11 Strength-Degree correlation, unfiltered scenario . . . . . . . . . . . 274

C.12 Strength-Degree correlation with RSP and RPP filters . . . . . . . . . 275

C.13 Nearest neighbor degree (weighted) without RSP and RPP filters . . . 276

C.14 Nearest neighbor degree (weighted) with RSP and RPP filters . . . . 277

C.15 Bipartite clustering (unweighted) without RSP and RPP filters . . . . . 278

C.16 Bipartite clustering (unweighted) with RSP and RPP filters . . . . . . . 279

C.17 Bipartite clustering (weighted) with RSP and RPP filters . . . . . . 280

C.18 Bipartite clustering (weighted) with RSP and RPP filters . . . . . . . . 281

C.19 Average weight as a function of the end-point degree without RSP and RPP filters . . . . . . . . . . . . . . . . . . . . . 282 
C.20 Average weight as a function of the end-point degree with RSP and RPP

filters . . . . . . . . . . . . . . . . . . 283

C.21 Market dashboard $\mathrm{HS} 2=01, \mathrm{Yr}=2000 \ldots \ldots$. . . . . . . . . 284

C.22 Market dashboard HS2 $=10, \mathrm{Yr}=2000 \ldots \ldots$. . . . . . . . . . 285

C.23 Market dashboard HS2 $=20, \mathrm{Yr}=2000 \ldots \ldots$. . . . . . . . 286

C.24 Market dashboard HS2 $=35, \mathrm{Yr}=2000 \ldots \ldots$. . . . . . . . . . 287

C.25 Market dashboard HS2 $=50, \mathrm{Yr}=2000 \ldots \ldots$. . . . . . . . 288

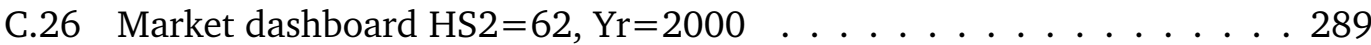

C.27 Market dashboard HS2 $=75, \mathrm{Yr}=2000 \ldots \ldots$. . . . . . . . . 290

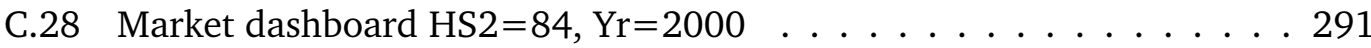

C.29 Dynamical distributions for selected industries HS2 . . . . . . . . . . . 292

D.1 Market dashboard HS2 $=20, \mathrm{Yr}=1995 \ldots \ldots . \ldots . \ldots . \ldots . \ldots 293$

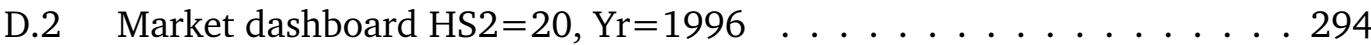

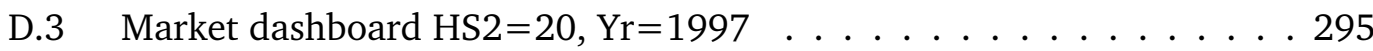

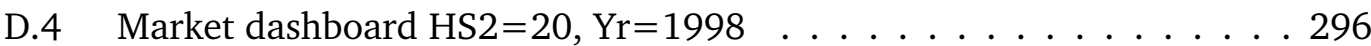

D.5 Market dashboard HS2 $=20, \mathrm{Yr}=1999$. . . . . . . . . . . . . . . . . 297

D.6 Market dashboard HS2 $=20, \mathrm{Yr}=2000 \ldots \ldots$. . . . . . . . . . 298

D.7 Market dashboard HS2 $=20, \mathrm{Yr}=2001 \ldots \ldots$. . . . . . . . . . . . 299

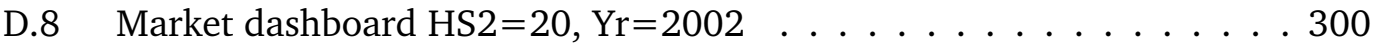

D.9 Market dashboard HS2 $=20, \mathrm{Yr}=2003 \ldots \ldots$. . . . . . . . . . . . 301

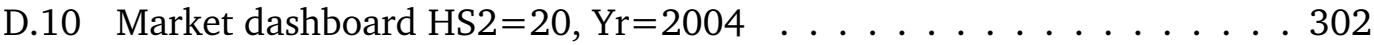

D.11 Market dashboard HS2 $=20, \mathrm{Yr}=2005 \ldots \ldots 303$

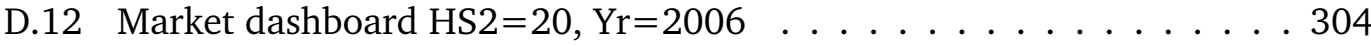

D.13 Market dashboard HS2 $=20, \mathrm{Yr}=2007 \ldots \ldots$. . . . . . . . . . 305

D.14 Market dashboard HS2 $=20, \mathrm{Yr}=2008 \ldots \ldots$. . . . . . . . 306

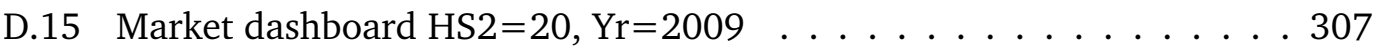





\section{List of Tables}

4.1 Example of the level of aggregation of products (HS6), categories (HS4)

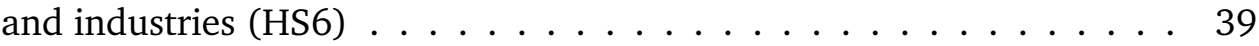

4.2 Example of country codes and equivalences . . . . . . . . . . 39

5.1 Summary of bipartite topological indicators . . . . . . . . . . . 67

7.1 Links and trade volumes by year for HS2 industries . . . . . . . . . . 93

7.2 Selected HS2 industries for the section . . . . . . . . . . . . . . . 95

7.3 Exporters $\mathrm{P}(\mathrm{K})$ regression model fitting, unfiltered . . . . . . . . . . 98

7.4 Importers $\mathrm{P}(\mathrm{K})$ regression model fitting, unfiltered $\ldots \ldots$. . . . . . 98

7.5 Exporters $\mathrm{P}(\mathrm{K})$ regression model fitting, filtered scenario . . . . . . . 100

7.6 Importers $\mathrm{P}(\mathrm{K})$ regression model fitting, filtered scenario . . . . . . . 102

$7.7 \mathrm{~S}(\mathrm{~K})$ regression model fitting, unfiltered scenario . . . . . . . . . . . 104

$7.8 \mathrm{~S}(\mathrm{~K})$ regression model fitting, filtered scenario . . . . . . . . . . . 107

9.1 Estimated coefficients of our gravity model for HS2=01 . . . . . . 157

9.2 Estimated coefficients of our gravity model for HS2=01 with $R S P>10^{-1} 158$

9.3 Estimated coefficient of our gravity model for selected industries . . . 161

11.1 Linear regression analysis of relative variables $x$ and $y \ldots 193$

11.2 Linear regression analysis of short-term variables $x_{s}$ and $y_{s} \ldots \ldots 4$ 
